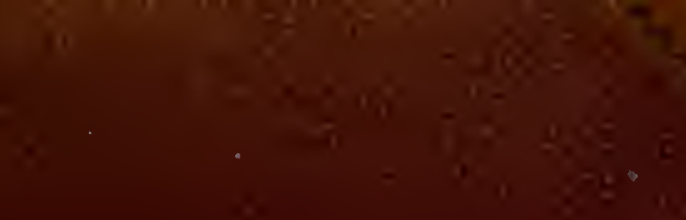


Natural History Museum Library

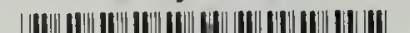

(III) (2) 

11
d
$B$

Dta 


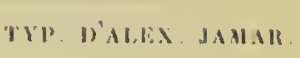





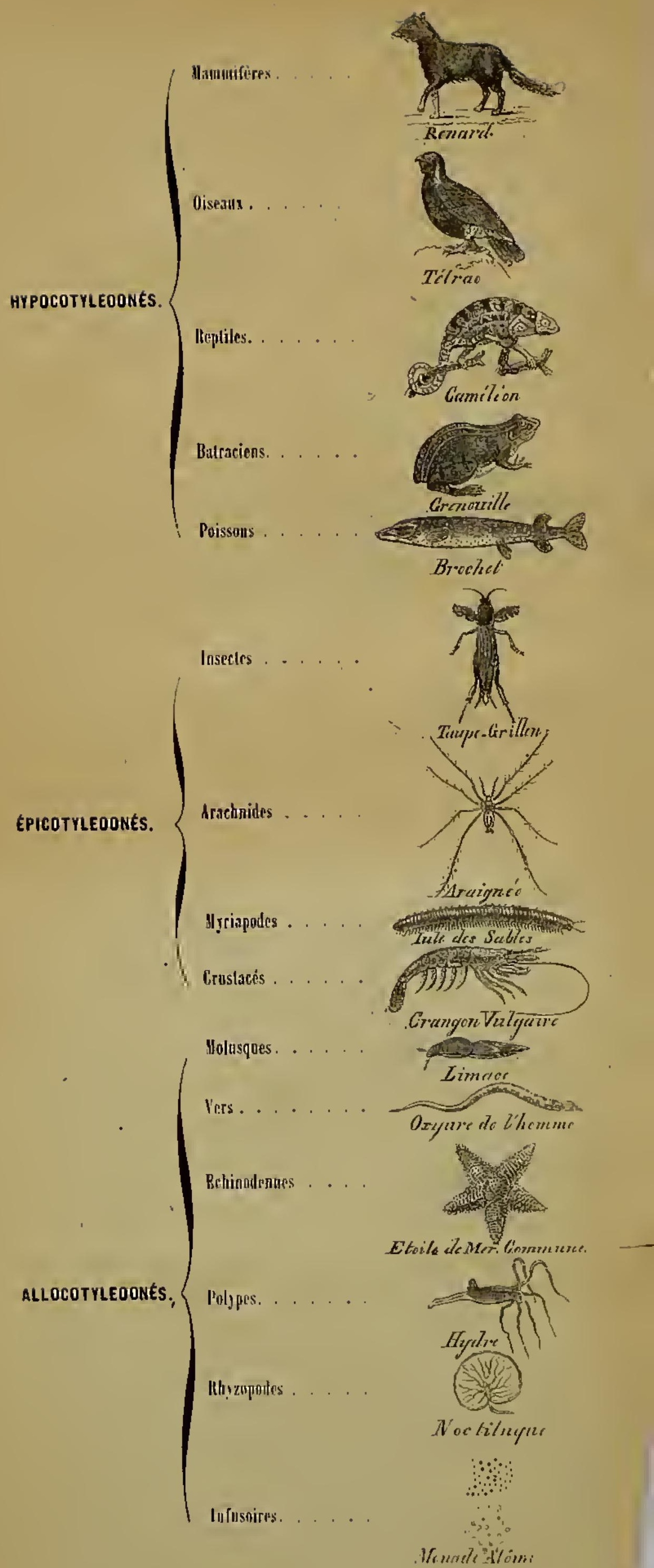




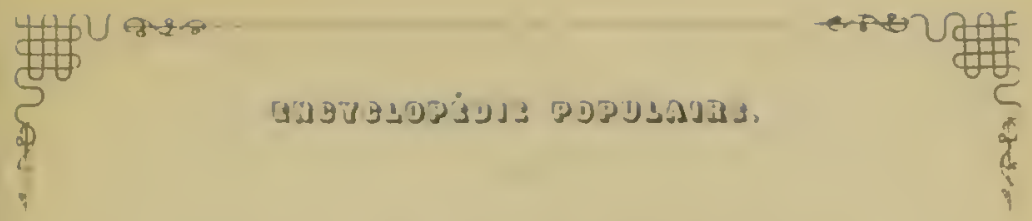

AMATOMIE OOMPAREE

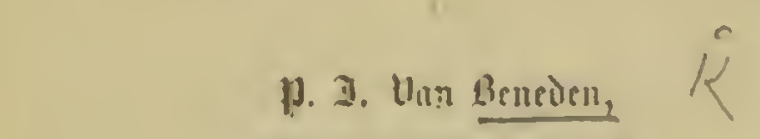

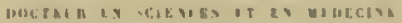

IT Kaht, DE I A

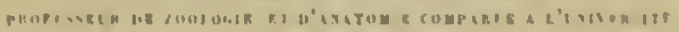

ur J.st vis, etc.., I TC

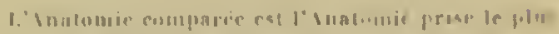
in griall quil suil possible

Fustevith

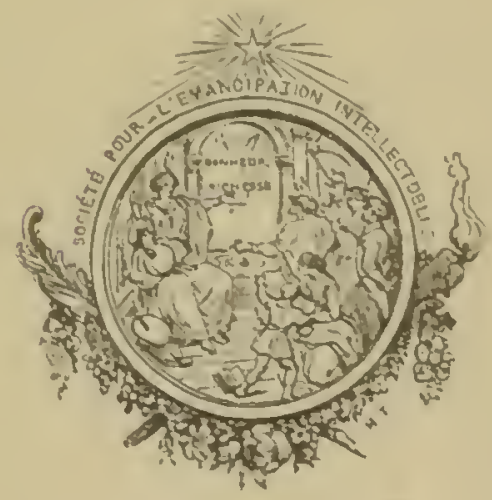

$\sum_{\text {है }}$ sociét

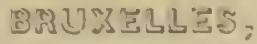

'émancipation intellectuelle, 



\section{ITR()I) (IT).}

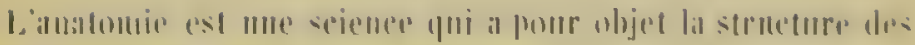

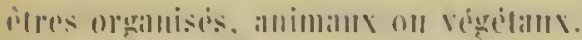

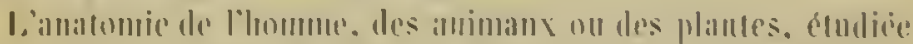

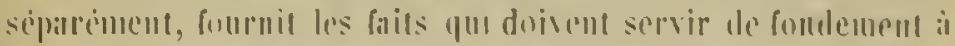

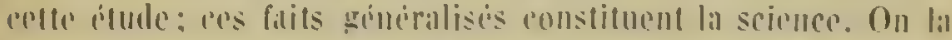

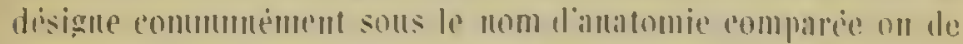

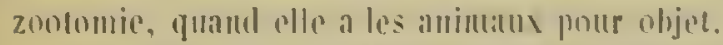

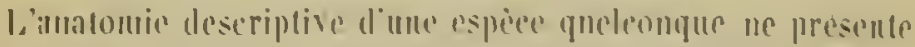

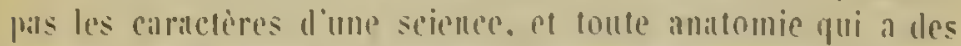

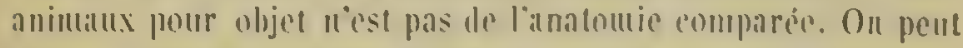
ímdier tomles les espices animales. comme on rtulie $1 \%$ homme.

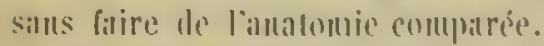

Juspu à la fin du sicele dernier, la structure de l'lunume a presque senle ocempe les analomisles: lanalomie clait comsidereso

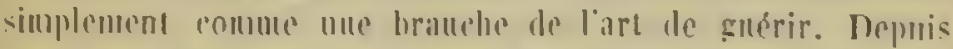

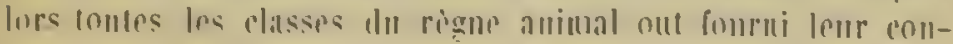


lingent de faits. of la seimee anatomiqun rst nece cost un produil du xise sichele.

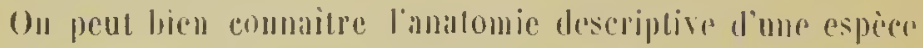

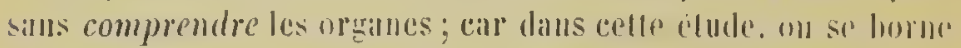
al on doil so bormer is lemp description pure el simple ande rimdication des rapports qu’ils out rutre cus.

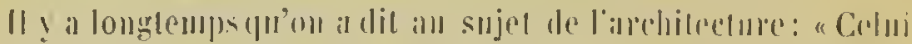

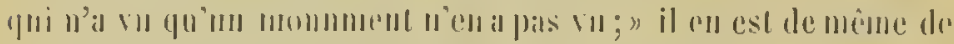

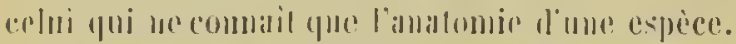

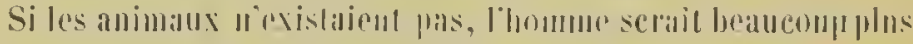

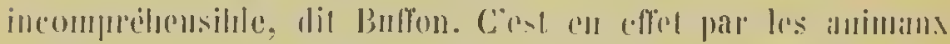

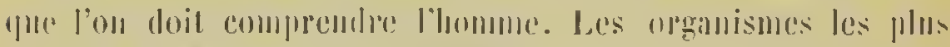

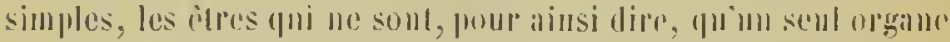

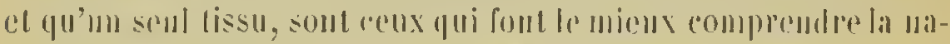
ture des organisitions si diserses el si compliques qui conrounent le segrue animble

L:Anatomie compare anua dour pour ubjet d'itudier les organnes at les modifieations puils subissent dians les diversen. classes dor regne inninal, de comparre les faits entre enx et darriver il lit commissance des lois qui president au développement el ì la coordination des divers êtres de ere regne.

A upremier abord, cette science parail d'une tendue effridyante

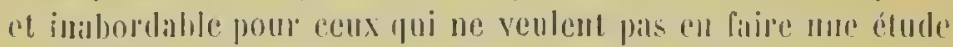
spreiale; que l'ou se rassure! Celui qui cemail loreranisation d'une esprece clevée, de l'lomme surtoul, connail bien vite l'organisation des diverses classes; comme celui qui a dmolie le style ogval en arediterture apprend aisiment a connaitre les divers styles dour il derive el qui l’oul precorde.

Ceest anssi une errour de croire qu'il est nicessaire de conmaitre d'abord l'anatonic de l'homme; rente connaissinter est

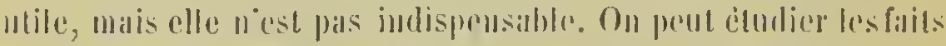

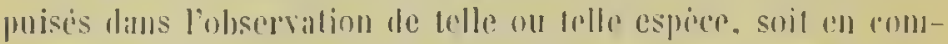
meneint par le haut de l'édolle, l'homme par ricmple. soir en prenant pons type an insecte ou mu ver; on peut aussi bien procéder dor simple an compose, que dor romposi in simple.

Vuici la marede que anens allous suive. Volre lont est.

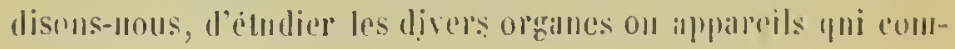




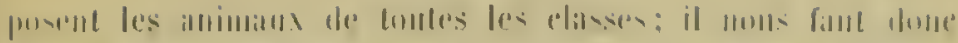

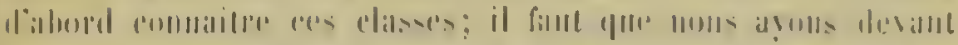

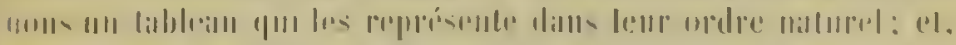

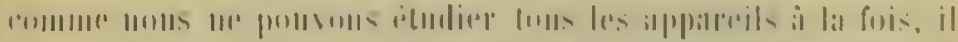

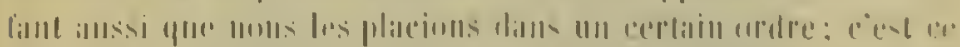

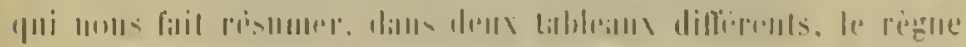

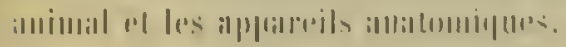

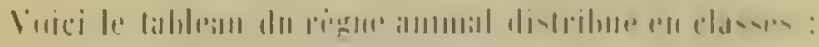

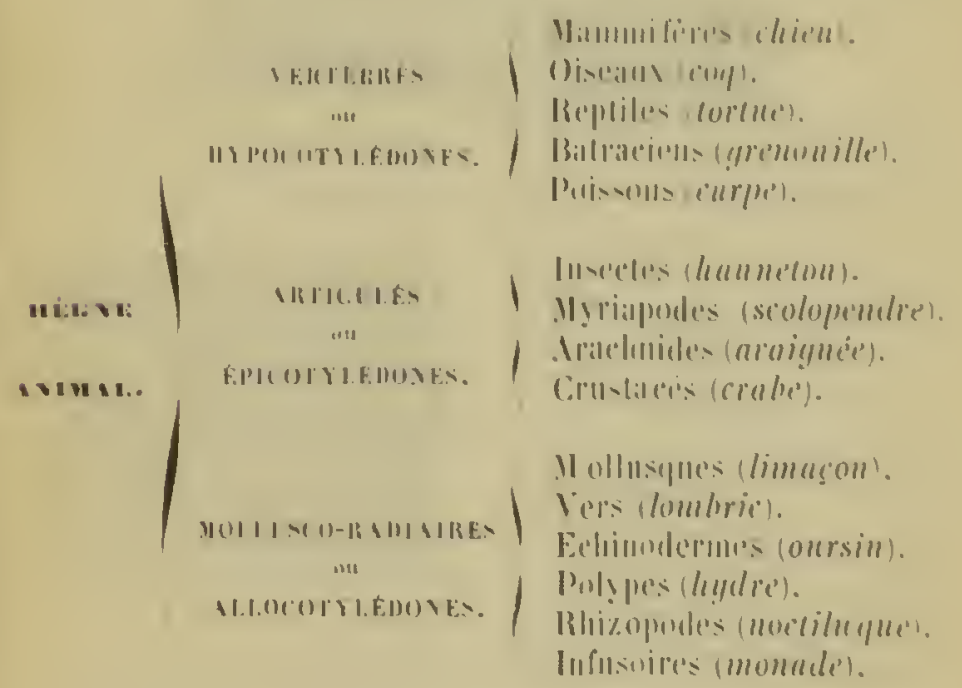

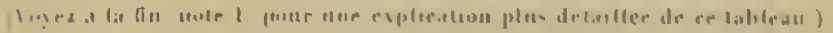

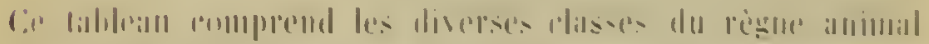

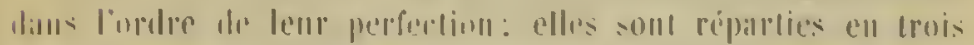

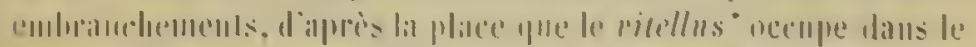

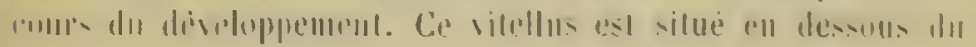

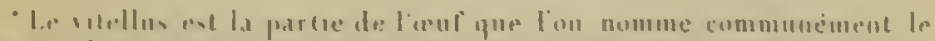

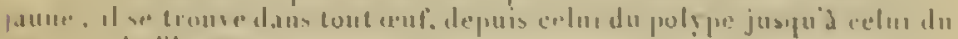

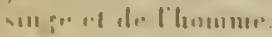




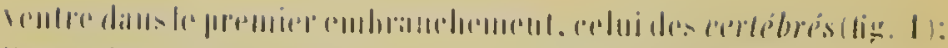

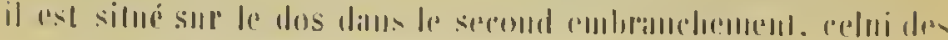

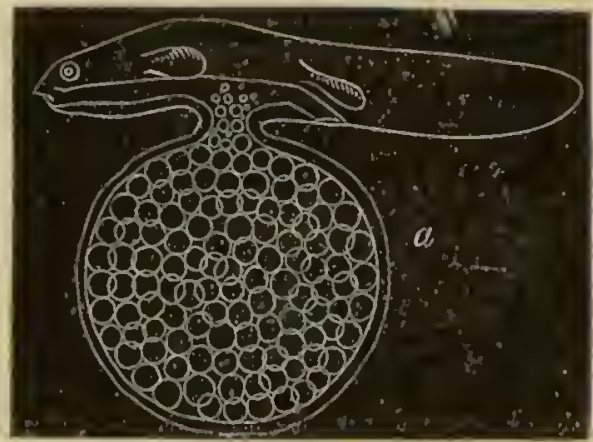

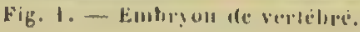

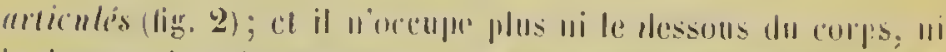
la desills, dalls les anlres ilmimall (liyg. J), qui sonl rrunis soms le nom d'allocolyleilomes; re sunl les mollusques el las rialialies ales anlemes. De li las mamis d"hyporolylélomes, éjucolyledlones el allocotyledones .

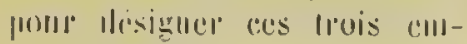
lorillelenenculs.

Colle elassiticalium, yne moms

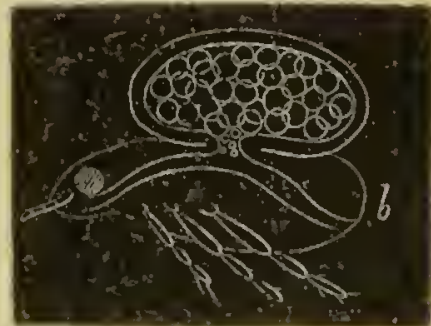

Fig. 2 - Embryon d'artirule.

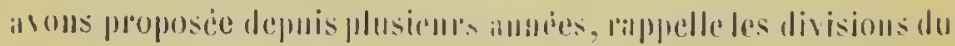

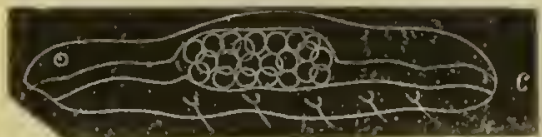

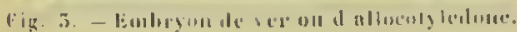
lime viunetal chablies lipros le mene orfrime s(m)s le minu de dicolylidomes, momocolylidones of acolylidomess.

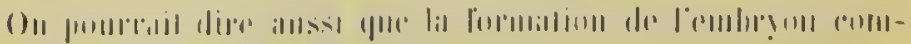

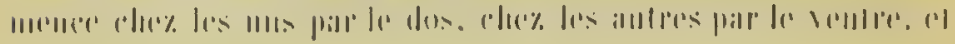

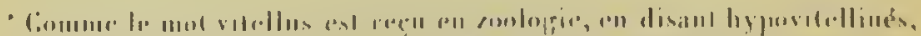

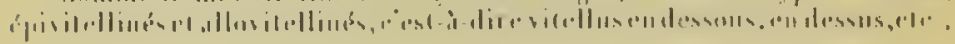




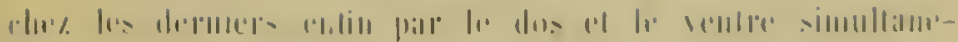
III'III.

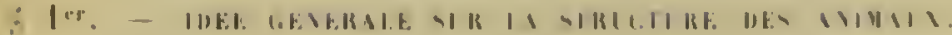

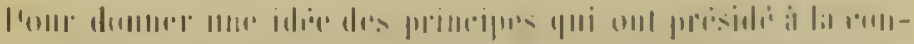

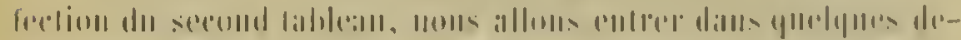

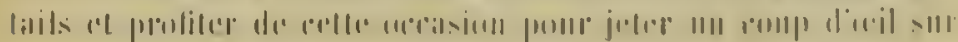

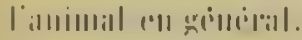

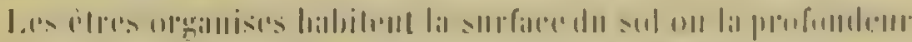
Ars a

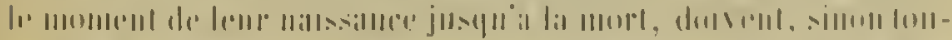

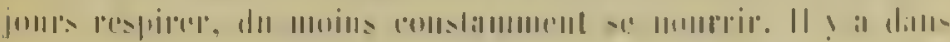
la11, IIII III-1)

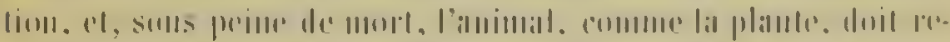

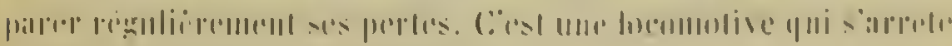

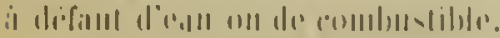

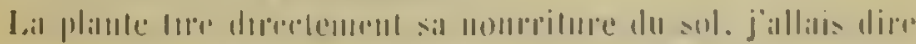

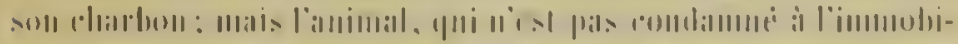

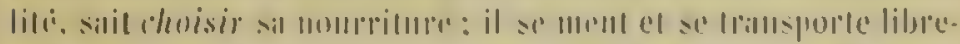

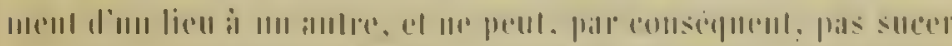

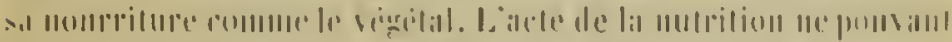

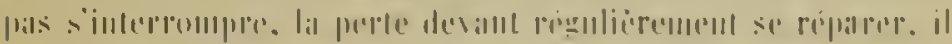

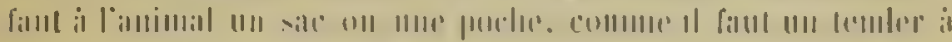

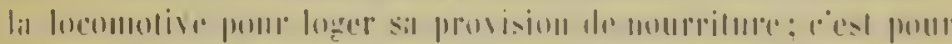

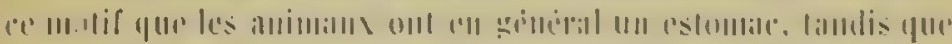

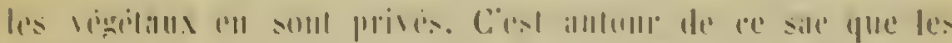

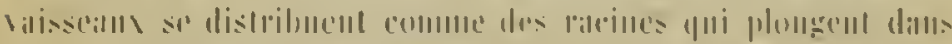

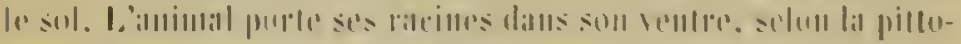

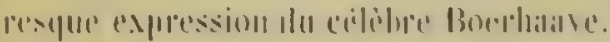

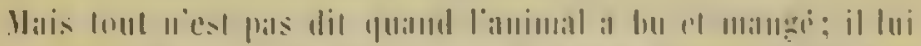

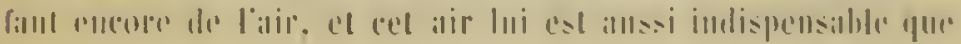

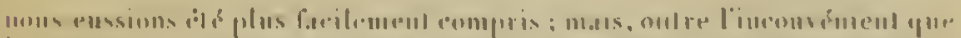

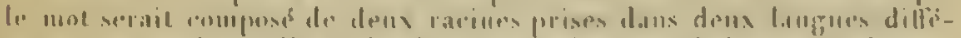

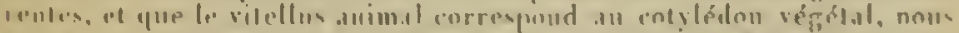

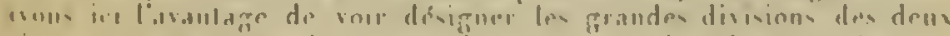

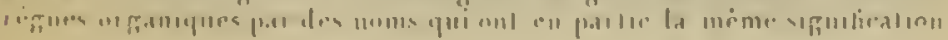




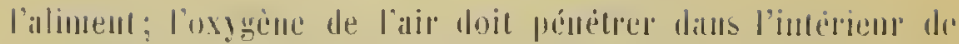

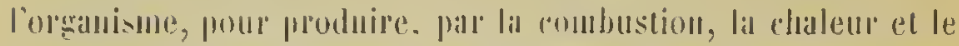

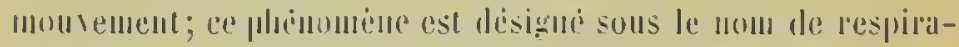
lion. Quand cet ali est reern dans une porde conformede de matmicre it mettre le salug ell contact arre lui, cette poche sappelle pounon ou trachee, on bicn branchie yuand linumal pespire dinls' l'eilu.

Lialinnent ct l'ilir élant introduits dans l'éeonomie animiale, un

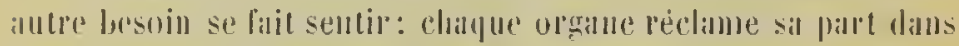
la distribution ale ees mattérialls; ce liest pas conmme llue loco-

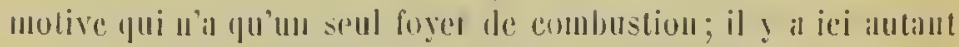
le foyers quily a d'organes distincts, et il laut pourvoir ì toutes les pertes dills loutes les parties du corps. La distribution de

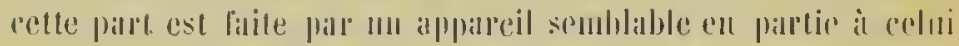

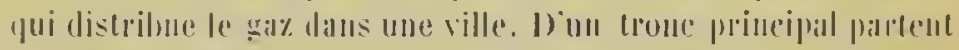

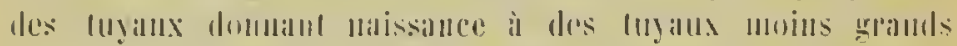

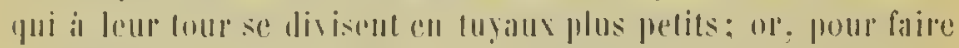

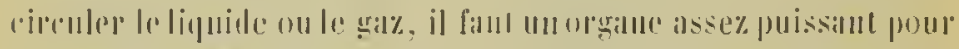

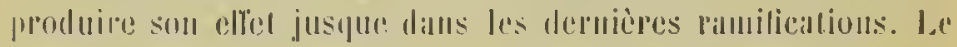
rexur, ens se contritelant, produit refle fore necessille pour

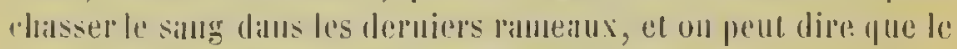

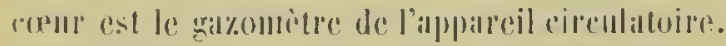

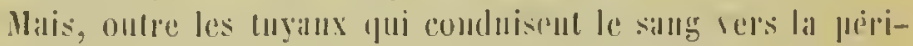

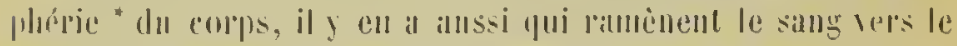
rour. Il an risulte un double courant : l'un du centre vers li rirconference et lautre de la circonlfirenee vers le rentre. Le:

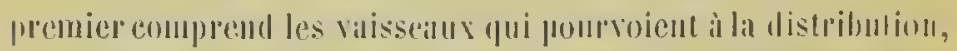

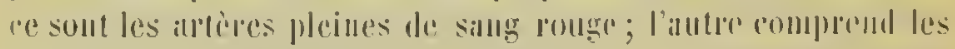

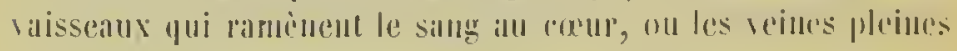
le silltg nuil.

Il caiste ainsi dans l'économite animale un momsement remlrifoğa a

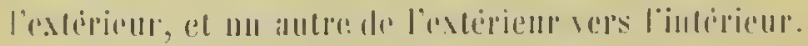

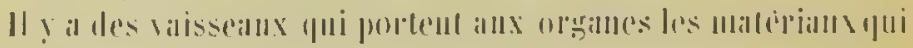

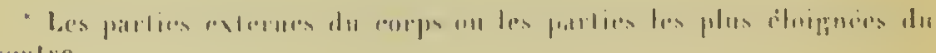




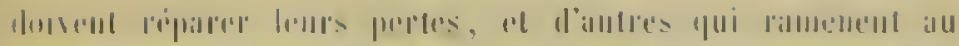

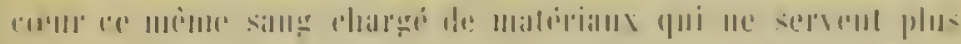

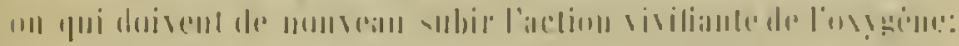

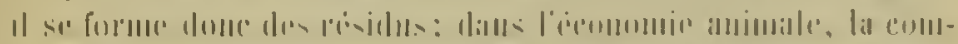

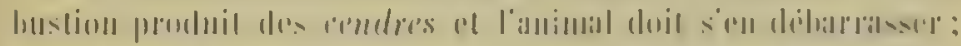

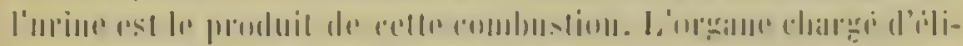

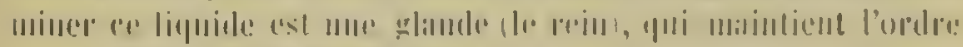

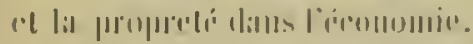

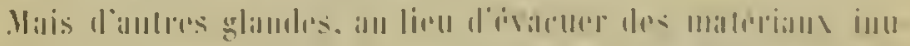

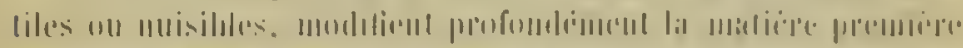

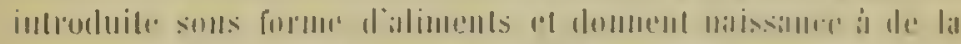

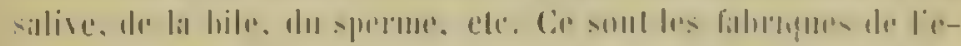

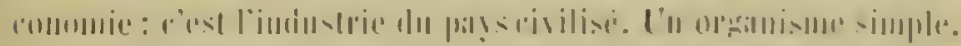

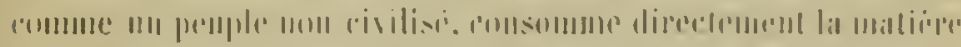

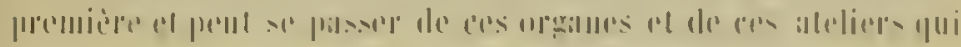

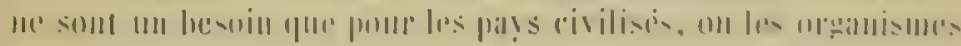
ilevis.

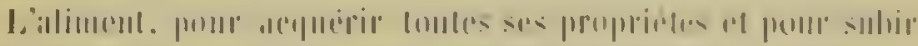

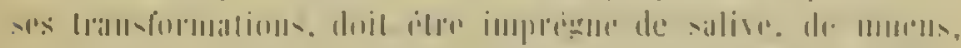

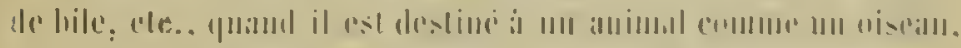

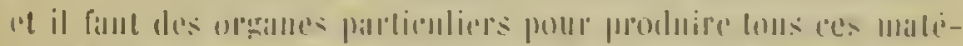

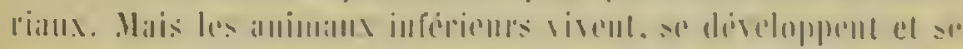

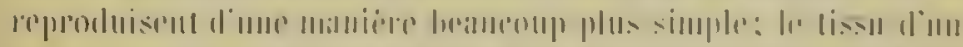
fuly

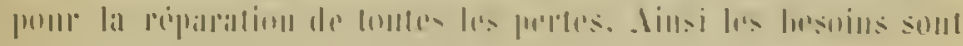

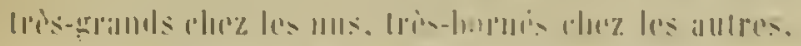

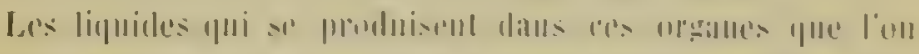

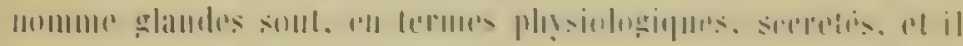

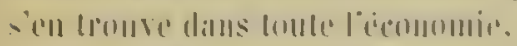

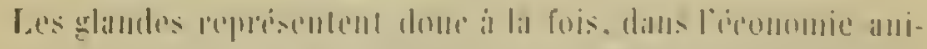

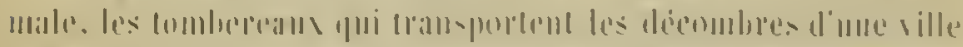

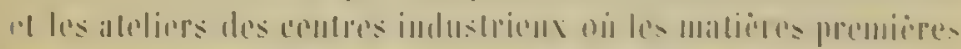

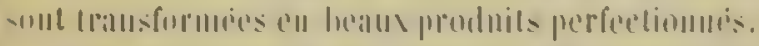

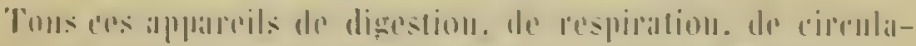

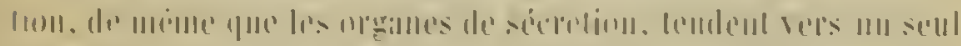

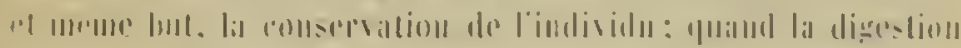

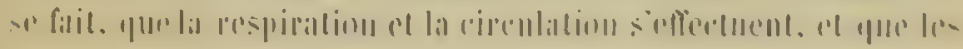




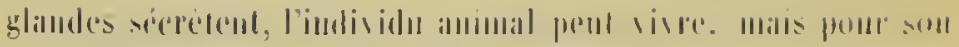

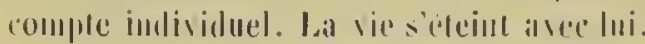

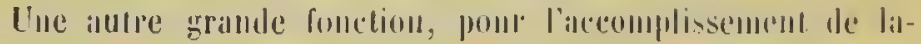

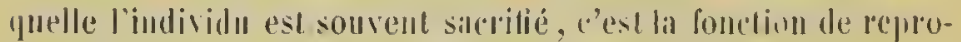

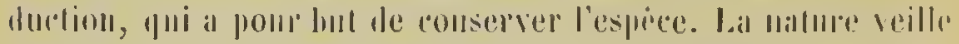

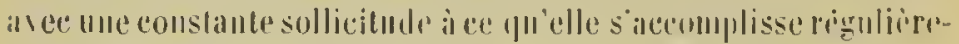
ment pt sans olsilacle; il ny a pas de soins dout l'acte pui doil assuller la conservalion de l'espre ne soil entomré.

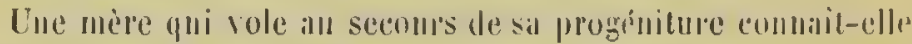

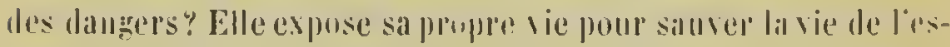

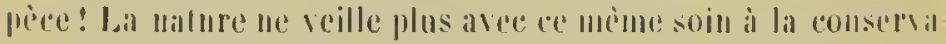

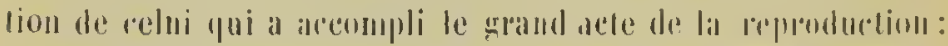

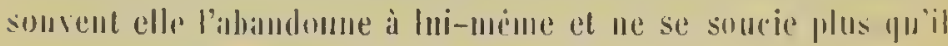
vive ou qu’il mante.

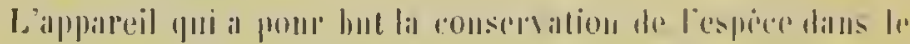

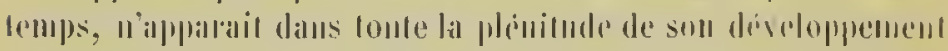
que quand lamimal asl complet on alulle. Lamimal a eu besoin

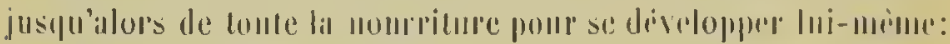

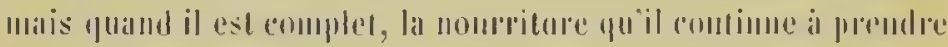

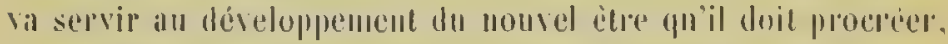

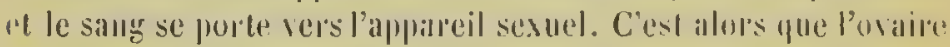

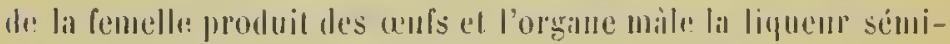

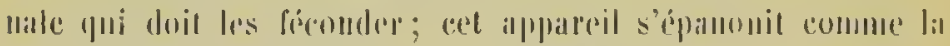

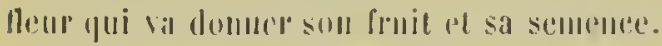

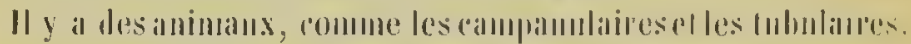
yni entrent en thopiason alosolument rommore les plantes.

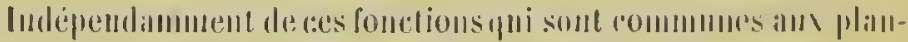

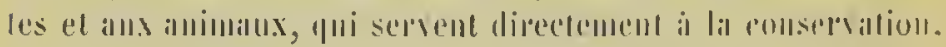

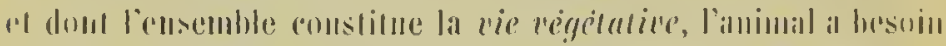

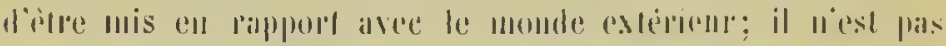

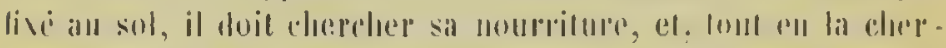

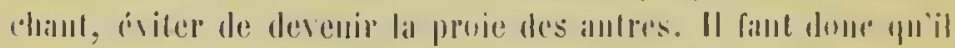

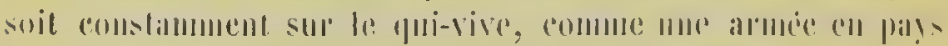

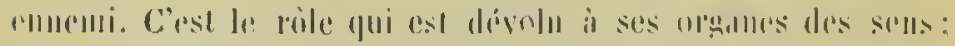

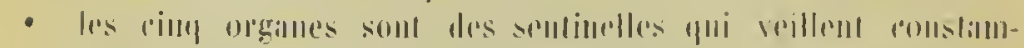

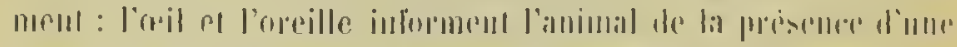

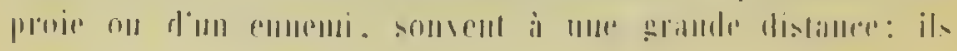




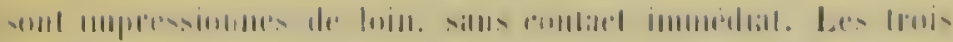

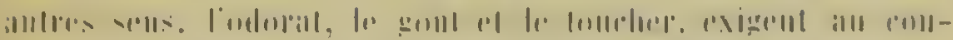

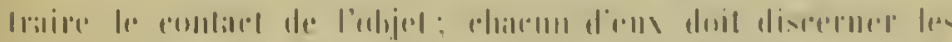

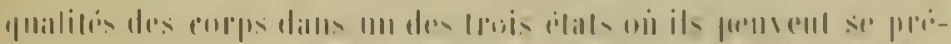

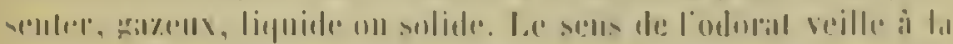

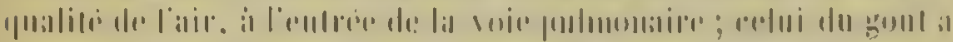

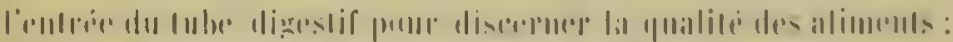

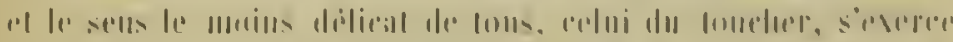

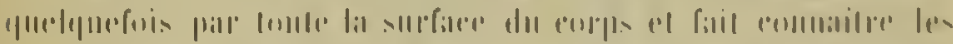

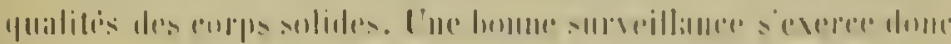

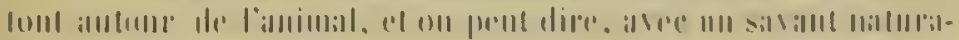

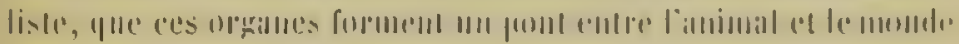
I'v tivicille.

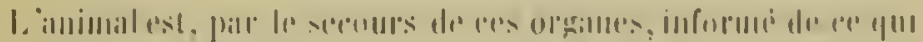

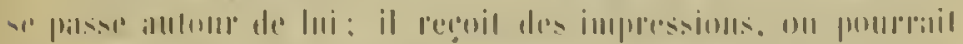
mane dire des a

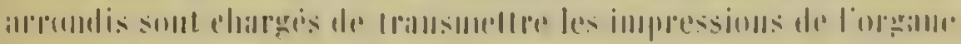

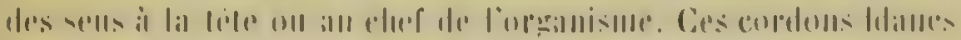

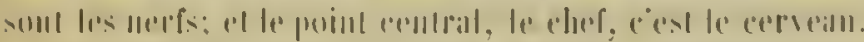

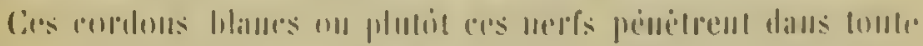

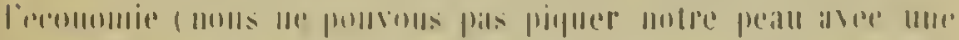

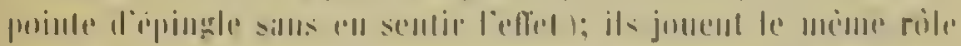

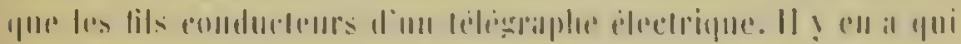

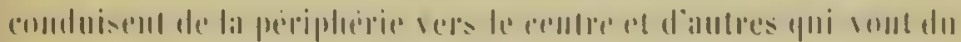

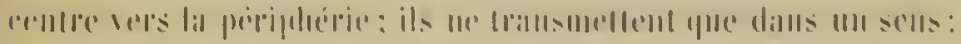

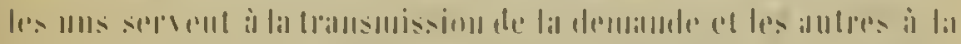

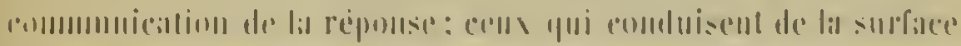

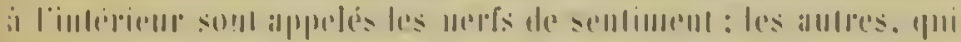

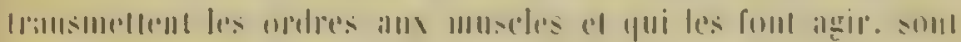

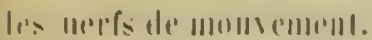

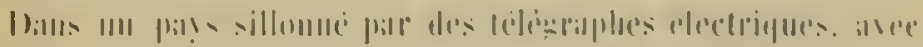

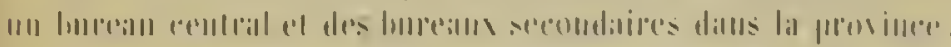

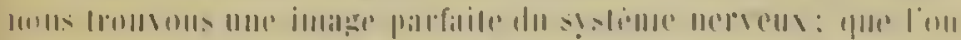

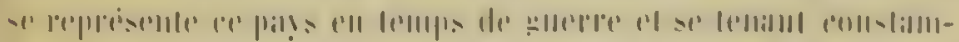

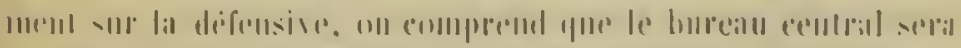

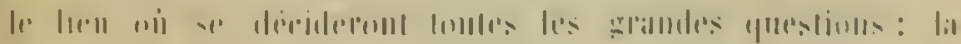

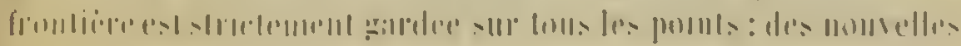




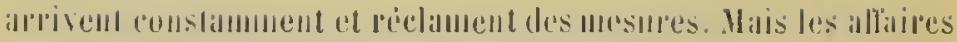

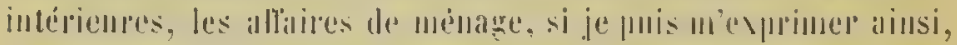

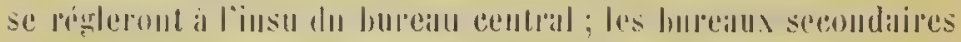

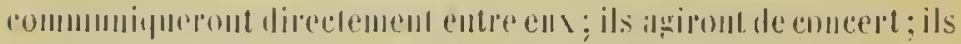

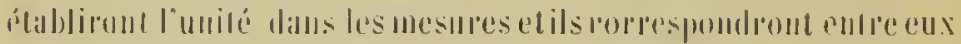

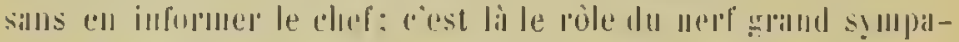

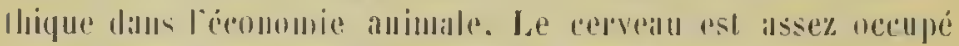

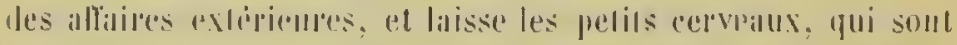

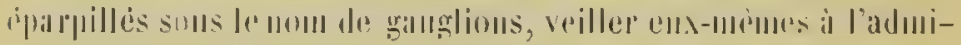

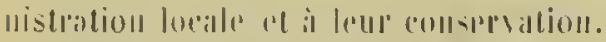

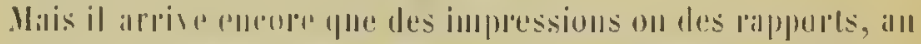

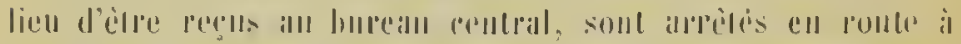

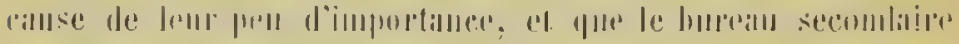

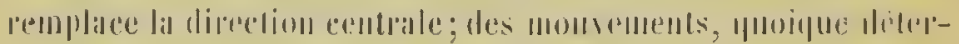

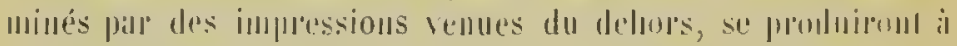

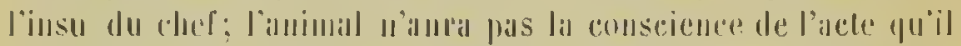

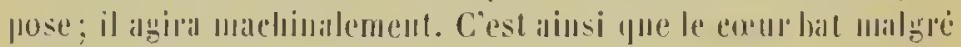
nous; que les fontelions de digestion, de respiration, ote. sade-

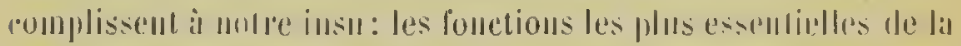
vir, nons ne sommas pas lihres de ne pas les aremmplir. C'est

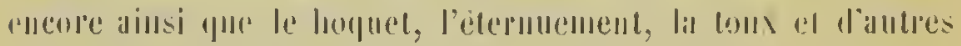

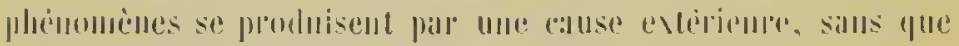

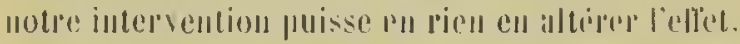

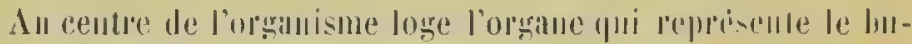

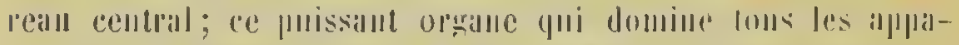

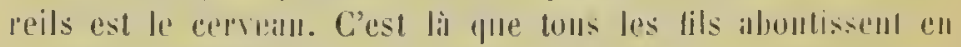

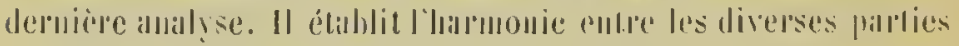

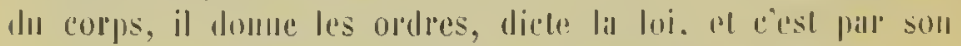

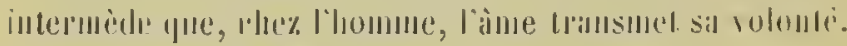

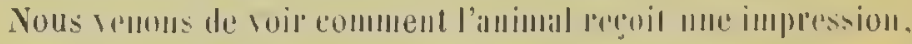

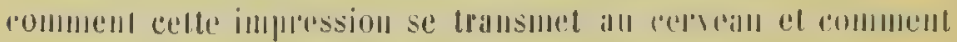

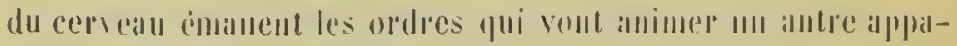
reil, celui de lis locomotion. C'est eet ilplinpeil pui peronet ì l'almimal de se mouvoir", de se thansporter d'un lien ì mu autre, de"

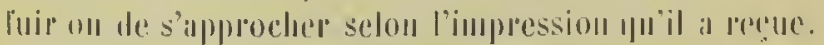

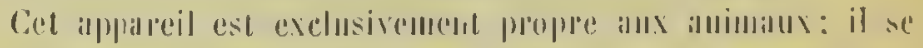

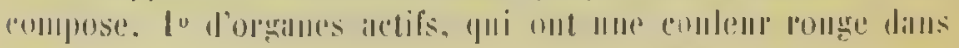




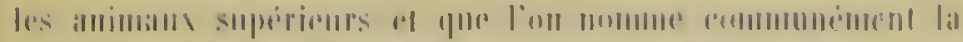

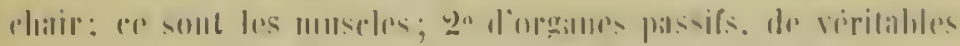

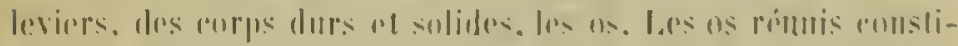

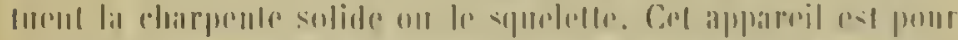

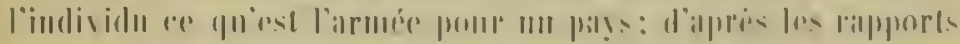

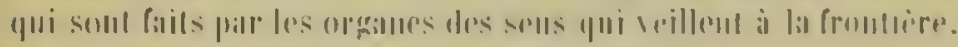

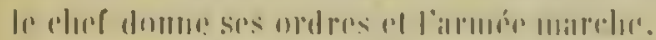

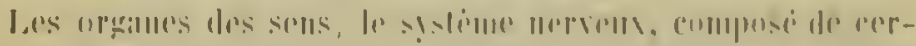

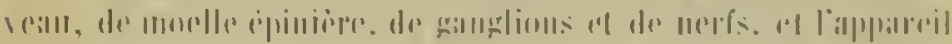

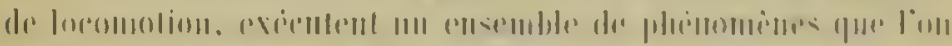

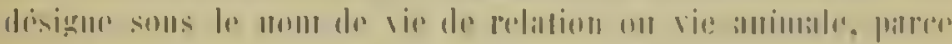

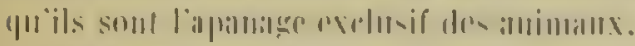

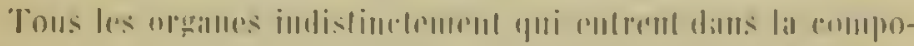

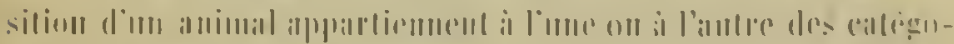

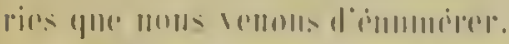

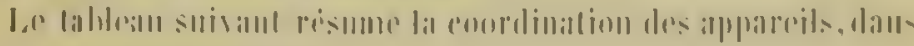

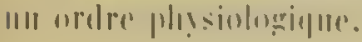

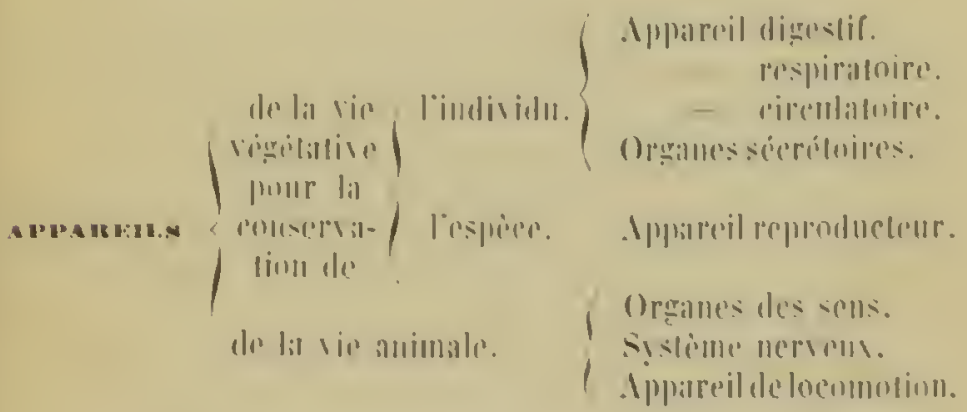

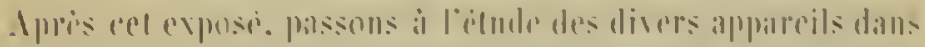

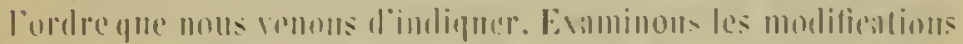

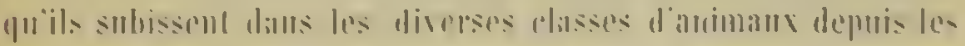

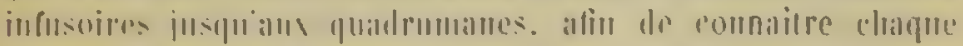

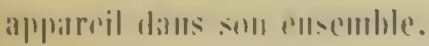

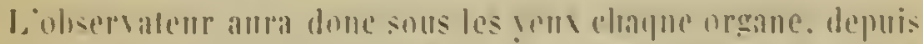

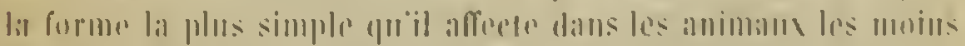

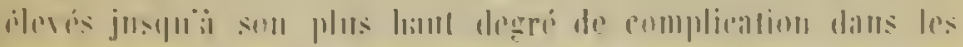

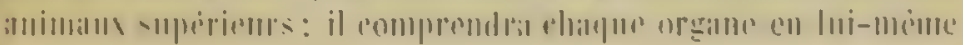




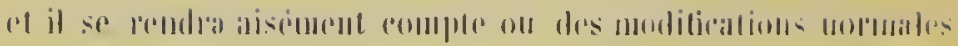

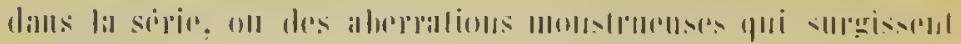
qunelquerois.

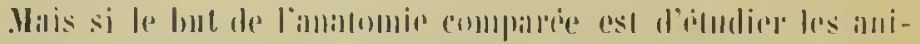

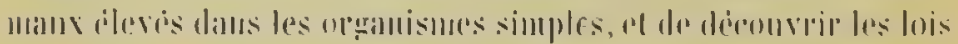

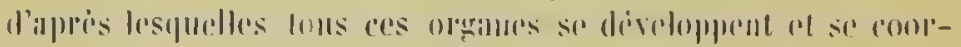

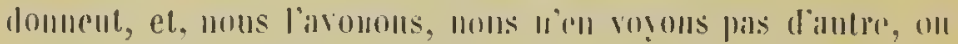

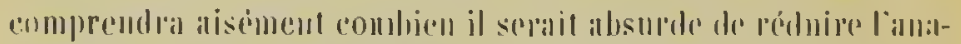

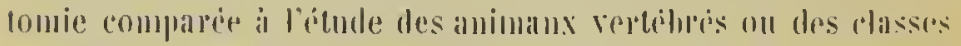

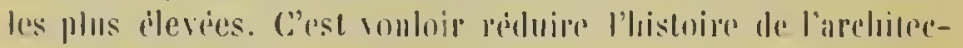

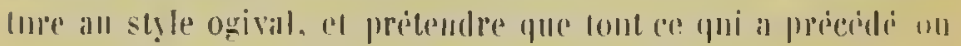

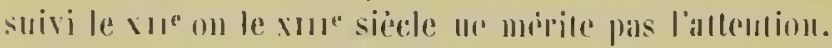

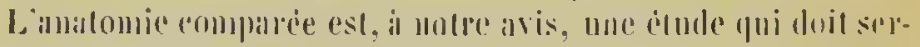

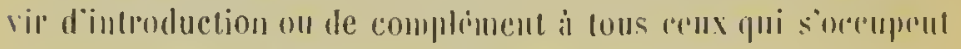

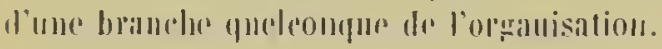

\section{S2. - COMPIHCATIOY HES ORGAVISULS.}

Tous les animanx ne presentent donc pas mu mome degredecomplication dans leur structure; tous virentel se reprodusant, mais

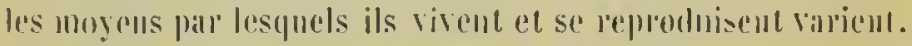

Qu'il me soil peruis de presenter ici nue complataison, alin de hien faire saisir la mature des complieatons qui distingnent les diverses classes d'animan. Cell, comparaison parfaitement juste a élé faite depuis longrtemps.

Les animanx les phos simples sont dans le meme cas qun le: peuplades chez lesquelles la civilisation n'a pas pènétré; quand il n'y a cutore ni eommerce ni industrie, chatem pouproit hui-

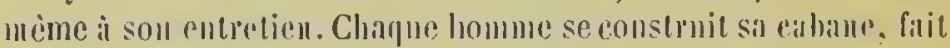
son rètenent, se nourpit par le produit de sil pêtele un de sil chasse, falurique ses meubles et ses nutils, en nul mol, chill'un se pourvoit et se suffit ì lui-mîne. Plus tambl des dedanges s'établissent avec les peuplades voisines, certains individus plus habiles construisent les uns les demempes, las mulres los outils, et insensihlentent la division du travail aneme la pere-

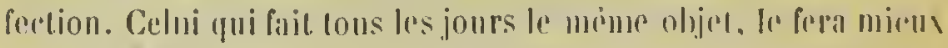
qu'un anyler qui se livere il divers travimx. Tont le mondre sail il 


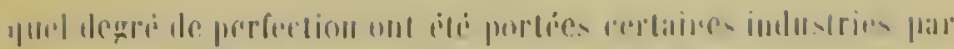

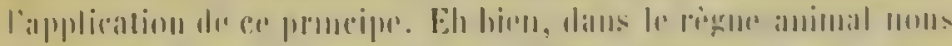

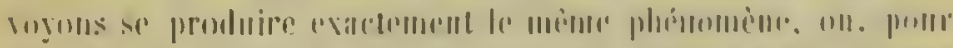

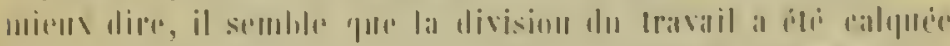

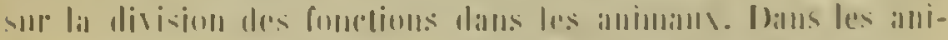

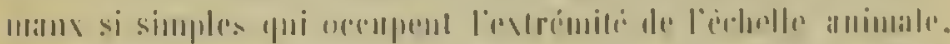

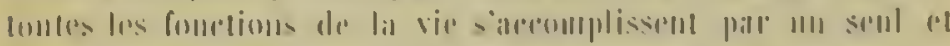

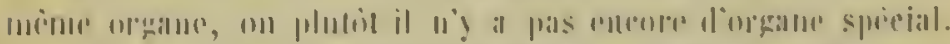

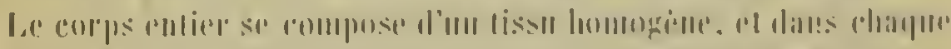

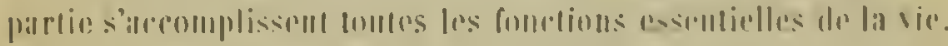

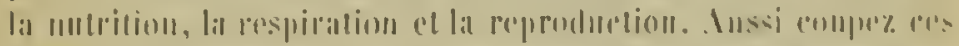

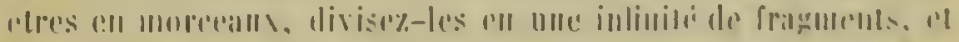

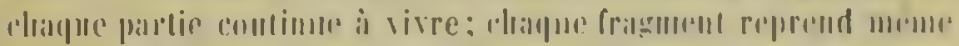

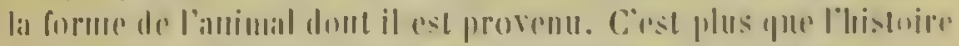

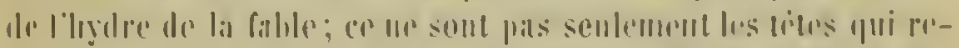

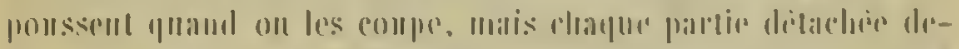

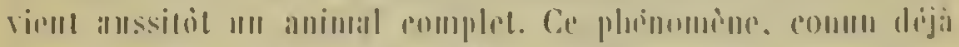

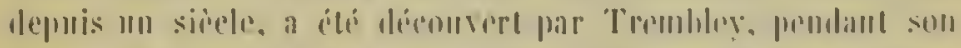

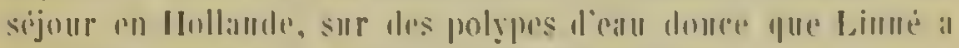

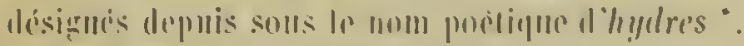

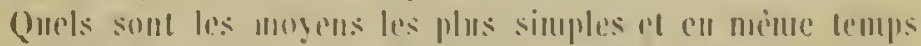

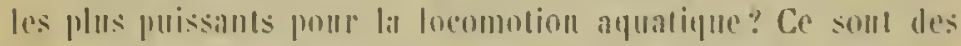

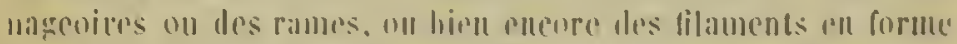

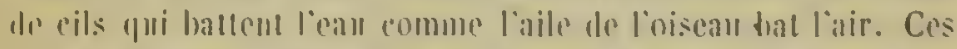
rils se trourent dans lous les aninatus infiricurs et serveut sourent seuls. a defaut de levior. dorsana locomoleur. Le torps. cul est quelpuefois comvert.

L'u dos evemples les plus remarquables de lacemulation des fomelions nous est fourni par ces eils; quand il neviste ni unscles ni morfs, quamd le corps me se eompose que diun seul lissu mun el homogrime, souvent lamimal mage avee autant le crilerite que le plus fringant poisson: son corps csl couvert

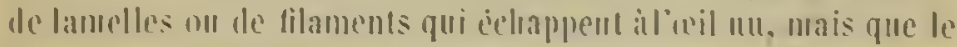
microscope mous montre sous la forme de rames eftiles ou de

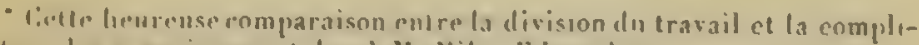

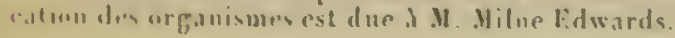


roues de bateau à vapeur ; éest ee fue l'on designe sous le nom de cils vibratiles.

Au lieu de rames, l'lıomme a fait des roues quand il a trouve le moyen d'utiliser la foree de la vapeur, et ees juissintes poues fout monvoir de petits mondes. Ici les avtremes se touchent. L'infusoire microscopique porte des cils en forme de soies, on en forme te rones chez les rolifires, et clee quelques animan. ıui ne scmblent formés que d'une getée vivante, an lieu de cils tout le corps est convert de lanelles et represente une rour vivante. C'est ce que l'on voit bien dans le singulier animal connu sous le nom de cydiple *. C'est encore un probleme que le savoir par guel métanisme ces lils ou lames se meurent; on aldmettrait rolontiers des museles plus mieroseopiques encore que les animax, mais la simplicite d'urganisaltion ne pernet pas l'admission de cette liypothèse. Quoi qu'il en soit, nous royols res cils sous l'unce ou l'autre forme servil ì la progression de l'allinial.

Ce n'est tontefois pas seulement la fonction de locomotion qui s'exeree palr ce puissant moyen. L'animal, souvent fisi: all sol dologe au milieu d'une masse d'ean, épuise bientot l'oxygéne par ses organes l'espiratoires; sous peine d'asphyxio l'ean qui l'entoure doit se renouveler. Qmel moyen plus simple que des lantelles ou des soles agissant comme la roue d'un batea à vapeur pour mettre l'eat en mouvement at pour changer le milieu ambiant? Le nouvean milicn apporte de nourel oxygime et l'animal sait respirer. Ces cils deviennent inutile's yuamd, pall un autre mécanisne, l'animal peut ehasser aree force l'eau sur' les branchies, comme daus les poissons.

Si l'ean yui baigne l'animal ne peut rester immobile, le liquicle intérieur ou le sang ne peut le rester davantage; le simn doit porter sur place, comme nous l'avous vu plus haut, les mattir'iaxx d'entretien. Le cour est chargé de ce rôle, ou bieu le viliseau lui-même se contracte pour clıasser le salng daus telle ou telle direction el pour produire le mouventent eirculatoire. Mais

- C'est un animal transparent, qui a la forme doun anf, el qui présend.

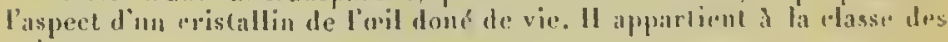
potypes 


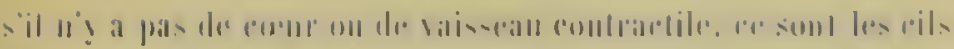

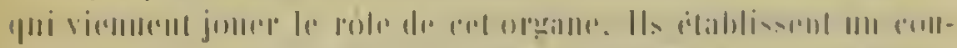

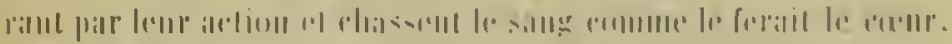

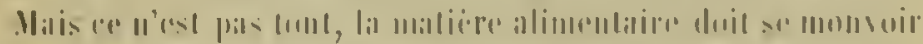

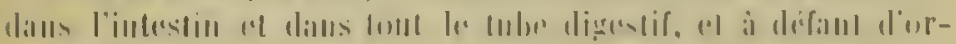

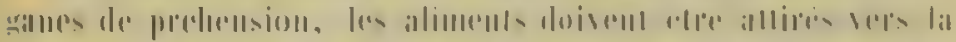
in)

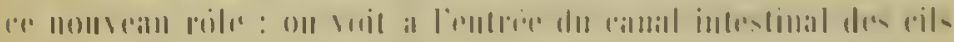

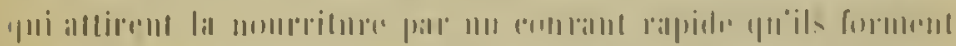

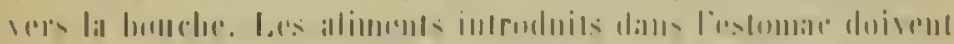

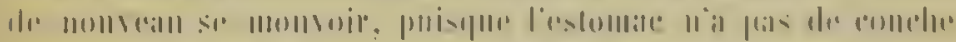

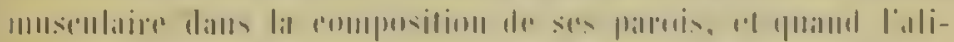

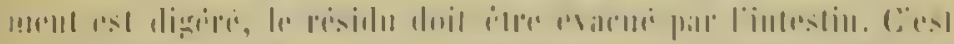
III IIII)

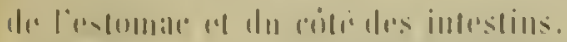

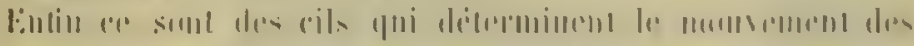

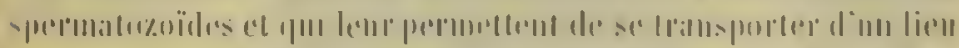
if IIII ilntre (0)

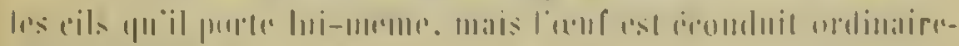

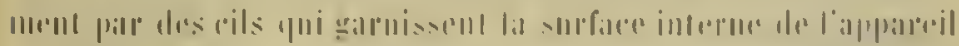

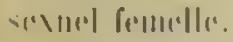

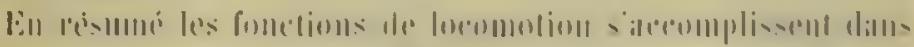

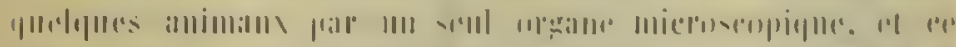

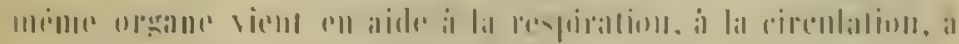

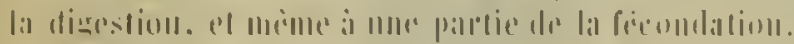

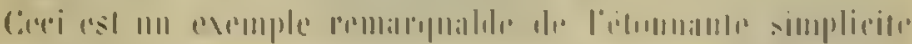

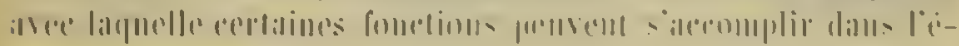
(2) momie animate.

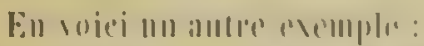

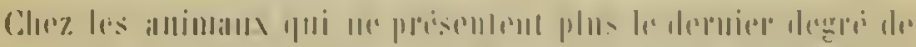

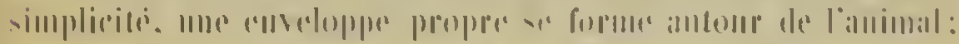
mor peralla

-

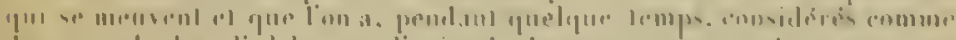

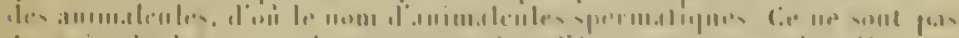

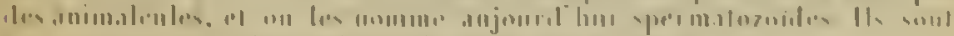

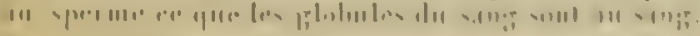


se folment les apparreils de digestion, de respiration, de secrétion, y compris meme la reproduction.

En eflet, ì mesure qu'on s'elève dans l'échelle des ètres, des vrointes pirticuliers apparaissent; les fonctions se linitent, des divisions s'ètablissent el on arrive de division en division à une complicaltion qui dénote un trasail d'anlant phus parfait el des produits d'autilut mieux élabortis, pue l'animial est formé d'un plus gramd nombre d'opyanes. C'est ainsi que la première mo-

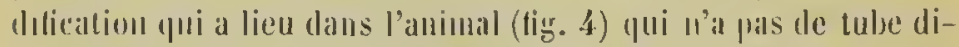

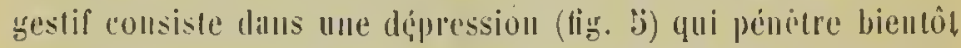
plus profondénent dans le tissu de l'animal (tig. 6) et qui finit,

Fig. 8.

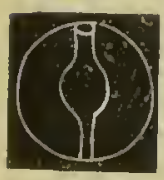

vig. 10.

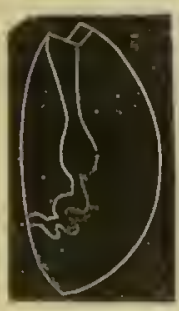

Fig. 6.

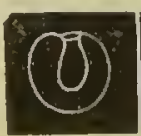

Fig. S

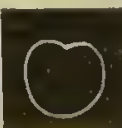

Vig. 4 .

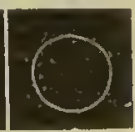

Fig. 7.

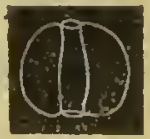

Fig. 11

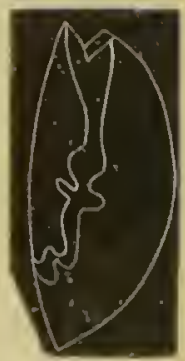

F"บralation du tulec digestir.

Pli prenant une plus grande extension encore, pall pelecel tout lo

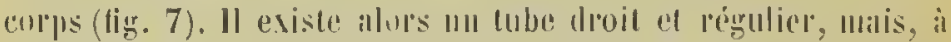

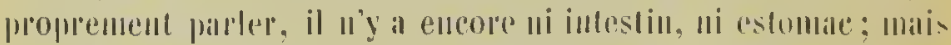
liembot le tube so dilate vers le milien (tigr. 8), le's alimems s"ale-

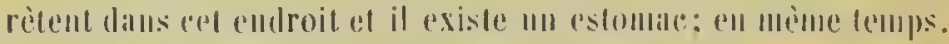

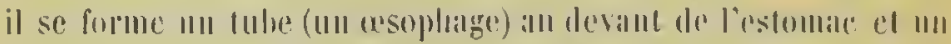

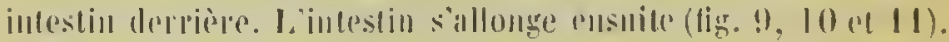

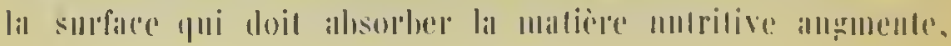




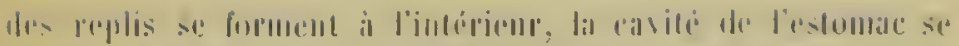

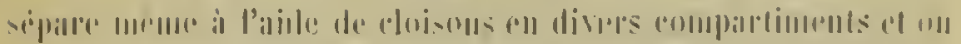

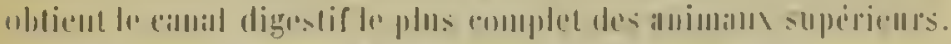

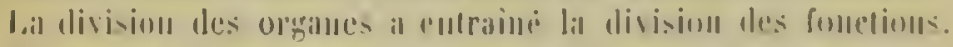

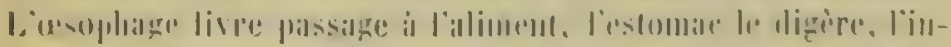

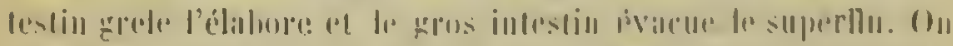

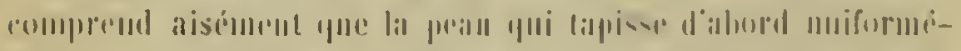

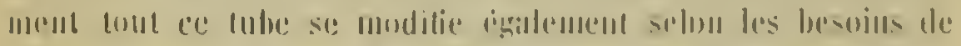

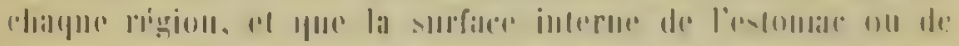

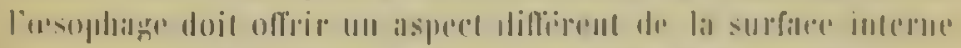

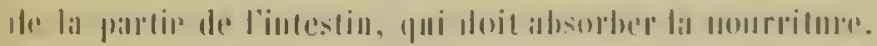

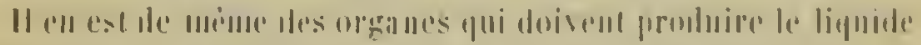

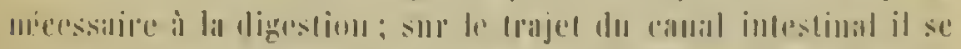

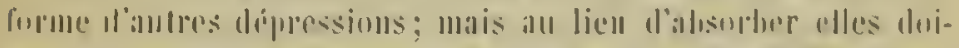

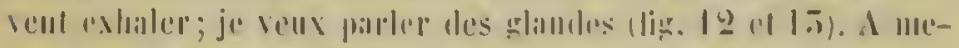
-

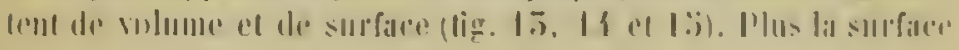

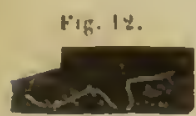

Fig 13 .

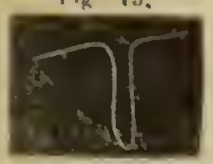

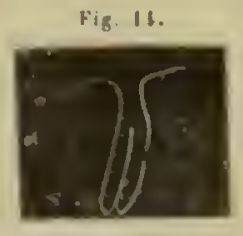

Formalion des glandes.
Fig. 16
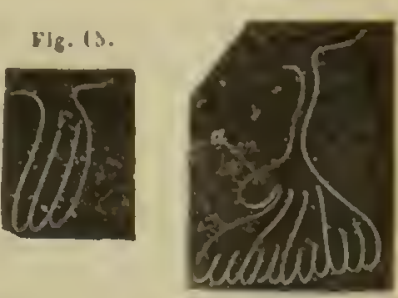

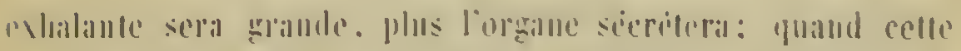

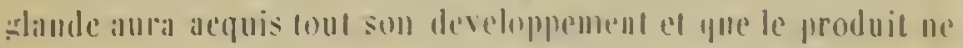

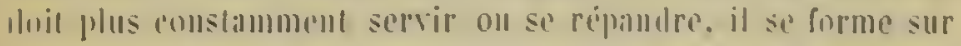

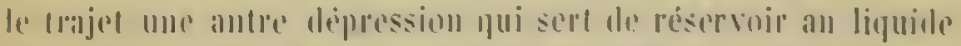

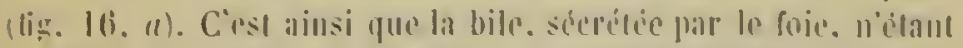

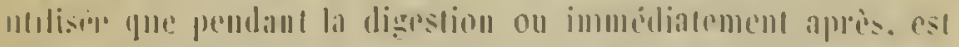

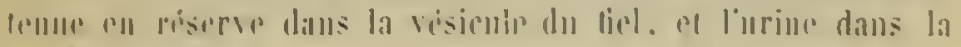
Arsole mrination.

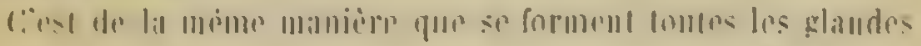


verritables, el si, daus les animanx inferienrs, libaboration des illuments saccomplit avec une aramle simplicilis, il faut, anl couIraire, dans les animan superrieurs des alantes particulieres pont'

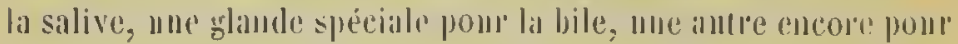

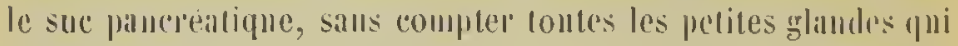
apparaissent st abondamment sur tonte l'etendne de l'appinleil digestif, lepuis la honche jusqu'ì l'anns. Il faut mème iri des glandes dont le produit est exclusivement destime a lubrilier la surface pour prevenue les inconventents du frothenent.

Eu somme. tous les appareits de la vic de conservalion. celui le la digestion conme cehni de la cireulation, celni de la les-

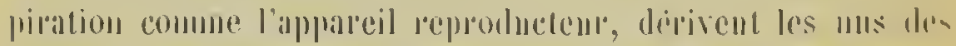

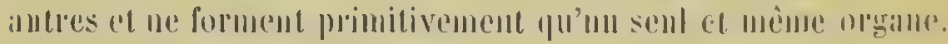
L'appareil sesuel sierète un germe comme le foiesterite la bile: la formation d'un nouvel individu el l'aleroissement de l'animal

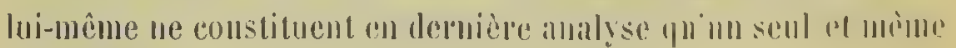
plı́nomène.

\section{SUDORDINAJBON JES CARACTERE、.}

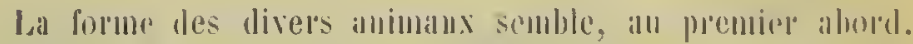

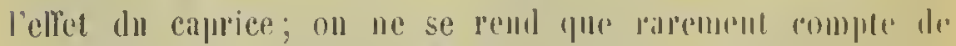
la bizarrerie des formes allecters palr les animand cependant, en y regardant de pres, on voil pue tout est snignenese-

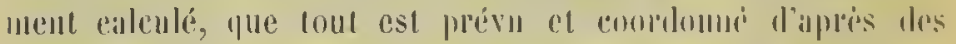

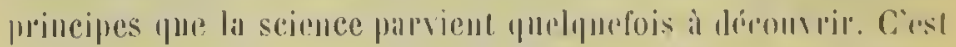

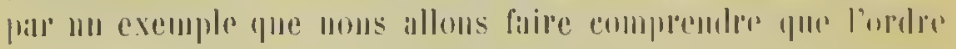

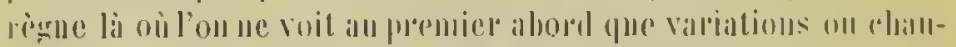

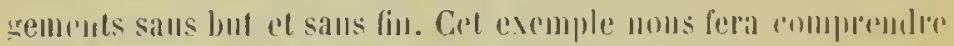

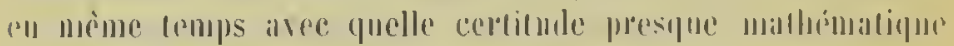

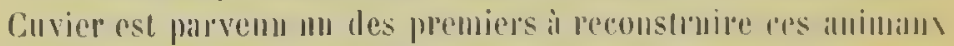

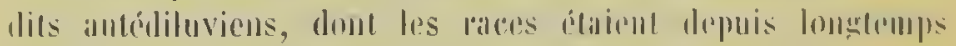

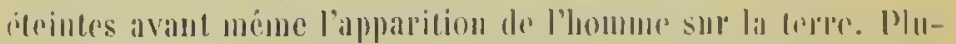

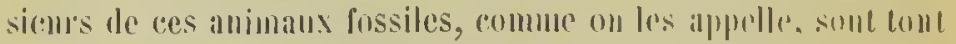

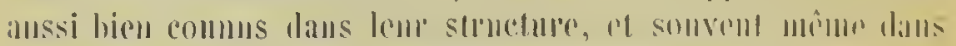

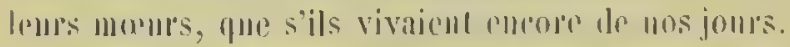

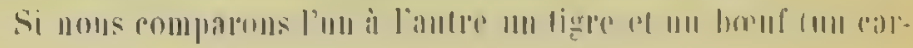




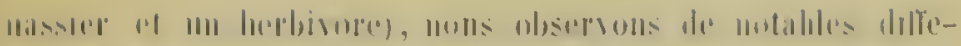

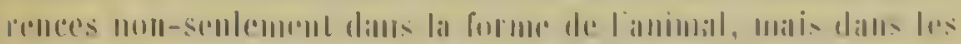

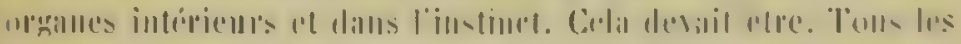

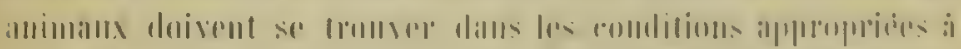

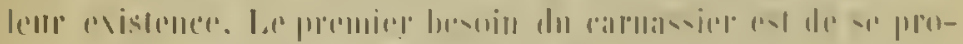

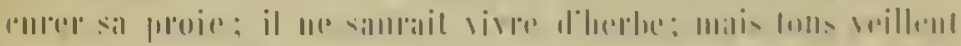

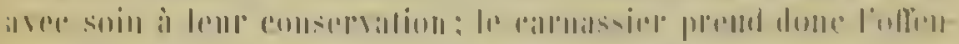

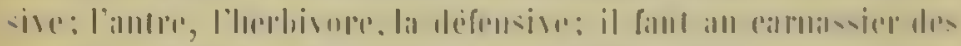
-

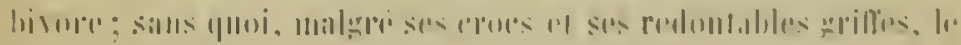

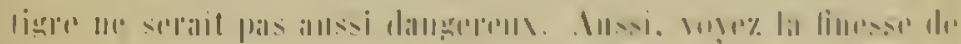

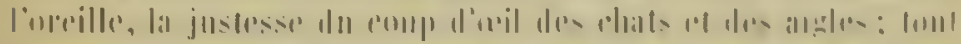

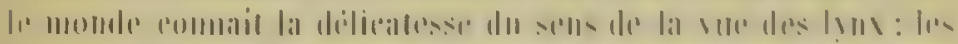

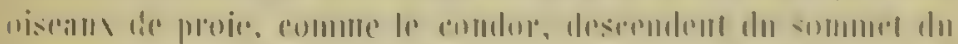

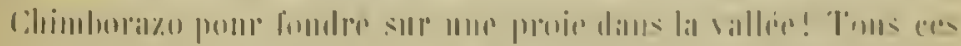

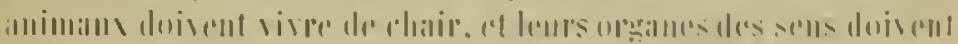
|

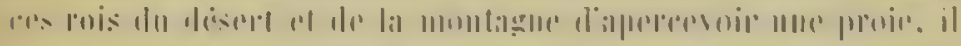

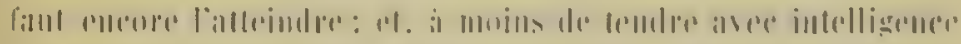

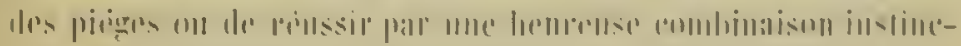

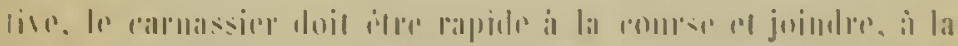

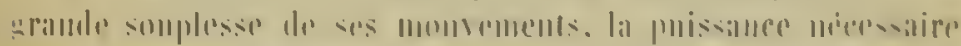

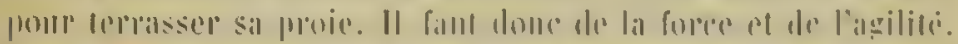

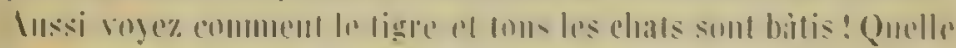

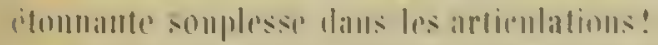

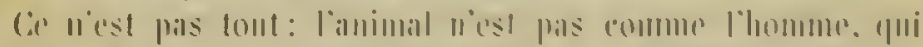

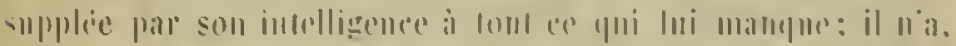

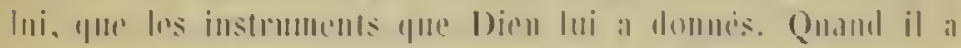

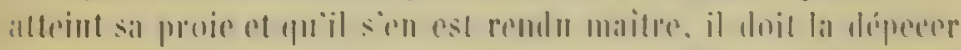

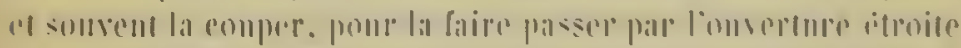
|

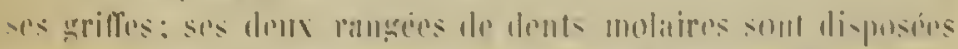
a

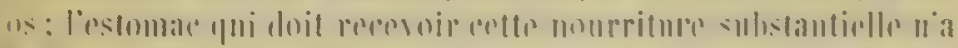

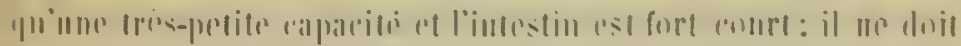

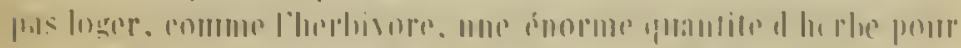

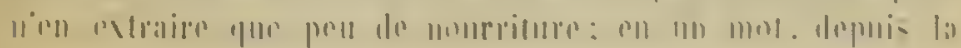


like juspu'il la queur, depuis les poils jusquand us, fous les organes indistinctement se ressentent de l'ohligitlou où se frouve l'alumal de poursuive sa proie mu de défendre son existence.

L'herbivore nous montre un contraste fraplpant sous fous les ripports; il se nourrit d'luerbe, lui, el tonte son altention doit porter sur le moyen de se la procurer. II u'illapue jimais: a quoi celal lui servirait-il? Ces lutles r'ntre l'éléplant el le rluinocéros sout des contes. Aussi l'luerbivore seul a des orgitlies de défense! Dieu a armé son front de bois elégants ou de: puissantes cornes qui le rendent aussi redoutable daus la leifense que les antres sont violents daus l'altaque. Les orgalles des seus u'oul que tout juste assez de puissance pour meltre l'animal sur ses gardes; l'instinct conduil en génćral l'herloivore dans la plaine ou dalls la vallée, oil il tronvera toujours mee nourriture abondante. L'herbe ue mancque pas; anssi l'herhivore peut vivre par troupeanx; le earlassier doit s"isoler. Le

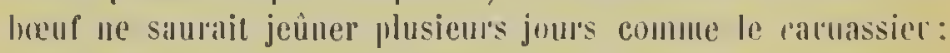
il ne perd pas de temps à gueller sil probe; il mange ou 11 rumiue. Ses membres aulérieur's ne servant ni ì altaquer ni il deperer une prove, ce sont des colomes de sustentation qui doivent simplement le porter et inclquefois le soustraire, par la fuite, à la voracité de son enueuri. Il ne fant done plus ui loights libres, mi ongles acérés, mais un pied large pour servir d'appui; aussi, an lieu de einn doigts, on en voil un on deux enveloppés d'un ongle énorme, qui a pris la fopme d'un sibot et qui est désigué communénıent sons ce nom. Le pied est fourelıu fuand il existe deux ongles, comme chez le bouf, et c'est le nombre que l'on trouve généralement clıez fous cenx qui st' nourrissent d'herdies. Le cheval u'a qu'un seul doigh ì chalpur pied avec un ongle énorme, et qui est devenu d'antant plus volumineux qu'il a reẹu a lni seul tome la nourriture qui est or". dimairement destime anx eimq doigrs.

Les dents, qui servent à la triluration des illimbuts, soml aplaties ì leur surface et agissent les unes sur les autre: compre une pierre meulière; elles ne sont plus trandhantes al pointues comme chez. Ies ealluassiers; elles ne doivent pas coul

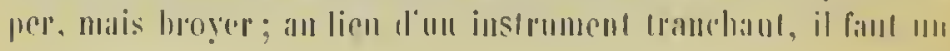




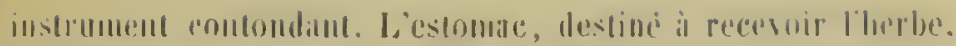

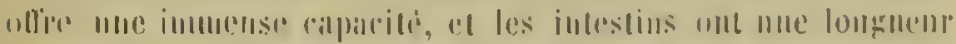

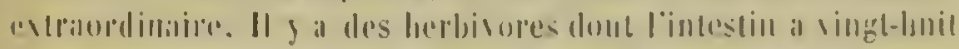

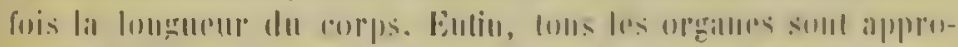

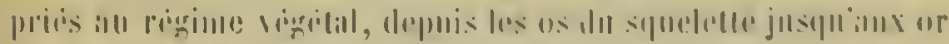

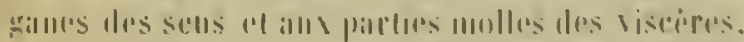

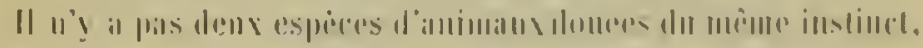

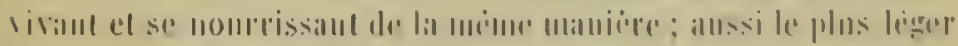

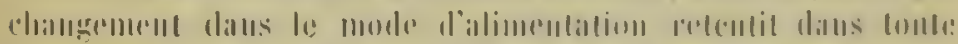

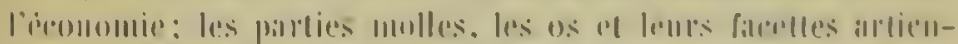

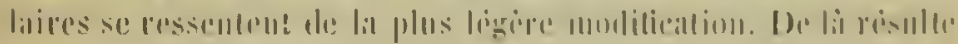

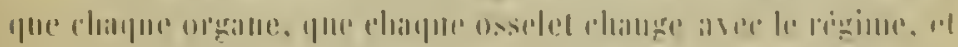

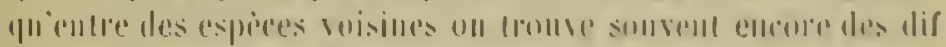

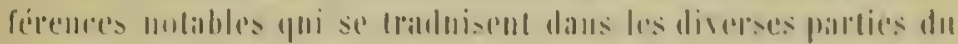
101018.

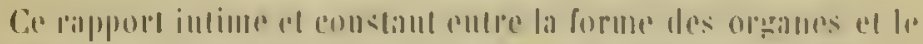

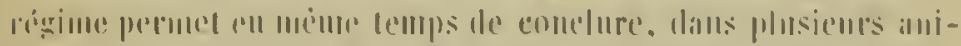

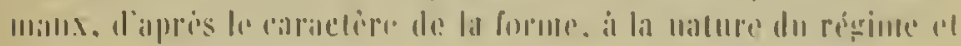

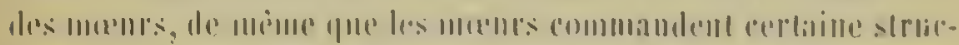

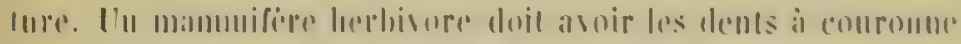

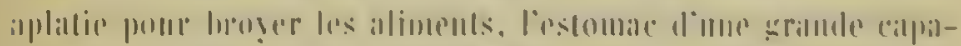

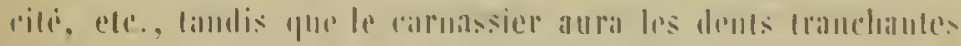

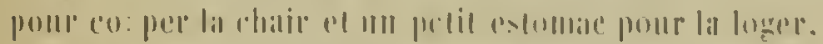

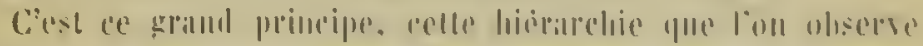

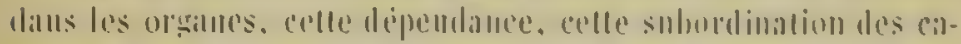

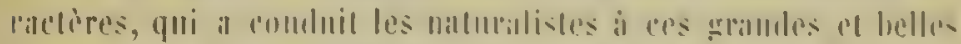

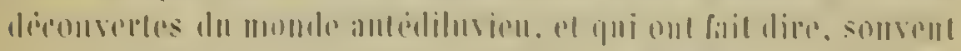

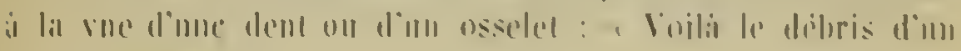
imimis I noแ10an!"

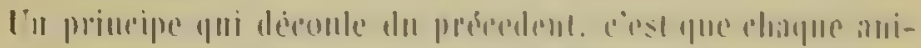

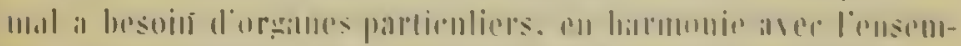

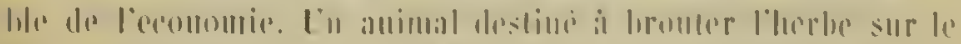

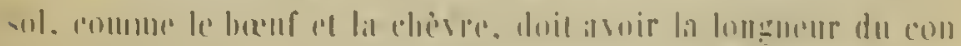

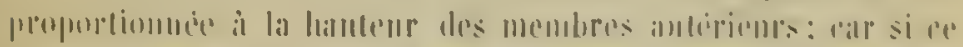

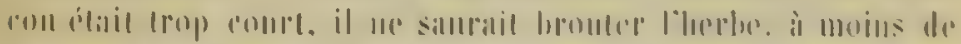

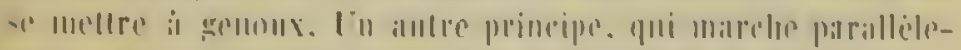

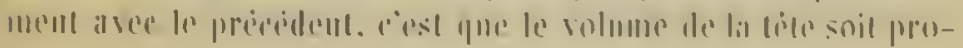




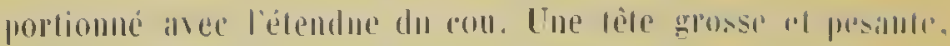
chargere de defenses a de dents voluminemsts, ne salurail èrre

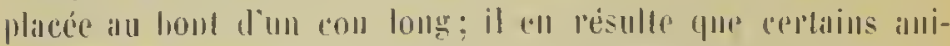

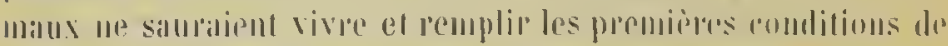

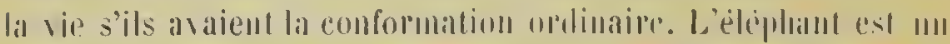

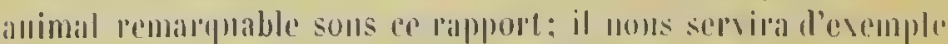
pour montrer avec quelle sigateite ef puelles bardies combinai-

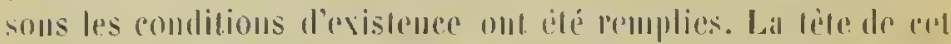
lerbivore est voluminemse; d'inormes difenses, de dis piods de long somvent, sonl implantres daus des alveoles : des dents

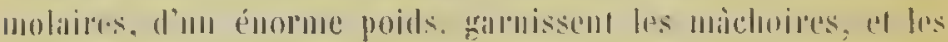

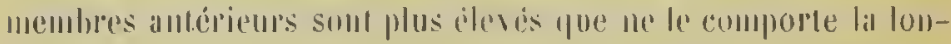

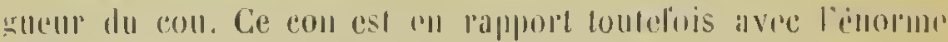

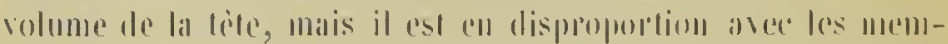

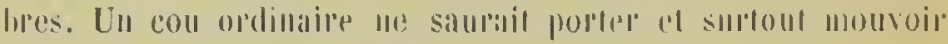
une si énorme masse, el s'il chait proportiontue à la hatutent des

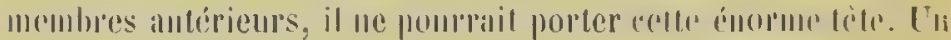

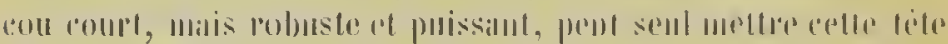

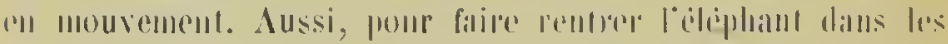

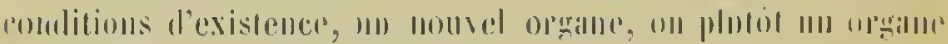

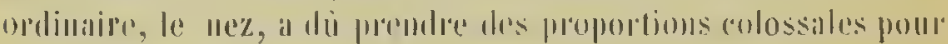

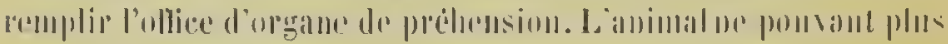

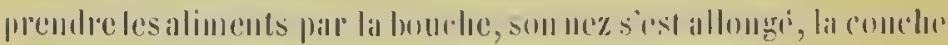

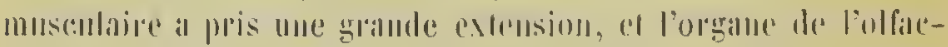

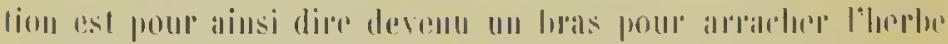

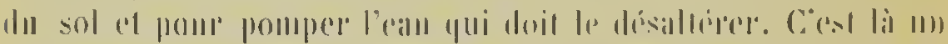

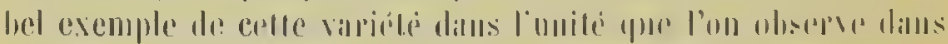

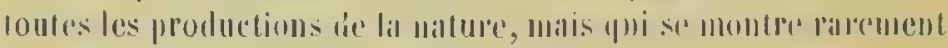

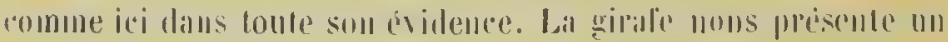

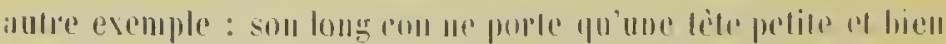

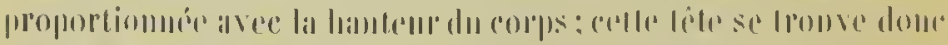

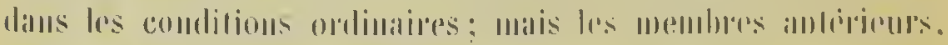

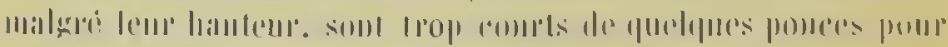

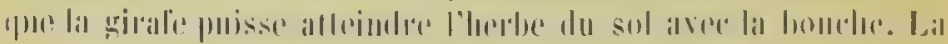

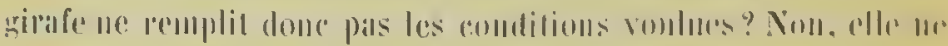

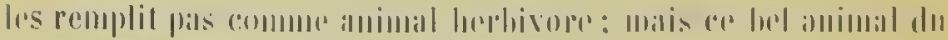

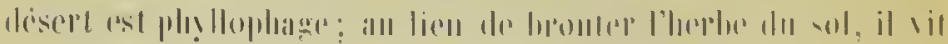




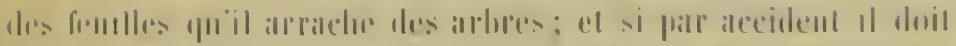

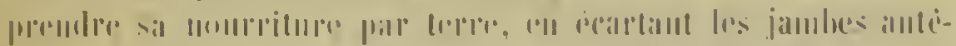

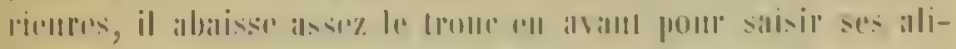

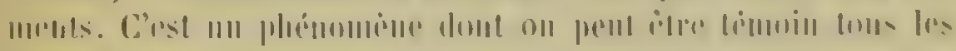

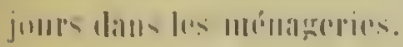

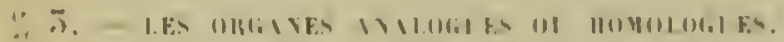

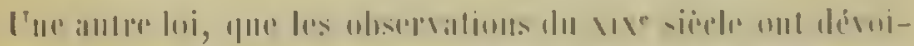

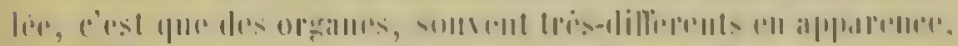

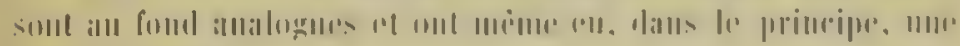
formur ielentiplure

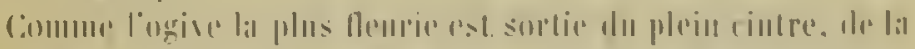

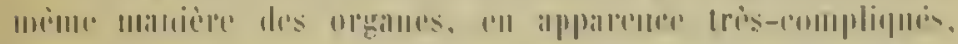

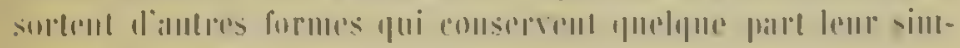
pliciti primilar.

Si nons a

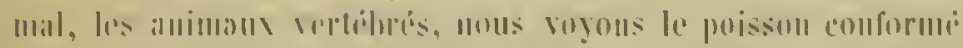

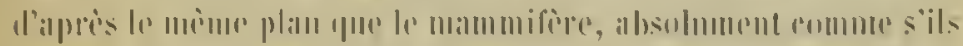

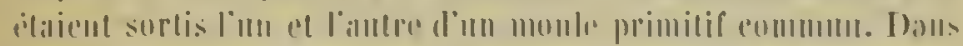

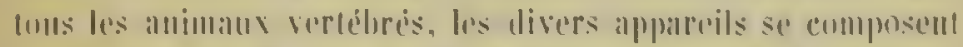

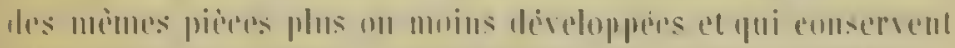

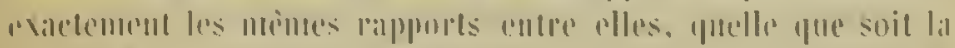

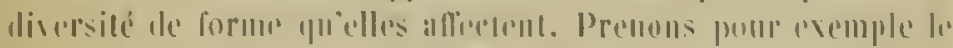

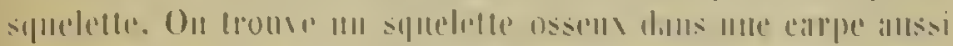

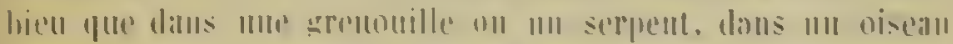

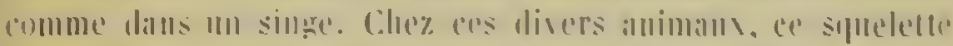

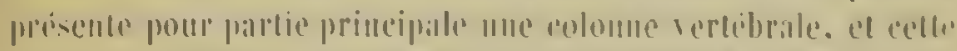

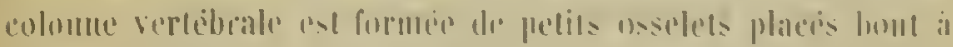

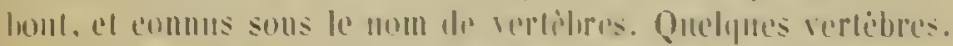

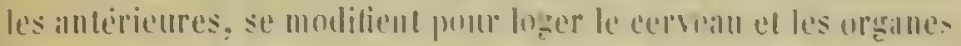
drssens, de de cette modilieation mait le erime. leerine humain. comme lo arine do poison. est forme par des vertébes legerement deformese. Le puisson el le singe, comme lodean, portent

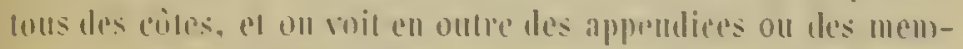
lores pui varient dapres le milien dans lepuel lanimal est appele:

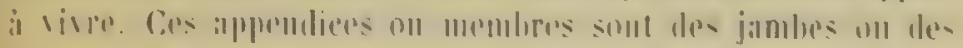


pattes daus les mammiferes qui simtent, qui courent, qui grimpent on tui fonissent; dans les clatuves-souris el les eisealux, as

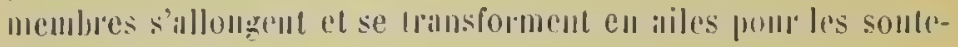

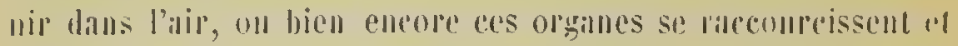

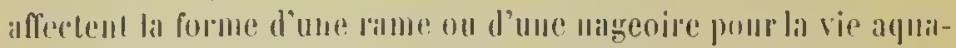
lique clecz les célaces, les phoques al les poissons. Ces palles.

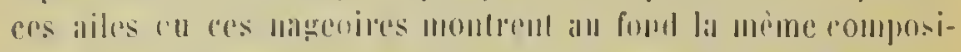

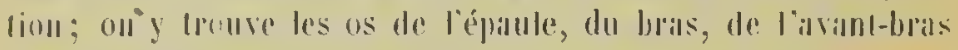

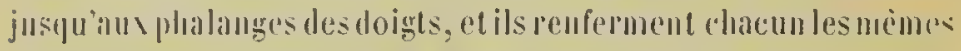
pieces, mais, comme on le pense bien, plus ou moins modifiées dians trun forme, selon les cxigenees du milien. Eu efrel. ces os sont grimels ou longs chez les uns, petils bu courts dhe les antres, rudimentaires ou tout ì filt raltilagincus

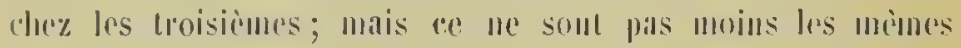
pieces qui doivent porter en anatomie te meme nom. C'est res rue lon appeclle des os analogues ou mieur eneore des homologues.

II en est ainsi de toutes les parties da squelette, depuis he simple fuisson jusqu'an pnadrumane et mème jusqüi l'homme.

Aimsi le crîne lumain comme celui de lout animal vertébré. non-scutement juesente toujours li mème composition, mais il est formé des mêmes os, il loge les mèmes organes, el ces os portent le mème nom, dopnis les poissons jusquand quadrumanes. Lu simge a des os frontiux, comme le bouf ethoiscau, le lézard el le poisson.

Mais si tous les animanx reptitures sont formés d'aluris un plan commun, peut-0u en dire antant d'un insecte ou des animanx arliculés? Evidemment nun! Les apticules sout formes

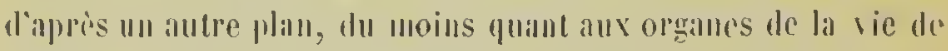
reliation. loinsecte a un squedelle, mais il est cmprumté ì lil

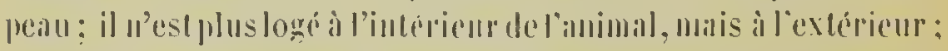
les ailes du hanmelon ne sout phos des palles transformbers, od les patles ne sont plus des appentices amalogues anx membres

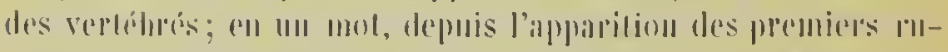

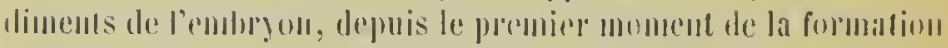

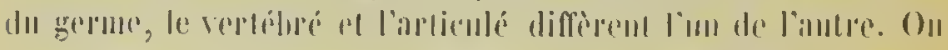

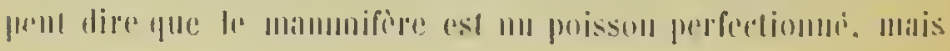




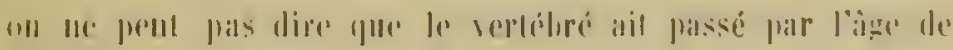
lialliculis:

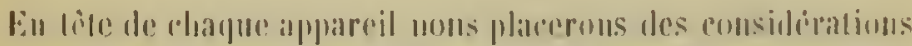

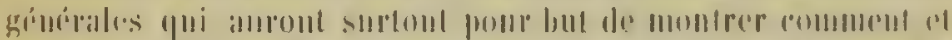

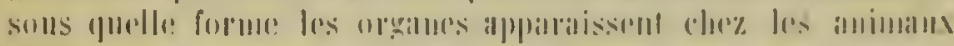

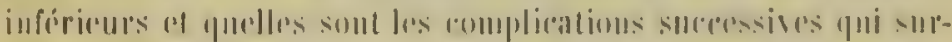

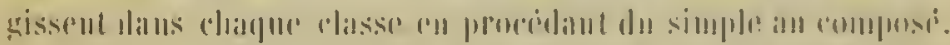

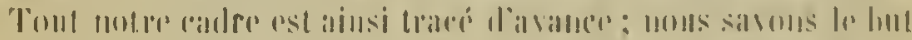

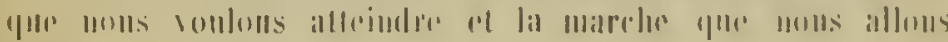
suivir. 



\section{MNATOME COMPPREE.}

\section{APPAREIS DE L.I VIE UE C.ONSLRVATION}

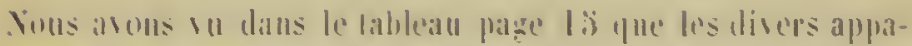

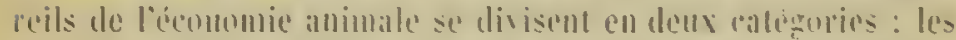

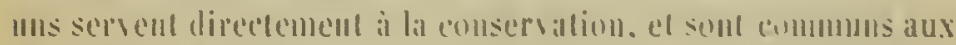

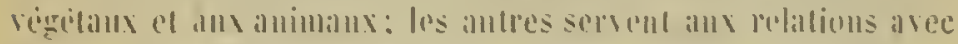

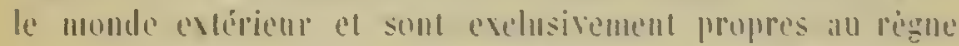

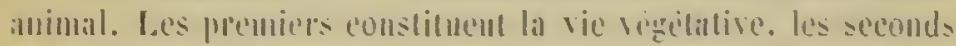
servent à la vir amimalr.

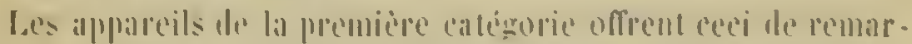

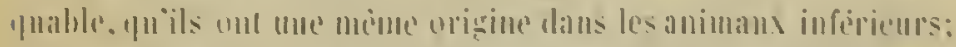

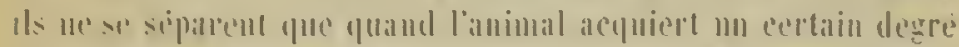

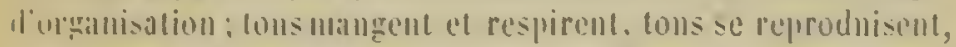

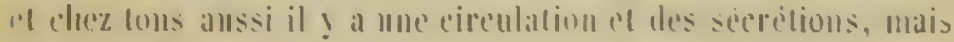


toutes ces fonetions peuvent s'accomplir dans un tissu parfailement homogène, quand il n'existe encore aucune division du travail organique.

Nous voyons les méduses recevoir l'eau du dehor's, charryéc de nourriture el d'oxygène, dans une grande eavité qui représente l'apprareil digestif et respiratoire, el de celle cavité s'étendeut, tout autour, des canaux qui représentent l'appareil circulatoire; el si nous considérons que l'accroissement d'un animal constitue au fond une reproduction, nous verrons toutes les fonctions de la vie végétalive s'accomplir dans les langs iuférieurs quand il existe à peine des organes distincts. L'lıydre est formée d'un lissu homogène, cl elle donne naissance à autaul de jeunes liyures que l'ou délache de fragments du corps mère.

C'est ce qui avait fail dire depuis longlemps à de Blainville que lous ces applareils dérivent les uns des autres et ne sont qu'une modificalion de la peau. 


\section{APPARLIL, DICALTHE.}

Les amimanx, n'élant point comme les plantes fixes all sol. portent gentribement une eavite qui sert de réservoir aus aliments et dans linguelle ce's aliments sulnissent les changements yui les rendent propres ì la mutrition.

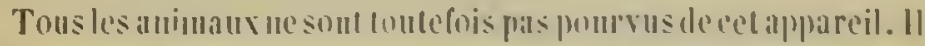

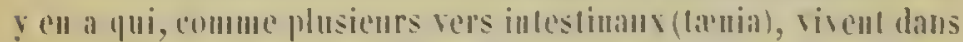
la monrriture de ceux qui les léberogeul el qui absorbent lenrs aliments par tonte la surface de la peau colérieure; ils noult pas besniu de poche pour transporter les aliments aree eus.

Dimtres. enmme les liydres. montrent une simple dépression de la peau daus une region du corps délerminee: la fonction se localise. Puis ahez d'antres encore. celle simple dipressinn

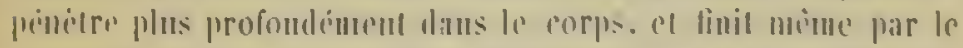

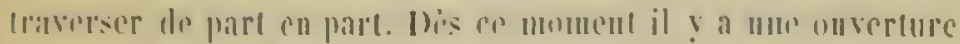


pour livrer passage aux aliments el une anto ourcelume pour l'evacuation du residu de la digestion; en d'antres terues il existe une bouche et un amus. La disision du travilil se manifeste ensuite dans le tube digostif: vers le milieu, il se dilate c'est là surtout que les aliments s'accumulent et l'estomite cest formé. Tout ce qui preciale l'estomare est eommu soms le nom d'osophlage, tout ce qui suit formor l'intestiu. Des etrangloments se moutrent ensuite, el le premier de ces iframlements ohlige l'aliment de séjourner quelque lemps daus l'estomac. Il est commu sous le nom de pylore. Jintestin, d'abord droit, n'olirant plus une surface assez gramdr pour l'absorption de la malicie uutritive, forme des circomvolutions, puis m antre étranglement

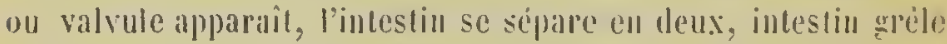
el gros intestin, el une divicion semblable se montre en avant, où nous voyous se former une cavile buccale, el une arrierebouche, au devant de l'osopliage. Des replis apparaissent pour fermer les fosses masales, te voile du palajs: III autle pour

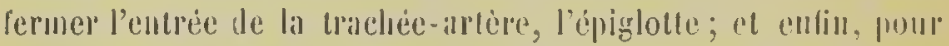
arriver à la plus grande complication, mous u’avous (pu à di iseo l'estomar lni-mène par quelques élranglenents qui élablissent les poches distinctes, et qui jonent claseun leur roble dans larele de la digestion.

L'appareil dont nous nous ocenpons est donc formé par unc rentréc de la pean; mais celle-ci, acenérant une destimation spéciale, se modifie daus sa composition. En effet, si la peau a l'extérieur sert à la defeuse de l'amimal of à lui comserver sil forme, la pean à l'intérieur est smotout destimér à absorber. puisque cest elle qui doit introduire la matièe nutritive on le? dugle dans l'économie. Dans la région ou cette absorplion devient active, elle se réduira prespue à mu résean vaseulaire; laudis que les autres parties de la peau, devenues inmliles. tendent à disparaitre. Celle pean perdal donc quelpues ('a-

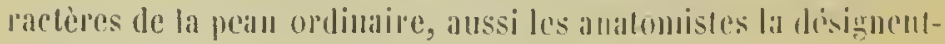
ils sous le nom de membrine mucyense.

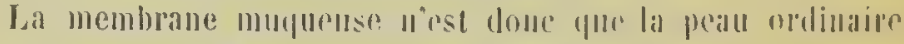
legerement moditire pour mu hesoin spreial.

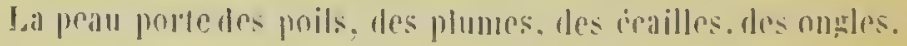


des eormes, des dents. Tuus des organes somt disignes sous le num enmmun de phaneres; ils sont bisiblis el forl distineds.

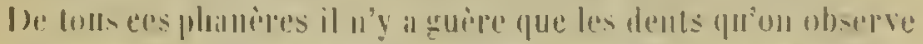

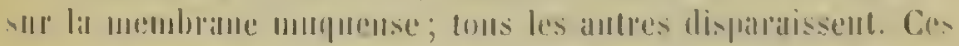

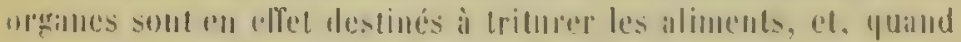

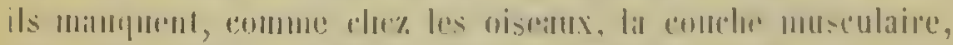

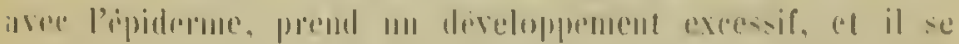

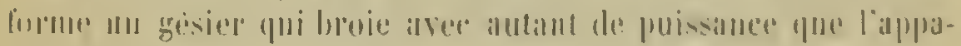
reil dentiaire.

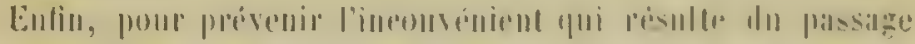

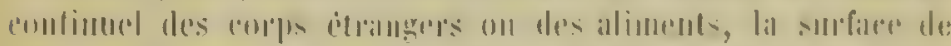

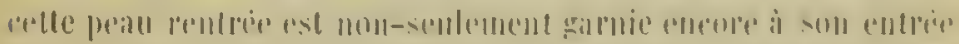

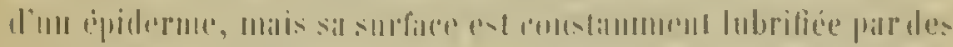

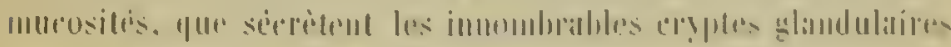
rephiludurs sill tolite la surfiter.

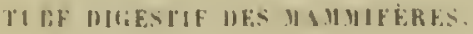

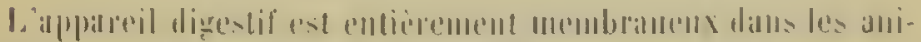

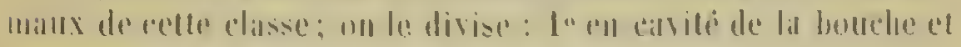

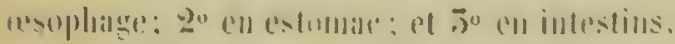

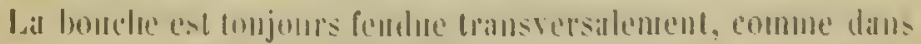

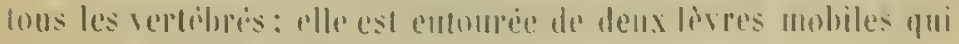
rhlt le moins deshoppres dians les riblacis el les momotrèmes. Ces derniers. ell ellet, se liaprophent des oi- rillis stits re rilppurt. Lal levire silpririmerer est bendur hills plusterurs ron-

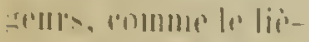
Ire (lig. I7), ains

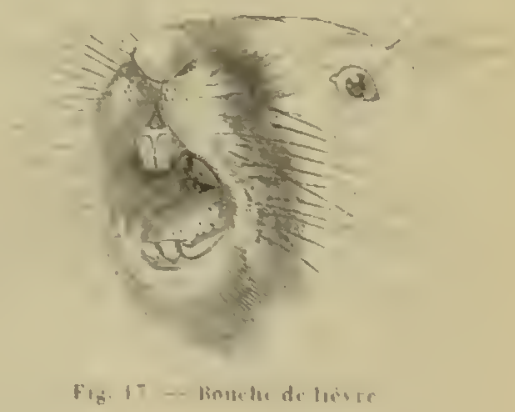

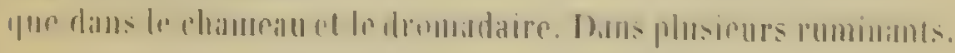


comme le houf, la lève supérieure śépaissit, se courre doun abondante mucosité el devicut un mufle.

La pein qui lapisse lin linee interne de la joue est poilue daus le lievre, comme la peau extériente.

Les poeltes des abajoues, qu'on trouve chez quelques mammifères, sout formes par une extension de la peatu des joues; on les observe seulement clrez des animaux i régime végétal. Presque tons les singes de l'ancien continent en sont pourvis; plusicurs rongeurs en portent aussi, el particulièrement le lianster ou lat d'Allemagne, qui peut loger dans son intérieur une quanlite considerable de griniu. Il y a aussi des rongeurs chez lesquels res proches pendent au dehors, Ces abajoues sont pour' ces animaux des greniers d'abondance.

Les phanères de li bouche les plus importants sont les dents; ils u'ont de eommun avec les os que leur dureté. Presque tous les mimunifères en sont pourvus; les deuts manquent dans ¡|uelques édentés (pangoliı, fourmilier), et elıez l'échidné parmi les monotrèmes. Les dents sont toujours implantées daus des alvéoles et portées par les os maxillaires et incișifs. Elles sout de Hature calcaire dans tous les animaux de celle classe, sanf

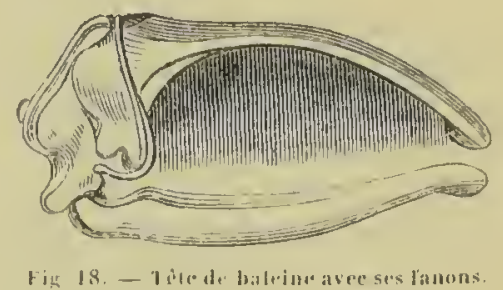

les baleines (fir. 18) et l'ornitlorliynque, qui ont des dents cornies. Ies dents servent gencralement à broyer les aliments, Inelquefois ì retenir la proie, ou bien encore, daus certains eas, elles servent ì la défeuse. Chez l'élépltamt, le marval el quelipurs antres, les dents se dévelopjent extraorlintiarement el premum r'll affel le nom de défenses. On distingue trois sortes

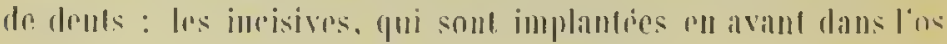




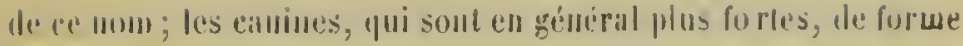

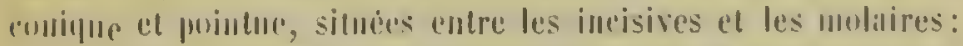

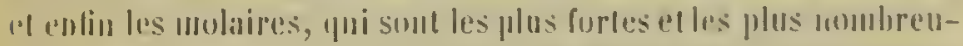

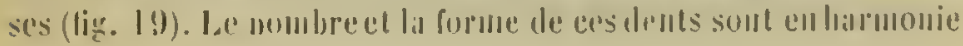

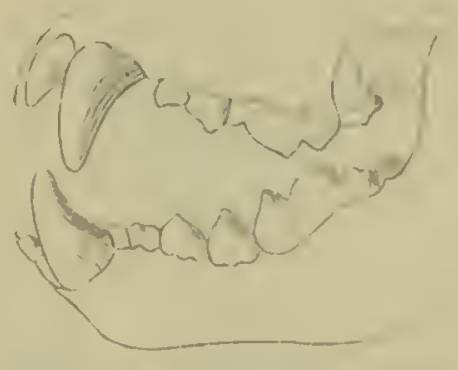

Fig. (9) - ystime dentaire d. carmassier

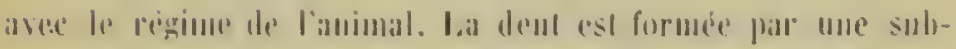

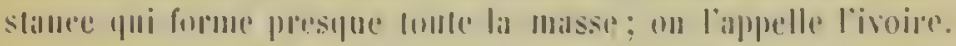

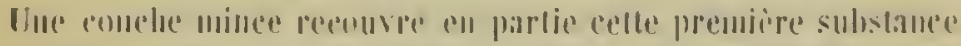

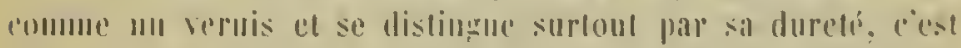

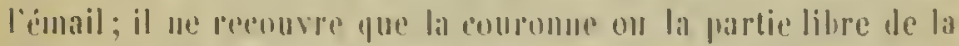
dent; les mammileres ont frumiralenent me troisieme sub-

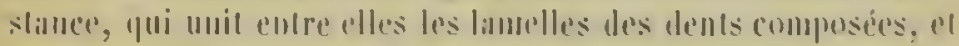

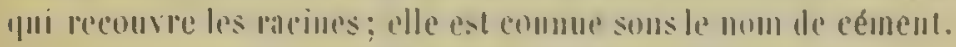
Chery les baleines, les lents regoivent le nom de fanons.

lal limgerue est un appendici lobre a sa parlie antérieure el illialde par sa biste, geniralement arrondie, quelquefois bilide: clle a un mourement intrinsique produit pir des musdes qui sunt disposes diapres un plan uniforme. Tous les manmiferes out la langrae mobile: elle est le moins developpre chez les ceita-

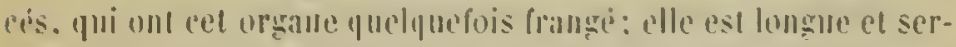
viunt doryane de pribension daus les ruminants, plus longue comere arroudic eomuse un ver dans les indentes fourmiliers (liow. 20). Ces leruiers animaus passent leur langue daus liu-

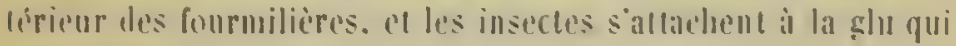
lit reenure. 
Eu dessons de la langue an voit, chez quelques mammiferes

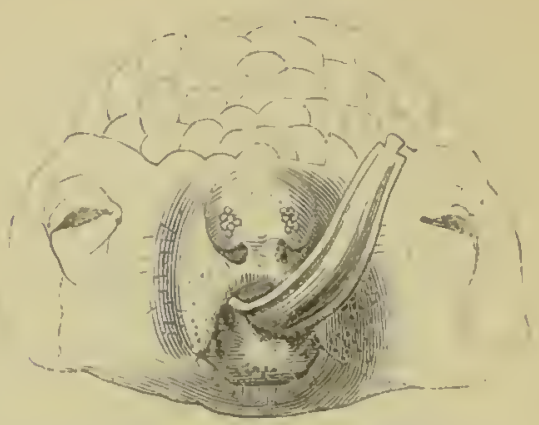

Fig. 20. - Pangulin numtrant si langue.

(ceptains simiens et chciloptères), une saillie qu'on a appelio langule accessoire (fig. 21).

A la face inférieure de la lamgrue, plusirmors manmmifères (calluassier's) out sur la ligne médiane mu cordon (ib)eux, puelquefois eartilaginenx, qui s'étend dans toute la longuene' de

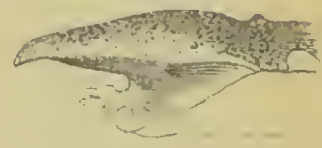

Fig. 21. - Langue de haptale. rel or'gane : e'est le ver.

Lal langue est souvent couverte de soies ou d'épines. quelquefois d'écailles (carnassiers, hérisson).

A la base de cet organe on voit généralement des papilles a alieiformes, disposées en V pnand il y en a plusienrs, el dout le mombre est très-variable.

La langue est le principal organe du sems du goult.

Le voile du palais est un repli de la pean interne qui sem surtont a boucher l'ouverture postérieure des fosses nisales. Il existe che\% tous les mammulères; mais daus les quadlumanes

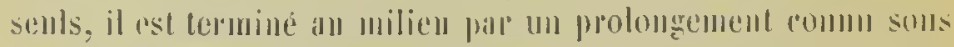

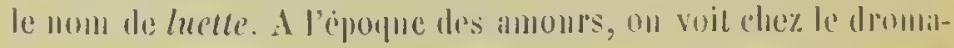

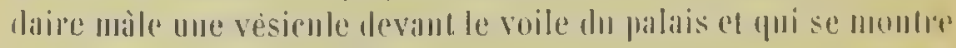

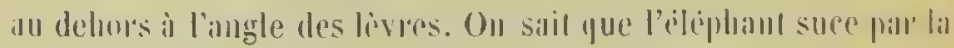

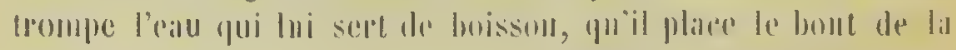

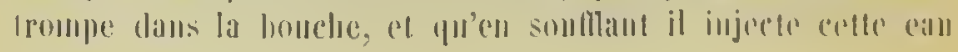


diuss sou gosier. II faut lone que léléplant avale ses aliuments

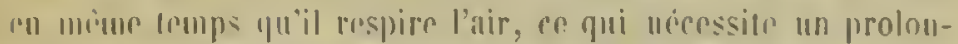

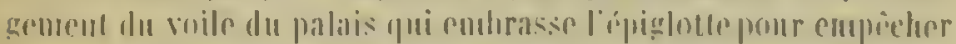

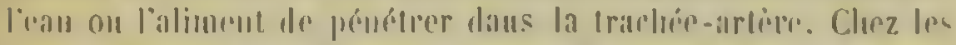

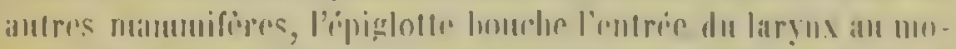

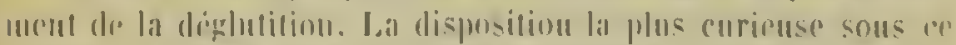

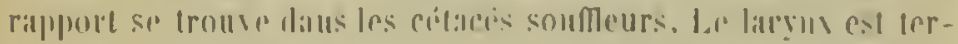

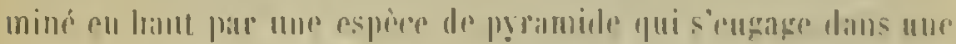

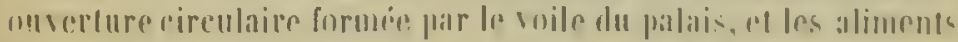

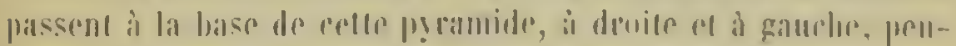
dallt que l'auimal respire (fieg. 220).

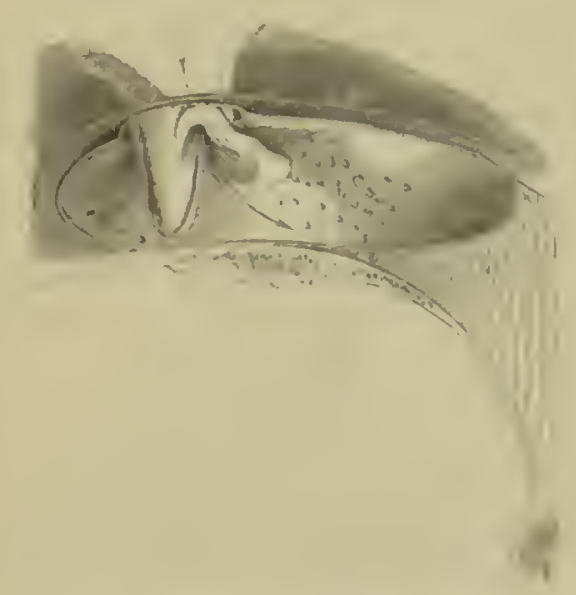

Fix. :2: I arona de nasenum

Lépigloto est une valvule qui sert it fermer louverture du laryux pendant la deglutition: elle evistr dans lous les mammiferes.

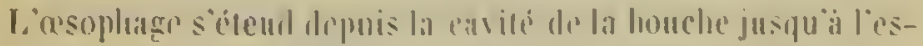

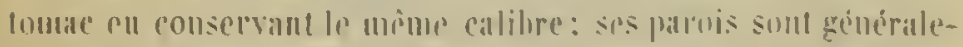

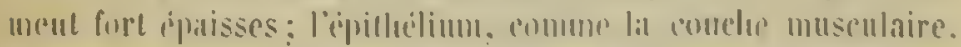

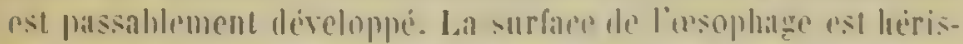

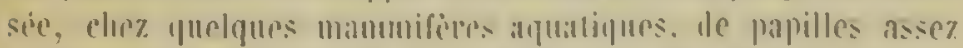

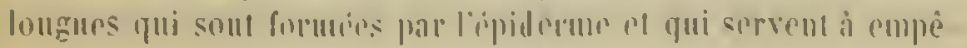


clier les aliments de rebrousscr chemin. I.e castor el la loutre nous montrent cette disposition.

L'osopliage s'ouvre dans l'estomac, aprés avoir traversé le diapluragme; che divers rongeurs, toutefois, il s'étend, en dessons du diajhluragrie, dans ta cavité abdominile.

lasophlage du cheval est terminé par un sphincter qui cunpêcle ret intinal de vomir ou de laisser l'evenir les alliments sur leurs pas.

ESTOMMC.

L'estomac est toujours membraneux; le plus simple parmi les mammifères est celui des carlulas-

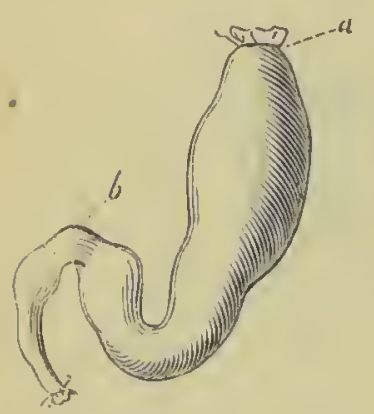

Fig. 8. - Hstomar de finuger. siers aquatiques on des plioques (figule 25). Il ne consiste que dans un léger renllement du tube digestif. On peut dire en géméral que, phıs l'animal est carmassier, plus l'estomac est simple. Cet organe présente souvent une cintancrure plus on moins profonde qui te divise en dens parlin's (divers rougeurs, figr. 24); l'estumale est hoursoufle diez quelynes puadrumanes, par la présonce de ballides

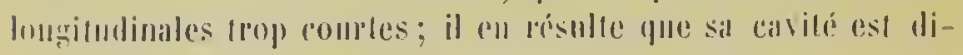

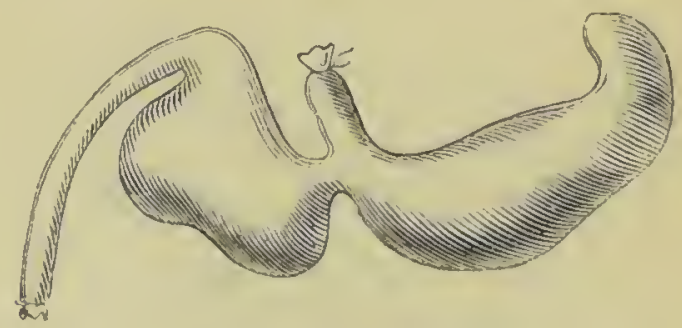

Fig. $2 t-F \operatorname{sinan}$ Ale hamstur

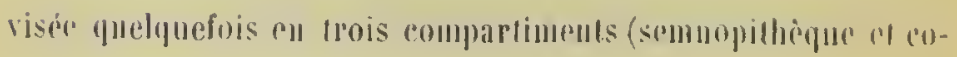


lobe) (fig. 2:3). Dans les cótaces souflleurs it est divisé en trois

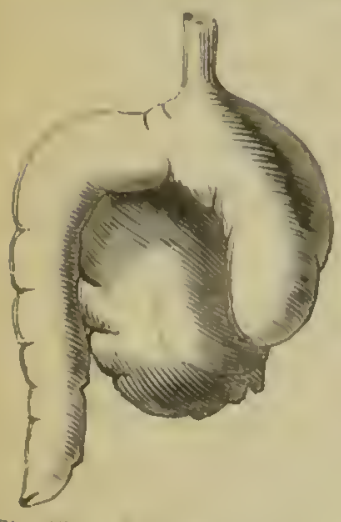

Fif. 2.5. - Fsenmar du daue.

-proches qui se suivent el qui sont nettenent sépuréces les unes drs au-

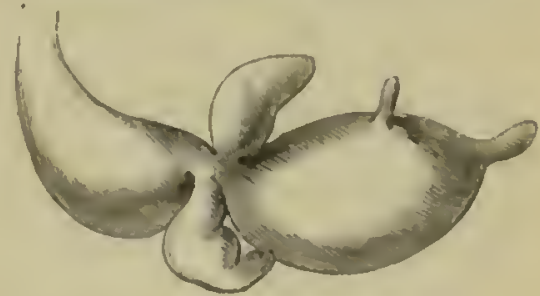

Fig. 26. - Hefumac de manalus ameriramus.

Ires; daus lo lanumin, liestomac pré-

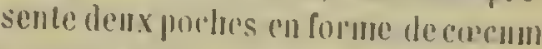
(fig. 26); mais cest dius les rumiunts que cet organe acyuiert

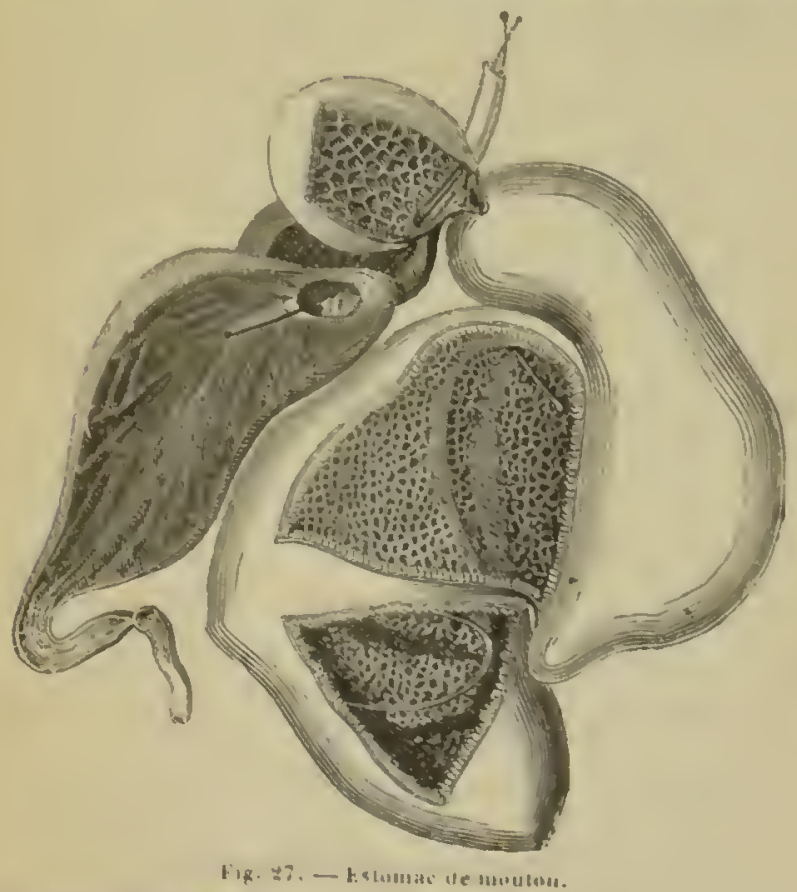

soll plus şimd dereloppement (fig. 27). II se rompose de quatre 
cavites: la panse, le bonnet, le feuillet et la caillette. La première est i clle seule plus grande que tontes les antres : elle est tonjours remplie d'luerbes. Le bounet est situé au-dessus de la panse; il est arrondi et porte daus son intérieur des replis en forme d'alvénles. Le fenillet se trouve au hout dı bonnet et prisente dans son intérieur des plis longitulinanx comme les fenillets d'un livre. Enfin la quatrième renferme la substance qu'ou emploie pour faire cailler le lait. Entre l'œsophage el le feuillet il existe unc gontliere formie pal deux lèves très-mobiles, la gontlière osoplatgienne; ces lères en śécartant forment mo

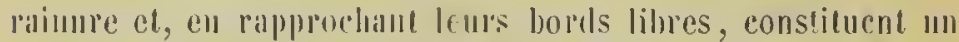
tube, qui conduit, après la seconde mastication, l'aliment de l'wsophage directement dans le feuillel; on a observé aussi cette gouttirice osophawiemne chez. l'arvicola amphibius, le muInt, le lemming el d'autres mammifioes.

Les chameanx, les lamas et le chevrotain de Java n'out que trois cavites dans lemr estamar.

On sait que les mammifires qui louninent, màchent leurs aliments à deux reprises différentes. Les licrbes, grossièrement divisées, passent d'abord de l'oesoplatere daus la panse, oì elles séjournent jusqu'ì ec que l'animal ait fait sa provision; elles reviennent ensuite à la bouche, et e'est alor's que commence la rumination. Celle-ci achevée, les licrbes arrivent parfaitement triturées au bout de l'xesoplatave, penètrent dans la gontlicre dont nous avous parte plus haut, et passent celle fois-ci directement dans le fenillet.

Dins la partic la plus déclive de la pause des claneans, il cxiste de grands alvéoles qui fout l'oflice de rescrvoils daus lesquels ees animanx conservent lemp provision d'eau. On sail que les claimeaux, appelés avec raison les vaisseaux du lésert. sont surtont preceicux à canse de leur grande sobrieté pendant leurs longs voyages dans les desel's.

Chez les célacis souflenrs, l'estomae est forme de trois cavites

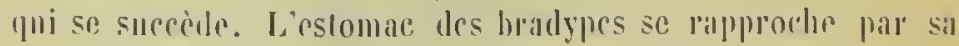
forme dre colni des ruminauts.

Pusieurs mammifères ont, daus l'ápaisserme mime des parois de l'estomac, un amas de glandules promant quelyurfois 
lit forme d'une grappe de laisin (castur. ele.). Clez le luir, ces Dindules sont rimnies, comme cho\% les oistanx, all devant de

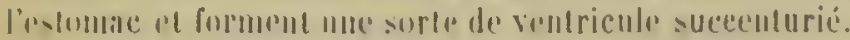

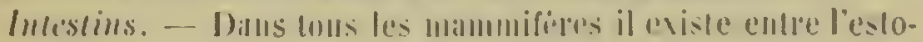

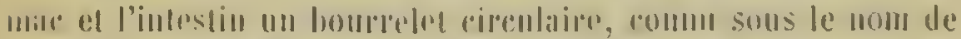

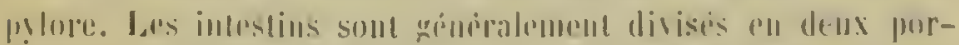

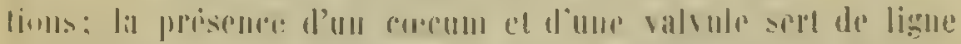

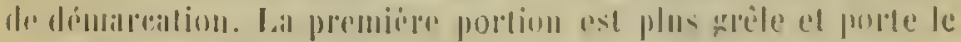

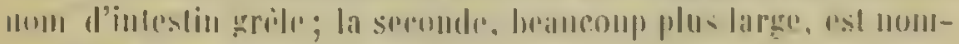
mince gros intrstim.

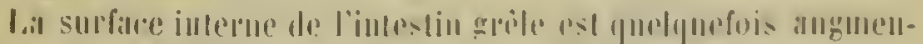

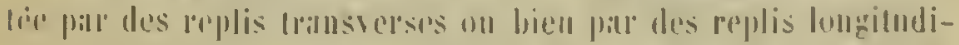
manx; che\% puelques manmuiferes (divers celactis) var roit en

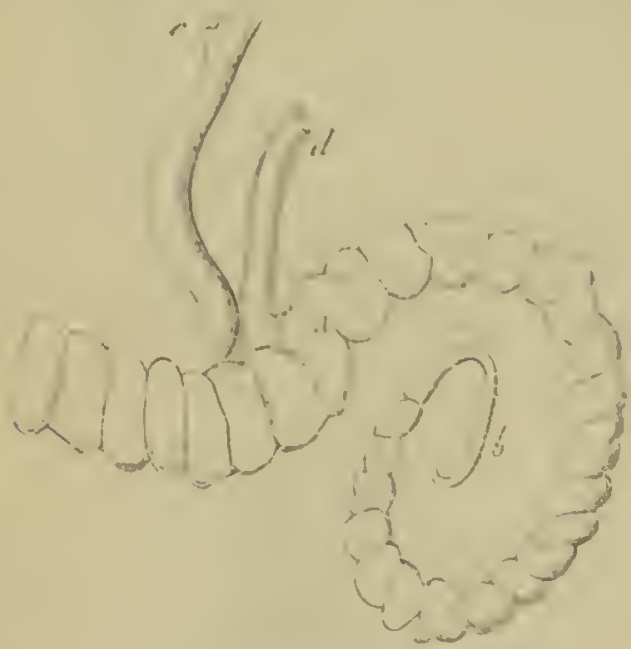

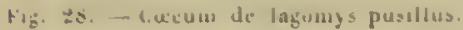

a firand coenum. - b Ippendices sermiforme. - petil cacan.

mime lemps les ulls et less alltes: dalls certains das ces replis folment des alsioles.

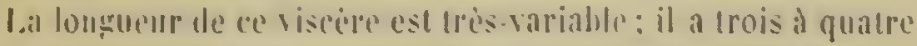

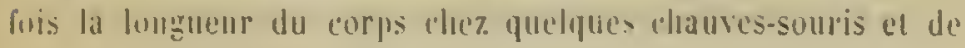




$$
\frac{29}{\sqrt{2}}
$$




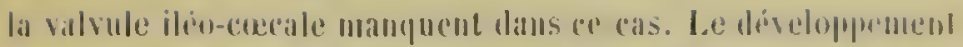

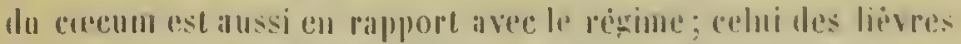
el des lapins est le plus volumineux ; il a juspu’à septrois la calpicite de lestomace el montre mo valvale spirale dans son intirienr.

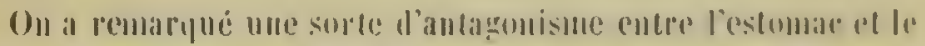

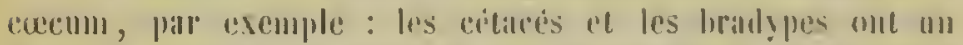

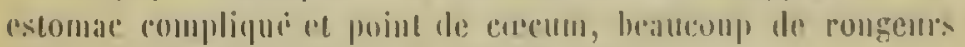

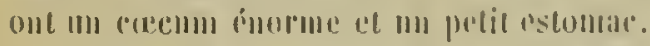

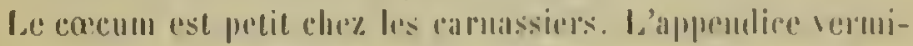
forme qui termine cet argane dans l'homme ne sobserve que daus l'orang-(1)utang et les yilbuns.

lal surface interme des intestius est sousent rouverte da

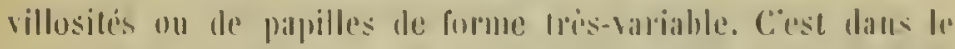
rlumoceros quelles sout le plus diveloppres. Ces villositio

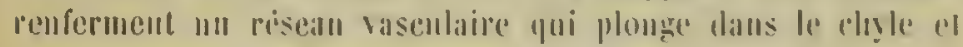
alosorle le principal produit de la digestion.

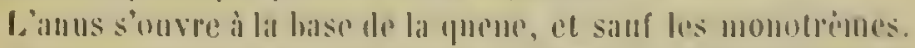

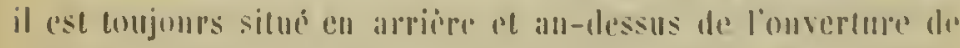
l'appareil genilo-urinaire. Clu\% les monotremes seuls il stour dams un ellatulue commere chez les oiseanx.

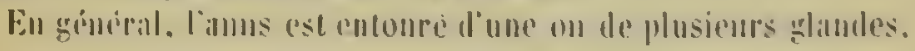

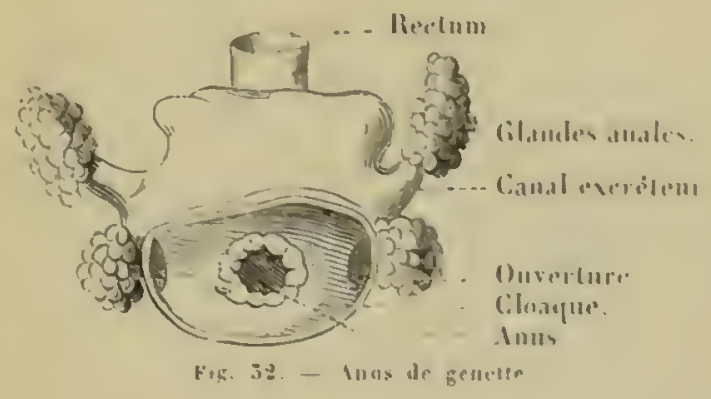

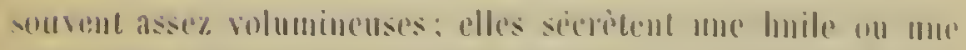

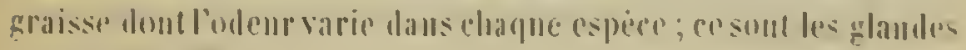

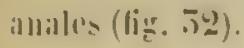


Chez les mouffeltes, la fétidité de ee produit est si grande, yu'ellè sert ì leur défense.

Clrez tous les manmifères, les intestins sont maintenus en place par le péritoine, qui se comporte partout de la même manière envers les viscères ablominaux. Les épiploons sont très-étendus et fortement chargès de graisse chè les manumilëres libermants, comme le birireau, la marmotte, les loirs, ele.

Cette graisse aboude surtout en antonne avant que l'anintal ne tombe dans son sommeil létharginue; elle lui sert de comlıstible pendant la dure de son sommeil et a complétement disparu à l'époque où il se révelle.

Glandes de Peyer. - Les plaques de l'iutestin commes sous le nom de glandes de Pejer se tronvent dims les divers ordles des mammiferes; elles sont tonjour's situées vers lin fin de lïntestin grêle. Dans les singes, Borlın en comple de six à seize. Elles different peu de celles de l'homme. Le cheval en a une centaine, le boenf une trentaine dout les plus grandes ont l'etendue le la main. On peut facilement élndier ces planues dans le mouton, eltez lequel elles sont répindues comme de petites îles dans tout l'intestiu grèle.

Les glindes de Bronner ne s'observent que dians le duodénum; elles sont toujours formées de plusicurs lobules en grappe de raisin; ehacnne de ces glandes est pourvur? d'un conduit exirriteur.

\section{OISEAUX.}

Loisean se nourrit conme le mammifore; celle classe renferme des caruivores, des frugivores, des insectivores, ele: mais l'oisean n'emploie pas les mimes mojens que le mammiềre pour réduire sa mourrilure. Tous les viseans sont pricí

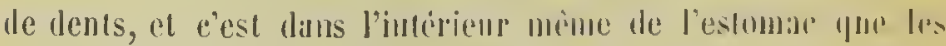

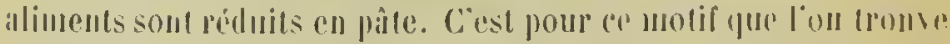

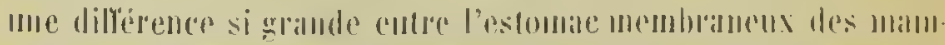

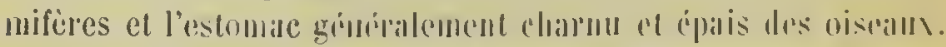

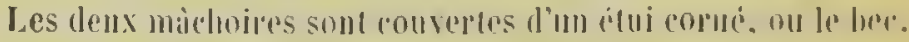

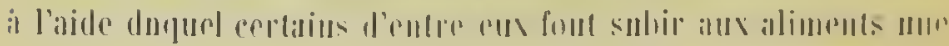




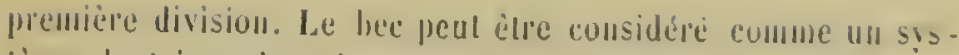
lème dentinire, dílus lèyuel les dents, de nalurec rorme, seradient fomdles les mutes dams les andrees.

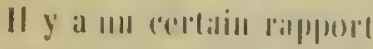

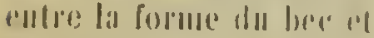
la nomrriture lailbiludelle des oistains.

Lal eavite de la lmurdur dithiere de aelle des Intam-

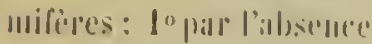
de livress an parr lialssence de dents: an lien d'un systime dentaide, i) se driveloppe uाI élui

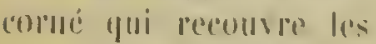
michoires: $5^{0}$ pall lialsserier de voile de piladis. "t fo cullin par labsence? d'ipiglolle.

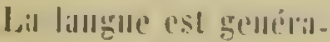
lement de forme litimcrulaire al sousent rolu-

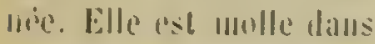
les prerrompets al les cil-

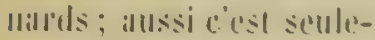
ment dials ans darmiars

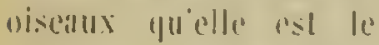
siruge da sens da gucil. Lal lamgue la plus romiall-

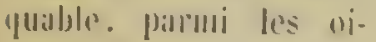
spame esl colle des pies:

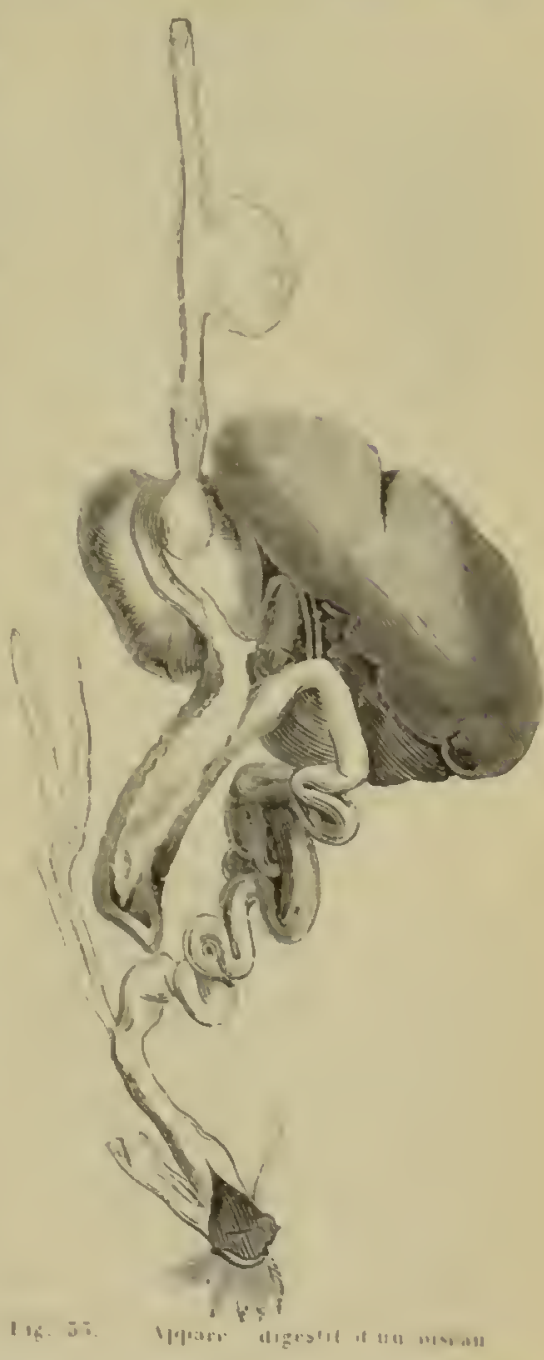

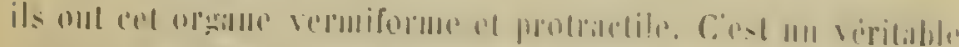

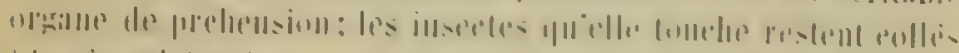

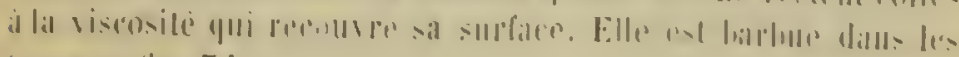
lonteills: (lip. 3 . 
Les fosses masales s'ouvrent daus la cavite de la bouche par une fente longitudinale, couverte de papilles cornees qui font l'offiee de voile du palais. Des papilles semblables remplacent l'épiglotte.

Chez le péliean, le cormoran el quelques autres oiseaux, la peau qui s'étend entre les deux branches de la màchoire inférieure se dilate fortement et forme une sorte de poche semblable à une abajoue. Chez l'otis tarda màle. un sac membraneux s'étend le long du con. entre la peau et la trachée-artèrc, et s'ouvro ell dessous de la langue (fig. 53).

L’oesophage des oiseaux est fort loug ct trẻslarge; sa eapaeité augmente générilentent par les plis longitudinaux y ui s'effacent et s'étenden! sur toute sa longueur. Les oiseaux, surtoul ceux qui se nour'rissent de poissons, avalent souvent une proic d'un très-grand volunte ef sins lia diviser.

A Sal partie inféríeurc, avant de pénćlier lans la poitrine, l'osophage se dilate et forme une poche comme sous le nom de jabot (fig. $5 \overline{5}$ ). Celte poche est double chez les pigeons et excessivenent développée dins la varićló de pigeon appelce grosse gorge. Après la période de l'incubation, il se forme dans les glindes du jabor III liquide blanchatre qui sert ì imprégner la nourpiture que le pigeon dome il ses petits. C'est une sorte de laclition.

Un peu en dessous du jabot, on observe uni seconde poche; elle u'rst pas lris-grande; elle est surtout caractirisie par les aryptes nui prodnisent un liquidenicessiare a la digestion. Cette seconde poche est appelec to remtricule succenturie. Les glandules de l'estomile sont réunie: iei au devant du gésier, a cause du robe noweran que les paroir

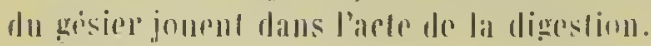


Cette seconde poclie s'ourre immediatlemeut dims une troisieme, qui représente l'estomac proprement dit. On la nonme

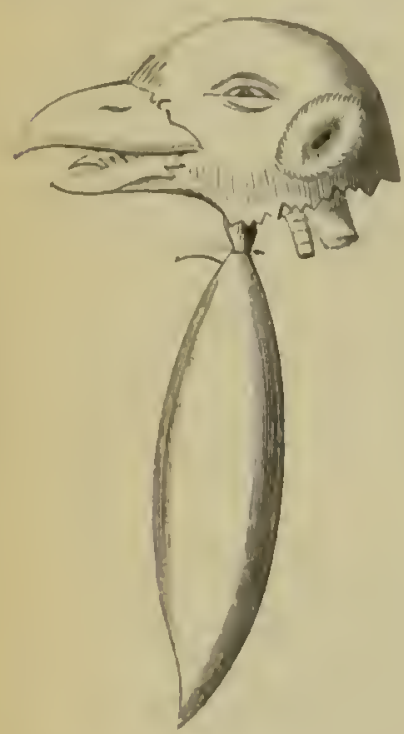

tog 3. - Intiarie miln.

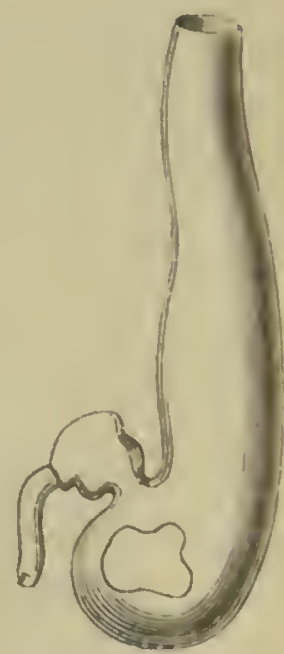

Fig 36 - Fstumac de heron.

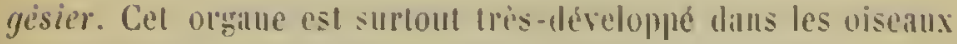
granivores aiusi que daus le canarl en les oies. Le gésier esl de

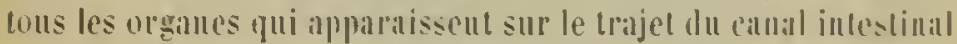
celui dout lat couche musculaire est lat plus développere, aliusi que la conche d"épiderme. C'est daus son intérieme yne les alinculs doivent itre broyis. Cet organe est conformo de matuiere is ce

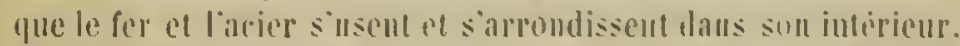
et les petits cailloux que les oiseams avileut fout loflice de dents daus l"interieur te celle poche.

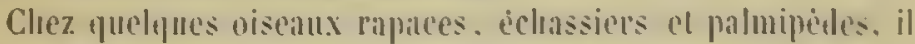

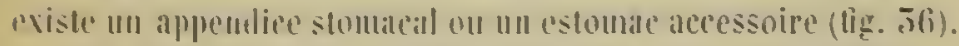

Tout lestomate ne consiste que daus un pelit appendier solus forme de coecum daus leuphone.

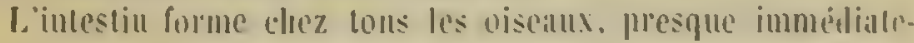


ment après la naissance, une allse qui loge la glande pancréalique ou lia grande silivaire abdominale. On ne trouve plus une frande dillérence entre l'intestin grèle et le gros intestin; ec dernier est souvent thès-court. Lal valvule qui sert de ligne de démarcation dans les mammifèresmanyue dans les oiseaux, etan lieu d'un cocum mnique on en voit alcux, sonvent non loin de l'allus. Ces deux coecmus aequièrent un très-grand dévelopjecment chez les oiseaux omnivores et hiploivoles. Chez l'autruche, il y a une valvule spirale dans l'intéricur. Certains oiseaux ont ell outre un coecun implail.

L'anus, au lieu de s'ouvrir dircetement au dehors, s'ouvre lans une poche commune arec le cantal everéteur de l'urine et de l'appareil sexucl. Cette poche, est désignce sous le nom de sloctque (fig. 57).

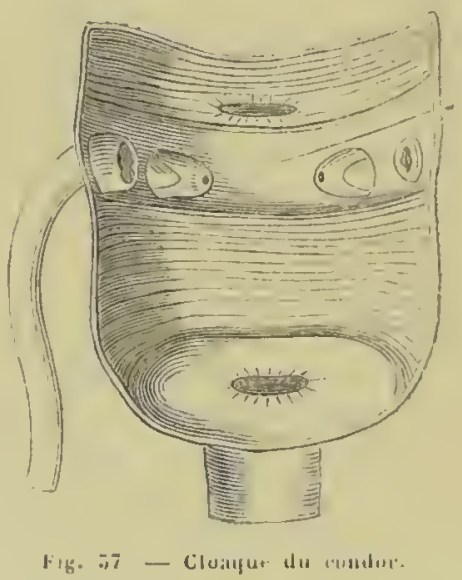

Au devant du rectun on voit souvent, surtout dans le jeun' îge, une hourse que l'on désigne sous le nom de hourse de fialnicius. C'est un orginte qui se développe pendant l'époupue fadilde pour disparaitre ou dimimuer après lil naissince.

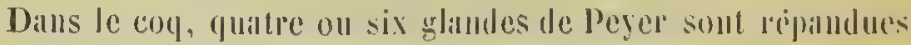
sur le trajet de l'intestio grèle. Elles se distinguent alutut pill 


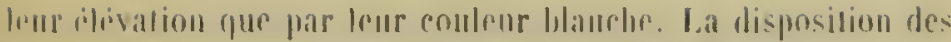

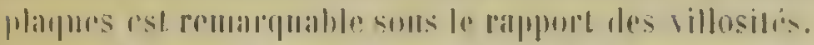

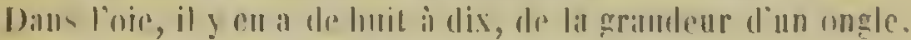
(1) les tronse de mene dams les camards.

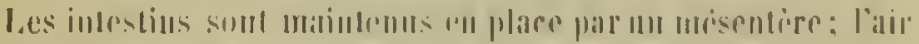

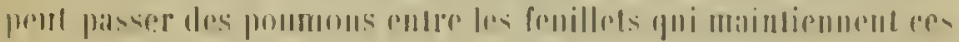

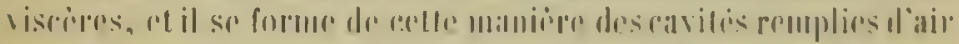

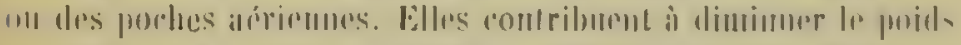

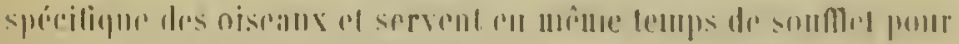
andiver la respiralion pulmonaire.

\section{REPTILES.}

Vunts II" Irom

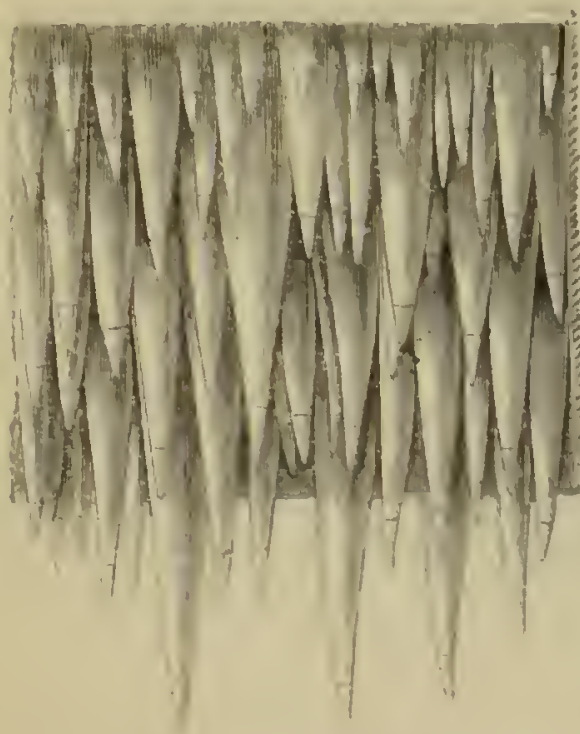

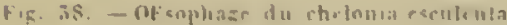

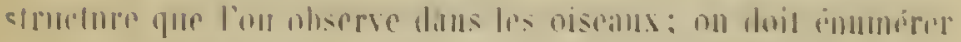

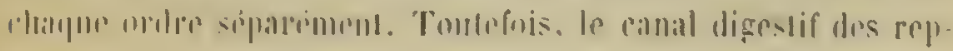


tiles n'offre point de grandes modifieations. L'estomae tend de plus en plus à se confondre avee l'œsophage; la division des intestinsen intestin grêle et gros intestin n'est plus si eonstante; souvent la valvule iléo-cœeale disparait, ainsi que les eœeums, et tout l'appareil digestif devient plus simple.

Les organes qui varient le plus sont les dents et la langue.

On trouve dans les reptiles, outre les dents maxillaires, des dents insérées sur le palais ou des dents palatines.

Chez tous les animaux de cette elasse, l'intestin s'ouvre dans un eloaque dont l'orifiee est transversal ehez les sauriens et les ophlidiens.

Les elıéloniens ont un bee corné et sont privés de dents eomme les oiseaux; la langue est peu développée; les fosses nasales n'ont pas de voile du palais et il n'y a phus d'épiglotte.

Tout le eanal intestinal est membraneux; on voit un seul estomae; dans quelques tortues marines, l'œsophage est hérissé de papilles eornées très-grandes qui empêelıent les aliments de rebrousser ehemin (fig. 58, p. 51).

Les sauriens eomme les ophidiens portent des dents; d'après leur insertion, on peut les diviser en incisives, canines et molaires; mais eomme en général elles ne servent plus à broyer les

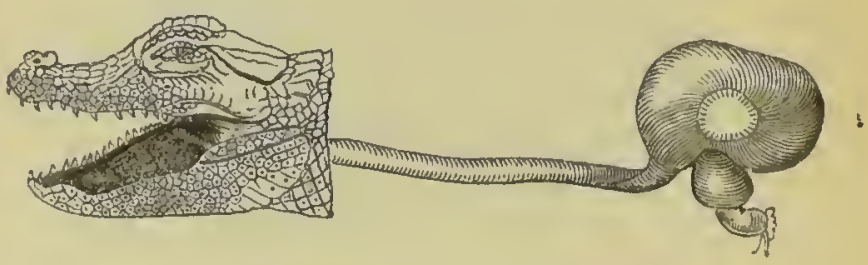

Fig. 59. - Langue, resophage et estomae de crocodile.

aliments, leur forme et leur insertion n'ont plus une grande importance. Les dents varient beanenup d'une famille ì l'autre: elles sont moins solidement enchìssées que dans les minnmirères, mais elles conserveut leur nature nsseuse. 
On voit des dents endrisscés l'une dims l'aulre chez les oru rouliles.

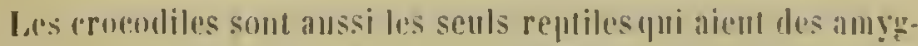

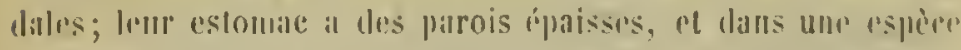

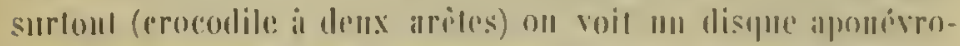

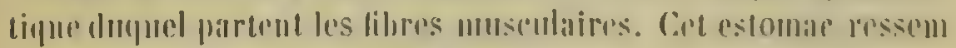

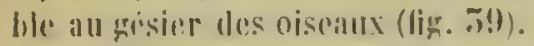

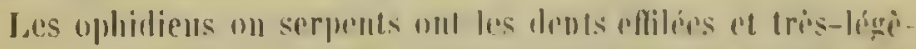

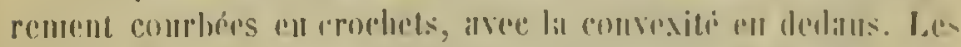

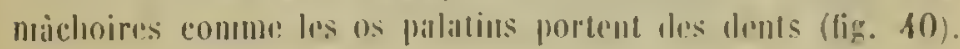

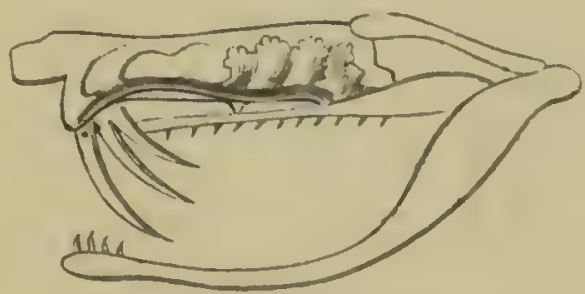

Fig. so. - Ttle de Irigonacephate

Certaines dents sont cremsées en canal ou présentent une gonutire of regoisent à leur hase du renin quelles versent dans lil phate par la pointe. Co sunt les dents renimeuses que lon ro

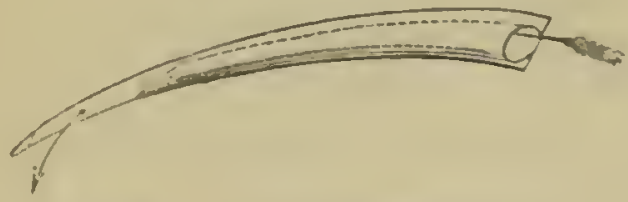

Fig. \$1. - bent venimeuse isolir.

connait à ce caractere. Le poison étant déposes an centre de la plaie forméc par la dent, labsorption en rist si rapide, que la mort simsuit inmuliatement. Il y a anssi des druts de rem- 
placement qui sont prìtes ì prendre la place de celles qui tombent ou qui se briscut.

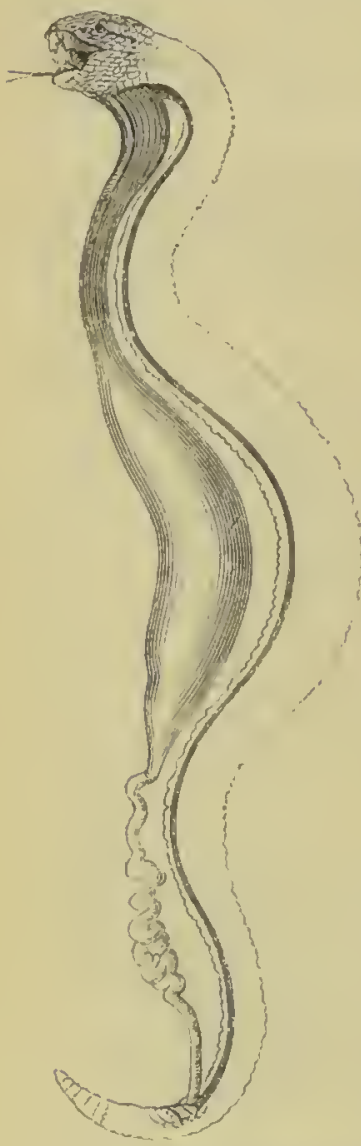

Hig. 12. - Tulse digestif de crolale.

L'osophage est très-large dans les ophidiens et se confond avec l'estomae; ils sont l'un el l'antre très-dilatables et montrent de nombreuses glandes mucipares. Le commencement de l'iulestin est toujours indiqus par un repli pylorique. Il y a peu de circouvolutions, quelquefois mème elles manqquent, mais en géuéral on distingue le gros inteslin par sa largeur (fig. 42).

Cliez le coluber scaber, on roit dans l'osophage des dents couvertcs d'émail; ce sont tout siunplement des apophyses autéricures des vertèbres, (fui traversent les parois digeslives (Jourdani).

Lal lingue des tortues est plus ou moins molle, mais peu déreloppée el très-peu mobile. Les croeodiles ont ine langue plus rudimentaire encore; elle ne consiste (qu'en ment inmolite el sans pointe libre. Aussi, les anciens loous avaient dẹji fait parl de celte observation, qu'en Égypte un petit oiseau (jluvier) prenètre sans erainte dans la gueule du crocodile pour' y prendre des insectes el des débris dont lanimal ne peut se débarl'asser. Ce fail curieux, raplyorte par It érodote, a cité conlirmé parle les uaturalistes qui faisaient partic de l'expéalition d'igypte.

Les lizards ont la langue molle, tris-molile et ligerement

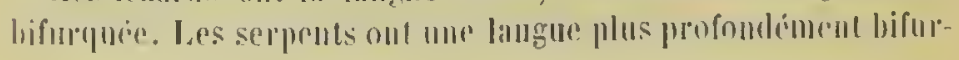




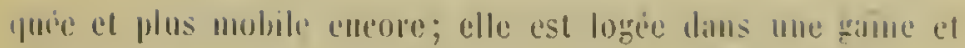

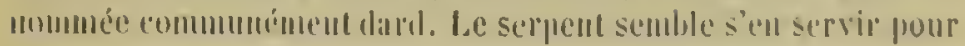
menliler.

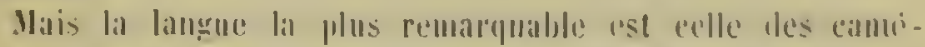

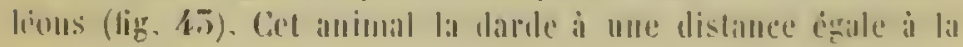

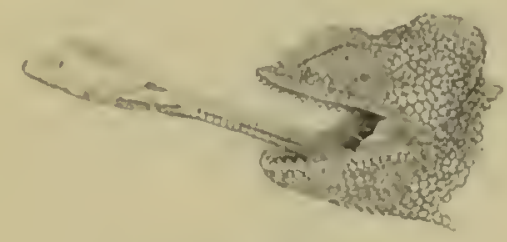

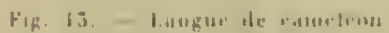

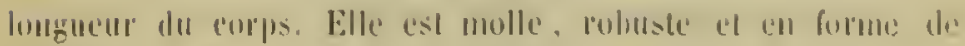
missille.

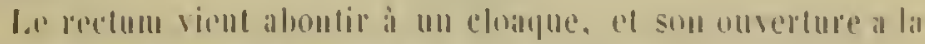
mêtur formor daus le's saturiens el les ser'mints.

Ou voil sousent des antis de gratise entre les mplis du propi-

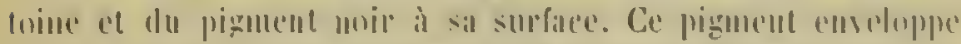

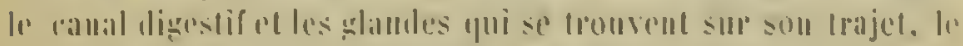

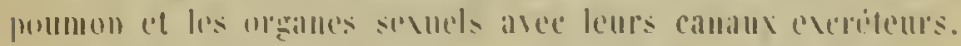

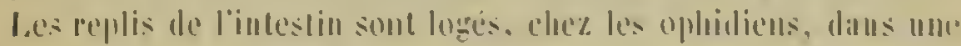

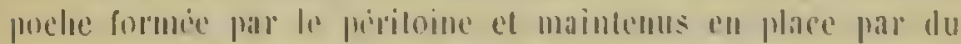

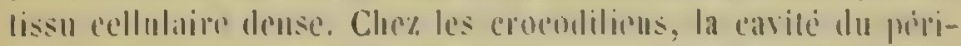

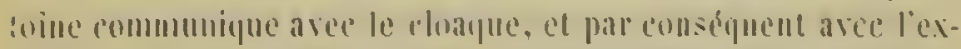

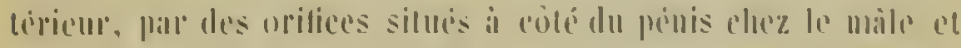

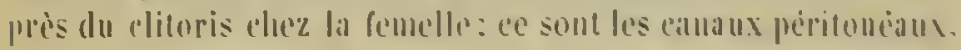

\section{BATRACIENS}

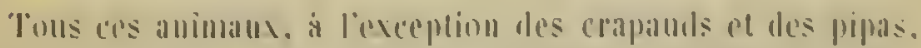
mot dis dents an palais: elles sont eourles de selries les unes

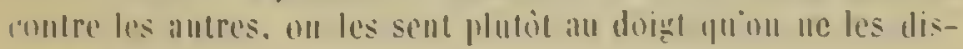
lingue il livil.

la langue des grenonilles est lixic a l'are du mentun. sal 
pointe est dirigée en arricre et l'animal la renverse sens dessus dessous, hor's de la bouche, pour saisil sa proie. La langue Inimpue entierement dians certains batraciens.

L'estontac présente suuvent la mène forme que celni des chéloniens. Le cana! intestinal est court: la partic anterienre plus

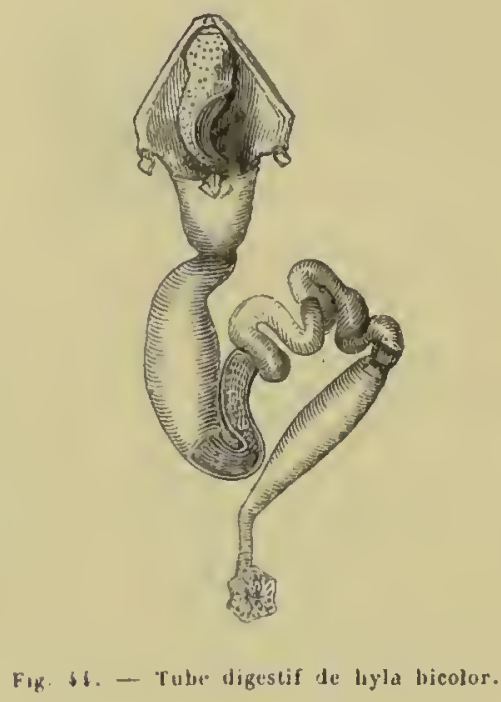

étroite, correspondant aux intestins grêles, fait deux ou trois coudes, tandis que la partie postérieure, qui représente le gros intestin, est très-courte. L'anus s'ouvre aussi dans le cloaque, dont l'ouverture est en $T$ dans les grenouilles et longitudinale dans les silamandres.

La grenouille à l'état de têtallost herbivore, et earnassière ou plutôt insectivore à l'àge atulte; aussi, dans le tètard, l'iulestiu est plus long que dans l'allimal adulte.

Quand le régrime elange avec l'àge, des modilications parcilles s'observent presine tonjours dans le tube digestif. 


\section{POISSONS.}

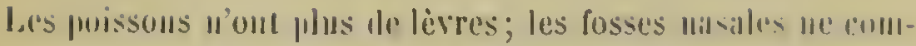

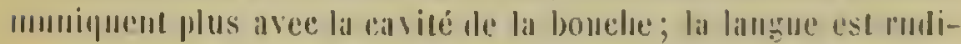

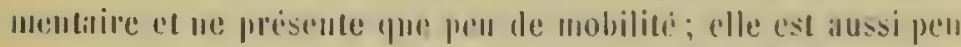
seusible, el on li trouse somsent couverte de dents.

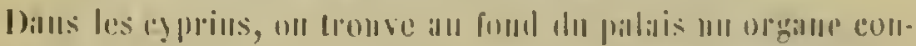

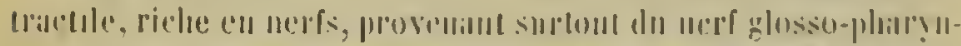

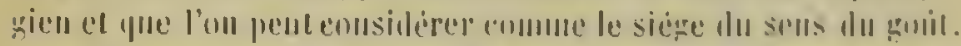

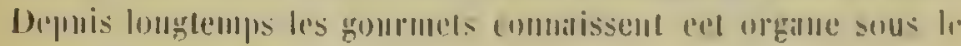
nom ale langhe ale earpere.

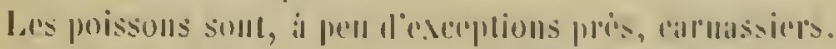

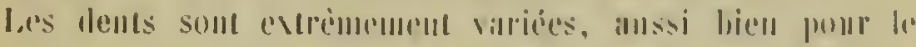

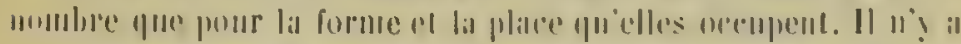

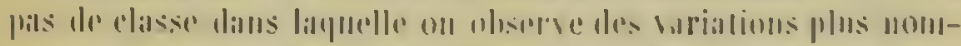

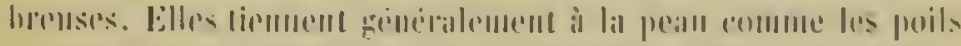

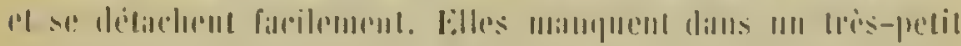

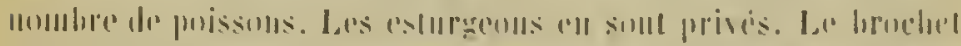

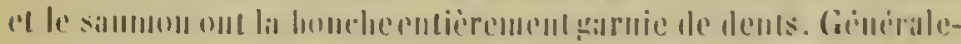

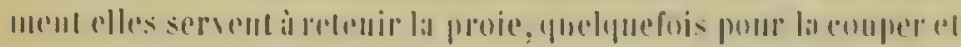

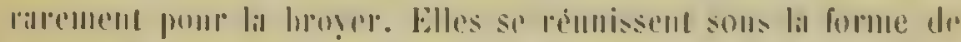

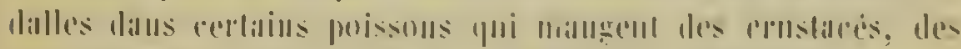

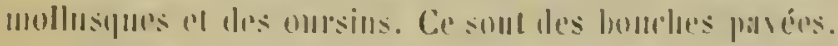

Dapres leur insertinn. on lis appelle maxilluires. inter-

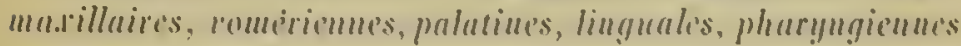
(1) linuchiul's.

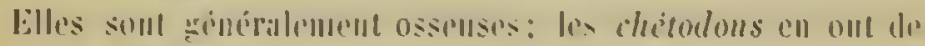

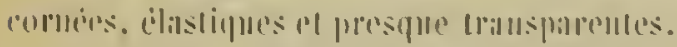

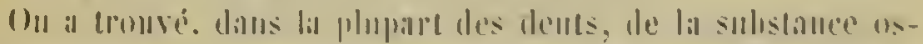

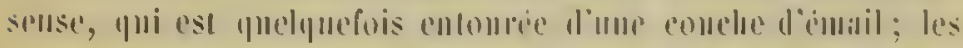

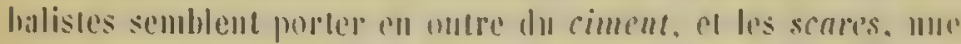

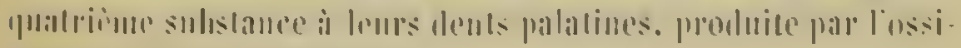

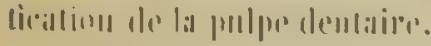

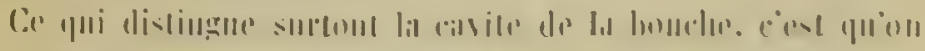
ilperente

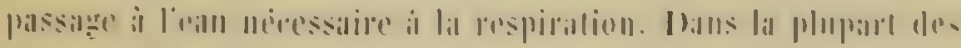


missons (esseux) on dérouvre de claque côté de la cavite luecale puatre fentes branchiales, et une lente extirieure, par oi l'eau écliappe. Dans les classes précédentes, l'aliment seul entro fiar la bouche, tandis que dans les poissons cette ouverture live passage it la fois et aux aliments et ì l'eau.

L'asophage eonsiste dans un canal fort cour', mais très-large,

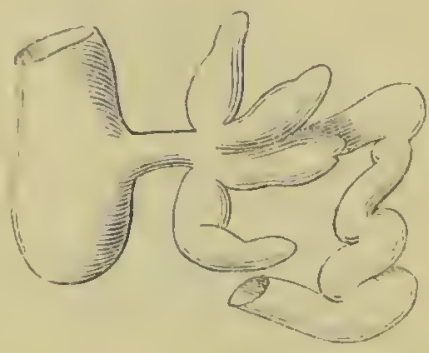

Fig. \$3. - Lstonac et recuns pyloriques de cantharus.

et il n'existe pas de ligye de démarcation nette entre ret oryann et l'estonac (fig. 4ij).

l'estonac est toujours membraneux, et montre sonvent un rul-de-sac très-développe; sa dimension est très-valriable.

Un repli pylorique séprire toujours la eavite de l'estomae de celle de l'intestin, de manièe que les aliments sont forcément alrrêtés pendant quelque temps. Danss la plupart des poissons, "un trouve au eommeneement de l'intestin des execums plus ou moins volumineux, d'autant plus petits qu'ils sont plus nombreux: on les appelle coeums pyloripnes (fig. $4: 3$ et $4(\mathbf{i})$. Depuis (pu'on a observé ces orgines chez les poissons pourvus d'un paneréis: (rsturgeour), on ne les regarde plus comme glande pancriatipne. Ils jouent sinls doute le même ròle que les cacums qu se trouvent sur le trajet de l'intestin dins les classes précilentes.

L'intestin est court et il est rarement plus renfle vers sil tor-

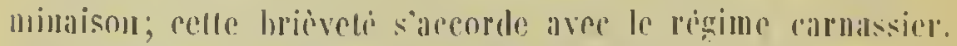

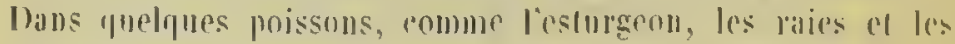




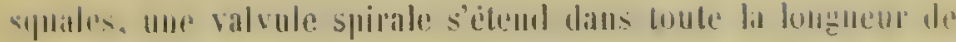

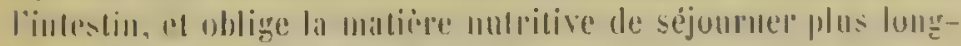
lemps dalls la cavité (tig. 4.7 ).

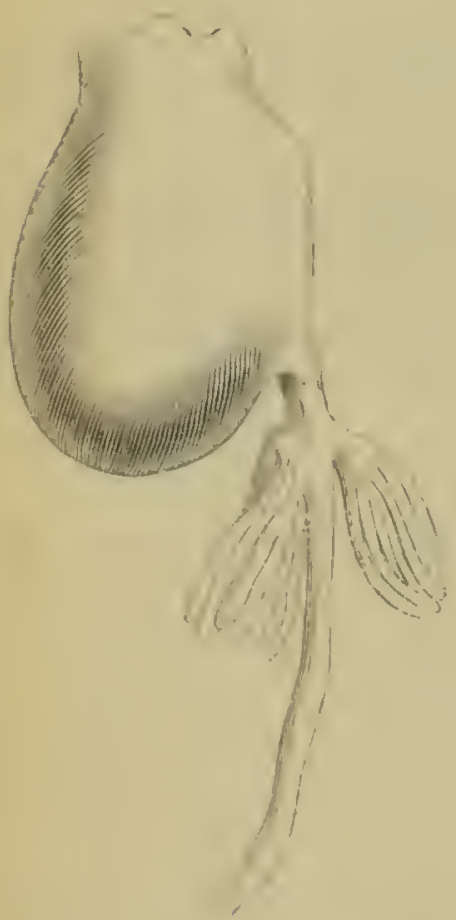

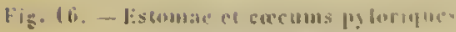
div fepturliguchus.

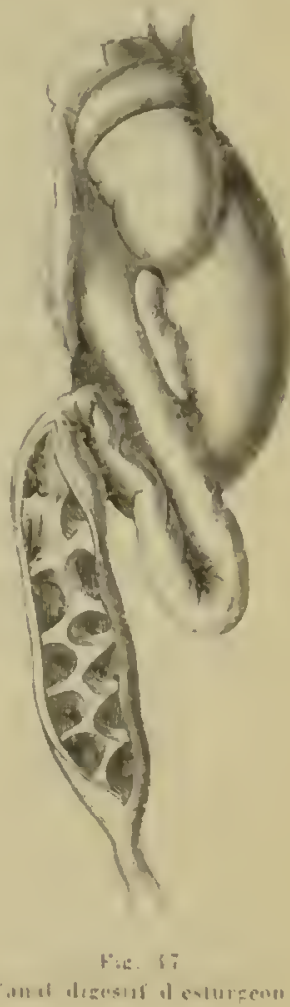

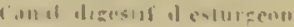

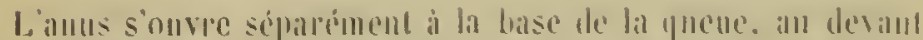

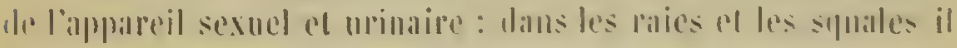

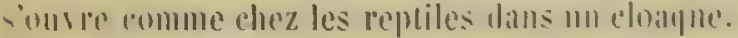

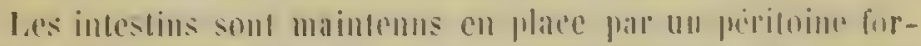
mant une membrame conlinue, of pueligurfois par de simules lorilles.

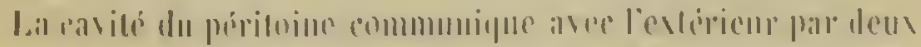

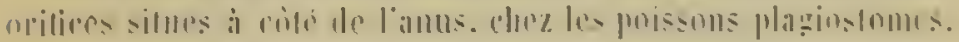


on par une seule ouverture, chez les branchiostomes. Dans ces derniers poissons, la cavité communique avec l'appareil respiratoire; dans les plagiostomes, elle communique avec la cavité lu péricarde. Il en résulte que l'eau du dehors peut baigner le caur de ces poissons.

\section{ANIMAUX ARTICULESS OU ÉPICOTYLEOONES.}

Dans les insectes, eomme daus lous les animaux articules, la bouclıe consiste dans une fente longitudinale, tandis qu'elle est transversale dans les ver tẻbrés, et au lieu de deux mâchoires qui agissent l'une de bas en liaut et l'antre de haut en bas, il y a au moins unc paire de mandibules el une paile de màchoires qui agissent l'une sur l'autre, de gaucle à droite el de droite à

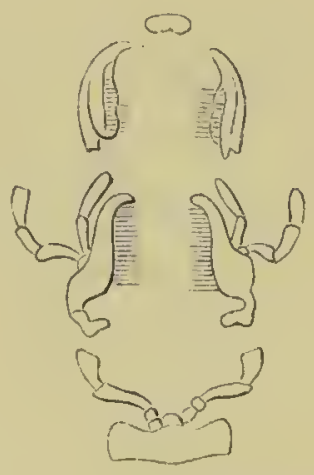

Fib. 48. -aices de Ja louche d'un caralu

gauche (lig. 48). Il y a souvent une livere en arant. at mir autre ch arriere, qui consistent, comme les antres pioces do lil houche, daus mue lame corne el mobile faisant partic du squelette. Entre ces denx lives on voit une pritire de mamdi-

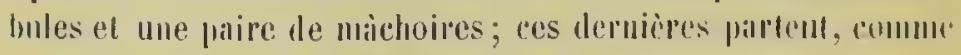
lil livere iuferienre, des palpes.

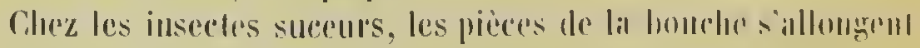

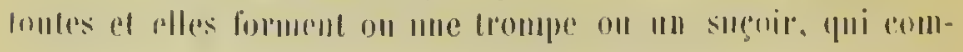


prend, malgré la diversité de sal forme, les mêmes pièces yue l'ou observe dims les hroyenrs. C'est ainsi que nous vọ̣ous, cliez tous les articnlés, le meme tyje varier presque a l'infini.

Les insectes ont le tulse digestif eumplet, commen du reste

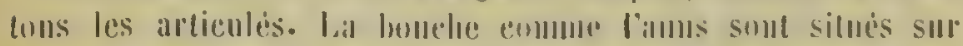

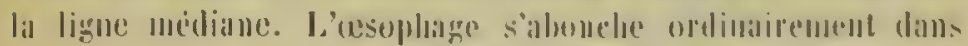

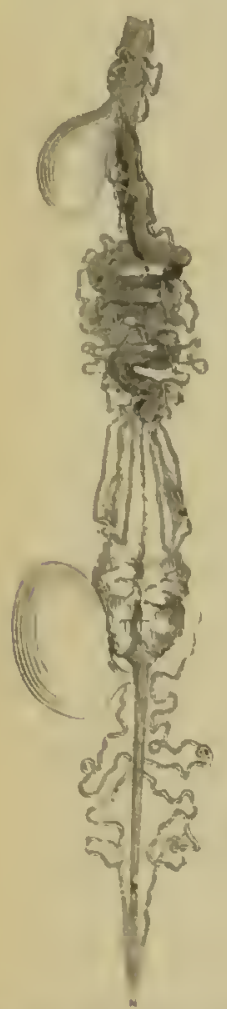

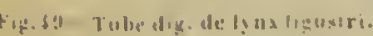

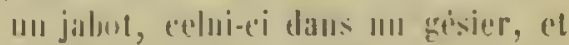

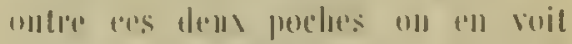
orlinilirement me troisieme. menume

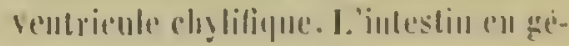

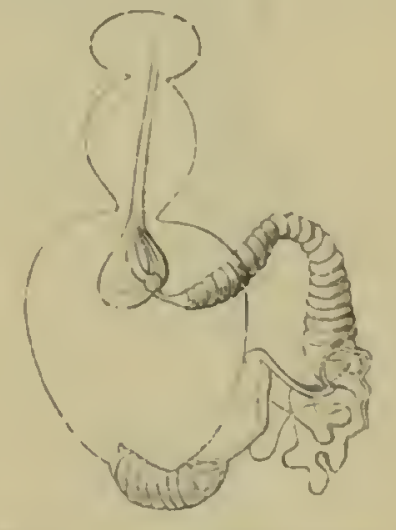

kig. so. - Tube thesuf at abealle.

méral est divisi end tem parlies eommo dans les animans vertebres: : la partic anliriemre represente lintestin grèle. la pilptir postirienre le gros intrim. La troisione fuchere ou le ventricule

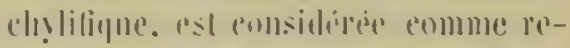

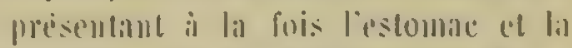

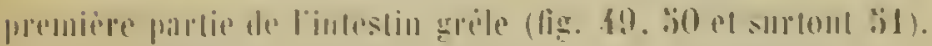

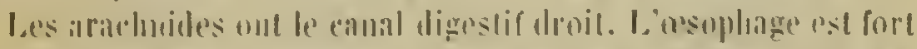

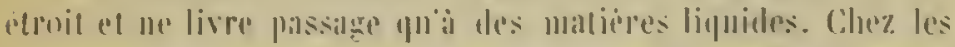

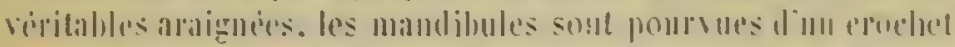

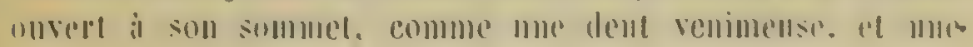


golande froduil de la mème maniere que chez les serpents du venin qui leur sert ì empoisonner instantanément les mouclıes ou les autres insectes qui viennent se jeter dans leurs filets.

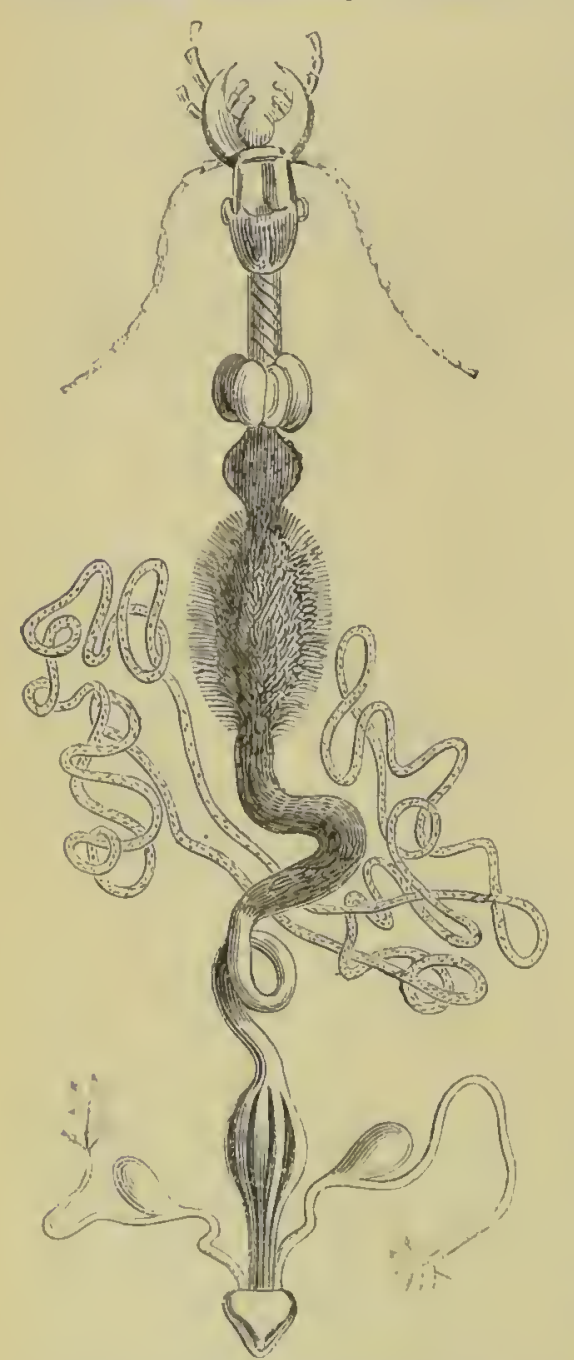

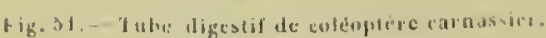

Cle'z quelques articulés, comme les cralses el les écrevisses, plusicurs paires de pièces viennent se joindre encore aux mandibules et aux mì-

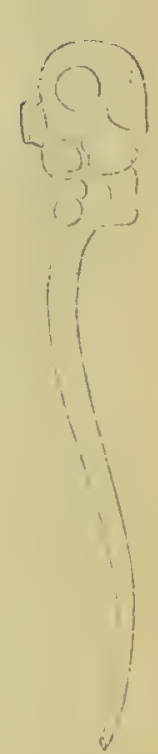

Fig 5z. Tule digublif d'écerisse.

choires; ce sont les piedsmilehoires.

Les crustilés, comme les erabes et les écrevisses, onl jusqu’i sil piares de pieres intont de la bonilic, IIII arsophate (rise-contr.

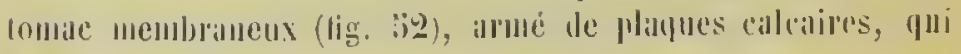




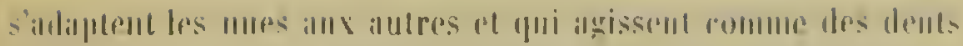

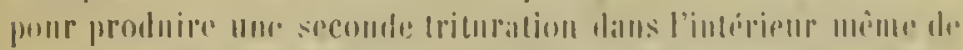

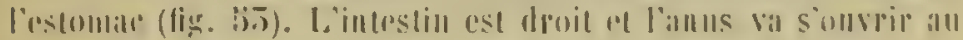
bunt de liabdumen.

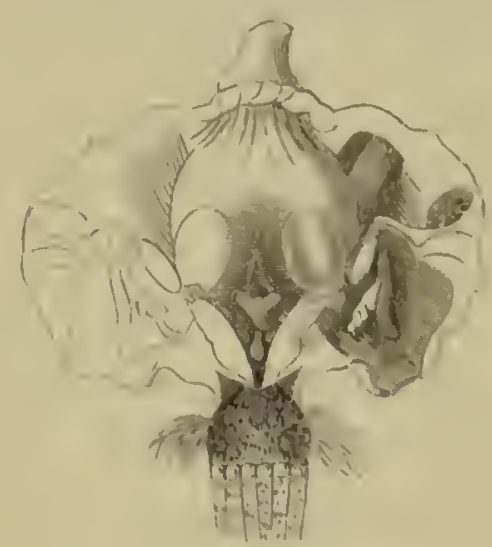

Fis. \$3 Exime de crabe, ouvert.

Dans les dromicrs erustaters, le tuhe digestif ne montre plus a

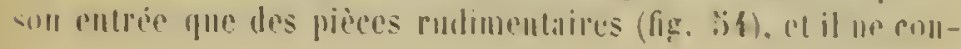

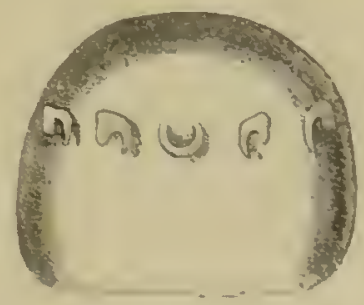

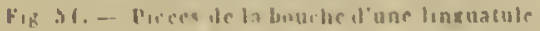

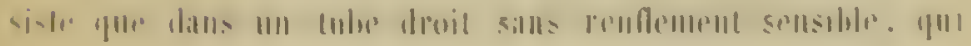


s'ouve ì la partie postérienre du eorps (hy. 5\%). Chez quel.

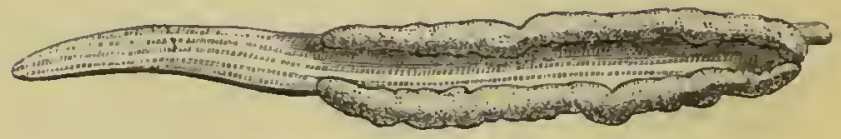

Fig. 55. - Tube digestif de linguatule.

ques-uns mime, ee sont les pattes qui, par leurs artieles basilaires, produisent la mastieation.

\section{ALLOCOTYLÉDONES.}

Le eanal digestif est eomplet ehez les mollusques, mais l'anus s'ouvre clıez quelqques-uns sur le còté du corps en abandommant. la ligne médiane.

Les cciphilopodes ont une bouehe cireulaire au milieu des bras

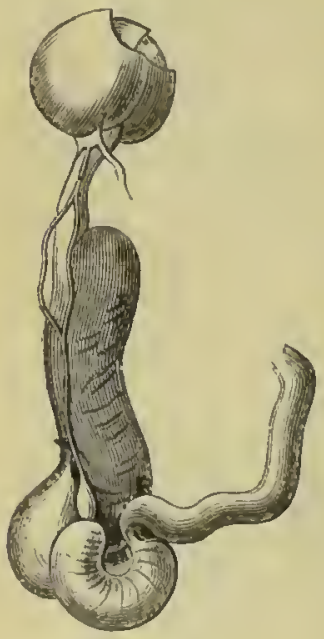

Fig. 86.

Tulue algestif d'argonaute. qui garnissent la tète. Ils portent un bulbe buceal, armé de deux forles mandilutes eornies en forme de bec: de perroquel, el d'une lame cornée qui recouvre le planeher. L'osophage, après avoir traversé le collier nerveux, se dilate et forme un jabol qui s'ibouehe dans un véritable gésier. A còté de ee gésier on apereoit un exeun spiral, el l'intestin, sans former de eireonvolutions, revient en avant pour aller s'ouvrir all-dessous de la tête. C'est un eanal digestif repliẻ nu milicu sur lui-nième (fig. \$56).

Dinns les gastéropodes, le bulbe bueeal est aruné, outre la lame cornée qui reconvre le plancher, de quelques pièces cornées implanties dans les parois et qui agissent comme des mandilutes (fig. :37-\$8). Il n'y 


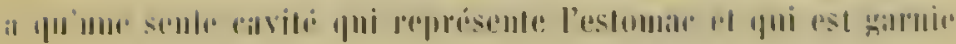

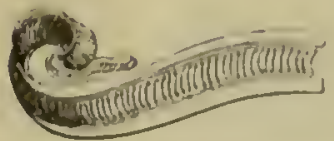

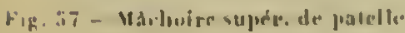

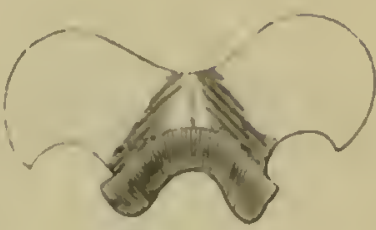

Yix. 58

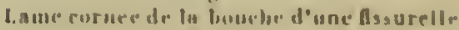

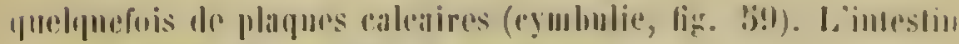
est long daus les limaers, il forme plu-

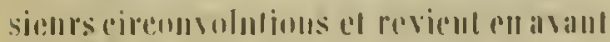
somrir à culi de lomserture pulmomaire, sul le low rel do bomelier.

Clin plusieurs gistíropotes, les ril-

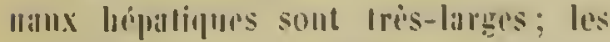
aliments pentent pimitrer dans lemr intéricur, sans que res paunax doivent itre cousieliris emunte analogues ì cems qui s'onverent daus la cilvited de l'estomac des acialephes.

Dans les acriphales, les moules pall

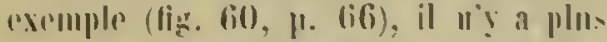
ancone piece soliele dams la bouche: liestomite consiste dias une calvite erensie an milien mene lu foic, et l'intes. lil, apris avoir formé uguelques anses.

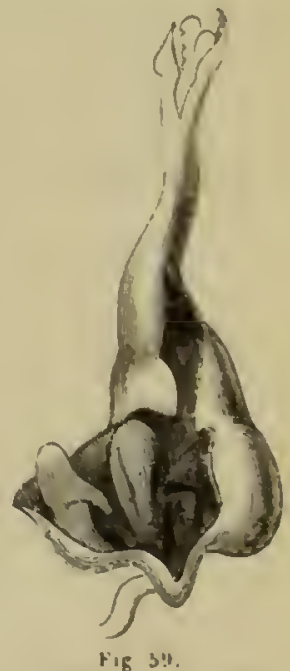

Tuler digestif de evouhulir se dirige ell arricre, passe intellessous III reur, el souvre à la partie du corps qui est opposée à la bouclie.

Lets tmuiciers (fig. 6l, p. (6i) el les bryozoaires out encore un canal intestimal complet, un estumac distimet, mais le mouventent des aliments est déterminé, dans les derniers surtout: par la pressence de eils vibratiles qui garuissent l'intérieur dı 
lube digestif. C'est par leur actiont que l'on voit les allincente

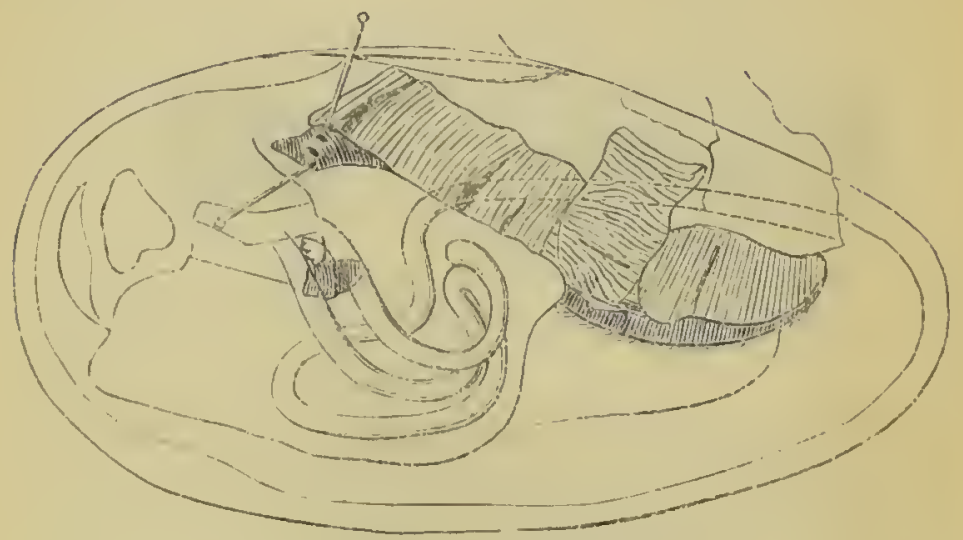

Fig. 60, - Tulne digestif d'anudunte.

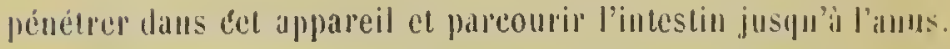

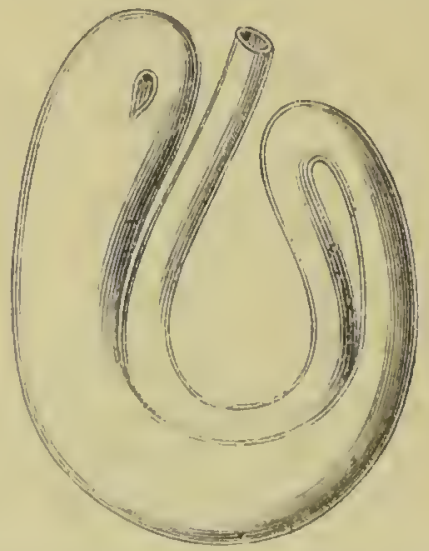

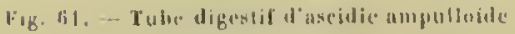

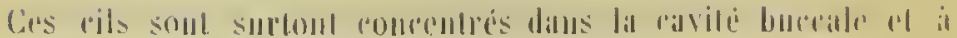
roriticer pylorinur. 


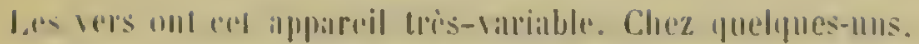

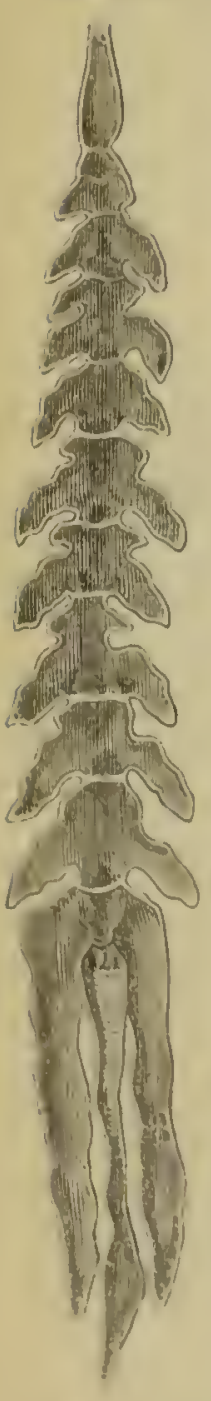

Pig $t$ ?

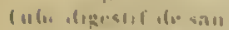

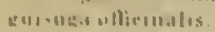

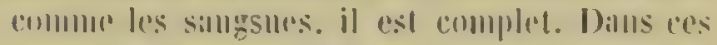

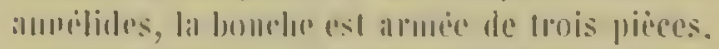

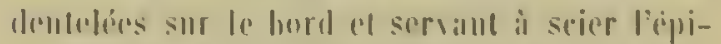

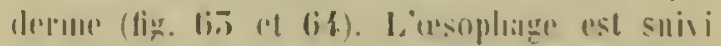

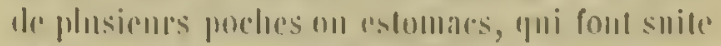

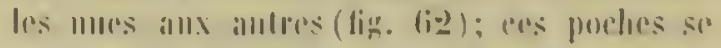

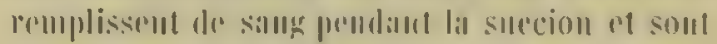

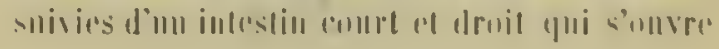

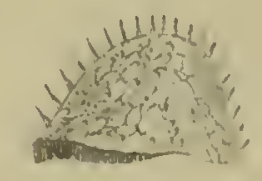

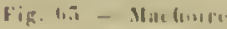

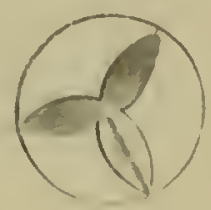

rip. fis.

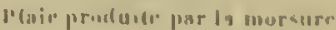

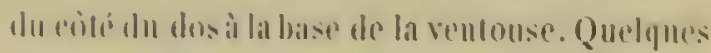

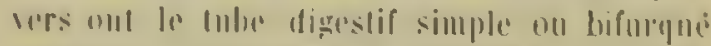

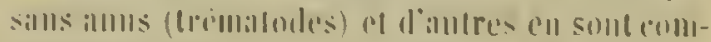

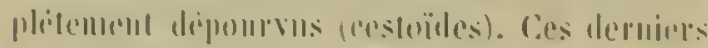

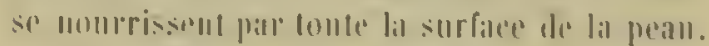

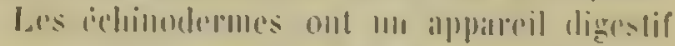

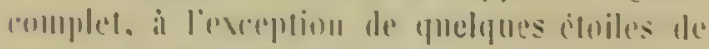

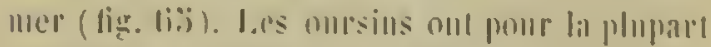

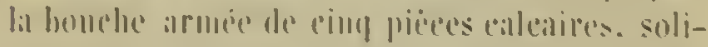

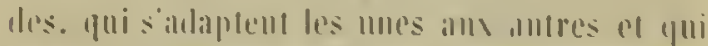

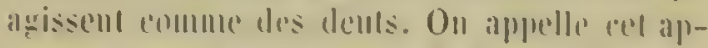

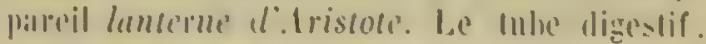

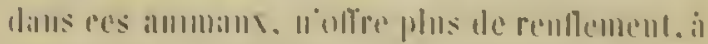

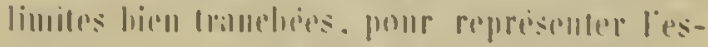

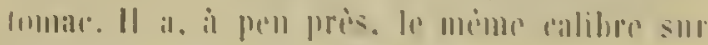

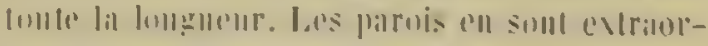

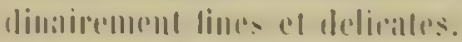

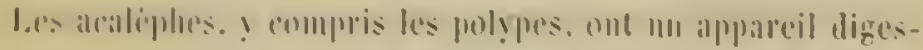


tif qui se sumplitic beancoup; il u’a plus de parois propres, el

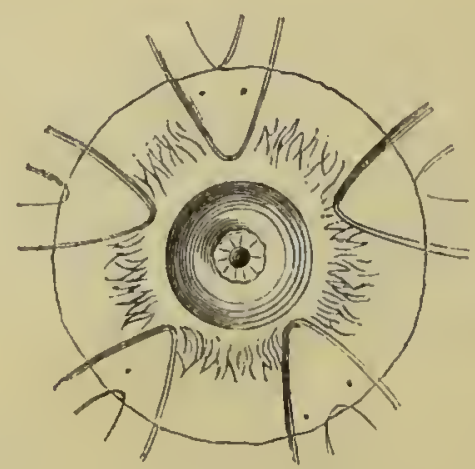

Fig. 65. - liunche d'asteric.

il consiste dans une cavité fereusée an milien du corps. L'eau clargée des aliments pé-

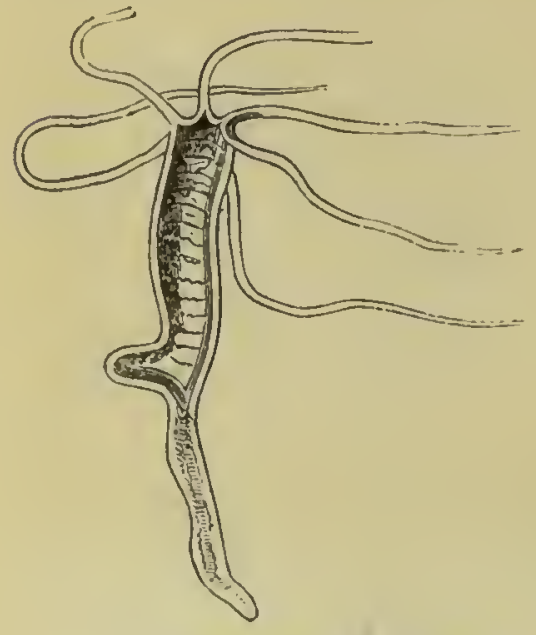

18. 60. - Carite digestive de l"hy dre. nètre par une on plusieurs ouvertures dalls celte grande cavilé.

Jes hydres (fig. 66) sunt pourvues d'une dépression au milien du corjus, dans laquelle viemuent se loger les aliments pendint quelque temps. Des bras crenx entonrent la bonche et communiquentarecedte cavile. C'est tout l'apparcil digestif, il n'a pas de parois propres. Lal mène noverture sert da bonche et d'anus. Anssi, pent-on retomruer l'animal comme un

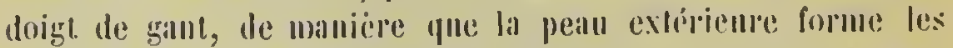
parois digestives, el l'animal contime a vive comme si rien 


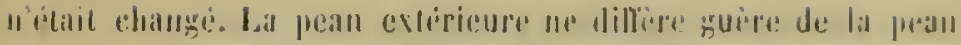

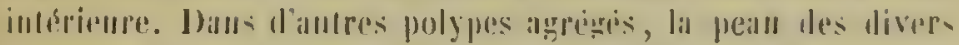

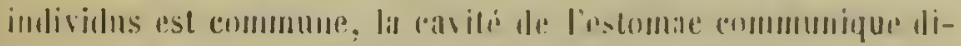

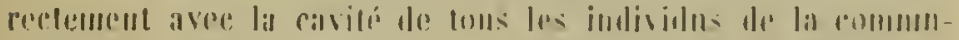

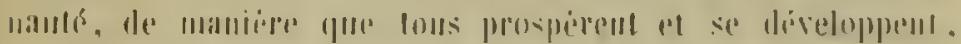

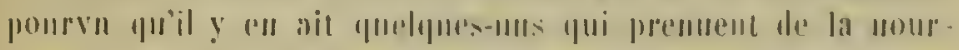

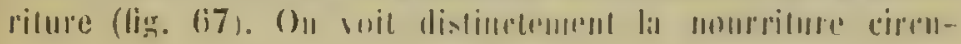

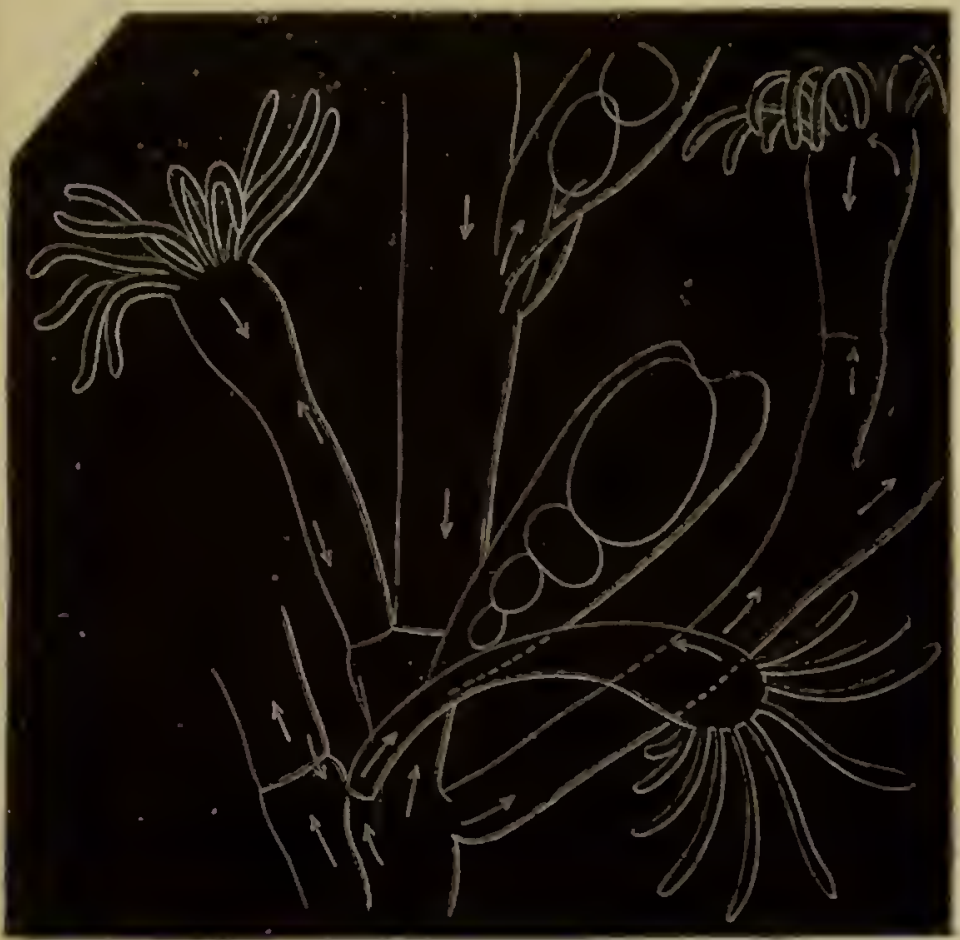

Fig. 67. - Couldé communde thua halecina.

ler el passer de l'estomate de l'un daus eelni de l'autre. C'est

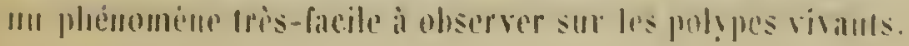


Les foraminiferes n'ont pas de canal digeslif. La pean peut former unc excavation qui sert à loger les aliments.

Clsez les infusoires proprement dits, la inatière alimentaire penètre bien dans l'intérieur de l'animal, mais le tube digestif u'a pas de parois propres, et les cavités qui logent les aliments ne sont que des vacuoles formées dans l'épaisseur des tissus.

Les infusoires proprement dits n'ont jamais de tube digestif complel, comme on l'avail supposé; il y ell a qui n'oul pas même de bouche; d'autres ont une bouclse et un oesophage, mais les aliments pémètrent eusuite dans le lâche parenchyme presique fluide qui remplit l'iutríreur da corps. Les fèces som ivacuées che\% quelpues-mus par un amms, situe ordinairement Ju côté opposé ì la bouche.

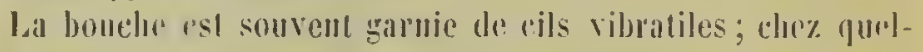
yues-uns il y a une sorte diappareil de mastication. 


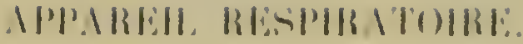

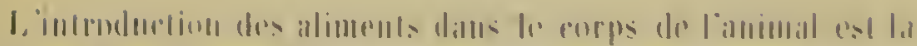

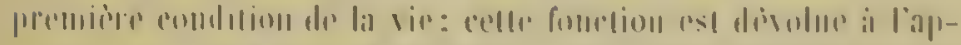

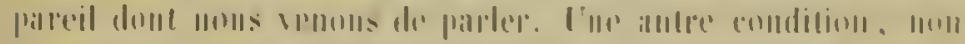

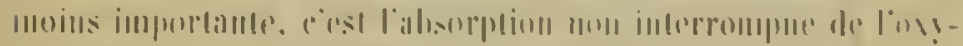

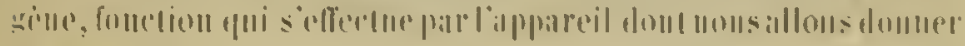

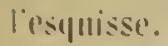

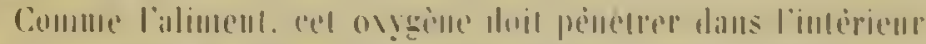

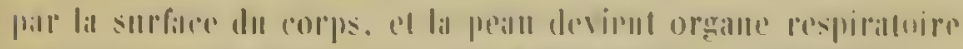

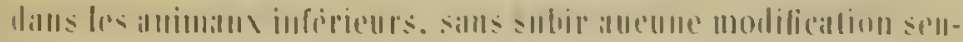
-il)lr.

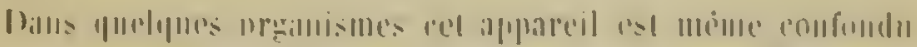

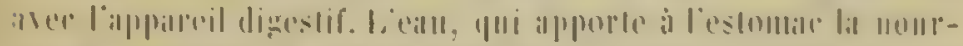

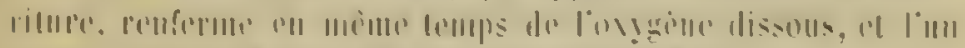

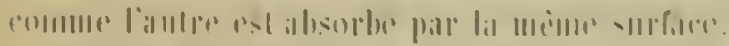

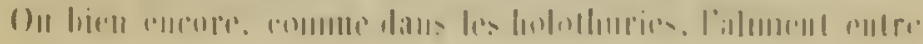




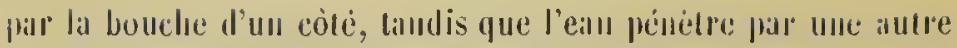

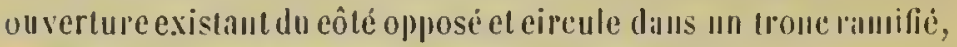
appelé arbre respiratoire ou appareil aquifére.

Dans des organismes plus élerés, la peilu, pour angmenter sil surface, montre des replis en forme de dents de peignr, ou bien des appendices sous la forme de tenticules qui fonlent dins l'eau el ahsorbent l'élément girzeus. Ce sont les branchies. On les trouve sous des aspects divers dius les animaus aljualiyues.

Eufiu dans des nrginnismes plus ilevis encore, elıe\% les anli-

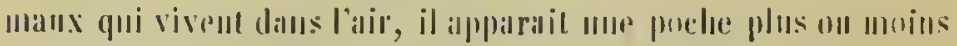
grande qui loge l'air, eomme i'estomac loge la nompriture, at yu'un désigne sous le num de pommon.

Le poumon, dills sil forme la plus simple. se prisente smus

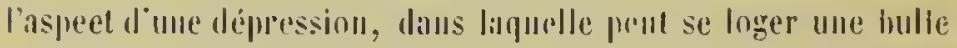

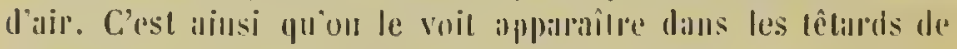

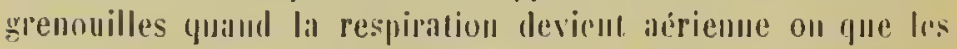
branchies diminuent. Cetle dipressinn an fond de la eaviti de lis bouclue atigmente insemsinlenent, at on roil enfin une pordue remplie d'air. C'est sous relte forme simple que le ponmon se montre quelquefois pendant tonte la vic de l'muimal.

La senle modificilion que subisse ensulte cente poche pulm.naire consiste dialls des replis (pui alphilrissent en dediuns die:

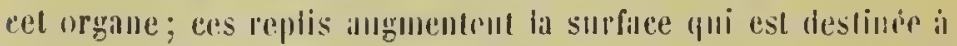

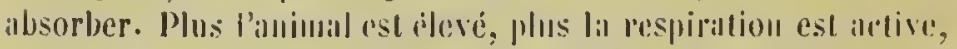
al plus lil surface yni doil ilisorluer est gymule. Aussi ces simples replis ue formant d'abord que de simpres brides. s'ibendent-its

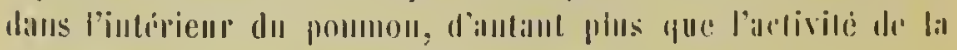
respiration est plus gralude, ch its se mutipritent a tel point alans

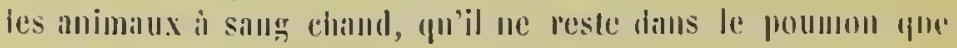

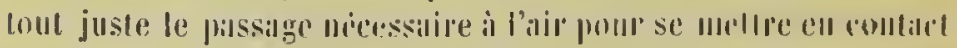

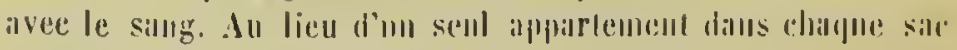

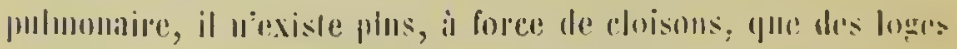

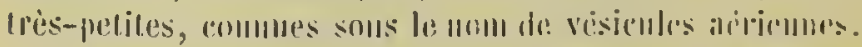

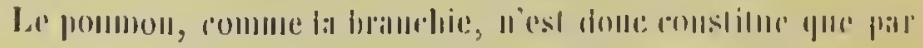

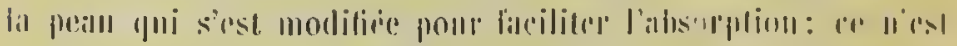
qu 
considerablement riduits, laissent à peine des traces de lenr existence, tandis que la couche vaseulaire a pris un grand développenent. C'est pour te motif que le pounou semble formé exclusivement d'un risean vascolaire.

C'est eneore ici le lien de dire ponrunoi il y a de l'analogie entre le pounon el les glandes. Dans les deux as, il s'agnit d'augutenter la surface pour aceroitre l'ativite de lorgane. Phus la surface est grande, ell effel, soil pour exhaler, snit pour al)sorber, plus ces fouctions d'exhalation ou d'alsorption servolt actives.

Le poumon reçnit l'air par un lube, appele bronche, et les bronches se remuissell ell un lube mique disigne sons le noul de trichée-irlère (fig. 68). Ce tube se modifie ensuite au laut on a la base: les anueaux cartilagineux, qui écurtent les parois el les emperdent de s’aftaisser sur elles-mimes pour lle pas ferner ainsi le passage ì l'air, se moditient dans leur forme; ils se séparent en plusieurs pieces portant des noms partieuliers, et leur ensemble est désigné sous le d’organe de la voix.

Liaborption de loxigene devant se faire par le sang, on trouve, outre les raisseaux orlinaires des poumons, el qui sont destinés à entretenir la vie le cet organe, des vaisseanx parliculiers qui sépanouisseul dans son pipalissur : de ceux-ci uaissent d’antres vaissonu qui ramenent le sang vers le caur après yu'il a subi laetion vivitiante de loovgrine. Coest l'artire el la veine pulmonaires, tandis que les vaissenux ordinaires sonl dpi- 
signes sous te nomi mpropre d'artère el de veine bronchiques, on de vaisseaux nourliciers du pommon.

L'oxygène ne vient donc en contact avec le sang qu'à travers les parois des vaisseaux; me vessie remplie de sallg noir devient ronge à l'aip par l'action de l'oxygène qui traverse les parois. C'est le même phénomène ici ; l'oxygène agit à travers les parois des conduits qui renferment le sang.

La respiration a donc lieu : $1^{\circ}$ par la surface du corps oll par les parois qui renferment les aliments et sans que rette surface ou ces parois subissent ancune modification apparente.

2 La pea s'étend, fornic un lilament, une lamelle, moe louppe, un feuillet baigné par l'eau, et il existe une branchie.

$\bar{\jmath}^{\circ}$ La surface externe ne remplissant pas bien toutes les ronditions, l'eau va se rendie dans des canaux propres, ramiliés au milieu du corps, et il existe mue artère respiratoire on mu système aquifère; au lieu d'ean, l'air peut circuler dans de pareils canaux qui forment alors les trachées.

40 L’air pémètre dans une poche unique on double, tapissée par un riclie lacis vasculaire, que le sang parcourt pour se renlle à la rencoutre de l'oxygene: c'est le poumon.

Ce sont les diverses formes sous lesquelles l'appareil respiraluire se présente dans la série animale.

\section{MAMMIFËRES.}

Cet appareil consiste, chez tous les manmifères, en deux ponmons; ils sont logés daus l'intérieur de la cavité thorachifure el toujours mettement séparés de la cavité abolominale palr la prósence du diapluragme; eelui-ci ne manque jamais dans les animaux de cette classe.

Clacun de ces poumons est entoure, comme cliez I'lonmme, l'une plevre; ils flottent librement dans la cavité thoraciane el n'ont d'autre adhérence que par le pédienle, formé par les honwes et pardes vaisseanx. Le poumou du côtr droit est en général plus grand que celui du coité gaturhe, et il rest divise aussi eu un plus grand nombre de lobes. Qurlques prommons onl jusqu'à

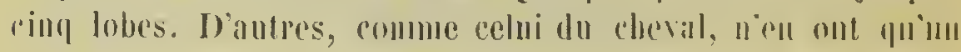




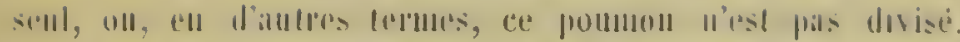

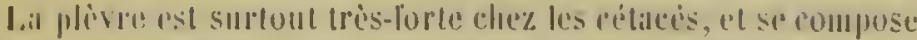

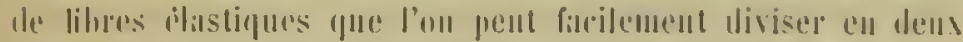

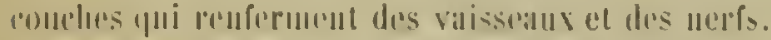

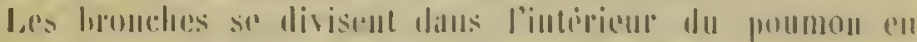

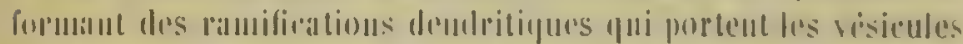

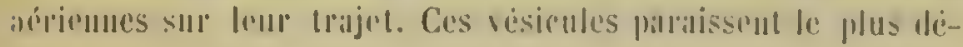

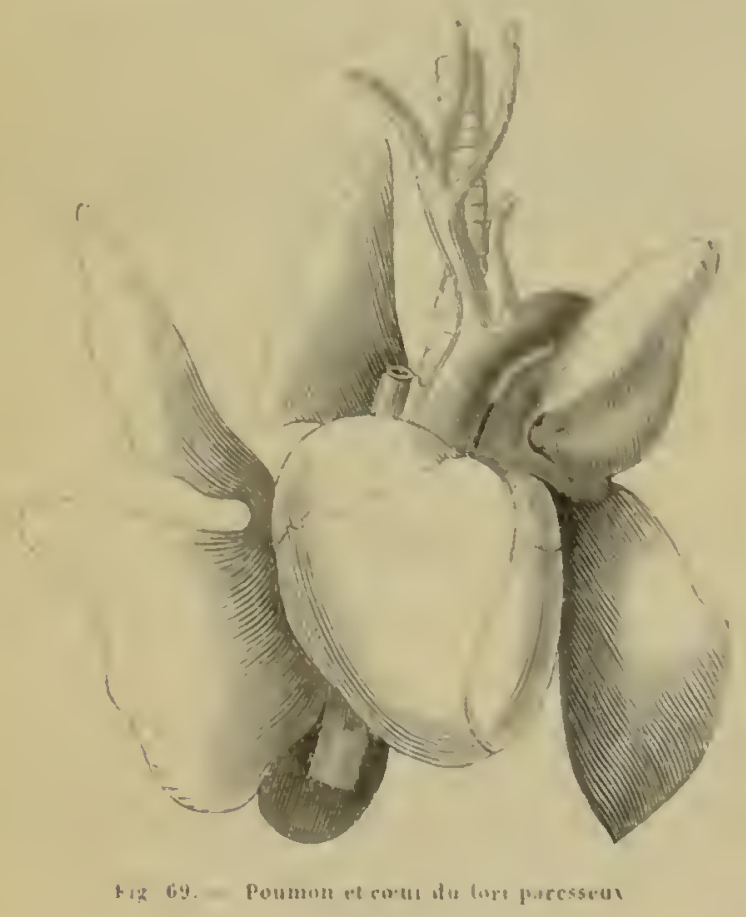

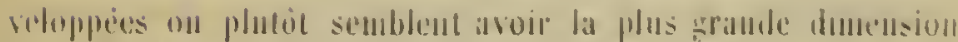

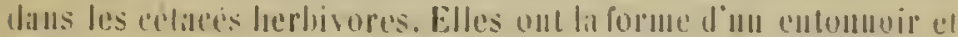
montrent sontent des doisons diats leur intritent.

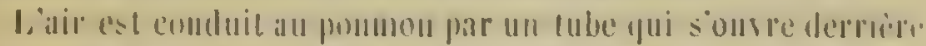

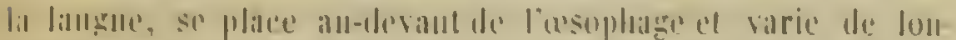

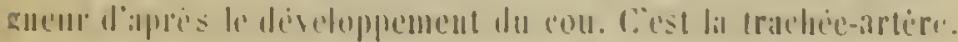


Dans la poitrine, cette trachée se bilur(que et les deux tubes, qui ronduisent directement l'air daus le poumon, sont nommes lornches. La trachée-artère est composie l'une série d'allneaux eartilagineux qui se développent dans l'èpaisseur des parois el qui empêtehent ce tube de s'affiasser sur lui-même pendant l'expiration.

Le nombre de ces anneaux est très-variable; le chameau et la girafe en ont jusqu'ì cent dix et an delà, tandis que les célacés n'en ont que de sept à douze.

Ces anneaux sont rarement complets de manière à entourer complétement le tube, et lil partie postérienre reste membrineuse.

Dans les bradypes, la traclıée descend jusq̨u'au diaplıragme, revient sur elle-même et alors seulement elle donne naissance aux brouches.

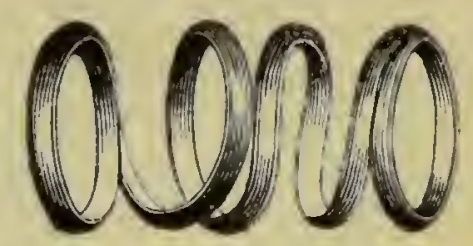

rig i0. - Cartitige bronehique dw dugong.

Dans le pedeles caffer, la trachée est divisée en deux dans une grande partic de son trajet.

Les cartilages des bronches du dugong soul en spirale (fig. 70).

OISEAUX.

Cet appareil subit diverses modifications importantes dans les oiseanx (fig. 71, 1. 77). Les poumons ne sont pluslibreset flottants dans la cavité de la poitrine, mais attacliés aux côtes et à la colonne vertébrale dans toute leur longueur. Le poumon étant l'organe le plus léger, le centre de gravité se déplacerait constamment pendant le vol, si cet organe n'était pas solidentent 


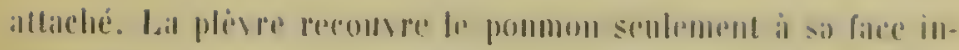
firinure.

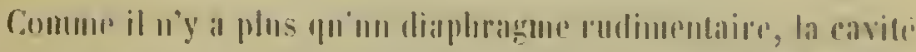

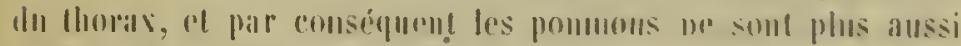

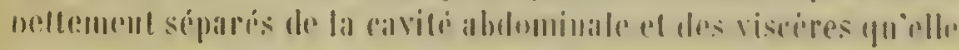

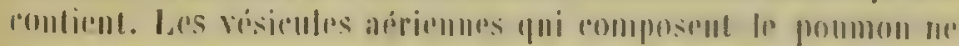

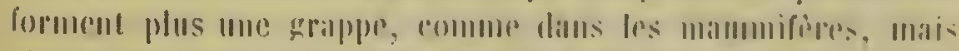

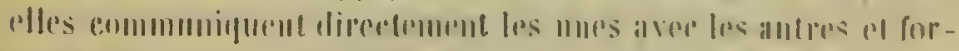

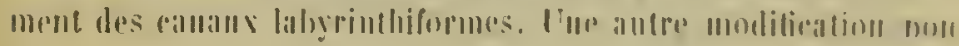

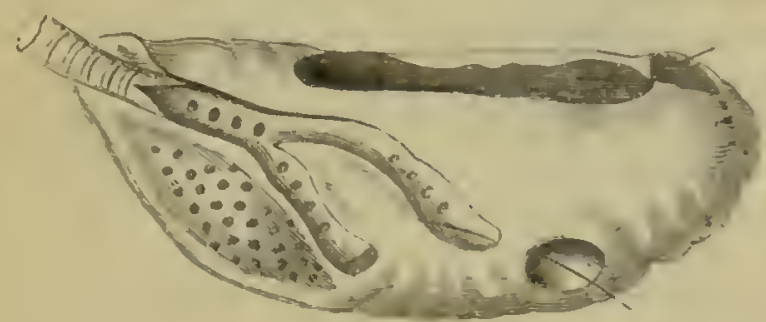

Fug it. Ipparal respirsuluire d) a nistams.

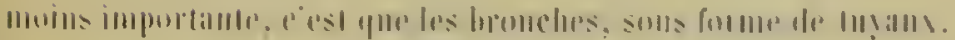

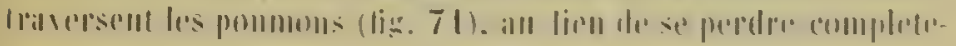

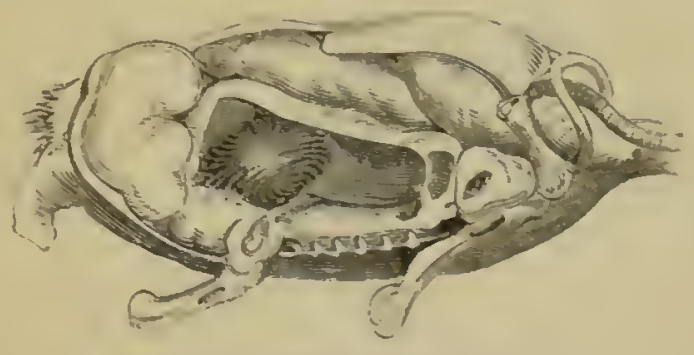

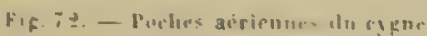

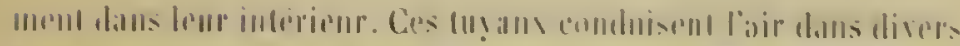

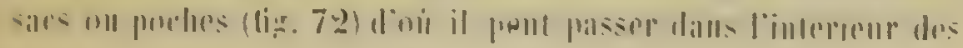


os. I l'exception de l'aptiryx, tons les oiseanx sont pourvus de ces proches aripunes; elles sont généralement inl nombre de einc] de elarque cote. Tout le corps de l'oisean se gonfle lor'syu'on insullhe de l'air par la trachée, el par la mểme raison, on voil l'air yu'on insufle cechapper de l'os de la euisse ou dulras fracturé si l'oiseau est mainteun sous l'eau. Ces sacs ariricus s'étendent chey. le fou (sula alba) tout autour alu corps entre les muscles et la peau, el eelle-ci n'est adlierente que par des filaments irrigulicrement disposes comme des fiusses membranes. Une énorme porlor recouve les muscles pectoraux daus ce mêne oiscalı, et útablit muc antistomose aćrienue entre les poches thorachiques et abdo-

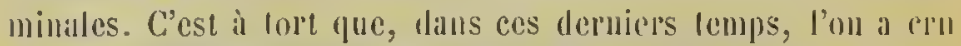
que daus les oiseaux l'air ne se rendiat jamais au nilieu du tissu crllulaire qui unit les muscles. Cuvier avait reeounu une dispusition semblable dans le parra cleuraria, ef recenment nous avous eu l'occasion de dissiquer un marabout, dans lequel nous avons vu se reproduire le même phénoméne. La poedıe, sous forme de saucisson, que cet oiseau porte à la hase du eou, se peuplit d'ail' par l'internide de res pocles aériemes sons-culinées. Lair rircule ainsi dims l'intérieur du corps des oiseaus. et on peut comparer les saes aériens à des souflets qui vienuent acciver la respiration daus les poumous.

la trachéc-artire est très-longue; ses ammeaux sont complerts

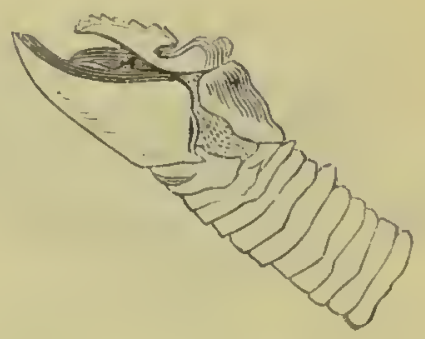

Hig. 73 - Largnì superietur du cygute.

"l ordinamenent osseux, Le nombre de ces anmeanx s'eleve jus-

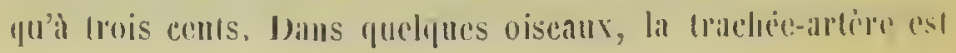




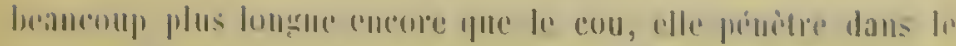

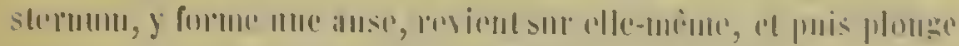

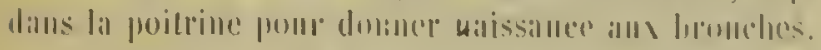

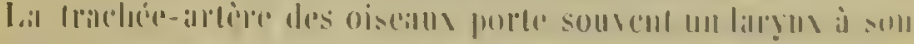

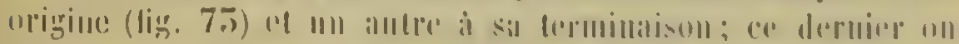

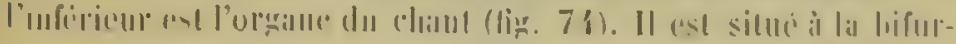

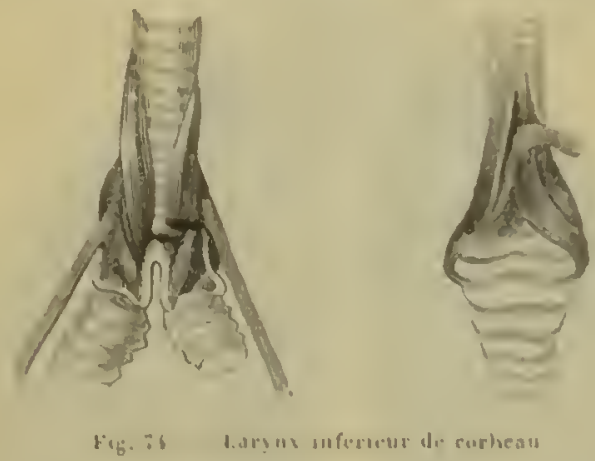

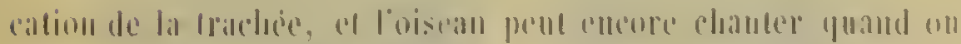

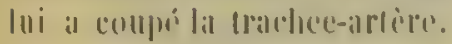

\section{REPTILES.}

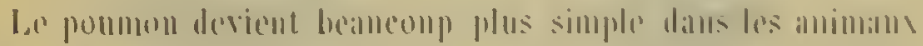

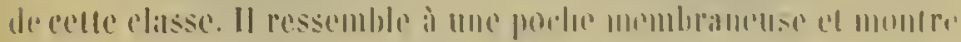

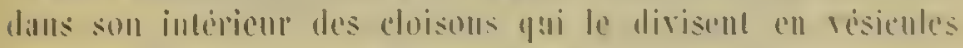

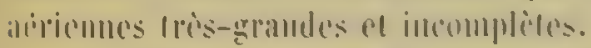

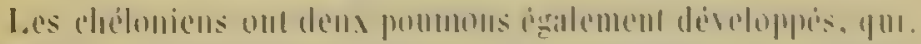

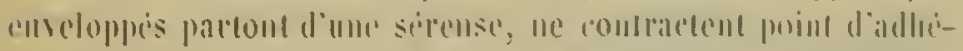

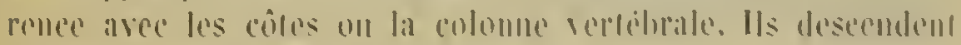
treis-bals dans la cavite abdominale.

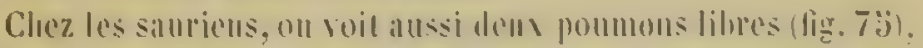

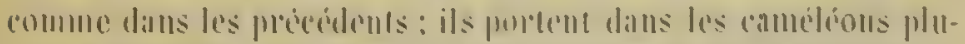

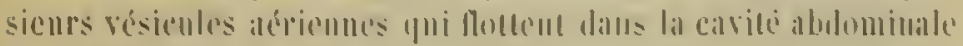

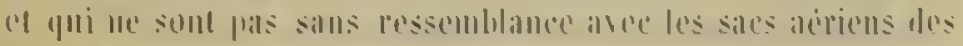
oistillis. 
Chezles serpents il l'ige adulte, on me trouve en géméal qu un seul poumon; it est fort long, étroit, libre el s'étend tres-toin dans la cavite absominale. La bronche continue dans le poumon

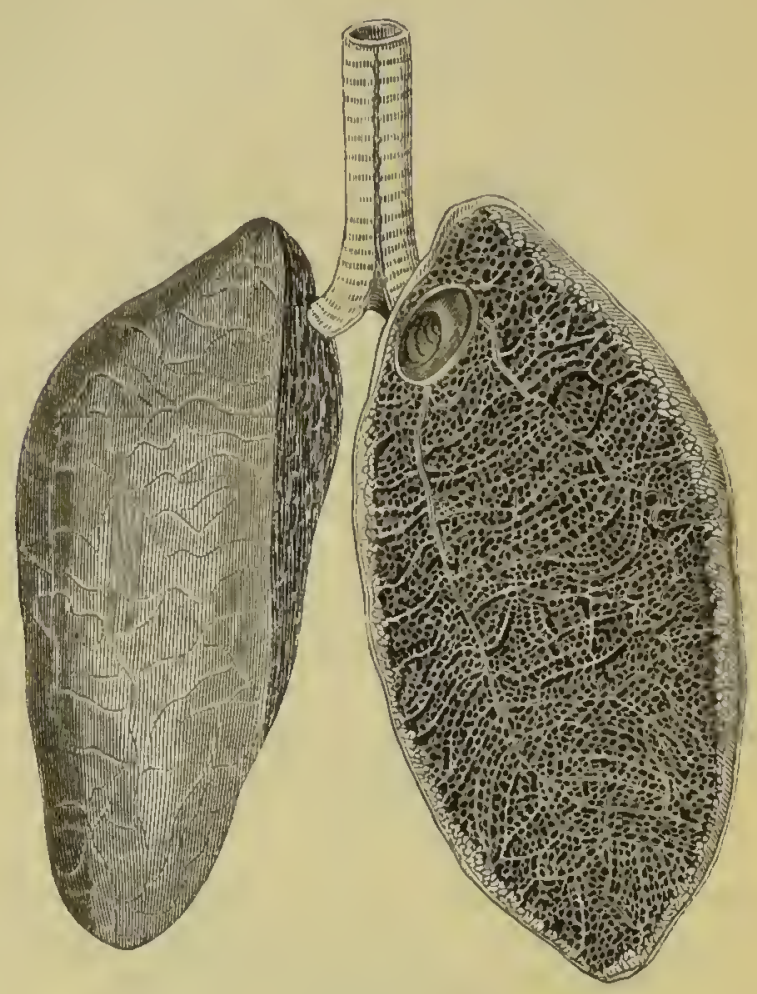

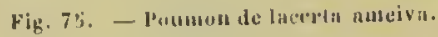

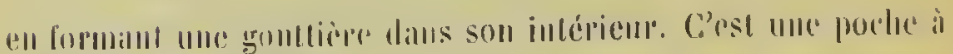
parois tris-illeompliels.

\section{BATRACIENS.}

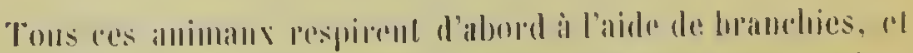

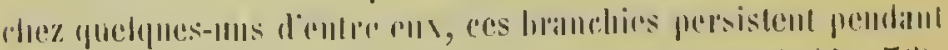

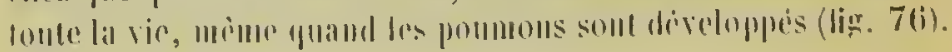




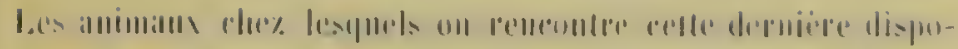

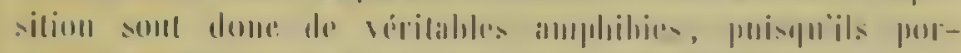

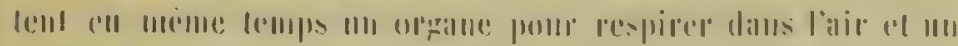

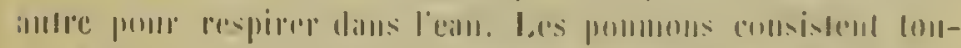

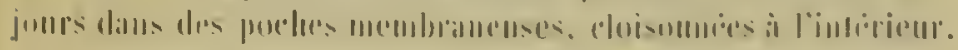

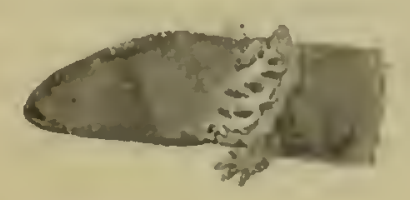

4 His. iti. Aranchios ale surene

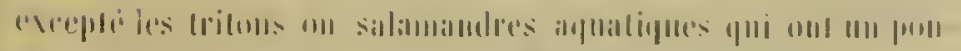

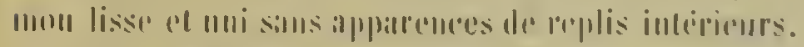

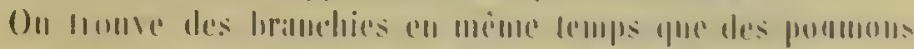

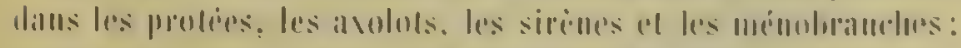
Alles somt

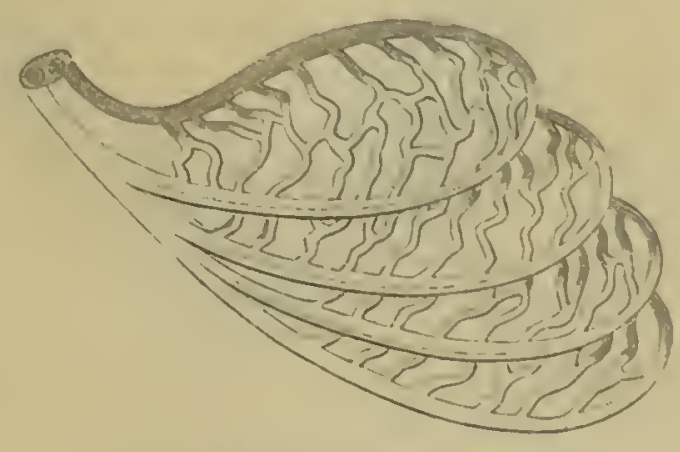

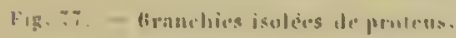

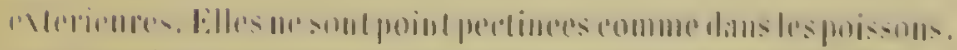

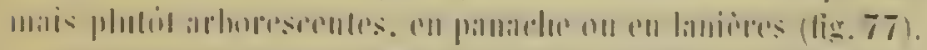

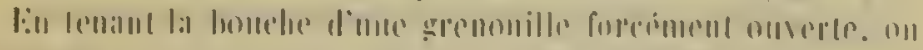

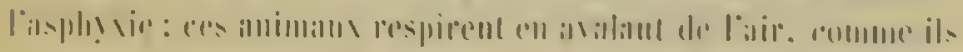


avalent des alliments, et en écartant les mâchoires on les cupêclie d'avaler l'air.

\section{POISSONS.}

Les poissons ont des marimes, mis qui ne conmminiquent plus avec la eavité de la bouche. Ces oryalles sont terminés en culde-sac chez les animaux de cette classe; e'est parl la bouche que pénètre l'ean qui doit céder son oxygène pour l'alcomplissement ale l'actc respiratoire. Cefte modilication est nécessitéc par leur

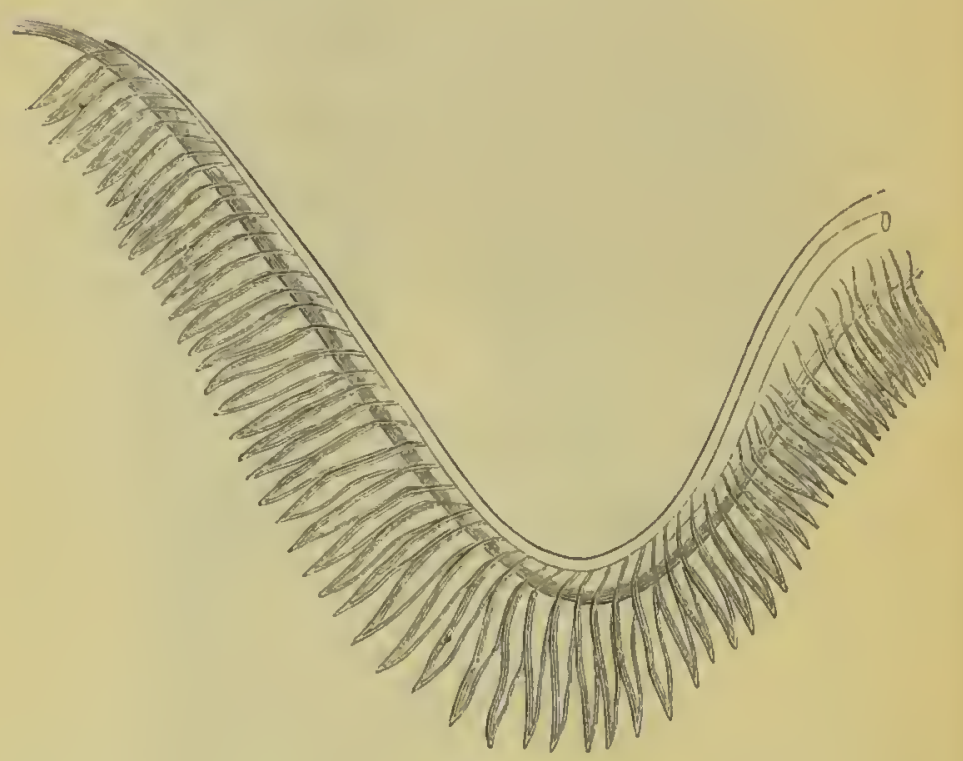

Fig. 78. - Are branchial de perehe.

genre de vie aquatique. Les branchies sout platòt des siles l'etompise, dont la face interne, cu devenant externe, se met plus facilencut en eoutact avec l'oxggine. Lin effel, l'eau ne peut pas

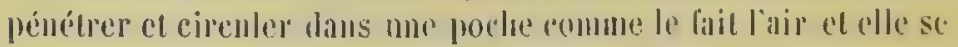
renourellerait diflicilenucut. 


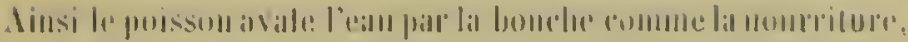

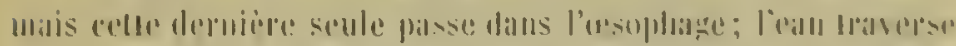

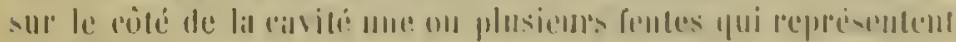

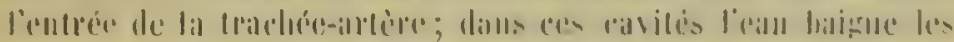

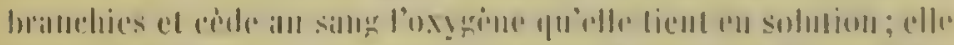

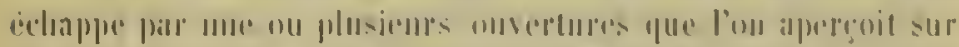

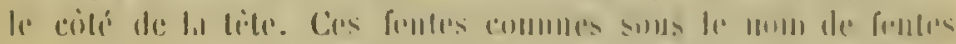

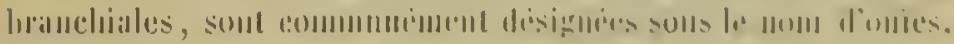

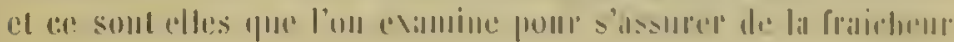
Alu joisst)II.

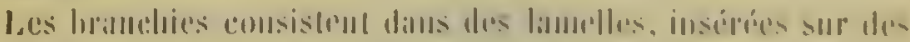

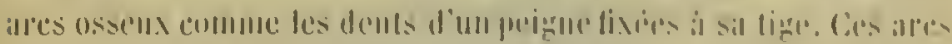

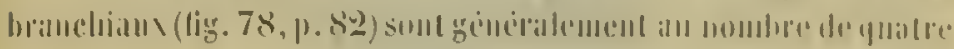

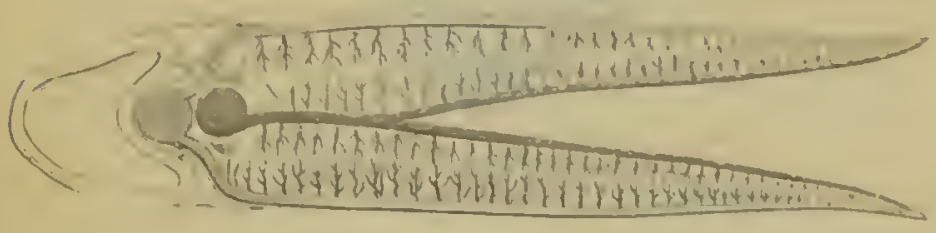

Fig. 79. t.ame brincluale de perebe

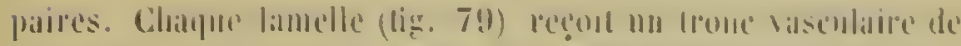

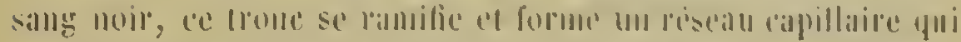

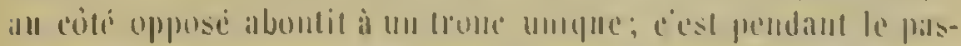

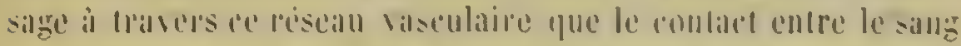

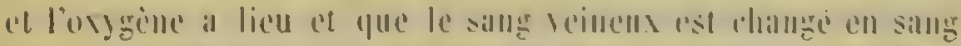

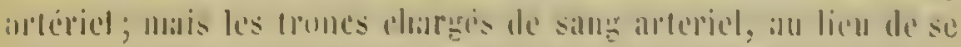

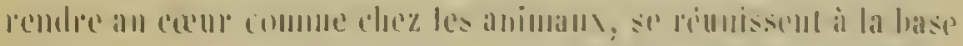

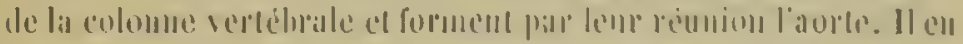

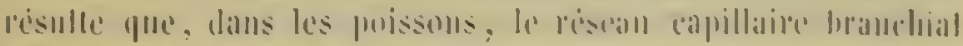

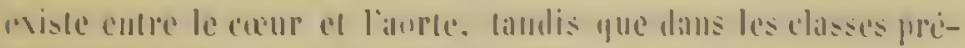

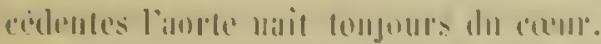

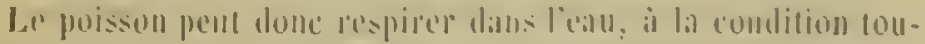

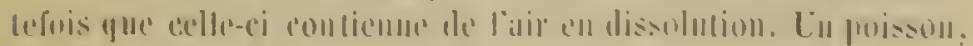

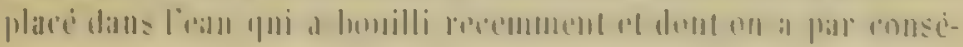


quent dhasse laill, meul. Quand le poisson sort de l'eill, les

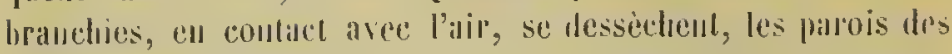

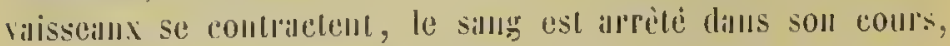
l'animal morult parr aspleysie. Il y a des poissons qui soptent de

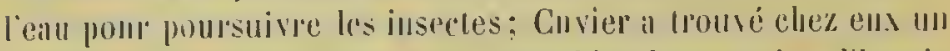

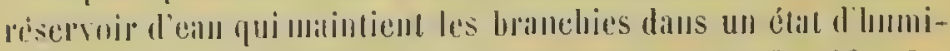
diti convenalile pendaut que l'animal est is terre (fig. 80). Ces

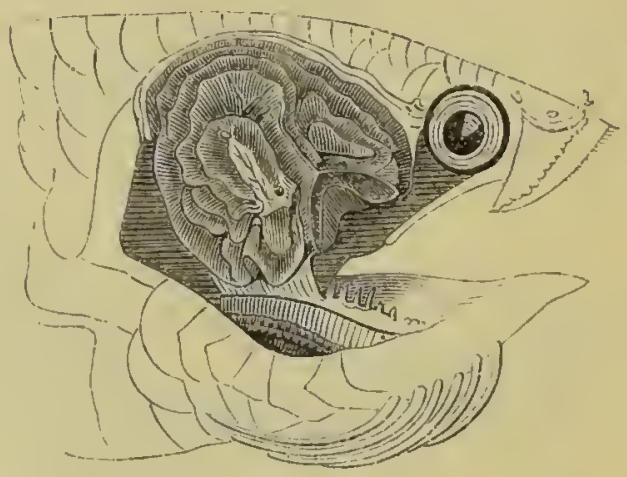

Hig, 80, - Inalors, Canaux lahyrialufurmes

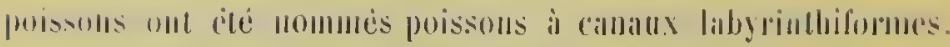

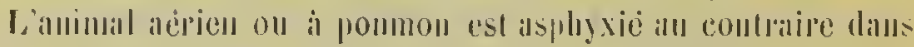

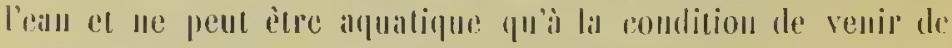

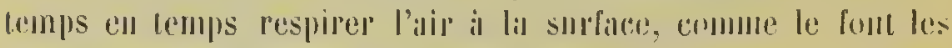
damphims, los balcines, les ploymes, de.

Chez purlynes poissons (phigioslones el my vimuides), les

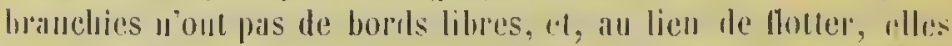

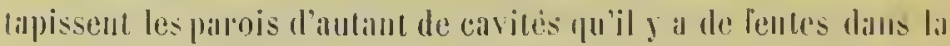

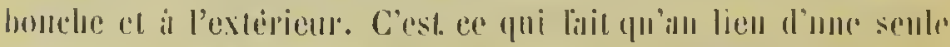

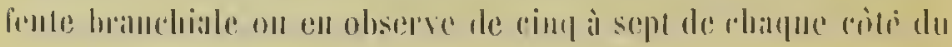
romp de res poissons.

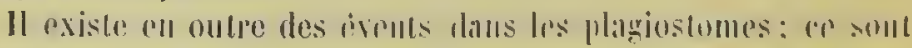
des mertures qui, silmes derrière les yrox, sont en communi-

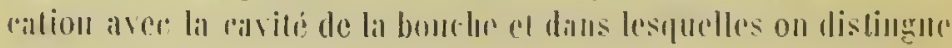




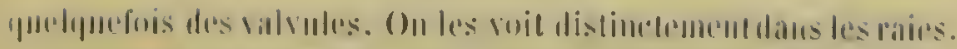

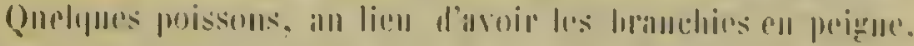

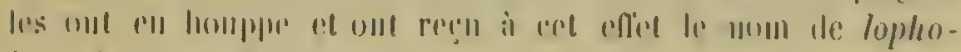

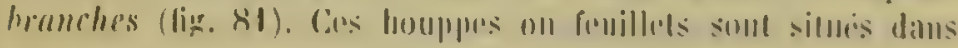

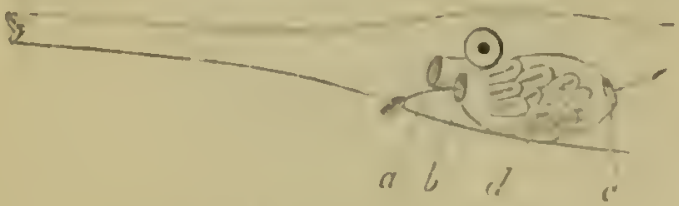

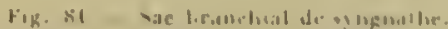

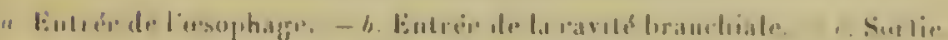
d. Houplers hionohiallen.

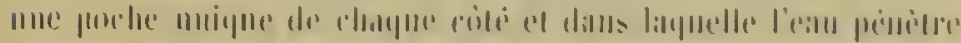

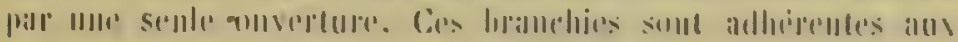

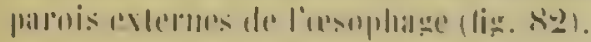

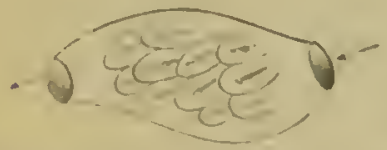

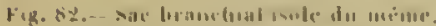
i1)

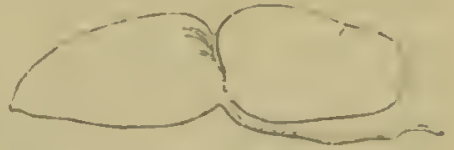

Fig. is

Pessic maluture it: rourpe.

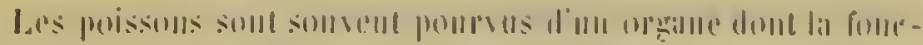

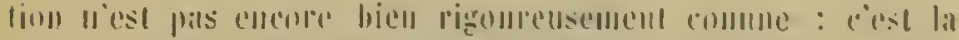

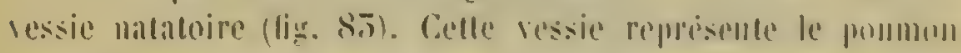

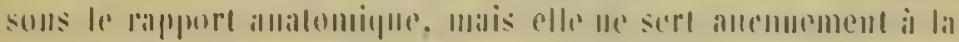

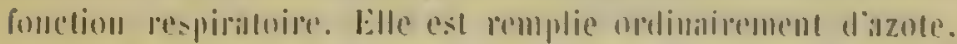

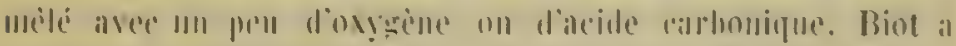

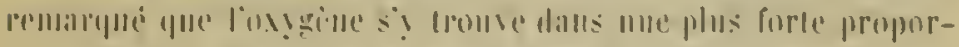

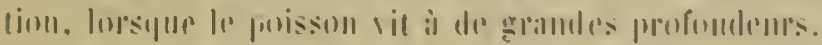

Ce Alum |

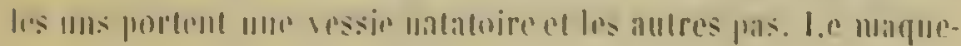


reau ordinaire n'a point de vessie natitoire, tandis qu une antre espiec trés-voisine en possède. Tout le genre polynème en est pourvi, à l'exception du polynemus paradiseus. Celle vessie derriente est toujours composée de deux membranes, dont l'une est interme el loujours fine, et l'autre fibrense el solide; entre ces deux membranes, on découvre souvent mu ganglion salıguin, tres-développé, que l'on a comparé quelquefois au thymus. Cet organe est tonjours altache it la colonne vertébrale comme le poumun ehez les oiseaux, el le péritoine le recouvre seulement à sa face inférieurc. C'est une poche simple, quelquefois étranshe au milieu et en communiration, par une sorte de trachéarlire sans ammeanx, avec la eavite digestive. Celte communcaltion a lieu ou dans l'oesophage, ou au fond mêne de l'estomac; quelquefois ce canal est complélement oblitére de maniere que la cavilé ne communique point arvec l'extérieur.

Si la ressie natatoire des poissons devait leur servir, comme on l'a supposé, pour augmenter ou diminuer leur poids spécilique, d'après le degré de compression exerec sur cel oryane pill' l'intermède des côtes, on ne trouverait pas chez quelques-uns l'entre eux cetle vessie cuferméc dans un élui complètencul osseux; du reste le mode d'articulation des cótes semble s'opposer aussi à celte supposition; cette articulation ll il pis lieu de manière à pouvoir comprimer la vessic.

Cette vessie mitatoire manque génciralencut chez les poissons qui vivent au fond de l'eau comme les pleuronectes el les raies; clle manque aussi dans ees excellents magenrs de la fimille des squales, commus sous le nom de requins.

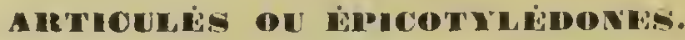

Les animaux de la classe des insectes respirent pall l'intepméde d'un appareil qui leur est propre el que l'on drigine sous le nou de trachées. Ces trachées existent dijì daus les larves. láabsence d'un appareil circulatuire a nécessité la formation l'un apparecil respiratoire particulier à ces allicoles. Voici sa composition.

Si l'on examine une larve d'un insecte, pall exemple te ver 


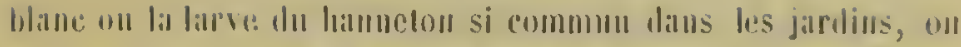
roil sur les filles, a prespue tous les immeam, mo playum

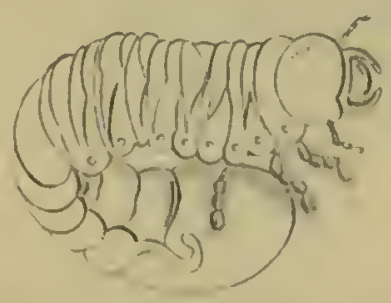

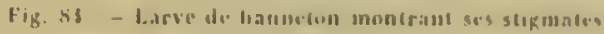

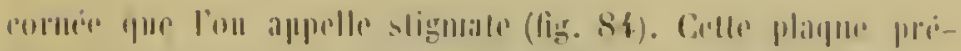

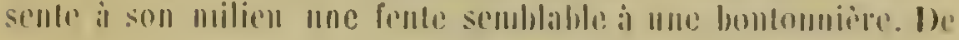

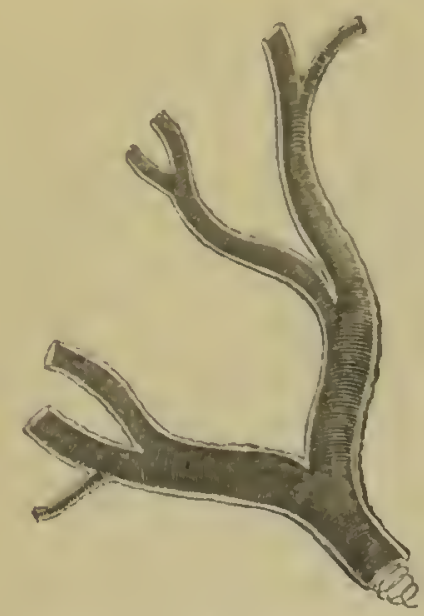

Fis. Sa. - Trachereale vanessa urtira.

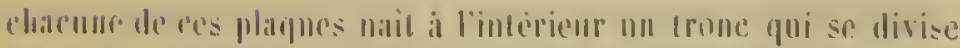

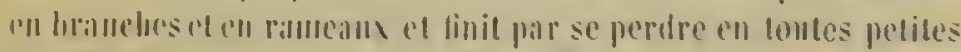

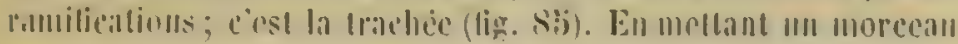


de celte trache sur' le porte-objet du microscope, on voil un fil, dispose en spirale, comme un ressorl, et enveloppé dans mue gâine (fig. 86). Ce fil spiral ticut les parois ćcarlees el jone dans ces organes te même rôle que les cercles cartilagineux de lal

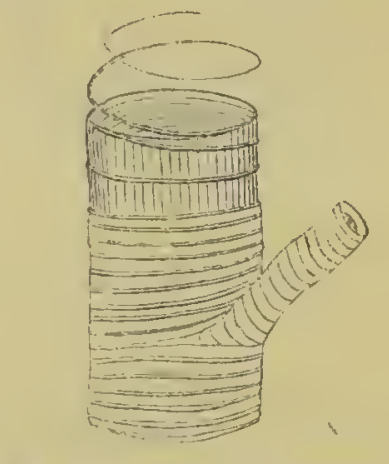

Y'ig. 8fi. - Trandice de hammaton.

Irache-altire des animaux superieurs. C'est de cette ressembance que vient leur unm de trache. Par la fente des stigmates l'air pémètre dians l'interienr du troue, el de li il passe ensuite daus toutes les br:aluches. On a dit que dans ees aluimaux l'ail rirenle à la place du silug, que e'est l'air qui va clecelere le sang, tandis qu'ailleul's e'est le saug qui va à la reucoutre de l'air.

Daus les myriapodes et une parlic des arieluniles, la respiration est igalement tracliécune.

J,es autres aracluides, comme l'araignce ordinaire ou les scorpions, ont ì la base de l'abdomen deux ou quitlte pelites feutes, qui s'ouvrent dans une poche, an milien de laquelle ou distingue un feuillet dout la surface eststrice; c'est pour ainsi dire une lamelle brauchiale daus un pounou. C'est aiusi que l'appireil respiratoire est eouformé clıcz les arachuides pulmonaires.

'Tous les articules, à l'exception des crustacés, sout aérieus: aussi les crustaces seuls portent des brauchies, et elles sout tresvalpialoles dans les amimanx de rette classe. Il y a des clustacés. comme les lernécens, qui u'ont pas d'appareil spécial; d'antres, ('omure les apus, ont des organes de loromotion foliacis qui servent a la locomotion el il lat respilation; d'antres encore portent de 


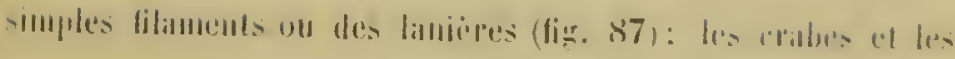

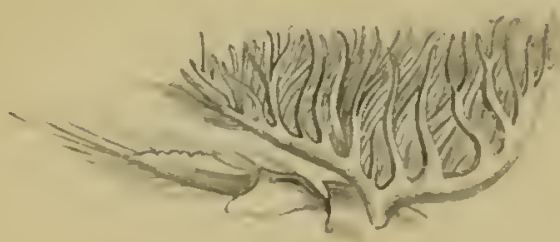

vig. $K_{i}$ - Branobue de sipuillo.

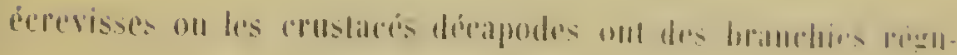

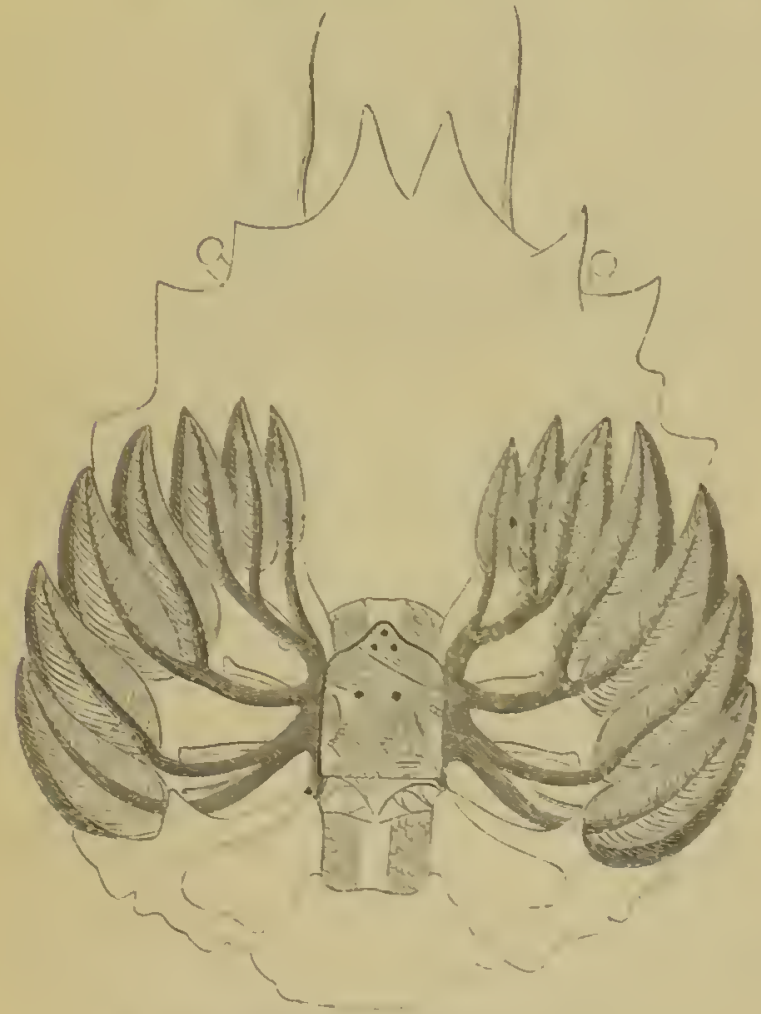

Fig. ș - Pranchics of corur te maia squinat.

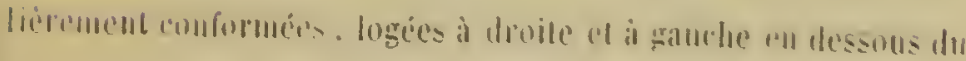


céphlalo-thorax on de la carapace (lig. 88), clles oeculpent une cavité assez spacieuse, qui communique au dehor's palr une larrar.

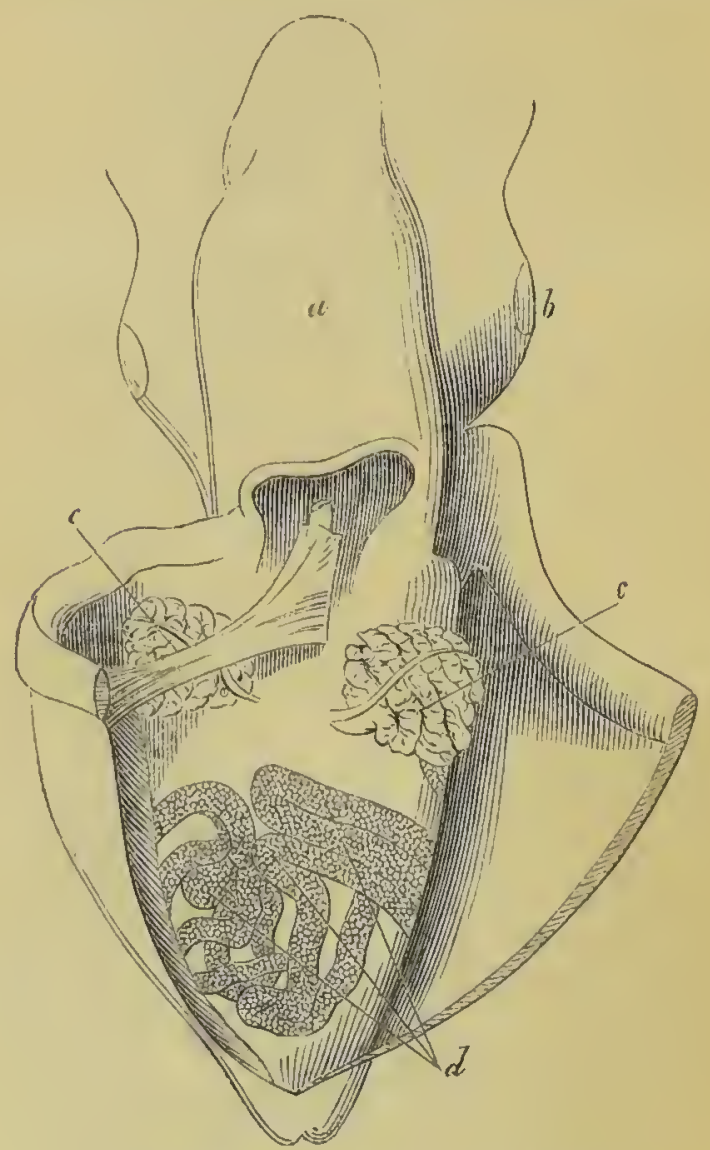

Fig. 89. - Brandio's de J'argonaule en place.

a Entonnoir. - b Youx. - r Branchies. - d Oridurle".

fente el qui s'étent de la premiere jusqu'i la deruière pailer

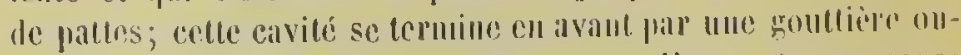

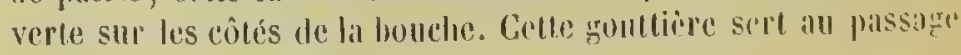
de l'eau de la lespliration. 


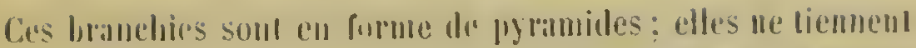

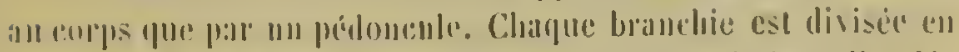

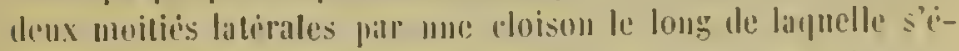

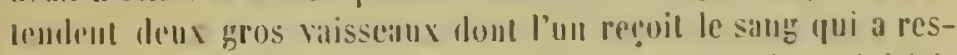

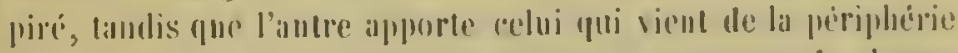

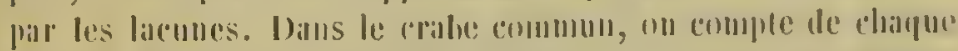

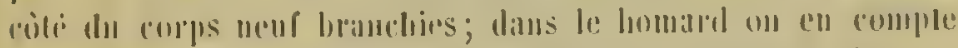
vingt-deux; les langousles en ont dix-lunit de chanper erite.

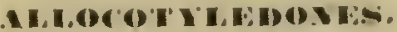

Mollusques. - Les cepplablepoiles ont is la fare inferiente du

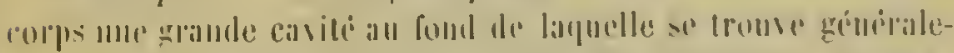

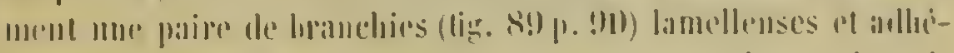

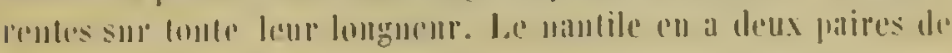
platpluce cobli.

Parmi les gasteropodes, les limades ont sur le berd du lonlrlier mon omserture qui mommminme ance une eaviti issez graude sur les

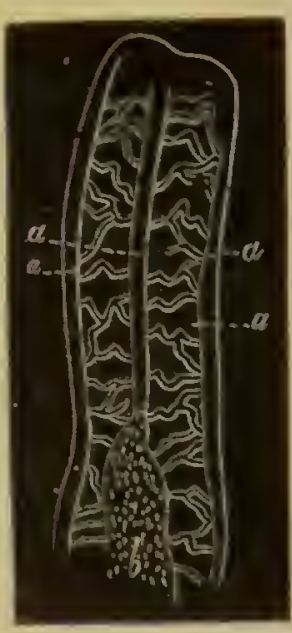

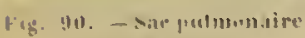
de lielis VIg!ra.

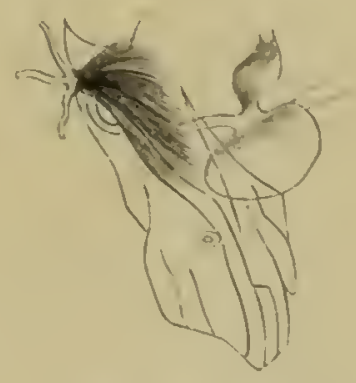

Fig. "1t-Banchic droitu el arur de rombule.

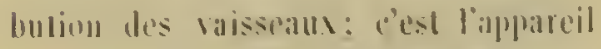

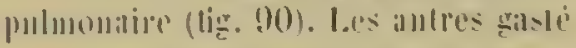

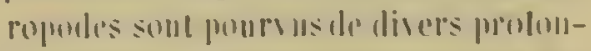

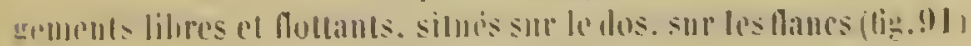


ou te long de leur disque elarmu, et puon designe encore sous le nom de branclies (fig. 92).

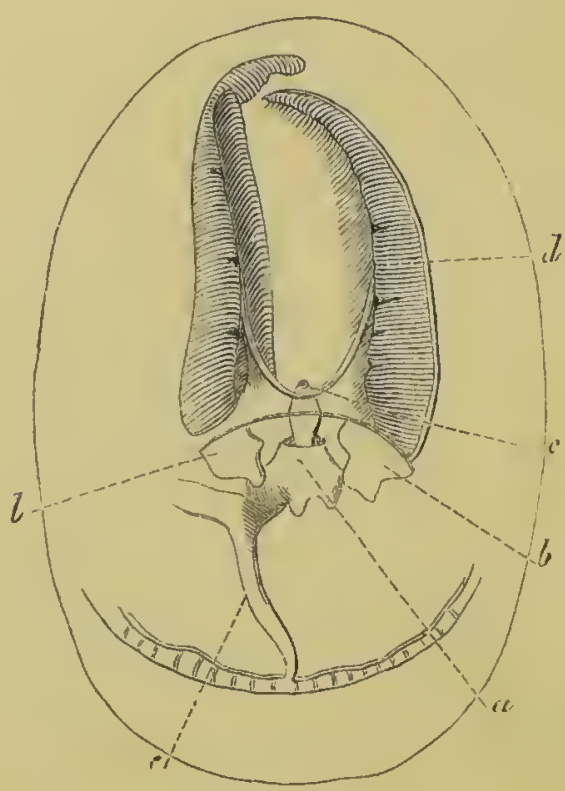

Fig. 92. - Branchies et ceur dt" fissurella.

a Cour. - $b$ Oreilletle. - c Anus. - $d$ Branchies. - edorte.

Les scéplales, comme les moules el les luâtres, ont deus paires de lamelles branchiales sur toule la longneur du rorps, s'étendant souvent depuis la bouclie jusqu'à l'anus. C'est la forme de ces organes qui a fail désigner ces mollusques sons te nom d'acéplaales lamellibranches. II. Valenciennes a constaté dans plusiemrs mollusques acéphales la presence l'une seule? Iranchie (lucines, corbeille, lelliua crassa). l.es branchies manquent mème, ou sout remplatres, llapres le meme savant, pir deux bourrelets longituliuan sins ancune lamelle sceondaire, dans le solen radiatus, dont il a fait le genenre leguminaria. Cos 


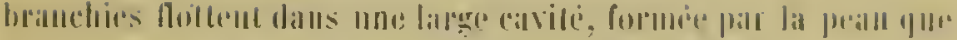

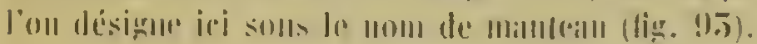

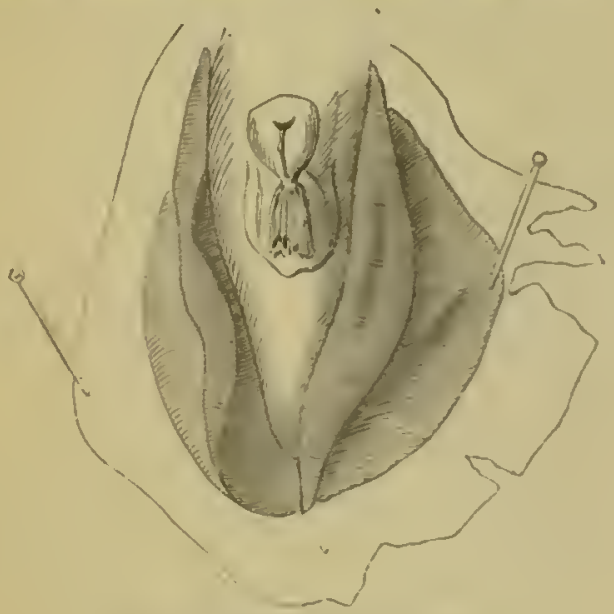

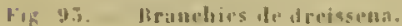

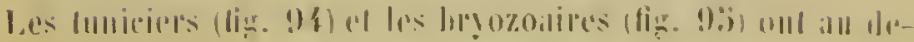

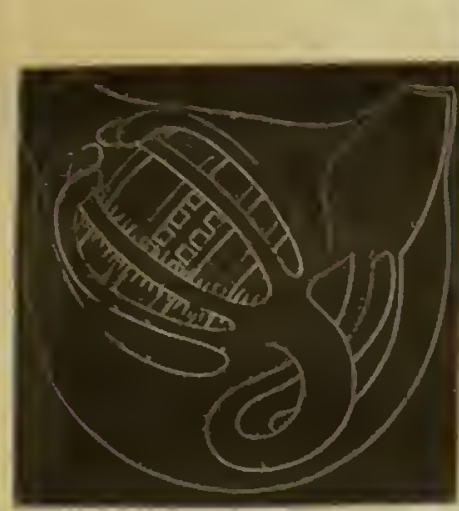

Fig. 96. जac hrancliat des asciolics.

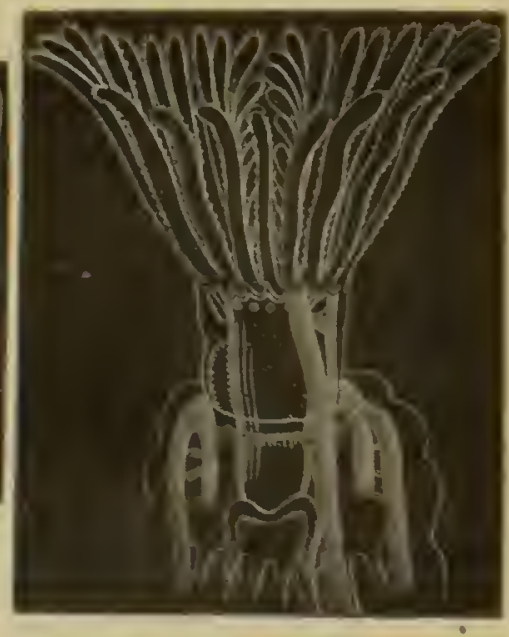

rig. 95. - Branchics de l'aleznnelle.

Iant de ha bondo dos vaisseau regulierement rilios. droils ed 
libres, comme des tenticules disposés ell entonnoir ou bien anastomosés entre eus el formant une cavité prébuceale. Les oryanes qui constituent l'apprareil respiratuire se montrent ainsi daus un citat de grlande simplicité.

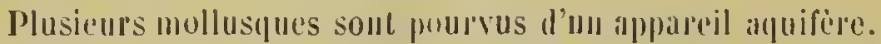

Vers. - Ceus qui oecupent la tète de cette classe unt des hranchies sous forme fle longhes lanieres qui recouvrent lat palptie anterieuse du corps (tig. 96), on bicu le milieu du dos; ces

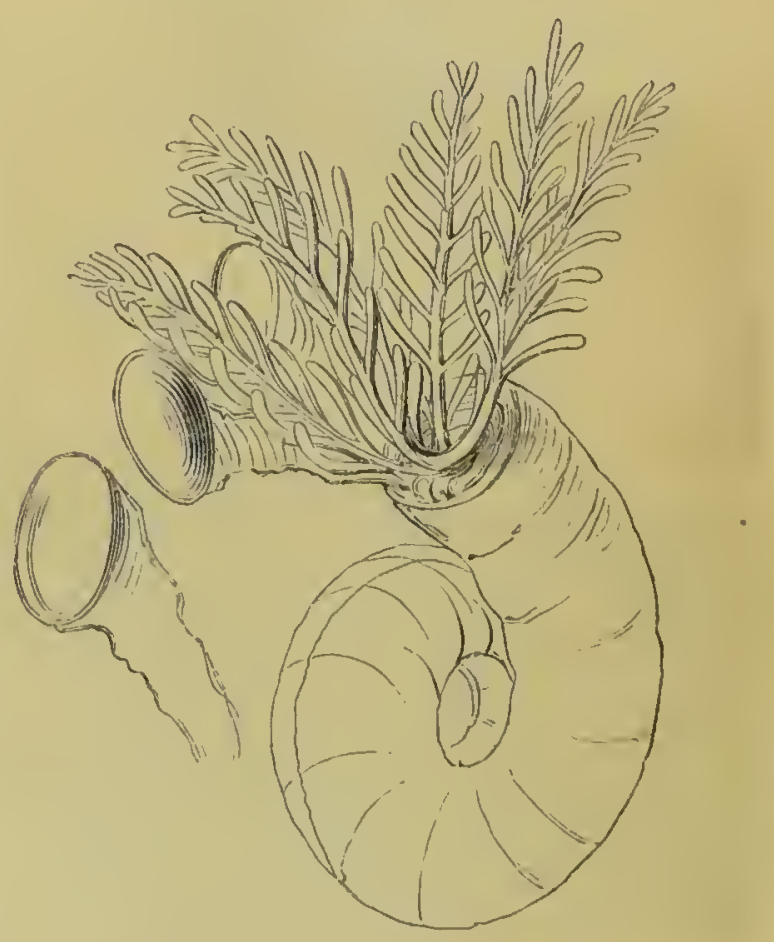

Fig. 96. - Branchies do spirwlue.

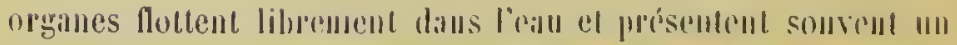

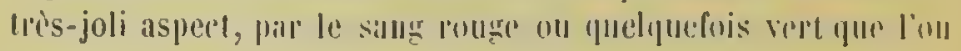




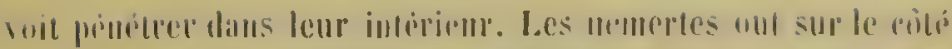
de la lite derly fosseltes, gialluies de cils viluritiles

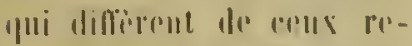
(o)

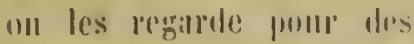

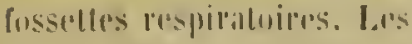
illiles vers, ret cins le plus gratud numbre, lisipirent pare thute la surfacer

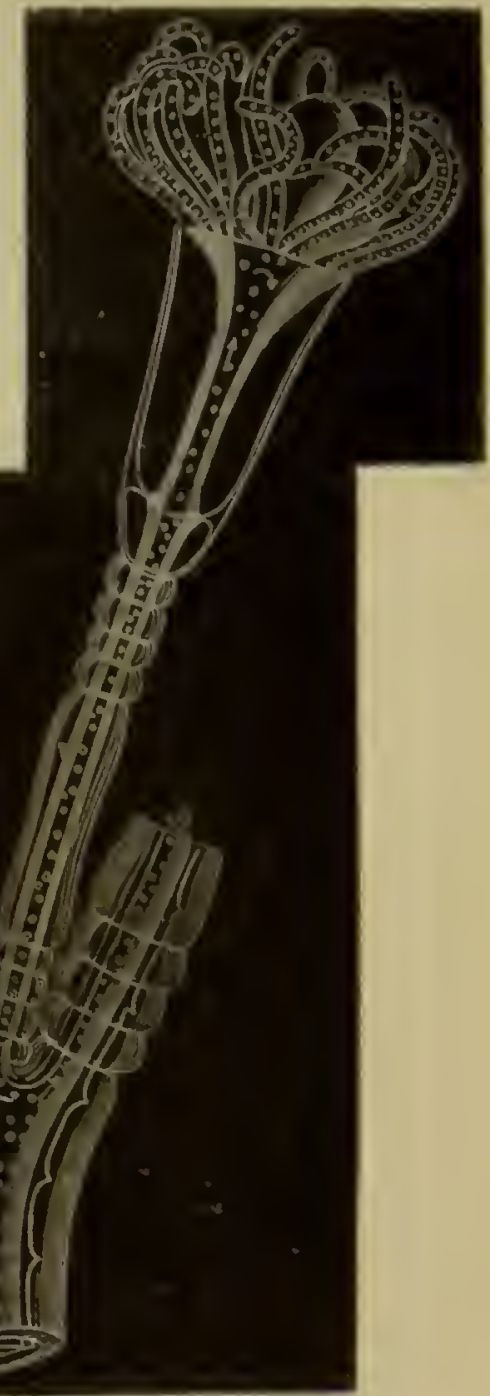

fig. 97 - Tige de campanulaite.

Ie la prau, aussi bien les sangsues que les vers intritinam. loappareil de circulalion on lapplareil aquifere quion a rru 
observer dans plusiems vers, particulièrement ehez les trematodes et les cestoïdes, et dont quelques-uns mème ont fait un appareil respiratoire, est un appareil séeréteur qui verse son produit an dehors, par le secour's d'une vésieule contractile. Cette vẻsicule est situèe ì la partic postérieure du corps et s'ourre directement au dehors; nous en parlons plus loin au sujet des oryanes de la séercition mrinaire.

Échinodermes. - Quelques-uns lle ces animaux respirent par des branchies qui entourent la cavité bueeale, ou bien par des traehies qui condnisent l'eau du dehors, comme les traches des insectes combisent l'air. Ces organes qui conduisent l'eau sont désiğlés sous le nom d'organes aquilères. On en olsserve dans des animanx de diverses classes. Dans les échinodermes, on voit encore l'eau s'introduire daus l'intérieur du corps par diverses ouvertures et porter plus directement encore que pilr un arbre aquifere l'oxygène aux divers organes. Dalıs les loolothuries, on voit de chaque côté du colpls un arlure respiratoire souvirip dans le cloaque.

Polypes et acrlèphes. - Il n'existe plus d'organe spécial pour laccomplissement de eet aete; l'eau qui apporte la nouldriture et qui la distribıe, ehez ces aninanx, renferme en même temps l'oxygène, et les deux fonctions de nutrition et de respiration se confondent. Dans les liydres, la cavité stomaeale s'étend dans l’intérieur des longs liras contractiles, ce yui n’a pas lieu daus les autres polypes; ectte disposition d'une cavité de l'estomac ayant des ramifieations dans l'intirienr du eorps et des appendices se reproduit dans les aealèphes.

Infusoires et foraminifères. - Enfin, dans ces animaux si simples, l'alssorption a lien par toute la surfice extérieure, et il n'y a plus guere d'organe spécial pour aneune fonetion. Les cils viluratiles qui recouvrent le eorjes ou ani entourent la bouche servent à la respiration, dans ce sens du’ils renouvillent. le milipu dans fequel les infusoires vivent, et par enuscinuent remonvellent l'oxygène.

Les cils remplissent iei la fonction de la cavité Horiachique. 
ANATOMIE COMP.RLEL. 



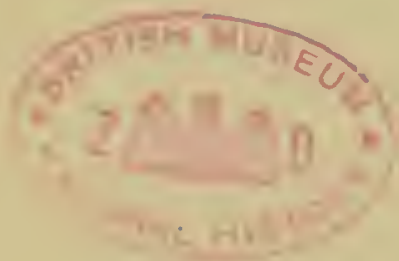



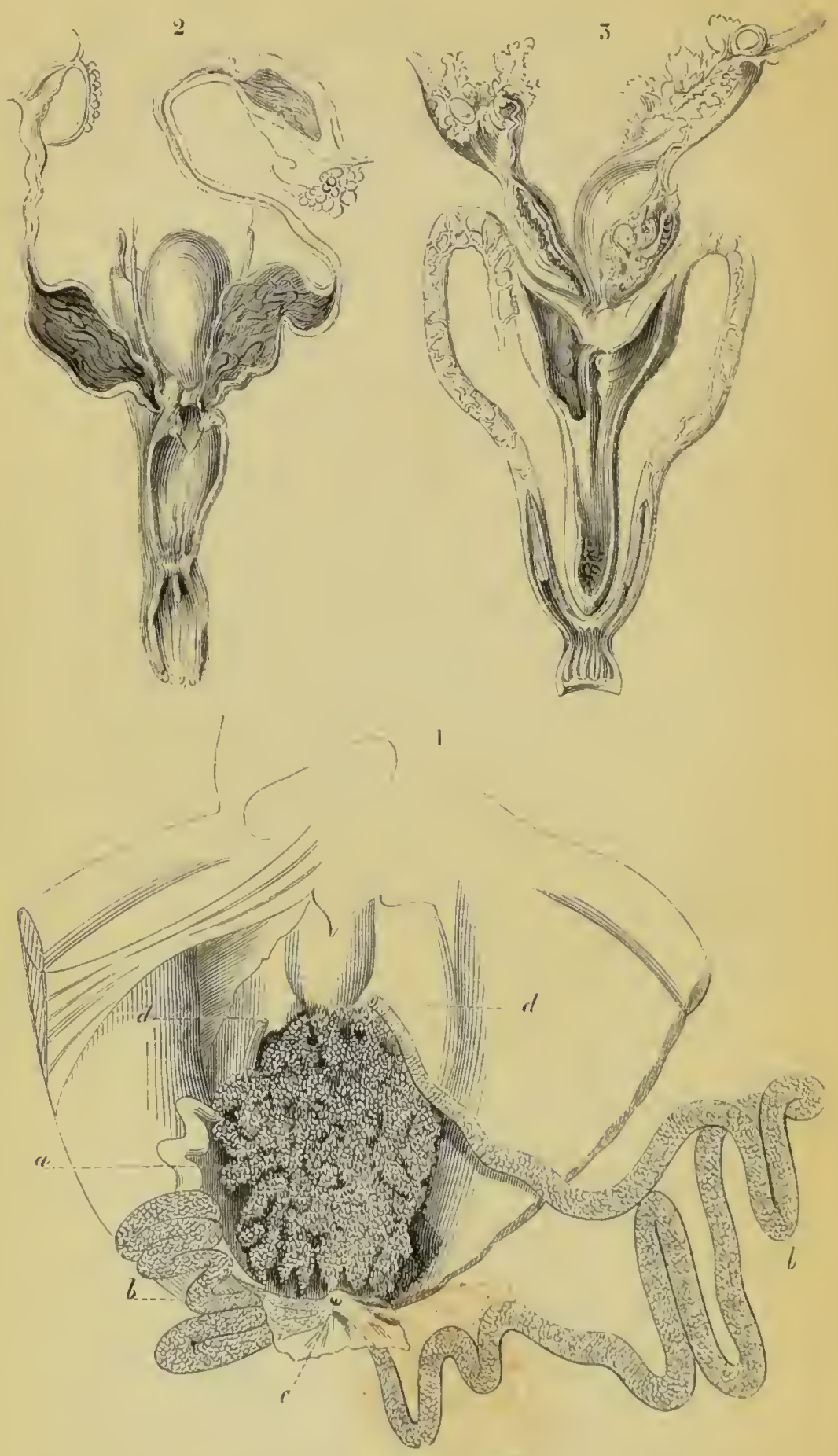

1. Aprareil femille diagonante. - 2. Appareil femelle de musterumanus. ה. Appireil femelle de kanguron. 



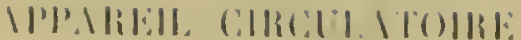

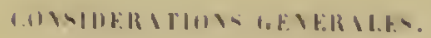

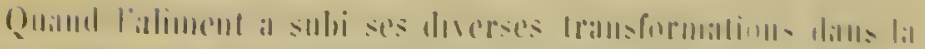

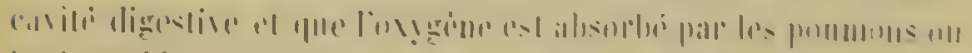

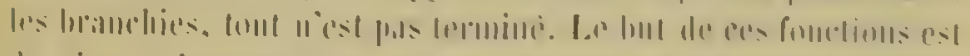

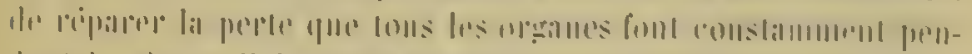

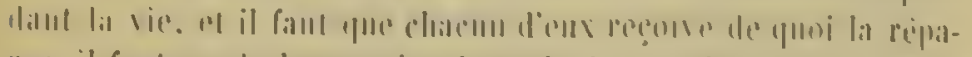

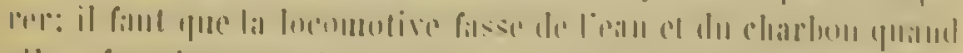

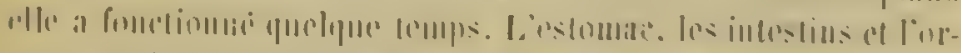

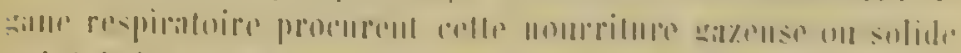

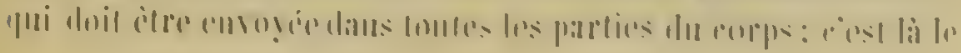


rôle de l'applareil qui nous oceupe iei (fig. 98). Nous l'avous déjà dit, dans les villes eelasires au gà, on distribue d'un point central le gaz à chacun des becs par l'interméde de tuyaux souterrins; ces tuyaux sont en tout semblables aux vaisseaux qui

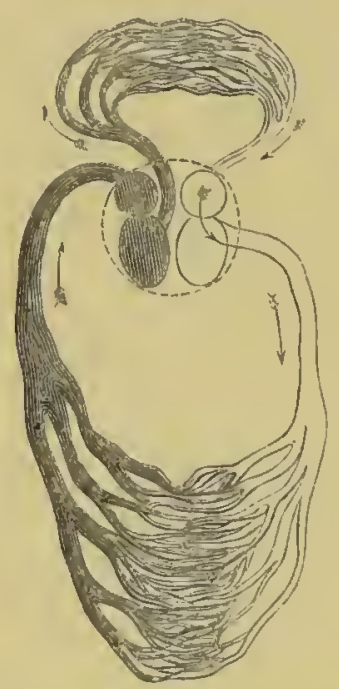

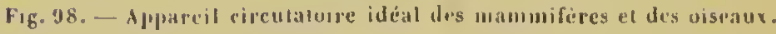

conduisent le sang dans l'eenomie animisle. I.e Luyau principal est fort gros, puisqu'il doit livrer passage ì toute la masse de ga\%, mais ceux qui en partent sont de plus en plus petits, et e'est ce qui donne quelque ressemblance avec un arbre, qui montre un tronc avec ses branches et ses rameaux; mais le gaz doit simplement être envoyé pour alimenter les becs, tandis que dins l'cicononic animale il faut non-seulement alimenter, mais il fau aussi éconduire les matériaux qui sout mis hors d'usage. Le ga\% lrûlé disparait dans l'atmosplière, les tissus brüles ou modifies doivent être élininés par des voies rigulières et determinces: la locomolive laisse tomber ses cendres en roule, dans les animanx il fant les icouduire par des canam; de la résulte la mé-

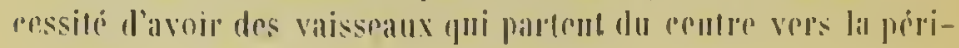




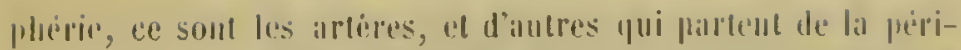
phrire vers lo rentre, on les veines; de lit inssi resultent des rourants an sens inverse : des allipes qui portent nu courant

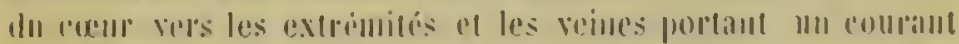

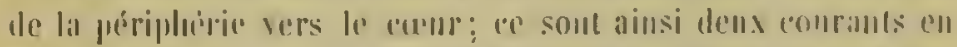

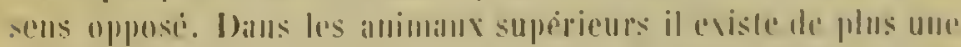

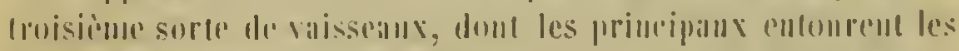

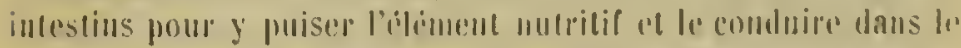

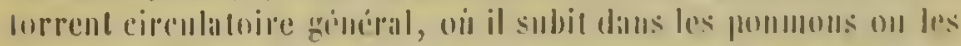

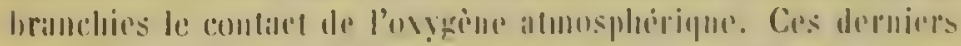

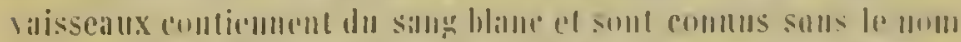

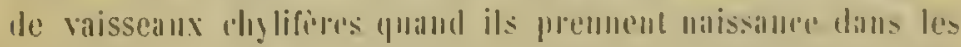

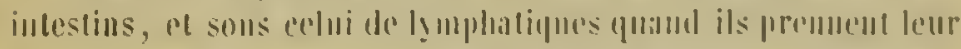

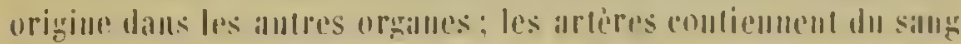

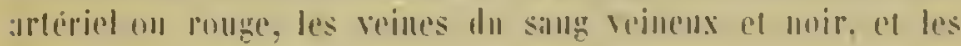
antres du silly hillue.

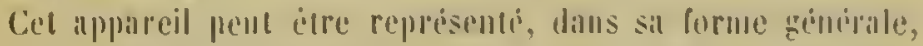
par mu tube recourhe en forme de C; vers le milieu, nu liger

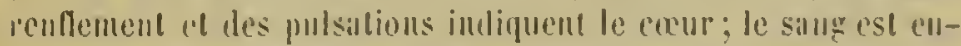

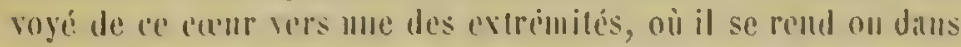
des lacunes ou daus un riseau capillaire; il revient de la mene mamiere vers mu antre trome qui commenee alsolument comme le premier a lini, et le lignide revient au meme point dou il

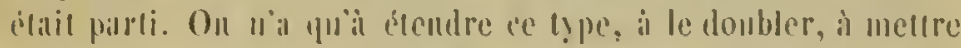

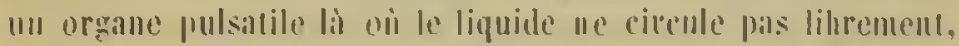
oủ il eprouve quelpue obstacle. et on se fail une idie de toutes les complicaltons qui peurent surgeir.

Mais il sen faut yne tous les animan prisentent un appareil rirculatoire complet: une eireulation est méessaire partoul. mais elle a lieu daus les animanx inferieurs sans un oryane spricial, sans le sccoms d’un liquide parliculier. Jetons un conp d'wil sur son mude d'apparition daus la série des animanx.

Dinls les organismes tris-simples, il ll coiste pis de liquide

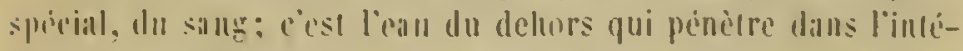
rieur somvent fral imbihition el qui ronduil elle-mème son

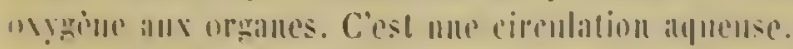

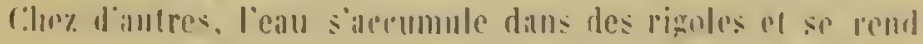


anx organes sans l'interméde de vaisserux; consuite la face inlepre de ees cavites se recouve d'une memhrane, ectle memhrane emprisonne un liquide propre, et il existe un raissean. Dès ee montent le liquide qui leprésente le sang peut suivre un cours déterminé; mais à cel effel il fant un organe d'impulsion. D’abord ce sont les cils vibratiles qui déterminent le cour's du liquide; ces cils se monvent ou lans les grandes lacunes du colphs ou bien i l'entrée el dans l'intérienr des vaisseanx; puis les cils drspalaissent et le vaissean devient contractile; il se resserpe el se dilate altermativement, et le sang est pousse dans telle on telle direction. Chez des anmonx phos éleves conere, la contuation so localisc, (clle n'a plus lieu que dans un endroil délepuniné; iri

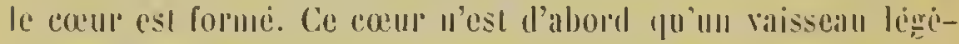
pement dilati, comme nous avous vol lestomac se former sur le lrajet du canal intestimal par la simple dilatation du lube; mais des él'anglements se forment bientòt dans son intérieur, de: cloisons surgissent pour former des eavilés distiuctes at le coen' montre me oreilletle el un ventricule. le vaissean so tord ensuite sur lui-mème, il se forme dans son intériem de unuvelles eloisons, et une conche musenlense apparait daus l'eprasseur de ses parois pour domner maissance à cet oryante

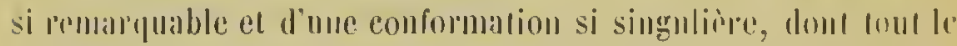

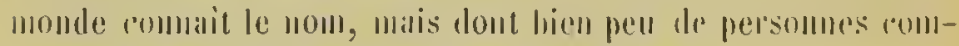
premucul le mode de formation : éest le cours.

Ce ecenr, dont nons venons de parler, est celni qui so déve-

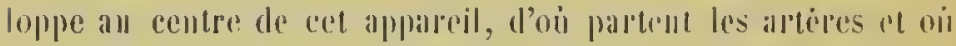

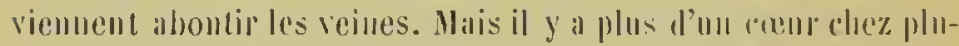

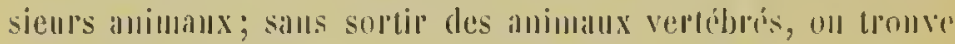
smr le thajet des rejues, et mème sur le trajet des valisseans

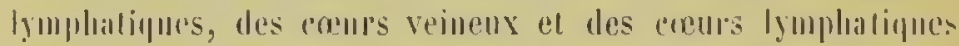

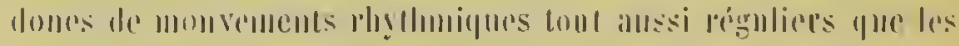
momverments du enore principal.

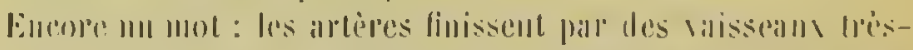

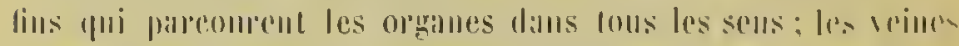

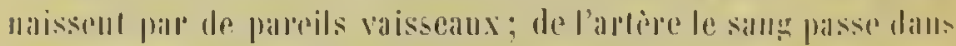

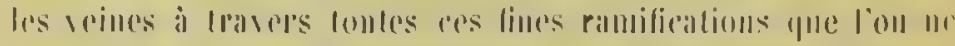

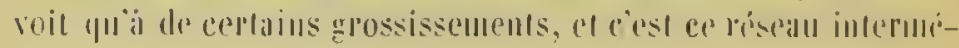




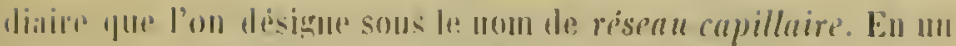

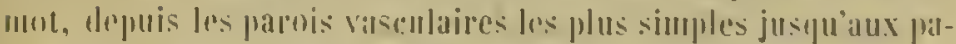

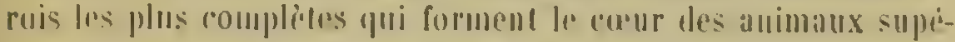

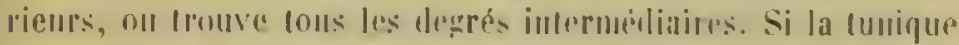

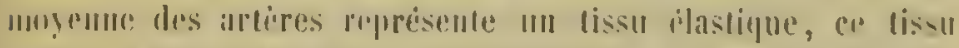

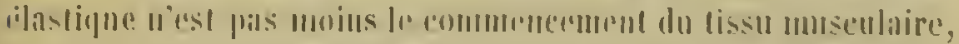

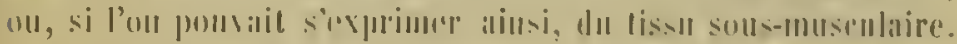

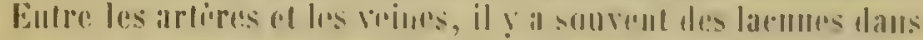

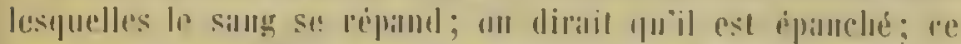

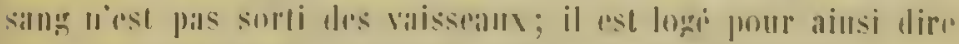

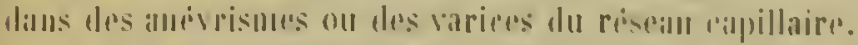

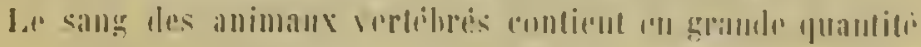

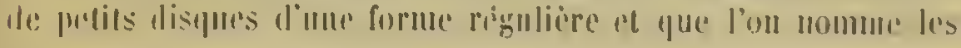

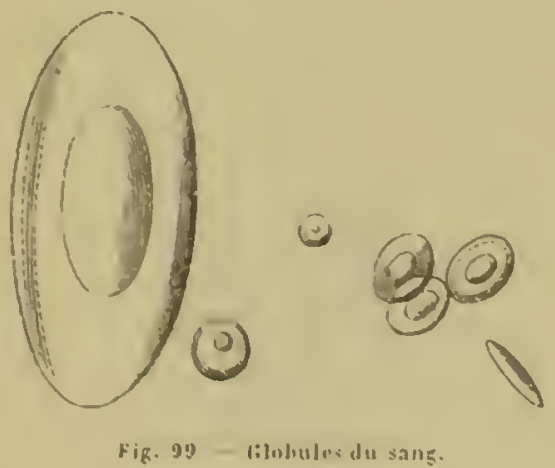

globules du samy (fig. !!!). Ciest daus les balraciens quils soul

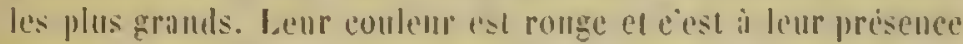
gure lo silng doil sa couleur dians les animanx vertibres. Le sillyg qui a perelu ses globules est d'un blatre jaunitre.

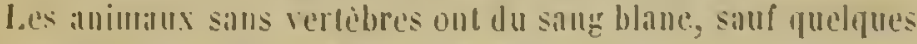

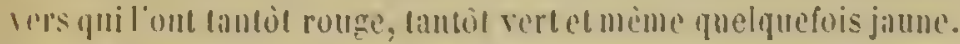
Mais la rouleur uest pas duc a la presence de glohules contme linus les vertibres: le liquide lui-meme est colore. Il y al assi

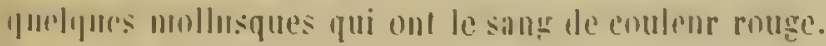




\section{MAMMIFEERES.}

Cet applareil to presente pas de moditications importantes

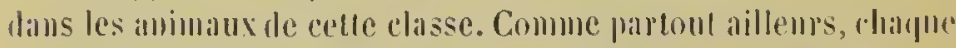

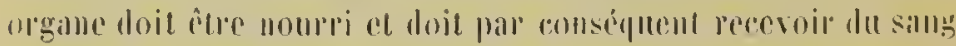

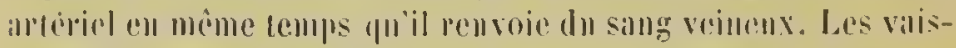

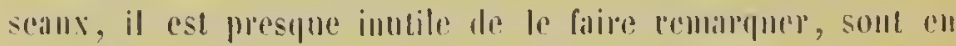

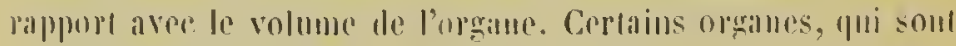

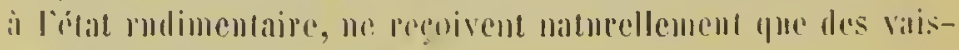

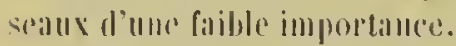

Ju rour oceupe i pen pres la mime place dams lous les

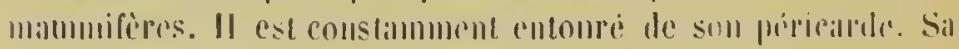

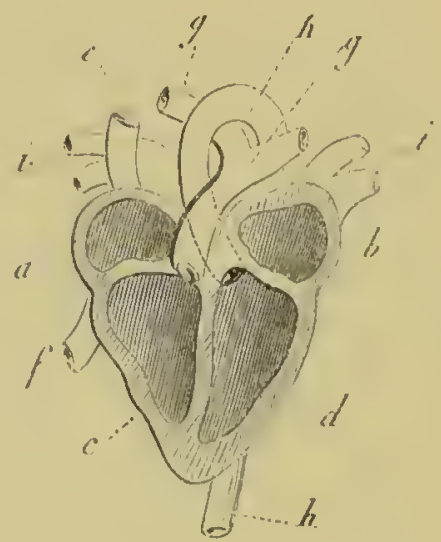

Yin. 99 bis.

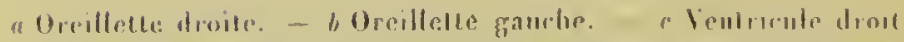

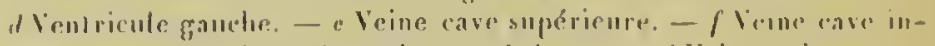

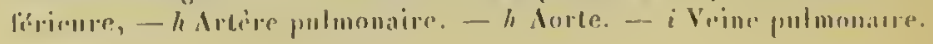

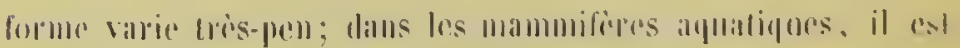

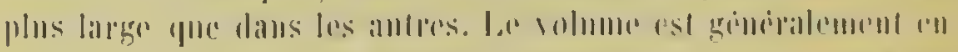

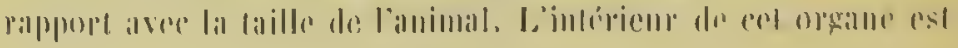




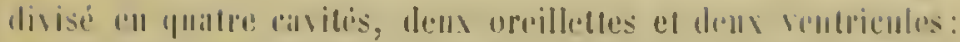

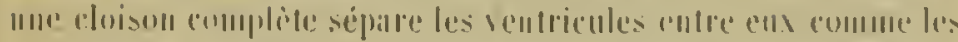

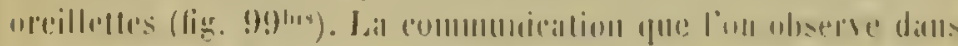

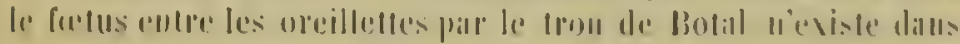

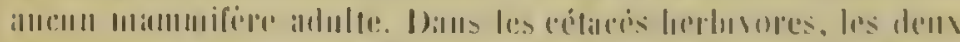

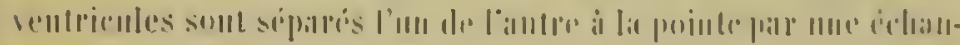

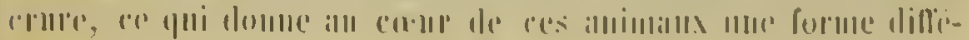

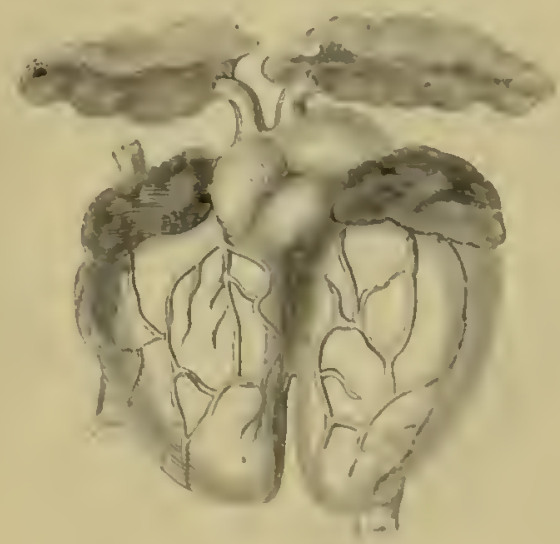

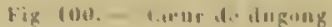

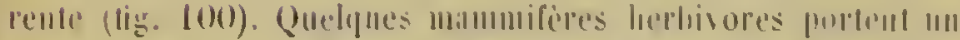

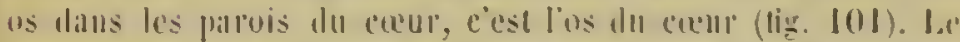

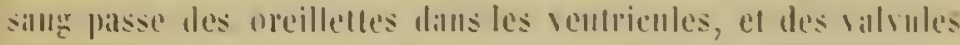

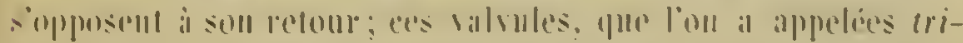

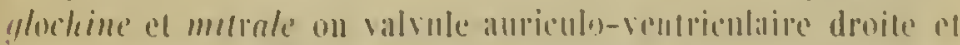

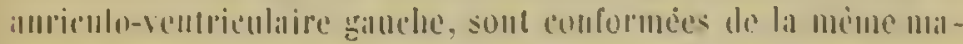
uiere dills fous les animans de ente clissede. siluf les muntofrimes.

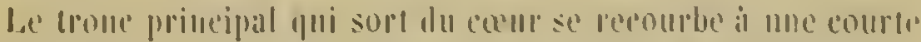

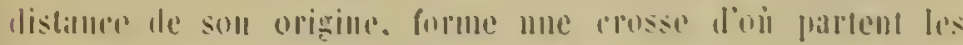

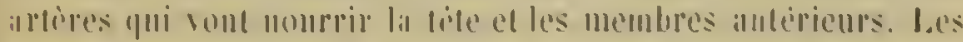

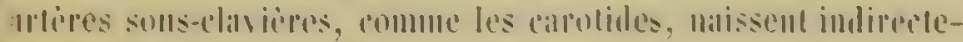

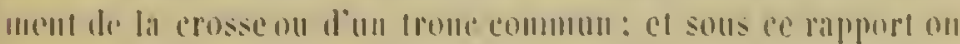


remaryue, chez les mammilères, certaines dispositions qui se reproduisent cluez l'homme à l'état d'anomalie. Ainsi le cas d'anomalie clez l'lonme est un élat régulier et normal dans certaius mammifères. L'artẻre saerée-moyenne, si peu développée chè. l'lomme, est ell général très-téveloppée chòz les manni-

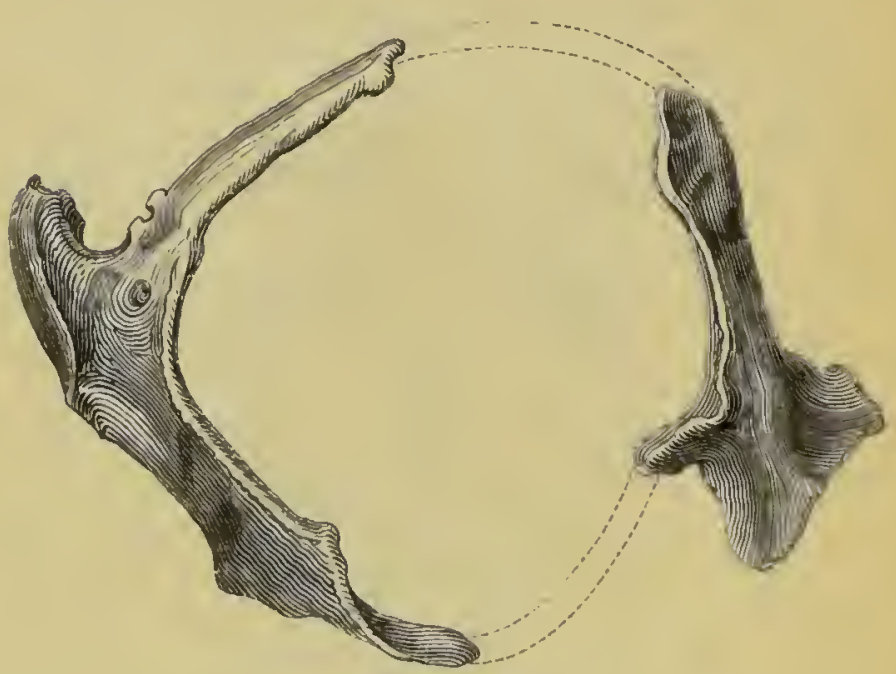

Fig. 101. - Os da cosur du beur.

fires et d'antant plus forte que la queue est plus voluminemse.

L'artère brachiale est allistomoséc alsec la fémorale dims le hérissoll.

La distribution vasculaire du tapis de l'oeil est très-variable d'une espèce à l'autre, foroique au fond la disposition soit la mème.

Afin rle ralentir le cours du sing, certinines artires se divisen en une infinté de petites altérioles et forment ce que lon alppelle des réseanx admirables (lig. 102). On en voit ainsi a lil base du cerveau de beanconp de mammières (rmminants et soli-

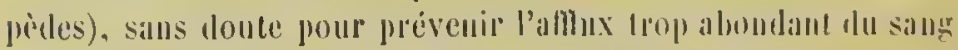

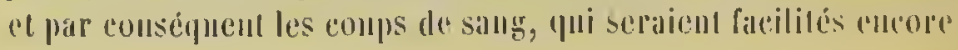




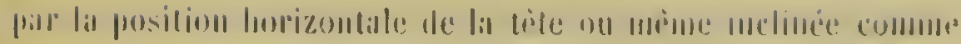

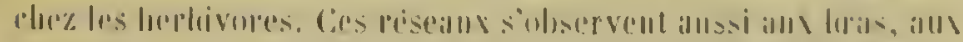

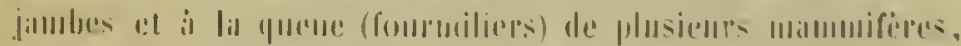

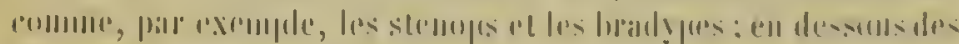

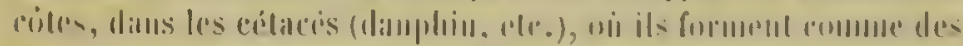

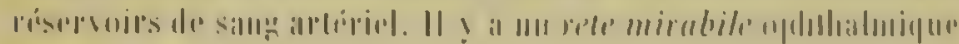
|alls: |r. |r|lil|.

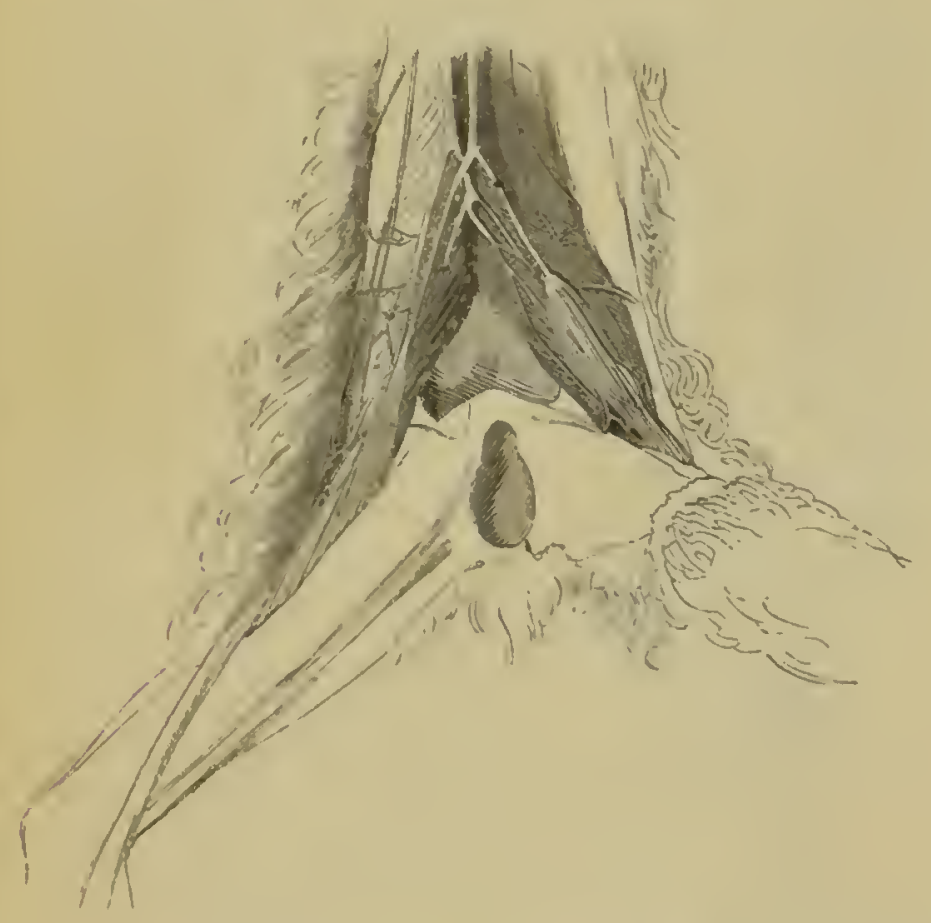

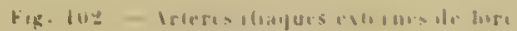

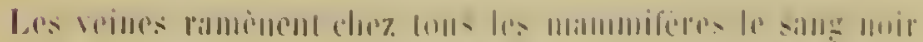

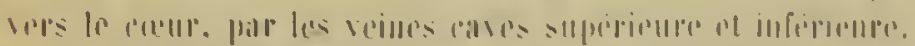

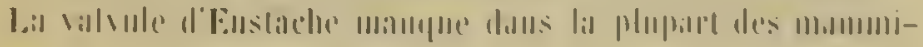
firis.

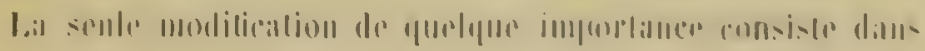


l'enorme développentent du systeme veineux abdominal des mammilères aquatiques et la présence d'une sorte de sphincter clıez res mêmes mammifères à l'entrée de l'oreillelle; ce sphnincter montre des filmes musenlaires striées, il empêche le sanyo d'arriver au cour pendant la submersion; il parait s'ouvrie it lis volonté de l'animil. Par ce moyen ils peuvent rester assez longlemps sous l'eau sillss s'ispliyxier.

Les veines de divers cèlacés semblent dépourvues de valvules.

On trouve des plexns veineux sur le muscle psoas, dans les dinphins; sur les reins, dans les phoques.

La veine porte, qui rimeine le

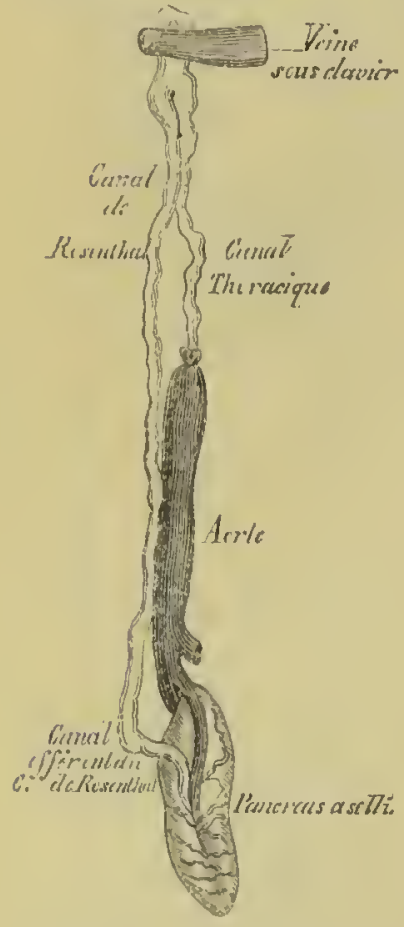

Hig. 105.-- Canal de Rosenthal. sinng des visceres abdominaux, pour le comduire an foie, est la même chez fous les mammiferes; 1) y remarque des valvules dlez. quelques-uns d'entre eux.

Le systeme lymphlatique se tomporte anssi de la même manière dans tous les aninaux de celle classe; les lymplıatiques de l'intestin grêle ou plutòt les vaisseaux "hyliféres versent leur produit dans des galnglions mésentériques éparpillés sur le méscntire, on rénnis ch une masse, quand l'intestin esl rourt, conme daus les carnassiers; res ganglions forment alors le pancréas d'A selli, et ils montrent généralenent plusien's camaux effirents. Les vaisseanx lactés des ploques sont tris-lalges, inl senl canal eflérent verse lent contenn dans le canal thorachicyur, éest

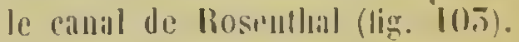

C'est il nn reservoil unique, le reservoil de Peequet, yur vieuneut abontir en génćal tous les vitisseans lỵphlattipures, 


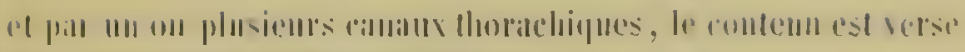

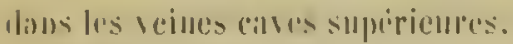

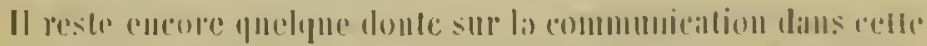

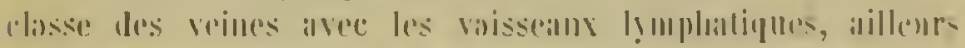

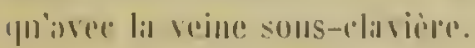

\section{OISEAUX.}

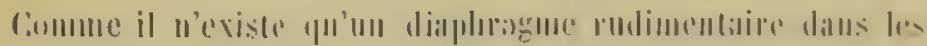

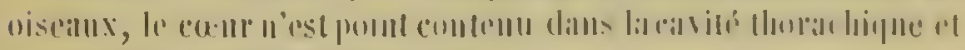
il desermu jusijutan milieu tes loles da fold. Il est entouri

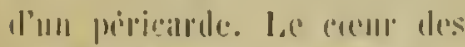
uiseimx ast pointu e't plus lon:

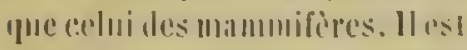
divise imteriemrement, rommor

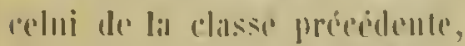

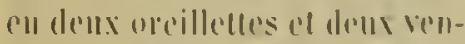
tricules à cloisons eomplites. mitis il est tonjouts faccile il

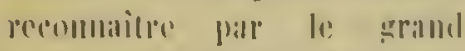

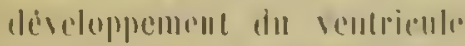

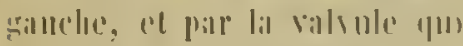

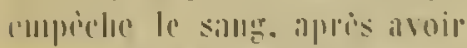
pémedre dims le vembicule, de

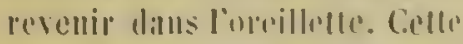
vidulule est moe bilule musinlaire qui silpplipue sur le’s pa-

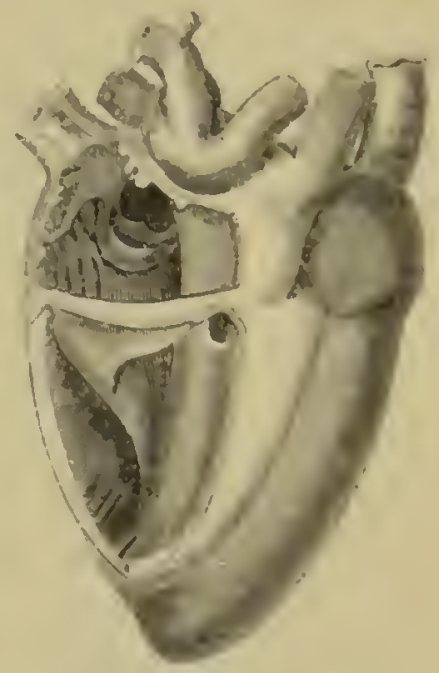

Fig. 106. - tarkar de r! gne

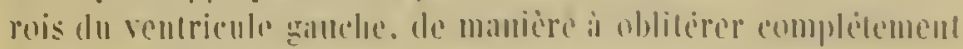

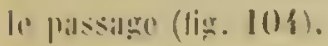

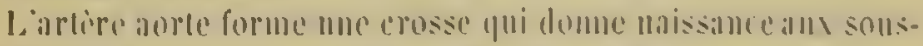

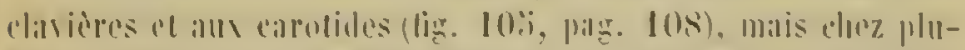

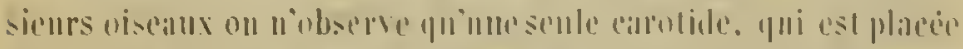

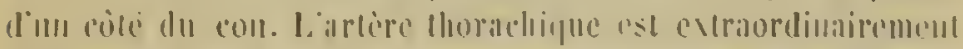

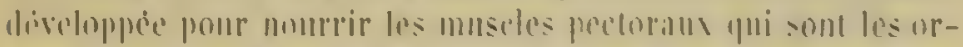

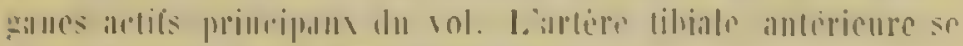

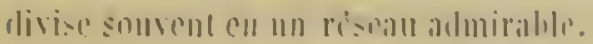




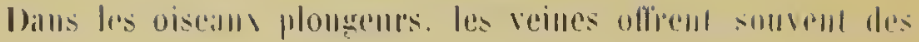

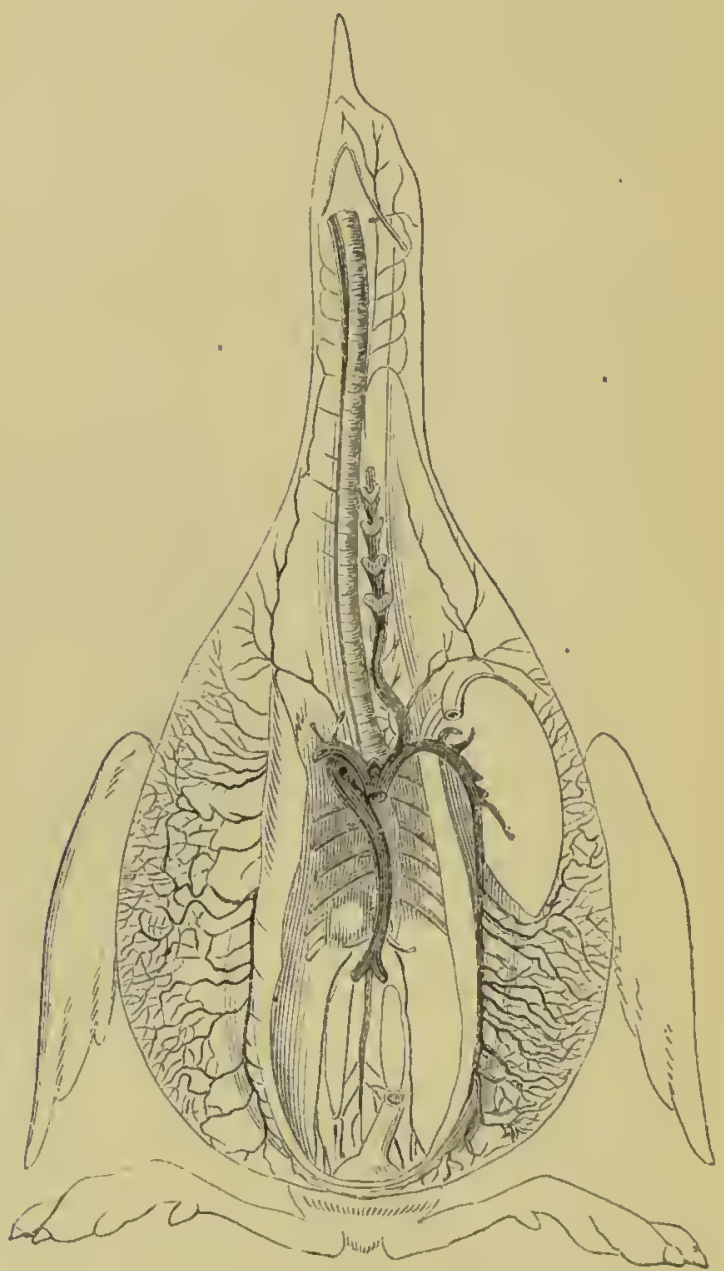

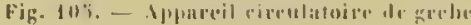

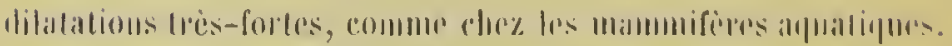

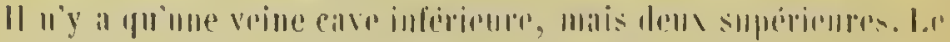


nomine (lo valumles lalls las somes plus allswi glandul 1ןte?

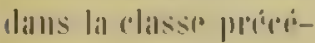
lents:

I, systime porte

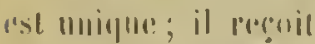
le siln des edtrimi-

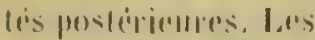
galliglimas lymplati-

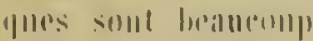

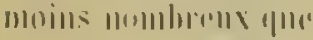

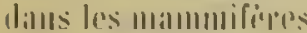
(4) la rallal thorachi-

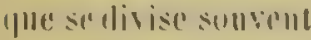
rll demx trones anas-

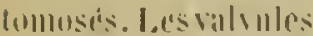
:(0)

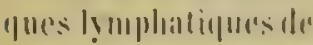
la rexion eamblales" r'muissint dills simls à pallols IIIIsrolenses porto former III fexerr lyוmbliati-

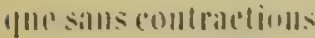
fhy laminues. Cost surtout daus l'antou-

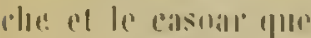

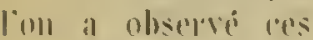
enells. L,elll inti-

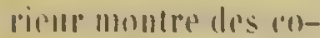
lomlles chillumes ot il rxiste lles valunles a lemrs nofifers. ()n ilait somperomeme lom "vislemor par allaloyic.

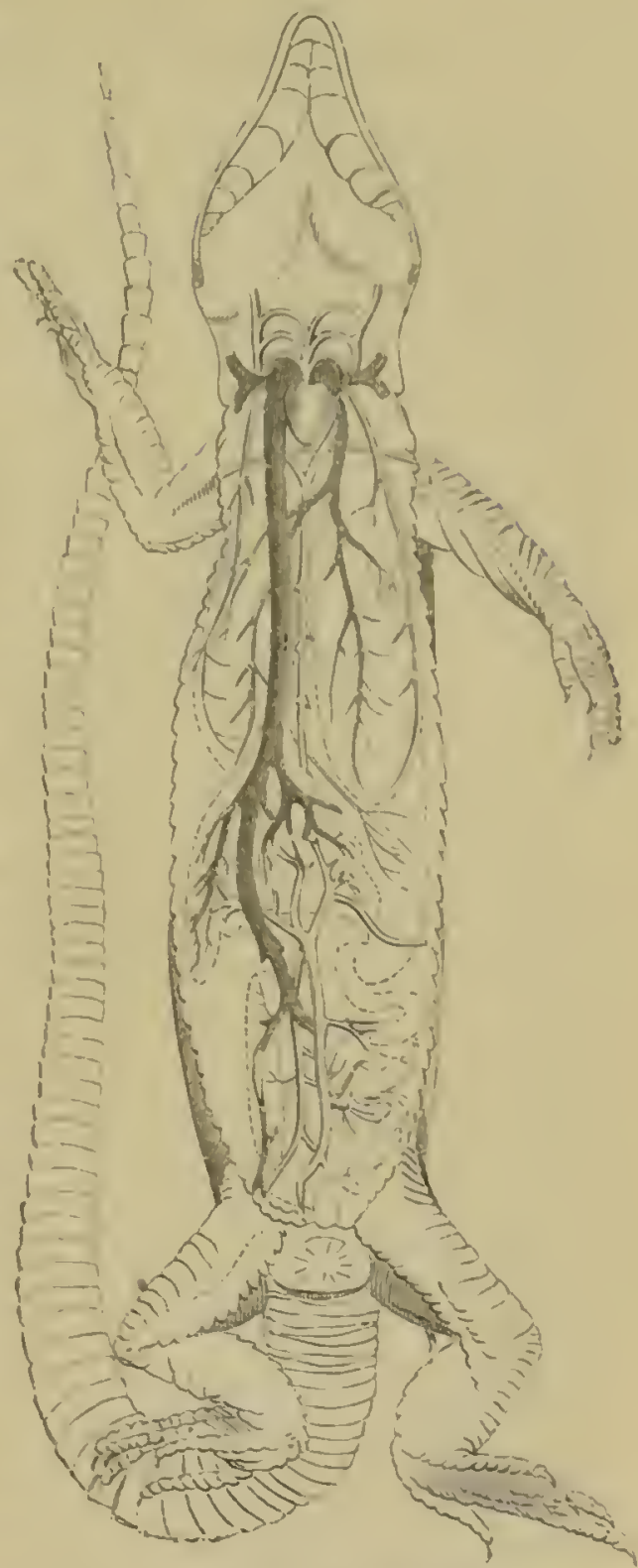

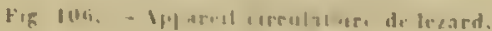


Lal cireulation des reptiles et des batlaciens a lien encore d'après le mème plan que celni qui cst suivi dans les classes préredentes, alvec celle dithérener, lontefois, que le singr ronge se mile avec le samg noir comme dims le foetus des animaux i sange chand. C'est la reprecsentation d'un aige fuctal (lig. 106, 1. 109).

Le cour est encore cutoure d'un prericarde, anyuel il est atlatché daus les chiloniens; il est silue à peu près à la mème plate; chez les chéloniens, crocodiles et ophidiens, il est toutelois situé un peu à gallucle et plus en arrière; daus les chéloniens il est très-largege, landis qu'il est long et étroit dins les serpents. On voit dins son intérienr deux oreillettes qui sont séparées par une cloison complite. Il cxiste deux valvules i l'entre des veines du corps daus l'oreillete droite; ces valvoles manquent à l'uriliec de la veine pulmonaire.

Lal choison interanticulaire tome naissance, en se prolongeant, ì unc vilvule semi-lunaire, plus développée du côti

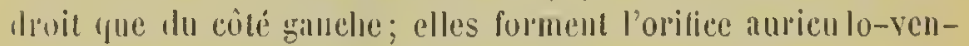
triculaire pour empècher le retour du sints.

latriere fulmonaire mat d'mu simus dams le ventricule drojt et montre pris de son origine deux valvules semi-luhiaires.

Les balraciens (fig. 107, p. 111) n’ont qu'un seul ventricule, tandis yu’il y en a deux daus les reptiles; tontefois ces deux ventricules ne somt séparés pill une cloison complète que dans les crocodiles. Ces derniers, sous ec rapport eomme sous bien d'autres, se lapprodhent done des mammifères. Malyré la présence des cloisons interanriculaires el interventriculaires completes, les crocodiles ont du samg uciéclans les artères pui maissent de lanortr vers le milieu du tronc (figr. 108, 1) 112). Clun' ces animaus, le camal artímel, qui s'oblitire dans les mammifères inmenliatement après la maissance, uon-sculement persiste ici, mais if prencl même un assez grand developpenent, in print qu'on l'a

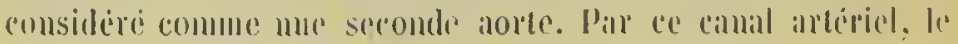




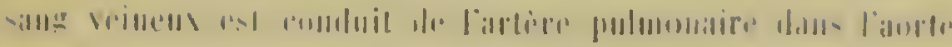

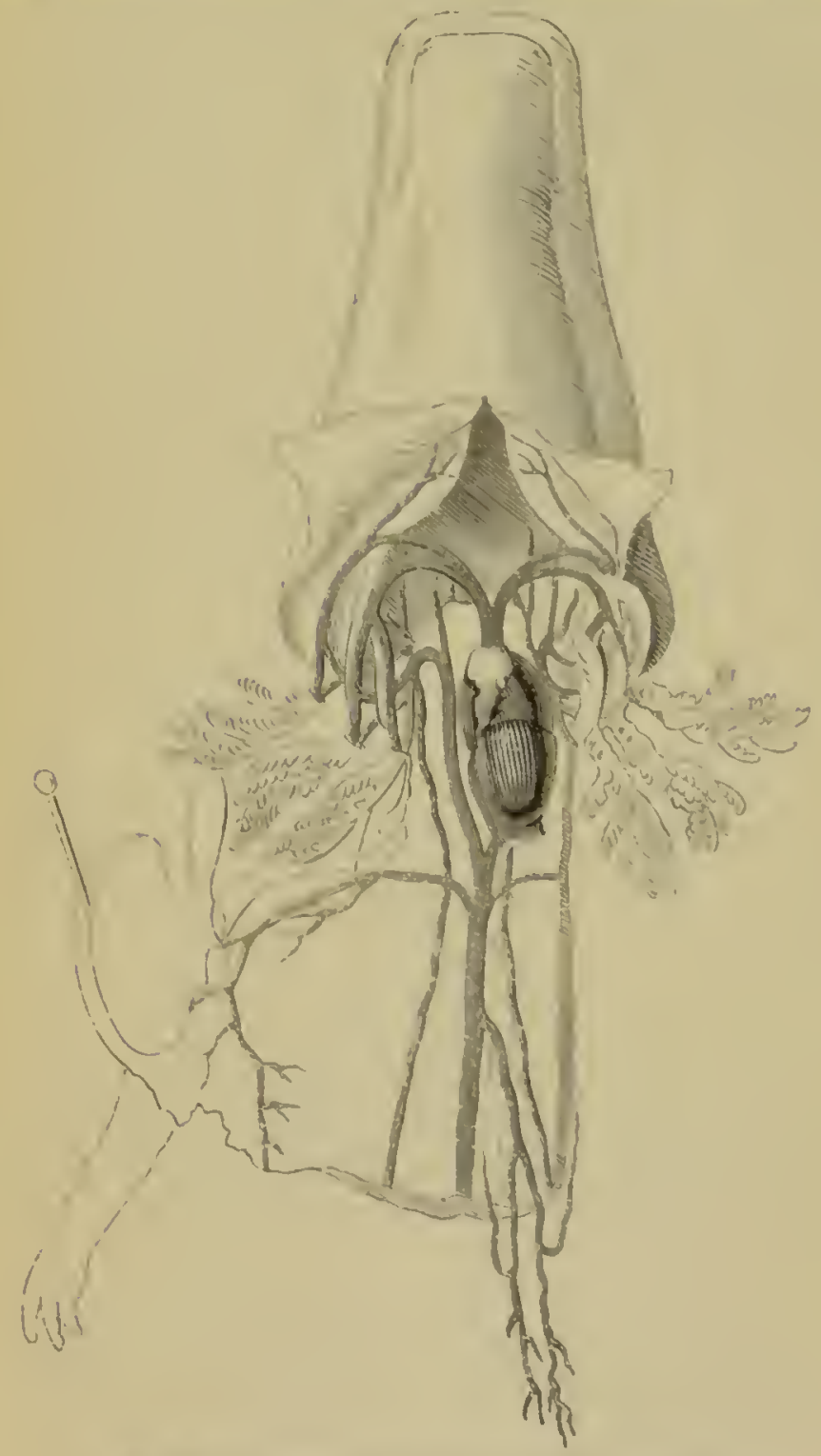

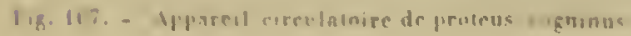


proprement dite. The autre disposition propre anx crocodiles, c'est que les deux bulbes de l'aorte el de l'artère pulmonaire sont accolés f'un à l'autle comme un seul trone, el que la paroi qui sépare les deux cavités est percée par ume ouverture; c'est comme un antre trou de Botal. Toutefois il y a trés-peu de sang

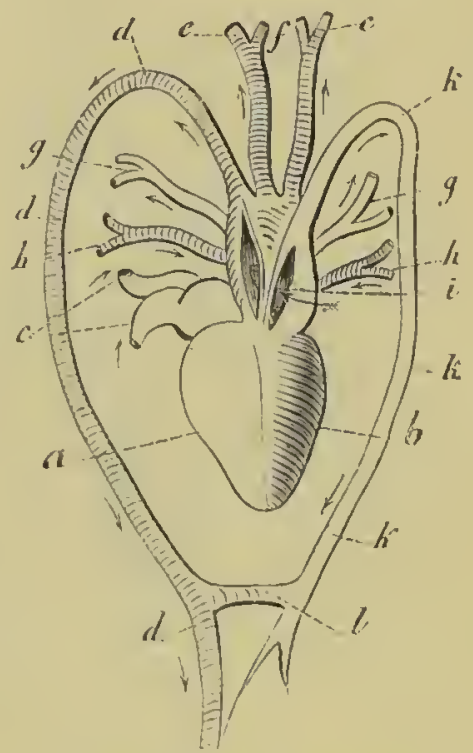

Fik. 108. - Cieur de protodile

"Ventricule dioit. - b Id. ganche. - c Veines caves. - d Aorte droitr.e Artéres sous-clavières. - f Artéres carotides. - y Artires pulmonaires. - h Veines pulmonaires. - $i$ Bulbe, montrant une cloison, percée par un orifice; la néche indique la direction. - li Cinal artéciel on aorte gauche rempli de sang noir. - IJonction de ce canal avec l'anter.

noir qui peut se mêler avec le sang rouge ì travel's iette ourelture, trop petite pour livrer passige à un courant de quelque importance.

Ins alderes ne presentent rien de remarquable. Liatere pul- 
monaire et liorte massernt du criti droit du ventricule; leulv

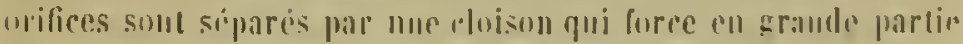

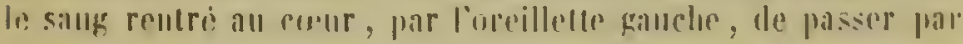

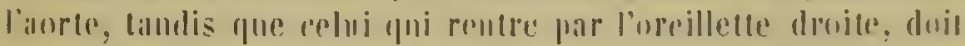

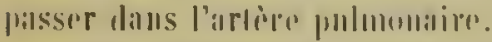

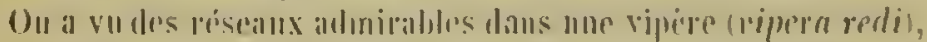

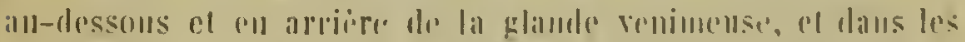

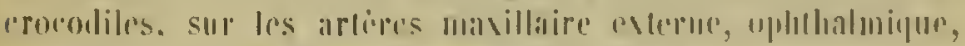
postérieure, otlumë̈lale ot masillaire interue.

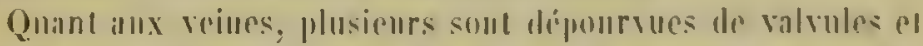

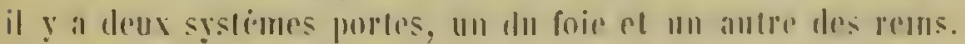
'Tonteforis, lo sycteme ponte rinal ue semble pas avoir l'impurlance pluysinlogiene de l'aulre.

I. a's vines du eorps almutissent à un simus contractile. qui, pourvo de filores musculitires, sonve dans l'n reilletle dioite.

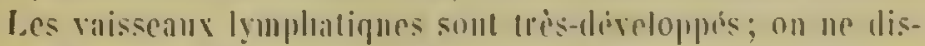
liugue guire de gangliuns; il y a un ganglion mersaringue dans les crnendiles; les vilvules sout ritres diuls les vaissenux lymphatiques de celle clasie, mais il y a puelepurfois de larges sinus oul varices daus lespmels la lymphe s'épanche, el sur le

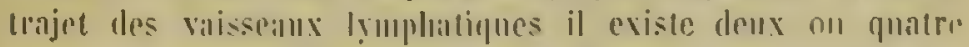
roums qui sont des camrs de en nom. Dans les grennulilles, par exemple, en plaçant l'animal le dos en haul, on aperẹoit de chaqque cóte, daus le pli formé entre la cuisse el l'abdomen, i une tris-pelite distance de la colonue rertebrale, un enur lym-

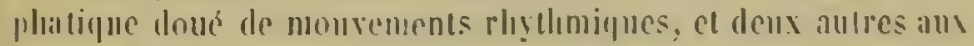
ipaules a la lanteur de la trnisieme vertibre cervicale. Ces rours lymplatiques commumquent arec les vejurs. Ils ont do dreourerts en méme temple a Berlin, pall J Iluller, et il Pavie.

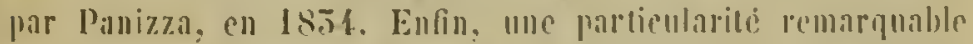
des vaisseaux lymplatiques, c'est qu'ils forment sumrent uur saline autunr des arlieses des veines, de sorte que les parois de ces derniers vaisseaur sont baigues dans la lymphe. II y a aiusi des vaisscaux rouges au milieu de valsseaux blames.

lars eours lymphatipues ont che observés lans les divers oriles de reptiles comme dans les hatraciens. L.eurs coutraclinus rlyblumiques ont éti remarquécs surtout dhez les chélo- 
niens et les batraciens. Ces cours poussent la lymplic daus les veines.

On dislingue dans leurs parois des fibres musculaires striées transversalement.

A l'entrée des vaisseaux lymphatiques dans les veines, ou voil des valvules qui empèchent le sang d'arriver dans le eocur.

\section{POISSONS.}

Cel appareil subit dans les poissons des elangements assez. uotables, tant dans la conformation de quelques organes que dans la marche qu'il fait suivre au sang (fig. 1(09).

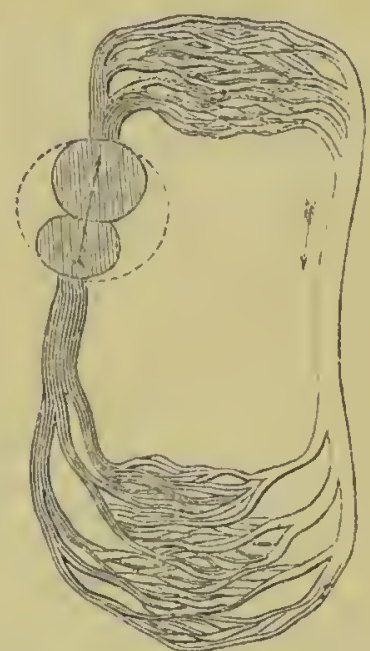

Fig. 109. - Ippareit circulatoire idéal dey poissons.

Le coenr est généralement situé dans une cavite distincte, en dessous des lames brancliales. Un péricarde l'entoure. II se compose de deux eavilés seulement, d'un ventricule tris-musenleux à parois très-épaisses et d'une oreillette sourent spaeicuse 
i partois minees el ponrve de valvules (tig. 110). Il ne contient yue du sang velueux. Lal cilvite, dians lapuelle le earur est sus-

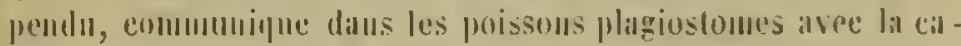

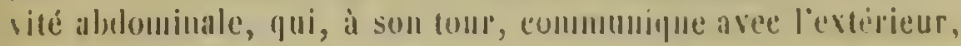

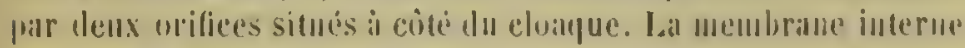
porte un épillsélium vilsıatile.

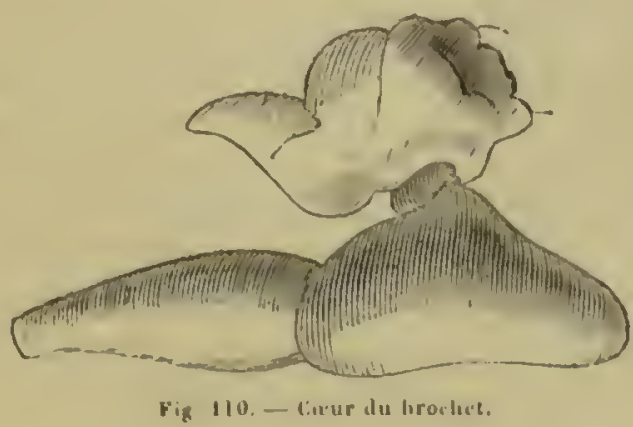

I.e troue prineipal arlipicl, pui mail de lextremite antériemre

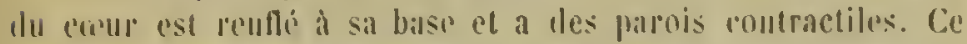

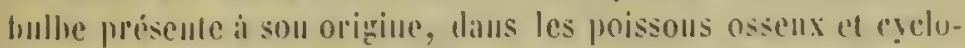
stomes, dems valuules semi-lumaires, tutulis que les poissums galmoides ot plagiostomes en out de leux a cimy rangées transversiles. Dans des deruiers poisons le bulbe arteriel est, pour allsi dire, la eontinualion du veutricule. Ce valisseau se llivise, il l'origrine de lappareil respiratoire. ell aulant de rameanx qüil y a le branchies, el le sang, après avejl traversé le lésean capil-

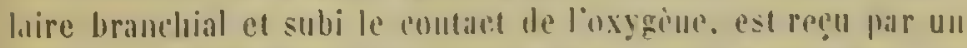
lamean, qui abandonne la brauchie et se remit a tous ceux qui proviennent des autres branclues. Cest par ectle réunion que se forme laurte. Elle est située immédiat tement an-tlessous de lit

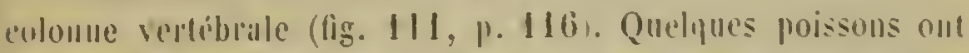

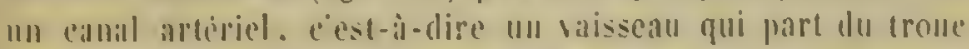

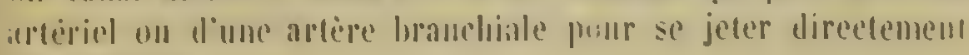
dims latorla.

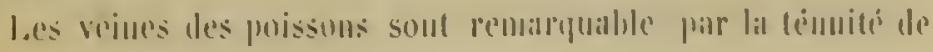


leurs parots, ainsi que par les sinus qu’elles forment sourent =ur

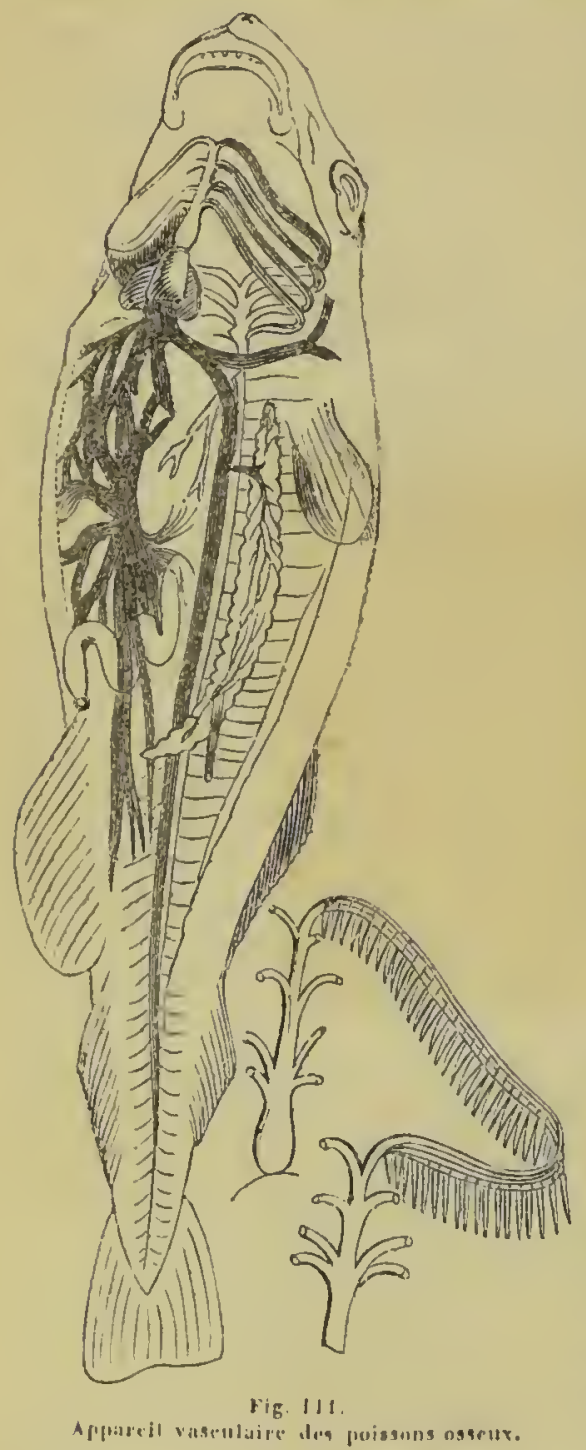
lenr trijel. Elles porlent ralrement des villvules. Il cxiste deux reines-portes comme dans les reptiles, mue pour le foic et mone antre pour les reins; mais il est évident que le systime-porte renal, si on neut lui donner le nou de veine-porte, ne remplit pas plus que dans les alutres verlébrés des fonctions analogues à celles que remplit te syslime-porte pui se rend an foice (fig. 112, p. 117). Lal rém!nion des reines qui rilpportent au coeur lo silly de la péripheriedome hilissance à un lalrye simis veincux qui s'ouire dans lorreillette muique de ces animatux. 11 y a diverses reines dont les parois sont coutractiles; ainsi, on troure yuelquefois un raenr à la reine-portc (mincliostimes el nuyxinoïdes), el dinıs un gramd nombre de poissous un comp veineur i) la base de la queue.

Dins l'anguille, où on a décousel' ce cuur, on distingue les 
rondractions rhỵliniqunes à travers l'épaisseur de la peau, salls falle subir ancene prefraralion all poissun. Chez les lorpilles

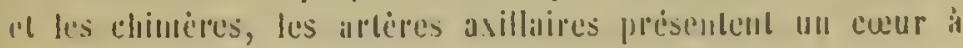
lenr origime.

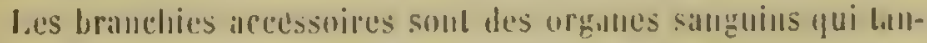

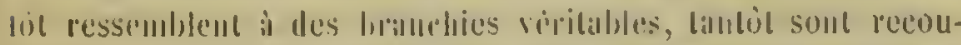

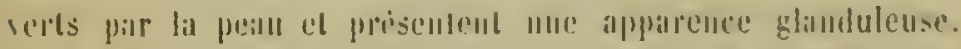

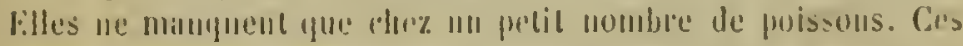

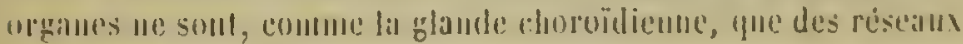
inluiliallis.

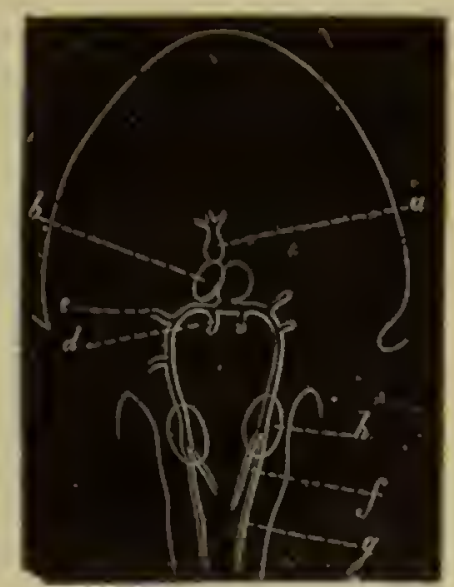

Fig. $11 \%$. M stine beineux di haudroie.

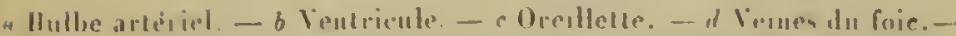

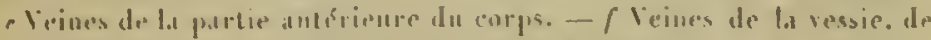

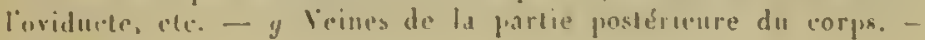
h, Ir.ius.

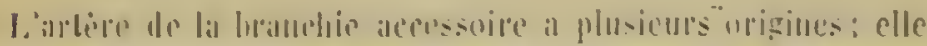

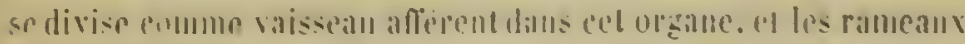

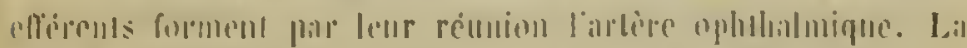
glande chornillienne est forme ell pallie par celle artere. lade

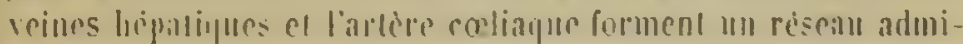


rable dans quelques poissons (lamna), aimsi que lat veine-porte ef les vaisseaux de l'estomac (Sq. v'ulpes).

Les poissous, comme tous les verlèbres, out le sing rouge, à lexception des hranchiostomes; dans ces poissons, si renoryuilbles sous tous les rapports, il est blane eomme diuns les anintanx salls vertebres; au lien d'm cour, ces poissons ont des vaisseanx contractiles comme les annélides.

Les vaisseanx lympluatipues des poissons se distinguent par leur plus grand calibre, l'alsence de valvules el la disparition les glamles; non-seulement les vaisseaux lymphatiques se rapprochent des veines par leurs caracteres anatomiques, mais les diflereutes sorles de vilisseanx se rapprochent ou se confondent.

Les vaisseaux lymphatiques recouvrent les vaisseaux songuins, rt particulierement les veines, au point de les cacher sous leurs l'anilicalions, surtout lorsqu'ils sont injeetés. lls se réunissen! en avant pour souvrir par deux trones daus les veines eaves supirienres; ils communi|juent cependant anssi avec la veine cave infirieure. Le eanal thoracinue est un lacis vascularire.

l'olmanu a pris diverses veines, surtout autour des branchies, pour des vaisseaux lymphatiques.

Marclial Itall * a déconvert le cour veineux, situé à droite et ì gaduclie dn corps de la dernière vertèlse caudale elez l'anguille. On voit ses pulsalions a l'wil nu el saus auemne préparation. M. Ilirtl " a tronvé, che\% presuue tous les poissons, sur le mème corps de vertibre, un urgane qu'il nomme sinus caudal (iig. $114,11: 3$ cl 116,1 . 11!)).

Ce sinus caudal ue présente pas toujours des pulsations; il esl double, el chaque partic communique avec l'atre par unc anastomose transversale à travers la colonne vertébrale (ligs. I I (i). Ses parois so composent de trois couches dout une cst museulaire. De clatque côté il est en eommunication avee le vaissean latéral loğć daus le canal du mêtue nom.

Ce vaissean oecupe toute la longuenr de la ligne latérale, el fournit sur son trajet un nombre très-rartiable de branches qui

- Critival and orperimentul lissuy on the rirrulution.

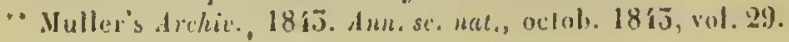




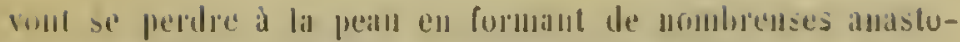
moses an milien desiquelles sont logies les reailles.

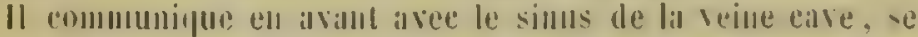

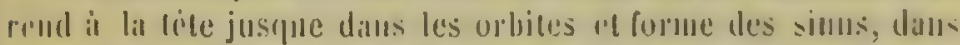

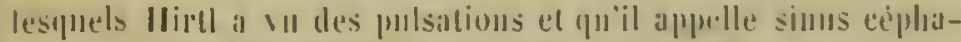
liques. Il contient un liquide blanc comme de la lymplue.

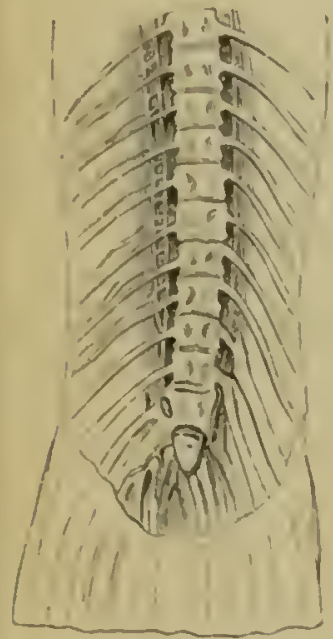

Fin. 116

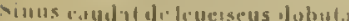
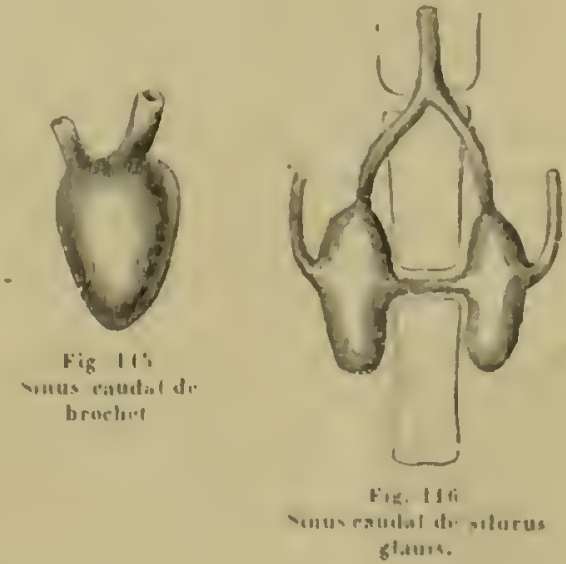

Le liquide contenu doit partio du milieu de la ligure laterale.

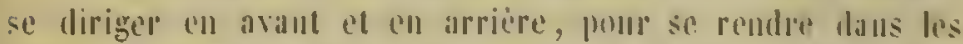

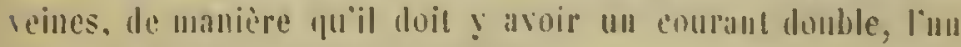
illant en avant el laulre en arriéc.

lliotl pense yut ret appareil est absorbant, el quon doit le

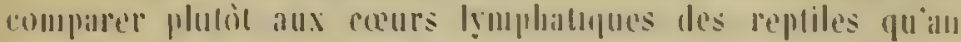

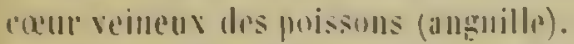

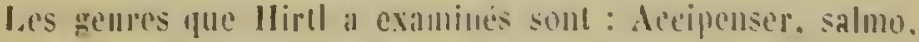
puea, ablamis, leuciseus, gadus, grobio, silurus, esox, cypri-

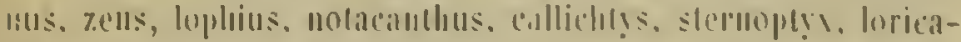
lia, "ry 
H. Gervais a iujecté les ralies entre lat peau el les muscles, el il a vu la miltièe d'injection pénetrer dius le coenr.

l.e cour de l'esturgeou est entouré d'un simus lyuphilipue llapparence grandulaire. (V. Siebold.)

\section{A ITTCULÉ OU ÉPICOTYLEDONES.}

Les animanx de cel embranchement ont le salug incolore el dejourvu de grolules réguliers; ils ont un cour"; quelquefois les artieres, mais pas de veines. Ia circulation reineuse se fail toujours fiar des lacumes.

Si on trouve encore des glohules, ils sont irreguliers el u'onl jaluais la couleur rouge.

Les vaisseanx lymplatiques ont completement disparu daus 1.) He les animanx salls vertebres.

Insectes. - Le sang esl généralement banc ou phutot incolore, at contient une faible quantili de corpus-

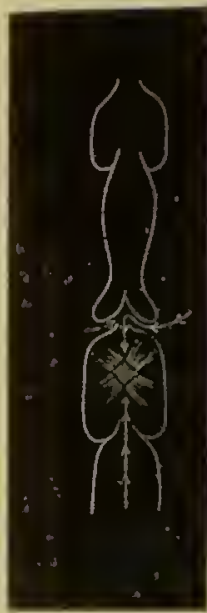

15. 117. liculd des insectes. cules ou globules. Il n'est pas contenu dans des vaissenux propres, mais répandu dans les lanités du corps de manière à baigner les divers orgatles.

Le loug du dos, on apereoit, à travers l'ípaisseur de la peall de plusieurs larves, un vilisseau qui se contracte pregulicrement d'alrriire en araut; éest le coml, lésigné aussi sous le noun de ruissemu dorsal (ligy. 117). Ce cour est divise par des cilranglements ell plusienrs clamulus, gémiralement au mombre de lumit chaque chamber présenle sul le còlé une ourerluce, qui laisse entree le sang du dehor's, mais ne te laisse plus soptir que pal lit chatubre pui precede. Celle ourerture est produite

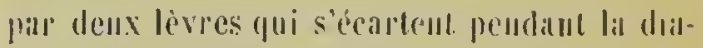
stole el se rilpprocheut pendant lat systole. Ide. sang passe loujours d'arriore en avant; les confluents veineux ahoulissent à la chambur postéricure; de lá le saug passe daus relle qui li précède pl arrive alosi juscqu'il l'antéricure rn passant 
par luntes les autres el en allententant sa masse de liquide de relui qui premetre par les uuvertures lationales de chaque comparrimenl.

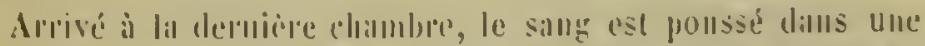

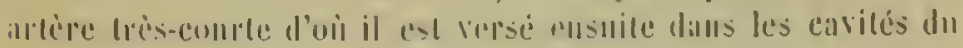

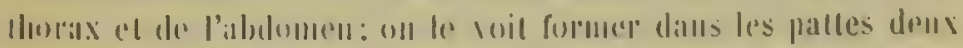

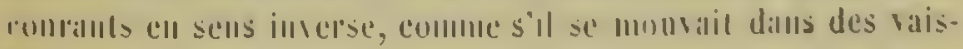
stillix.

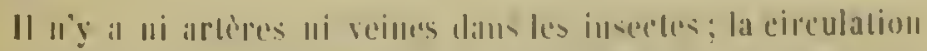

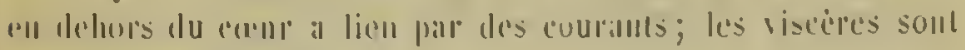
biligules dills: le sillig.

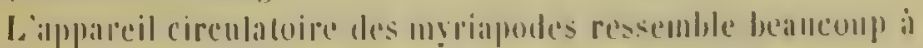

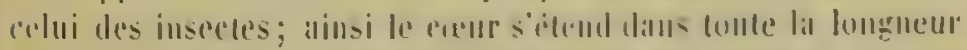
du corps be long du des; il se disisere alls si en chambres pui cor-

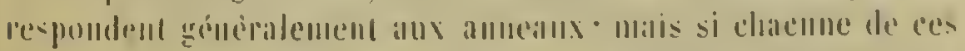

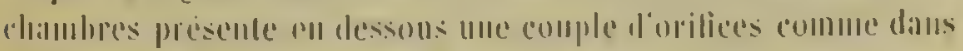

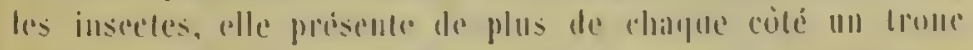

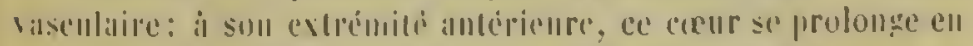

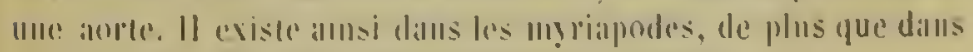

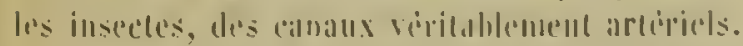

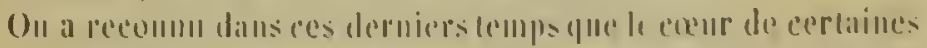

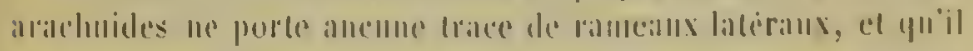

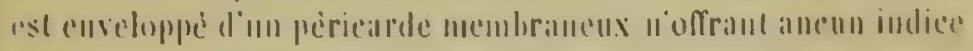
de merforaltion lationale.

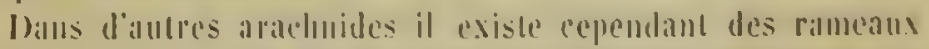

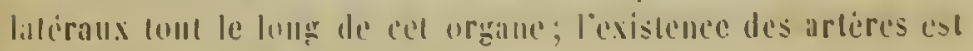
IIII filit mis hor's de loult doule.

Plusicurs arachuides semblent anssi dépourvos, mon-senld-

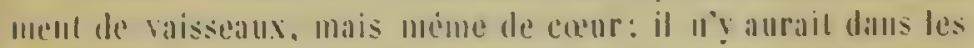

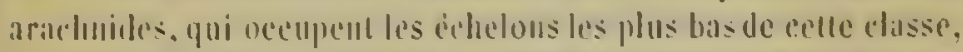

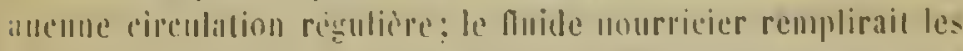
inderstires du conps el me scrait mis en mouvement que par les

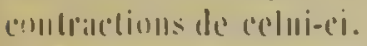

Dalls les cruslates derapodes. lappareil circulatoire est assez

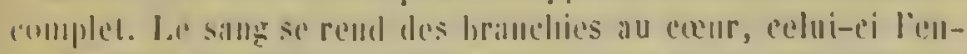

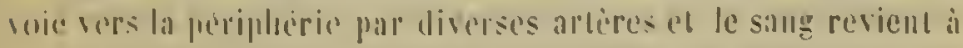
III latge sinus, silue a la bilso des branchies, en passant par lee 
lacunes et lesiuterstices yui existent eutre les viscères (fiz. 118).

Le cour est enveloppé d'un siuus qui recoit d'abord le salng les branchies et qui n'est ni une oreillette ni un prericarde (tig. 119, 1. 125).

Tout cet appareil, et même le cour, semble manquer dans quelques crustacés des lerniers ordres. Il occupe toujours, quimal il existe, le milieu du corps à la partie postérieure du céphalo-

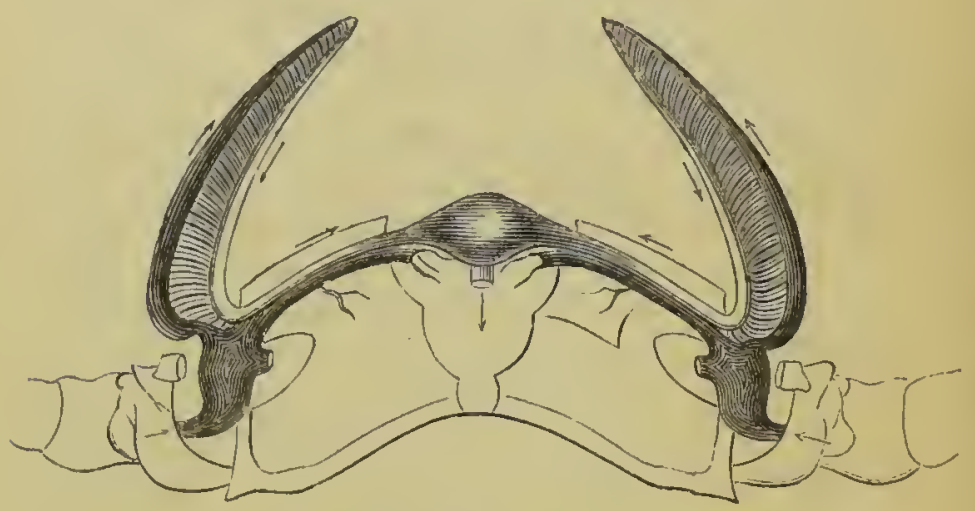

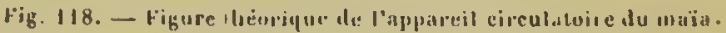

llorax. Dans les crabes el les ecrevisses il a une forme calrece, ses parois sont assez épaisses et on aperêil dans son intérieur ues colonnes charmues. Les viisseanx yni aloutissent au cour, comme ceux qui en partent, sont ponrvis de valvules qui empêclient le sang de revenir sul ses pas.

Le cour contient du saug qui a passé par les branchies, et des artères distinctes le conduisent du earm ver's les organes. Ces artires manfuent seulement dans les ordres inférieurs, el les veines, au contribe, manquent dans tous les animaur de la lasse.

Si unus pronous pour exemple les crubes el les ecrevisses. nous voyous les divers commuts veindex se roncentrer it la base des piltes, se rendre ensuile aus branchies, reveruir de lid

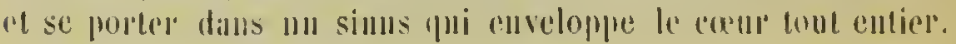


APP.ARE.H. CIRCUI.ITOIRF.

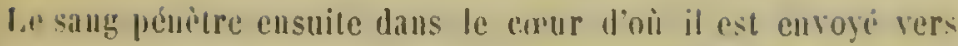

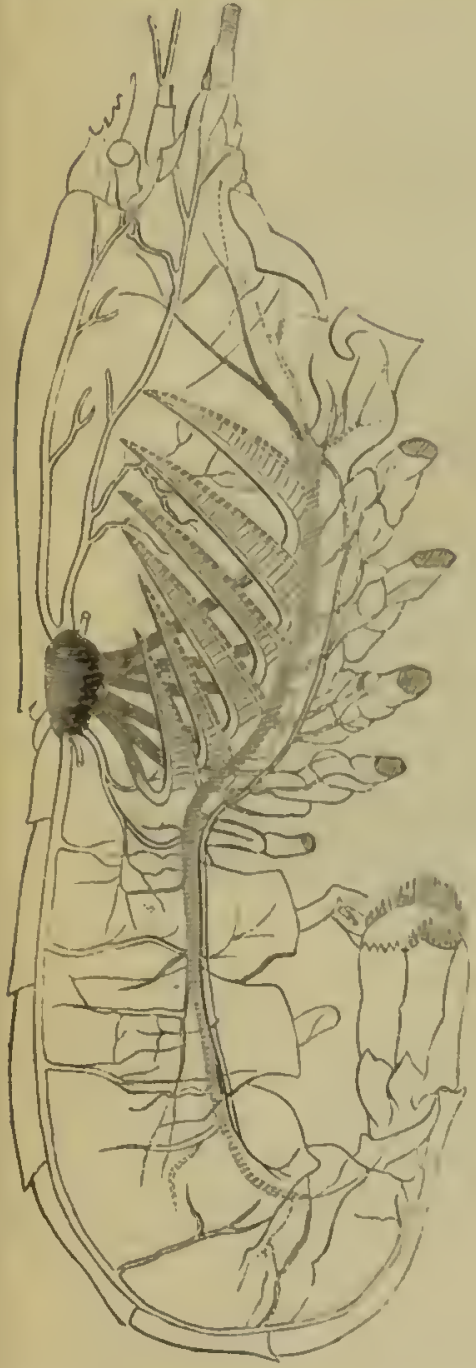

Fig. (19. -Appareil circulatoire du homard.

lat perijpléric plat plusieurs iatiòres.

EII somme dour, l'apjlitreil cirrulatuire est incomplat dalls lines less artirulis. puisinue les veines manqueut ('t ylle la circulation reilleuse s'effectue par des liarlliles.

Mollusyues, - On a iru. jusques dints ces derniere anues, que les mollusques out tous unn-soulement un corur, mais aussi des artirecl des veintes; cest uncerreur: le dernier ordre, les bryozoaires, est prives de rour el de toute trace do vaisseaux; les aures solt géuéralement privés de reines. Le sang esi, à quelques exceptions pris, incolore.

On peut cousiderer te cour des cephalopodes comme compose de trois poches (fig. $120, p .124)$ : deux oreilletles et un ventricule, unais qui sont séparées toutes les trois; il y a unc oreill lette isolée contenant du sillg veineux, à la base de chaque branchie, et uı ventricule, isolé aussi, ntais situésur la lig̣ne uédiane entre les deux branchies. C'est ce qui a fait dire que ces animaux ont trois cecurs. I.e ceur principal reçoit le sang qui revient des 
branchies; il est par conséquent à sang artériel; ses parois sont ipnisses et musculeuses; l'intérieur est tapissé de colonnes charnues, et l'orifice des vaisseamx est garni des valvules qui cmpêchent le retour du sang. Ces cocurs sont doués de coutratctions rlıytlımiques.

Il y a plusieurs arlères qui partent du cour et qui vont porter le sang aux organes; elles ont les parois assez cpaisses.

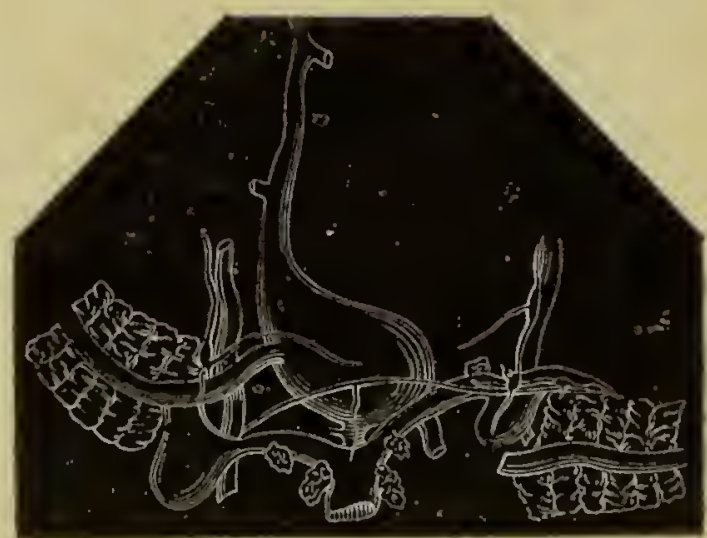

Fiq. 120. - Apparcil circulatuire des céphatopules.

Les céphalopodes ont aussi des veines, car le sang veiueux revient par des veines eaves aux cours branchiaux; ces vaisseanx communiquent tous directement avee les cavités qui logent les viscères ; a insi, sans produire aucune lésion, le liquide injeclí s'épanche dans ces cavités. Il y a done aussi lles lacunes dans cet appareil.

Les gastéroporles ont un cœur doué de pulsations rrigulières; il se compose de deux cavités qui se suivent (fig. 121. 1. 12J); la première, l'oreillette, reçoit le sing provenant de l'appareil respiratoire; de l'oreillette il passe daus le ventricule, et celui-ei l'envoie vers la péripherie par une forte artère aorte qui se di- 


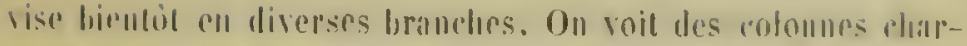

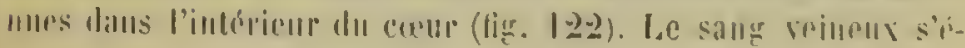

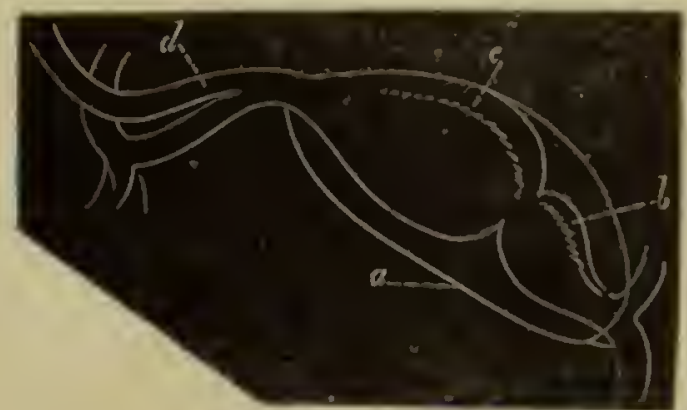

Fig. 121, - Corur d'ligal.

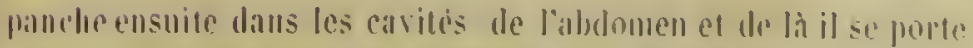
dins les bramdies ou les poumons pour y suhir le contand do l'oụyume.

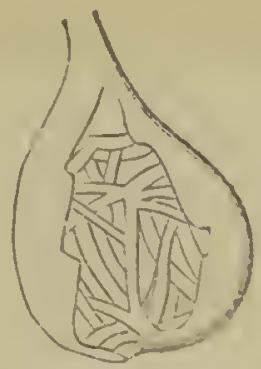

Fig. Iมz. - Ventricule dhyale ouvert

Qundques gasfiropodes (chilomsi oul deux oreilledes latériles: le rour est lratlerse par le rectum.

I. sang est ronge ou rougeitre dans les plamorbes, landis qüil est blane dans les alltres animau dre ce grompe.

Dans les mules, parmi les arephales. dems orcillelles reeni- 
vent le sang qui revirul des loranchics, el elles le versent dans III ventricule à parois:

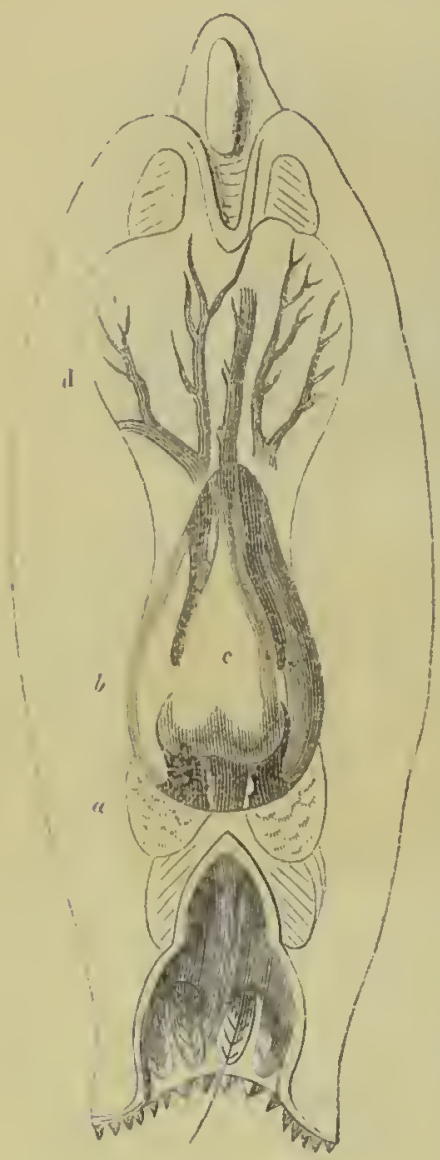

Fig. 123

Appareil circulatoire de l'anodonte.

"Rectum. - " Oreillettes. c Ventricule. - d drtères. musculeuses silué sur le reclum (fig. 125). Ce ventlicule chtoure l'inteslin comme une hague entoure le doigh; il existe des arlères qui portent le sang aux organes, mais les veimes manquent. Le sang veinenx revient aux branchies jar des lacumes. Dans d'autres acepllales, le cocur est isoli (fig. 124. 1. 127) ct lle coniractc aucune adhérence aver: les intestius.

Liapareil circulatoire le plus r'cmarquable est celui des luniciers. Dans ces mollusques, ou olserve un vaisseau i) contractions rlythmiques; il communique d'un côté avee le réseau eapillaire de la branclic et de l'autre avec la cavilé qui loge les viscires. Cer valissean se contracte pendanl quelques imstants de droile à ganclic, puis le mouremeut sarrète lorusquement, el pen de temps apris les pulsations recommencent; mais au lieu de se coutracter le moureau de droite à gantele, il le friit dars un sens opposé, et il pousse le liquide dans une aultre direction (lig. 12:i, p.127).

II n'y a pas de vaisseanx dans lintirieur du corps, si ce n'esl ceux qui forment le réseau branchial (iog. $126 \mathrm{p}, 128$ ). I'ur 


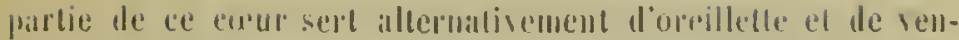

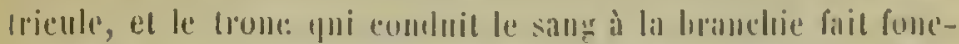

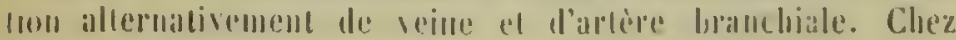

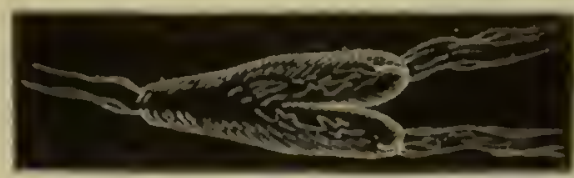

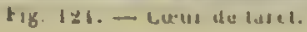

quelques-uns deotre cur (ascidies), M. Milue bolwarels a obspipe dil silng ronge.

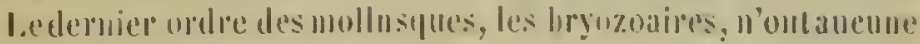

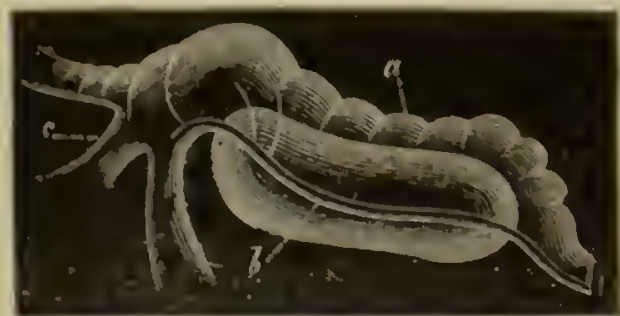

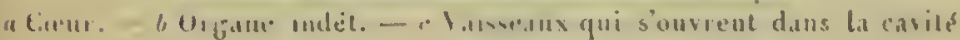
perigistrique.

Fig. 19s.- Cieur d ascidic.

Irace d'alpareil éréulatoire. L̈n liquide remplit la eavité périintestinale; il se meut parl laction de cils viluratiles, se rend dams le's appendices droits qui forment la couronne branchiale et

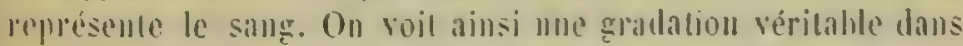
col appareil, depus ces derniers juspluas cephalopoles.

lirs. - Cet apparesl presemte des modifieations profondes dians les animam de eelle classe: il ! en a beaucoup yni nom pas de traces de valisealux, tandis que diutres ont un resealu

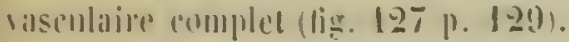

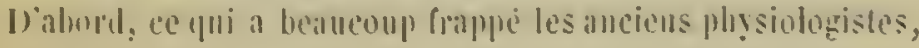


r'est que le sang est rouge dans beaucoup de rers, et on al mème cru, jusques dans ces dernières ammées, qu’il était rouge

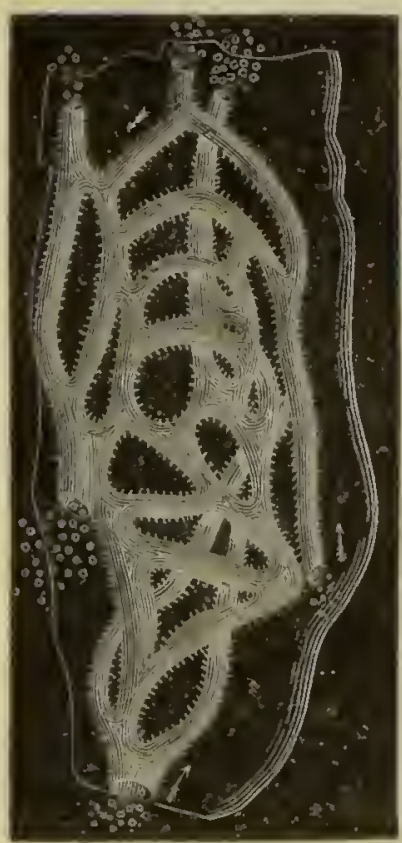

Fit. 126 .

Parois du sac bronchialde l'ascidic aw z̧ulloille. daus toutesles anmélides. Malgrré l'identite de couleur avecles animaus vertébris, eette coloration n'est pas due ici à des globules, mais c'est le liquide lui-mème qui cst louge, comme il est vert, jaune el ineolore dans d'autres. Un phénomène très-curienx observé d'abord par Delle Cliaje, c'est que le sang est vert ou rouge dans le même ver, l'apris l'aceumulation du liquide dans les vaisseaux. Dans le sang de quelques vers, mous avous ru des globules liguliers mais contpétement ineolores; la grande maforité des annélides n'a toutefois pas de ees glolules dans le sangr.

Chez plusienrs anmélides, on roil dans tonte la longueur du corps des lrones vasculaires contractiles gui jouent le ròle de ecur. Dans lil singsue, on en distingue un le long du dos, un autre le long du ventre, et puis sourent encore deux latéran (fig. 1297 p. 129). Comme tout le vaisseau est eontractile, le can n’est point encore locilisé. Toutefois, clè quelyues annélides, on roil tris-bien le corur se former; sul le trajel du trone principal il apparaît une ou quelques dilatilions vilriqueuses, dans lesquelles se concentrent les contracliotis rlythmiques. Ces vaisseaux longitudinanx communicuent entre cux par des anastomoses et sont parfaitement clos.

Chez quelques vers on roit une partie des vaisseans, par cxemple le vaisseau dorsal, ètle contractile, landis que les alutles ne le sout pas; il apparait quelquefois des vesicules eontractiles sur leur trijet, comme dans les branchellions. 


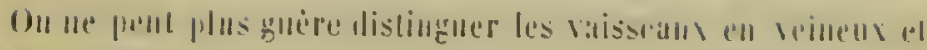
rilliteriels.

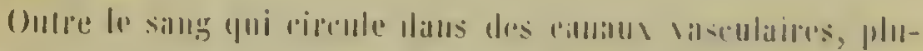

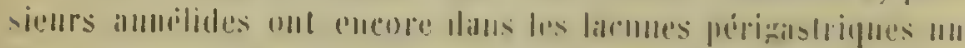

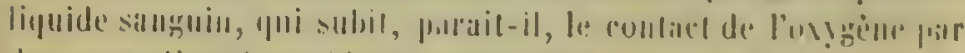

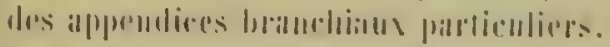

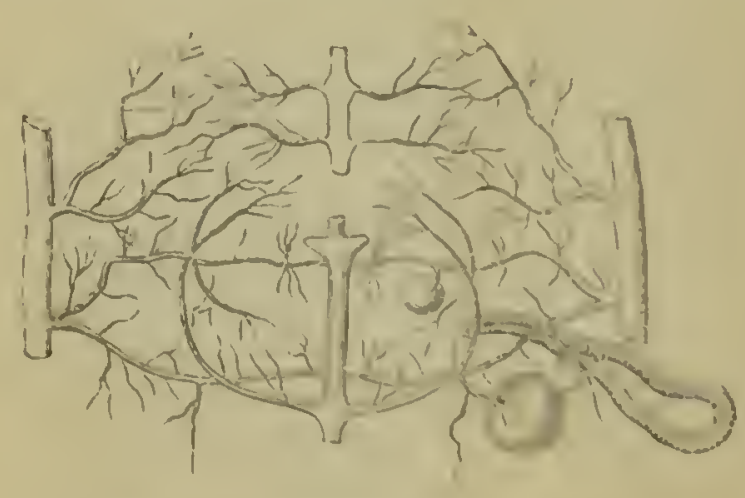

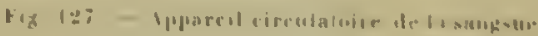

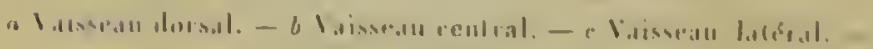

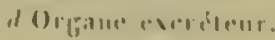

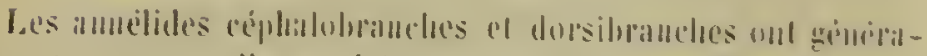

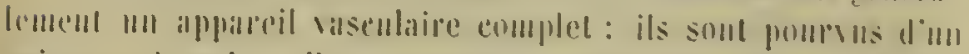

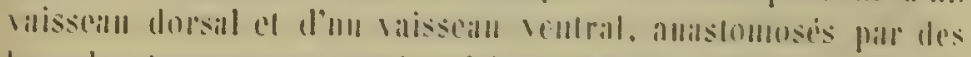

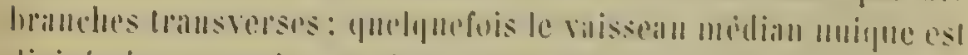
divisé plus nu moins e'l dem dromes distinets (ligr. 128).

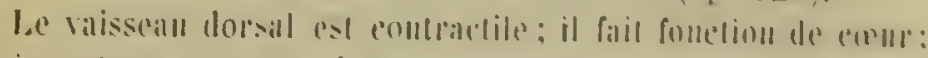

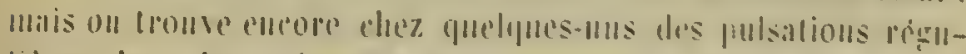

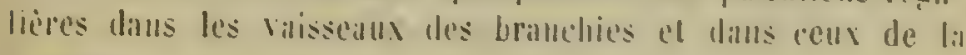
trompe.

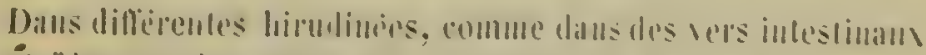

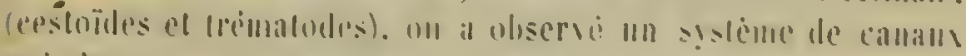

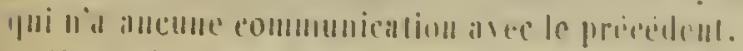

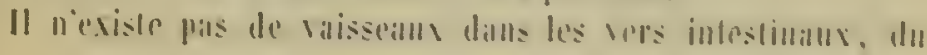


moins pas dius les trémitodes ni daus les eestoidles; e'est à tort que l'ou a pris puur tels des canaux excréteurs. Ces prélendus vaisseaux s'ouvrent à la partie postérieure du corps, et

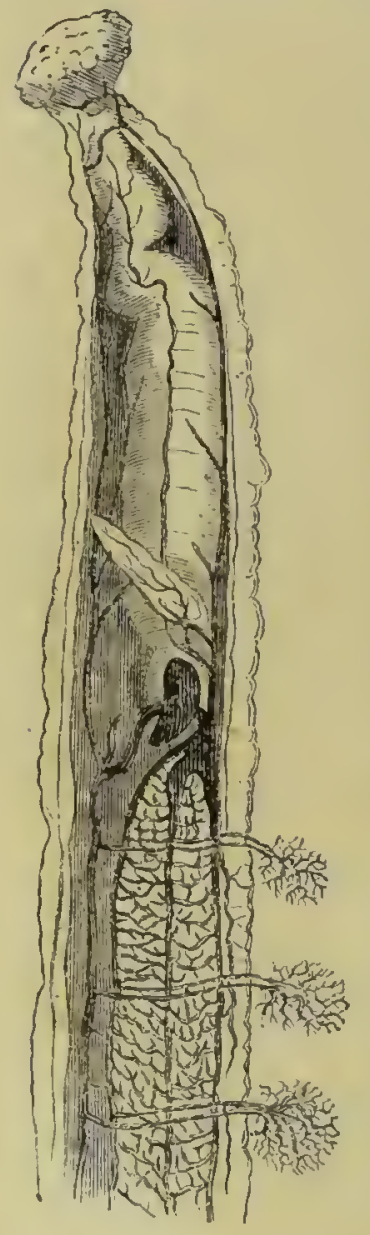

Fig. 128

Arésicule des pétheurs: appareil circulatoire. aboutisseut a un organe pulsatile situé dins cet cudroit; le contenu suil loujours la mème direction dans le mème vaisseau.

Eihinodermes. - Les éehinulermes sout pourvus d'un systime vasculitire singuiu, qui comprend un tronc illtériel et un trone veineux; ce syslème est isolé. Il parait y aroir aussi un rudiment de corur. Dans les étoiles de mer, par exemple, il existe trois anneaux vaseulirires; denx, autour de l'ouverture de la bonelıe, un sous la peau du dos, et entre eux on voit uı cocur allongé qui part de la plaque madréporique pour se rendre à l'ouverture de lis bouclie.

Acrilephes et polypes. Il I'existe plus d'ilphatreil eirculatoire proprenent dit, à cantutux fermés et sills communication au delsors. On voit au eontraire cliez beaucoup d'icalèphes, des eanaux partir de la cavité qui reçoit l'eau, se ramifier et s'illastomoser dans l'ejıais. seur les tissus (fig. 129 p. 151). On semble en outre avoir observé dans quelques aealiphlies de véritables vaisseanx situés le long de ees eanaux.

Dans les polypes qui vivent elt comInunauté, les estomaes les divers individus qui conıosent une colonie sout en eommunication; le liquile pent passer de l'estomac de l'un it celui de l'autre (royes lig. 67, p. (i9), el ce passage a éte pris pour un 
mourement circulintoire. Il l'est dans ce sens que le liquide charrlin en mène trmps la nourriture el losygène.
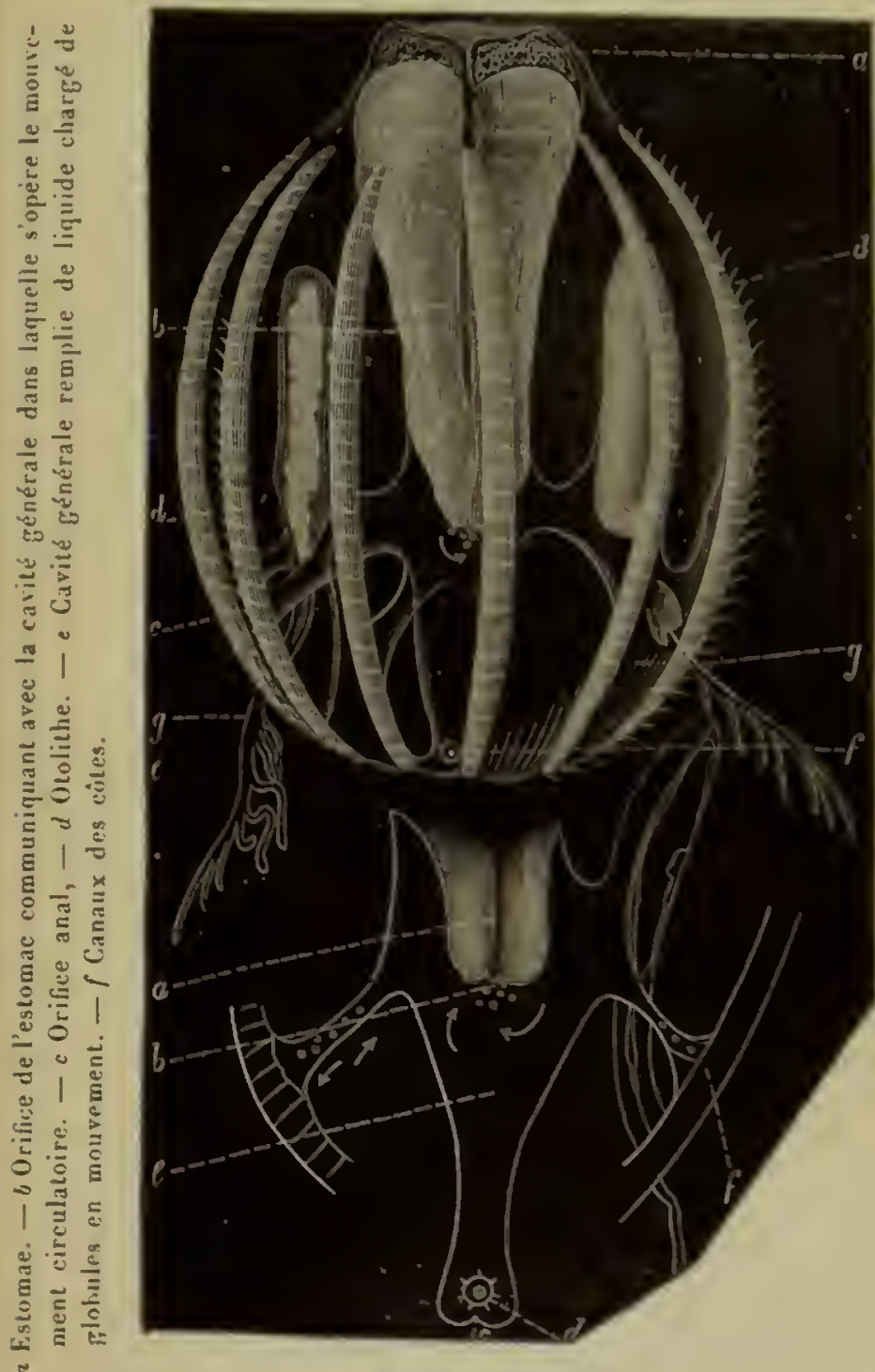

Fi: $\mid z !)$ - Rerne pileus 
Dans le cydippe pileus (figure 129, p. 151), il existe une cavite digestive distinete et une large eavité eireulatoire qui est en eommunication directe avee la premiere; à certains intervilles, le cul-de-sae de l'estomae s'ouve el laisse echapper un liquuide clitl'ge de globules; ee liquide se meut alors dans eetle

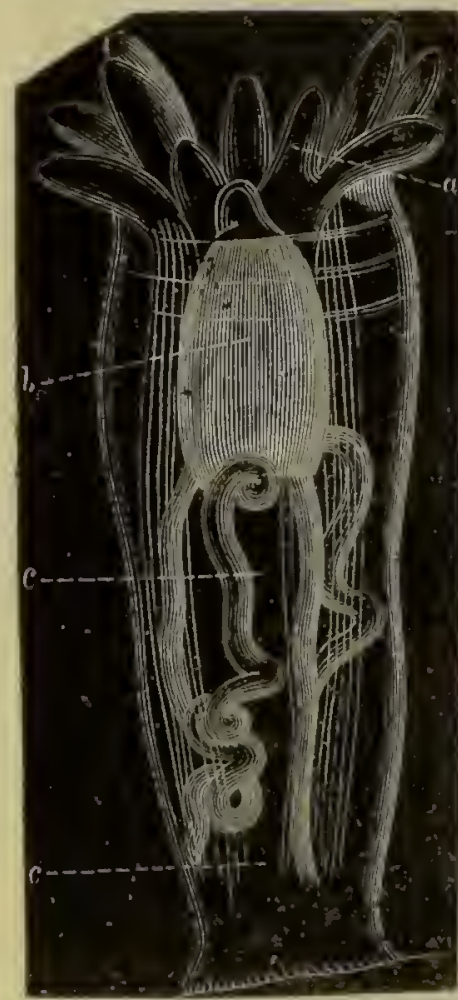

Fig. 150, - drtinie.

"Tentirules. - b Estomanc. c Civilé générale. eavilé el se rend dans les ealmaux longitudinaux sous formuc de côles ct dont la face exterice porte d'cinormes cils viblatiles; c'est le premier exemple, cu remontaut l'échelle animale, d'un lipuide envoyé dans des (anaux propres servalut ì lil l'espiliation. Il n'est pas doutatux, en effel, que ces huil rótes des cydippes ue reprísentent l'applareil de respiria(ion).

Les aclinies (fig. 150) selllifent aroil la cavité digestive sćparce compléteneut de la cavité grénćlale du corpes, el la miltiere mutritive doit daus ce ras passer a lravers les parois: il 11 'y a pas de virisseaur; toml le sally baighe le cantal alimentaire comme dians les bryozoaires; ou voit le sing entrel' daus les tentacules et revenil ensnite dans la eavité générale.

Les lygdres mresenteut te mène momenent eireulatoire. avee entle différence que la ravite des bras communique directement, nou avec la cavilé périgrastripue, mais avec lal dariti de l'estontac elle meme, comme daus les acaliphes.

Infusoires. - Ces organismes simples, etant entièrement permubbles a leau qui les entoure, u'ont évidemment pas besolu 


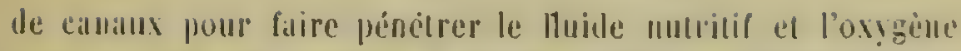

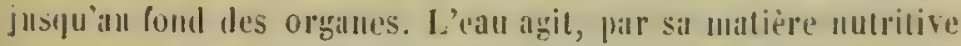
rel par son oxygène, sur la pealn exteriente el sur le parenchyme dil eurps.

Daus le loxodes bursariu, ou init encore un mourement circulatoire et, chez plusienrs antres infusoiles, on distingue une on diverses car ilcis dont les parois sont loués d'un monvement de systole et de diastole.

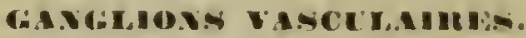

Quelymes orgalla's doul les fonclions soml encore problema-

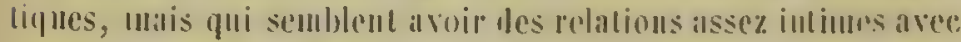

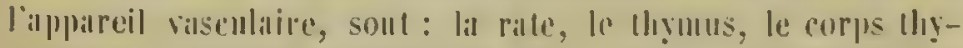

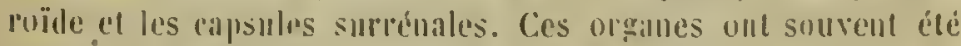
désignes improprement sous te nom de glandes; ils ront pats le callal excreteur.

\section{II. I'T:}

On trouve une rate dans tous les animanx vertebris, à lex(eption des brauchiostontes; elle manque au coutraire daus lous le's animilnx salls verlibres; son volume est generalentent eu ralpport aree le volume du foie: elle est plus developple dans les eallilissiers que dills les lerbivores.

Les roppuseules de la rate sont des simes ou des varicosites lymphatiques; ou les nbserve daus les carnassiers comme daus les lerbivores. Des vaisseanx sauruins sont distribués sur leurs parois.

On roit me rate étroifement unie à lestomile, chez tous les utammifires, el surtoul it la premiere poche yutind il y ell al plusients, pall exeluple. dhez les rumullamls. Lal rate est simple el barie dalls sil forme selon les ordres. Les simiens out me rale

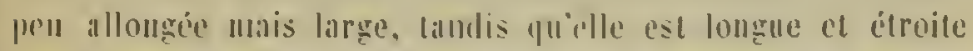

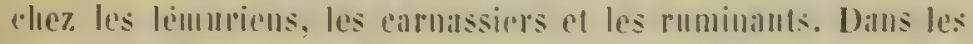
daupluins. on trome des ralles alecessures dunt le mombre silive quelequefois jusqu ì dis-seju, comme il a ete reconnu dans le IIIIrstuill. 
La rate est formce de deux lobes, dans les monotrènes, et. elle affeete la forme d'un ' $T$ dans les marsupiaux.

Les oiseaux out tous une rate proportionnellement petite; elle est en géuéral de forme splıérique, rarement comprimée; sa eouleur est foncée; elle est située daus le voisinage du foie; clle est extrèmement petite dims le eoueou. Quelques oiseaux ont des rates supplémentarires.

Oı a reeounu aujourd'luni cel organe dans tous les reptiles et les bitraciens; si siluation varie selou les divers ordıes salls qu'elle abandanne toutefois le roisinage de l'estomac. Il y a alussi quelques modilieitious daus la forme el le volume.

Les chéloniens et les sauriens ont eet organe assez grand; cluez les ophidiens, il est souvent adherent au paneréas; il est petit et arroudi elıez les batracieus.

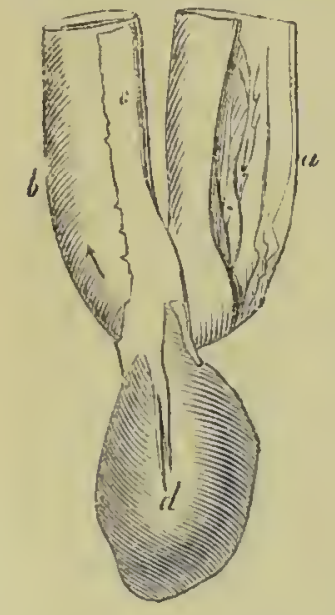

Yig. 131. - Pancrèus et rate de hrochet.

Tous les poissons, y compris les lépidosiren, ont une rate; on l'a reconunc alussi dans les eyclostomes. Chez quelques poissons pligiostomes, ainsi que elıez les esturgeons, eet organe se compose de plusieurs rates aecessorres, réunies par des vilissea ux singuins, On roil une lal te nniIue dans les poissous ossenx en général; elle est simple, de eouleur brume ou quelquefois rouge, de forme variable, mais toujours gorgée de sang. Comme dims less elasses préeédentes, on la trouve dans le voisinage de l'estomise (tig. 151).

Il est à remarquer que le seul a lumal vertébré, dépourvu de liate, est aussi eelui qui n’a pas le sang ronge, fait qui

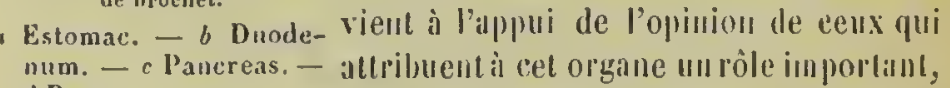
"Rate. $\quad$ soit dims la formation des globules du sing, soit dans leur destruetion. On prétend en effet avoir constaté que les veines afrérentes sont plus elıaryies de globules que les efferentes, d'un il faudrait conchme que la ratr sert a lil destruction des grobules qui sunt mis hors d'usigun. 


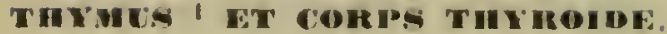

Ces organes oul efli reonnms dans lons les ordres do mam-

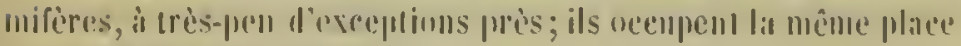
que dans l'espece: lommiaine.

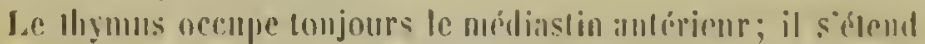
en bas juspuan cour el en laut ses comes font somvent snillio lours de la cavité llovacique. Il parail mampuer dans les marsupiills.

Le llymus u'aequiert lont son développement que clicz Ins mammifieres. C'est vers la fin de la periode fortisle pure cet organe

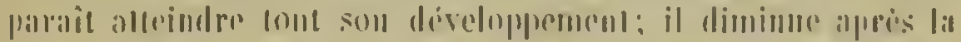

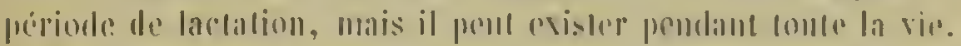

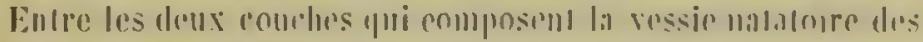

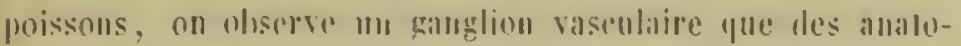
mistes ont compare an llygmus.

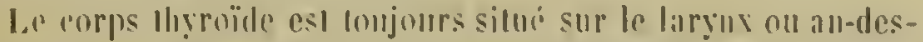

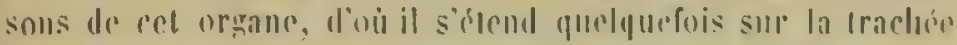

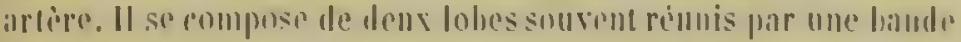

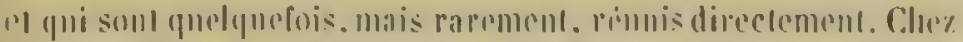

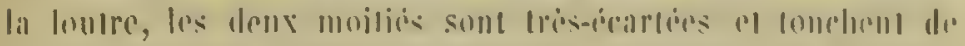

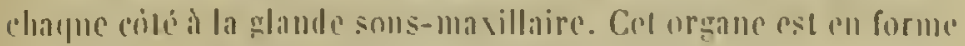

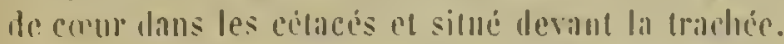

Dans les oiseaux, comme dans les repliles, on Iroure il còti

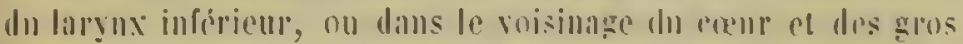
Irones vasculaires, des organes pun volmminem, arrondis on

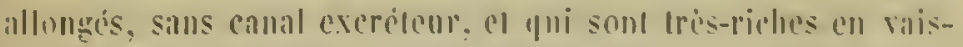

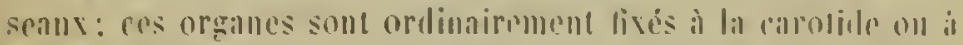
la veine jugulaile: ils correspondent au llymus et an corps llsỵrnïle.

On Irouve enenre des traces de ces nrganes diuns les hialritciens, mais il n'ell existe plus chez le's poissons ed le's animam silns rertibres.

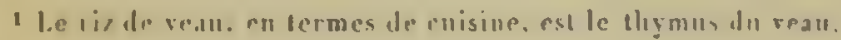




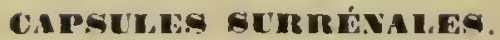

Ces organes ont biti olservés anjomrdluni daus les diverses plasses de vertélurés. Ils sont silues, cllez les mammifires, en delans ou en ivant des reins, ont une forme variable el montrent gréméralement dans leur composition une substance medullaire et une substance corticale; leur volnme est valrable, el sonvent, an moment de lat misissance, ces organes sout plus prtits qu’à l’ảlat ildulte.

Dans les oiseaux, les capsules surrémales sont grises ou d'mu jaune dore; elles sont logées ell avant dans l'épiassenr des reins: elles I'ont point de cavité el sont souvent en contact avec le lesticule on l'ovaire.

Dans les reptiles el les balraciens, ces organes existent géurilialement, mais souvent, comme dims les salimandres, ils sont formés d'un amas de gramules jaunes pliters ell dessous des rins.

La présence des capsules sirrénales a été constitéc chez los puissons ossenx et les plagiostomes; ce sont souvent des grilmules arromdis de conleur jaune, ou que lquefois tles bandelelles itroites qui reconvent les prins ou les urctipes. 


\section{APPAREILS GLANIUUIAIRES,}

L.es divers applareils que nous rennns d'esquisser sont chargés d'assimiler la matière nutritive, de la convertir én sub. stance propre de l'aumimal. Il appartieul à la phşsiologie de faire commitre comment celte assimilation a lieu. La commassance de l'apriareil est seule du domaine de l'aniatomie.

Il unns reste à parler daus ce chipitre l'une série d'appareils inui sont clargés, les uns, dëliminer rles substances devemues iumtiles a l'économie animale, comme l'urine; les autres, au contriaire, doivent produire certaines matières nécessaires il l'entretien de la ric, comme lia bile. la salive, ele.; dautres eufin ont poul destimation speciale de produire de nonseanx individus; ce sont les neganes qui pourvoient ì la procrẻation: ils perpetuent lespece el forment un appareil particuliev connu sous le nom l'appareil générateur; nous en parlerons dans mu autre (hapitre.

Tous ces orgnnes, qui sécrètent on produisent une substaner particuliere, sont désignes sons le nom de glandes. Elles sont

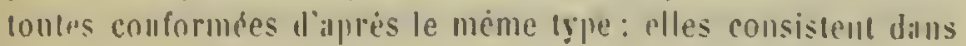


une portion de peau rentrée, un crypte, et leur surface est en géneral d'autant plus étendue que le produit anquel elles donnent naissance est plus abondant. Ce repli de la peau pénètre quelquefois profonlément dans les tissus. Les glandes sont siluées el s'ourrent anssi bien dans la pealı ou la muqueuse qui forme. le canal intestinal que dans la peau externe ou la pean propreInenl dite.

Le type de ces organes, quels que soient leur forme, leur volıme, leur situation et leur coulen', est une vésicule termine ell cul-de-sac, ou un cecum simple ou multiple, froit, flexueux, ramitié et nontrant quelqurfois des anastomoses (fig. 152).

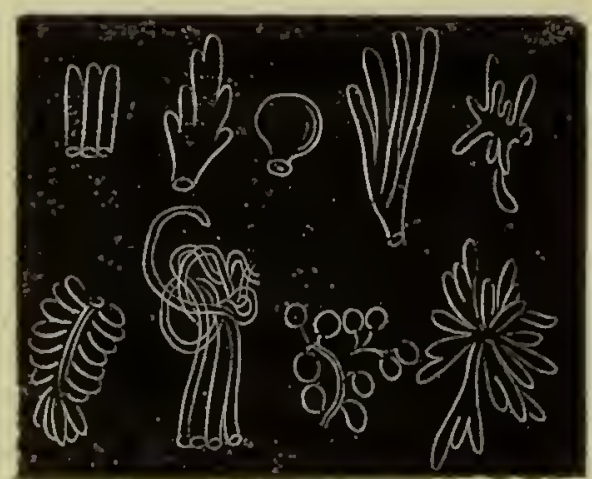

Fug. 132. - Diverses formes de glandes.

La plupart des glaudes, quelle que soit leur complication daus les classes supérieures, se montrent dans l'une ou l'aulte clivision du rigne animal sous leur forme primilive, pendaut loute lil vie le l'animal; e'est ainsi que les glintes salivaires sont formees dans les insectes par de longs tubes, qui enveloppent lo cillal intestinal.

Le caractère d'une véritable glande est de communiquer au delors suil directemenl, soit indirectement, el de répilludre son produit par un orilice. Quelques-uns de ees organes ue soul pourvis que momentanément d'une issue.

Entre la glande el l'orifice extérieur, il exisle cu gimiral un

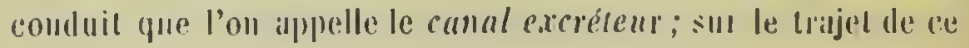


condrit on roit souvent une dépression. men wisiente pui sert de lépoot au produit (fig. 135). C'est ailusi que l'urine n'est pas évacué à mesure pu'elli se produit; elle s'accmmuls dans la vessie. C'est de la même manitre yue la bile est recueillic dalls la vésicule du fiel, pour pénétrer dans l’iıtestin pendiant l'acte de la digestion.

Avant de parler des glandes particulières qui se trouvent a

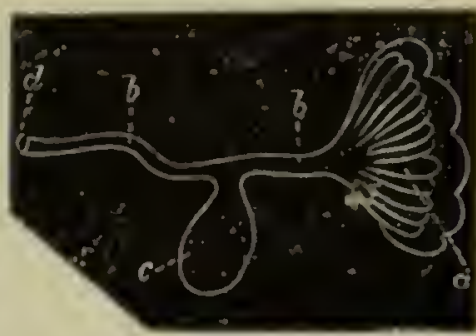

$815.133 .-4$ lanuer.

a Tubes séréteurs. - $b$ Canal excrélear. - Eésicule de dépuis. - dUrilice.

l'exterieur de l'animal, nous dirous un mol das trois glandes principales qui jonent, par l'action de leur produit, un role important dans le phénomène de la digestion.

\section{GLANDES SALIVAIRES.}

Ces organes yui, comme l'indique leur uom, prouluisent la sa-

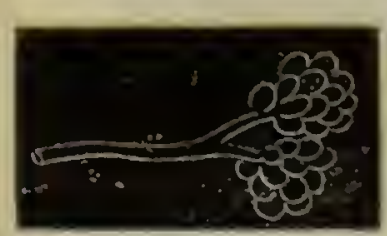

18. 136. - Glande siltiare. live, sont au mombre de truis dams le: plupart des mammifires; on les appelle les parolicles, les soms-maxillajres et les sublinguales. Elles s'ouvrent séparénent daus la cavilé de la louclic. Chez les phonues, ces organes sout peu dereloppes, et ils mampueut daus les cétacés. Dans quelęues rongeurs et éleulés, ils alleigueut, an contraire, le plus grand developpentent. Cest tantòt la parotide et tantot li sous-maxillaire qui est la plus volumineusu. On trouve quelquefois une visienle sur le trajet du canal exiriteur de la glande sous-maxillaire.

l.es oiseamx moutrent orlimairement les trois glandes saljvaires a litat rullimentaire. La parolide scule acipuiert cliez. quelques-mus un grand développement.

Cliez les reptiles el les hatraciens, on me tronve guere ces organes que daus les ophidirns; nais le promil change de dusti- 
Iration. le canal exeréleur s'abouclıe à la base de la dent crense fies serpents venimeux, et la glande, au lieu de salive, protuit le venin.

Les glandes salivaires manquent chez les poissons.

Les insectes ont souvent des glandes salivaires assez développées; elles consistent torjours dans uii coecum simple, isolé el libre dans la cavité du corps. Ce cœecum s'étend parfois jusqu'à l'extrémité postérieure de l'animal et présente unême des circonvoluions. Il y a deux paires de ces ylindes che\% certains insectes;

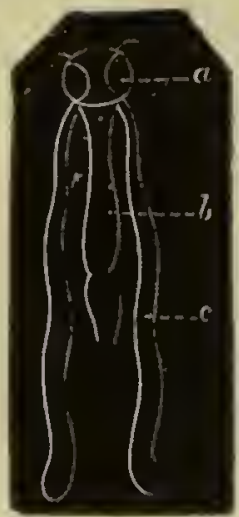

Fig. 135.

blandes salivaires dinsecte.

$n$ Tète. - $b$ Canal digestif. -

c Glande salivarre.

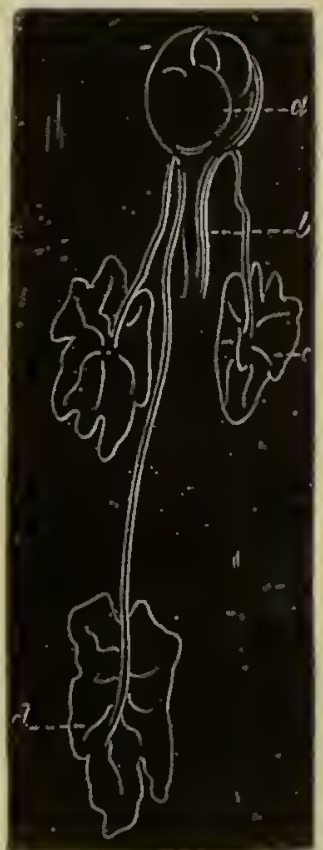

Fik. 136. - Mollusque vephatupario.

a Cavilé buecale avec les miclioir cornées. - $b$ le coinmeniement de l'osophage. - c Les grlandes salivaires antérieures. - d Les glandes salivares postérieures.

elles s'abonclıent dans la cavité de la bouche, sur le còlé de la languette. Dans la larve du bombyx, qui produit la soie et qui est communément nommée ver à soie, les deux glandes salivaires s'abouclseut à une petite ourerture conmune, siluée au milicu de là lèvre inférieure. Le produit de ces glandes se durcil au contact de l'air pour former min fil de soie. 
Dans presque loutes les arachuides, on roil sur le côté de la eavite de la bouche des ceacums glandulatess yue l'un doil regarder conmuc des glinules sulivaires.

On trouve encore des fimdes salivarres dims les mullaspues céplialopodes (lig. 156, p. 140) el dills les gastropudes; elles ont le mene aspect que daus les animans superienrs el se sfoparent en divers lobes, qui, de cluapue cólé, disment naissance à un camal excréteur commum : celui-ci est mince el forl lomg. traverse avec l'usophage le collier tesuphagien ponr somsir

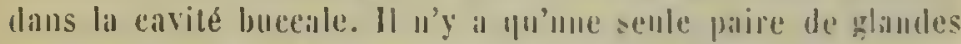

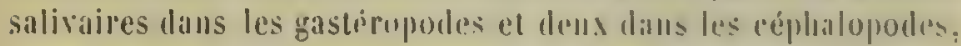
unc en avill el l'autre en arricie dis collice nervils.

On me trouve plus ces oryanes eliez des animalu platers phos

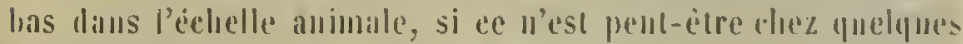
amimaux de la classe des vers, el peut-étre aussi elhez quelpues relinoderines.

\section{FOIE.}

C'est la glamele lil plus volmmincuse el en méme temps la plus impontante, musqu'on la roil encolo, chez les animaus imférieurs, quand les autres out diji entiemenent disparu. 'Tous les vertébres montrent un s!stèmeprorte du foie que l'on ne retionse plus ehez ancum invertibré.

Tous les mammiferes ont un foic tlès-grand, logé dias la cavité abdominale, immédialement eu dessous du diaphlagme el du còté droit du corps. Il a une surface supérieure convexe, en rapport avec le diaplirague, el une surface inferienre cuncave, en

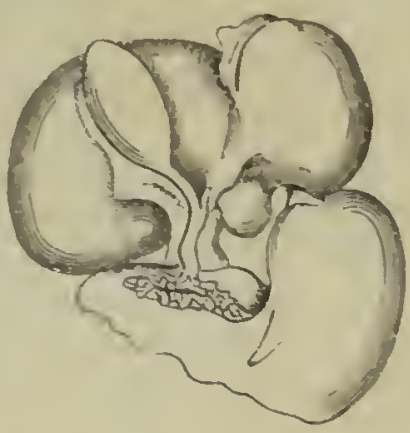

Fig. 13i - Fole el estemar d urangolltang. Unvernos. lapport avec les untestins (tig. 137). Cost daus les mammifires il estomac composé, que le foie est le moins leveloppe, el rlez

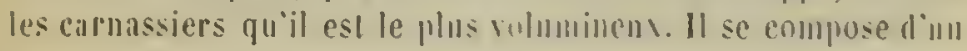


lobe primeipal qui maintient l'organe en plaee par le liganent suspenseır, el de quelques lobes latéraux dout le nombre est variable.

Sur le trajet du canal excréteur, on tronve généralement une

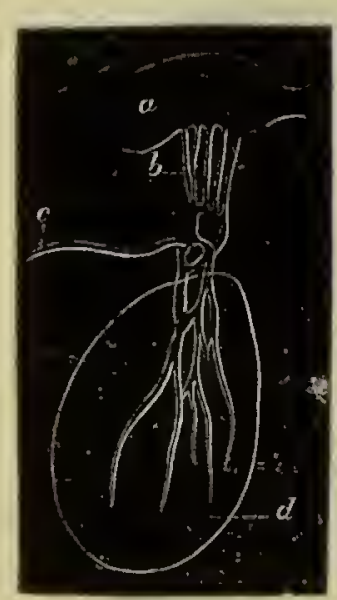

Ejg. $158 .-$ vescule walase avec canal hépatique et plusieurs canaux excrébleurs du python d'apres Poelman.

antestin. - b Canaleholédoque. - c Canal hépalique. $-a$ Vésicinle de dépòt.

vésicule de dépôt, la vésieule du fiel, qui manque toulefois dans plusienrs espiees appartenant i divers ordres ${ }^{1}$. Un camal unique eonduit ordinairement la bile dans l'intestin, nou loin du pylore.

Dans les oiseaux, le foie est toujours voluminenx; il est d'un brun rougeâtre et très-eonsistant; il est formé surtout de deux lobes qui entourent en parlie le eœur.

La vésicule du fiel manque anssi quelquefois comme dans les mannifères, el on voil dans ee eas deux conduits excréteurs s'abouclıer sẻparément daus l'iutestill?

Les reptiles (fig. 158) ont un foie eomparativement plus volumineux que dans les deux elasses précéleutes; sa forme est trìs-variable el cliange avee lia forme du eorps. Les ophidiens ont le forie le plus allongé. Cet organe n'est souvent pas divisé en lobes et prisente des élancrures sur son bojd libre. Le eanal hépatique est quelquefois d'unc lougueur remarquable, et la vésicule du fiel manque rarement.

Le foie des poissons est tonjours remarquable par la grande quantité d'lunile qu'il contient; il est volumineux et s'étend sonvent dans toule la longueur de la cavité abdominale. Sa eouleur

'Elle manque : Parhydermes : éléphanl, tapir, daman, rlinocéros:

Célacés : slellère, balonnes:

Huminants : cerf, thamean;

Vilentés : ai (elle existe dans l'muan):

Rongeurs : érethison el coendon (se trouve dans le pore-spic).

z La vésicule manque dans lo concont, plusimurs permuncels, la pintade. la freliontle, quelques pigerus of liantruche. 
est très-variable; on voil des poissnus à foie jaune, brun, noiràtre, verditre et neêne d'une belle coulenr rose un ruuge de minium. Il est sonvent divisé en deux lohes tris-longs mnis a la hase par une conmissure. Cel organe n'est pas toujours daus la fartie troite de la eavité abdominale. Ial vesicule dn fiel manylus larement; il en existe quelquefois thur et l'un volmue comsill. laible. Celle vésicule est souvent isulire.

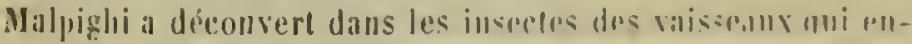
lourent le caund intestiual el qui abonclient dans le ventrienle elyylifiutue; ils portent le nom le cel anatomiste (raisseanx de Ma]. pighi) et reprisentent le foie daus eelte (lasse d'allimaux (fin. 130). Ces vaisseanx sont ell nombre tresvariable; ou en voit ale deu. a liuil; ils soll toujours repliés sur l'intestin. Ils sont terminis en cul-desae, el le bont est quelquefois reune on visiculenx: lantòt ils s'inserent tous sépariment, tautò ils se réunissent pour former un ou plusienrs camaux lıcpaliques. On remarque anssi quelquefois des réservirs lien earactérisés qui font fonetion de vésieule hiliaire.

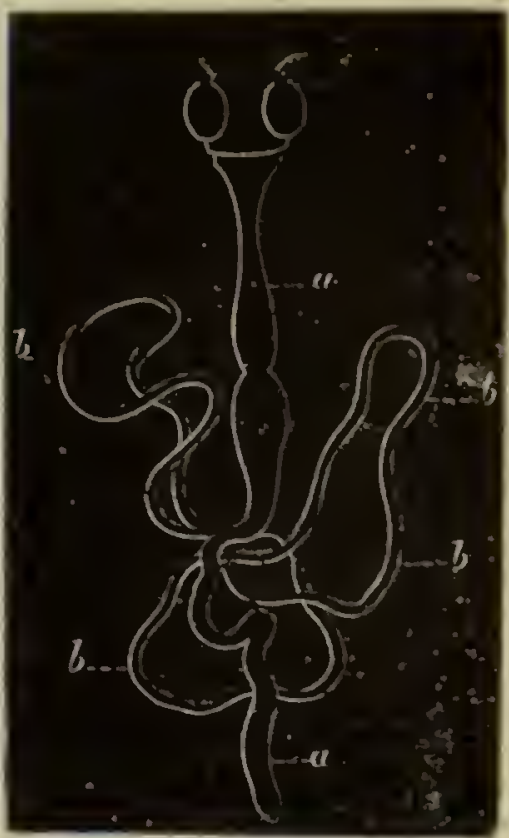

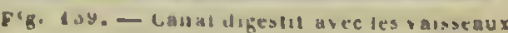
de Malpighl, chez les Insectes.

a Canal digestif. - b Vaisscanx de Malpighi.

Dans tous les insectes sans exeeption, dit II. Leson Dufour. les vaisseanx hépatiques s'abouclent uniquement dans le ven. tricule elıylifique, et, dans tous, la sécrition biliaire est incontestable.

II est probable que Ious les articulés, sans dislineliou, snut purvus d'un foic; mais cost surtuut dans les crustaces, tels yur 
les crabes el les écrevisses, que cette glande acquiert un grand développement; ell effet, le foie forme dans ces articulés deux masses assez volumineuses dans lesquelles on distingue, mème à l'œeil un, les coecums ramifiés et pleins de bile. Ce foie entoure l'estomac, il remplit une grande partie du cephalothorils et il verse son produit par un court canal, tres-pres du pylore. Ce foie a très-peu de consistance, les parois des coecums étaut extraordiuairement miuces et déliciltes.

- Les céphalopodes ont tous un foie très-volumineux, de couleur jaune, formant une masse arrondie, entonréc d'un feuille péritonéal. La bile se répund géméralement par deux canaux liépatiques qui se rémuissent en un canal commun près du r(x)enIII spiral.

Le foie est encore volumineux dains lous les gastéropodes; il est souvent d'une coulcur terrense eltoujours neltement séparé du canal intestimal. Il se compose de plusieurs lobes distiucts "qui versent leul produit par deux ou llois cananx excréteurs dalls l'intérieur de l'intestin.

Dans les autres ordres de mollusques, cene glande devient de plus en plus sinule; elle existe encore comstanment dans les acéphales et euveloppe si étroitement l'estomac qu'ou we peut guère isoler l'un de l'antre. Le foie verse la bile dans la cavité de l'estomae par de larges orifices. Chez plusicurs tuniciers, le foie ne se compose plus que de cryptes peu nombreux entourant l'estomac ou le commencement de l'iutestiu, el ne formant plus un organe d'un aspect glatululaire. C'est à peine si on en voil encore des traces daus les bryozoaires.

Parmi les vers, on trouve chcz les annélides géméralement ın foie à l'état rudimentaire.

On voil, chez quelques animanx inférieur's à cenx qui précélent, les parois de l'estomac ou de l'intestin pourvues de cellulas coloriées, dius lesquelles se produit salus doute la bile nécessaire i) la digestion des aliments. C'est la deprieiere trace d'un orginte hépatique.

Dans les étoiles de mer, claque rayou loge mu crecum volumineux, pourvu d'un grand nombre de vésicules qui lui domen! l'aspect d'une grappe de raisin el dius lesquelles il se, forme un 
linuide jaune yni est verse dans l'estomate ordinairement pall ma druble conduil : e'est le fore.

\section{PANCREAS.}

Le pancrias on glande salivaine ablominale existe chez lous les mammifies. Il presente: tonjunrs un aspect blanchatre claez les animanx de celle classe; il se compose de plusieurs lobes,

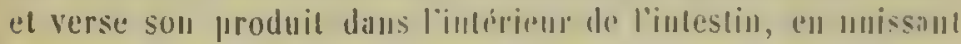

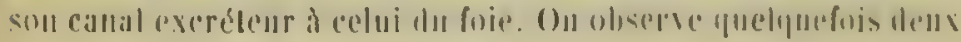

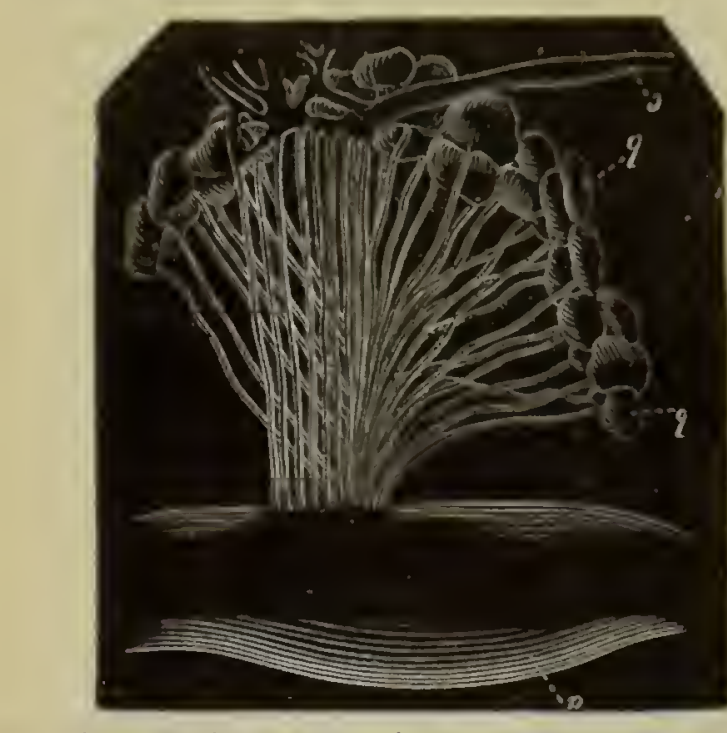

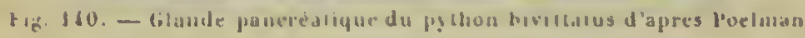

$a$ Intestin. - b Pancréas. - c Canal hépatique.

canaux qui s'ouvreut sciparement dans la cavili du duodénum. Ou n'a pas recounu encore de rapporl eutre fe volume on les modificalions de cet organe, el le riģme de l'animal.

Le pancréas est tris-volumineu cluez les oiseanx: il est tonjours de conleur bianchat tre el situé dans l'anse forme par le duodemum. Il se compuse quelquefois de demix lobes acenles lans le sens de lenr longucur. Le's condnits excritcurs sont au nounbre de deux on de troiset s’ahouchent séparèment dans l’in- 
testin. Il cst à remarquer que lc grand volunc du paucréas compense clıcz les oiscaux le faible développement des glandes salivaires. Il cst à supposer que le produit du pancréas cl la bilc agrissent séparément sur la malière nutritive, puisque nous voyons daus l'autruclıe le canal cholédounc s'aboucher près du pylore et le canal du pancréis à trois piets de distance du pré. cérlent.

Claude Bernard prétcud que Ic canal pancréatique cl le canal cliolédoque se contractent de la manièrc la plus évitente avec une forme rhyllimique dans les oiseaux.

Le pancréas est facile à découvrir chez tous les reptiles et les batracieus (fig. 140); il al souvcut une teiule jaunc; il est géuéralement moins dévcloppé chę les reptiles aqualiqu's que cliez les terrestres. Cet or sane contracte aussi, chez quelques-

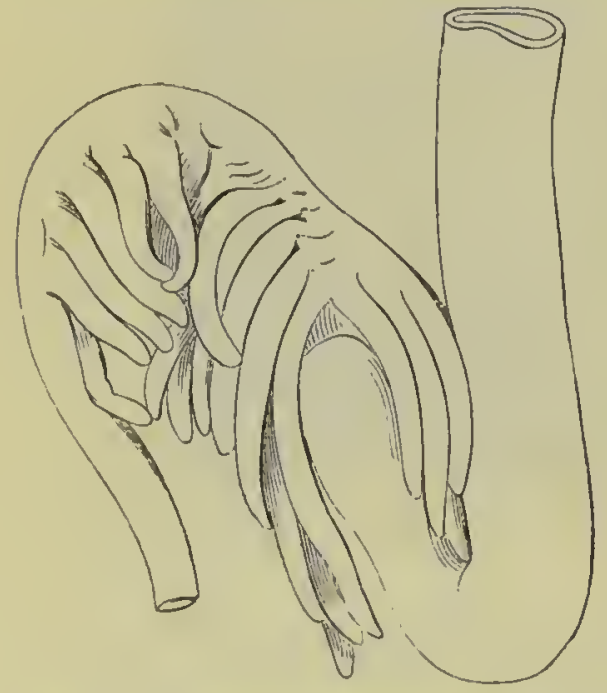

Fig. 116. - Estomacel coreuns py toriques de salmo fario. uns de ces animaux, ulle union assez illtime avec la rate. l.e canal excréteur est rarement touble el il s’insìrc généralemeut vis-à-vis dul cilnal cholédoryue.

Dalus la classe des poissous, il existe un pancréas vérita ble dans les sturio.nicns, les poissons plagiostomes (les raies el Ics syuales), et dans III grand nombre de poissous osscux, indépendantment des coecuns pyloriques '. Il a un aspect glandulcux dans ces poissons comme

IOn en a vil dans le salma salar, clupea harengus, fiadıs, cillarias cottus scorpius, perca fluviatilis, pleur. platess., pl. maximus, belone fongirostris, et cyprin. brama, daprés Brokmann. 
dalls les vertébrés smpérieurs; ; ussi le lịnide pincréalique est versé dins l'intestin par un eallal unique i une petite distance din pylore.

Cluez les poissons osseux, ou tronve an benciral des corcums antour du pylore, que l'on nomme coreuns pyloriqnes et que Ion a longlemps consideres comme representant le pancrias (lig. 161). Le nombre el le volume de res cueums sont tres-va. riables; ils sout d'anlint plus volumineur qu'ils se trousent en plus petil nombre. On en soil depuis deux, trois ou quatre, jusqu'au delit de ceut. Ils s'albouchent dians l’iulestiu par plusieurs ouvertures; on les trouve gémeralement pleins. Dillus l'autres animanx de cetle classe, ceux qui termineul la sirite des vertebris, les caemus pyloripues et le pancréas mimpuent complétcmeill.

I.es senls amimaux sans vertibres cluez lesquels on observe uII pilucrias, somt les mollusques céphialupodes. II existe it lit base du foie el sur les conduits hépatịues, des tubes glamdu-

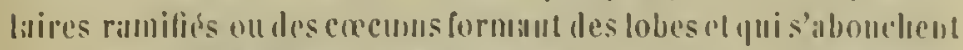
par disers urifices dans le cantal excritenr du foie: cette glatule, yui a cle vue depuis louglemps, represente évillemment le panirliss.

\section{GLANDES SPËCIALES.}

Il existe dins les mammifires, comnte dins d'autres classes,

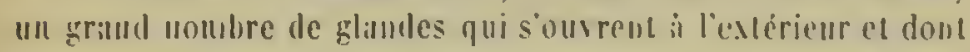

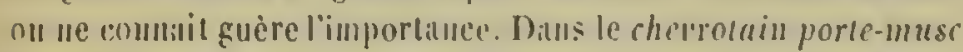
comume dims le rall el un grind unmbre d'iutres mammiferes, il soure an prépuce une sclinde assez volmunimense, qui. comme la glande anale, a une odeur specifique. Toul le moude

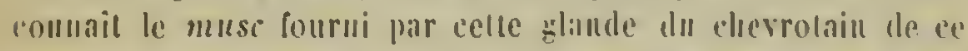
unm. Les antilopes el les eerfs out atu-tlessous de. l'neil mue plande formée pir un auas de eryptes, dont le produit coule comme des limrmes, mais qui lia cependant rieu de commun avec ce lipuide: éest le larmier. Léléphant a une glaude qui souvre an milien de la joue; le chimea en a une derrièr loreille. I,e preari en a une suorme an milieu.et en arrière: 
dı dos. Daus les musaraignes, on en voil plusieurs sur les flancs, qui sécrètent aussi un liquide musqué. Quelques chauvessouris et la marmolle souslik ont un sinus de la peau dans lequel s'ouvrent divers cryptes. Les desmans en ont sur le côté de la quene. Plusieurs antilopes en portent dans le pli de l'aine. Dans l'ècartemeut des doiğts des chamois el à la racine du sabol lles rennes el d'autres ongulés, oll trouve aussi un amas di cryples. La prélendue glande venimeuse du mâle des ornithorhynques, siluée sur la cuisse, appartient aussi à celle calégorie.

Les oiseaux, surlout les niseaux aquatiques, ont à la base et

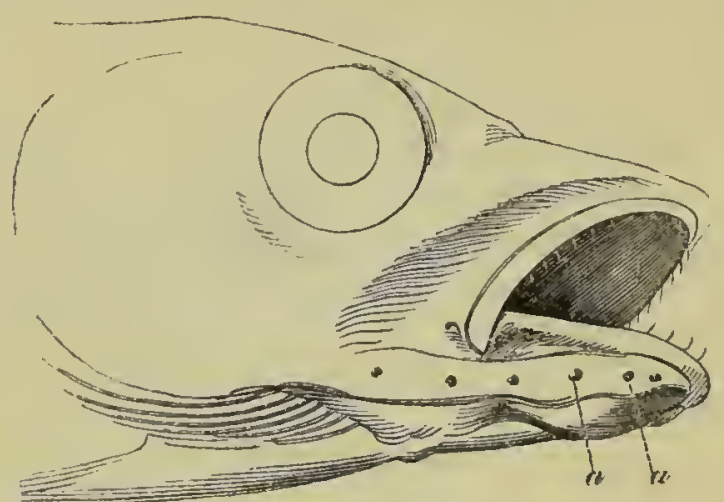

Fig. 112. - Tete de merlan.

a Cryples culanés.

au-dessus du coccyx, une glande assez volumineuse qui porte ce nom ; elle sécrète l'luile qui doil rendre les plumes inıerméables. La bourse de Fabricius n'esl pas une glaude, mais un organe létri à l'étil adulte, propre seulement d̀ la vie embryonnaire.

Dans les reptiles el batraciens, il existe encole plusieurs glandes cutanées. Les crocodiles en ont une vers le milieu et entre les deux branches de la màchoire inférieure; les chéloniens out quelques glandes anales comme les ophidiens; mais plusieurs sauriens ont des glandes qui s'ouvrent régulièrement 
sur les cuisses et dont le nombre est constant dans claque espere? on les appelle pores fimorilux.

Cependiunt les galumes les plus remarquables sont celles que lou ulserve sur lat mugne ou all cou des erapauds el des sil:a-

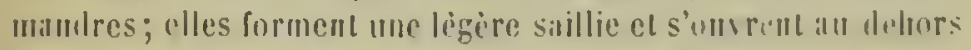

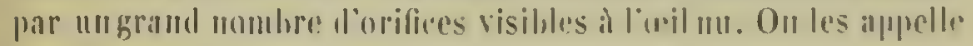
it tort piarotides.

Presiuc lons les poissons onl des cryples cultules, silues arer

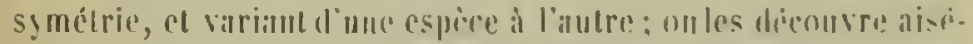

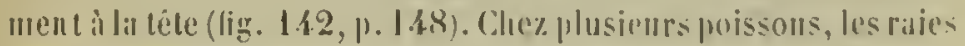
pir exemple, ees cryples soul formes de tubes tris-longs, que lan prot metlre it nu silns difficulte el qui sout silués eu dessous de la pean.

Comme glandes culaners dinls les auimatux sills vertibres, il fillt menliouner surtout les glamles surres les pucerons, les filieres des araigmes tileuses, (ole., ele.

Dans les mollusques céplabliporles, on voit une glande remplie d'une substance noirc el d'une lémitr evtrime: elle s'ourreau delors à coté de l’amus. Ciest la hourse du noir. File est logeco en partic dans le foic cliez quelquues espices, ce yui l'avait mème filit regarder conime la vesienle ilu fiel. Clecz. d'autres, elle 11 it alucun rapport aree le foic, el clle souvre toujours a l'extérieur pour réplandre son prodnit au

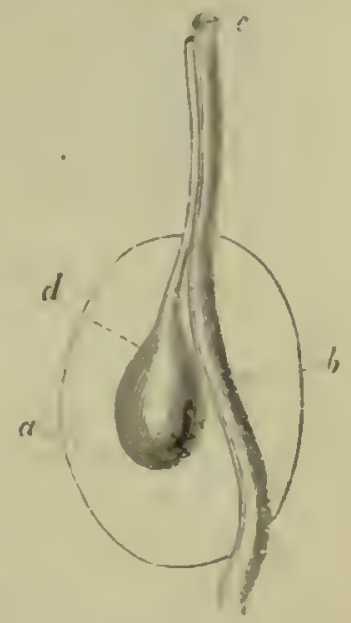

Fis 163.- Vesicule du noir des cephalopodes.

a Foir. - $b$ Intestin. c. Inus. - d Vésicule du noir souvrant it collé de l'anus. dehors. C'est une glande spieiale qui sert it lat defense de linimal (fig. 145). 


\section{APPAREIL, URINAHE.}

L'appareil uriuaire existe sans exception dans tous les vertébrés el se trouve, sous des formes diffélentles, daus un grand nombre d'animaux sans verlèbres.

Daus le cour's du développement, on voit daus les embryous des manmuiferes des organes, desigués sous le nom de corj)s de Wolf; ces corpsse modifient etperdeut leur juremier aspect, sauf dlans la classe des poissons, où ils deviennent les vépitables reins. Dans les batraciens, une partie de ces corps semble se modifier encore, quand toutefois la nasse principale conserve soll caractère primitif.

Les batraciens font ainsi la transition : dans les vertébriss allatoüliens, le corps de Wolf se transforme en épididyme; dians les batraciens, une partie du corps de WVolff reste rein, une autre devient épididyme, el dans les poissons, le corps de Woln reste avec tous ses caractères pour remplir les fouctions de roills.

Sous le rapport de la forme, de la siluation el mème de la 
rouleur, les reins ne présentent guìre de modifieations dans lomle la classe des mammicires. Ils affectent partont la forme de haricot; lantot cet organe se compose d'un seul Inbe, tantót au contraire il est formé de plusieurs, au point que la surface présente l'aspect de dalles, conme dans les dauphins. Les divers mammireres aquatipues, les loutres, les ploques el les cétacés,

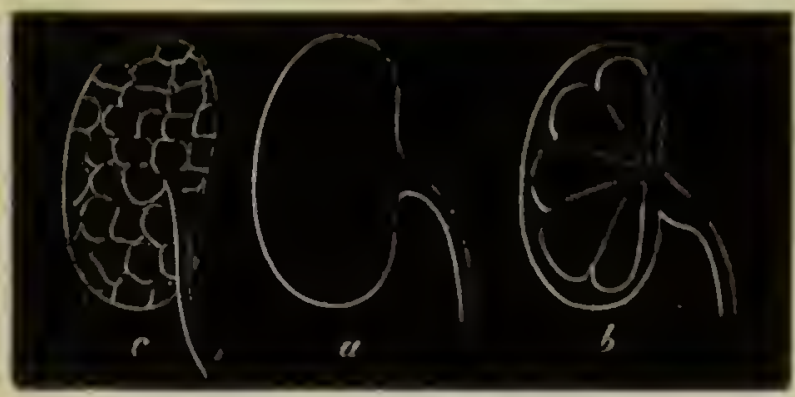

Ib. Is - It totas.

a Ilein non divsé on simple. - b Rein divisé en lobes. - c llein très-divisé.

ont cet organe le plus divisé (fig. 144 c). Dans le cochon, il n'y a ancune trace de division a l'exlérieur, tandis qu'il est bossole dans le bould.

Cot organe est tomjours domble; il est situe dans la cavité abdominale en dessous du diaphrague et couvert par le peritoine a si fire iuférieure. On dislingue loujours mue conche corticale, qui entonre tout l'organe comme une écorce, et une substance médullaire qui est formce par les ececums ou les cabaux secreteurs. Les corpuscules de Milpighi 'se retrouvent daus tous les animaux de cette classe.

Ces canaux secriteurs se rüuissent ordinairement en groupes el versent leur produit par me papille dans une première poche, le calice; de li, le produit passe dans le bassinet, qui est le riceptacle de toute l'urine sécrélée; le caual excréleur, l'uretire, nest pour ainsi dire yue la continuation du bassinel;

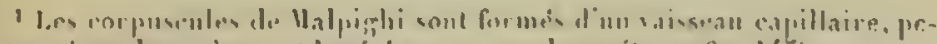

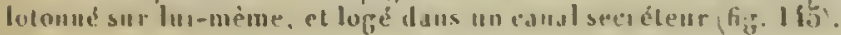


il descend le long de la eolome vertébrale et s'ouvre dans le col de la vessie. Cette poclıe de dépôt ne nanque jamais et ne présente que peu de différenee dans les animaux de cette elasse. De la vessie, l'urine passe, elıez les mìles, dans un eanal conmun qui reçoit en mème temps le produit du testieule el que l'on désigne sous le nom d'urètre. Ce second camal répand l'urine au delıors.

Les oiseaux ont deux reius eomme les mammiferes, natis leur urine, au lieu d'ètre liquide, est tonjours solide; cette urine est évacuée avec les fèces sous la forme d'une pite blanelıe qu'ou voit à la surfiee des exerénents. II I'existe pas de ressie.

Les reins sout situés daus la eavité abilominale, derric̀re les poumons, et aceoles comme eux à la colonne vertébrale; ils s'enfoncent dans les exeavations du saerum. Iseur eouleur est toujours terreuse, ou d'ull rouge-brun foncé. Leur forme a compléteneut changé et ils se présentent sous l'aspect d'une bande allongée, découpée en dehor's en plusieur's lobes.

Les conduits exeréteurs des lobules se réunissent de claque eôté eı uı eanal commun pour fornier les uretères. Ceux-ci sont contractiles, passent derriere le reetum et s'ouvrent dans le cloaque en dedans des orifiees des organes génitaux. On trouve aussi les corpuscules de Mappighi chez les animaux de eette elasse.

Les reius des reptiles el des balraciens ne sout pas saus analogie avee eeux des oiseaux. Ils sont aussi nu nomlme de deux el logés dans la eavité abdominale dout ils ocenpent toute la partie postérieure. Ils sont très-allongés, surtout ehez les ophiduens. Quelquefois ils se touehent sur la ligue médiaue et se eonfondent mème parfois en une seule masse à la partie postérieure. Souvent ils sout eomposès d'uu grand nombre de lobes séparés les uns des autres par des ciclanerures. Les canaux sécrèteurs sont orlinaircment flexueux et rarement droits. Les eorpuscules de Malpighi sont volumineux, surtout chez les batraeiens.

Le eanal de l'urètre est souvent très-long et s'ouvre dans le eloaque, soit isolément, soit en se réunissant aux conduits des organes génitaux. 
On il constaté dans les canaux everéteurs de l'urine, clee quelynes batraciens, la priscuee de cils viluratiles sur une partire de leur longreur (li⿳. 14ij).

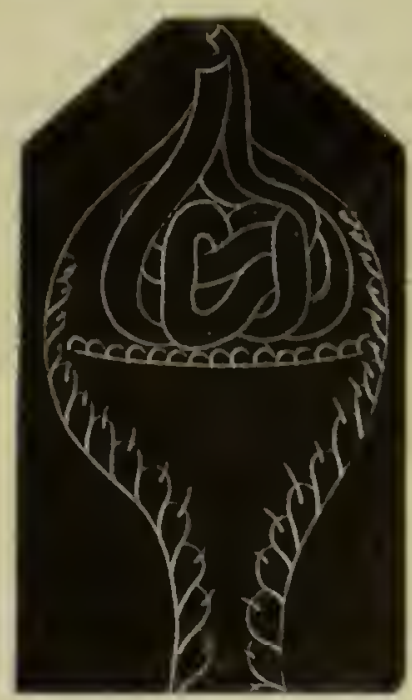

Pig. Ids. - liurpureubes de Malpight de la grenoulle

II existe gencralement une vessie daus les rephiles biritables comme dere les batraciens, mais elle ne reçoil jamais directrment les uretires et elle sousre dans la paroi anterienare du doilpule.

Tous les poissons sont pourvus de reins. Ils sont silués clie? les plagiostomes dans la partie postérieure de la eavite alulominale. Ils sont eomposés de plusieurs lolues. qui envoient se. pirrimeut leur canal exereteur vers mu point commun oi comunener l'urelìe. Il existe, comme dams los reptiles, une ressie, mais qui na pas de enmmmication aree lometere el qui soure ru avat daus le cloaque. Dans les poissous nsseux, les reins sont ordinairement disposes comme deux longes rubans, de couleur rouge ou lirune. seitendant daus lonte létendue de la cavité abdominalr, entre les vertihres et la vessie nataloire. Ces organes ont entueral tris-pen de eonsistance. Ils sont separis l'un de l'aulle, ou quolquefois remus plus ou moins completement sur 
leur trajet. Les uretères s'unissent souvent en mn seul cantal, qui s'ouvre dans le corps de la vessie. Cetle vésicule de dépôt paraît manquer rarement daus les poissons. Ia vessie répand sols contenu par un court eanal de l'urètre, qui s'ouvre derrière l'anus.

Plusieurs animaux sans vertèbres produisent de l'urine; on a conslaté la présence de l'urée elıez plusieurs d'entre eux, mais il y a peu d'organes qui sont positivement reconnus pour des reins.

Dans les insectes, les vaisseaux de Malpighi out domne de l'acide urique à l'analyse climique; on a tromvé de's calculs "uriuaires dans leur intérienr, aussi peut-on les regarder peutètre comme faisant simultanément fonctions de foie et de rein; mais a vant tout ce sont des organes biliaires.

On trouve des organes semblables dans les arachnicles et les

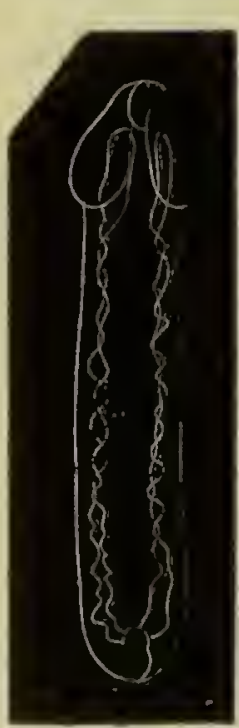

rig 1\$6. - Appareil monaire de cestojide. myriapodes, et qui doment sans doute naissance au méme produit.

On u'en a pas encore reenuu dans les crustacés, loutefois on voil certains organes autour du canal intestinal qui pourraient bien, apris une étude approfondie, être recounus pour un appareil urinaire.

Dans les mollusques cepplalopodes, on voil ì côté de l'auus, un canal exeréteur provenanul d'une glande qui coutient le noir, connu sous le nom de sépia. Depnis longlempıs, ('el organn a été regardé comme le rein, mais lout récemment ou a crul recounaître le reiu diuns le's corps spongienx qui recouvent la veiue cave de ces mollusques. La plrésence de l'urée diuls ces appendices el leur rapport avec les vilisseaux sont des colditions favorables i celle déterminatiols.

Dans les gasterropoles, on voil byilement une glande dont le canal exeréteur accompange le rectum el

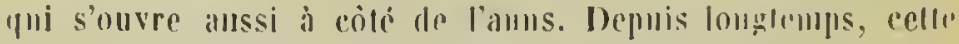

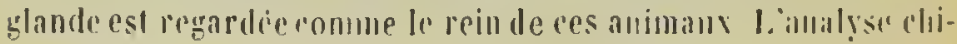


niique a révile la présence de l'acide uripue dans son iutérienr.

Dims les mollusques acephales, ou tumait depuis longlemps III orana dout ha malure est problémalique el que l'on désigne sous le nom de poumon de Bojinus. On! a découvert des concrélions qui contiennent de l'acide urique, de manise pue ces animanx seraient encole pourvus d'un rein, d'après quelques antrurs.

Eufin, on doil regarder comme reius los canaux exeréteurs qui parcourent loute la longrueur du rorps dans les vers trématodes el cestonjles

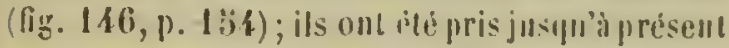
pour un apjarejl cirrulatuire; ils s'our reut souvent à l'exteriour par l'internuede d'une vésionle pulsatile, el les canaus portent de longs cils vibratiles qui ue se menvenl yue dans un seul sens (lig. 1.77). Cet appareil rexiste dans les di

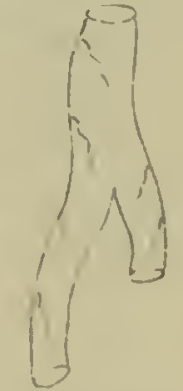

rig. $157-1$ innal

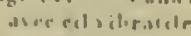
des sere. vers ordres de vers, depusis les himdindes jusqu'ams planaim, il les rolifires. 


\section{IPPARELL GENERATEUR.}

Tous les appareils dont nous avons parlé jusqu'ici eoncourent i un seul et mème but: au développement ou à la conservatiou de l'indivilu; l'animal peut vivre par l'accomplissement de ces fonctions. Mais un but plus élevé doit être alteint et pour lequel souvent tout l'organisme est sacrifié, il faut cll effet pourvoir ì la conservation de l'espèce. L'animal, comme tout être organisé, n'est appelè à vivre que pendant un certain temps; comme la plante, il est ué, il doil mourir'; sans la reproduction, lia surfoce du globe scrait bientôt complétement dépeuplée. Cet appareil conserve l'espèce dans le temps.

Ceux qui parlent encore de génération spontanée ou d'auimanx se formant de toutes pièces, doivent prendre rang parmi les naturalistes qui font provenir les poissons de la boue des étangs; nn eonçoit que celte doctrine ait pu régner du temps d'Aristote, mais de uos jours l'olservation en a fait justice.

Le génie de l'lomme a inventé des machines bien admirables, mais quand clles sont usées et mises hors d'usage, il elt faut d'autres; Dieu, en créant ees autres marlines, qu'on apprillr plantes el animaux, les a doures clles-mêmes de la faculté do se reproduire et de perpétuer ainsi sur notre plancle ces formes 
infunies, telles qu'elles sont sorties de ses mains. Tous les efforts de la mature sembleut eoncomrir à ce but supreme : de conserver la vie à la surface du globe; l'iulividu u est rieu ell presenee de l'espree. Les individus somvent disparaissent aussilól que le développentent de la progénilure esl assure. la animill, en géusial, ue semhle vive el se dévelupper yue pour se reproduire, car à peiue? a-l-il alleint l'age idulte pl complet, pue

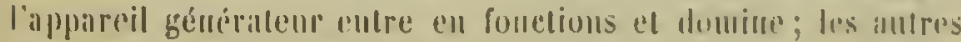

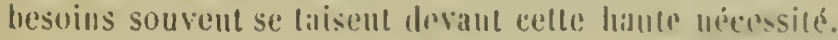

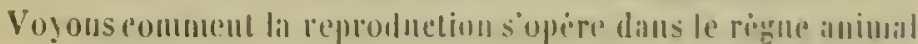

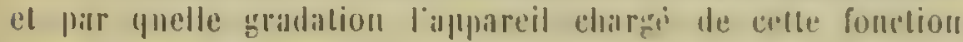
flisse suceessivement.

Cousidirio dans lout le rigne animal, la reproduction st eonfond d'alord avee l'aceroissement l'animal, ay anl atleint un certuin volume, se divise eu divers fragutents yui deviomuent bientôt chalun un iulividu compled, semblable il celni qui teur

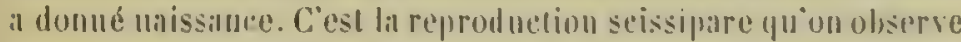

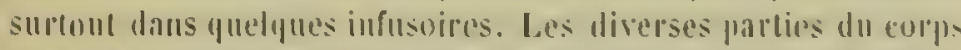
se sépareut les mues des autres, poutiment malgre ceda a sae. croitre, el devienuent toutes semblables entre elles el at eelu dout ils proviemuent.

Daus les polypes, art aceroissement se locillise; il se forme

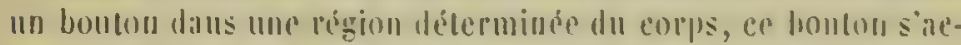
croil, des tentacule's apparilissent el bientit re boutou ressemble all polyje qui lui al dommé maissance. C'est la reproduetion pal gemme ou par bourgeon. II pousse des hourgenns sur le corps de lanimal comme sur les allitres.

Ditns doulles animaux plus ibevis, an lien diun boulon forme par eoutinuation des lissus, il applaralit, dialls une région du corps, une cellute isolée qui prisente less le principe un earactère d"individualité, e'est l'auf : la reproduction alors est ovipiare.

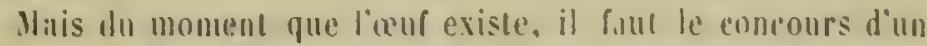
autre produil qui le rende ficond : il faul le produil d'un urgatue mile ou d'un testiente; le contide d'une liquenr spermatique

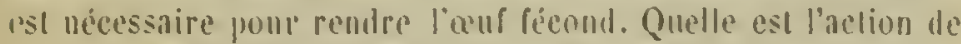
relle liqueur male sur lixuf? Ciesl ce que lon ignore: lout re 
que l'on sail, c'est que le eontiacl est indispensable pour produire lis fécondiltion. Il y a done trois modes de reproduction el à la ligueur seulement deux, ear les repruductions scissipare el grmmipare diffèrent bien peu l'me de l'autre; ees modes sont : la reproduction ovipare, la reproduction gemmipare el la repunduction scissipare.

Pour celte première reproduction, il faut des sexes; elle est sexuelle. Il n'en faut pas pour les deux autres; ces reproduclions sont agames.

Ces sexes peuvent se trouver réunis dans un seul et même iudividu, e'est ce qui constilue l'hermaphroditisne, et cet étal l'lıermaphrodite est complet quand l'individu se suffil à luimème; il est incomplet quand, comme daus les sangsues, deux individus, pourvis chacun des deux sexes, agissent réciproqueureut l'un à l'egard de l'autre, a la fois comme mile el comme femelle; après la copulation lous les deux produisent des oufs ficonds.

Dans lous les animaux supérieurs, les organes sexuels sont norlés sur des indivilus distincts et l'espèce se comprose de miles el de femelles.

Ainsi, tous les vertébrẻs et articulés ont les sexes sẻparés, tandis que plusicurs mollusques, III grand nombre de ver's out les sexes rémnis; les acalèplıes el les polypes ont souvent les sexes portés par des imdividus distincts eomme dans les animanx superieurs.

On peut dire d'une manière générale que les animaux qui sont fixés au sol comme les plantes, sont hermaplirodites cumme relles-ci, el que ceux qui jouissent de toute la libertés de teurs mouvements sout à sexes sipares; ces derniers peuvent sculs aller i la recherclie les uns des autres, pour accomplir le grand acte en faveur de l'espèce.

On peut considérer comme une transition de la réunion des sexes ou de l'luermapluroditisme à la séparalion des sexes sur des individus differents, l'exemple des salpa, qui agissent comme mâles ou comme femelles, selon lent ige. D'antres herunaplurodites sembleut agil comme miles ou comme femelles, selon la saisun. 
Lis siplaration des sexes pent ètre considére comme une division du travait, la femelle el le mate ont à actomplir das devoirs dillerents sedon les circonstances dints lesquelles ils soult plictes.

Dills plusinurs animanx, les deux modes de reprodnclion soml rénnis; la meme espece est mipare el bemmipare. l.'ligdre d'eau douce produil des gemmes en éle, des aenfs en antomute.

L'imlividu qui produil des gemmes, s'il vit dans des comlilions difrerentes de celni yui est ovipare, meisente une forma sourent bien differente, el connue on voil des distinctions entre les mâles et les femelles, onl en roit aussi entre les gemmipares el les ovipares; eclle différence le forme dalis une seule del mene espice a produil des plünomenes bien singuliers at doul on ue s'est rentu comple que dans ces dernieres anmés.

C'est ainsi qu'nu individu gemmipare, provenant d'uı auf, proluit un individu ou loute une géneiration qui ne lui ressemble pas; el si, comme cela arrive dans quelques animaux, l'animal esl alterna livement gemmipare el ovipare: si celui qui provient d'un auf donue un bourgeon el celui qui provient l'un bourgeon un a'ul, il y a denx formes qui altement, ed la fille, au lieu de ressembler à sa mere, ressemble à sa gramd"nere. Les indwidus ovipares produisent des individus gemmipares, ceus-ei de nouvean des nvipares, el ainsi de suite; de maniere que l'iulividu ovipare ressemble sa grand'ınère, oripare comme lui, mais pas a sa mere, qui est gemmipare.

C'est ce plténomène que l'on a désigntés sous le nom de génération alternante (Steenstrup) el que nous considérons romme un simple phénomene de double reproduction ou de digénèse.

Nons avons ru la division du travail daus les appareils; nous voyons ce principe s'étendre ici jusqu'aux individıs; nous royons les individus spermatogines, des individus ovigènes, les individus pliylogines (ceux qui produisent des bourgeons), 't il y a meme, daus les classes inferieures, des colonies composies d'individus chargés, les uns de manger el de boire, les autres de respirer, d'autres de conduire la colonie (Ies rameurs), 
ceux-ei de jiroduire des oufs, ecux-là de former des gemınes, el on voil elıque animal affecter une forme qui est en rapport avee ses folletions. C'est eetle diversité dans la forme qui u'avail pas permis anx naturalistes de contprendre les singulières colonies flollantes réunies sous le nom d'acalèphes hydrostatiques.

Y a-t-il des animanx qui subissent des mélamorphoses el d'autres qui ı'en subissent pas?

L'opinion la plus fausse règne encore aujourd'lut dans la science au sujel des métamorphoses. On répète encore tous les jours, dans les ouvrages de zoologie les plus recommandables, que tels et tels animaux se distinguent des autres pal leurs métamorphoses, en oubliant d'ajouter que ces phénomènes dépendent de l'époque de l'éclosion, que l'embryon pareourl tous les elangentents de forme par lesquels il doit nécessairement passer, tantôt daus l'œuf ct tantôt seulement après sà sortie de l'ouf. Un animal queleonque peut-il se développer sans mélamorphose? Évidemment non!

A l'époque où l'on croyait que les animax se trouvellt tout formés el en miniature dans leur ouf, on a pu dire par oppositicn que les grenouilles subissent des métamorphoses; depuis que l'observation nous a appris le eontraire, que le jaune sc transforme en substanec de l'embryon, ecla I'est jlus exacl. Tous les animaux sulyissent des métamorphoses; elles sont plus longues el plus étendues elıez les uns que chez les autres. Chez quelques-uns, ees métamorphoses ont lieu plus tôt qur ehez les antres, elles ont lieu tantôt dans l'œuf, tantót après la sortie de l'œuf, voilà la différence entre eeux qui subissent des métanorphoses complètes el cenx qui n’en présentent pas.

\section{COMPOSITION OE L'APPAREIL.}

Cet appareil est formé, eomme toutes les glandes, de eocums plus ou moins complets, de eanalicules anastomosés qui en forment la partie principale; e'est le testicule daus le mile, l'ovaire dans la femelle. L'un el l'aulle est pourvu d'un eanal excréteur. qui recoit le nom d'oviducte dans la femelle, de eanal déférenI ou de spermitlucte pour l'organe mâle; sur le trajet de l'un el 
le lantre canal excriteur, on Irouve souvent me vesieule de

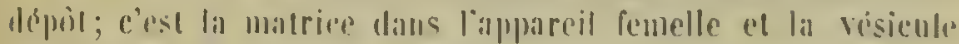

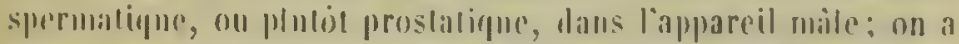

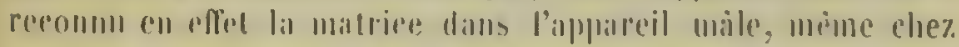

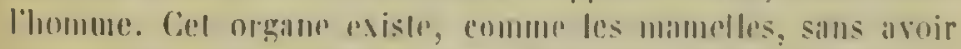
incme destination dins ce sese. Enlin, mo dernier canal con-

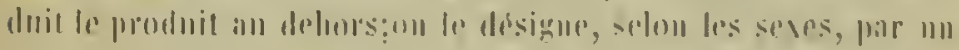

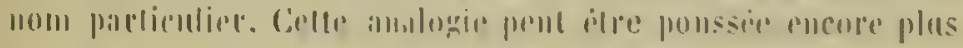

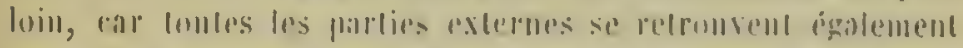

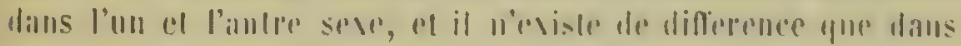

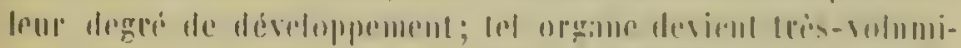

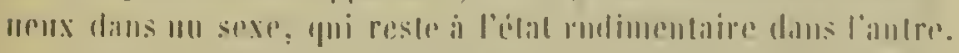

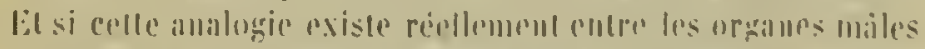

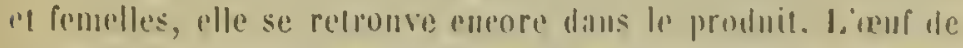

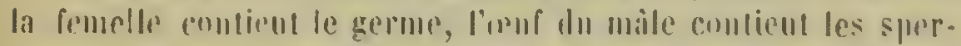
matozoüles qui doivent féconter ee germe fonrui par loregane femenle.

Il y a une rpoque de la vie embrymuaire oi lo sese nest pas eucore recombiassible; le lesticule el lovale ue peuvent ètre distingués l’un de l’antre. Le point de départ d’un appareil màle ct d'un ilpareil femolle ost exactement le mème; les différences ne se manifestemt que daus te conrs dn développement. Jes sexes sont somes sur la route de lorganisation.

Celte epoque de la vie emhrụnuare persiste jusquì l’àge adulte dans quelques poissons, au point que cerlains animaus de ceuc clisse citaleul consiléris encore dans ces deruiers lemps eonure ulayant qu'un seul sese. Le testicule des miles est tellement semblable a loorare des femelles, gur le microscope, montrant les spermatozoïdes dans les uns el les aufs dans les nutres, peut seul les faire distinguer.

le testicule produit la liqueur ficondante, l'ovaire produil des nufs qui doivent ère ficontés.

Disons un mot de l'un el de l'autre de ces produits.

\section{EUFS.}

I.es aufs moulrent une grande différence entre eux, el ce n'est 
qur depuis qurlques années que l'œuf a écé reconnu dans les mammifies.

Les nufs sont sécrétés par l'ovaire, comme le snerme par le testicule. Cette sécrétion a liêl

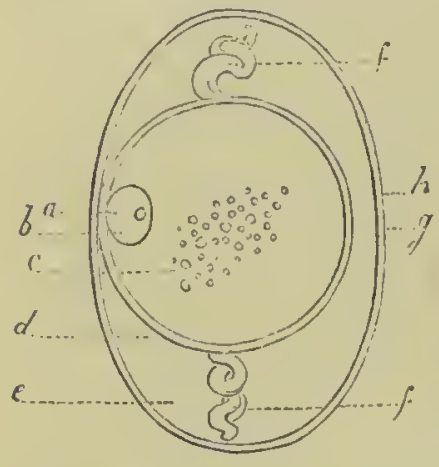

Fig. 168. - oEuf d'nisenu.

"Tache de Wagner. - bésicule de l'urkinje. - r Vilellus. d Membrane vitelline. - e llbumen. - $f$ Chalaze. - g .Iemhrane de la coque. - h Coque. indépendamment dı coït. O॥ voit des perroquets femelles, isolés depuis des années dans des cages, pondre des oufs, inais ces oufs sont stériles.

Ce qui distingue l'œuf des le principe, c'est qu'il est isolé et jouil, dès le moment de sou apparition, d'une vie propre. Le bourgeon est une partie de la mère, l'œuf un produit.

L'œuf prisente des parties essentielles el des parties accessoires; les plarties essentielles sont, en procédant de deliors en dedans : Ia memlırane vitelline,

le vilcllus, la vésicule germinative et la tache de Wagner. Les parties accessoires sont, en prartant de la membrane vitelline, le blanc ou l'allumen, les chalazes, la membrane de la coque et enfin la coque (fig. 148).

Les premieres parties ne diffirent guère que par Jenr volume ou leur quantité, les autres diffèrent entre elles sous lous les rapports.

Les parties essenticlles se trouvent dans tout œuf; les parties accessoires ou de perfectionnement peurent toutes manquer. Ainsi, la coque peut être membraneuse, calcaire, cornée (fiğ. 149), flergamentacée et même elle peut manquer, comme l'albumen.

Un embryogéniste disting̨ué a émis récemment l'avis que l'ouf débute par la membrane vilelline el que c'est, par conséquent, la partie la plus essentielle; l'olsservation directe prouve le contraire. Dans des animaux inférieurs, on voit la vésicule germinative quitter le lieu où elle s'est formée, s'envelopper ensuile de glohules vitellins et enfin de la membrane 
vitelline. C'est la vesienle germinative qui est le noyau de formation (fig. 150).

licul est tress-volmuineux quand il doil eontenir loute lat nourrituré nécessinire au développeutent complet del'embryou, comme dans le's viseanx; l'our est très-petit, ilu contraire, quand if ne renferme que la nourriture des premicers jours el que l'embryou eontinue son crolution aux dépens din sin! ale lat mèe, commedans les manmilères.

Les enveloppes de l'uuf som minces et ldilicates, yuand celui-ci llest pals exposé à ètre comprimé el pecul etre déposé en

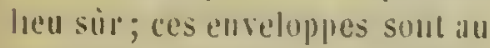
contraire calcaires on cornés, quand l'unf est couve dans un uill ou expose à des diangers.

Dius les animaux superienrs, ceux a silny chaud, le nombre d'auls poulus par un animial est tres-petit; lat progentuiture est cotourée de lant de soins, le la

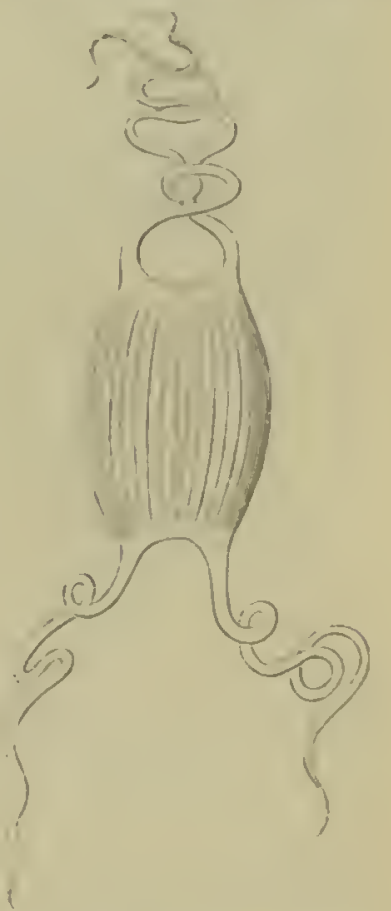
Fig 169.-OEuf de poisonn plagiosiome. part de la mire, que claque aeuf produit un jeune qui a des

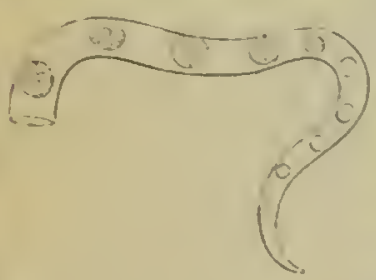

Fin isn. - OEurs de nemaloijle un ou qunlipurs couples dieurs, les reptiles en prondent des classes, les aufs sonl souvent aballdomnes à eux-mèmes, el le nombre en est d'atutant plus grand, qu'ils ont moins de clances le se trouver dills des conditions filvorables; les mimmifires produisent souvent un auf il la fois, ceux yui unt une paire de mamelles; les oiscaux pondent elantees de vivre; dans les autres 
centaimes, les poissons en géméral des milliers, et les vers inlestinanx, dont les ceufs el les embryous trouveront si diflicilement leur destimation, e'est-it-dire l'animal ou l'oryane qui doil les nourrir, en produiroul par cent mille.

On lit parfaitement dans le nombre, le volume el la forme de l'auf les clances de vie du futur embryon.

Tous les auimaux produiseut des aufs; ils sont done lous ovipares; mais chez les uns, les oufs sont évacues avant que l'enlsı!̣on ne soil visiblemrent dévelopur, comme che\% les oiseaux par exemple; ce sont les ovipares proprement dils. L'enlıryou accomplit son évolution après la ponte de l'wuf, ou après sa sortie de la mère. Les aufs sont couvés dians un mid, e'est-àdire yu'ils sont maintenus à la temperature du corpis de l'oiseau; ils peuvent également ètre convés par une chaleur artificielle dans unc couveuse.

L'ouf des mammifères, an lién d'ètre déposé dans un uid, s'altache à la matrice de la mère; il est couvé dalus l'interieur du corps; la matrice correspond au nid de l'oisear. l'embryon, ell eontractant allterence avec la mère, se nourrit de son sang à l'aide d'un placenta el d'un cordon ontuilical; il sort du corps de lil mère comme le jeune oiseau sort de son nid ; il est tout formé en venanl au monde; c'est unt animal vivipare.

Au lieu d'ètre pondu, l'auf peut continuer a sejjourner dills le corps de la femelle, el l'évolution entsryomaire s'elfecluant comme si l'axuf filat pondu, l'animal est ovovivipare; l'embryon me contracte pas d'alliérence avec la mère. On peut obliger certains animatux à retenir leurs oufs, et d'uvipares qu'ils sont maturellement, on les rend forcement ovovivipales.

Il y a des ovovivipares dans presque toules les clisses du règne animal, à l'exception des oiseaux. C'ét daus la clisse des mannnifères que l'on trouve les veritables vivipal'es, et de loules les autres classes, ce sont certuins poissons pligiostomes, les émissoles, par exemple, qui s'en approclient le plus.

C'est à l'embryogénie à donner les détails de la formation embryonania'e. 


\section{SPERMATOZOIDES.}

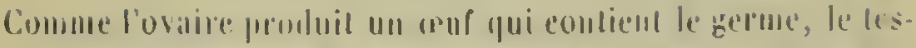

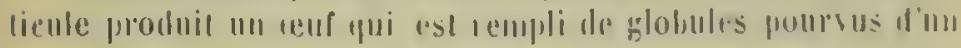

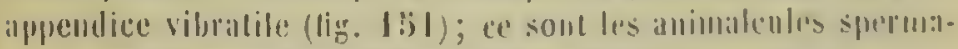

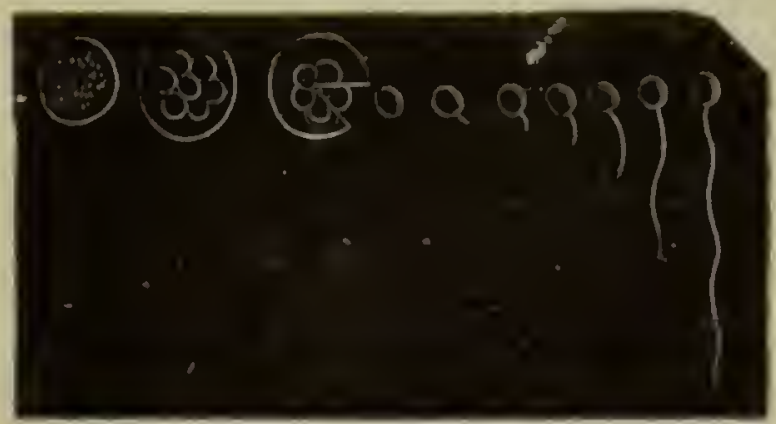

Fig. 1is - Formation dess spermaluzades.

lipues des ameiems, les spermilozoïles des modermes. On en

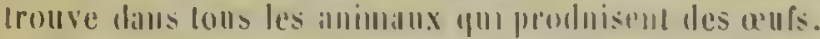

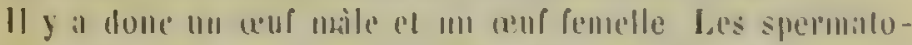

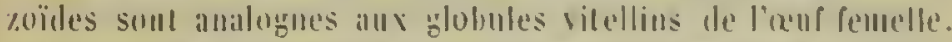
Ces glohules viloflims devienment lihres dills les mailes eat ils for-

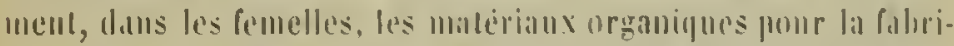
rallion des embryous. Les spermaluzüldes formand lessence le la liqueur mile, commo les ghobules du simg formentlessence du sille dalls les animaux verlibris.

Ce ne sunl evilemumbil pas des animaleules parasiles.

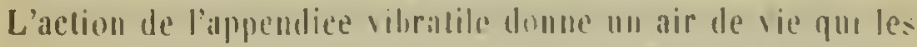

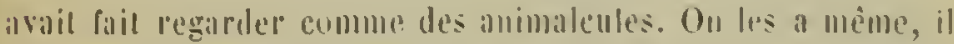

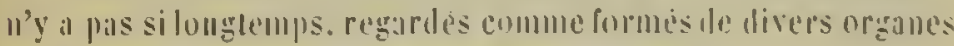

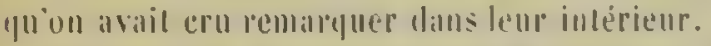

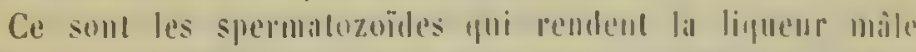
ficonde : le liquide yui a perdu ces eorps ne produil plus ancun roflel sur les auls.

Pour produire celle fécomblian, il me faul aulre close que

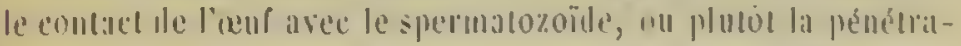


tion de eelui-ci à travers les enveloppes, soit dans l'intérieur del'ovaire, soit dans l'oviducte ou la matriee, soit liors du corps de la mère. On peut produire la fécondation artificielle comme dans les plautes. Chanue espice a une forme de spermatozoïdes १ui lui est propre (ijg. 152).

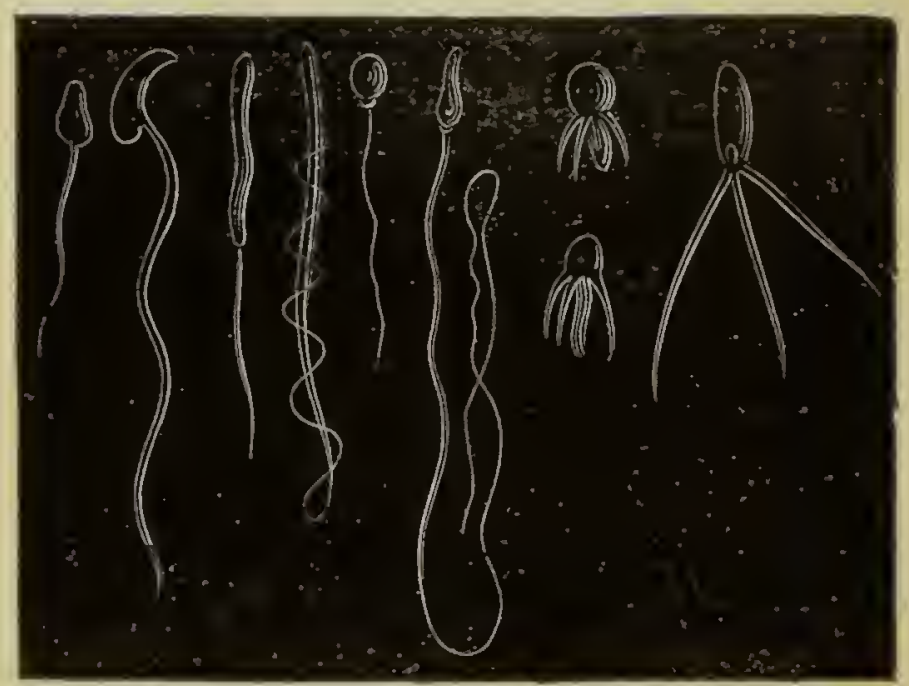

big. 152. - Spermatuzujiles de l'lumme, du rat, du cot, Ju hombinator, de la loche, du colimaçon, de crabe, d'écrevisse et d'ascaris.

Les lifférences principales que l'on remarque entre eux eonsistent dilns le développement plus ou moins grand de l'appendiee vilratile qu’on a désigné sous le nom de queue. Il est généralement unique, plus ou moins long, quelquefois immobile et triple; mais la disposition la plus remarquable est eelle que. uous montrent les cíphalopodes. Aussi bien que le vitellus s'entoure d'une eoque el de blaue fouruis par l'ovilucte apris sa sortie de l'ovaire, aussi bien le spermidlucte forme quelquefois une gaine propre autour des spermatozoïlles, el eeux-ci soul reufermís lans un tube qui est laneé far le màlt: sur le corpss de la femelle et éclate eomme une fusée. On l'appelle spermatophore (fig. $1 \$ 3$ et $1 \$ 4, p .167$ ). C'est par ce singulier moyen que la fècondatiou s'opere eliez ees aluimanx. 
Cliez le eyclops eastor, un tube cylindrique, rempli d'un liquide spermatique, s'échappe de l'ouverture sexuelle du màle immédiatement après l'embrassenent; le mile suisil ce tube el

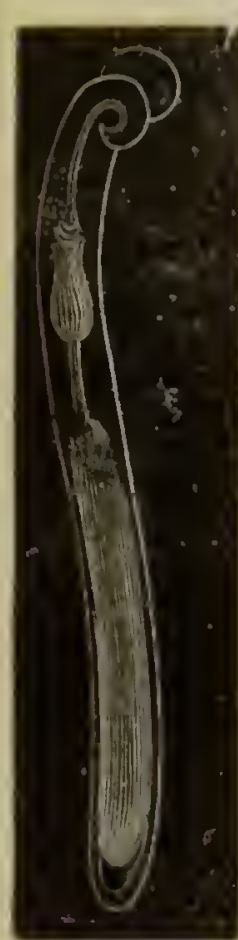

rig. 13.i

Spermilopliore de

Ia scielin.

M. Eidwards.

le esolle contre le ventre de la femelle, au-dessous de lin villie.

I.es spermatozoides sout des organes de l'économic qui survent is la fécondalion; la durce de leur vie est plus nu moins longuro

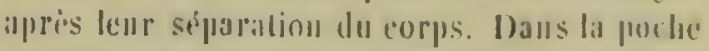
copulative des insectes, la vilialite a consorve peradint plasieurs mojs. Nums crogons qu'elle peut se conserver des amuses clue\% les lingualules el cleez plusicurs vers.

MAMMIF ÉRES.

Appareil mile. - Cet appareil compreud: le lesticule, te calnal déférent an le spermidncle, la vé. sicule séminiale, le canal ijaculaleur, la vésicule prostalique, le pénis avec le cinlal excréleur commun qui le parcourt dans lonle sa Inngueur, el enfin des glandes qui

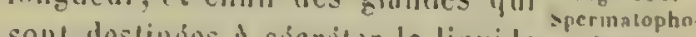
sont destineses al sécriter le liquide re de eyclop. qui doil lubrilier les surfaces.

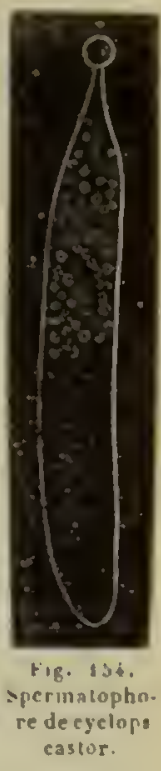

Le testicule est toujnurs dnuble cher. les mammiferes; dans le jeune igge, il est loge dans l'abdomen oủ il reste pendaul

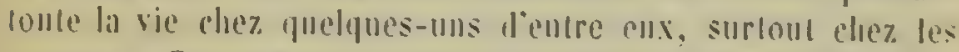
lougeurs. Cel organe est toujours arrondi el envelnppe de plu. sieurs membranes, quand il est silue a lextérueur. L'iuterienr se complese de cillanclrès-fius, a roslomosés en tre eur. quion appelle vaisseaur séminifires el qui comnouniquent areclecanal excréleur (fig. I:ii). l.ẹpididyme ne manque jamais dans celle classe.

l.e cantal deférent est tris-lung el se rend at la hase du canal 
de l'uretre, en montrant la vésicule seminale pres de sil termimilson. Cetle vesicule seminale n'existe pas consiamment, mais

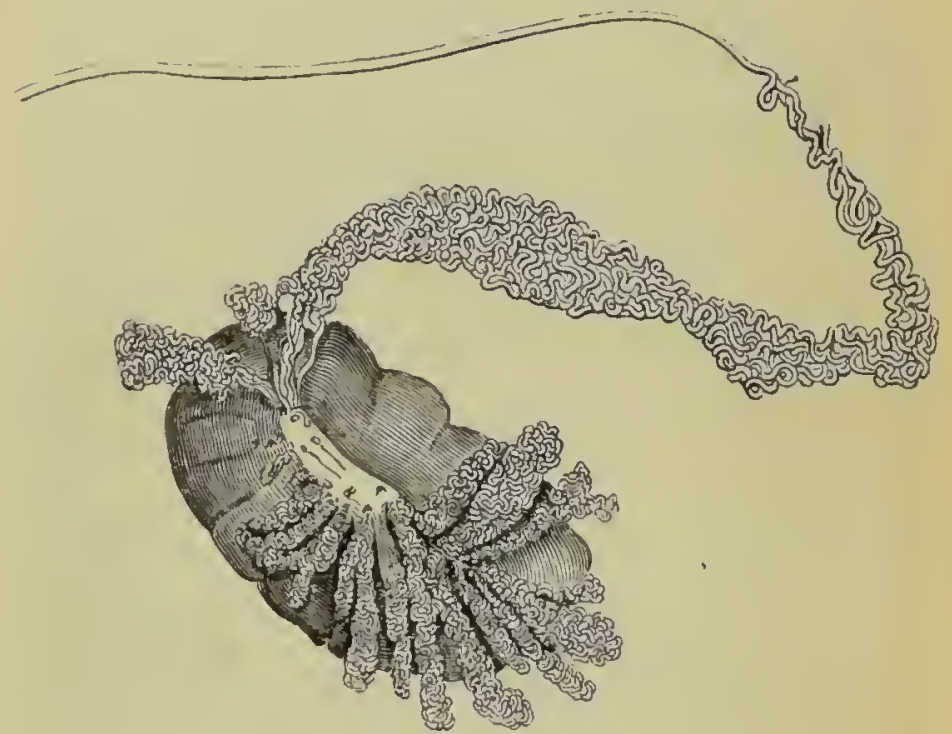

Fig. Ij3. - Testicule injecté au mercure

on trouve toujours entre les deux canaux une petite poche qui repriscule la matrice mâle (figg. 156, p. 169).

La vésicule sèminale, en effel, manque clac\% beaucoup de carnassiers el de marsupiaux, cle\% les monotrèmes, les célacés el les rongeurs. Il y en a trois dins le cheval et une très-grande lans le lièvre.

Un cantal commun, le canal de l'urètle, conduil ensuite au Jehor's, à travers le pénis, l'urine et la lipueur spermatipue. Il ! it un pénis dans tous les animaux de celte classe, mais dalls chaque espèce il a une forme particuliere. Le gland qui termine el organe est quelquefois couvert de soies ou nreme de petites écalles. Un os est logé dans l'intérieur lu pémis clač beaucoup de mimmifères, e'est l'os pénial. Clıez quelines mimmifères, comme les singes el les quadrumanes, le pénis est pen- 
dant; landis pue d'antres, comme fes ruminatuls, le portent dans

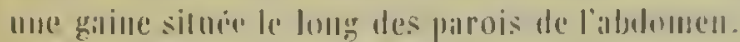

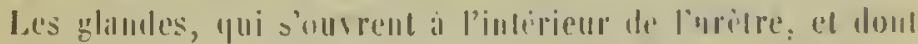

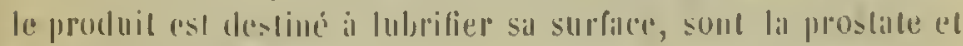
les plandes de Cow per. Lal prostilte est somvent mullilubée et Irìs diveloppée dansplusients: rongertes a inserti. vores. ()II ell compul. Irois paires dians la lıérissoll (") illtanl dialls le rilt. Lal grlatule de Cowper ariste dalls la

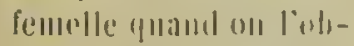
serve dills: le mille. Elle est tris-meriludte dallis le ril el les lliallres-soutris en garmiril: Iris-pelile, ill ronlrilire, dians li chliall rel nulle dians le chien.

Appareil femelle.11 se compose diul

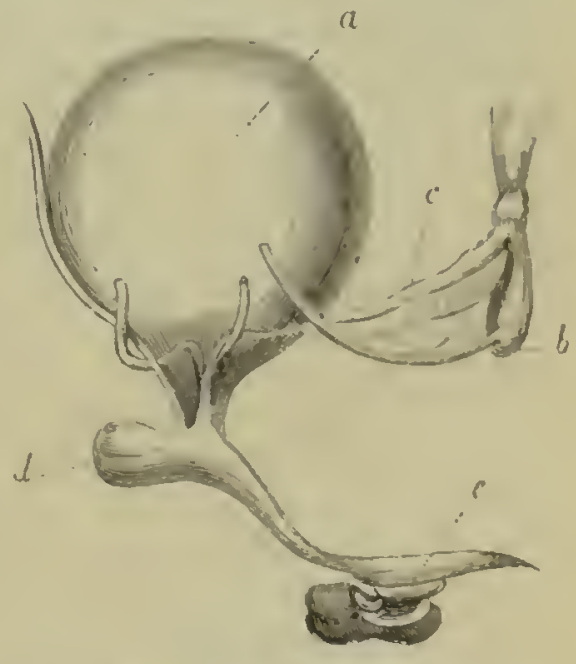

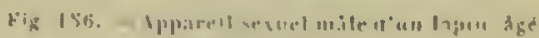
if Irois urus

"Vessie urinaire. - b Tislicule. - r Canal délérent. - d likrus milr. - I'éuis.

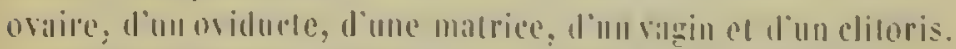

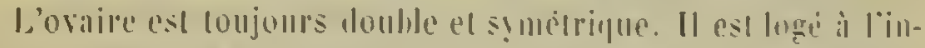
lérienr. Sa formeso papproche le celle du lestienle, à moins que

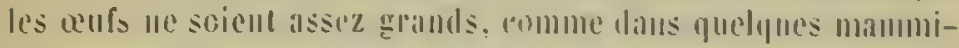

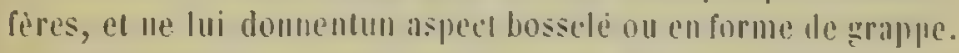
C'est losare qui produil l'oeuf, comme le testicule produit les spermatozoinles.

J.'euf, arrice ì sa malurile, quille lovaire, péuétre dans loviducte el va se leace dias la malriec pour un temps variable dans chargue espder. Ces deux oviductes sunvrent sumvent dans

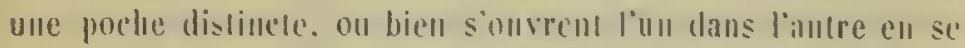

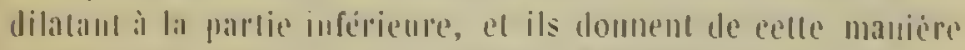

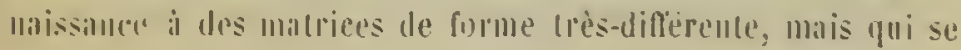


rapportent à un seul et même týpe. C'est la partie iıférieure de l'oviducte qui se sépare plus ou moins neltement el qui devient matrice. Comme l'oiseau couve l'ueuf dans son nid après la ponte, le manmifère couve son ouf dans la matrice. L'ueuf de l'oiseau, au moment de la ponte, eorrespond à l'œuf du mammifére au moment d'entrer lans la matrice. L'époque de la ponte correspond au monent où l'œuf quilte l'oriducte. L'oviducte se tcrmine superieurement en entonnoir, et eet entonnoir est plus "u moins bien silué pour reevoir les oufs mùrs qui se déticlıent de l'ovaire. La matriee est simple dalls les singes, les cheiroplères el quelques élentés; elle est bicomue dans les ruminants et les cétacés, divisée dins les earmassier's et donble dans li plupart des rongeurs. Le vagin s'ouvre directement en dessous llu canal de l'urètre. Clıez jlusieurs mammiferes, il existe à l'entrée du vagin une valvule qui a été désignée sous le nom de membrane de l'llymen.

Le elitoris ressemble eı généril au pénis du màle. Il est trèsnnilud el pereé comme le penis cliez le maki el le lori, simple dans les mammifères monodelplıes, double ou platô bifurqué dalıs les didelplies; le clitoris de la loutre porle un os pénial.

Chez lous les mammifires, l'embryon ne sijourne pis dins la matrice jusqu'ilu développenent eomplet de ses principaux organes; les mammiferes de l'Australie, que l'on nomme didelplies ou marsupiaux, portent sous le ventre une bourse, dans lilquelle se trouvent les tetines des manelles; les petits quittent la mitriee quand ils sont eneore à l'etat embryonmaire, ils séjourıent dans eette bourse un temps déterminé, entés sur les retimes, el dans celle seconde matrice ils prennent leur développement complet. Ces singuliers animax maissent pour ainsi dire deux fois, el ils peuvent rentrer eneore dans cette poctie sous-abdominale aussi longtemps (qu'ils sont trop faibles pour se défendre.

Leur appareil sexuel se compose de deux ovaires el de deux oviduetes, d'une maltrice assez petıle el de deux valgins quis'onvrent dans un canal uretro-sexuel unique (fig. $1: 57$ ). Quelyuefois la matrice (macrop). Beneuii) s'ouvre directement dans le eallal urétro-sexuel (Paclman). 
Les mâles ont un pêtis dirige en arriere pendant le repos, et le ghand est divisé en denx parties, correspondant aux deus vagius.

l.es mnnotrèmes oul ì drnjle el ì fanche un nvaire liosecté comme celui des niseanr, el III ovidurte qui se lible ì sa piortie infsrienre aviant de prometrer dans le callail uríIro-sexinel. C'est l'ilppiareil sexuel des nisialus, mais an lie'u d'itresimple conme dians cenr-ci, il est double (ligg. fisis).

l.es mamelles sout l'apanage exclusif de la première classe du règur animal; cest it cinse de la présence de ces oplandes quinu les appelle mimmifieres.

Eilles sont formers, eomme les autres glandes, de corums ramiliés qui s'abouchent it un on quelques camaux exere-

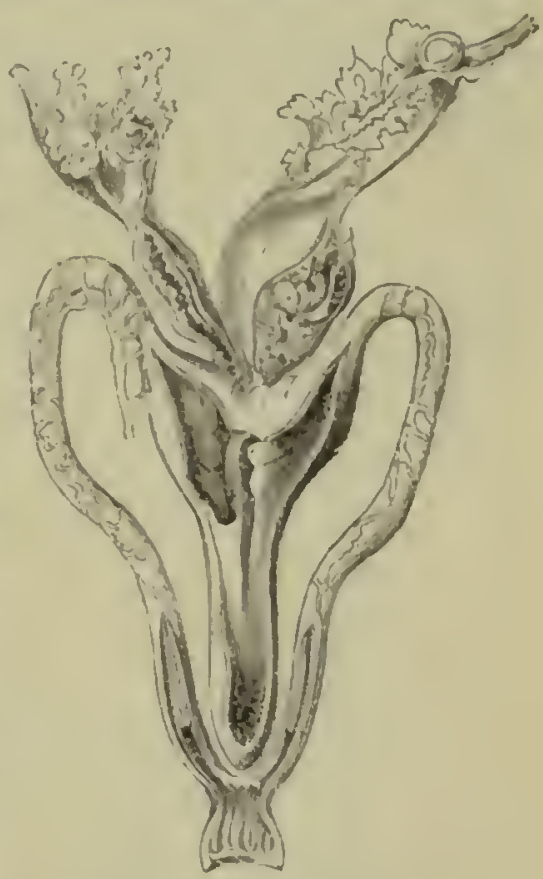

Fig. 157, - Apparesl femelie de kanguroo. teurs. Ce camal est terminé par une papille (le mamelon ou le tetiu), à l'exception des monotrèmes.

Il existe un rapport entre le nombre de pelits que l'animal produit à la fois et le nombre de mamelles; on voil une paire de mamelles pour un jeune.

Ces orgaues varient beaucoup quant i leur situation et leur nombre; les mamelles sont : pectorales, abdominales on inguinales, selnu qu'elles occupent lin proirine, l'abdomen ou le pli de l'aine. Quelques mammiferes portent ees trois sorles à la rois.

Elles sont silures à lextérieur dans les divers ordres de 
ınammiferes; dans les marsupiaux, elles sont silucies au fond de la loourse (fig. 199 ).

Les mamelles sont peetorales dans les quadrumanes, les

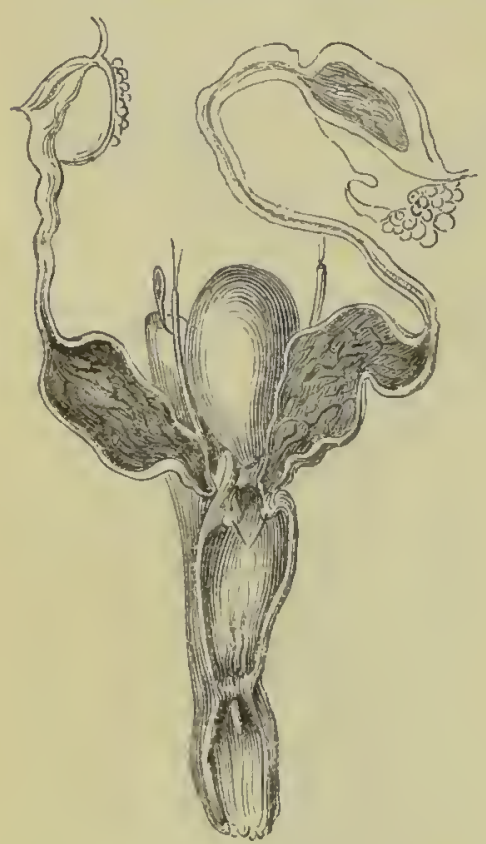

Fig. 158. - tppareal fimelle d'ornithorlignque. eheiroptìres, les bradypes, les ećlacés lıerlivores el l'éléphant. Chez les carnassiers el les rongeurs, elles sollt surlout abulomiuales; les ruminants et les paehydermes les ont souvent inguinales.

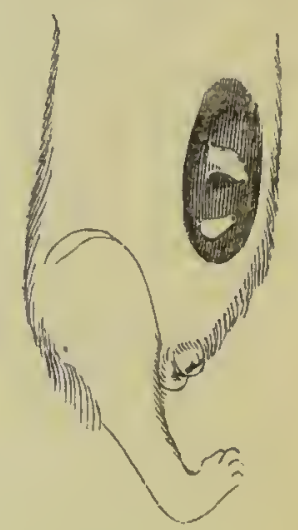

Fig. 159. - Finurse m mamrlles de didelphis opossum.

Les telines du eoïpon sont situées, au nombre de quatre, sur les flanes, le long d'une ligne qui partirait au-dessus de l'œil et se dirigerait ver's les lanehes. Elles sont siluées sur l'épaule chez le pore-épie.

Les cétaeés véritables portent deux mamelles à côté de l'anus.

Oiseanx. - Les testieules sont doubles, de couleur blanelie, de forme arrondie el de grandeur très-variable selon l'époque de l'année. Les testicules des coqs sont au moins quatre fois plus grands au printemps qu'co hiver. Ces organes sont toujours logés dans l'iutérieur le la cavité abdominale, en lessous de la parlie antérieure des reins. Ils sont composés de vaisscanx ou 
plutol de lubes seminifires tres fins, comme dans les mammifires.

Les camanx excrélenrs forment iो leur sortic un reullement

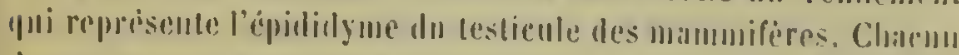

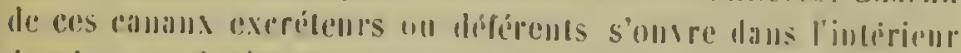

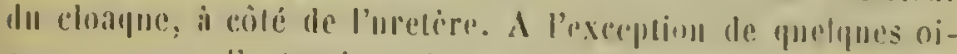

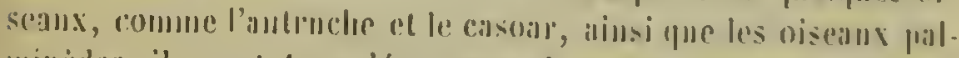
mipredes, ils sont lous dépontrus de pénis veritalule. Cet organe, quand il axistre, présente le long le sid face inférionre une

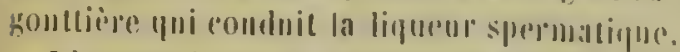

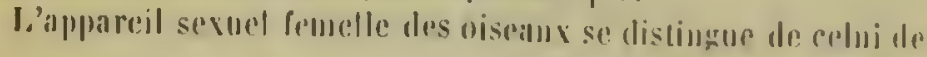

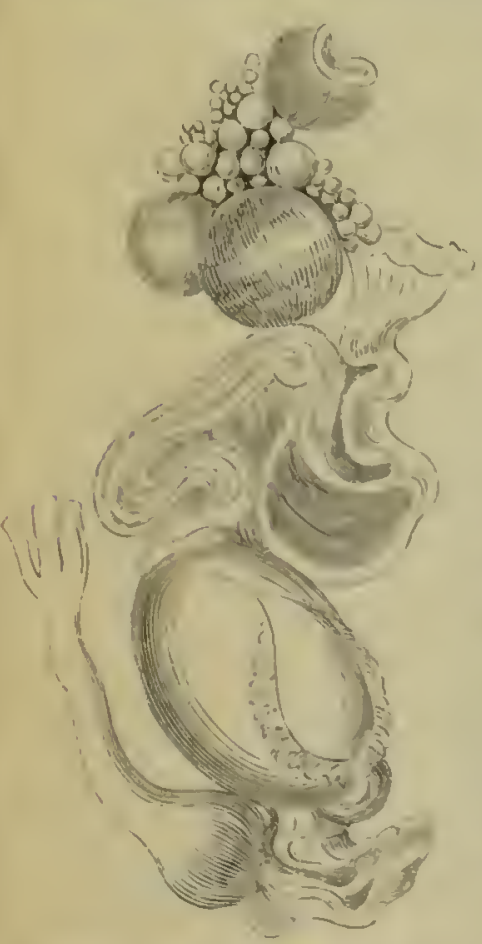

Fig. 160 . Organes génilnux de poule.

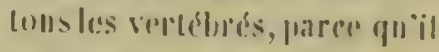
ne se compose que l'un sinl ovaire el d'un senl oviduele.

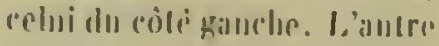
est de boune heure atrophia: (lig. 1 (io). Cel oraire est silurs à la meme place qua Ie lesticule ganche dims le mile: it a 1111 asppect hosseptí ou promel

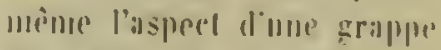
de raisill. II est healucoup plus déseluppé a lipopue des anomors el contient des emes: i) Ituss les degris de disinleppeneme. Loviuncle differer a)sa romsidierablement de diamelre: il est termint suprerientement en entonmuir el reçoil lienf mur qui se délache de lovaire. Cel ouf ne cumsiste que dans le jaune. L.ps parois de loviducle sécribent diathord le blance on lallumen qui dwil envelopper le janne en, a sa partie inferienre, an milien dinn renllement qui est une sorte de matriee, un

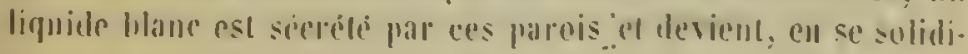


liant, la conuille de l'ouf. Ce canal excréleur s'ourre aussi dans le cloanue, el on trouve un clitoris dans les fenelles dont les màles porlent un pénis.

Les oiseaux, à l'époque de l'incubation, perdent les plumes de l'abclonen, el les vaissenux de la peau prennent un développenent extratordinilite; il en résulte une angmentation eonsidéralble de tempéralure dans celle régrion.

Quoique les oryilnes sexmels soicul toujour's eaehés, dius les miles comme dans les femelles, on chitre cependint les oiseans anssi bien que les mammifies, pour rendre leur chair plus dílicate. Le con devient clapon apres cette operation, el la poule poularde.

Parni les viseaux, les pigeous sont pourvus d'organes qui He sont pals silns quelque analogie avec les mamelles. Après l'éclosion, il se forme lans le jabol mue secrétion assez abondante d'un liquide lateseent qui imprège la nomritnre que lis mère dépose d'aboral daus son jabol avant de la dommer à ses petits.

Reptiles. - Les lesticules ne sont généralenent pas placés a la même hauteur dins la eavilé abdominale, et ils different en mème temps entre eux par la forme. Ils sont situés an-devant ou au-dessous de la paltie antérieure des reins. On y reconnait eneore les eanaux séminirères. Le canal déferent est en générial très-long el s'ourre dans le cloaque. Les tortues el les crocodiles oul un penis simple, loge dans le eloaque; les autres sanriens el les serpents ont un pénis double, logé en dessous de li peau, à côté du cluanue, et qui se déroule eomme un doight de gallt.

Il existe deux ovaires dams tous les reptiles, it la mème platee oi se trouvent les testicules daus les miles. Ies oviductes sont an nombre de deux, el contrairement i ce qui se roit dans less deux classes priceidentes, ils sont placis an cóte exterme des ovaires. Ils sont souvent bien longs el décrivent des circomvolutions. Ces ovales sont ereux el les aufs se developpent dalls leur inliricul.

Batraciens. - Les deux testicules sont encont de forme alrrontlie, de couleur blantele el sont silues au fond de lia cavile 
abdominale. On recommait eneore des canaux siminifires dams leur iulérieur. J.e canal déférent se coufond daus un eanal

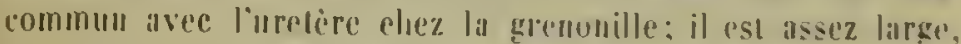
descend le long du bord externe des reius "t s'ourre dius: le éluayne. Il ñy a pas de pénis, mais il existe une pir.

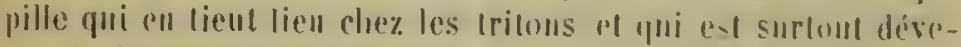
loppe a l'ppotue des amours. II y a somsunt des glamles qui s'ousrent directement dans le rloapue et pue loun a comparées al Ia prostate.

L.es ovaires sout aussi au mombre de deux; ils sont tris-rolumineux, surfout a l'ipoupue des monours. Ils comsistent dans dea

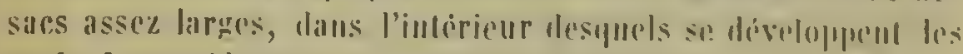

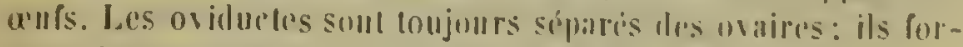

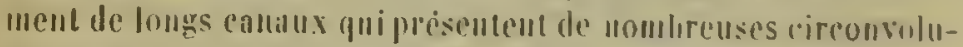
tims el qui sourreut dans la parni dorsale du rloalyue.

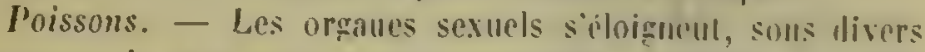
rilpports, de ceux des elasses précrideutes. Quoiqu'll y ait des

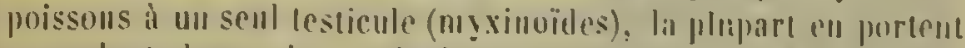
rependant deux; le testicule est toujours tres-voluminemx.

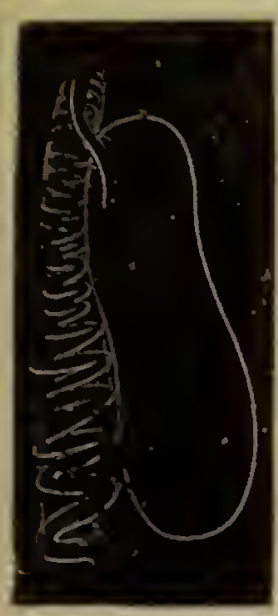

Fin. $\mid 61$. - Testicule et eanal deférent de raic. nceupe sourent tonte l'élendue de la ciavitio aludominale et varie beaneoup de conlenr et de cousistance. Il ressemble, dans quelques poissons, par exemple lesauguilles, tellement

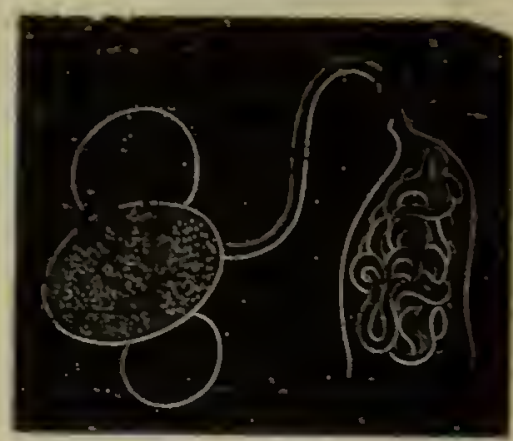

Vig. (68. - Torpedo. diapries Mullet. a Teslioule. - $b$ pididyme.

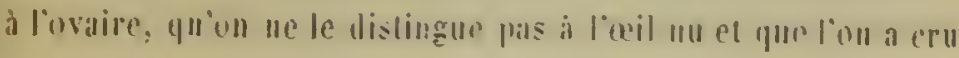


mudant longtemps que ces poissons u'avilient qu'un senl seve. Cet organe est treux (fiğ. 165̄), très-long, intestiniforme clıe\% quelques-uns; les spermatozoides se forment dans l'inlerieur. Il est compose de gramulations Irès-fines. On recounaît aussi dans plusieurs poissons les cinnax semminiferes qui sont anostomosès tutre eux et qui se reeunissent ell un canal dẻférent.

I.es testicules sout elltourés en partie jar le péritoine, et ils ont en onle une tunique propre, qui enveloppe les canaux sperIIIatiques.

Les poissons plagiostomes ont les testicules composis de cauaux ou plutót de cloisons qui dıisent leur sulsstance en cavités plus ou moins gramles; chatente de ces cavilés renferme une vesicule, qui est l'emplie de cellules dans lesquelles on irouve les spermilozoïles; des canaux affércnts assez larges aboutissen

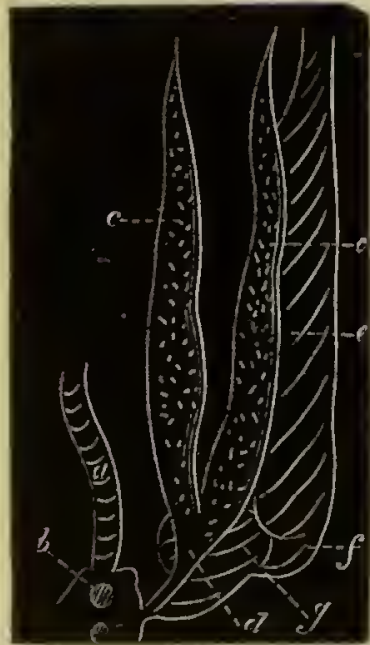

Fig. 16j. - Apiarcel mảle de trigla.

a. Reclum. - b. Anus. $r$. Testicule. $-d$. Canal déférent. - $e$. Reius. f. Urelèrs. - g. Vessie.

à un eanal déférent, après avoip formé uII épididyme composé de nombrenses circonvolutions (fig. 161 et 162 ». 175). Le canil excreteur s'ouvie dans le cluaque conjointement avec le canil excriteur des reins. D'autres poissons n'ont pas de traces de canal déférent, la liqueur spermatique est directement évacuée par une ouverture siluée derrière l'inus, ou bieı encore la liqueur tombe dans la cavité abdominale el se répand ensuite au deliors par une ouverture post-auale (eyclostomes). Les deux callaux défejrents se réunissent souvent en un canal commun qui s'ouvre eusuite dans une pelite pilpille sifue derrière l'inus (pleuronectes).

Ice calral defirent des esturgeous s'ouvre dans la eavilé abdominale, à sou origine, comme loviducte des rlisses superpieures, et s'abouche dans l'uretère.

Laes miles des poissons plagiostomes portent ì l'extérieur 


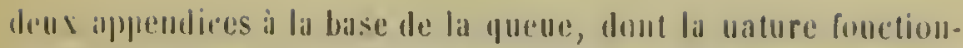

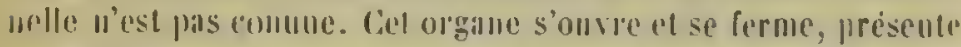

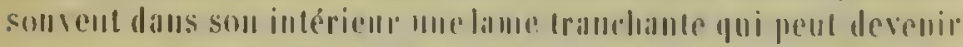

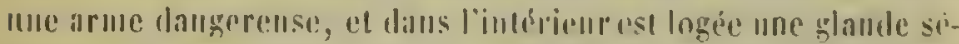

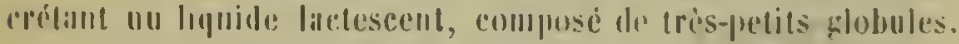

l'ovaire des poissons est sumvent un sac, dans l'intérieur

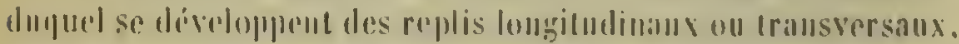

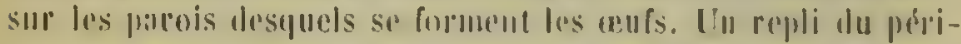

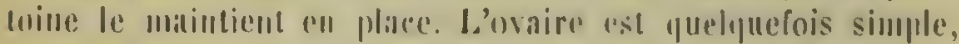
mais plus sousent cependaut domble en toujours lrès-tolnmi-

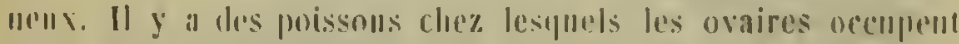

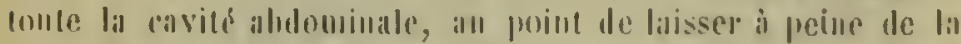
place pour les antress viscrires. loviduelo mangue quelquefois at

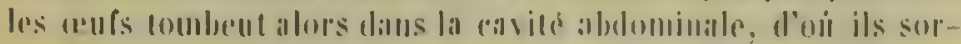

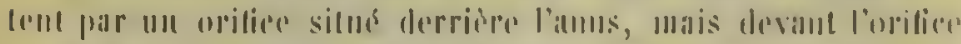

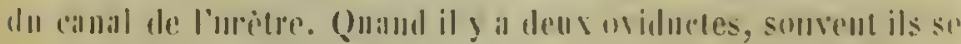

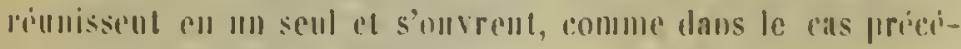

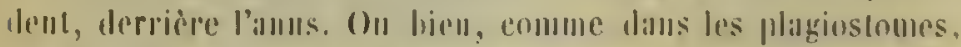
nii l'ovaire ast eomparativement petil, el quelquefois unique, les

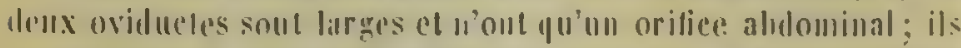
presenteut sur lemr trajet, eleez, les nvipares, une forte glande

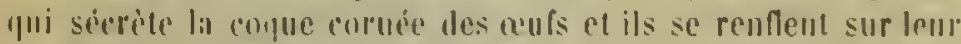
Irajel pour formene me matrice véritable dans les ovo-viviparres. romme les mustelus; avant de somsir dans le cloaque, le. dex oviduetes se reunissent en donmant masssance à une sorte औ. vagiı.

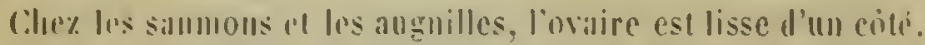

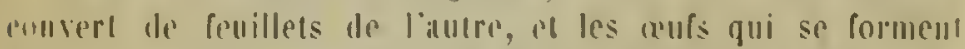

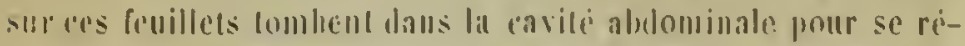

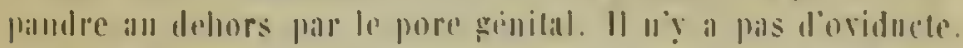

les esturgateons out loviducte ousert dans la cavite abdominale pal" un entomunir. comme le canal deferent dans les miles: le's maires sont tris-voluminem et laissont lomber le: urufs dans la eariti ahdominale. l.es ovidueles souremt ensuilu

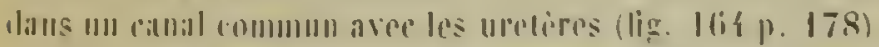

Cliez les syugnallues ol les hippoempes, poissous lophobran-

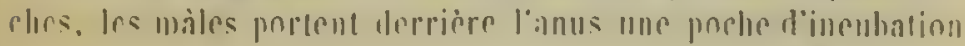


formée par la peau et dans laquelle les embryons achevent leur développement.

Insectes.-Tous lesinsectes ont les sexes séparés; quelquesuns, comme les aheilles ou les fourmis, ont, outre le sexe male

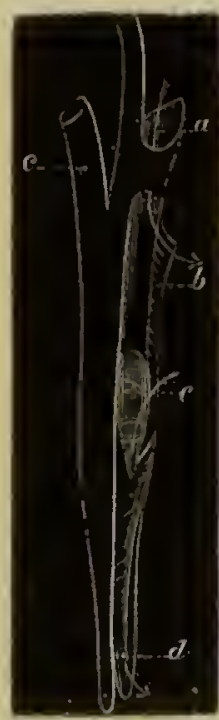
e.t femelle, le sexe neutre, et l'espèce se eompose de trois sortes d'individus. Ou dit que les neutres sont des femelles, dout l'appareil sexuel est dans un arrêt de développement.

Les inseetes sont ovipares, tous s'ae-

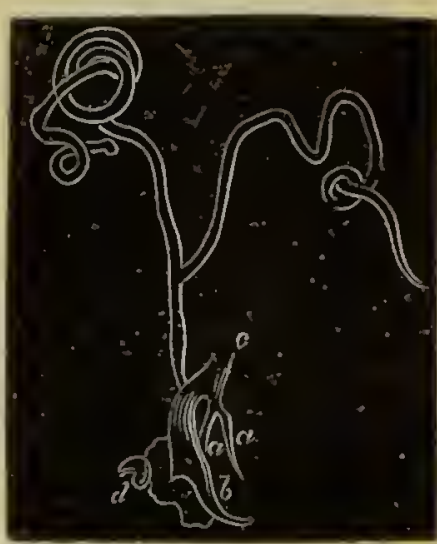

Fig. 165. - Appareil màle de cirabs.

a. Muscles. - b. Pénis. c. Canal ejaculateur. opposé.

couplent; les pucerons el quelques autres sout en outre gemmipares pendant une partie de l'année.

Les femelles portent souvent au bout de l'abdomen un orgills? particulier qui leur permet de déposer les oufs daus les licus les plus convenables à leur développenent. Ces oufs ont des formes très-variées.

Les deux sexes présentent souvent de grandes différences.s. non-seulement dans la coulcur, la taille el la forme, mais dans lous leurs organes. Ainsi, dans le ver luisant, la femells est aptère, ressemble à une larve, séerc̀te une pulpe plısplınreseente, tandis que le mâle est un inseete ailé, et qui ue semble avoir atcun rapjort d'organisalion avee sa femelle. 
Les coufs sont fécondes avint la ponte.

Appareil mâle. - Les testicules sont donbles et syuétriques; ils consistent souvent en un tuhe flexueux tris-long qui se pelotonne quelquefois smo lui-mine, mais que l'un pent aise.

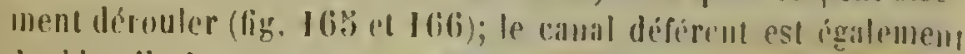
louble; il n'est que la conlimalion du valssean séminifire; les deux cimante se réunissent en un canal gjaculateur qui sonver ler. riere l'anus. loes camaux léfirents se dilintrul quelynefois pour former une vésicule séminale, et l'ou trouse habituelfement sur le trajet du conduil éjaculatemp deux careums sim-

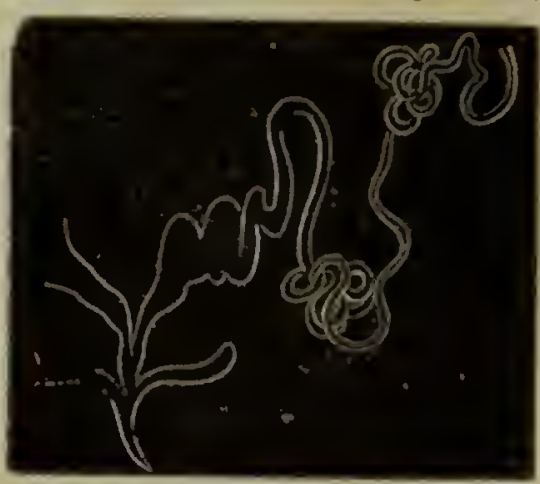

Fig. 166.- Ijpareil male dinsecte. ples, qui reprisenteut la prostate.

lies enmanx séminiferes qui eonstitnent le testicule varient considérablement en nombre, forme, volumer, foudue, etc., au moint de ressembler à des eorps tris-différents et d'affecter parfuis la forme de neurs.

A l'extrémité postéricure de l'abilonen, ou voil les organes de la copulation qui sont exIraordinairement variables d'une 'spice à l'antre. Outre le pénis, les mile's portent des pièces cornées en forme de stylets ou de piuces, qui varient beauconp et qui servent d reteuir la femelle; on roit souvent des organes pareils sur les autemues ou sur les pattes.

Dins quclques insectes, il y a une intumeseence dans le penis qui fait qu'après l'accouplement, les organes de la femelle, revenant brusquement sur eux-mèmes, saisissent le pénis el l'arrachent au mile. C'est ce qui a permis a lluber de s'assurer Iuela reine d'une ruche d'ibeilles qu'il observisit, était fécondée ì son relour. Elle portait l'orzane nòle asec elle.

Alpareil fimelle. - II existe toujours un ovaire double: sa forme varie extraorlinairement, tout en conservant toujours le type glandulaire. Cliez plusicurs de ces auimanx; nn 
voil des tuhes ellilis at terminés en pointe s'ouvrir dans unr civité commune el montrer des oufs en voic de developpemenl sur toute leur longueur'; ce sont les ovaires (itg. 1 (67). Ils s'ou-

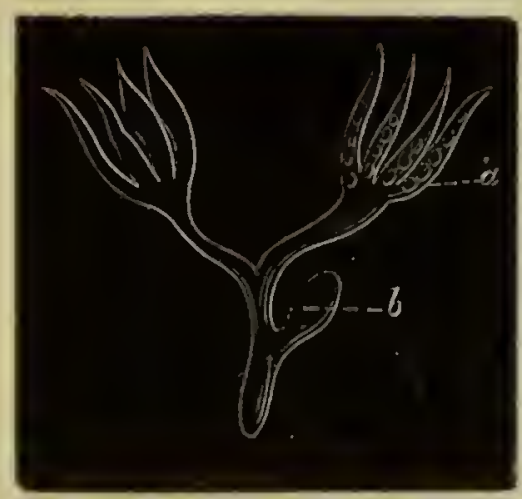

Fig. 167. - Apprateil femelle ansecte.

a. Ovaire. $-b$. Poche copulative.

vrent directement daus m॥ oviducte, el ces deux couduits excréleurs s'abonclient cusuile daus un vagin uniģıe. On remarque dans les insecles sur le trajel du canial sexuel femelle une poclue qui est remplie souvent de liqueur mâle; c'est la vésicule copulative. Le sperme s’y conserve longlemps sans perdre sa faculté fécoudante. Perudint liaceouplenent, la verge dis mile s'introduil dins rette poclue pour y lejposer le sperme.

Sonvent l'appareil femelle exterienr est très-compliqué: O॥ voit des appendices à l'extrénité postérieure du corps, qui servent ou comme organe le défense. ou comme organe particulier pour la distribulion des oufs (oviscapte el aiguillon).

Les pucerous sout agames en élé el se reprodnisent par gemmes; ell antomme, les appareils sexmels se développent dans tonte une grenérution, et au lieu de domer des gemmes, ils sont "vipares.

lal femelle du grillon, pemalint l'accomplement, monts: sur le mile, qui fait saillir une capsule transpiarente, remplie de sperme; celte capsule entre dilns l'orllice vaginal et la femells se retire en l'emportint, une parlic de la capsule restant delırs. Le unâle n’a pas de pénis. (Élırard.)

Myriapodes. - Les myriajodes out les sexes séparés commo tous les varis articulés; les oritices génilinx miles sunt situes daus le sepliène segment, les orifices de l'appareil femelle daus le troisiène, du moins si mons prenous pum ex(mple les jules.

l'appareil màle dans ces deruiers esl compose dr deux longrs eordous, garnis de emeums lateraux sur une grande parlie du 


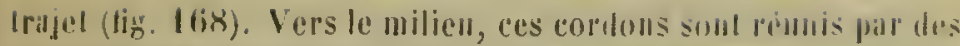
athislomoses. Iacs anaux deforents sont collies l'mu contre l'ilutre et se siplarent loul pris ale lobilice. lamplareil femelle est rencore plus simple (liz. 16!)). Jo'ovaloe esl forme d'un sac: unique lris-allongé, yui s'ćlomel dans loule la lomgunar uli corps, at los ants se revoluppent simulamennent dans lonte l'étendus. Tout pris des orifices, il se forme alems canamx exeréleurs par la lofmrealion du ramal muigue; ils se rement a l'axlirieur aux leux orifices. Il existe un répelate du sprerme.

Jes orilices de cel appareil s'ouvieul ì la pirtice postericure clece les scoloprudies; il u's aurait chr\% los lillobbiros yu'un seul testicule d'apros Trevirions, el un soul orilice erinilal; fol los femelles namratent de meme quan seut ovaire

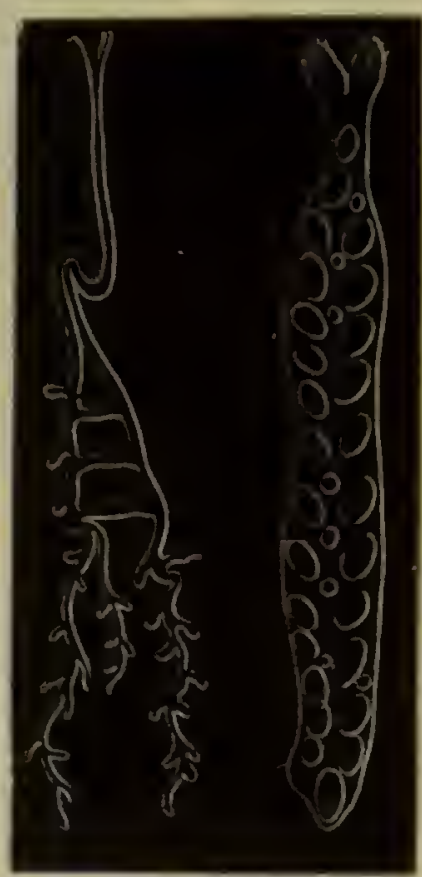

Fig. I6s. - App, Fig. 169. - Alls. male de iule. fenclle de lule. s'onviant dans le dernier strrment du corps. Sur le còté de l'extrémité de loviduele, on découve anssi une résicule spermalique.

Arachnides. - Lesaraigneses véritables ont un appureil sexuel mále très-simple (fig. $170 \mathrm{~N}, 182$ ). 11 consiste en deux tubes plus ou moins flexueux, logés en parlic antre los lobes du foie; chacun de ers lubes continne sous forme de canal deferent, souvent tress-grile et qui vient souvrir dans une fente transverse, situe i la base de labulomen. Les palpes de ees miles sont profondenent modihes el aejuièrent souvent un grand rolume. On a mème cru que ces organes contenarent le testicule dans leur intérieur. Les araignces recneillent la liqueur mále à l'aide dre ces palpes, et elles les appliquent ensuite sur l'orilice de l'appareil femclle. 
L'appareil femelle des araneides est lout aussi simple que relui dr's miles; il existe denx ovaires lï̀s-simples, de forme

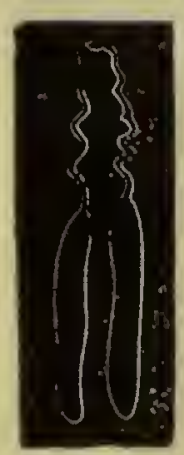

rig. $170 \ldots$ Apjareil male ovale, qui s'abouchent daus mu ovidncte, lequel s'ourre ì sou lour dlans le viugin; l'orifiee de cel appareil est situé à la base de l'abdomcu, entre les deux saes pulmonaires. II existc deux réservoirs séminaux ì eôté l'un de l'antre.

Crustacés. - Tous les clustacés, à l'exceptiull des cimpiperdes, nut les sexes séparés. Les parasiles mème ne font pas exerption.

L.es spermalozoïles des mìles sout souveut enutemus daus me capsule (spermatophore) gui loit éclater dans le voisinage de l'apparcil sexuel fomelle. V. Sicloold a vu lc mâle, dans le cyclops castor, coller ce spermatoplıore à eòté de la vulrc.

Cliez presque tous, il y a une poelie inembatriee que les femelles portent derrière ou cn dessous de l'appendiee de la queue our de l'abrlomen. Chez les erustaeés supérieurs, les erahes et les écrevisses, les oufs sont eollés à l'aide d'une substance visqueuse anx appendices sous-abdomiuaux ; dans les erustaeés inférieurs, les lernéens el d'antres, ils sont logés dans une gramle ct longue poelie pendante (fig. 181, p. 186).

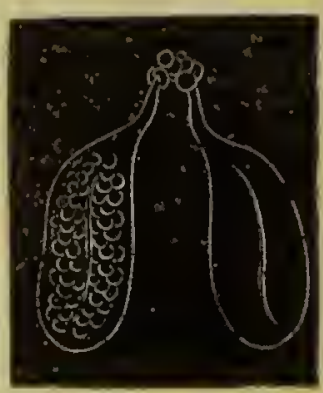

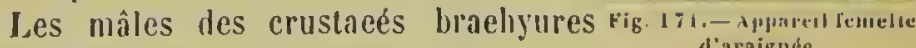
deviennent beaueoup plus grands qu: les

femelles, tandis que eelles-ri devicnn nt des eentaines de fois plus grandes que les mâles dans les erustaeés parasites de la famille des lernéiles. Chez ees derniers, les miles vivent en parasites sur les femelles et ne les quittent pas.

Plusieurs entomostracés (daphués, eypris) se reprolıisent par gemmes ct sans coneours de sexcs; on is vu plusicurs gènérations agames sc sueeéder les unes anx antres comme daus les puccrons jarmi les insectes.

Appareil mâle. - Cct appareil ue differr que tris-lígìre. ment dans les divers ordres de erustacés. I.e lesticule cousiste 
(tig. 172, 175 a 17.4) ('n un lube plus on moins nexuenx, ol-

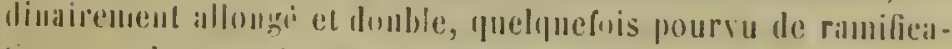
lions, el dims punclpues cits les denx lesticules communiquent

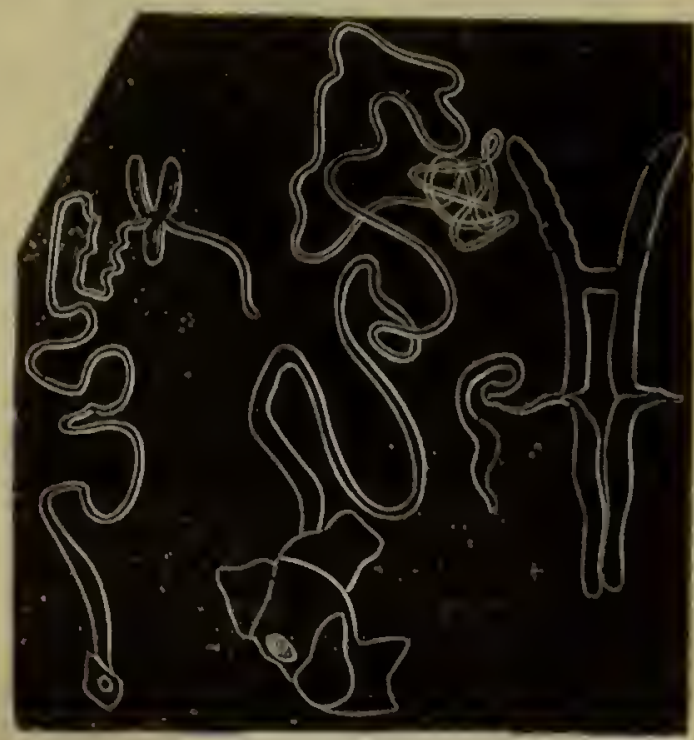

Fig 178

Fił. $17 ;$

tion 471 .

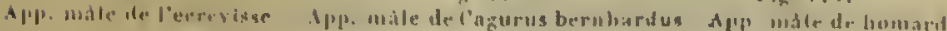

par une anastomose. Dans le homard, drux cocums assez larges se réunissent de chanue còlí en un canal deféreul : Ies antérieurs sont reunis par une anastomose assez laree, les autres soul siparés dins loule leur largenr. Dans l'ecrevisse, on voit trois lohes glandulaires se remur el douner maissance à deux canau

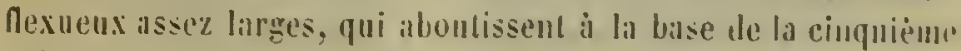
paire de piltes. I.es deur testicules somt des tubes termines en cocums tres-lougs, entortillés el sans communication l'un aree: lautre daus les pagures. Dans fouscus murarius, les deux leslieules soul séparis aussi, mais ramilies au boul; dans le genr. anchorella (lemeru). Ie testicule cousiste dans un lube asse?. large, divise an lohes dermine par un conurt caunal dérérent. A l'ipoipue du fria, on distinge bujours aisement le spermiducte par sa coulenr blanche (lig. 175). 
Le canal déférent se déroule souvent, comme un doigt de gant, pour servir de pénis, ou lieu le premier appendice abdominal,

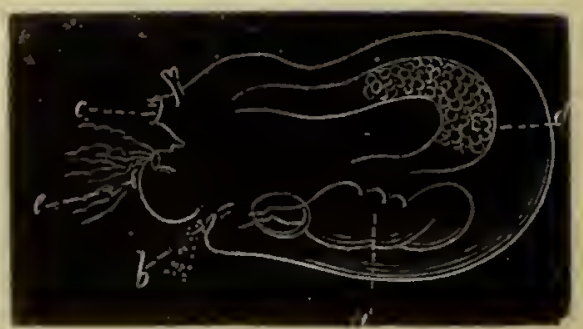

rig. 175. - Aneforetla rugosa mâle.

a. Testicule. - b. Orifice. - e. Bouche. - d. Intestin.- e. Anus.

quelquefois mème le second, se modifie en se crensant daus une partie de sa louguenr el fail l'office d'organe copulateur.

Il se forme des spermatophores dans plusicurs animaux de celte classe; ils affectent des formes différentes el qui se réu-

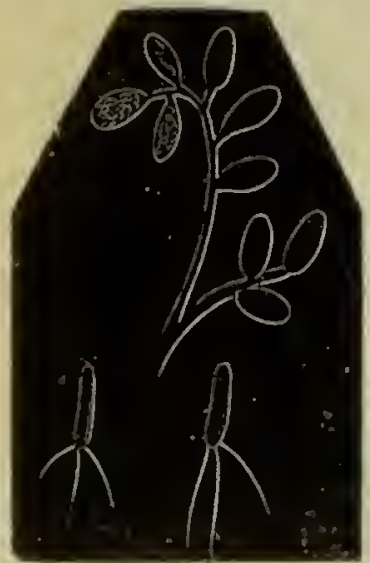

rig. 176 .

sprermatozoidis de galathea st rigosí.

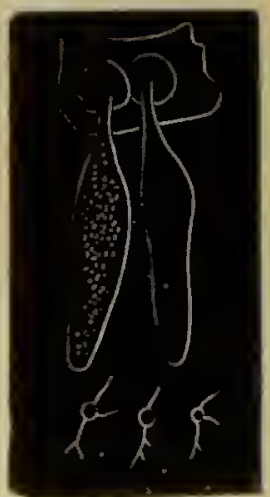

Fis. 177

Spermatophore de pasurus.

uissent quehurefois en grappe; nous en voyous un exemple cu-

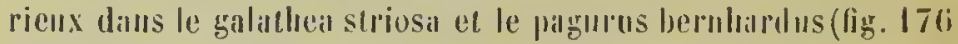
et 177).

Le teslienle est unique: dans les lingualules el cousiste taus me longue proche à parois minces; denx cananx deférents con- 
duisent le sperme i deux poches dans lesquelles est logé, do

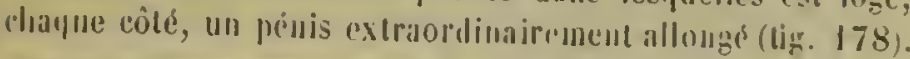

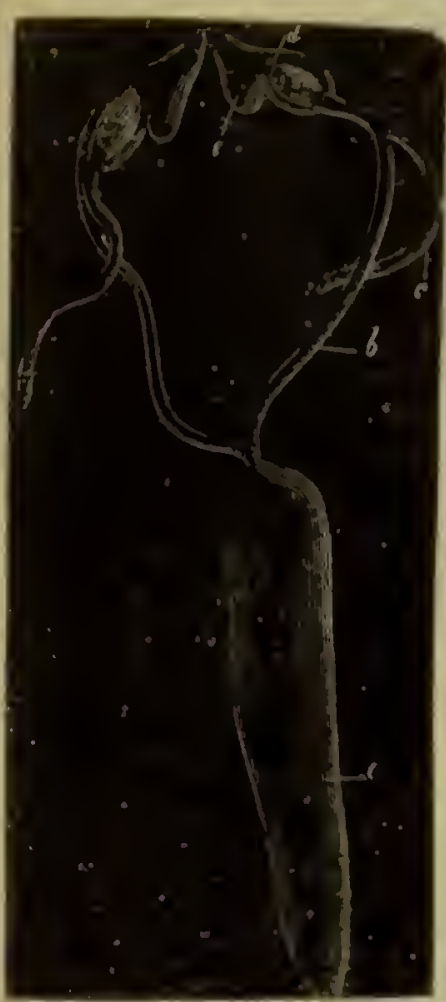

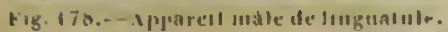

2. Testicule. - b. Canaux déférents. c. Fourel du pénis d. P'oche logenut le penis.

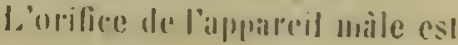
- ilué usun foin de la bouche, sur la ligur me miane.

L's ovaires soll formes, daus les crmstacés decapodes, de qualtro

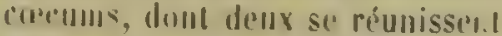

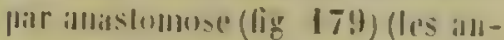
lérimars). Ils almoutissent i un canal revritemr, un sagin, qui s'ou-

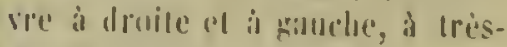

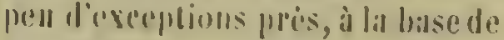
lat lonsidume paire de pilles. Daris les crille's, il $y$ in de flus un or-

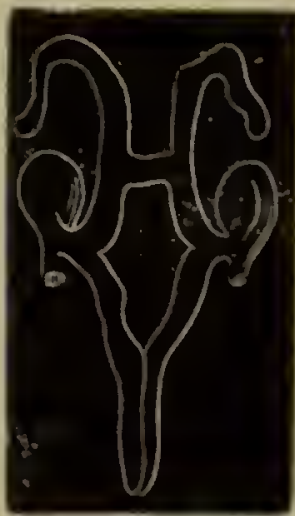

Hif. (79. - Ippareil femelle de moila.

gane sous forme de porlye ou de glandr', silué près de l'origine (dil vagin et sur la malure duquel on li e'st pas d'aceord.

Cet appiareil est logé en graule pirlie en dessous du cephat-

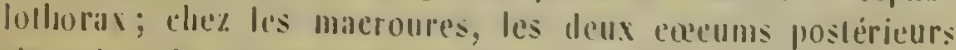

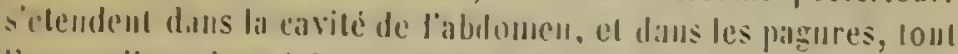

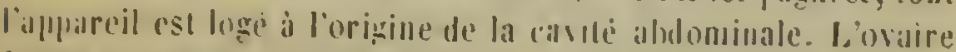
forma une masse romposie de lovis lohes comme le lesticule

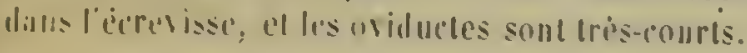


Dans plusicurs autres crostiteis isoporles et amplipipodrs, l'ovaire consiste en demi tubes simples qui s'ouvrent séparémecut it la base de la cinquieme paire de palles (fig. 180).

L'ovaire est ramifié dans les limules el souvre de chaque colé il la base de lil premicre paire de paltes abdominales, pross de la ligne médiane.

Outre les deux coecums qui forment l'ovaire, on trouve chcore souvent une glan-

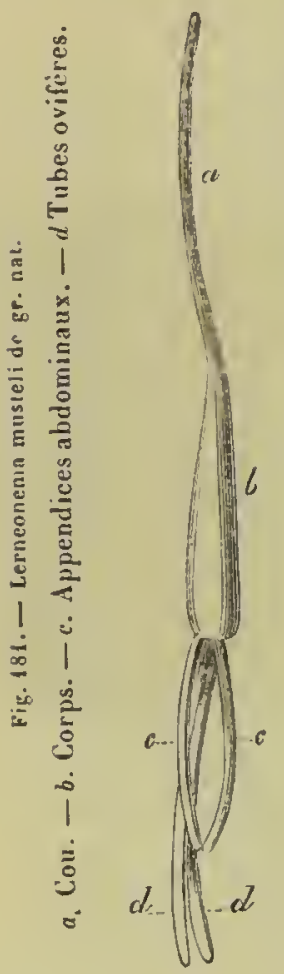

de accessoire, destinie a secriter, selon lonte apparence, une. enveloppe aux oufs.

Les crustaces re-

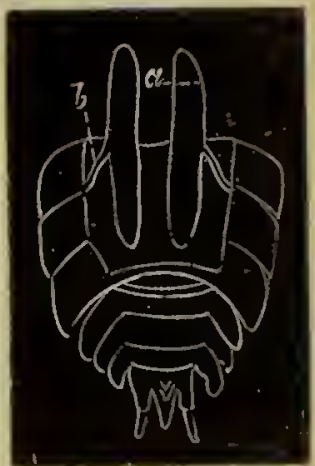
mellẹs portent, en gé- a. Ovaire. b. Oviducte. ucrial, leurs aeurs jusyu'au monent de l'éclosion, soil dians de lougs tubes, conme chez les lerrirens (lig. 181), soil le long des appendices illdominanx, auxiquels ils sont solidement atlachés à l'aile d"une maliche visquense.

Les ovaires sont logés dams l'intericur des paltes dians les pycuogonous (figure 185), mais nous ignorous si les aenfs sont contemus dinns les camanx gilsIro-vasculailes, ou s'ilssout situés ell delıors; nous nc savous pas davallage par où se fait lá ponte. On ne connail pas l'organe mâle.

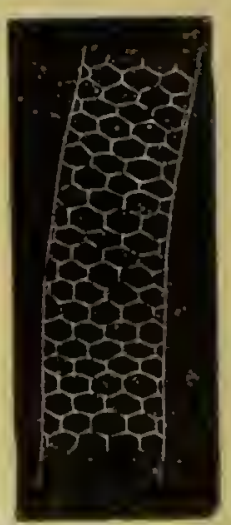

Fig. 182. - Tutse oviferen nossl.

L'ovaire des linguatules est double en avant el consiste dialıs III tube long, à parois minces, qui s'étend dans toute la longnrul du corps. Il y a deux oviductes pairs, deux vésicules copu. 


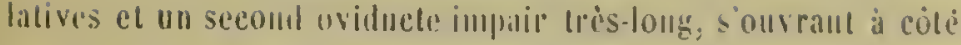
de. l'allus (lig. 184).

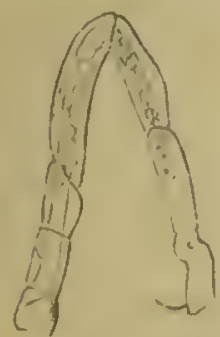

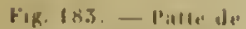
(1) romgunetr.

Cimhiprides. - Les seves sont rimisis; ce sont pent-ritre les semls hermilphrodiles do celle chissie.

l.e testienle est formé d'un cordon bline sinueux plus ou moins l'amilice, silué te chaq̧u' còté sur les fialles.

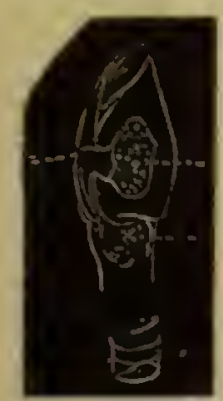

Pig. 185. - Ananile lians. a. Oraire. - b. Orisin - Povhre commune.

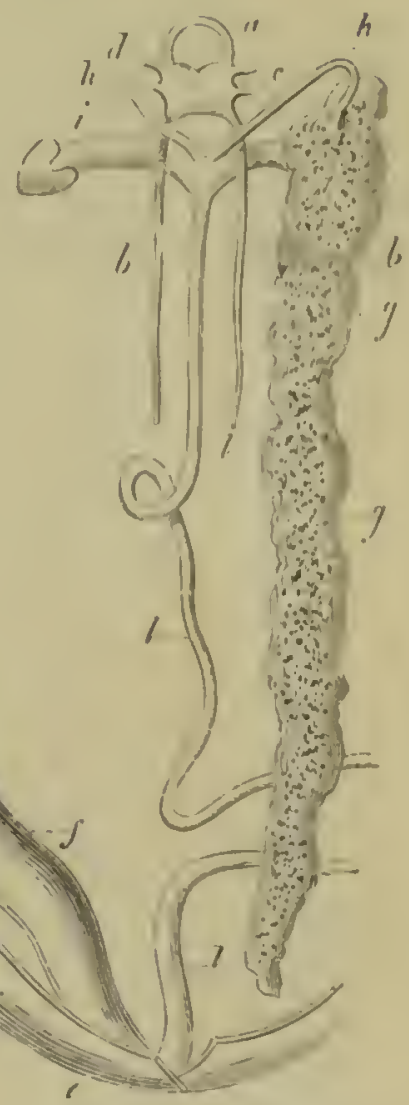

Fig. 184. - Ippareil fewullle de linguamle.

a. Collier minplugin. - b. Cordons nerveux. - c. lianglion. - d. Filets mervell. - f. Reclum. - g. Ovaire. - h. Premier ovidarte. - i Visicule eopulative. - I. Second oviducte.

Les spermatomoildes ont la forme ordinaire, un disque et un tris-long lilinmellt.

Lovaire est silui en dehors du corps (fig. 18:i), il la partie la 
phus basse chez les balanes, à la partie supépieure el dalls l'iuLirienr de la ligace des anatifes.

Les oufs sont de couleur bleu de eiel dans l'analife ordinaire.

Dins le pied, les weufs ont encore leur vésicule germinative et ils sont très-petits; ils se forment lá; on les voil tneore en. lassés dans les coecums de l'ovaire.

Ils passent par un canal unique, silué en dessons entre les deux valves, el s'ouvrent là dans une poche à droite et à gauehe qui renferme des spermatozoïdes. Les reufs fécondés se logenl

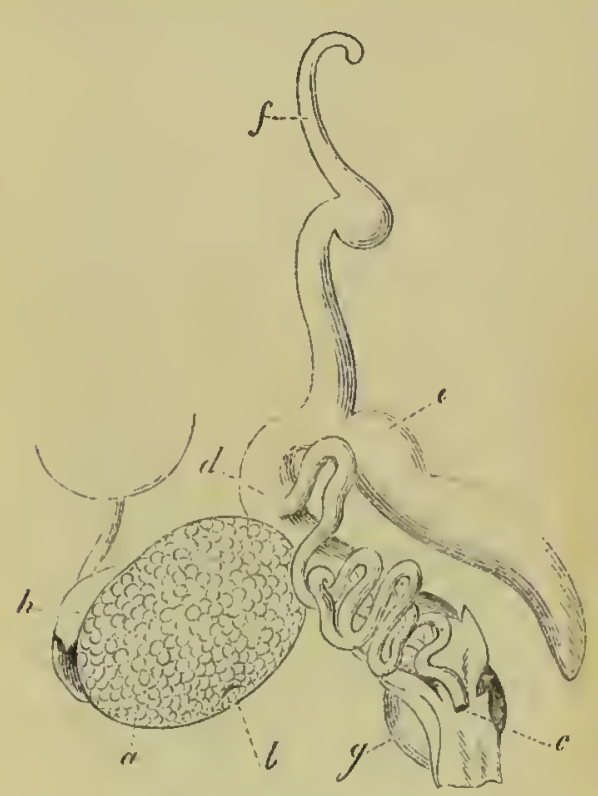

Fig. 186. App. mâle d'uctopus vulgaris.

a. Testicule. - b. Orifice. $c$ - Orifice du canal déférent. - d. Canal déférent. e. Glande de Needham. - f. Canal éjaculateur. - g. Glande. - h. Membrane. ensuite elltre deux feuillets silnes en dedalls des villves, et se distinguent aisément par leur belle eouleur blene.

Ils sont vivipares; on roil des embryous tout formés dans les œufs encore renfer. unés daus ces ovaires comme dans les lernéens.

Mollusques.-L'appareil reproducteursu. bit les plus glandes inodifications dius les animauxde celte classe; d'une complication assez grande daus les cephalopodes, il se réduit daus les bryozoaires ì son dernier degré de simplicité. On trouve tantôt les sexes séparès, tantôt riunis; el dans l'ordre le plıs élevé, les céplıalopoules, si quelques máles se distinguent à peine de la femelle, d'autres montrent une diffirence assez grinde.

Les mollusques soul ell gélléral ovipares; ce u'est que dans 
les deux dermiers ordres qu’il existe anssi une reproduction par germmess.

Céphaloporles. - Tous les céplablopodes ont les sexes sceparés; les individus des deux sexes se ressemblent souvent, alu point qu'ou a de la perine à les distinguer à l'extérieur.

L'appareil mòle est furmé d'un lesticule unique, logés an fond de la cavild aludominale (fig. $18(i)$; il est splerique el forme

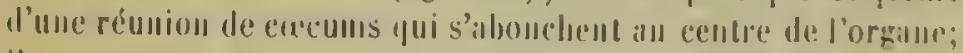
il est entouré d'une memlirane propure. Le canal deferent n'es fas la contimation des vaisseanx séminirères; il existe, comme dians l'appareil founclle des classes suprérieures, une solution de rontinuite entre la glande et le canal excriblenr. Ce canal defé-

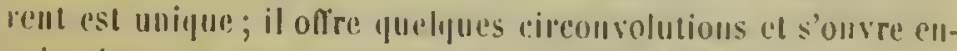
suite dius un canal plus langa sur le trajel doquel on décourre

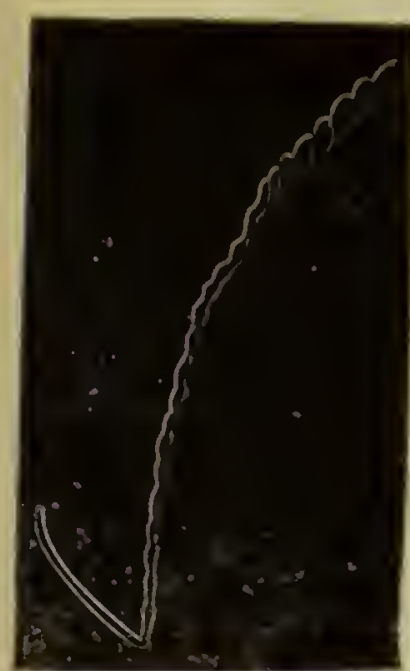

Fig. 187. - Spermatuphore de loctopus vulgaris.

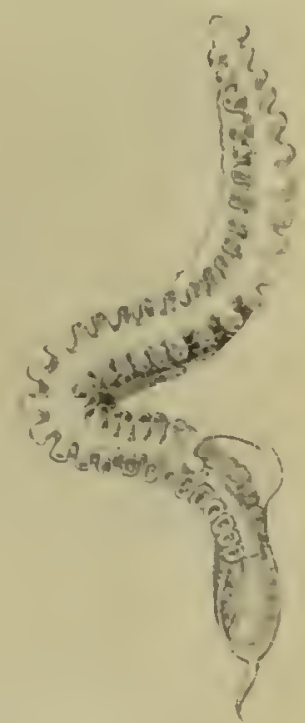

Fif Isis - Bras cupulsieur de iremnelopus riviaceus portant le spermatophore

souveut deux glaudes assez fortes, dont lil supérieure porte In nom de Neelliam. Elle produit des gaimes membrameuses autour des spernatozoides el elle donne ailsi maissance aux sperma- 
topliores (fig. 187), qui, pendant longtcmps, ont été désignés sous le nom de corps de Needham. L'orifice de l'appareil se trouve au côté gauche de l'animal, à la lauteur à peu près de l'anils.

Dans quelques céphalopodes, le spermatophore va s'unir à l'un des bras du màle qui l'a produit (fig. 188); ce. bras est conformé de manière à se détaclier périodiquement, el e'est par sou

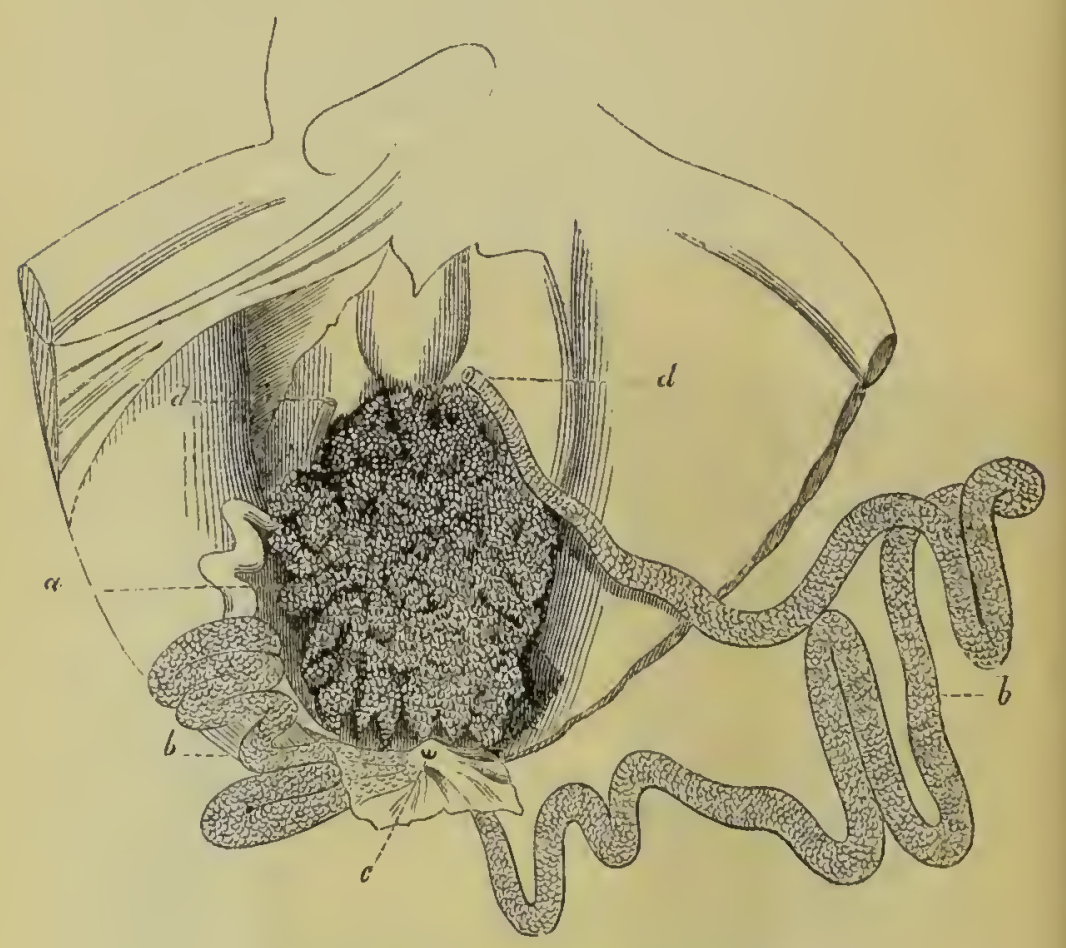

Fig. 189. - Appar. fem. d'argonaule.

". Ovaire. - ". Oviducle. - e. Commencement. - d. Fin.

iıtermède que la fécondation s'opère. Ces bras, chargés de spermatophores, ont été désignés sous le nom d'hectocotyles; on les prenait pour des vers parasites. II y a peu de temps, on les prenait pour des mâles complétement défigurés; on sait aujour- 
"loni, surtout depuis les traviax de II. Muller, que l'hectocolyle n'est que le bras du male chargé du spermalophore el qui s: sépare du corps pour aller opérer sur me femelle. I.e bras conlinue un certain lemps à vive apres sa sépallalliun (liz. 188).

L'appareil femelle est forme d'un ovalire uniplue, sitme it la mème place on se loune le lesticule (lig. 18!); il existr

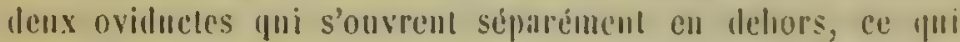
permel de distinguer à l'exterieur les sexes enire enr. Sur lo'

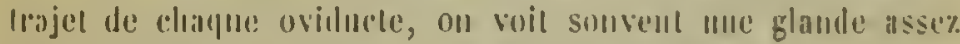
forte, destinee is secréter les enveloppes iles arufs.

Gastiropodes. - Les uns out les seves sejparés comme les patelles, les autres somt hermiphrodies comme les liunaces el les colimacons, mais l'termaphrodisme est incomplet. Il parail

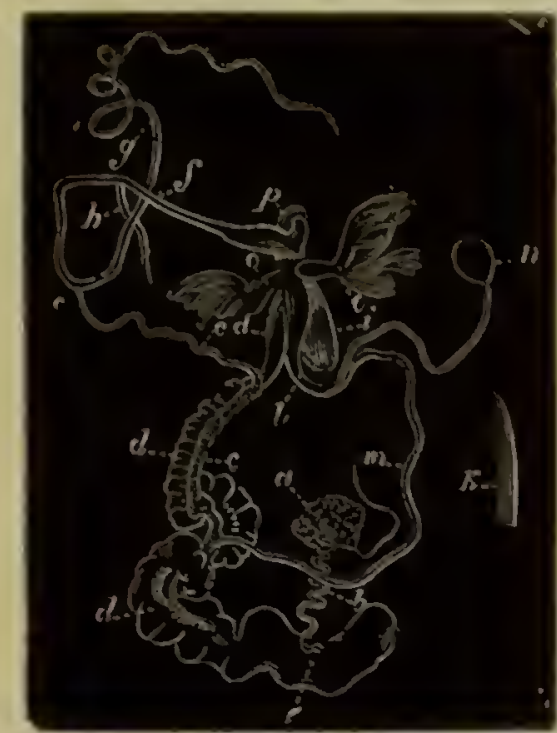

Fix. 130. - Organes de la genération de $1^{\circ}$ hotlis aspersa.

a Testiculeovaire. - h. Canal pxréleur eommun. - c. Canal déférenl. - d. Oviductr. - e. Glande des envelnppes. - f. Pénis. - g. Fonet. - h. Memhe rélracleur. - i. Poche du dard. - k. Dard isoli: - I. Giande el conduil de la vésieule copulalive. $-n$. Vésicule copulalive. - o. Proslatr. $-p$. Onverture commune.

que, penlaut lancle ste laccouplemeul, les deux individus agissent 
simultanément comme màles et conme femelles, qu'ils fẻcondent et qu'ils sont fécondés.

L'appareil sexuel des colimaçons, que nous prendrons pour type est très-compliqué, et malgré la ressemblance que l'on observe souvent entre les coquilles, l'appareil générateur montre des diffírences assez notables. Il n'y a guère d'appareil sur la signification duquel on ait été mons d'aceord jusqu'en ces derniers temps; les divers organes ont été le plus diversement interprétés par les anatomistes les plus distingués. Cuvier ne partageait pas l'avis de Swammerdan, et ni l'un ui l'autre u'avait maison.

Dans le tortillon' formé en grande partie par le foie, on voit collée sur eet organe une glande dont l'aspect el la couleur contrastent ordiuairement avec le foie (fig. $190 a$ ); c'est à la fois le

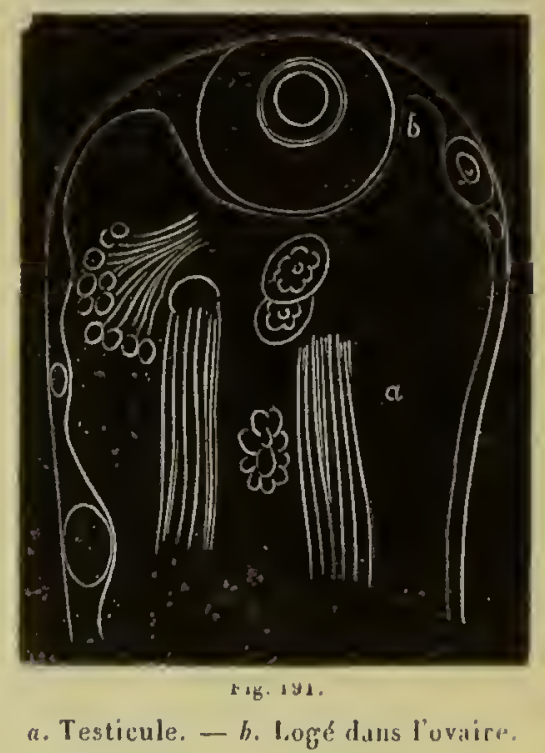

testieule et l'ovaire. Il est formé de deux cocums emboités;

I On appelle tortillon la partie postérieure de l'animal qui est enronJée el tonjours cachée dans la coquille. 
colai du malion reprisiole le lesticule, l'aulre l'ovaire; les sfermalozoides so formentan nolien des uufs, mais sans venir

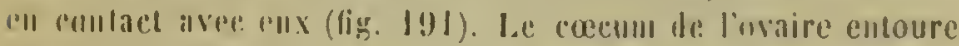

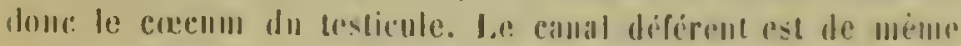

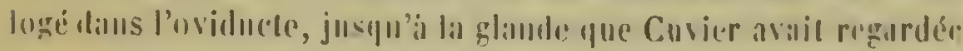

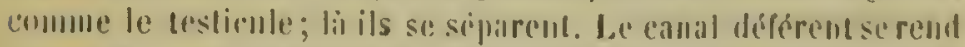
all prus, il est mince ct fort grêle; l'uiducteest beaucoup plus large et se rend direchmont ou indireclement à mue mome cavilé

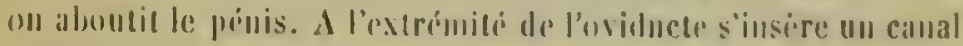

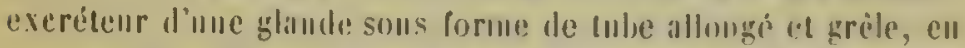

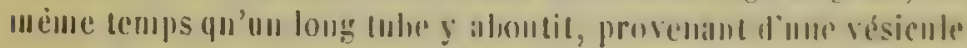
") ("opulative.

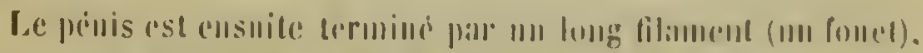

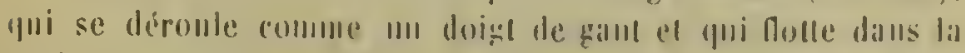

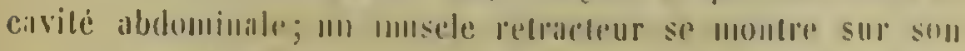
Irajel. A cote de lexarimite de loviducte, on buit encore la

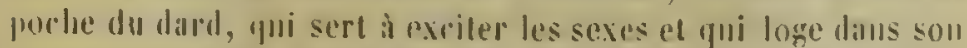

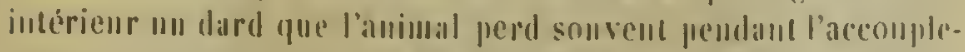
ment; el dans colle poche du diral s'oube en outre une double

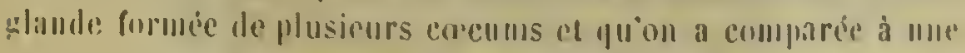
prostate. Le diud est mu orrane solide de la consislance du cristallin de l'uil. Xons lavous rejuresente separement.

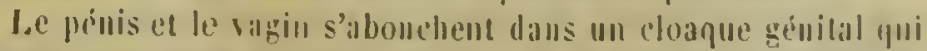
souve du cotri troil de l'amimal a la base des lemlateules (lim. 1!) 2 ).

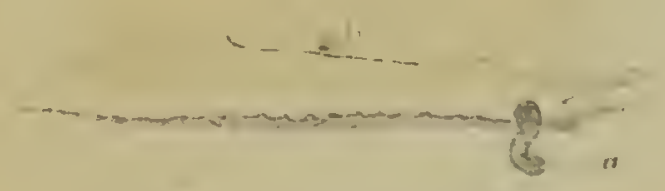

Fig 19:. - I.imare montrant le penis.

a. Iernis. - b. Orifice pulnonaire.

Cel appareil debloulle el placé sur deux individus formo liaplareil sexuel des gastéropodes a sexes schpares. - Dilus 
l'appareil femelle ainsi isolé manque souvent la glande qui produit l'enveloppe des æufs, el on me recommit ordinairement pas le réceptacle du sperme.

Les mollusques acéphales sont aussi en partie hermaphrodites, elt partie à sexes séparés, mais les deruiers sont beauroup plus rares que les autres. L'uppareil, dans l'un el l'autre. sexe, est très-simple, el souvent, saus l'examen du produit, ou ne saurait distinguer les màles des femelles.

Le testicule comme, l'ovaire consiste dans une eavilé, où s'abouclicnt diyers tubes ou lobes, avee un court eanal excréIeur qui s'ouvre sur le côté à la base des hranchies (fig. 195). Ou ne distingue ui glande secondaire, ni aucun organe de eopulation. Ces organes sont aeculés contre le foie el se distinguent

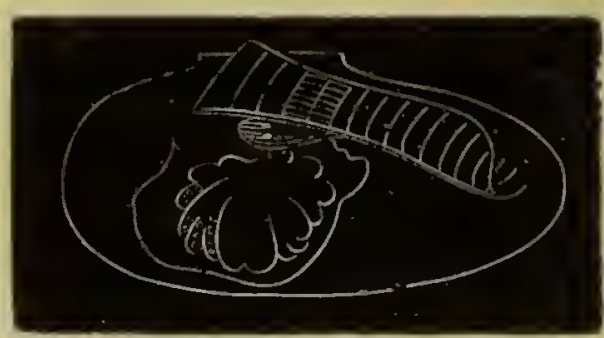

Fig. 193. - Anodonte (appareil seruel avec ouilice).

facilement à l'oeil par une couleur différente. Chez quelques acéplables liermaphrodites on distingue aussi le testicule de l'ovaire par la couleur. Ces organes sont logés ordinairement dans le pied, ou, quand le pied est petit, ils se répandent mème dans le inanteau.

Les aufs sont mis en contact avec les spermatozoïdes, quand les sexes sont séparés, par l'intermèle de l'enu.

Clier quelques-ins de ces mollusques, comme les anodontes, les embryous sijournent un certain temps dans les branchies, comine daus une matrice, jusqu'à leur parfait développement. Il ell est de même des huitres.

Tuniciers. - Les aseidies simples et composées sont liermaplurodites; voici ce que nous avons vu surl'ascillic ampulloülde. Le testicule et l'ovaire ne forment en apparenee qu'un seul et 
même organe, qui est logé dans nue anse intestinale près du rectum. Cet appareil est double el occupe le milieu du corps. l.e testicule forme une sorle de cadre antour de l'ovaire; il est l'un bialue lacleseenl, tamlis que l'ovaire est noiratre. Cel organte mile est composé d'une infinté de conrts coecums entortilles qui ne somt pas saus offrir de l'allalogie a vec les canaux seminifires des animinx superieurs. $d$ la suite de l'engorgemen Ilı lesticule, la liqueur mile se fraye un plassage par trois ou

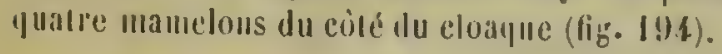

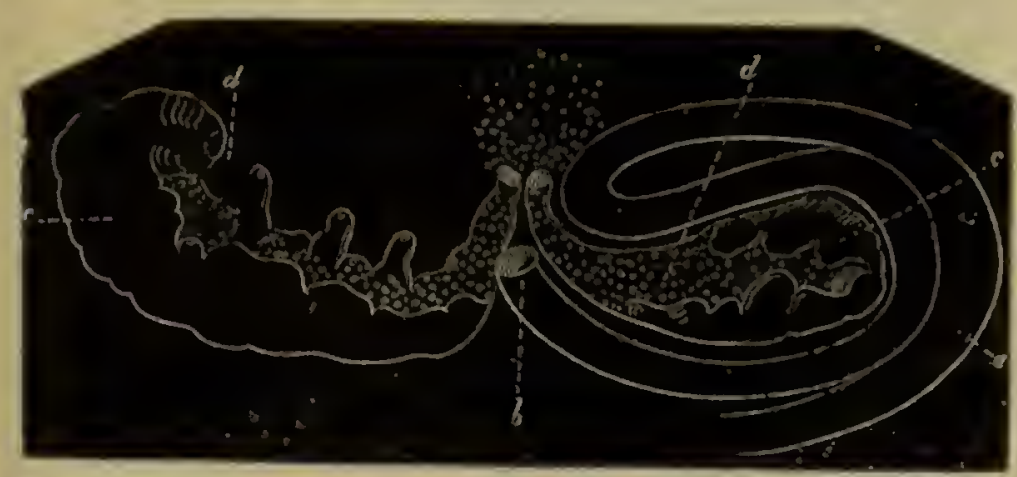

Fig. 196.- Appario sextel complet d'asudia.

a. Inse intestinale. - $b$. Inus. - c. Testicule. - d. Ovare.

Lat plupart des tuniciers se reproduisent anssi par genumes. Les bryozoaires sont pourvus d'un appareil sexuel Iris-dis. lime, mais comme tous vivent en communate, el que le sangr

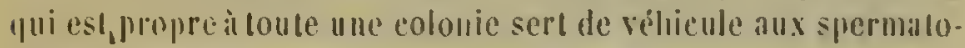
\%uiles, il est sans importance que les sexes soient réunis ou sépares. Fu tous eas le teslicule el l'ovaire sedeveloppent séparímeul. Cies organes ue sont guere distincts que lorspüils sont gonfles par leur produil. Le lesticule est silur doez plusieurs bryozuaires lerriere le cul-de-sac de l'estomac; les cellules mäles se developpent dans sun intirieur, el lursque les spermatozoilles out aequis à peu pris leur leveloppement, les parois se dechirent, "l ils se repandent dins le liquille qui baigne le eanal digestif.

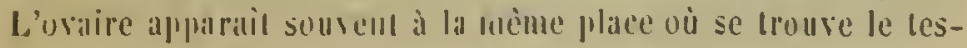


lieule; comme lui, Il n'est bien distinel que lorsqu'il est rempli l'aufs; mais il est it remarquer qu'il y al mI moment où les cufs mâles, avant la formation des spermatozoïdes, sont tout à fait semblables aux oufs femelles. Les œufs ichappent lorsyu 'ils sont mirs et se répandent dans le même liquide commun qui a l'eçu les spermatozoïdes. C'est ainsi que les oenfs sont fécondès avant la ponte sans aecouplement quand mème les sexes sout sipares. Nous avons vu les oufs se developper aussi en dedaus

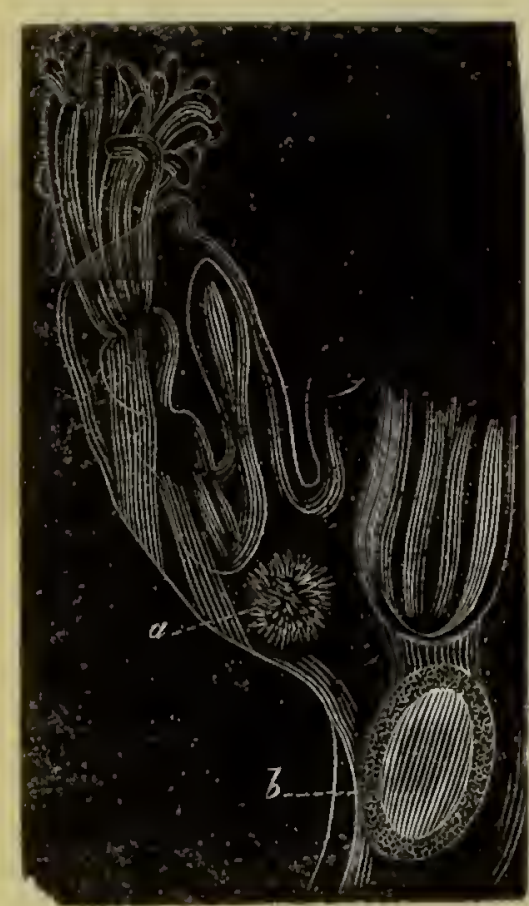

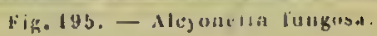

a. Testicule. - b. Ovaire. de la peau non loin de lin couronmede tentacules. Nous avous vu, dins une colonie de bryozoaires, desindividus mailes, des individus femelles et des imdividus liernaplirodites. Ces trois sortes d'illdividus s!ul un mème piel reproduisent dans le rigne animal la vingl-troisieme classe des plantes de Linne ou la polyganie (fig. 105).

Tous les biyozoaires se reprodnisent anssi par gemmes.

Vers. - La reproduction jar bourgeons est assez commune dans celle classe; souvent les bourgeons atherent lougtemps ì la mère, et (III voit des gloupes formes le plusieurs individus appaltenant aे diverses gènclations. Ou voit diuns celle classe la reproduction scissipare el gemmipare se confondre. Tous les vers ont en outre mu apprareil sexuel. Chez les uns res sexes sont siparés, (hoz, d'autles ils sont réminis sur un senl el mème individu. Daus re dernier eas, la fécondation s'opire sunvent par acrouplement riciurngue, mais il y en a aussi plu- 
ANATOMIE COMPARELE 




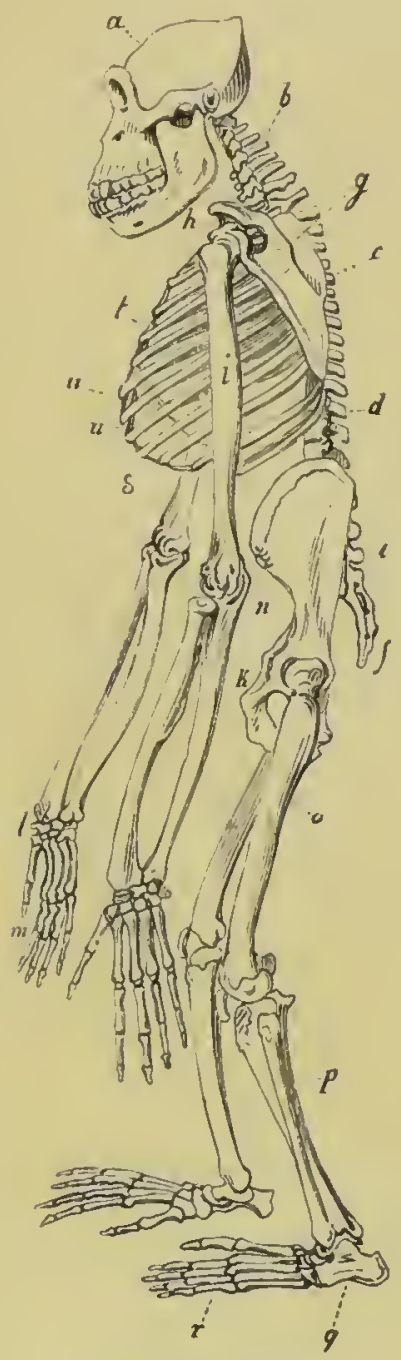

squelette do gorille

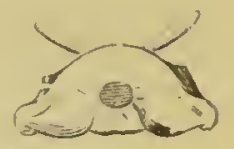

Dolte cartilagineuse du sepia officmalis

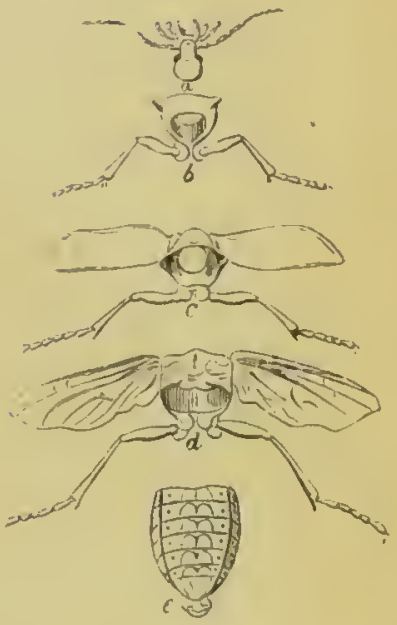

squeietle cutane du calosoma 


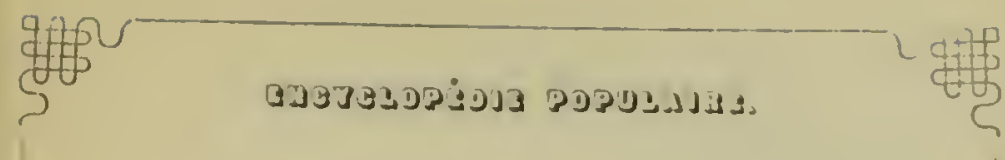

ANATOMIE COMPAREE

r.n

\author{
J. 3. Uan bencien,
}

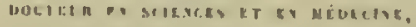

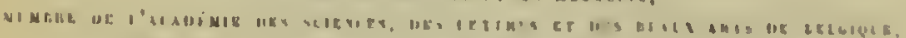

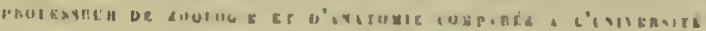

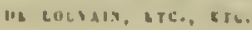

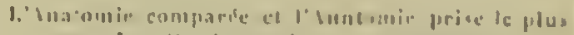

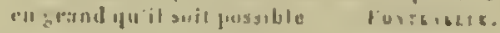

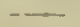

| 1 |

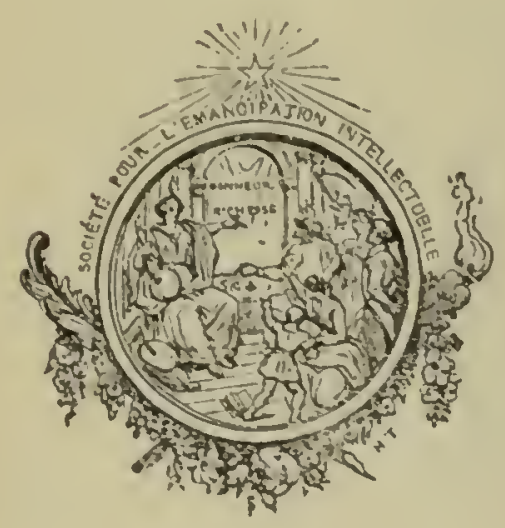

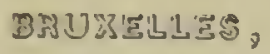

Société pour l'émancipation intellectuelle,

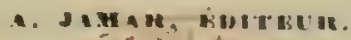

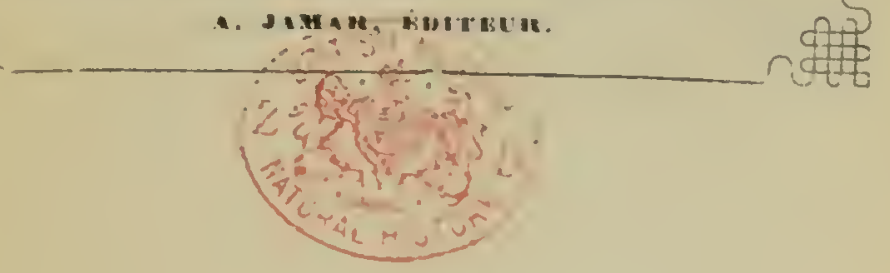



sieurs qui se récoudent eux-mèmes par un accouplement solitaire.

Dans aucuue classe on ne trouve les moyens de reproduclion plus puissauts, ni aussi varies, ui les germes plus nombreus. Ce sont les derniers animax que l'on aurait dù invoquer pour sontenir le système de la sponteparité ou de la génération sproutance.

L.e's vers à sang coloré, et qui sout brauchiferes, nut les sexes sipares, mais leur appareil de reproduction est le plus simple. de tonte lil classe; ils se trouvent avee raison a la tite le celle classe par tous les antres appareils.

l.es testicules, comme les wivires, prisentent la forme glaudulaire la plus simple et ne consistemt souvent poe dams un sac ou un tule sur les parois lesiguels se développrut les aends daus les femelles, les spermatozouides dius les miles. Ces organues sout logís dins la cavite peri-intestinale, on les roit à preine loors la saison du frai. Il u'y a pas d'acenuplement et puand le produit est mîr, les parois se rompent eu répindant les auls nu lo sperme daus le liquide qui baigue le canil digrestif. Il u'y a point de canaux excréteurs.

los némitoïles out aussi les sexes siparés'applareil male consiste daus un canal seminière très-long, qui s'étend dans la louguenr du corpss (lig. 196). Liextrémiti

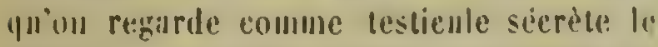
sperme, une autre partie le combluil an dehors, éesi le cantal defirent; a l'eaterieur (1) voil un ou deur pénis cormés qui soml lingis dius une cavite à còté de lian!us. Quelques miles portent des rentonses a cote de l'appareil sexuel pour micux s'allacher is la femelle.

L'alpialreil femelle est tris-alevelnppé tout cul conservant son type simple. II s'omve susvent en arant ou vers le milien du

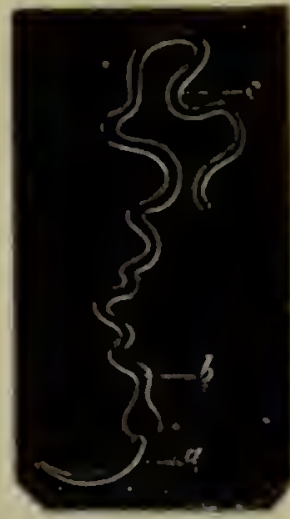

Pig. 196 - Ipy. male de nemutuido.

a. Psinis. - b. Canal defirent. - r. Testiule. rorps. On y dislingue un vaguiu, sourent deux oviducles, un

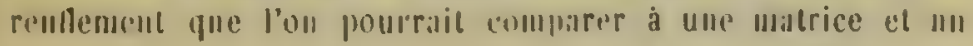


Honble ovaire sous la forme d'un long tube simple C'est a l'evtrénité que se forment les vésicules germinatives, el plus loin: plpiraissentles globules vitellins qui les enveloppent lirus-

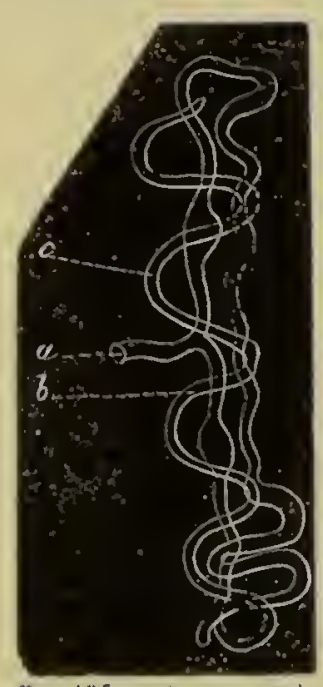

Fig. 19i.- Ajp. lem de nématuìde quement pour compléter l'œuf (fig. 197). C'est un pliénomène reconnu maintenant cliez les cestoïdes, les planaires et les trémalodes.

Les échinorlynques laissent tomber les cufs et les spermatuzoïdes dans la cavité gémérale, où on les voil se nouvoir; à la suite d'une leggere pression, nous avons vu les ceufs sortir par deux ouverlures dıslinctes siluées à la partie postéricure du corps.

Les sexes sont séparés dans les némerliens; le testicule et l'ovaire ont la forme. l'un sae qui s'ouvie à côté de l'anus. II n'y a pas d'alpendice copulateur (fig. 198 ct 1!9).

Les liirndinées nu les singsues sont lier a. Orifice fem.-6. Ovi- mapluroulites incomplets. Ces vers portent durle. - c. Ovaire.

en lessous du corps, vers le liers antélifur, deux ouvertures siluées sur la ligne médiane à une courte

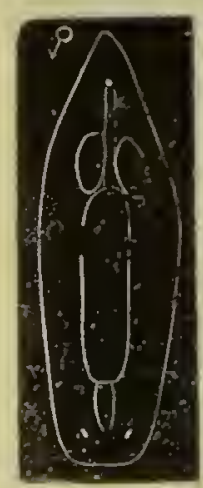

fing. 198. isiunghitus frm. distance l'une de l'autre (fig. 200); l'aulérieure est l'ouverture mâle, l'autre l'orifice femelle. Lialpalreil niâle consiste en plusieur's paires de testi. cules (fig. 20l) placés sur le côté du corps et unis entre eux par un canal dérérent; celui-ci, non loin de la poche lu penis, se pelotome sur lui-mène et forme me sorte d'epillilyme. Le pinis est simple, flexible el loge dans une galine musculaire. Le nombre de testicules varie selon les espices.

L'apprareil femelle consiste en denx

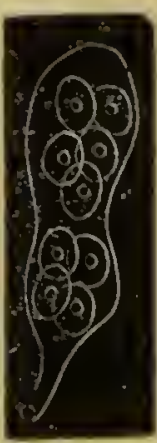

rig. 19V Asmitance dinoplibilus. ovaires, deux ovidnctes, un vagin assez. fong et une vulve, 
silme, enmme nons venons de fr dire, à une pretite distance en dessous de louverture du prinis (fin. 202).

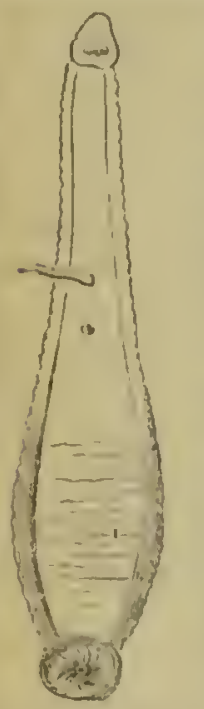

Flg. $800 .-11$ a 1100 pis rerax.

Dants les restoñdes. Ies sexes suml rémis a s'onvrent l'un ì cooti le l'itulru (fir. 205). II existe min lesslienle, composis de vésiculus transparentes. mu raual dériprout, faisinl fomelinn allssi de visicule spermatipue. sons la forme diun vilissean simple el minjur, Iris-10m: et logé dauss lal parlie antérienre du conpls. Ce vaissean se dironle an boul eomme III) dojge de gant et forme unt prinis sombent couvert l'aspériless. I.a vulve s'ouvre à còle; on voil un long vagin qui s'ilcul jusqun a la pidrlice postirieure da corps; il purle an lonl mo vésieule eopulative. Les vésicules germinatives et les globules vitellins se forment dans denx glandes distimcies qui s'abouclient an mime endroit el oul'on voit, par un monvement brusque, le vitellus se precipiter autonr de lal vesisule dugerme el completer instintamement l'auf. Ces aufs so logent dans me malriee, mais ils ne sont eviacues que pour antant que les enreloppes du corps se dechirent. Le vagin ue sert yu’a

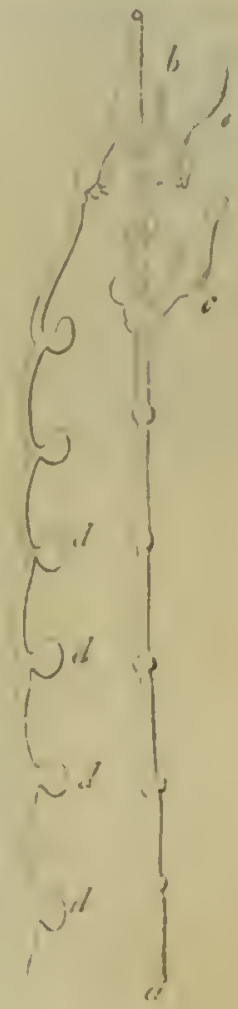

Fik. \pm 11 .

II mojis vorax

a Systeme lervent. -b. Vénicule seiminile. - c. Ipl. fermelle. - d. Teslicules. - e. Ménis. l'entree des spermatozoides el pas à l'evacuition des aufs.

Il y a ficondation solitaire.

Dans les trématodes, l'appareil est au fond le mème, mas souvent le testicule est composé de vaisseaux seiminifères anastomosés et logés vers le milieu du corps. L.es oviaires sont donbles et très-grands; ils s'êteudent souveut dans la longeneur du 
corps, consistent cu canaux ranifies, qui, à l'aide de denx ovi-

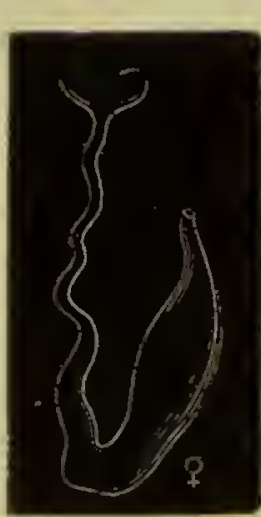
ductes, versenl lour produil dans une sorle de matrice qui s'onvre à còtè du pénis. Les résicules germinatrices et le vitellus se forment aussi séparémeut.

Les planiares sont liermaphrodites; les leux appareils s'ouvrent en dessons l'un de l'aulre à une très-courle distance. Ice lesticule est formé de deux coecums asse\% larges, du miticu desquels uail de chaque còté un canal déférent qui s'ouvre dans une vésicule sémirale unique, située au milieu; il exisle génẻralemeut un péuis. L'ovaire est doulale Fig. quz - Memupis commı le testicule; il est forme, de clıaque virax. Ipp. fem. isulé. côté, d'un caecum assez large, du milieu duquel ıaîl l'oviducle: ce canal excréteur s'ouvre dans le vayin,

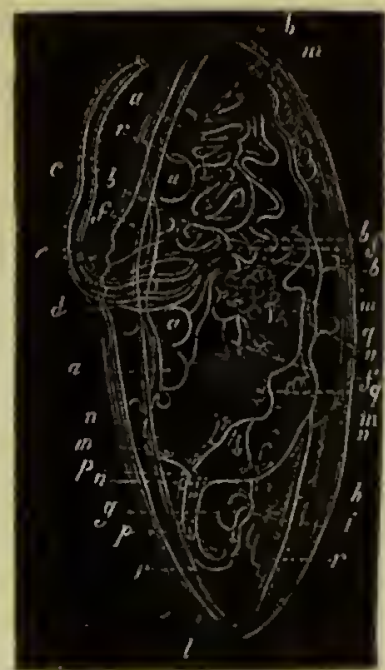

Fig. 203. - Appareil sexuel de ceswîde.

a. Testicule. - b. Canal déférent. - e. Pénis. - d. Burrse du péuria. c. Ouverture du vaguin. - $f$. Vaggiu. - g. Vésicule séminale. - h. Germugine. - i. Germaducte. - . Endroit où les globules vitellius sout versés dans le germuducte. - m. Vitellogène. - n. Vilelloductr. p. Oviducte. - $q$. Malrice. - r. Cananx longitudinaus.

sur le laijed duquel on voil souvent un autre organe glandulanre. 
Xoms ne serions pas smpris qu'il y olit aussi dans frs phanaires une fécondation solitaire comme dans le groupe precrident, d'antant plus que, dapres 0 . Schmide el schultze, il existe un organc spécial pour la formation du vilellus el des vesicules germinatives (fig. 204).

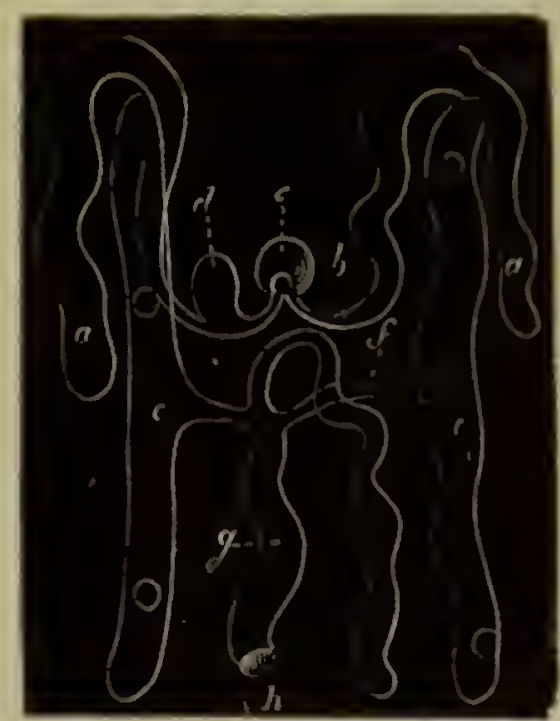

fig. zos. - Appareal sexuel ideal de planare.

a. Teaticule. - b. Canal défésent. - r. Vésicule séminale. - d. Pénis. - Ovaire. $-f$. Oviducte. $-g$. Vagin. $-h$. Oritice.

Les planaires d'ean fouce n'ont, en géueral, qu'un pore :énital, tandis que les planaires marines en ont deux, un, male, devant el l'autrc, fenelle, derrière.

Échinodermes. - Tous les échinodermes ont les sexes séparis, mais souvent on ue distingue les sexes entre enx qu'à l'aide du microscope. Le testicule et l'ovaire occupent la mème place, présentent la mènre structure et ils ont jusqu'à la mème conleur. Ia reproduction par gemmes n'est pas conuue dans celle rlasse.

Alépoque du frai, les organes génitanx acquierent un énorma.

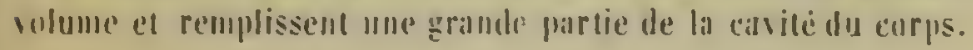


Dalls l'asteracantlıion rubens, l'espèce eommunc de nos còtes, chaque rayon loge deux grands ovaires qui eonsistent daus un sale plus ou moins spacieux, dont toute la surface est couverte de lobes. Les oufs se forment sur les parois internes. Chaque

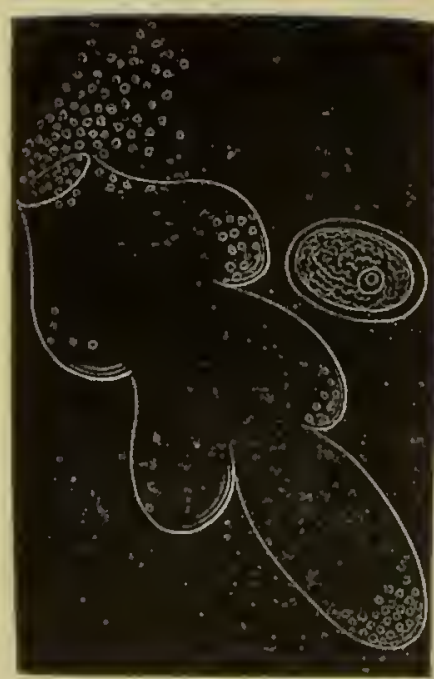

Fiog. 20j. - Ovaire d'ustérie (asteracanthion rubens). ovaire a III) eanal exeréteur qui s'ouvre du côté du dos près de l'augle rentrant (Iuller el Troschel) el qui répand son contenu par plusieurs pores. Il existe donc dix ovaires dans ces animaux, et dans chacun d'eux se développent des milliers d'œufs (fig. 205). Le lesticule est conformé de la même manière el s'ouvre au mène endroit. L'eau sert de véhicule pour la féeondiation.

Les oursins ont cinq testieules ou cinq ovaires formés de coecums ramifiés, qui s'ouvrent séparément dans le voisinage ou autour de l'anus.

Le testicule et l'ovaire des holothuries sont formés de eceums très-lougs, fort nombreux et flotlant dans la eavité du corps; il u'y a qu'un seul eanal exeréteur qui est situé entre les tentáeules buceaux (fig. 206).

Polypes. - Les polypes et aealephlies ne forment qu'une seule elasse. Ils offrent les trois modes de reproduetıon : par seission, par gemmes el par cufs. Les sexes sout en général portés sur cleux individus.

Plusieurs de ees animaux n'ont élé eonnns, jusque dans ees dermiers temps, que dans le jeume âge, d'autres seulement ì l'état adulte; e'est pour ee motif que l'on n'a pas reconnu plus tôt leurs affinités. On connaissail les individus agames dans les polypes, et les individus sexués dans les acalèplıes; il manqquait une forme dans l'un el l'autre groupe.

Dans quelques elasses, les individus sans sexes ou à sexes sont semblables; iei ils difrerent souvent les uns des autres sous 
tous les rapports, paree qu ils sivent dans des conditions diffirentes.

On remalnue me analogie frippante editre ces animama les

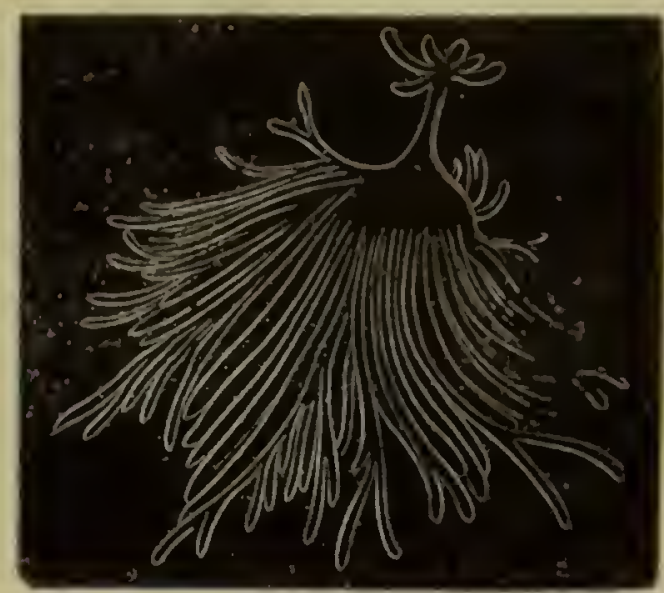

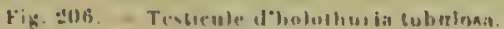

plantes. On voil sorlir le la graine vegzitale un iudividu sans scxe, soms la forme d"un bonrgeon: colui-ci prodnil mu antre bourgeon et pe second produil une troisiome gimiration sums la mente forme; InI arbre est forme ainsi d'un nombre considerillic d'indivilus qui vivent agrigis. Mas apres un cortain nombre de geineralions agames, daulres lontous apparaissent at devirmuent des flenrs on des hourgerons ì seves. Ia neur. on rffel, produit ionjonts nue semence el le germe fimelle est tonjours fironde par un germe mile. Il ya douc aiusi alternativement des individus sans sexes sons me telle forme, mil'antres iullividus i sexes sous une antre forme, el l'espree se rompose dimlividus lifferents.

Cost exactememt le cas de plusieurs polỵpes, les dampanmlaires, les lulumlaires, elc.

II sort dr l'ouf des indivilus polypes sins seves, qui produisent des lourgeons, doi sorteut plusieurs aulres genérations de

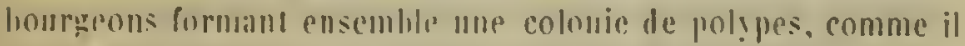
se forme dans le righe vigetal un arlure. Quand celle colonie a 
atteint un certilin développement, il pousse des bourgeons différents, d'où sortiront desindividıs qui ı'ont presque pas de rap-

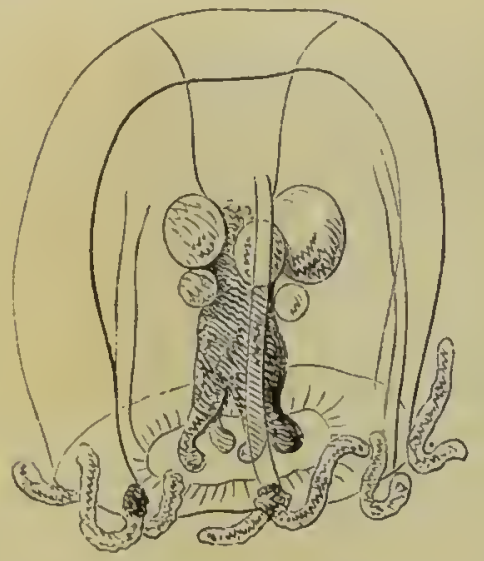

Fig. 207. - Cọteis donnaut des bourgeons. ports par la forme avec les géuérations précédentes. Ce sont les bouryeons floraux, et ce nouvel auimal, comne la fleur, porte un sexe dans ses fiancs el produil des aufs. Nous II'avons done jas la moinure différence à signiller eutre ces phénomèues des deux règnes. II $y$ i plus : les organes floraux ne sout pas indispensables pour la production de la graiue, l'esscutiel c'est l'étimine et le pistil; le calice, la corolle el les autres parties peuvent manquer sans porter aucune perturbation daus l'accomplissement de ce grand acte. Eh bien! dans ces mêmes polypes, le mème pliénomène se reproduit. L'enveloppe animale et li gaine vivante penvent disparaitre, pourvu que les oufs et les spermatozoïdes arrivent à terme, pourvu que la conservation de l'espèce soil assure. Peu importe si le male ou la femelle devienneut adultes, s'ils s'affublent de leur robe de noces ou non; quind l'œul est fécondé el quanil il cst mis à mêne de se développer, le lunt est rempli.

Nous avons, dans celte classe, des amimaux réduits à l'étal de testicule on d'ovaire.

Les béroès out ell didins el le long des côtes un testicule daus les iniles, un ovaire daus les femelles; ces orgilles s'ouvrent ell aviall. la'un el l'autre a la forne simple d'un sile.

Les meduses ou du moins la medusil aurita est gemmipare tans le jeune àge, scissipare à un ìge plus avancé el ovipare à l'étilt adulte. Les sexes soul séparés. Les lesticuleset les ovaires out le minıe aspect el sout logés tout autour de lia grande cariti. digestive dius linquelle printre l'eall. l,un el l'autre se répanal par déliscence. 
D'autres méduses (cyleis), sous leur lorme adulte, produisent les bourgeons ì la base de la tige eentrale, et les embryons leviennent directement méduses sáns passer par la phase polypiaire (fig. 207).

les campinulaires et les lubulaires sont gemmipares d'aboril, elovipares plus taril sous lent" forme adulte. Les sexes somt sijarés. L'applareil de génération est disposé comme dans la medusa iluriti.

I.es actinies on andmones de mer ont les sexes séparés el tre sont qu'ovipares (fiğ. 208). l.es organes sexuels ont lil forme de rubans situés en dessous on en dlhors de l'estomac; les wenfs tombent dians la cavite commune el somt évalemés par la benclie. Les denx orgines mile sexuels.-b. Esat fenclle sont conformés de la meme manière.

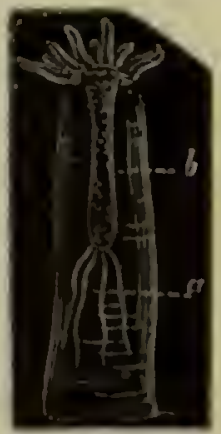

Fig. 208.-Actinle.

Las hydres doment naissance à des bourgeons mobiles et

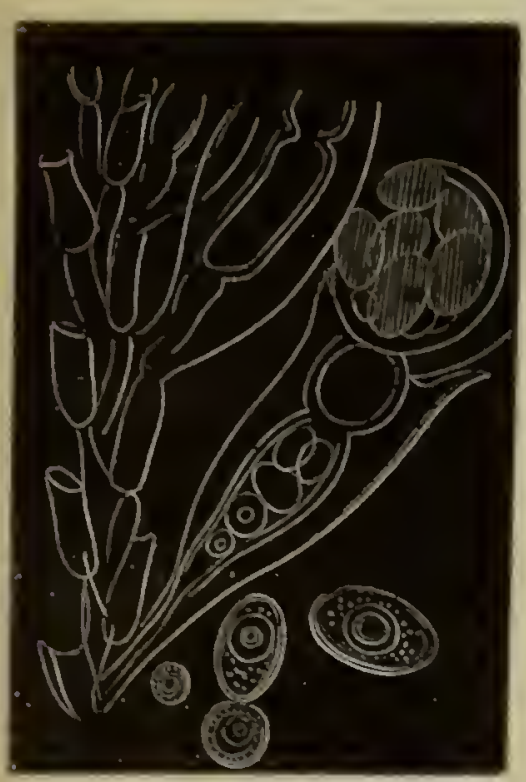

Fip. 209. - Serlularia cupressobiles fenuelle. portent les deux sexes sur le meme indivilu. Toule. lois, d'apres notre interprétation, lovisac comme le spermisac sont deux individns distinets, mais qui sont arrités dans lear développement. Ils sont rédnitsl'un et l'antre à l'étalt de gaine. C'est la fleur rédnite il l’élat d'étanine ou de pistil.

Daprès quelques observations, des colnnies entières de polypes ippartiendraient à l'un ou à l'autre sexe; tous les individus se. raient exclusivrment màles ou femelles. Co'st ee que Eral a observi dans le verelillum eynomorinm ell'allẹnniun ; Krolın dill'aroir remarq̨ué 
lans les sertulaires. Ce serait la monocie de limné dans le règule animal (fig. 209).

Ni les organes sexuels ni le mode de reproduction ne sout bien conuus dams les rlizopoles.

Infusoires. - Les animaux appartenant véritablement à celle classe n'out pas d'organes sexuels; du moins jusqu'à présent on

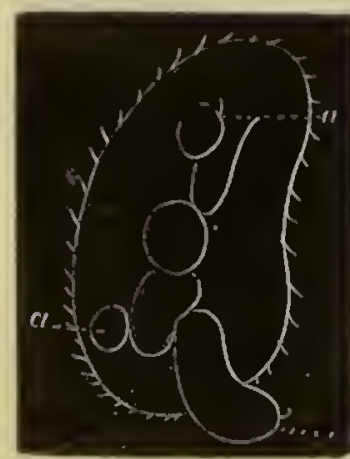

Fig. $210 .-$ Loxudes bursaria.

a. Vésicules contractiles. -b. Embryon qui est sur lo point de se détacher. ne les connail pas. Leur reproduction a lieu généralement par scission et quelquefois par gemmes (fig. 210). Celte scission est souvent très-simple: l'aninaal se divise spontanément en plu. sienrs parties, quelquefois il se désagríge, il se décompose physiquement, mais bientôl on aperçoil que la vie continue dans chaque fragment et que claqque parlicule qui s'esı délachée affecte bientôl la forme de l'individu dont elle est provenue. Ce moyen de reproduction est extraordinairement rapide.

Outre celle reproduction par division, qui parait avoir lieu régulièrement pendaut plusieurs génćrations, ol a vu des iufusoires donner naissance à de jeunes infusoires d'une autre forme et décrits sous d'autres noms; on en voil un exemple dins les vorticelles; celle reproduction, qu'clle ail lieu par gemmes ou par spores, représente le norle de reproduction ordinare, commun à plusieurs classes des raugs iuférieurs.

Il paraît que la multiplication par division s'épuise au bout de quelques générations et qu'il faut alors une nouvelle souche provenant d'un bourgeon ou d'un spore. 


\title{
ANATOMIE COMIIMLEL.
}

\author{
APIARLILS DE LA VIE III: ISLLATION
}

Les vightitus portent les divers appareils flont nous avous parlé jusqu ì présent aussi bien que lés animan ; les uns ed les: itutres se nourrissent el se reproduisent; anssi lansemble des phemonènes que présentent ces appareils est lésignti sous le $110 m$ de vie végélative. II nous reste á parter maintenant de la vie anmale ou de la vie de relalion, qui est exclusiventent pro. pre aux animalus.

Celle secunde partie comprend l'apparcil locomoleur, l'aphpareil ou le systime nerteux, ell'apparil de's organes des se'ns. 


\section{APPAREIL LOCOMOTEUK}

Cet appareil sert surtout au transport de l'animal d'un lieu dans un autre et à produire certains mouvements dans l'iutérieur même de l'animal. Il est exclusivement propre aux animaux, et on observe sa présence jusque dans les rangs inféricurs du règne aninal.

Les moyens de locomotion sout très-différents dans les gralldes divisions du règne animal; on tronve des os et des museles dans les animaux supérieurs, et des organes d'une extrênte sinplicité dans les rangs inférieurs.

Les muscles sont les organes actifs de cette fonction; les os ou les autres parties solides sont des organes passifs, des leviers, comme nous en employons constamment quand nous voulons faire un certain effort.

Dans les derniers degrés de l'échelle animale, ce sont des eils mobiles ou de petites soies, qui agissent comme les planchetles d'une roue de bateau à vapeur el qui font mouvoir l'animal en prenant leur point d'appui dans l'eau. Ces cils sont tris-petits: on ne les aperçoil qu'à l'aide du microscope; on les appelle cils vibratiles, parce qu'ils sont dans un mouvement continuel.

Les organismes microscopiques que l'on conıait sous le ॥om d'infusoires, et qui se développent en si grande quantité dans 
toute eau renfermant des débris organiques, jouisseut de ce urode de locomotion; ceux qui ont des cils disposés en cercle autour de la tète, et qui produisent un mouventent semblable à celui d'une roue en mouvenent, out été nommés pour celte raison rolateurs. Placés longtemps parmi les infusoires a cause de leur taille mieroseppique, ils en sont aujourd'lua hien eloigncs. Un grand mombre l'animaux ont, à l'age embryomiatire. des cils pour preniers orgalles locomoteurs el qui disparaissent quand les orginnes ordinaires de celte fonctrou se sont développés.

Cliez les limaçons, la pean tout entiore est contractile, et dans l'abseuce de parties sulides servant de levier, les mouvements sont tris-leuts et l'animal avance en ramp̧aut. la coquille dans laquelle les limaçons s'abritent ne leur sert que de pro. lection.

Dans les insectes et les arliculés, lélément museulairu se sépare de la peau pour former des bandelettes distinctes: cetle peau se durcil, devient cornce ou calcaire, se divise en segments mubiles les uns sur les autres el devient un squelelle extérieur. C'est doure un squelette formé aux dépens de la peatu. C'est pourquoi on l'appelle rulané. Dès ce moment l'animal peut se mouroir avee rapidite; la course, le saut, le vol deviennent possibles.

On peut done dire qu'il existe un squelelle dans le lanneton. mais il u'est point iuterne; an lien d'ètre composé d'os et de former une charpente, ce squelete est exterue; il est formé aux dépens de l'enveloppe de l'animal.

Eufin, dans les classes supérieures, il se forme des muscles et des pièces solides, de natnre calcaire, qui sunnissent entre elles par des liganients et qui forment la charpente de l'animal. Par le secours de celte clarpente, qui est le squelelle, les animax penvent alleindre de grandes proportions. L'appareil tocomoteur est ici formé d'os et de muscles.

APPAREIL LOCOMOTEUR DES VERTEBREES OU DES HYPOCOTYLÉDONES.

L.es mnseles ou la partie aclive, el les os ou la partic passive, 18. 
sont toujours uellement séparés les uns des autres daus tous les animaux supérieurs; mais, tout distiıcts qu'ils sont, ils se trouvent toujours dans une dépendance réciproque; tel mouvement exige tel ou tel muscle en même temps que telle ou telle conformation des os. Il en résulte que l'on peut juger des muscles par les os et des os par les museles.

\section{SQUELETTE.}

Le squelette joue dans l'économie animale un triple rôle: il sert d'abord à soutenir les diverses parties molles qui composent l'animal; il sert ensuite à protéger les organes les plus imfortants de la vie; et enfin les diver'ses pièces qui le composent servent de levier aux muscles. C'est en considérant les os suus ce triple point de vue que l'on parvient ì se l'endre conple des variations infinies qu'ils présentent dans leur forme et dins leur développement.

C'est surlout dans l'étude du squelette que l'oul découvre clairentent l'uniformité du pliı suivi daus la couformation des animaux qui composent un embrabeliement. Le squelette est conformè d'après un seul et même type, depuis les poissous jusqu'aux mamnilères, comme s'il sortait d'u॥ seul et même moule; mais comme clıque espèce a un rôle particulier à jouer dans l'économie de la nature, que certains animaux sont destiués pour la vie aquatique, les autres pour la vie aéricmue ct quelques-uns même pour une vic souterraine, que les uns vivent de chair et les autres d'herbes, ces organes se sout successivement modifiés, dans le cours du développement, pour les besoins particuliers de clıacun d'eux. Peudaut l'àge embryounaire, c'est une matière première qui est la même dilus le poissoll comme dans le mammifère; mais, daus le cours du développement, elte subit un travail particulier, selon l'usage auquel elle est destinée. D’après cela, on concevi'a aisément que les mémes os se trouvent partout, dans la tête le l'oiseau comme daus la tête du poisson, peu importe la consistance des pièces ou la difference the leur volume ct de leur nombre.

Celui qui conuail la composition de là tête d'un animal quel- 
conque, comme le médecin comaint les os de la lète de l'lımme et leurs rapports, peut aisément déterminer les os qui forment la tète dı poisson ou de tout autre vertébré, en ayilnt egard aus prineipes suivants :

$1^{\circ}$ Les mèmes os eonservent les mèmes rapports avee les mimes organes; ainsi la partic supérieure de lunbite est toujunrs formée par l'os froulal; le tron qui livre passige a la moclle est tonjours formé dans l'oceipital; ees tleux os, qui par Id sont très-ficiles à reconnaitre, conservent partnut les mrêmes lipports avee les autres os du erime, comme le parietal, le touforal, cte., el la déterminalion en est lonjonrs aisée. l.es mèmes nerfs criniens traversent les mèmes os dians l'lonme et dians tous les vertébres; meime dans les poissous. II sunit lone de se rappeler les rapports daus une seule espece pour les commaitre dans toutes les autres. Ce principe des rapports est d'mu immense seeonrs dans la determination des organes dont nons nous oceuperons.

2. En compariut la tète d'un poisson avee celle d'un mammifere, on est toul surpris de voir un si grand nombre d'os enIrer dans la composition de la premiere, et on desespère bien rite de acenuririr dans un poisson les os aln squelelle d'un singe. Celle diférence provient surtont de ce que dans les manmifères aldultes plusienrs os, qui étaicul d’abord síparés, se sont réunis pour ne former qu'me senle pièce, tandis que ces mèmes pièces. dans les poissons, restemt séparées pendant loute la vie de lanimal. Il filnt done choisir, pour ćtablir celle eomparaison, III futus de mamnifire tomt les os sont entore tous séparés; ilors ou decourre aisément le's partics analoğmes. Le poisson présente, ì l'état permanemt, me forme passagire du mammifere.

$3^{\circ} \mathrm{On}$ il reconnu une loi de billancement dans le squelette, le liqquelle résulte qu'un os ne prend jamais un grand developpenteul sins qu'un autre se rapetisse dans la mène proportion; ce qui explique un grand nombre de modifications dont on ne se rendrail gule compte silns eela.

Ainsi, quand les inembres se raceoureissent on sitroplient.

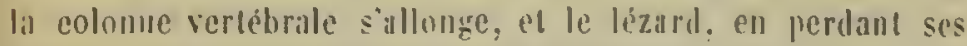
palles, acquiert lil forme el la longueur dn serpent. Quand, an 
lieu de cinq doigts, il "'y en a plus qu'un seul, comme dans le. cheval, ee doigt unique devient énorme, ainsi que l'ongle qui le termine.

En résumé done, Ijous trouvons partout iei la variété dans. l'unité ct l'unité dans la variété.

Le squelelle se compose toujours d'une série de pièces eonnues sous le nom de vertèbres, et qui sont jointes entrc elles pour constituer l'échine ou la colonue vertébrale; c'est la partie essentielle de la eharpente osseuse.

Celle colonne vertébrale est termince en avant par la tête, qui se compose du cràne el de la face.

Lc crâne eonsiste d'abord dans une boîtc cartilagiulıuse; dans l'épaisseur de ce cartilage se dépose du plıosphate ealcaire dans divers eentres qui eonstituent autalıt de points d'ossification; c'est aiusi que se fornent les os du eràne primitif. A ce crìne viennent s'ajouter d'autres pièces qui we passent pas par l'état de eartilage el qui ne lui appartiennent pas. Dece nombre sont diverses pièees dont nous parlerons tout à l'heure.

Le crâne est formé par la modification de quatre vertèbrcs, tandis que les os de la faee proviennent des appendices latéraux auxq̨uels les vertèbrcs crânieunes donnent naissance. Les os qui vienuent se joindre aux os du cràne $\mathrm{cl}$ de la face forment avec eux la tête osseusc.

Les autres pièces latérales des vertèbres sollt l'os hyoïdc el les côtes.

Enfin deux autrcs paires d'appendiees eomplètent le squelette et servent principalement à la progression : ce sont les deux paires de membres, les antérieurs qu'on appelle tlorachiques el les postérieurs ou abdominaux.

Colonne vertébrale. - Cellc partie du squeletle est toujours formée de la même mauière, en ce sens que ec sont diverses pièces solides qui se meuvent les unes sur les autres et qui sont toutes formées d'après le même type. Quel est ce type?

Il eutre dans la eomposition d'une vertèbre trois éléments correspondant au triple but que remplissent lcs os; la partie priucipalc, c'est le corps (fig. 211. 1), qui sert surtout a tenir les diverses parlies en respect el qui fail l'office de charpente. 
Ce corps est surmonté te deux pięees disposées en toil, formant un are el servant at la protection des organes les plus dissentuels à la vie; c’est te second dément (fig. 211, 2 el (i).

L.e troisiène élément sert ì fumruir des allaches aux museles el se léveloppe l'autant plus que le mouvement sera plus éleudu; celte partic est tonjours eu rilpport avec la furce des musclies; elle se developpe en dessus et en dessous. el oul la désigne sous le mon d'upophyse épinerse (fig. 211, 5 at 7).

Sur le côlé du corps il se diviloppo d'antres pices qui leviemment apophyses transterses (fig. 211, 4) 0u iótes (idem, b), "l qui premuent quelquefois mu develuppement prodigien.

Toutes les vertibres se mollitient d'aprè la région quelles ocenpent el d'après la différenee du ròle qu'elles ont d jouer ; e'est ainsi que les verlibres lombaires, qui doivent unir le traiu de devant à celni de derriere, se dislinguent par le grand développeneut

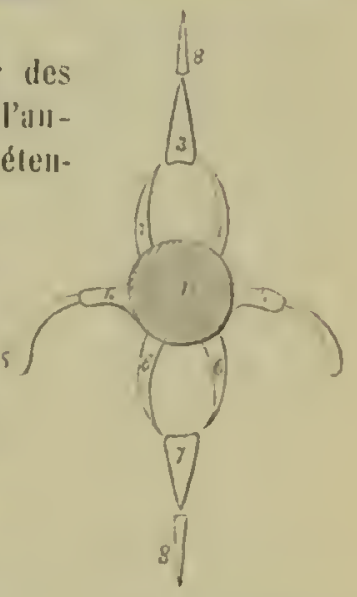

Hig 211 - Ine vertilire iddir d'apres $18.0 \times \mathbf{n}$.

1. Corpis de la vertibre.

2. Meuripopliyses. - 5 Yeurépine. - F. Parapophyses. - J. Plemrinophyses. - 6. Hamapophyses. - 7. Hamepine. 8. Diapopligses. du corps te la verlibre. Les verlebres eràicmes, destinces ì protéger le cerveau, moutreut un développement extraordinairi lans les parties latérales; l'are, qui sert il la protection, absorbe' pour ainsi dire les deux autres élenents de la verlèbre. Fontin les apoplyyses épineuses sont extrimement développées vers le milieu de la colonne vertébrale, où les museles sunt le plus volumiueux el cil s'exerce la plus grande foree nuseulaire. Dans la rígion de la queue, où les muscles sont faibles, vers lo boul. par exemple, et où il n'y a plus de moelle à protéger, la verlèhre se réduil an corps en perdant ses deux autres ilements, el le corps de la rerlibre prend l'aspeet d'un osselet allongai. conme une plaalange des doigts ou comme un osselet du stermun.

C'esl dans les poissons que le cerrean est le moins volumi. 
ueux el que les vertèbres crànienues out le moins perdu leurs caraetires de vertèlores.

C'est vers le commencement le ee siècle que, presque simultanément, eu Allemagne el en Franee, des naturalistes philosoplies out comparé le erâne à une série de vertèbres. II a fallu une trentaiıe d'années pour faire aceepter celle idée.

I.e erâne est toujour's composé de quatre vertibures (fig. 212): Ine postérieure, formée par l'os oceipital, el qu'ou appelle verlèbre occipitale; une moyenne, formée en dessus par les parié-

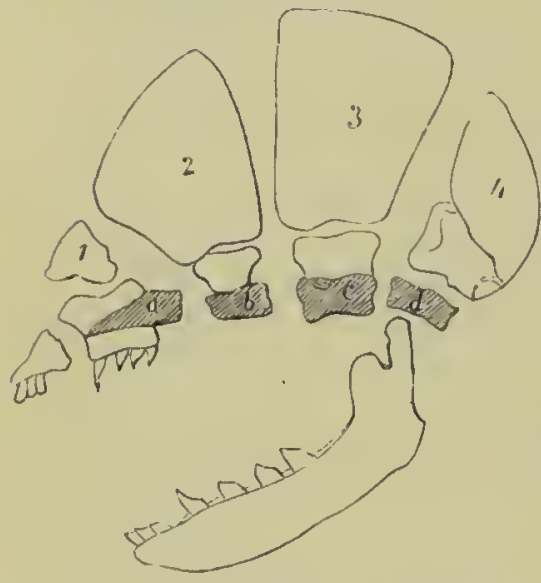

Fig. 212. - Téte idćale, divisér en quatie vertibres.

Le corps de la vertèbre antérieure représ. sentée par le vomer (a); eelui de la vertèlire frontale, par le sphénoide antéricur (b); le corps de la troisième vertébre ou de la pariélale, par le spbénoüde postérienr $(c)$; celui de la quatrième, par $I_{d}$ partie basilaire de l'ucejpilal (d).

1. Vertèbre nasale. - 2. Vertèbre frontale. - 5. Vertébre pariétale. - 4. Vertèbre occiputale.

comprend les petiles ailes el forme la purlie infiriure ol late rale de la vertèbre frontale; la moitié postérieure eomprend les grandes ailes et les apopliyses ptérygoïdes, de maniere que. toutes ces pièces, étant séparées, furment lutil osselets distiucts thins les poissons el un seul dans l'lomme.

L’os temporal mérite aussi une mention spéeiale. Il se comlaux, en dessous par le sphénoüde postérieur, el sur les côtés par les temporaux, c'est la vertébre pariétale; la vertèbre appelée fronlale est furmce en dessus par l'os de ee nom, el en dessous par le splténoïde antérieur. Il est à remarquer que le splıénoïde elıez l'ıonme adulte est le résultat de la leéunion de plusieurs points d'ossification ou d'os distiıets, el qui, dans les poissons ell général, restent lous séparés, mème à l'àge adulte. Ainsi le corps du splıénoïle est divisé en deux, une moitié antéricure el une moitić postérieure; la preniciere (n) 
fose de puatre pièces distinetes, li portion écailleuse arec l'apmilyse \%yomatique, le cerele du tympan ou le lympanal, le mastö̈dien el le rocher. Le tympanal reste toujours séparé daus les ovipares el forme l'os carré; il se place cutre la mildonire iuféricure el le erìne. Dans la derniere elasse des verlébrés, lous ces os restent distincls el se séparent mème les uns des alltres; il y en a yui restent lixés au erine el d'autres qui s'en déIacheut pour se joindre aux pieces le la face. Eufiu la yuatrieme verlibre, on la première en commençant la colonne vertébrale en avant, se compose l'un corps qui est comu sous le non de vomer, d'un are supérieur formépar les fromtaux antérieurs par l'etlumoïde et par les os propres du ne\%, et d"un are infirienr forme par les maxillaires supérieurs el par les intermaxillaires.

Si nous apereevons mue si grande miformite dans la composition des verlebres, nous ne trourons pas une uniformite moins grande lorsque nous eomparons entre cux les extrémilis ou les membres des animanx vertélırés. La eomposition est toujours la mime au fond, que ce membre serve ì la course, ill vol ou d la nage; ll u'y a que les diverses proportions qui varient. Linsi, dans le hras ou la jambe d'un mammifere, dans l'aile ou la palle d'un oiscan, on dans la nageoire d'un poisson, on trouve l'abord une ecinture qui unit le membre au squelelle : c'est l'épaule pour le membre thorachique, te bassin pour le membre abdominal (fig. 215). Celte ceinture se compose le deux ou de trois picees. Ite membre lui-mème montre ensuite une seule pièce, un os long, qui est l'humirus ou le fémur: ensuile dem piè. res, le radius el le cubitus en arant, le tibia el le pérone $\mathrm{en}$ arrière; vient après le carpe on le
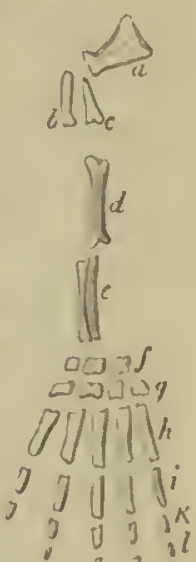

Fig 213.

Mrubre thorachique idual.

a. Omoplate. b. Clavicule. -c. Coracoidiell. - d.Ifumérus. - e. Radius el culbitus.-f. Procarpe. - g. Ilisocarpe. -h. Métacarpe. - i. Ihalann gees. $-k$ Phalangines. l. Phalangeties. tarse, qui est formé de deux rangées d'osselets que l'on peut considérer eomme formées, la supérieure oll procarpe de trois os, el l'inférieure on mésocarpe de quatre; le mciacarpe on 
le mélatarse, qui se compose, dans un membre complet, de cinq pièces, et qui est suivi enfin de ciuq doigts, dont quatre se composent de trois phalanges placées à la lile les unes des autres, et dont le cinquième, l'interne ou le pouce, llen a que deux.

Les trois rangées d'os, siluées entre l'avant-bras ou la jambe at les plıalanges des doigts, out èté lésignées dans ces derniers temps sous Ic nom de procarpe ou protarse, mésocarpe ou mésolarse el mćlacarpe ou métatarsc, dénominations qni doivent ètre acceptées.

Comme le corps des animaux articulés est formé de plusieurs scgments semblables que l'on a appelés zoonites, de mème le squelctle des animaux snpérieurs est composé de vertèbres, qui sc ressembicut toutes quaut à lenrs éléments, el que l'on appelle parties homologues.

De la mème manière, clıcz Ics animaux vertébrés, les membres antérieurs se composent des mêntcs éléments que les memlores postericurs, et tous les os sc répètent exactement dans chacun des appendices.

Dans ces derniers temps, M. Gervais a émis une opinion d'après laquelle les membres sont envisagés sous un point de vuc nouveau : au lieu d'un appendice simple, chaque menbre est composé, d'après le savant professcur de Montpellier, de cint appendices soudes complétement à leur base et séparés seulement au bout; ces cinq appendices appartiendraient, quaut aux membres antérieurs, aux quatre dernières vertèbres cervicales et à la première dorsalc, parce que ce sont ces cinq vertèbres qni fournissent les cinq paires de nerfs pour constituer le plexus brachial. En d'autres termes, Ic membre antérieur d'un animal vertćbré différerait des cinq paltes des écrevisses, par cxemple, en cc quc dans ces dernières, ces appendices sont séparés dans toute leur longueur, tandis qu'ils ne sont séparés, lans les vertébrés, que jusqu'ì la région métacarpienne, ou même ne sout pas séparćs du tout, comme dans les cétacés.

L'omoplate el l'iléon correspondraient à des portions de côtes soudées entre elles, tandis que la clavicule et le coracoüdc en „vant, l'iselion et le pubis en arrierc, ainsi que l'os marsupial, ne seraient que des cottes sternales. 
Ces vues thériques, quand même elles ne satisfunt pas comlplétencut l'esprit, ne taissent pas que de rendre les serviees ins servilut de liens entre les livers faits.

Tous les os dus squelelte n'appartiennent pas au squeletle interue; il y en a qui dépendent de la peau ou du squelelle cutamb, et d'antres qui font partic du squelelle viscíril. Il eutre doure trois sortes d'os dans la composition du squelette.

Tuns les ns qui constituent la boite crindenne appartiennentils au syurlette proprenent dit ou au squelette interne?

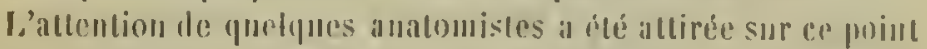
daus ces deruirers temps. Les as du syuelette interne sunt

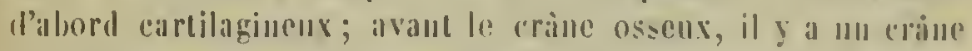
carlibgineus; c'est le crime primitif; puis les pieces du squelette cutané, qui ne passent pas par fétat de carlitage, vienurnt

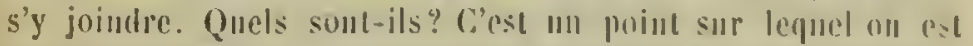
toin de s'entendre el, si fou s'en rapportait à quelelunes analomistes, loute la nomenelature des os du erine de quelenes classis devrait ìtre moditié.

'Tous les animanx veptélorés nont pas un squetelte ossenx; les os, avant d'itre solicles, ont passé pour la plupart par l'étal de cartilage, at, avant l'ítal de cartilige, its ont été ì l'étal tibreux; c'ost ainsi que l'ou trouve d’abork chez l'embryon une corle filirense qui represente le premier puliment de la coloume

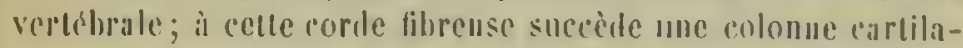
wineuse, el cutin celle eolonne carlilağineuse devient solide et se divise en un grand nombre de pieeres connurs sous le nom de vertebres. Depuis les derniers des poissons jusqu'alu primales, nous royons la mème marche.

Hais ee qui n'est que temporaire dans la plupart de ces animaux devient permanemt cluez quelques-uns, et la coloune vertébrale, comme tont le squelette, est représentẻe elez certains poissons par une simple corde fibreuse, sans os el sans cartilig ge; che\% d'autres, le squelette est plus avancé, les carlitages se sont formo's, mais ils ue vout pas jusqu’a leitat d'os; il y a léjà un cortain nombre le poissons ì squelette cartilageineux, if "y en il que bien peu sims carlilage. Enfiu, les carlilages se transforment on lout nu en partie en ns, el dans quelques ré- 
gions du corps, des os d'une origine différente viennent se joindre à ceux-ci pour compléter le squelette.

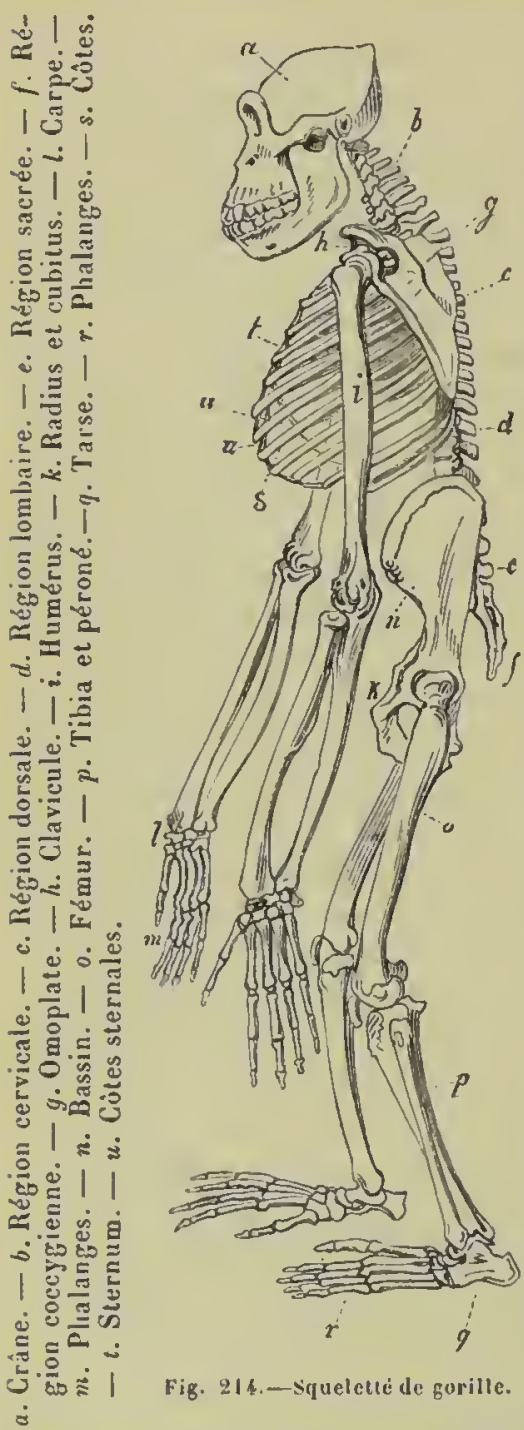

Mammifères. - La colonne vertébrale est divisée en six régions, savoir : la crâuienne, la cervicale, la dorsale, la lombaire, la sacrée el la caudale ou coccygieune (fig. 214).

La région crânilenne est formée par le crâne. C'est une boîte osseuse qui loge le cerveau el dont les quatre vertèbres qui la composent sonl soudées entre elles. La verlèbre occipitale (fig. 215) se distingue toujours par la présence de ses deux condyles articulaires. Dans le jeune mammifère, on trouve uIl occipital inférieur $a$, ou le corps de la vertèbre, deux occipitaux latéraux $b$, ou l'arc supérieur, et l'occipital supérieur $c$, ou l'analogue de l'apophyse épineuse. La vertèbre pariétale est formée en dessous par le spllénoïde postérieur, par une partie du temporal et par les pariétaux, entre lesquels on trouve quelquefois un pariétal impair ou un interpariétal. Le cercle du tympan est généralement soudé avec.

les autres parties du temporal, la portion écailleuse y comprise. La vertèbre frontale comprend supérieurement l'os de ce nom 
el en dessous le splnénoide antérieur. Comme l'os frontal forme toujours la voute de l'orbite el que l'vecipital livre loujours passage a la muelle, ces denx picees sout tonjours faciles à recomlailre el les autres par conséquent aussi, puisıue les rapports restent lonjours les mitmes.

Euliu la vertebre nasale eomprend le vomer et les maxillaires supérieurs, qui sont tris-léveloppes cliez lous les animan de celle classe.

On tronve dans les mammiteres les mèmes os de lia fice que eliez l'homme: l'ahmoüde est toujuurs silué en avallt eutre les froutaux: le romer forme la eloison de la eavite des fosses ua-

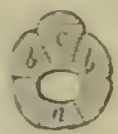

Fif. $21 \%$.

Virlohre vecipitale idrale, vede face.

a. Corps basilaire ou occipi t.al inférieur.b. Occip. tatérain. - r. (1ecipit. superieur. sales; le maxillaire supérieur, élaul l'os le plus volıntineux de la face, donne inserlion aux dents cantues el molaires; l'illtermaxillaire on l'os incisif, est placé en arant eutre les précédeuts pour former le bout de la face; les os propires du nez, forment la ronte des fosses nasales el sont refoules loill en arrière daus les célacés viribubles, à cause du déplacenent des "tariues; les laerymanx, situis ell dedans de la caviti orbitaire, livrent passage an combil exereteur de la glande de ce nom; l'us mialaire, on l'os de la pommelte, forme la partie antérienre de l'areale zygomalique'; entin les palatius enntinuent en arriere la voute du palais, forme en grande partie par les maxillaires. La mideloire inferieure ou le maxillaire inférieur ne forme, d létal adulte, qu'une seule piece, paree que les deux branclies se soudent de bonne lieure ell avant. Dans les didelphes, eet os purte ell arriere une apopliyse assez forte qu'on ne trouve que dans ees mammifires australiens.

La région cervicale compte sept vertíbres, quelle que soit la longueur du con; les seules exeeptions sont: le lamantin, qui u'en a que six quelquefois, el les bradypes, qui en ont lutil ou neuf. Daıs les cétacés véritables, elles sont ntinces et aplaties souvent eomme une feuille de carton; dans le clameau ou la girale, elles sont tress-longues. Les delx premières vertèbres, l'allas el l'axis, sunt toujours distineles des autres el clles sont plus volumineuses. II uy a que leux uu trois exeeplions deetle 
règle. Les vertèbres de cette région n'ont pas de côtes et par conséquent ne portent pas de surface articulaire pour ces os.

L'axis lla pas d'apopliyse odontoïde dans les cétacés. Le corps de l'atlas reste toujour's à l'état de cartilage dans plusieurs marsupiaux, et quand il s'ossifie, il reste séparé.

Les apophyses épineuses sont nulles ou peu développées, mais il existe souvent dans cette région des apopliyses èpineuses inférieures, chez des carnassiers, des pachydermes el des ruminauts.

La rég10ı dorsale comprend autant de vertèbres qu'il y a de còtes et on les distingue au grand développement de leurs apophyses épineuses comme à la fossette articulaire des côtes. Le nombre est très-variable, on en compte de dix à vingt-trois. Le nombre le plus commun est douze ou treize. Les monotrèmes out des apopliyses épineuses inférieures à quelques vertèbres dorsales.

La région lombaire esı sans cóles, mais le corps des vertèbres est souvent très-développé et il existe des apophyses transverses très-grandes. Le nombre est variable de deux à neuf. On en compte ordinairement de cinq à sept.

Chez quelques mammifères, par exemple, chez l'oryctérope et le lièvre, il y a des apoplıyses épineuses inférieures sur quelques vertèbres de cette région.

La région sacrée, formée de trois ou quatre vertèbres, se distingue des autres régions, parce que les vertèbres se soudent plus ou moins entre elles pour ne former qu'une seule pièce.

La région caudale varie à l'infini, quant à la forme et quant au nombre des vertèbres; on ell trouve jusqu'à quarante, et comıne chez l'homme el les orangs, quatre ou cinq. Les dernières vertèbres perdent toutes leurs apoplyyses et se réduisent en un osselet allongé et arrondi comme une phalange.

Les mammifères à quene longne, et surtout les cétacés, montrent des arcs vertébraux inférieurs ou des os ell $\mathrm{V}$, pour abriter les vaisseaux; ces os montrent souvent leurs deux picces simplement réunies sur la liggue médiane, et sont toujours placés sur le point de jonction de deux corps vertélnaux. 
Les vertibres soul arlieulées cutre elles, surtout parle corps, au moyen de disques fibro-eartilagineux qui sont quelquefois recouverts de lanelles ossenses; ees lamelles osseuses sont trisdéveloppées dans les célacés, el e’est anssi daus ces mamnifères anpuatipues que l'on voil les disques les plus ćpais; l'espace entre les eorps des vertebres est surtuut tres-yrand à la base de la région caudale, tandis que les vertèbres de la rigion eervieale sont souvent sondées entre elles. La surface articulaire du corps des vertèbres est en général aplitie; toutefois, dans les ruminants surtont, les vertébres de la région cervieale sont consexes d'mu côté el concaves du còté opposé.

L.es còtes som divisées en vraies et en fausses, selon qu'elles s'articulent directement avee le sternum. Lecur nombre est trèsvariable eomme le nombre des vertibres dursales. Elles sont divisées en denx moitiés, l'une supúricure, qui deseend des vertèbres on les còtes vertélbrales, el une antre yui part lu sternum et qui va à la rencontre de la précédente on la còte stermale; celte dernière est gíniralement a l'élal de cartilage. Les cotes s'artienlent an général par la tele avee mue facelle articulaire, formée par deux corps de vertélures; ot par leur Iuberosité avec l'apopliyse trausverse.

Les còtes sont aplities el étroites, à l'exeeption de quelpues éleutis, oil elle's se recouvroul de manière à ne pas laissel d'espace intercostal.

Le stermum (fig. 2l(i) est forme d'une scirie d'osselets assez semblables, ell giméral, aux dernières vertébres de la réngion taudale. Quelquefois toutes ces pièces se réunissent el forment une sorte

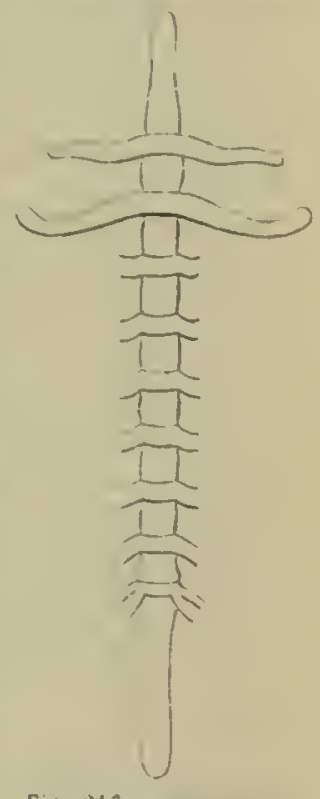

Fif 216 . - vernum ideal de mammifere. de bonelier. Ice milien du sternum porte me érète dans le: mammifires qui ont les muscles pecloram trè-diveloppes. comme les cheiroptires ot les tanpes. 
Tous les mammifères ont deux paires de membres, à l'exception des cétacés. Les antérieurs sont unis au squclette par la ceinture de l'épaule, les postérieurs par la ceinture du bassin.

L'épaule montre toujours une omoplate qui s'unit au sterIum par la clavicule, quand celle-ci est très-développée; l'apophyse coracoîde de l'omoplate, qui cst toujours distincte dans le jeune âge, se soude plus tard, mais conserve un certain développement dans les cheiroptères. Les clavicules existent dans ceux dont les membres antérieurs jouissent de mouvements trèsétendus, comme clez les quadrumancs; elles s'atroplient dans les carnassicrs et les rougeurs, et disparaissent complétemcnt dans les pachydermes et les ruminants, où les membres ne jouissent plus que du mouvement d'extension el de flexion.

Le bassin se eompose de trois os dans les mammifères : l'iléon, l'ischion et le pubis; un quatrième os vicnt s'y joindre Jans les marsupiaux, l'os marsupial, qui se place au devant du pubis dans l'épaisseur des parois abdominales. Dans lcs eétacés, quoique les membres postérieurs manquent, le bassin est encore représenté par deux osselets placés au milieu des'muscles, ce sont les isclions.

Au milicu de toutes les modifications que subissent les membres, soit pour voler, ou pour fouir, soit pour nager, pour courir, pour grimper, on reconnait toujours très-bien le plan primitif. C'est dans les cétacés qu'ils sont le moins dévcloppés et e'est dans les clieiroptères qu'ils le sont au contrairc le plus. Les doigts sont toujours formés par deux ou bien par trois phalanges, qui sont d'autant plus volumineuses que leur nombre est moins grand. Dans les cétacés, le nombre de ces plaalanges dans les doiğts est beaucoup plus élevé. La dernière phalange varie bcaucoup d'après l'ongle qu'elle doit porter. Elle a souvent une forme singulière.

L'humérus cst très-court elız les mammifères aquatiques, ainsi que elıez les fouisseurs, comme la taupe; ces derniers se distinguent en même tcmps par le grand développement des a pophyses de cet os. Il est très-long et grèle chez les paresscux. Cet os présente souvent deux trous à son cxtrémité iuférieure; l'un traverse le condylc interne et l'autre est formé par l'ab- 
senee de eloison entre la fosse de l'olécrane el lil cavilé eoronoïdienue. Cliez quelyues cheiropteres, il existe une rotule au conde.

Le radius el le culjitus forment l'avant-bras; ce dernier est souvent rndinentaire. Le earpe se eompose de plusieurs os disposés ell deux rangées el dont le nombre est viariable; les os de la rangée supérienre ou du proearpe sont géuéralement plus granits. C"est dins le carpe de lis taupe qu'on trouve le nombre l'os le plus élevé.

Le metaearpe (fig. 217) varie heancoup, anssi bien sous le rapport du nombre de pièces qui le composent que de sa longueur. Ce monbre eorrespond en général au nombre de doigts; il n'y a qu'un métacarpieu prineipal très-long et fort daus le's ruminants el les solipedes. C'est le canon dles vétérintiares. On trouve sonvent des stylets à eôté qui sont dès métacarpieus rudimentaires on arrètes dans le comrs du développenent. II existe cinq mélacarpiens, en général, puamel la main est complete.

Le nombre de doigts est tris-rariable; il n'en existe jamais plus de cinq à l'état normal; on roit quatre, trois, deux et méme un seul doigt dans quelques mammifires, par exemple, daıs

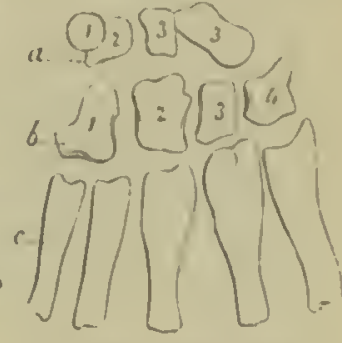

Pig. 217.-Carpe idéal

a. Procarpe montrant :

1. Pisifurme. - 2. 'yramilisl. - o. Semi-linaire. -3 '. Scaphorde.

b. Misocarpe.

1. Oscrochu ou miciforme. - 2. firand os. 5. Trapezoüle.-1. Traprize.

c. Métacarpue. les solipedes; le nombre de denx s'observe surtout clie\% les ruminants. Dans ce dernier ordre, on déconvre eneore souvent deux doigts ludimentaires, qui, plus ílerés que les autres, ne touchent pas le sol, et sont quelquefois nommés ergots. Ils out anssi lenrs plualanges et un ongle.

Le lémur est en général court dans les mammifires aquatiques, les solipides el les ruminants; on le reconnail toujours a sa tète, au col el aux trochanters. On troure généralement une rotule. La jambe est formée principalenent par le tibia; le pé. roné est souvent faiblement déreloppé. 
Le tarse (fig. 218) montre deux rangées d'osselets; les deux

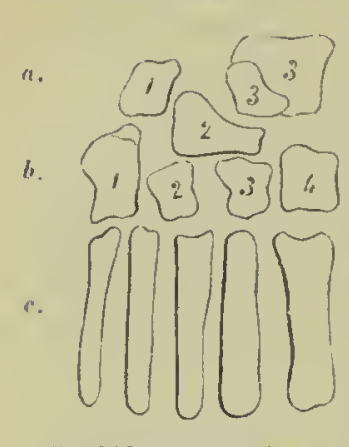

Fig. 218. - Turse ideal

a. Protarse montrant:

1. Calcanéum. - 2. Scaphoide. - 5. Astragale. 4. Mésotar'se.

1. Cuboïde. - 2. Cunéiforme moyen. -5 . Petit cunéforme.-4. Grand cunéiforme.

i. Mélatarse.

principaux, l'astragale el le calcanéum, formant le protarse, sont extraordinairement dévcloppés chez les tarsiers.

Le métatarse comme le métacarpe, est quelquefois constitué par un os principal très-allongé, et les mênies variations que l'on observe dans les phalanges des doigts se reproduisent dans les os des ortcils. Dans les marsupiaux, on trouve souvent un développement très-irrégulier dans les os du métatarse et des doigts.

L'os hyoilde est formé par une pièee médiane, le colps, qui correspond à la stelnèbre, el par deux paircs de cormes dont les antérieures représeıtent les côtes. Elles sont génćralement ossifiées dans les animaux de cetle classe, et c'est par celle première paire que l'os lıyö̈le s'unit au crìne par le roclicr du temporal. Le corps de l'os hyoïde est extraordinairement développé dans les singes alouattes ou stentor's.

On trouve un os pénial dans la plupart des mammifères. II est canaliculé et contribue à la formation du canal de l'urètre; sa forme cst extrêmement variable et diffère l'une espèce à l'autre. Il est volumincux, surtout dans les carmassicrs.

II cxiste cncorc quelques os isolés, mais que l'on n'observe que dans certaines espèces; ainsi le eoelıon a un os propre et des muscles particulicrs dans le groin; la taupe et le fourmilicr ont aussi de ces os qui appartiennent au squelelte viseéral.

Oiseaux (fig. 219). - Le squelettc des oiseaux présente de notables modifieations; plusieurs os se remplissent d'air et deviennent pneumatiques; ils ont une singulierc teudanee at se souder les uns aux autres. Le crine des oiseaux adultes n'est formé que par une seule pièce.

Sous le rapport de lcur pneunaticité, les os se divisent en troiseatigories: $1^{n}$ Iesos toujours pneumaliques qui sont : Ie ste 
IIIIm, l'lumérus, les vertébres pectorales et les cervicules; 20 les os quelquefois pueumatiques: les clavicules, les omoplates, les
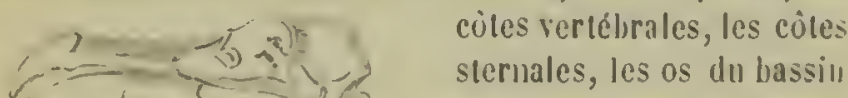
el le sacrun; $5^{\circ}$ les os qui ue soul jamais jueumalipues : Ies os de l'avantluras, de la jambe, de la milin el des pieds.

Eı grinéral, les os du eraine se remplissent diair alinsi yne les vertebues rervicales, l'lum meruselle fémur. L'ail' s'iutroduil dans les parois dı crime par les fosses masales el par lia trompe d'liustaclie, el dans les is du bras el de la cuisse par les pocles acriennes de la cavilé llırachique el abdominale.

Dills quelyues oiseanx, par exenple cliez le fon el le marahoul, l'air ue cirenle pas senlement dans l'inlérieur les os, mais comme nous l'avous vu daus la description de l'appareil respiratoire, l'ail entoure les os el circule au milieu des muscles el entre la peau el les aponérroses.

La colonue vertébrale est diviśéce en régions, comme dans les mammifères; la région lombaire manıjue. C"estla région cervicale yui a le nombre le plus constant de vertebres, el la région caudale le nombre le plus variable dans les mammières. Ces ré- 
gions présentcnt précisément l'inverse dans les oiseaux : c'est la région caudale qui varie le moins et la région du cou, au contraire, qui var'ie le plus.

La têtc des oiseaux se distingue par la mobilité de la face

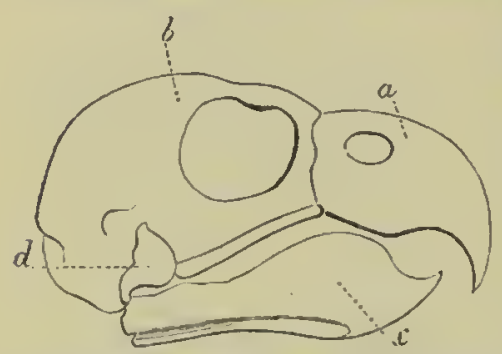
sur lc crâne (fig. 220); une clıaine d'osselets est situéc à la base du cràne el produil l'élévation de la face ou de la màchoire supérieure en même tempss que la màchoire inférieure s'abaisse.

Le crâne est divisé, dans Fig. 220. - Tite de perroquet, vue de profil. le jcune âge, en quatre vertèbres comme dans lous les a. Face. - b. Crâne. - c. Maxillaire animaux de cel embranclıcinférieur. - d. Tympanal ou os carré.

ment. La vertèbre occipale ue porte qu'un condyle articulaire el se compose de quatre pièces. La modification importante de la vertèbre pariétale cst celle que subit l'os tympanal; au licu de former le cercle autour du trou auditif, cette pièce s'abaisse, repousse la mìchoire inférieure, et à cause de sa forme prend le nom d'os carré ou de tympanal (fig. $220(d)$. La face montre deux autres os qui sc modifient notablement; le premier c'est l'os palatin (fig. $221 c$ ). Les fosses nasales s'ouvrent dans la cavité de la bouche par une fentc Iongitudinale; les os palatins, au lieu d'ètre placés en travers, se placent dans le sens de la fente el forment Ic bord de celle ouverturc; ils s'étendent d'avant en arrière. Le second os, placé au bout du précédent, unit le palatin au temporal et forme une partie de la cluainc dont nous parlons plus haut (fig. 221 b). C'est l'apophysc ptérygoïde interne des mammifères qui se détache ici ct forme un os à part que l'on désigne sous le nom de ptérygoïdien.

Les autres os de la face des mammifères se retrouvent ì peu près tous, mais plusieurs d'entre cux sc modificnt considérablement. Ainsi les maxillaires, qui dans les mammifères étiaicnt les plus volumineux de la face, deviennent très-petits, tandis que les intermaxillaires sont très-volumineux ct forment presque 
toute la mandibule supéricure. Les os masaux sont séparés l'un de l'autre par les branclıes montantes des intermaxillaires, qui rejettent les marines en dehors.

Le nombre des vertebbres de la région cervicale est en rapport avec la longuenr du cou; on en eompte depuis dix jusqu'd vingt. trois. Ces vertibres sout les plus molibles de la colonne vertébrale. Les premieres vertibres (1) les autérieures se meuvent seulement eu arrière (les quatre ou einq premiires), landis yute les dernieres ou les postírieures se meuvent seulement en avant, de là provient le pli foré du cou de plusienrs oiseaux en forme d's. Pour allouger ou étendre le cou, l'oiscau n'a qu’i diminuer la courbure.

La rigion dorsile se distingute par son munobilite; les apophyses épineuses sont larges et coupeses carrément. Ite nombre

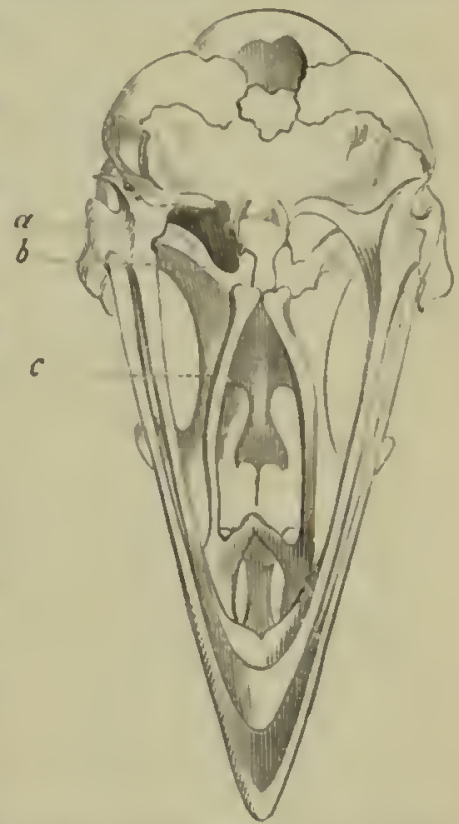
de verlebres varie de sept à Fig. 221 - Tite is poule vuepar la base. onze. Souveut les premières a. Os tympanal ou carré - $t$. Ptédorsales portent des apoplyyses rygoidion interne. - c. Palatin. epuneuses infeirieures, comne les dernières cervicales.

Les vertibres de la région sacrée sont unies eutre elles et ue forment aree les os iliaques qu'une seule et mème piece; il y a de neuf it vingt-leux vertébres qui concourent à la formation du sacrum.

Les vertèbres de la région caudale diminuent considérablement en rolume. Elles conservent quelque mobilits. La deruice vertibre est la plus volumineuse de cette rigion el elle affecte aussi une forme toute particuliere, devant fournir allache ams pennes de la queue. Le nombre ordinaire est de sept, mais il vilrie de six à dix. 
Les vraies eôtes s'attaclıent aux eorjs des vertèlıres et aux apoplıyses transverses; elles portent des apophyses partieulières

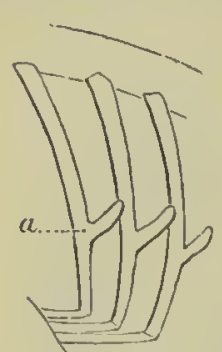

Fig. 222. - cotes d'oistau avec l'apopliyse, a.

(fig. $222 a$ ) vers leur tiers supérieur el qui sout dirigées en arrière et un peu enl haut; ces apoplıyses reeouvreut la eòte suivante. Les eôtes sternales forment avee eelles-ei des angles aigus qui s'ouvrent à mesure qu'on approche des eôtes postérieures. Elles sont de nature nsseuse eomme les côtes vertébrales et s'artieulent avec eelles-ci et avee le sternum.

Les dernières vertèlıres cervieales el les dernières dorsales ont des eôtes qui ne s'articulent pas avec les eôtes sternales. Le nombre des côtes varie peu; il est le plus souvent de sept et ne va pas au delà de onze.

Le sternum des oiseaux (fig. 225, $a$ et $b$ ) est en général trèsgrand el de forme earrée ehez les oiseaux qui on t le vol rapide; il présente au milieu une forte erête que l'on connaît sous le ıom de bréchet. Au devant on apercoit une saillie, que l'on nomme l'épisterıum el qui est tantôt simple, tanlôt bifurquée. Le bord postérieur présente ell gélléral une double éclianerure, d'autant plus profonde que l'oiseau est plus mauvais voilier. Dans les rapaees, ees éehanerures disparaissent souvent. Le bréehet disparaît eomplétement daus les oiseaux eoureurs, comme l'autruehe et le easoar, et le sternum prend la forme d'un bouelier.

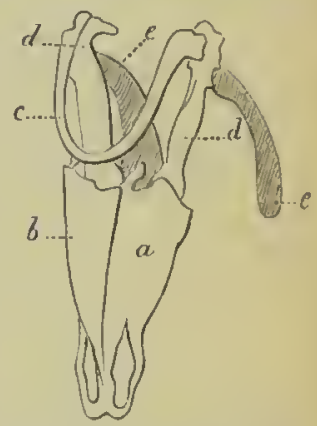

Fig. 225._- Sicrum d'uisean avee crinture de l'epaule

a. Sternum. - b. Son brérhet.-r. Clavieule. $-d$. Coracoide. e. Omoplate.

La ceinture de l'épaule est formée de trois pièees distineles, qui manquent rarement dans les oiseaux (fig. 225); ee sout l'omoplate $(e)$, le coracoïdien $(d)$ el la clavicule $(c)$.

L'omoplate est toujours très-allongée et placée parallèlement à la colonne vertélırale. Il n'y a point d'épine, ct l'apophyse acromion est pen ou point développece. 


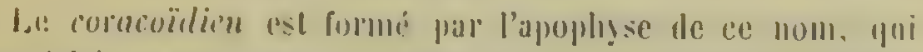

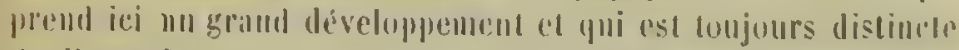
de l'omplotate. Il s'arlicule avee le horl latiral cl antirieur du stermun el fournit III appuni solide a l'ípaule. C'est l'os qui donne le plus de solidite ì l'aile.

lal clivicule se distingue par les dens

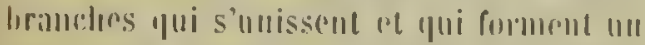

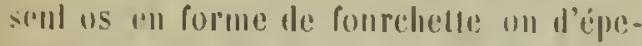
ron. Fille est silmie all devint des rom-

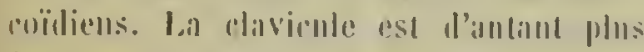
forte pure le vol est pilus rapicle.

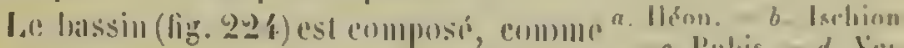

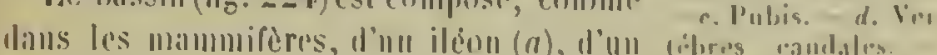
ischion (b) at l'un pullis (c); ces trois os e. Firmur

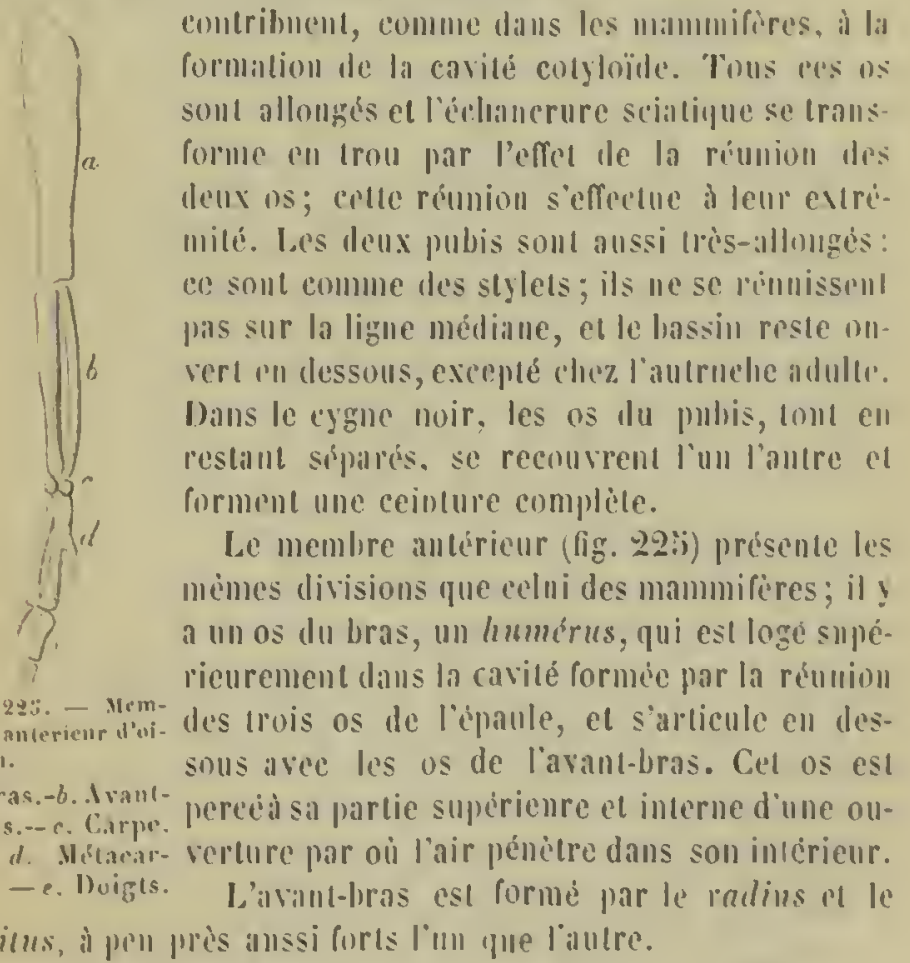


Le earpe ne eompte qu'une rangée de deux osselets; les os du métacarpe se réunissent de bonne heure; ils sont aussi au nombre de deux et portent ehaeun un doigt, dont le médianl est le plus long et montre deux phalanges, placées au bout l'une de l'autre. Il y a aussi un pouee rudimentaire et quelquefois même un petit doigt. C'est le pouce qui porte les pennes bàtardes; le earpe et le métaearpe, les pennes primaires; el l'avant-bras, les pennes seeondaires.

Le membre postérieur (fig. 226) des oiseaux montre d'abord

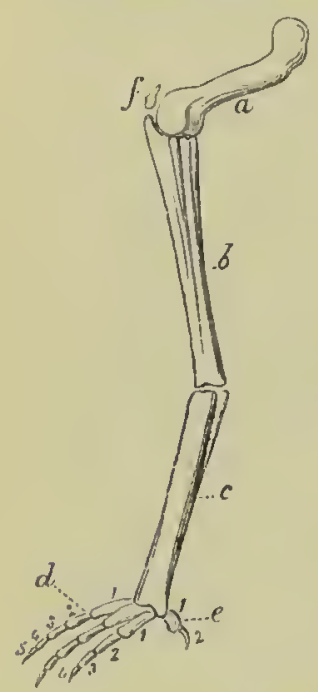

Fig. 226. - Membre posterieur d'oiseau.

a. Cuisse. - b. Jambe. -c. Tarse. - d. Doigts. - e. Pouce. $-f$. Rotule. l'os de la cuisse ou le fémur; c'est un os long, eylindrique el droit; on le reconnaît faeilement à la tête et au col, il n'a qu'un seul trochanter. Il montre pris du col l'ouverture qui livre passage d̀ l'air qui pénètre dans son intérieur.

La jambe est formée d'un tibia et d'un péroné. Ce dernier os ne descend jamais jusqu'au tarse. En général, le tibia est plus long que le fémur.

On trouve en général une rotule.

I.e tarse et le métatarse sont réunis dans les oiseaux et ne eonsistent que dans un os unique très-long, qui forme ee que l'on appelle eommunément la jambe ou le tarse de l'oiseau.

Les doigts sont au nombre de deux, de trois ou de quatre; il n'y en a jamais cinq à l'état normal. Le premier est dirigé en arrière et désigné sous le non de pouce; il eomprend deux phalanges; le seeond, qui est plaeé en dedans, comprend trois phalanges; le triosième ou médian, quatre phalanges; et le quatrième ou externe, cinq (fig. 226). Par ee moyen on connait les doigts qui manquent dans eertains oiseaux et on s'assure que chez l'autruclie d'Afrique, qui n'a que deux doigts, ee sont les doigts médians qui seuls sont restés.

L'os lıyoïle est composé d'un eorps qui eonsiste dans un 
osselel grîle, allongé el muni d'une paire de eorıes qui sont trìsallongées el qui sont formées de deux ou de trois pièees plactés bout à bout. Le corps de l'os liyoïde présente souvent ell avant un osselet qui soulieut la langue, e'est l'os lingual, el yuelquefois il y en a eneore un autre ell arriere, l'uro-liyal. Cet os lingual se trouve lléja dans quelques mammifires.

licpliles. - l.e squelette subit ici des modifieations notables; il présente des différenees d'un ordre à l'autre; une ou deus paires de membres manqueul clez quet(jues-nns; elıez. d'autres e'est le steruum qui manque, et cliez d'autres encore ce sout les eôtes, si ou réunit les batraeiens aux reptiles.

Région crânienne. Les os du crìne restent séparés pendant toute la vie de l'ani-

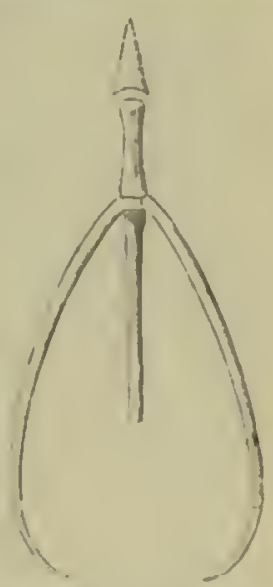

Fig. 2ะ7. - 0s hyoĩde d'viseau. mal. Ces os sout generalement durs. On divise trés-facilement le erinte en qualle rerlibres.

La vertẻbre oceipitale est formée géuéralement de six pièces: l'occipital basilaire, qui produit principalement le condyle artieulaire unique; l'oceipital supérieur et de chaqque còté deus occipitiux latéraux; souvent les deux occipitaux latéraux concourent également d la formation du condyle oceipital.

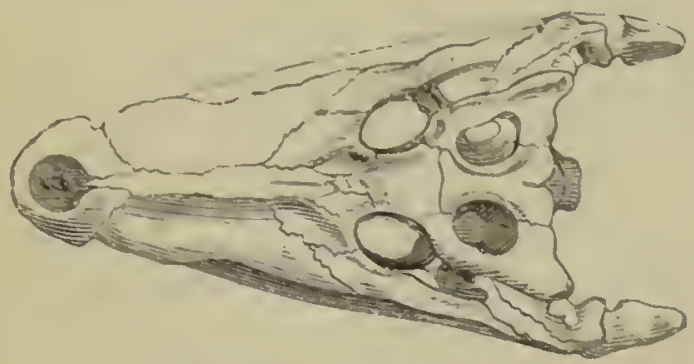

Fig. 29s. - The de crucollile.

Les parietaux sout doubles ou quelquefois impairs. Le spluc- 
noïle forme toujours une selle tureique. Chez les ophidiens, le mastoïdien et le tympanal sont placés l'un au bout de l'alutre el ficartent fortement les deux branches de la michoire en arrière.

Le frontal est tantôt pair tantòt impair, el montre quelquelois en avant un frontal antéricur, en arrière un frontal posté-ricur, et sur le bord de la cavité orbitaire des os sus-orbitaires, qui dépendent de la peau.

Chez quelques reptiles, la boite crânienne n'est pas complétemcul ossifiée.

On retrouve la plupart des os de la face; il n'existe quelque-

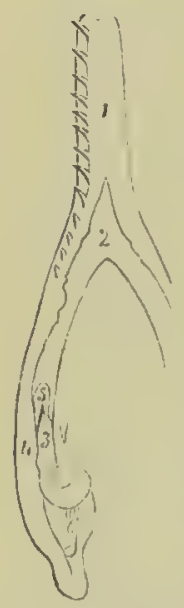

Fig. 229, - Machoire inferieure de crocodile.

1. Le dentaire. -

2. L'operculaire.

-5. L'angulaire. -4.Le surangu- lius, se recombissent dans les crocodiles complémentaire. comme dans les mammifères; ils occupent les laire. L'articu- mêmes places et remplissent les mèmes foncvertébrés. lions, comme daus lia première classe des

L'ellmoüde est formi de mème d'une lane cribleuse, d'ailes latérales, de comets supérieurs el d'une lame verticale; mais il demenre en grande partie eartilagneur. 
Denx ficie's paraissent representer le bas de sa lame verticale uu vomer; deux autres, quelques parties de ses anfractunsilés supérienres.

Le frontul ocempe la mème place que daus les nummiferes, mais ses apopliyses anté et post-orbiraires sont des os distincts.

L'occipital reste divisé en quatre parties comme dins l' fulus.

Sphénoülal. - Le corps du sphélloide est à la móme plater ct remplit les memes fonclions que dans les mammifires, mais il u'est point séparé du sphéuoüle antéririnr.

l.es grandes ailes demeurent toujours séparées du corles de l'us comme daus les foetus de celle elisse; elles embrasseut ume nrande partie de l'espace des ailes orbilaires. Il ne reste de vesliges de relles - ci que de petils points d'ussilieation libres dans la membrane yui ferme eel condroil.

Les ailes plérygoiles restent toujours séparies du corjus de l'os, comme dans heancouj de mammiferes, et somnissent "ntre elles en dessous pour prolonger le tube nasal, commedaus les fourmiliers.

Los de la enisse tympinique ou le tympanal donne la facelle pour l'artiéulation de lin mielioire inférienre.

Le unasloülieu s'étend un peu plus en arrière.

Le rocher se prolonge dans les os roisins.

Il reste entre la caisse el le jugal un os qui ne peut répondre yua la parlie zygomatique dn truporal; et entre lasile pléry. goille, le jugal et le maxillaire, un autre os qui répond, mais issez faiblement, à une apophyse plérygö̈de externe dı splıenoïle, qui serait entièrement détaeltée de son os principal, ce: yui n’arrive jamais chez les mammifies.

Toules les diférences essentielles se rélluisent done à celte lernière distinction et à la division du frontal.

Colonne vertéhrak. - Eille est diviséc en régions dans les crocodiles comme dans les mammiferes, landis que chez les ophidiens elle ne présente plus de régions distinetes. Généralement les verlebres caudiales ont des ares vertebraux inférieurs nu des os en V attadiós entre le corps de deux vertibres.

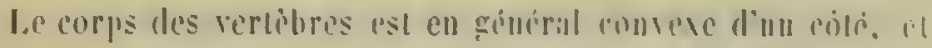


concave dı côté opposé; mais tantôt le côté concave est cn avant el tantôt en arrièrc.

Les vertèbres des geckos sont biconcaves d'après les obser. vations de II. Gervais.

Les chéloniens ont la colonne vertébrale divisée en plusieurs régions : la région cervicale est la seule qui jouisse d'une certaine mobilité; la région dorsale est complétement immobile; ses apoplıyses épineuses et les côtes, au nombre de huit, se recouvrent de plaques osseuses cutanées et s'étendent en largeur, s'engrèuent les unes dans les autres et formcut la carapace de ces singuliers reptiles. La partie inférieure de ce bouclier ou le plastron est formée par le sternum et les côles steruales. Les pièces qui unisscut la carajace au plastron, et que l'on a longtemps prises pour des côtes steruales, appartiennent au squelette cutané. La pièce médiane (entostcrnal de Geoffroy-SaintHilaire) rcprésente seule lc sternum; les autres pièces paircs correspondent aux cótes sternales. Les vertèbres de la région caudale sont petites et mobiles les unes sur les autres. Lcs crocodiliens ont sept vertèbres cervicales, douze dorsalcs, cinq lombaires, deux sacrées el une trentaine dc caudales. II n'y a plus de régions distinctes chez les ophidiens. Le nom-

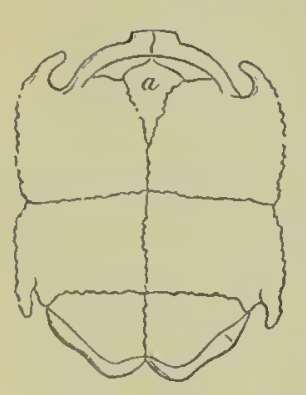
bre des vertèbres est très-grand et elles portent prestue toutcs des côtes libres ct flottantes.

Sternum. - On s'est mépris sur la signification des os qui composcntle plastron des tortues. Ce plastron a la mème composition dans tous les chéloniens; il sc compose, comme nous vellons de le dire, d'une pièce impaire qui cst le sternum

Fig. 230. - Plastron de tortue grecquo.

๙. Sternum. - Les autres pièces paires représentent les cóles sternales. véritable (fig. $250, a$ ), et de quatre pièces paires qui sont les tôtes stcruales ou abdominalcs. On a reconnu une pairc de pièces de plus dans des chćloniens fossiles.

Le sternum des crocodiles n'a noul plus qu'une sculc piecc osseusc, allongée, librc antérieurement, ct cnchâsscéc dans une 
lame cartilagineuse en arrière (fig. 251, a). Celle-ei reçoit les

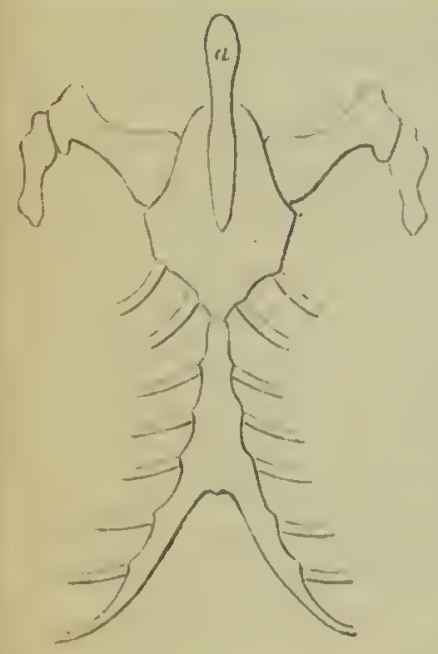

fib. 231 .

Sternum des crocudites. a.

l'épaule.

Les crocodiles, qui se rapprochent par tant de carncteres des mammiferes, portent sur les còtes les mèmes apopluyses que nous avous signalées dans les oiseinn.

Les cotes des dragons soutiennent la peau des flanes; au lieu de se recourber autour du thorax et du ventre, quelquesunes d'entre elles se dirigent comme des bras directement en dehors.

Ceinture de lépaule. - Elle se compose génélalement d'mne omoplate, d'un os coracoildien et d'une clavieule. Celte clavicule manqque daus les camélérus el les (rocodiliens. L'épaule et le sternum manquent chez quelques sauriens et eliez tous
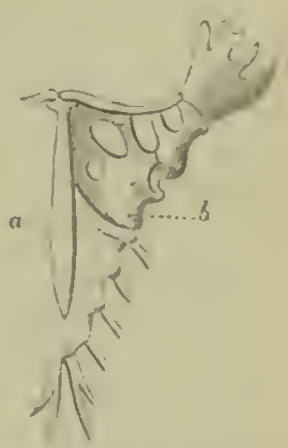

Fig. :3ะ. - sternum de lezard.

a. Sternum. - b. Ceinture de lépaule. les ophidiens. Les chéloniens ont une ompplate avec un long acromion, et un coracoidien distinct, qui coneourt arec l'os 
préeédent à la formation de la cavilé gléuoïle (fig. 255).

Dans les erocodiles, l'épaule est composée de deux us : l'onno-

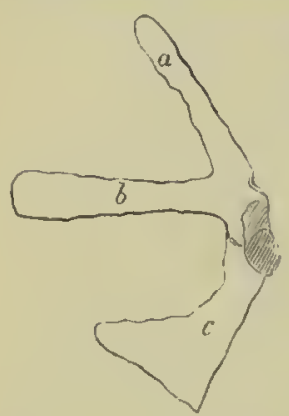

Fig. 233. - Ceinture de l'epaule de lortue.

a. Omoplate.-b. Acromion. - c. Coracoïdien.

la eeinture antérieure.

plate et l'os coraeoïllien. Ces os se ressemblent beaucoup entre cux; on a même dit que les crocodiles out deux omoplates. La clavicule manque.

Ceinture du bassin. - Elle manuve complétement dans les oplidieus, et sc trouve réduitc à l'état rudimentaire dans plusieurs sauriens. Le bassin est formé en général de trois os qui concourent tous les trois à la formation de la eavité colyloïle; chez les crocodilles, l'os que l'on a eonsidéré comme pubis ne eoutribue pas à former eelte eavité; celte eeinture ressemble beaucoup, surtout chez les chéloniens, ì

Les membres se divisent comme dansles elasses preeédentes; il y a un os unique dans le bras et la cuissc, deux os dans la composition de l'avant-bras cl de la jambe, le radius et le cubitus, le tibia et le perroné; plusicurs osselets dans le carpe (fig. 254) el le tarse (fig. 25:i); plusicurs aussi dans le métacarpe el Ic métatarse; el enfin uı nombre variable de phalanges dalls chacun des doigls.

L'os hyoïde est très-varié dans les tortues seIon les genres cl même selon les espèees (fig. 2506). Il est plus compliqué que dans les crocodiles.

On y trouve le corps, qui, tantòt unique, cst tantôt constitué par plusieur's pièees; sur les côtes il portc deux, quclquefois trois paires de corncs, et en avant, au-dessous du corps, ct non cu avant comme dans les oiscaux, se trouve l'os lingual.

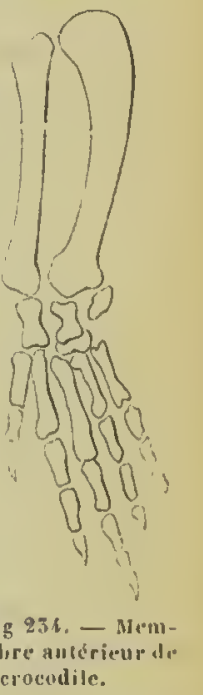

L'os tryoüde est des plus simples dans les crocodiles (fig. 257). Le corps consiste en une large plaque en forme de houclior, 
ilyaul une cehanerure sur le borl snperieur el unc paire de cornes sur le coite. Ial corne est en forme d'eipuerle ol a une piece

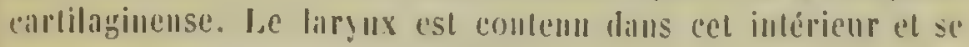
tronve représenté jial dles points.

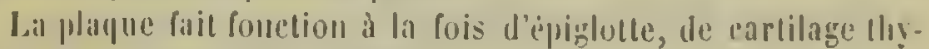
roilde el de corps de l'os ligoïde.

Batracions. - Ce qui distingue surtunt la tète des balra-

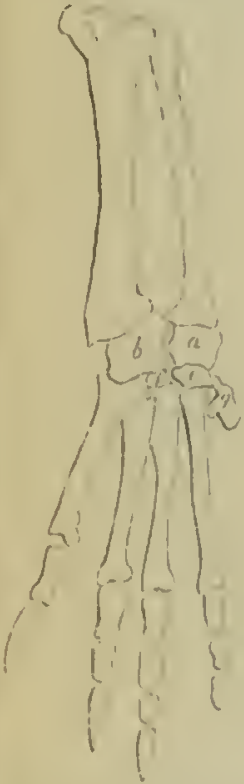

Flg. 435. - Mevobre posierient de crueodale

a. Calcantum. -

b. Istrafale. e. Conbuinle: $-f$. Cirneiforme. - $y$. (1s surnuméraire.

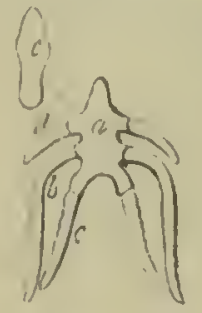

fำ $236-(1)$ lismide de torture

a. Enrps de los lijuile. - b.c. d. Ciornes. e. Os lingual.

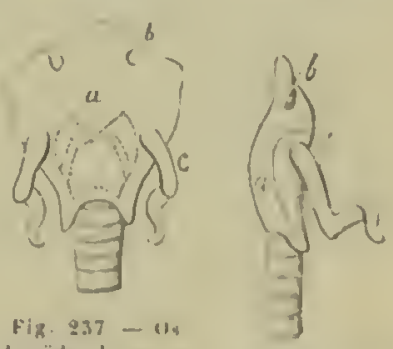

lageñd. Je reroen dile s a de :acer.

a. Cor pis. -

b. Eihanerurt。

- c. Curiars.
Fix. 25x.- 1 inclate vu do protil.

ciens, e'est que la vertèbre oceipitale pré. sente deur condyles artienlaires, el yue looeeipital hasilaire el le supérieur manquent genéralement.

Le eorps des vertebres présente des ilepressions coniumes semblubles à celles des poissons, et ordinairement elles sont rentplies d'une substance gilalinense. Cliez quel. ques-uns lit face antérieure est convere, la face posterieure concare. l.es apophises transverses sont tris-developpées diez les batraciens anoures, el surtont celles de la verlébre sacrie (fig. 259). Lil deruiere redidre est tris-longue el en forme de salure dans ces animaux.

les còtes sout en géneiral rudimentaires. on bien elles manIur'ul complélement.

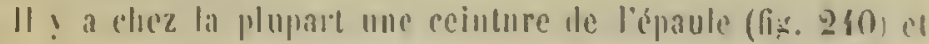


une ceinture du bassin, un sternum formé de plusieurs pièces

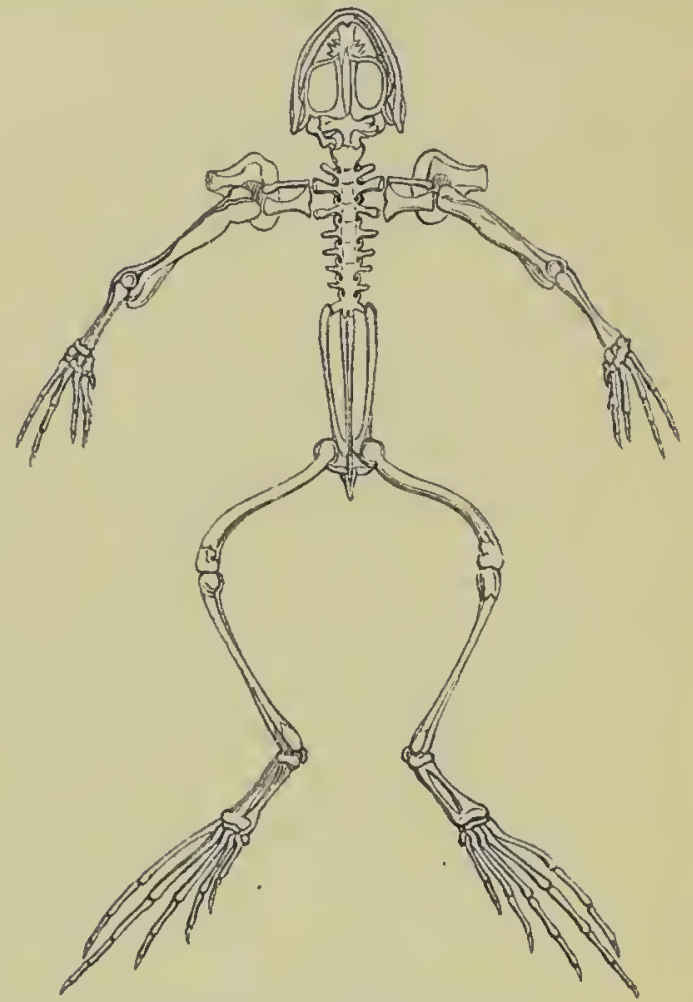

Fig. 239. - Squeletle de grenouille verte.

el des membres divisés comme dans les classes précédentes,

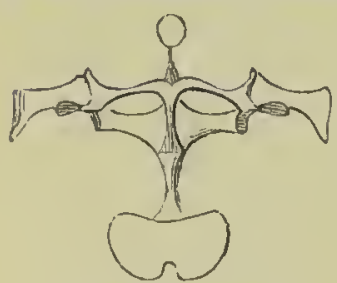

Fig. 250. - Ceinture de l'epaule. montrant exactement les mêmes os, depuis l'humérus ou le fémur jusq̨u'aux phalanges des doigts.

Dans les grenouilles, toutefois, le tarse est formé de deux os allongés comme les os de la jambe ou de l'avantbras (fig. 259).

Poissons. - Le squelelte se compose des mêmes parlies que l'on observe dans les classes précédentes; mais, chez quelques uns d'entre eux, une partic du 
squelelte reste à l'état de cartilage; clıe d'autres c'est le squelette entier; cliez d'autres chcore tout le squelelte se rédluit à

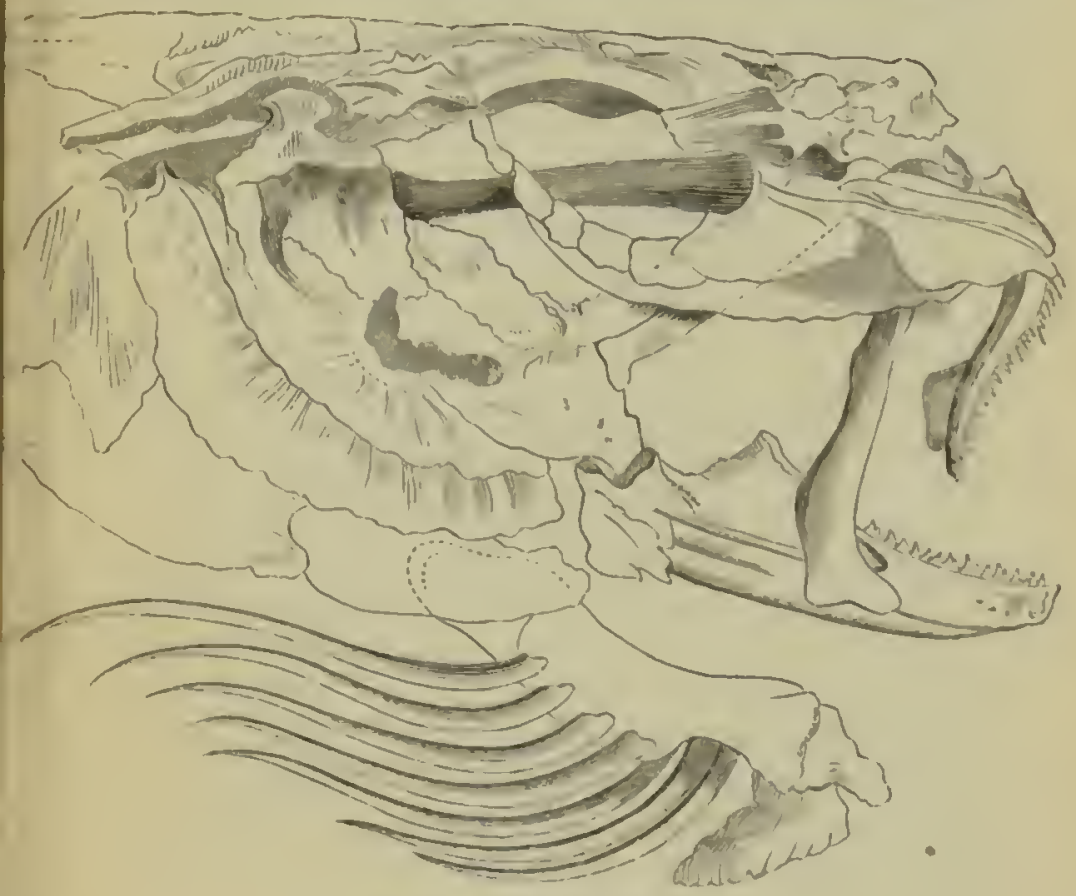

Fig. zl1. - Tele de morue.

une corde fibreuse représentant, dans le premier age enbryonnaire, la colonne vertébrale. Les os sont quelquelois si peu développés que plus d'une fois des poissons ont élé pris, par des maturalistes de grand mérite, pour des animaux sans vertèbres.

Nous parlerons d'abord des poissons osseux (Teleostei).

Région crânienne. - Les os du crime se séparent les uns des autres el forment qualre rertèbres qui conservent les mèmes caractères que dans les classes précédentes. La vertẻbre occipitale est composie de quatre ou de six pieces; la rerlebre moyenne montre en haut les pariélaux, en bas le corps du sphénoïde postérieur, sur le còté l’aile du sp̣hénoîde, le rocher el le mastoidien. La vertebre frontale montre en haut les os frontaux for- 
Inant les parois supérieures de l'orbite, le frontal antérieur el le frontal postérieur, comme dans quelques reptiles. Un sphénoïde antèrieur, el, clıez quelques-uıs, de petiles ailes eomplètent celte première vertèbre. Enfin, pour compléter la boîte erànienue, nous trouvons, pour la quatrième vertìbre, l'elhmoïle et le vomer. Il existe ordinairement vingt-six os dans la composition du crìne (fig. 241).

La face est composée d'un nombre beacoup plus grand de pièces, el il est à remarquer que les os qui forment la voùte du

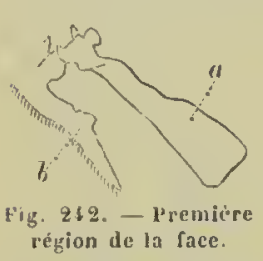

palais sont rejetés icei à drnite et à gauelıe, puisqu'il n'y a plus de palais proprement dit.

Une première région te la face eomprend le maxillaire el l'intermaxillaire (fig. 212), qui sont ell général mobiles sur le cràne et placés l'un à eôté de l'autre. Le dernier est a. Maxillaire. - situé en avant, forme la partie supérieure de ४. Intermaxillaire. l'entrée de la bouclıe et porte les dents.

Une seeonde région, située en arrière el sur le eôté de la plécédente, est formée de trois os: le plalatin, le ptérygoïdien interne et le ptérygö̈dien externe (fig. 245); la troisième règion eomprend les deux pièees du temporal qui se sont détaclıées du cràıe, le temporal (jortion ceailleuse) el le tympanal, le jugal

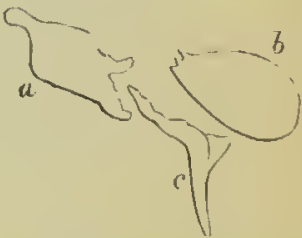
et une quatrième pièce que Cuvier désigne sous le nom de sympleetique

Fig. 243. - Deuxième région. (fig. 244). Enfin la quatric̀me région eomprend les quatre osselets de l'opereule, l'operenlaire, le préoperculaire, l'interopereulaire et le suboperculaire (fig. 245).

La cinquième et dernière région comprend souvent quatre os, colrespondant au maxillaire inférienr : le dentaire, l'articulail'e, l'angulaire, l'oper'eulaire (fig. 246).

Au-dessous de l'orbite et sur les tempes on voil souvent de petits osselets, qui, plaeés superficiellement, déplendent de la peau et sont appelés sous-orbitaires el surtemporaux.

La eolonne vertébrale ne présente pas de régions distinctes: 
les vertibres ont te corps concave des deux côtés, el l'esplace

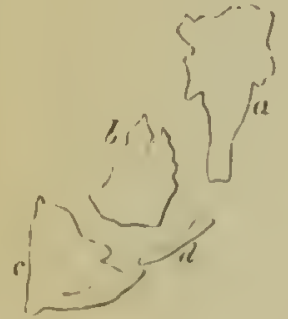

Fig. 茫. - Truisiome rigian.

u. Temporal. - b. Tympanal - $r$ Jitgial. d. Symplectique.
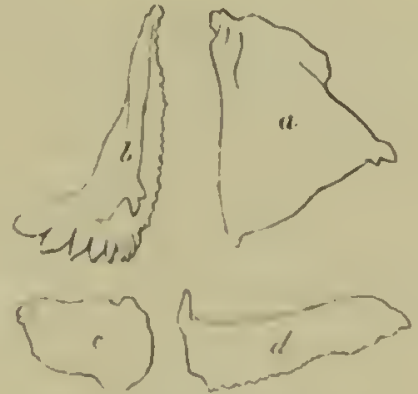

Fig. 26 ' - Qualrusine région.

6. Opereulira. - 6. Dresperculaire. - r. Incomperiulairs. - d. Sils. "pascrulains.

laissi cutre les diverses verlibres est rempli par une substance

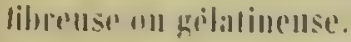

Less vertidnes presentent sonvent un are supérienr pour la - moelle épinicere el un are inféricur

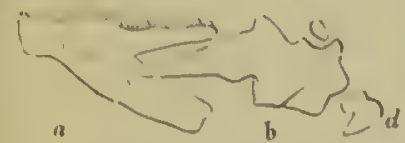
pour les vaisseanx. Le's coles se présentent comme tes appentices deilaches tes apophyses qui forment l'are verlibral inférieul. Elles manFig. : เ

n. H,unline - b. Arliruquent complétement dans quelques laire. - e. Ingulare d. Operowloire. poissons.

L.es apophlı̣ses épineuses présentent sourent a leur extrémites des osselets particuliers quui soutieunent les rạons des nageoires impaires; ce sont les os interépincux.

Derriere la lite on roil gílẻralentent une ceinture osseuse à laquelle s'attachent les nageoires pectorales; ce sont les membres antérienrs. Les deux picies supérienres qui s'altachent au crinte, correspondent i l'omoplate; ell tessous de celles-ci los long, qui forme presine entierement la ceinture, correspond i l'humérus el it la claviente: liapris d'autres anatomisles, l'os long et pointu qui part vers lo tiers inférieur de ce ternier, el se dirige en drssons el en arriere, est le coracoì- 
llien; vers le milieu de l'humérus on voil deux os qui correspondent au radius el au cubilus; en dehors de ceux-ci on voil une rangée d'osselets, qui correspondent au carpe, et au haut desquels sunt tcs rayons des nageoires qui correspondent aux loigls.

Les membres postérieurs sont beaucoup plus simples et ne sont pas toujours situés derrière l'ablomen; its peuvent même venir se placer au devant des membres pectoraux. On ne trouve pour ceinlure et os de la jambe à la fois qu'un scul os de forme triangulaire, engage au milieu des chairs, et portant sur te bord posterrieur les rayons ou doigts qui soutiennent la membrane de la nageoire.

Pour compléter le squelette, nous avons encore à parler d'un appareil très-compliqué que l'on voil au-dessous de la cavité de la bouche et qui appartient an squelette viscéral; il est formé en avant par l'os hyoïle, an milien par les arcs branchiaux, en arrière par les os pharyngiens. L'os hyoïle csı formé de chaque côté par cinq pièces, doul la plus grande porte les rayous branchiostéges, qui ne sont pas sans analogie avec les côtes.

Les ares branchiaux sont au nonbre de quatre ordinairement, et chacun d'eux est formé de six osselets placés bout à bout et formant un cercle autour de la cavite buccale; le bord convexe ou cxterne présente une gouttièrc qui loge les vaisseanx branchiaux.

Derrière ces quatre arcs branchiaux, it existe enfin deux os mobiles, souvent garnis de dents, qui sont situés à l'entrée de l'œsophage el que l'on nomme les os pharyngicns.

Chez la plupart des poissons, une partie du crâne prinilif persiste pendant toute la vie à l'état de cartilage el représente lc cràne primilif. On voit fort bien celte distinction chez le brochet. On détache aisément, dans une tète bouillie de ce poisson, les os qui n'ont point passé par l'ètat de cartilagc el qui n'on par conséquent pas la mème signification que les autres. La loîte cartilagineuse est au-dessous d'eux. Ces os, qui ne proviennent point de la boitte cartilagineuse, sont analogues à ceux qui entrent dans la composition crìnicnne des vertébrés supérieurs, et on s'est mis à douter si tous les us tu crâme appar- 
liement réltentent au squelette interne, ou s'ils ne font pas plutôt partic du sipuelente cutane.

Les poissons pligiostomes ont le sipuelelle dartilagineus comme l'esturgenn; la bnite crinieme n'est pas livisée en diverses pieces, et des foutanelles persistent pendant toute la vie. On reconnili encore les vertèbres dans la culonne vertélurale, des ares branchianx al des membres.

Chez les hrialdhiostonies, lunt le spuelelle: est repré-

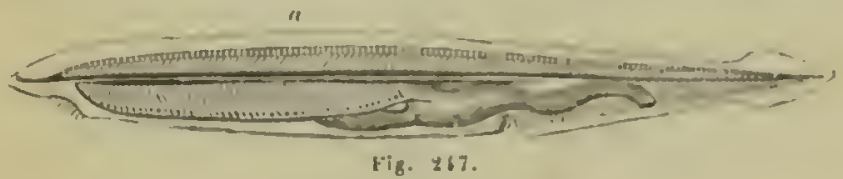

a. Ciorde dius sile.

seuté par une simple corle fibreuse qui acenpe lia flace de la coloute vertóbrille, c'est-ì-lire, dlı colpls les vertibres (lig. 2.47). C'est la colonne vertólurile la plus emhryommaire. Lal corle dorsile des myvinoülles est gilatineuse, avec un filed fibreux an centre. C'est une organisilion un peu plus avancie que la précelente. Cethe corde dorsale existe che\% lous les ver-

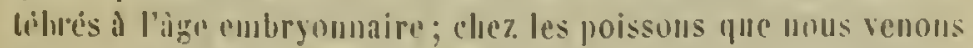
l'énumerer, rlle prersiste pendant loute la vie de l'animal; cley.

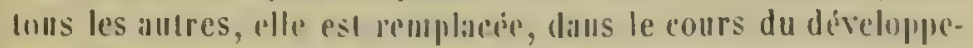
ment, par le corps des verlibres.

Mollusyues. - Ilors des animanx verlibrés, les mullusques apholoporles som les seuls illirz lesquels on observe un squelolle interne. II consiste en diverses piedes qui restent a l'élat de carlilage el dout la struclure ofre les mémes (aracliers que celui des animbur supérieurs.

La pice principale est la boile crimienne (fig. 248). Elle loge le cerveatl el fournit deux prolongements sous forme d'orbiles pour y loger les inormes yeur de res mollasyues. L'kesophage passe à travers celle boite

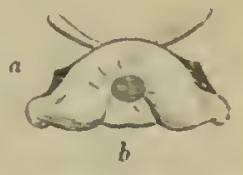

Fig. 288. - Boile ear lilagineuse Je septil allicunalis.

a. Orbiles. $b$ Orifice jourle prassage de linsophage. inec les candux excreburs des glandes salivaires infiricures.

Ou Irouve cusuite une autre piece sur la nupue que l'on a pu 
comparer à des areeaux supérieurs des verlébres; elle est formée de deux plaques qui représentent, anx yeux de quelques anatomistes, la colonne vertébrale; c'est dans le calmar que celte pièce acquiert le plus grand léveloppement. Dans les seiches on observe une piece à trois apopliyses il la base des bras, une longue lame le long de la nageoire, el enfin des pieces dites artieulaires à la base de l'entomoir el auxquelles s'adaptent des caltilages du mantean.

II existe trois cartilages dans les poulpes, douze dans les

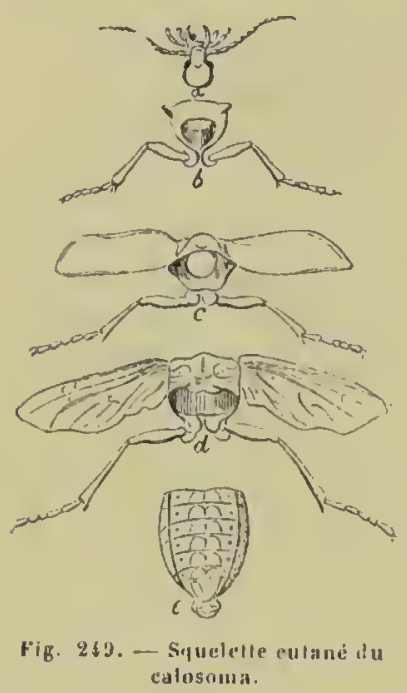

a. Tète avec les antenues, les mandibules, les michoires et les palpes. - b. Proilhorax, avec lapremiere paire de pattes. - c. Mésolliorax avie: ine paire d'ailes et la seeonde paire de palles. - 1 . Métathorax avecla deuxirme paire d'ailes et la troisieme pritie de pattes. - c. dbdomen. calmars el theize dans les seiches. Squelette cutané des articulés. - Cliez les animaux artieulis. il existe un squelette, mais il es! uniquement formé par lil peau; c'est pourquoi on l'appelle squelette dermique ou cutané.

Dans les animaux vertebrés, il est intérieur et ellveloppé par les museles; dans les articulés, il est extéricur, et les muscles sont logés en dedans.

Il est corné ou calcaire daus eet enibranchenent, et renferme uu principe ehinnique partieulier eonnu sous le nom de chitine.

Les picees ne se joignent pas i laaide de ligaments, comme dans les vertélırés, elles se soudent par juxtiposition; on les nomme apodèmes.

Uil animal de cet embraute. ment est toujours formé d'une série d'annealux (liy. 24!) joints ensemble el qui se modifient d'autant plus profondément que les appendices qu’ils portent sout plus développés. Les myriapodes out, comme les larves en général, les appendices el les anneaux semblables culre eux. 


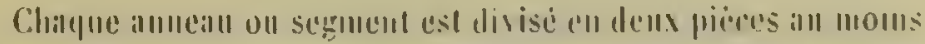

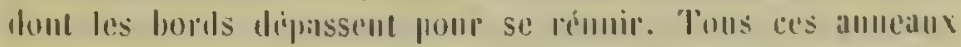

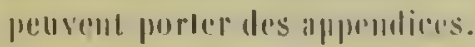

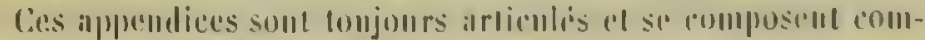

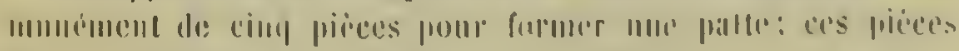

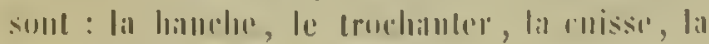

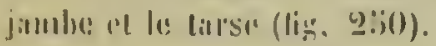

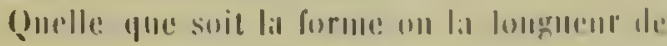

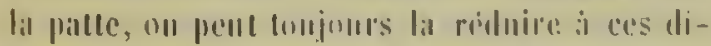

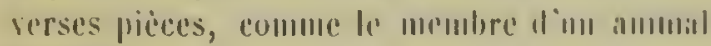
vertebri.

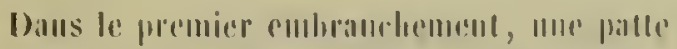
pent se transformer en aile; diuls les animians aldicules, les alles sont les appendicus differeuts, qui ue deviemuent jamais des palle's. I.es

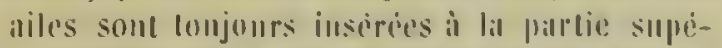

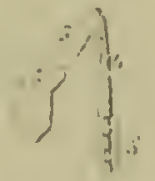

Fist $: 0: 10-10.1116$ 4t un urlecult

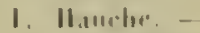

2. Trosthatere - 5. (inisu.

1. J.1131\%. i. Tuse.

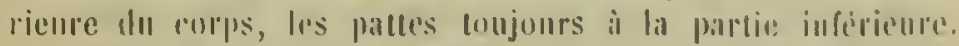

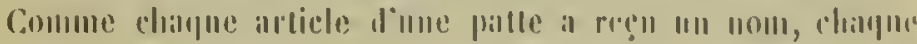

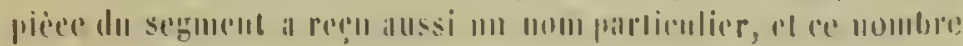

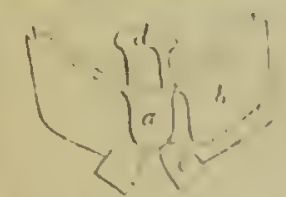

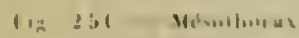
4. "litistus.

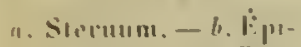
stermum. - E. E.11mire. - 1, linllio1.15. - e. Lit hilliche.

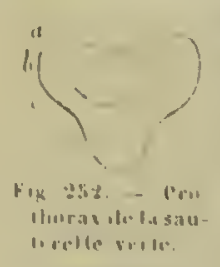

a. Iי⿺辶卬 - b. $\therefore$.

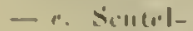
liruा - 4 l liustsculcllum. peolls silever, dillis less sonme'lls les plls compligunis (eris qui purtent des pialless ol des ailes), i duall re

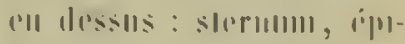

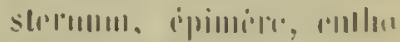

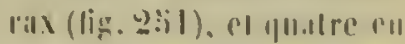

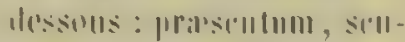

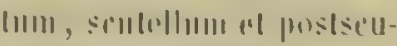

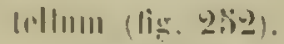

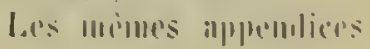

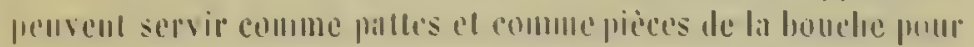
lil milsticiltion.

II 11 y a jamias plus de deur paires de membres dams les ler-

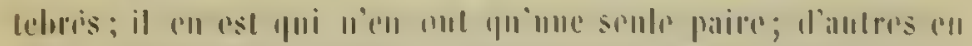

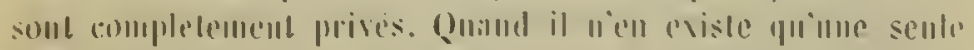

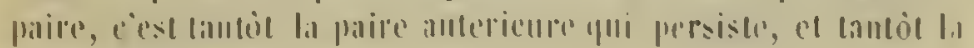
paire moliricurr. 
Il y a au plus ileux paires d'ailes dans les animaux articulés; elles sont toujours iusírées sur le deuxièmı et le troisième anheau du tlorax. Conme pour les menbres des verlébrés, une paire manque quelquefois; d'autres fois elles manquent toutes les ileux.

Les pattes des articulés sont an nombre de deux paires dans quelques crustacés inférieurs; elles sont au nombre de trois dans les insectes, de quatre dans les araclınides, variables de deuxà sept dans les autres crustacés parasites et libres, et entiı au nombre de plusieurs centaines cliez les myriapodes.

Il n'existe plus de pattes lıors des articulés.

Dans tous les insectes la bouche se compose de six pièces

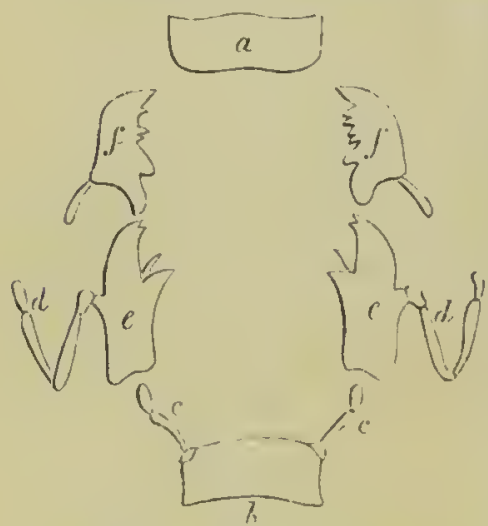

Hig. 255. - Piece de Ja bouche de l'hydroplitus piceus.

a. Lèvre supérieure. - b. Lèvre inférieure. - c. Pillpe labial.- $d$. Palpe maxillaire. - e. Machoire. f. Mandibule. (fig. 255): deux impaires: la lèvre supericure el l'inférieure; denx paires : les mandibules et les mâclıoires. Les màclooires et la lève iuférieure portent en outre des palpes. Toutes ces pièces u’appartiennent pas au squelclle cutané; il y en a qui doivent itre rapportées au squcletle viscéral.

Ces six pièces de la bouche s'allongent pour former une trompe ou un suçoir cliez les insectes suceurs; elles restent courtes el disliucles dans les insectes broyeurs.

Cliez les mollusques, il se

forme généralement dans l'épaisseur de la peau des pièces soliles, ordinairement calcaires, et que l'on appelle communément coquilles. Il n'y a que chez. les oscabrions qu'elles présentenI de l'analogie avec le squelette des animaux articulés.

Celle coquille n'est forméc en général que d'une ou de dem. pieces qui servent plutỏ à la protection de l’animal quı’à la loromotion. 
'Tontes les enquilles, quelle qu'en soit la forme, penvent se rédnire à un còne diversement enroulé (fig. 254).

11 n'y a pas ule mo daus les mollusymes : la mine coquille contimne à erojIre prendiant la vie de l'animal.

Les mollnşues qui vivent en colonie, comme les bryozoaires, et qui ont aussi une enveloppe calcaire de protection,

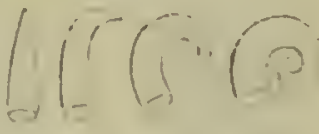

Fig. this - Coquilles suni-

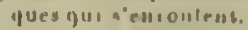
forment souvent des tiges ramifiées, semblables a des plantes, et que l'ou a appelées polypirars.

Quelgues mollusques (lestmiciers) présentent, dans la composition le lenr squelette culane, me sulstance semblable, sons le rapport chinique, à la cellnlose vigúlale.

Plusien's vers ont, comme les cestö̈les, des corpuseules ealaires de forme arrondie dans l'épa isseur de leur peau.

Beauteoup de vers i sang rouge portent des soies de forme ures-variéce (tig. 2:3i), quelugefois en si grand nombre yu'elles formurnt une sorte de feutre sur le dos de l'animal, romme dans l'aphrodite. Ces suies deviemuent qualquefois lamellenses ot se recunrent comme les ćcilltes (lig. 2:ili), pial' exemple daus les polymés.

D'autres vers ont le corps revêtu d’un spithélimm vibriltile (les turbellaires des an. leurs), et portent souvent laus l'épaisseur de la pealu des corpuscules en forme de hitomets qui semblent carileléristiques de ces animanx.

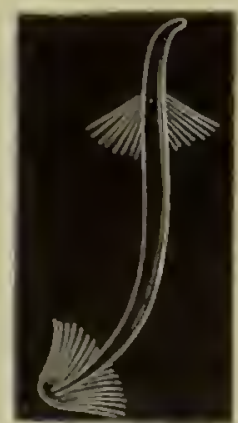

Fig. żis. Sirio da inmbricus complanalus.

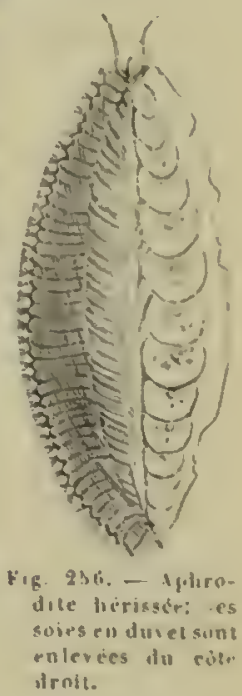

Le sifnelptte cutané se rédnil dans qunelques échinodermes el phusieurs polypes il des spicules doune forme constante qui iuerustrut la pean et qui permeltent de distimguer les principans

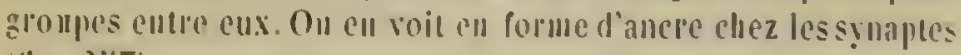
(1i: $2: i 7)$. 
Cils vibratiles. - Chez les animanx inférienrs, la locomotion aquatique a sonvent lieu à l'aide de poils très-fins, pui batlent

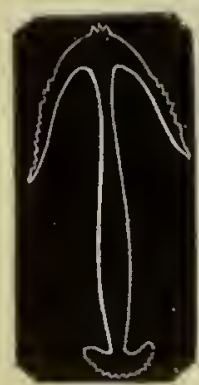

li.ig 257.

- siricule de synaple.

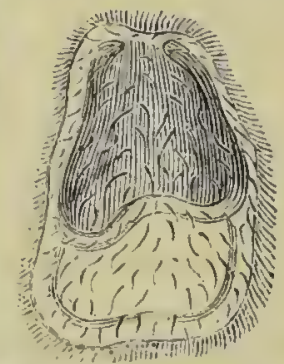

Fig. 258 - Jeunc alcyonelle, couverte de cils vibratiles.

l'eau comme les planches d'unc: rone de bateau à vapenr el que l'on désigne sons le nom de cils vibratiles ( figr 2:38).

On ne distingue res organes qu'à l'aicle du microseope. On voit des cellıles de forme. variable former un épilhélium, el au bout de clarpue cellule, des poils hyalins terminés généralement en pointe. C'est un épithélium vibratile (lig. 2!j9).

La plupart des infusoires, les ver's turbellaires, lesembryous le vers à sang ronge, les embryons de mollusques en général, soul pourvus d'un épillélium vibratile, chez les uns sul' loute la surface du eorps, ehez les autres dalls quelques régions selllement.

Cel épillélium vibratile existe dans les animaux supérieurs el même elıez l'lomme, non pour la locomotion de l'individu, mais pour la locomotiou de certains produits qui sans eux ne pourralient être mis en mouvement. Le produit màle, le spermatozoïle porte luiınême le eil viluratile pour se monvoir, mais dans l'appareil femelle ce sont les cils des trompes ou des oviluctes qui déterminent le monvement de l'œur.

Dans d'autres eas, les cils sout évidemment destinés à faire mouvoir les linjuicles qui baignent les surfaces que ces eils reconverul.

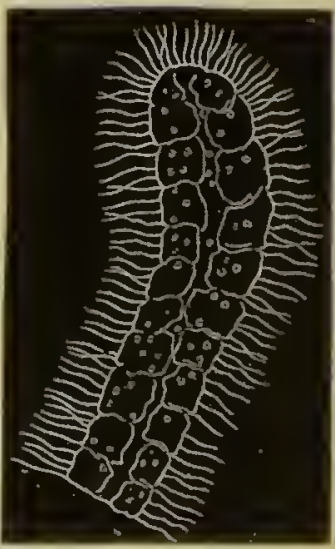

Fig. $259 .-$ Tontacule de byozicate, couvul l'un ipullélium sibratile.

On a compraré aver raison le monvement viluralite de la surface d'une membrane a l'ondulation dim champ de bé ballontio parle vent. 
MUSCLES.

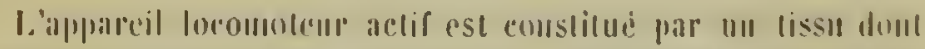

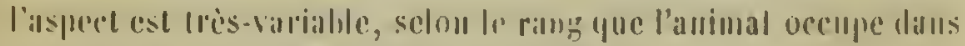
lis série, ret yu'oll momme lissu musculatire.

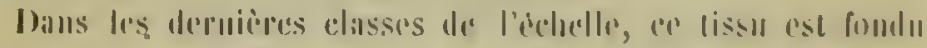

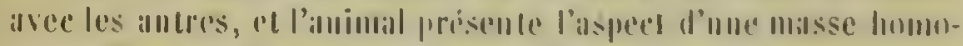

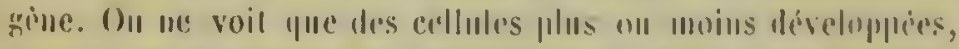

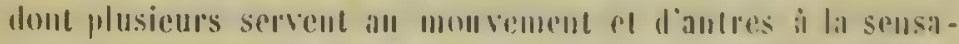

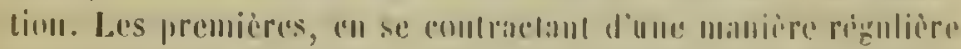

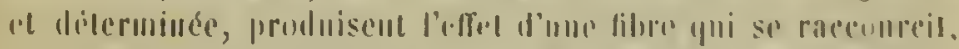

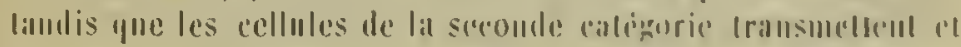

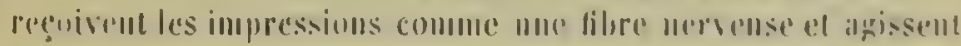

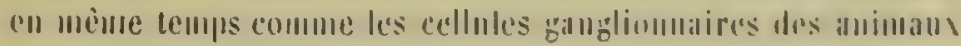
superieurs.

Quind l'orginisme se compliyme, an lien de cellules displo.

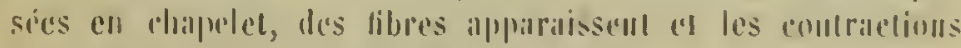
gagment ell foree el coll pricision.

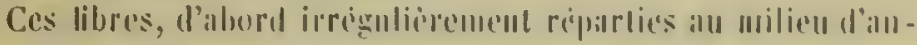
tres lissus, se groupent amsule emsimble, conslituent des fais-

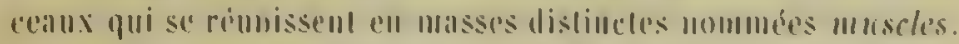

Dans les classes inferientes, ces libres sout loujours blan-

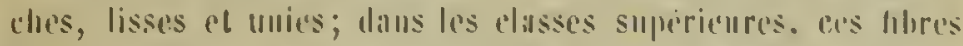
lisses persistent dans les appliareils de la vie vigriblive: mais les apprareils de la vie de relillinn sont poursus de fibes stribes.

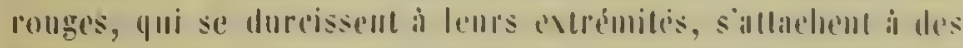

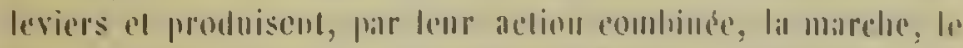
sant ou le vol, el lous ces mombements varies yui distiusucul les animaus superieurs.

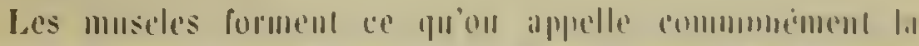

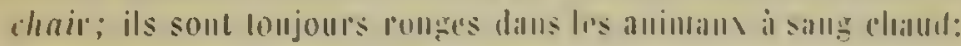

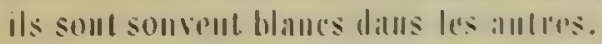

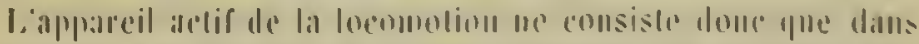

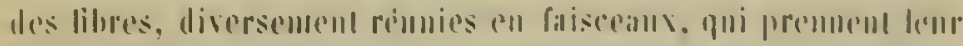

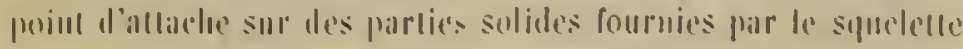

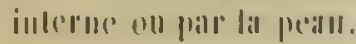


Clıaque groupe te faisceaux a reçu un nom particulier; on el compte plusieurs centaines dans un animal.

Le squeletle avec les muscles forment le véritable appareil locomoleur.

Il est inutile de faire remarquer que le cadre restreint dans lequel nous devons nous renfermer ne nous permet d'énumérer que quelques modifications du système musculaire dans les principales classes du règne animal.

Dans les classes inférieures du règne animal, y compris mêne les articulés, l'appareil locomoteur est fourni par la peau. Daus le premier embranchement, qui comprend les classes supérieures, on trouve un appareil de locomotion spécial qui leur est propre. En général, les muscles de la pean persistent jusque dans les rangs les plus élevés, même avec les parties solides ossenses ou cornées; mais ils ne servent plus ì la locomotion proprement dite; ils servent plutôt à la protection.

\section{VERTÉBRÉS OU HYPOCOTYLÉDONES.}

Mammifères. - La disposition des muscles a lieu dans toute la classe des mammifères d'après un plan uniforme. Ils sont partout faciles à reconnaître, si on les connait dans une espece quelconque. Les muscles des membres somt ceux qui presentent le plus de variations.

Le peanssier, ou muscle de la peau, acquiert un grand développement chez les mammifères en gẻnéral; il enveloppe souvent tout le corps, conme clıez le hérisson ou le pore-épic, qui ont la faculté de se rouler en boule, ou bien il prend seulement du développement le long de la colonne vertébrale, par exemple chez le chien et le chat, pour faire le gros dos.

Les muscles de la colonne vertébrale des cétacés se prolongent en avant el ell arrière saıs présenter de modifications importantes, et ils se groupent à la partie inféricure de lis région caudale comme à la partie supérieure.

Le diaphragme existe chez lous les manmifires; quelquesuns, conme les chameaux, ont une partie du centre aponévrolique ossifiée. 
I."'s muscles de l'abdomen, surlout le grand oblique el le "rraml troit, sout el général tris-volumineux clie\% les mamoifires, el s'élentent quelquefois sur toute la filce inférieure du lronc. Le pyramilial est surtout développé cliez les marsupianx. ()n ne voit pas les intersections tendineuses du grand ilruit ab-

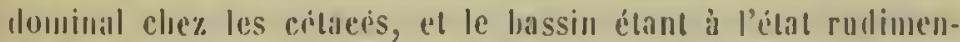
laire, les intersections postérieures de ces muscles offrent llassez notables morlifications.

Le musele gramil dentelé est lien développé cliez les animaus de eclle elasse; il s'altache orlintirement à loutes les éoles el jusqu'aux apopliyses trausverse's des dernieres vertébres cervicales.

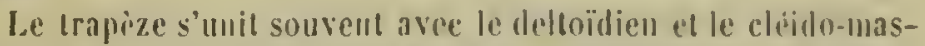
toïlien, pour former une la gre hande musculaire sur le cou el l'épaule, surlont quand la clavieule manume.

Dalls la plupart de's mammifires, on voil un muscle éleudu uepuis l'acrominn jusqua l'apoplyse transverse tles promière, deuviome el quelqurfois qualrième el cinquiènte verlèbre exrirale, et quelquefois alussi jusqu’i l'apopligse basilaire; c'est l'acromio-basilaire.

Le pectoral est surtout developpe ehe\% les mammifires fouisseurs et ehez les cheiropteres; le petı pectoral manyue ordimairement avec lis clavicule. Les eléiroptères out un muscle partirolier destine à teulre la mombrane te l'aile e't qui šinsère sur l'humerus.

Les unscles qui meuvent la michoirt inférieure. le masseler, le temporal et les deux ptẻrgoüliens sont géuéralement tris. léveloppés cltez les nammilères; le digastrique manque quelfuefois, at souvent il n’a lüul seul ventre.

La queue se nteut ordinilireucut it laide de luit muscles: leux relcveurs : le sacro-coceggien supeśrienr et l'inter-épineux supéricur ; quatre abaisseurs: l'iléo sous-eaudien, le sacro-souscandien, le sons-eandien inferieur et le pubo-sous-caudien; el entiu deux latéranx : lischio-caulien el l"ulertransversiare.

Oiscaux. - les unscles des oiseaus ont ell général une rouleur plus foncre que ceux drs mimmifires, el ils se distinsatul, imlipeudaument de leur couleur. par leurs tendons lui- 
sants, qui sout souvent ossifiés et séparés entispoment de la portion clairmus.

Quelques oiseaux, par exemple les coqs de bruyère (genre titrio), ont les museles de la poitrine palles et foncés. Les externes sont pour ansi dire noirs, tandis que les profonds sont toul hlaucs.

Jee peanssier ou les muscles cutaurs sont souvent très-déveluppes dans eertines rejurions du corps; ee sont eux, en effel, qui relevent les plumes de la léte pour former une lıuppe on qui font dresser les pemnes de la quene pour faire la roue. Dans les kakatoès, nous voyous les exemples les plus remarquables de la luppe; les pans et les diudons nous fournissent des exemples remarqualiles de la roue.

Les membres antirieurs des oiseaux devenant les seuls et uniques orgaues de la locomotion aérienne, les muscles pectoraux qui abaissent l'aile deviennent tellement volumineux qu'à emx seuls ils forment souvent ume masse plus considérable que tous les autres muscles du corps réunis.

Le long antérieur lu cou prend naissance sur les crêtes des corps des vertèbres dans l'intérieur de la poitrine el envoie des haudeleltes à toutes les vertíbres du cou.

L'os ptérygoidien et l'os tympanal out un muscle particulier, formé de deux portions qui les élèvent et les tirent en avant; on reeonnait encore les tempraux, les masseters, les ptírygoïdiens et le digastrique; les temporaux sont souvent formés de trois ou de quatre portions distincles.

Les muscles abdominaux manquent souvent ou sont peu développés, à cause du stermum, qui recouvre une partie de l'abilomen.

Le diaphragme est formé de quelques chefs charnus venant des côtes el dı sternum; le centre est toujours aponévrotique.

La queue de l'oiseau, surtout de l'oiseau rapace, porte luit paires de muscles comme eelle des mammiferes : deux ell dessus, qui sont releveurs; quatre en dessous, qui sont abaisseurs, el deux sur les eôtés.

Reptiles. - Les reptiles ont en général les muscles de lı 
peall tris-pen développés, el clie\% plusieurs d'entre enx on n'en voil pas meime de traces.

Un muscle peanssier s'étend sur toul le ventre et sur les côtes lles ophilliens; il s'insère en partie sur les còtes el en partie sur les écailles.

Cenx qui out les mâchuires tres-mobiles, ainsi que les os palutins, comme les scrpents, out de nombreux museles, parmi lesquels ou distingue eneore facilement les deux prirygoiliens, le temporal el le masseter, el yni forment deux grandes masses, dont l'exterue est la plus voluminemse. A ees muscles, que l'on troure avee les mêmes caractères daus les diverses elasses. viemtent s'ajouter quelgues nouveanx muscles qui agissent sur les os palatins el les maxillaires.

Chez les serpents venineux. nne partie du musele temporal comprime la glande venimense an moment de la morsure al pousse le venin daus la dent.

Le musele digastrịne est eneore distinet.

Conme il y a une grande différenee dans la mobilité des verlibres, selon les reigions ou selon les ordres, il existe une diversité très-grande dans les museles du dos. Cliez phrsieurs d'entre cux, les muscles du dos ef de la queue se divisent par tranehes, comme on le voit daus les poissons en général.

l.es muscles inter-osseux manquent chez. les chéloniens, mais aequitrent un grand développenent chez les ophidiens, et emlurissem sourent plusieurs côtes dans ees reptiles.

Les muscles abdominamx s'úfendent jusqqu an trone, et on roit le muscle droit se eonfondre aree le sterno-lyyoülien. Les interseetions tendineuses que ee musele présente eorrespondent a nombre de eòles.

Un diaphragme rudimentaire existe ehez les reptiles et les baIraciens; on le recomnait surtout eliez les chíloniens.

l.es museles perdent en général leur conleur ronge ehez les repuiles comme eliez les batraeiens.

Poissons. - I.e systime musculaire se simplifie considérahement chez les poissons; il n'y a plus qu'un petit nonbre de. musples a signaler, mils quelquis-uns prennent un dereloppement execssif el forment presque tout le eorps du poisson. 
Faisons remanquer d'alord que le poisson frappe en gencíal l'eau par des flexions alteruatives du trone et de la queue, et que les apophyses épineuses supérieures empêchient le mouvement daus le seus vertieal; c'est pour celil que nous voyous, de ehaque eôté du corps, Ilepuis la tête jusquu’à la uageoire eiludale, un énorme muscle qui représente les fassecaux du sacro-spinal. Ce musele est remarquable par ses lames aponérrotiques qui le divisent en autant de conclies de fibres lu'il y a de vertibres; elles sont souvent disposées en zigzag, surtout à la partie postérieure, el elles rappellent la eomposition anmulaire des animaux articulés. Ce sont elles aussi qui donnent souvent à la elıair des poissons tin aspeel feuilleté, surtout quand la gèlatine les lames a èté dissoute par la enisson. On l'appelle communément muscle latéral.

Ce musele est divisé en une bande supérieure, qui représeute, d'après Cuvier, l'épineux du dos; en une bande moyenne, qui représente le lonğ dorsal avec le lonbo-sous-caudien latéraldes mammifères à queue, et en une bande inférieure qui répond, dans la partie qui règne sous la quene, au lombo-sous-eaudien iuférieur.

Sur la ligne mẻdiane, en dessus el en dessous, on volt un muscle longitudinal qui rappelle en dessous les museles drouts abdominaux.

Les nageoires paires et impaires ont un grand nombre de petits museles, dont on compte trois sortes differentes dans la Ilageoire caudale.

Il n'existe pas de muscles eutanés proprement dits, mais les muscles adlièrent en général intimement à la peau, par exemple clıez tous les poissons plagriostomes. On sait eombien ces poissons sont difficiles à éeorelıer.

Les rayons de la tète du lophius piseatorius portent des muscles abdueteurs, des flèchisseurs el des extenseurs, dont les premiers surtout sout très-dẻveloppés.

L'opereule à un muscle externe qui le relève et un internc quil'abaisse; mais les muscles les plus forts le la tète sont ceux des mâelıoires. Ils constituent la masse charnue de la jone et semblent representer à la fois le temporal et le masseter. II I'y a ni ptérygoüdien ni dignstrique clıer. Ies poissons. 
C.es ares branchiaux se meuvint par de nombreux pelits mus. eles, dout quelques-nus s'insèrent à la base du crine el agissent romme clovatenrs, landis qu'mn muscle très-fort part de la face inférienre de la colonue vertébrale, s'éteme à la parlie superienre des ares branchianx el tire cel ippareil en arriere. Eusfin un unsele s'élend de l'os loyoüle an pharyngien inférieur êt agit comme antigoniste du précrélent.

Les museles des poissous sout rll gínéral blancs ou jaunatres; (quelpuefois ils sont roses on mêne ronges, commer dans le sanmon el le llon. Centinus poissons, par exemple l'esturuneon, out quelynes museles rouges enume des muscles d'animan à sang chaul, el la conlent parait meme varier dans lo mine prisson, selon des circonstances yue l'on n'a pun apprécerer jusqu’i préseut.

\section{ARTICELS OE EPICOTYLAOYES.}

Tous les genres de vie se rencontrant dans l'embranchement

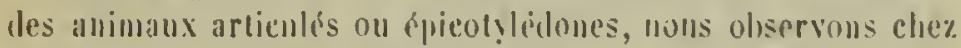
eux un systeme musculaire tris-développe; mais, comme il n'existe plus le squelette interne, les muscles doivent s'inserrè sur les segments cormes ou calcaires dn squelette colanė.

Les museles sout formés de fibres encore parfitement dis. lincles, incolores on légerement janmitres, et montrant toules des stries Iransverses, meime quelquefois le's fibres organiques.

Les muscles sont insérés généralement sur des prolongements du têt, qu'Audouin a desigunés sons le nom d'apodème el qui s'allongent quelquefois comme les tendons. Les veritables tendons manquent.

Les muscles se rendent en général d'un segnent à celui qui le suit et produisent ainsi le monrement des preres qui se suivent. Dans la région thorachique qui fournit insertion aux paltes el aux ailes, outre les muscles des segments, on troure encore cenx qui meurent ces appendices, el la masse musculaire y devient très-considerable. L.e ș̣stime musculaire est d'autant plus diveloppes que les segments ou les appendices sout plus mobiles. C'est ainsi qur, dans les derniers articulés. les lernéiens para- 
sites, qui ont l'abdomen atrophié el eomplétement immolile, toute traee de fibres inuseulaires a disparu.

Quand les segments du corps sout lous semiblables, eonme chez les larves d'insectes, les museles sont disposés de la même manière dans loute la longueur le l'animal; mais après les métamorphoses, les appendiees étant survenus, des différenees considérables se remarquent sous ce rapport entre la région thorachique et la rigion abdominale.

\section{ALLOCOTYLÉDONES.}

Mollusques. - I.e systìme museulaire est encore trìs-développé dans les mollusques; il est formé de filores lisses el nou striées qui se réunissent en faisceaux distinets et qui prennent orlinairement leur insertion sur la eoquille. Souvent aussi les fibres s'entre-ervisent et tendent à se fondre dans les autres tissus.

Les eéphaloporles présentent les museles les plus développés; on en voit deux paires entourer l'entonnoir et s'insérer sur la boîte eràmienme el deux autres moins volumineux qui permettent à ees animaux de eontrater l'entonnoir avee foree pour faire jaillir le eontenu de la bourse du noir. Les bras sont formés de fibres museulaires longitudimales, eireulaires et rayounantes qui en font un organe d'une fexibilité extraordiniire.

Les gastéropodes ont un pied qui est museulaire dans toule son étendue, et dout les fibres s'entre-eroisent ent lout sens. II y a dans les hélix, par exemple, uı musele rétracteur du pied qui va s'attaelier à la eolumelle de la eoquille, et un seeonl musele columellaire qui s'unit antérienrement aux parois museuleuses de la eavitéde la bouclı. On trouve ensuite les muscles propres des tentaeules el un muscle de la yerge.

Plusieurs mollusques aeéplales montrent encore trois museles bien distinets, deux transverses addueteurs et un longitudinal rétraeteur. Le musele transverse postéricur est ee petit eordon blance et asse\% dur qui reste attaclice a une des vilves des moules apris leur cuisson. 
Cillez les tuniciers, ce systeme se simplitic consillérulfement, misque nous ue trouvons plus que des libres musculaires isi)lines, les mes circulaires, li's antles longiludinales, en dessons he lis peall.

Nous pouvous en dire autant des bryozoaires. Des filres musculaires isoles se gronpent ensemble pour former de: fiasceanx, mais ees libres ne s'unissent point les unes anx inl lies. Il y a des museles réllateteurs de l'animal qui ront sinsérer d'un roté a ha hase de la courome tentachlaile el de l'antro coutc a la paroi interne de la loge. La's cordons musculaires qui composent ces faisceaux sout tres-lours.

Vers. - Les vors nous moulrent mu s!steme musculaire bean. coup plus simple que eedni de's mollnsigues. Chur les vers ì san: ronge, une conche musculaire, formice de denx on lruis plans de fibres, les nnes circulaires, les antres lungitmdinales, tapisoe? la pea dins lonte sa surfice, et dome an rep la meince sull. plesse dans toute son étendne. Quclquefois ees couches un labres forment a l'intérieur du corps des cloisons, qui le divisent ('Il intiull de loges.

On tronse encore des fibres musculaires lapissant la pean dhe\% les vers les plus simples, et on roit les ventouses des ténias c.xelnsivmm formies de tilmres musculanires lisses.

Clez phosieurs rers toutefois, le munvement du corps un des appendiers qui le conrument a lien par la contraction de's cellules yni le comprosent. Celis se roil distinetement eluez plu sieurs tremalodes el quelipues cestuides.

Echinodermes. - Les édunudermes ont encore des muscles distincts. Les loolothuries ont une conche de tibres circulairas

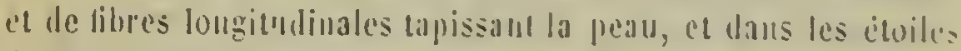
de mere comme daus les ontrins, on roil des muscles qui dou-

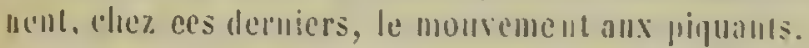

Polypes. - Euliu, chez los polypes, on roil emere des monrements tris-rigulieres el distincts, qui sont dus à la contraclion de edlules ef qui se distinguent it peine des antres rellules

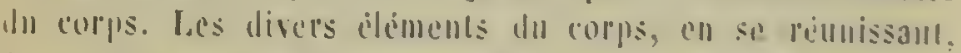
forment une masse homogeme qui fail a peime supposer qual entre des chéments diftérents dalts sa romposilund. 


\section{APPAREIL NERVEUS}

Cet appareil, désigué plus conmunémeat sous le nom de systeme nerveux, se eonipose surtout de trois parties distinctes: $1^{\circ}$ de nerfs qui transmettent les impressions ou les ordres; $2^{\circ}$ de wanglions qui les reçoivent, et $\bar{\partial}^{\circ}$ de commissures qui établisseut des communications entre les ganglions et qui produisent l'lalrmonie dans l'économie animale.

Les impressions reęues du delıors par les organes des sens sont communiquées au ecrveau, soil directement, soil iudirectement, par les ner's; les ordres qui émanent du cerveau sout trinsmis par les nerfs scublables qui marchent parallèlement a ceux-ei : les premiers nerfs sont nommés nerfs de sentiment; les seeonds, nerfs de mouvement, ec l'ensenible de ces orgenanes, qui établis sent ainsi le rapport avec le monde exterieur, constitue le sỵstème nerveux de la vie animale.

Mais il existe en outre des ganglions et des nerfs qui prési-. dent au mouvement de divers organes qui sont soustraits il l'empire de la volonté, comme le cœur, les intestins, ete., et dunt l'animal ne peut ui activer l'action, ni ralentir la marche. l.e cour bat sans notre intervenlion; ce sont les nerfs et les 
gallghtions yui forment le systeme du grand sympathinge ou de lis vie végélative.

II y a très-peu d'animanx cliez lesquels on liail pas l'our III systime nervenx plus ou moins développé.

Cel apparcil u'est fout couformé sur le uème plan dans les divers embranchements du riggne aninal; il est dillicile de re. enmailre daus les amimaux sans verlébres les parlies analogues des vertébrés. On tit : "le cervenu d'un insente, le cervein d'un mollusque; " maris il u'est pas démontré que ce cervean correspoude à célui des animaux supéricurs.

I.c systeme nervenx se manifeste dans fes anmanx infericul: sous une forme très-sintple : on roit dres ginglions, rémnis entre enx par des commissure's, entourer l'usupharge sous forme de collier, el de ces ganglious on roit miltre lous les merfs. Ce collier se trouve dans la plupart des amimaux silns rel libres.

Dans l'embranchement des artientés, les ganglions inferieur':

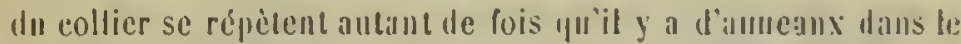
eorps, et de lì résulte une chaine ganglionuaire qui s'itemal daus coute la lougueur de l'aurimal.

Quind ces inmeanx sout sembables cutre enx, les ganglious te sont aussi, mais comme les anneanx thorachiques sont gemirialement plus developpés que les antres, a cause de l'inserlion des patles et des ailes, les trois paires de ganglions correspondantes sont modifiées anrsi. Dins innelques-nns de ces animanx, les anncalnx sont serres les mns contre les autres, comme dinns les crabes, et la ednane gangliommaire prend l'aspect d'une iloite. Ce n’est au fond cependant que bil mème disposition.

Ces nerfs appartiennent a la vie de conserration; ils établis sent les rapports entre l'animal el te monde extericur.

Mais dejia dans ces classes peu clevées, le longy du camal in Irstimal, on voil quelyues ganglions qui distriburnt leurs tilet:

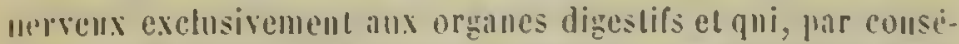
ynent, président à la vie de conservation ; ces gangtions, arer les nerfs qui en maissent, lepresentent le grand sympathique des animan. superieurs. IIs sout en relatiou aree les ganglions du 
collier par une double commissure, el se trouvent déji dans des mollusques qui n'ont qu'un simple collier osophagien. O॥ les désigue sous le nom de stomalo-gastriques ou de ner's splanclıniques.

\section{ANIMAUX VERTĖBRÉS OU HYPOCOTYLEDONES.}

Le systeme nerveux est exactement conformé d'après le même plan dans tous les vertébrès, depuis les mammifères jusqu'aux ploissons. Il est formé par une moelle épinièr'e, un cerveau ou encéplaale, des nerfs el un système du griand synıpallique.

Ia moelle épinière, qui esı la partie principale, consistc dans un loug cordon blanc, logé dans l'intérieur du canal spinal, et se compose de quitre filisceaux intimement unis entre cux; de celte moelle on voit naittre autant de paires de nerfs qu'il y a de vertèbres, el toutes ont deux racines, une antérieure et une postérieure. Les célèbres olsservations de Bell ont fait connaitre que les racines antérieures des nerfs spinaux président au mouvement; les postérieures, au sentiment. La section des p̨renièr's produit la paralysie, la section des dernières l'insensibilité. Il parait ell ètre de même des faisceaux qui composent la moelle épinière; les antérieurs sont des faisceaux de moulvement el les postérieurs des faisceaux de sentiment. Cellc dis. linction semble s'étendre jusłu'aux animaux sans vertèbres. La chaine ganglionnaire des articulés cst formée de deux conclies superposées; la couche supérieure, quand l'animal est placé sur ses palles, correspond à la couclıe du mouvement, l'autre à celle du sentiment. En plaçant l'articulé sur le dos, les rapports sollt les mèmes que dans les vertébrés.

Nous trouvous ces doubles faisceaux jusque dans les bris lles mollusiques céplıalopodes; lans clıacun de ces appendices pénètre un cordon nerveux droit naissant du bord antérieur des ganglions oesoplaggiens, el un autre cordon ganglionnaire, s'anistomosant de distance en distance avec le précédent, s'étend aussi dans toute la longueur de cel organe el jone, à ne pas ell dnuter, le röle d’un nerf de sentiment, ou plutòt du graud șin- 
pithinue. Cés neres, avee leurs ganglions, existent indépendamment des autres el u'oul de rappult avee eux que par des rommissures qu'ils euvoient. Ils penient agir le concert sur les nombreuses vantouses yui garnissent les singuliers bras de tes animaux.

Lal moelle est généralement renfléc ì l'endroil oủ uaissent les uerfs (fui doivent se rendre aux membres.

Celle inoclle est toujours erense a liage enbryonuare, el te ventrienle yui en résulte ne disparait que dans les vertébris supuerienrs.

Le cerveau est forme par les ganglious plus ou moins volumineux qui se développent sur l'extrinité antérieure de l: moelle; éest la présence de ces ganglions yui a détermim. le.

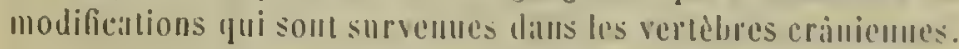
Il y a Irois divisions principales dans l'encéplaale : les leémisplères, les lubereules quadrijumeanx el le eervelel; celle division correspond at la premicre division yoi se manifeste dins le cours du développement: on voit en effet d'abord clee\% les embryous trois visienles qui, plus taru, se divisent et dont te nombre s’élève jusqu't cim.

Dins le cervedu on distingue en a vant denx ganglions, ordi mairentent les plus volumineux, el pui ne sout pas sans avoir des rapports arec l'imlelligence; ce sont les lúmispheres. leur commissure est designte sous le nom de corps callems (n) inésoluhe.

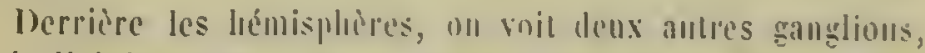
Iui, divisés souvent par une raimure transverse, donnent naissance à quatre éminemes : ece sont les lubereules quadrijumeans oul les lobes optipues. Ils portent all-dessus l'eux li glande pinciale.

Le eervelef forme la troisieme parlic el occupe loujours dia ligrne médiane. La cavilé de la moelle pénetre en avant au-des. sous du eervelet, el forme ce qu'on appelle le quatrieme ventri. rule; clle passe ensule en lessons des lubercules quadrijumeanx, cl donne naissance, dausles lémispheres, aux ventrienles lathraus el en dessous an Iroisieme ventricule. los lohes da ervelet ont yuelyuefois une forte commissure, que lon dessignte? 
sous le nom de pont de Varole, mas qu'on ne remeontre que dans les vertébrés supérieurs.

Outre la glande pinéale que l'on voil au-dessus des eorps quadrijumeaux, on trouve aussi clans les diverses classes de verlébrés la glande pituitaire ou l'hypopllyyse, qui est toujours logée à la mème place en dessous du cerveau et aequiert son plus grand développement dans la classe des poissons.

A la base du cerveau, naissent douze paires de nerfs crâniens qui doivent se rélluire à quatre paires, correspondant aux quatre vertèbres crìniennes. Nous avons vu plus haut qu'à charjue vertèbre corresponll une paire de nerfs.

I.e eerveal, comme la moelle épinière, est entouré constamment de trois enveloppes, sans compter l'élui osseux formé par les vertèbres; ces enveloppes sont: la dure-mère, l'arachmoïde el la pie-mère.

Les nerfs consistent dans des cordons blancs qui longent souvent les vaisseaux el se ramifient en filets de plus en plus minees pour se perdre dans les organes. On ne voit pas de lifférences entre les nerfs de sentiment et ceux de mouvement. On peut dire qu'il n'y a pas d'organe qui ne reçoive des nerfs, saufle cerveau et la moelle, et ici encore les vaisseaux conduisent quelques fines ramifications du grand sympathique.

Le grand sympathique consiste d'abord en autant de paires Je ganglions qu'il y a de vertèbres; des ganglions plus ou moins nombreux, qui président au mouvement du cœur, de l'estomac, les intestins, des vaisseaux, etc., apparaissent dans le voisinage le ces organes; ils sont unis au système nerveux de la vie animale par l'intermide des commissures et des nerfs spinaux el cérébraux.

Nous trouvons le type dı système nerveux des vertélırés dans le branchiostoma, ce poisson si remarquable sous tous les rapports; le centre nerveux céphalo-rachidien consiste en un cordon allongé qui représente la moelle épinière; il n'y a pas le ganglions propres pour rę̧résemler le cerveau, et les nerfs spinaux naissent comme les nerfs cràniens sur toute la longueur te la moelle par deux racines distinctes, une antérieure et une jostérieuro. 
Le système nerveux est uniquement formé de deux éléments anilominues, de fibres urvenses el de corpuscules ganglionnaires; ces derniers penvent souvent étre consillérés comme l'origine de cerlaines fils'es.

Une fibre nerveuse se réduit iın tolal à un cỵlintre axial, qui, suivant les circonstances, est enveloppé par une écorce simple. Dans les pétronyzous, celle couche manque; alle n'est donc pa: isolante.

Mammifères. - Ia monllo épinière (fig. 260) occupe prosquue

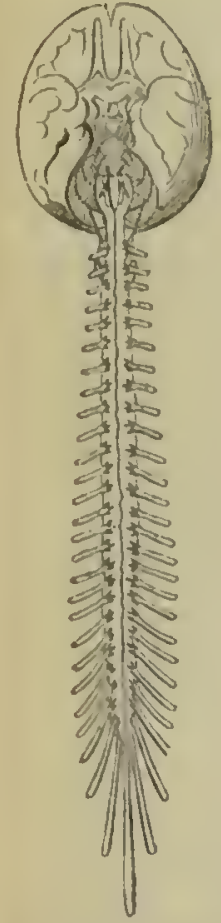

Hig. afiv. - simis nemestrina.

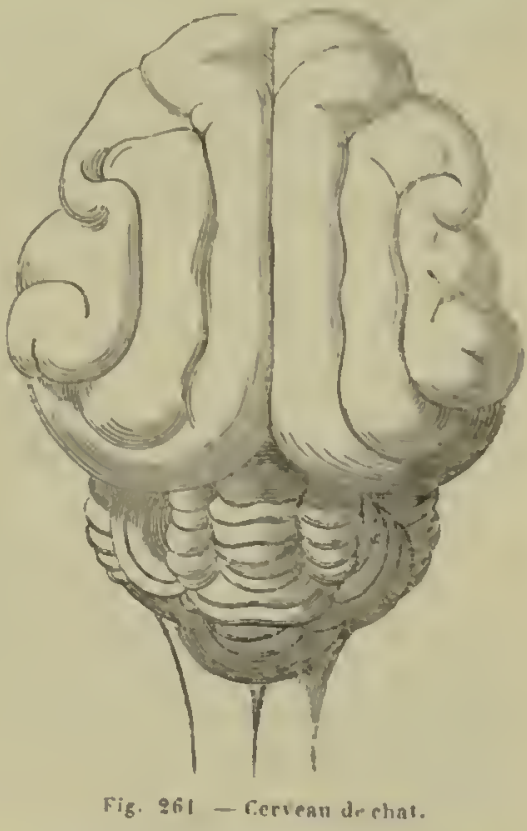

coute la longueur du canal verlébral. Fille est prolégée par les ares vertibraux el les autres enveloppes; tautôt elle se termine dans la re. giou sacréce tantòt dans la région lonuaire; lans un petit nombre de unm niferes, elle ne sétend pas plus loiı que le milieu de la région dorsale. C'esı dans léchidné el le hérisson quiolle est le plus courle. Le camal central de la moelle ue sulusiste que cher qurlques mimuifives. 
La moelle presente toujours un renflement dans les points où maissent les nerfs qui se rendent aux membres. Ce renflement est assez considérable elrez les kanguroos a eause dugrand développement des membres postérieurs.

Le ecrveau se distingue toujours par le grand diveloppement des hénisphlires (fig. 261); ils reeouvrent souvent les tubercules quadrijumeaux et même quelquefois te eervelet. Le corps calleux ou la commissure de eette première praire de ganglions est très-développé dans tous les manimifères monodelphes et n'exisle plus qu’á l'état rudimentaire dans les autres. Ce qui distingue surtout le cerveau des mammifẻres à l'extérieur, c'est le pont de Varole ou la prolubéranee annulaire, et à l'intérieur la voûte à trois piliers. Les tubercules quadrijumeaux sont toujours petits et divisés en quatre éminences. Ce sont tantôt les intéricurs et tantôt les postérieurs qui sont les plus volumi-

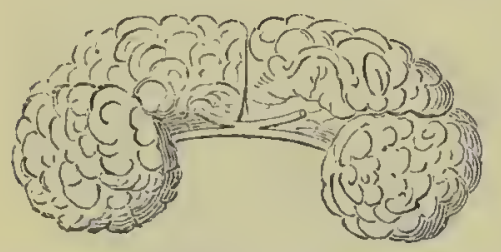

Eig. 26iz. - Cerveat de delphinus phnerena. neux. Le eervelet est grand el pourvu de lobes latéraux.

On voit chez beaucoup te mammifères des lobes au devant et en dessous des hiemisplières qui donnent uaissance aux nerfs olfactifs. Ce sont les lohes de ee nom. Ils sont creux et leur cavité communique directement avee tes ventricules latéraux. Derrière ta protubérance annulaire, on voil généralement sur la moelle allongée des saillies que l'on désigne sous le nom de corps irapézoüdles.

Les lıémisplıères des mammifères présenlent à leur surface des circonvolutions, qui, pour être trìs-variables d'un groupe à l'aulre, ne sont pas moins colıstantes daus une espece. Elles ue sont point l'effet du hasard; il y a un prineipe qui préside à leur arrangenent.

Les quadrumanes, les carmassiers, les céla-

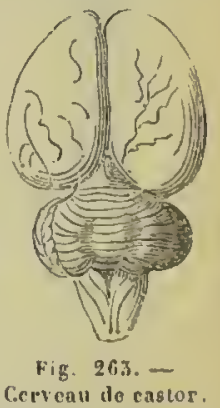
cés (fig. 262) et l'ćléphant ont de nombreuses cireonvolutions; les rongeurs (fig. 26.5) en onl peu; l'ouistili parmi les siuges 
llen a pas, ainsi que le microcebe parmi les limuriens.

D'apris .I. Camille Dareste, il y aurail dans les mammirères me relation constinte entre le degré de developpenent des rireonvolutions du eerveau et la taille de l'inimal, de maniere que taus tous les groupes, bomme les ouistilis parmi les quadrumanes, les espeees de predite linille auraient le cerveau le moins pontru de circonvolutions.

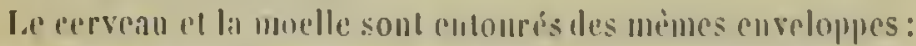
pir-mire; araclanoücle, dore-mere el etui ossenx. La faulx, forme par lit dure-mire, ainsi gue la tente du cervelet sont solusent assilices.

Le eervean remplil complétement la lovile crimienme.

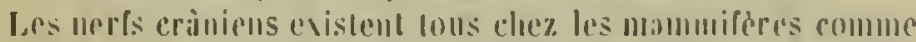
(hro. l'homme, al lexerplion de la premine paire, qui mangue

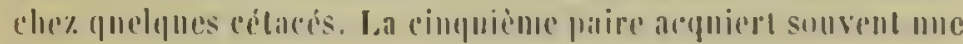
zarimle importinter.

Las autres uerfs de li moelle aussi lien que le granil ș̣mpallique ne présentent guere de modificalions importantes a signiler; ils sont disposis comme chez l'lomme. On pourrait faire remaripuer toulefois que, daus la plupart des manmiferes, le grand sympallique s'unit intimement daus la rigion eervieale avee le pneumo-gistrique, el que souvent des ganglions te la l'inion cervicale se enufoudeul les uns dans les autres, de manicre a ne plus trouver sept ganglions correspomlant aux verlibres cervicales. II n'y en a sullsenl que trois.

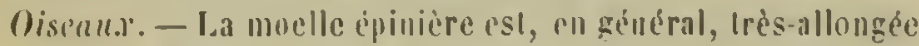
i) éause de la louggueur du enu; elle est fénéralement crense daus toute la longueur. I.es faisecaux supririeurs de la moelle s'écarteut, au milieu du reuflement forme' i) l'origine des ner's yni se rentent aux patles, el forment le simus rhomboidal, qui est earactéristique de la moelle des oisenux. Celle-ei remplit toute la longueur du caual spumal.

I.es hemisplieres du cerveau ont une forme triangulaire (lig. 26.4); on distingue a peine it linr surface les traces de rircouvolutions. Les parois de ces lıémisphères sont très-minces. ()॥ voil en avaut les lobes olfaclifs dont la caviles est anssi en (a)mnunication avee les ventricules latiraux. Les Inhercules 
quadrijumeaux occupent les flancs du cerveau; le cervelet se réduit à la portion moyenne ou au ver. Le pont de Varole man-
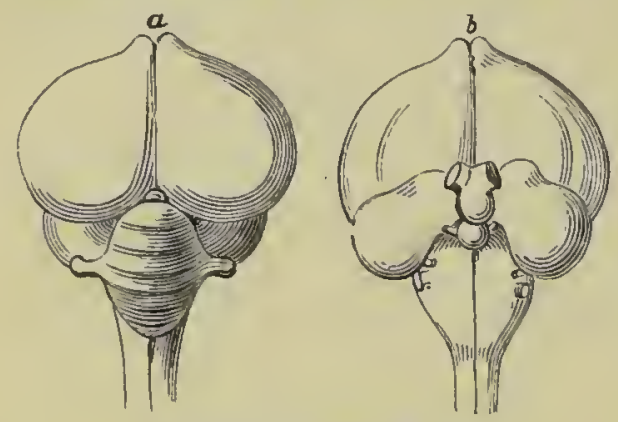

Fig. 264.-Cerveau du coq.

$a$. Vu en dessus. $-b$. Vu en dessous. que, ainsi que le corps calleux. Le cerveau remplit la cavité lu crâue; il rst entouré des mê. mes membranes ordinaires.

Les nerfs cràniens se relrouvent tous, el les mêmes nerfs se rendent aux miêmes urganes. Les nerfs optiques sont souvent très-gros et lamelleux. Le nerf lingual de la cinquième paire manque.

Les nerfs vertébraux ne présenteut rien de particulier.

Le grand sympathique est très-distinct; les ganglions de la région dorsale se voient facilement le long de la colonne vertébrale; mais Jans la région cervicale, ce nerf́ péuètre dans le canal vertél,ural formé par les apopliyses transverses des vertèbres.

Reptiles et batraciens. - La moelle épinière est très-longue, par exemple, cliez les serpents, el fort conrte dans les batraciens anoures, comme les grenouilles et les crapauds. Le ventricule persiste pendant toute la vie. La moelle surpasse le cerveau en volume. Il existe des renflements assez grands dans la moelle à l'origine des

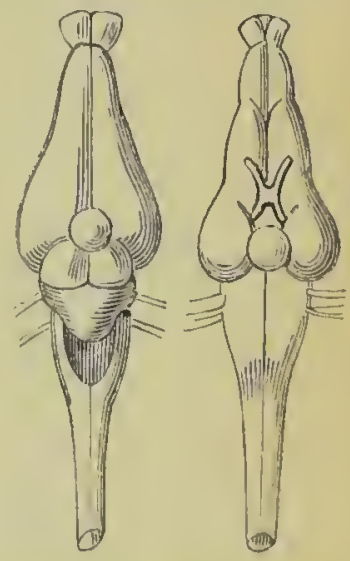

Fig. 265 - Tegtudo graca. nerfs des membres. Chez les oplidiens on a observé aussi des renflements à l'origine des autres nerfs.

Le cerveau (fig. 263) a quelque ressemblance avec celui des oiseaux, a vec cette différence toutefois que les corps quadriju- 
nreaux sont silués ì la même place que elré les mammifères, e'est-à-dire endessus entre les hémisphìres et le eervelet. Les latriciens anoures ont un eervelel rudimentaire; il ne eonsiste que daus un mince cordon place ell travers eomme un pout sur le quatrième ventrieule. Le eervelet est le plus développes dans les erocodiles.

Les nerfs eriniens, comme les neris vertébraux el le grand sjmpallityon, ne présentent rien de partienlier. Les ganglions le ce dernier sont plus délieats et sont édehés ehez quelques. mus dans le canal vertébrat. Il présente des amastomoses avec If Irijumean, l'ahduclenr, le facial, le glosso-pharyngien, le pneumogistrique el l'lypoglosse.

Sons divers rapports les batracieus se rapprochent plus des poissons, el les reptiles véritahles, des verléhrés supérieurs.

Poissons. - La moelle oceupe la longuenr du canal spinal, a l'execption de qquelques poissous, comme le poisson lune el la landroie, qui ont une moelle extremement eourtc el elsez lesquels la queue du cheval eommence à la hauleur des premieres vertèbres. Elle est géméralemrent ẹlindrique el ereusée par lè ventrieule; elle a quelquefois la forme d'un ruban. On distingue facilement les quatre eordons qui la composent. L.es nerfs naisseul tous par deux raeines. Flle se termine ehez les poissons usseux par un renflement ginglionnaire.

Le cerveau des poissons ne remplit pas la eavité de la boite crinienne. Entre la pie-mère el la dure-mère, il existe une substance gélalineuse, entenant dius ses mailles une grande quanlitéde graisse, qui remplit l'espace entre le cerveau et les parois du crine. Le cervenu des poissons se distingue eneore de celui les autres elasses, par les ganglions qui snot à peu près écialement diveloppés el qui se suivent contme un chapelet (fig. 260 (i). Le nombre de ees ganglions est très-variable.

Le cervelet se distingue toujours facilement paree qu'il est impar el qu'il termine posterieurement l'encéphale. Quelquefois il ressemble, eomme dans les batraeiens, à une simple commissure siluée eonme un frout au-dessus du quatrieme ventrieule. Le cervelet est au enntraire très-développé dans le llon (thynmus vulgaris). Au devaut du cervelet on voil sou- 
vent deux anties paires de ganglions que l'on peut regarder eomme les corps quadrijumeaux el les liémisphères, el au de-

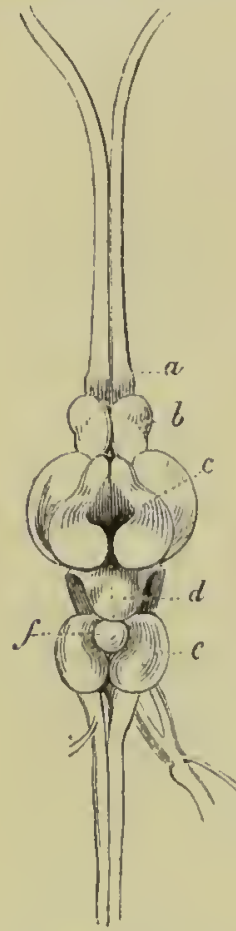

Fif. 266 - vericau de carper.

a. Lobes olfarlifs. b. Ilémisplùì's. c. Tubercules quadrijuneaux. - il. Cerdu pneumogastrique. - f. Ganglion impair. velel. - e. Rianglion

vant de ees deruiers on aperẹoil souvent les lubereules olfaetifs. Eı dessous o॥ trouve les lobes inférieurs dont il est difficile de reconnaitre la signification. L'lıypopliyse est en général très-développée dans celle classe.

Il n'est pas rare le trouver dans quelques poissons un lobe impair el médian, derrière les hémisplıères : c'est le lobe du troisième ventricule.

II y a souvent derrière le cervelet des renflements que l'on désigne sous le nom de lobes postérieurs; ce sonl des ganglions qui existent à l'origine de la rileine pos. lérieure des premiers nerís spinaux ou dı pneumogastrique, quelquefois aussi i l'origine du trijumeau.

Le cerveau des poissons est tris-peu volumineux relativement à la moelle épinière et aux nerfs. Souvent le cerveau uc semble en être qu'une dependance.

Ce sont les poissons plagiostomes qui ont proportionellement le eerveiu te plus volumineux parmi les poissous.

Les merfs olfictifs sont tris-volumineux; ils ont un renflement a leur latse et quelquefois deux, ou bien ils porlent te renflement au bout ì l'entrée des fossies nasales.

Les ner's optipues soul aussi tris-voInmineux; souvent ils s'entre-croiscml complétement el passent l'uu all Iravers de l'autre (fig. 267) ou bien l'un au-dessus , le l'autre sans fecliange de filme's (fig. 2(i8). Ils ue se eroisent pils chez le bolellostomi. Ces nerls eonsistent che\% quelpun's poissous c'n une membrane plisscie. 
Les merfs des muscles de l'oeil manquent rarement: ils se rendemt anx menes museles pue dans les elasses prededentes.

laes nerfs trijumean el puenmo-gastripme sont llune grosseur remarquable; le premier, untre les branchu's principlales, fouruit encore un lroue pui passe a travers la voute du erime el s'élend jusquadux dernières vertebres caudiales. Ide seenud souscul phas volumincux eneore que le prócédent, ontre: les urers qui se romlent a l'appareil branchial, a lestomac, an erenr el a la ressio natatoire,

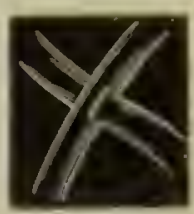

1ig. \pm 69 fournit une branche importanle qui se loge dans le camal lallio ral en dessous de la ligne ot qui s'íteme dalls loulde la lon-

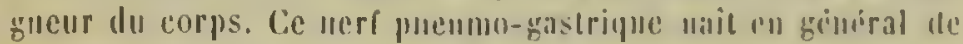

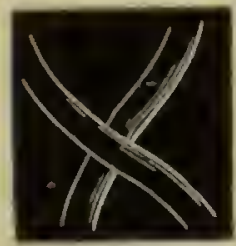

Fig zlis. deux raciues distinctes dee les poissons usenr.

Les nerfs aconstipues somt ordimairement tressdereloppes, tindis que le facial manqque.

Un nerf facial nia ite olserve que cluez lis

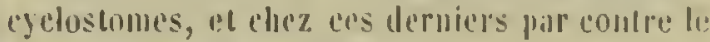
glosso-pluar!ngien mintume.

Les glosso-plaryngiens fournissent une branclie assez forle qui se remd alu premier are bran.

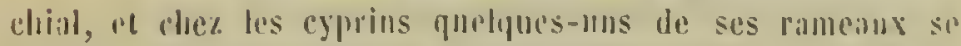
perdent daus l'urgane que lon considire comme le sirgio du sems du gavit.

Le's nerfs lypoglosses semblent mampuer dans les poissons, tandis que l'ateessoire de Willis criste encore at s'muit par des rimous avee le puemo-gisirique.

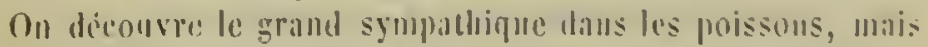
lis merfs et les ganglions sont phos temires et partant phos dif-

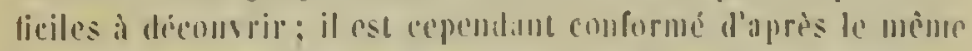

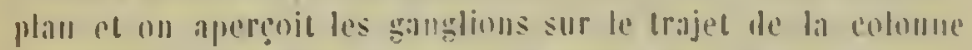
vertebrale. Cost dims les poissons ossem que res uerfs sont le phus divolopues; ils le sout beancoup moins dans les pliginstones el on doute mène de leur existente dans les eyelostomes. 


\section{ANIMAUX SANS VERTĖBRES.}

Il est assez remarquable que le systène nerveux des animaux sans vertèbres se rapporte à un seul ct même type légèrement modifié, et qui s'éloigne considérablement du premier embrancliement; si on admet encore un cerveau dans ces animallx, c'est plutôt par les fonctions qu'il remplit que par son analogie anatomique.

Le système nerveux se compose ici des mêmes éléments : ganglious, commissures et nerfs; ce sont les mèmes parties que l'on observe clıez les vertébrés. Les ganglions sont des corps arrondis contenant des corpuscules particuliers d'où partent des tilets nerveux; les commissures sont des neris qui vont d'un gangliou à l'autre et qui les mettent en relation; les nerf́s sont les cordons qui partent des ganglions et qui y aboutissent, et qui se perdent dans les organes oubien y prennent leur origine.

Les modifications du système nerveux daus les animaux sans vertèbres portent sur le nombre de ganglions, leur séparatiou ou leur coalescence, ct la place qu'ils occupent au milieu des autres organes.

Il n'est pas sans importance de faire remarquer que si la plupart des animanx sans vertehres ont encore des neris et des ganglions de la vie organique (grand sympathique), ces nerfs et ces ganglions sont placés chez les articulés au-dessus dı tube digestif el cliez les mollusques au-dessous.

Ce que nous venons de dire est sans doute applicable encore aux vertébrés; chez eux aussi il n'y a que ganglions, commissures et neris, mais ces diverses parties ne sont pas nussi bieı connues dans cet embranchement. Les dernièrcs reclıerches microscopiques fout entrevoir que d'ici à peu de temps nos colnaissances sur cet appareil pourront notablement s'accroitre.

\section{ARTICULES OU ÉPICOTYLÉdONES.}

'Tous les animaux de cet embranchement ont un collier uer-veux autour de l'œsophage (fig. 270) et un chapelet de ganglions 
fartant de la partic inférienre du collier pour s'étendre dans toute la longueur du corps (fig. 271).

Ce collier est formé de deux ganglious situés an-dessus de l'uesophage (les ginglious sus-resophagiens), el de deux ganglions sous-oesophagiens. Ces quatre gauglions sout unis entre cux par des commissures qui forment un cercle complet.

Ces ganglions sus-cesophragiens que l'ou a comparés an cerveau, dontuent maissalle aux nerls

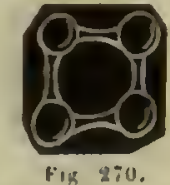

Collier aerveun. qui se rendent aux yeux ou nerfs opliques, aux antennes et à

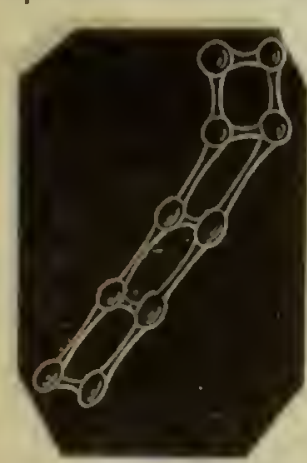

Fig. 271. - Collier ner veux asec clishe gan= Rliunnaire.

d'autres organes de la lète; les granglions sous-asophagiens douneml surloul maissance aux uerfs qui se remlent aux pièces de ta bouche.

Des deux gauglious sous-usophagieus partent dens commissures qui se remtent inx preniers ganglions du llorax; elles sont phacées daus laxe du corps landis queles prenières commissures sout trausverses; nous arons ainsi des commissures fongitudinales el des conmissures Irausversales.

Comme le corps de lous ces animaux est divisé ell anneaux, on doit compter que chaque anneau posside sa paire de ganglions, el, si les anneaux sout semblables entre eux, les ganglious le soul aussi.

Chaque auneau posside eu effet sa paire de ganglions; ils sout lous unis en avant el en arriere par une commissure longifudinale el une comnissure transierse. L'ensemble de ces ganglions furme la chaine ou le chapelet double silué en dessous Itu caual digestil el que l'ou a cherché en vain à comparer à li moetle épinière des vertébrés.

Quand les anueaux sont bien distincts les uns des autres, les yanglious le sout aussi; c'est ce que l'ou roit ell général dans les arlieulis qui ont lo corps allongé, comme les larves (fig. 272). ()uand au contraire les anneaux sout peu distincts, qu'ils se confondent entre eux et que te corps se raccourcit, les ganglions 
sel'approchent, les commissures longitudinales disparaissent, ct les diverses paires de nerfs, au lieu de former une elaine, donnent maissance à une masse nerveuse centrale qui affecte

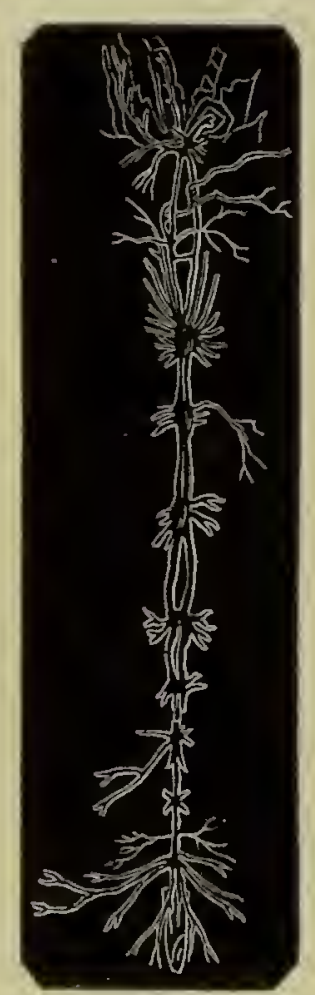

Fig. 272.--Systeme nrrveux de palinurus ỵuadricornis. sonvent, par les ner's qui en partent, la forme d'une étoile (fig. 275).

De même que les comnissures longitudinales disparaissent el que les gan-

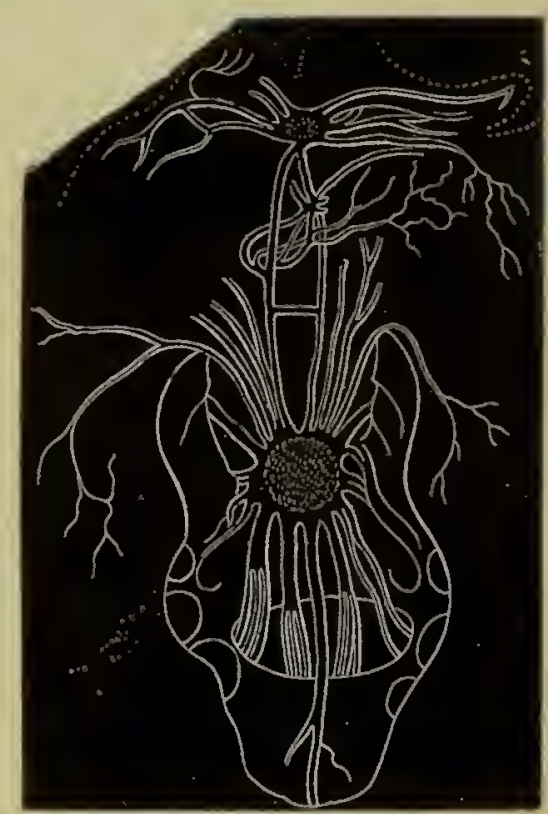

rig. 273 . - Systime nerveus de maïi squinado.

glions se rapproclent d'avant en arricre, les gauglions lattémax ou pairs se ripprochent par la disparition des commissures transverses, et au lieu d'une claine double on ne voit plus "ju'uir chapelet simple.

Dans tous ces animanx la forme du corps tradnit parfilitement la disposition du systime nerveux.

On trouve aussi des ganglions et des nerfs yui agissent independamment de la volonté, qui se rendent surtout à la bonche. à l'asophlare el à l'estomac, el qui remplissent les mènes fonc- 
tions que le graml sympallique des animanx supérieurs; on los apjuclle stomulo-gustriques. Ils maissent par des commissures des ganglious sus-aesophatiens on du cerveau.

Des reclserches intellixentes out fail commatre que la claaine ganglionnaire est forméc de deux falsceanx juxtaposés :

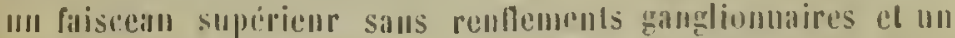
faliscean infériema i ganglions; le supérieur correspond, à ce yu’il parail, au filisecan anlírieur de la mordle: ol meristlerail an montement landis que l'anlle correspoudait an sen-

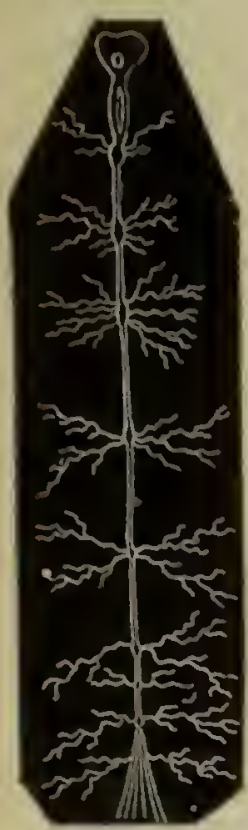

Fig.

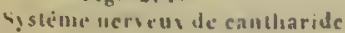

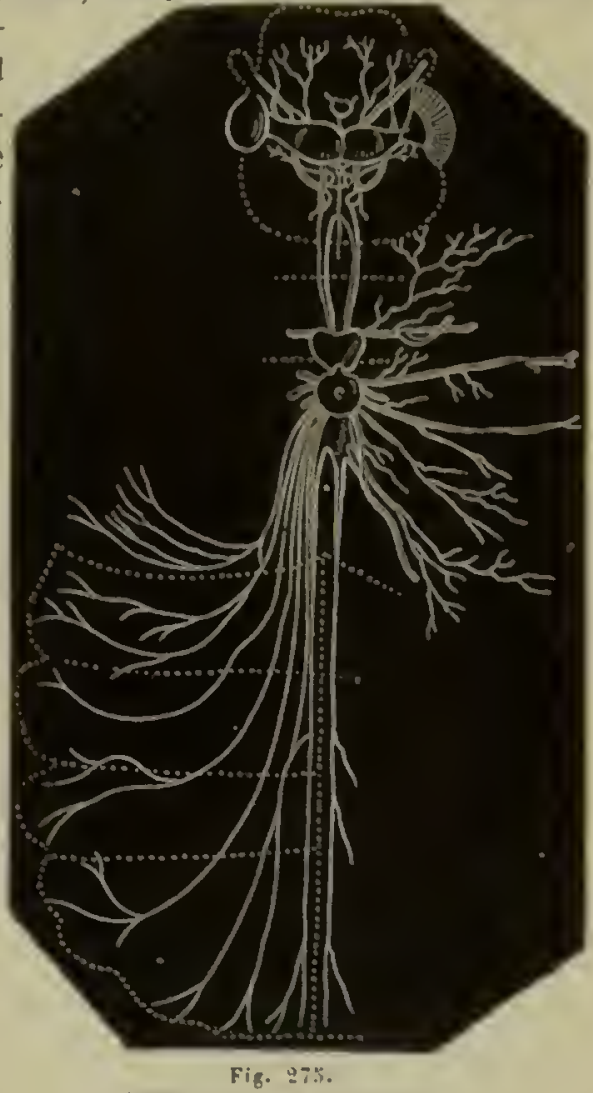

Sy stene uirveus de hannelon.

limeut. Les merfs maissent géméralement des gruglions, mais

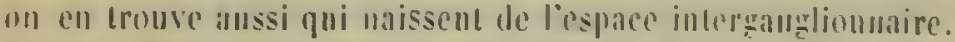

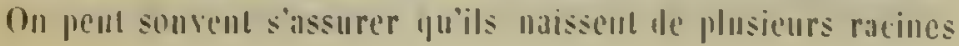


transverses et longitudinales, ee qui rend eompte de la simultanéité d'action des divers segments du corps.

Insectes. - Les irsectes orrt souvent les deux ganglions sus-asophilgiens réunis en unc seule masse qui forme le cerveau. La elıaiuc ganglionnaire est toujours distincte, et, à l'élat adulte, les trois paires le gauglions, correspondant aux trois anneaux tlırachiqnes, sont toujours les plus développées et les muius régulières (fig. 274 el 27j).

Les ganglions cervieaux dounent naissance aux nerfs optiques,

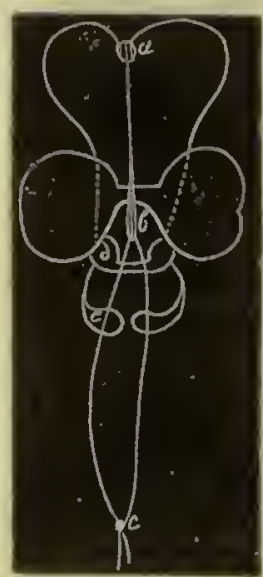

Fis. $276 .-$ Nerfs splanchiniques.

a.b.c. Ganglions médians. -

d. e. Ganglions pairs. pour les yeux a facelles comme pour les yeux simples, et aux nerfs des antenmes. Les pièces de la bouche el les palpes recoivent les nerfs des ganglions sous-oesophagiens.

Le système stomato-gastrique consiste souveul en un cordon nerveux impair porlant trois ganglious el co deux autres paires de ganglions qui tirent les uns et les autres leur orivine des ganglions sus-cesophagiens (fig. 276).

Les ganglions viscéraux des insectes sont unis au systeme nerveux animal par des commissures qui se rendent toujours aux ganglions sus-oesoplagiens.

Arachnides. - Ces articulés montrent en gértéral un collier nerveux, formé supérieurement par uır ganglion cérébral; inférieurement, les ganglions du collier sont réunis en une seule masse avee ceux de la chaine (fig. 277). Les seorpions, qui ont un corps allonge, montrent la chaine gangliomnaire étendue dans toute la longueur du thorax et de l'abdomen et les eommissures longiludinales très-développées.

Mlyriapodes. - Les anneanx étant très-nombreux el le corps excessivement long, le système nerveux est composé d'un grand nombre de ganglions et la elıaine est très-développée en longueur. On a signalé dans ces articulés quatre sortes de racines aux ıerfs : une longitudinale qui s'ètend jusqu'au collier, 
mue autre qui suil la mêtue marelie, mais seulement jusq̨u’an ganglinn priceslent, une troisiène transverse qui se reml dans Ir ganglion voisin, et me quatricme qui tire directement sou origine du gangrion d'on le nerl sort. Nons n'oserions dire que crei ne repose pas plutôt sur des vones théoriques. Ces articulés. possident comme les precéWemls mu systeme stomatogastrique double, l'nu pair el l'intre impair.

Cirustucess. - Ide systime nervenx des cristices dós.apodes est tres-remarquable; le corps des uns riant trisallongé at celui des antres très-court (tig. 272 et $27 \bar{n})$, nous voyous clairement la demoustration le ex yne uous venons de dire phis

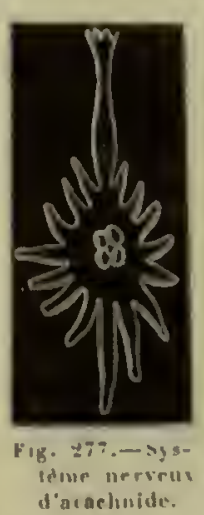

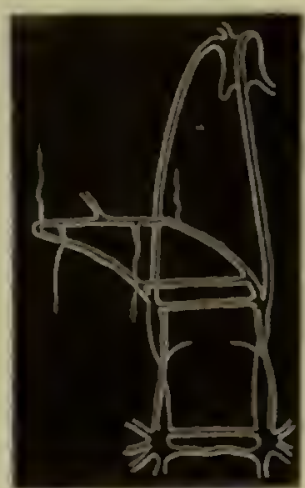

Fig. $\geq 78 .-$ sistinue net inut des apus. lıant.

liécevisse possède me longue chaine gangliommaire plemdue dias tomte la longuenr du thorax el de labilomen; les cralus an contraire ont tons les ginglions rémis en dessons du coiphiaIotloorax sons la forme diune etoile. Dans les uns el les autres les ganghions sols-asophagiens sont élojgues du cervean, de manière que les commissme's laterales du collier nervonx smlul tris-longues.

Dius les crustacés iuférieurs, les deux corlons longiludiun trudent ì s'éloiguer l'un de l'antre, les ganglions diminuent ì mesure que les anneanx seffacent on mêne disparissent, el la chane ganglionnaire sóloigne du ! pe des arlienlis.

Les crmstaces, surtont les supericurs, possident des ganglions ('l des nerfs stomalogastriques (lig̣. 27s). Il mait en géméral, des (m)m missures lengiludiniles du collier, un cordon qui se rend aux parois de lestomate où il aboutil à mu gamglion d'où partent divers lilets nervenx pun les piorois alu camal intestiual.

Parmi les crustacés suceurs, on en voil pui ont la chaine ginglionnaire rémue en avant, comne che? les arliculis, el 
écartée en arrière, (diclelestium) (fig. 279), comme Jaus beaucoup de verset d'autres dont les deux moitiés sont complétement

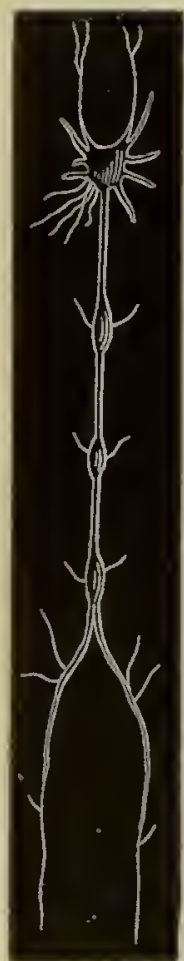

Fig. 279. - Systeme nerveux de dichelestium sturionis.

separées dans toute la longueur (achtères et Penella). Cetle dernière disposition se montre aussi dans les pentastomes, où l'on voil de

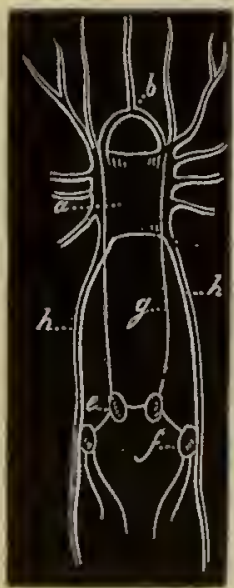

Fig. 280. - système nerveux complet des linguatules.

a. Ganglions sousasoph. - b. Col. lier. - c. Chaîne ganglionnaire. c. $f \cdot g$. Ganglions et nerfs sympathiques. plus les ganglions complétement disparaître, et la chaine présenter l'aspect d'un cordon (fig. 280). On distingue encore, dans ces articulés si simples qu'on les a confondus jusque dans ces deruiers temps avec les helmintles, des ganglions stomato-gastriques (fig. 280 e.f).

\section{ALLOCOTYLEDONES}

Mollusques. - Le système nerveux des mollusques diffère de celui des articulés par l'absence de chaîue ganglionnaire; tout le systeme est réduit à un collier d'où partent les nerfs qui se rendent ì la périphérie du corps.

Si l'on considère les ganglious sus-œsophagiens comme représentant le cerveau, plusieurs imollusques sont privés de cerveau, puisqu'il ne se trouve souvent au collier que les ganglions inférieurs. C'est ce que l'on voit dı reste déjà chez quelques articulés.

On aperçoit généralement un système stomato-gastrique chez ceux qui ont encore une tête distincte.

Céphalopodes. - Quoique ce système ne consiste que daıs un collier ordinaire d'où partent tous les nerfs du corps, il est cependant assez compliqué, ainsi que uous allons le voir (fig. 28I). 
Le collier nervour est luge dims me boite cartilaginemse qui

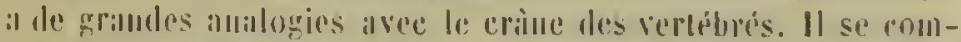

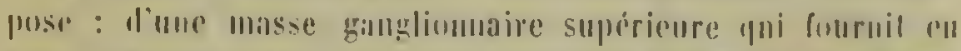
aviul loss uerfs olfactifs al, sur le coili, los unerls uplingues el les commissures du

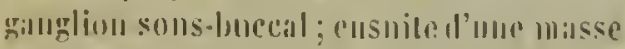

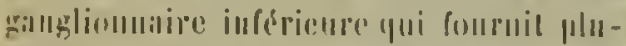

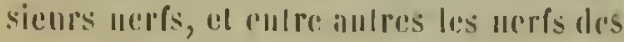
liras of le nerf acoustipur.

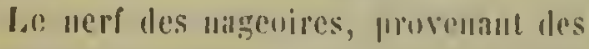

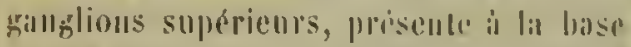
de cos migeoires un gan:lim volumine

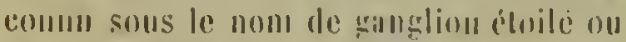
frallylion de lit pille d'uire.

Ide: systrme stomato-gilstrique est tresleveloppe; il est formé par mon on pal denx gillghlions, quisont siluris all-dessons de lil cavile lutecile, el palr un autre gaul-

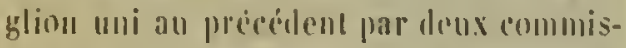
sures, el dui est placé sur los parrois do lestomac. Cors gillylions sont asser volmominens. Tont lo systime nervens se fail remarquer par une cendince parti-

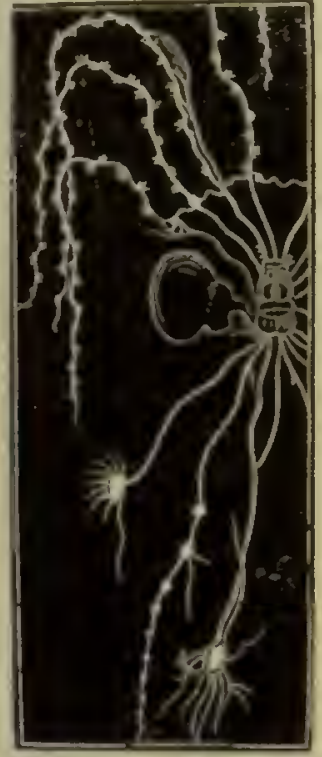

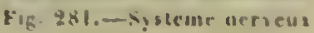

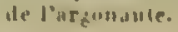
culiere i former des rentements gunglionuaires.

Outro les nerfs splancluniques dout nous venous de parler, on voit encore dans ees mollusques des ner's qui se rendent anx loranchiors et an corur el qui the soul pas sans antologie avec le merf puemmo-gistrifue des animanx superrieurs.

Claque bras comprend un nerf gamglionnaire anquel est accole mu nerf ortinaire provenant directement dn boril anté-

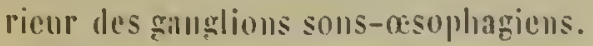

Gusterropodes. - Le collier est lonjours complet (fig. 282); generalement on voil den ganghlions au-dessus de l'msopluage qui foumissent le merf optique el le nerf des tentacules inférienrs, el plusicurs gandions sons-nsoplagiens rémis les uns iny antres el formant quelquefois une masse assez volumineuse. l.esmerfs aeoustiques missem de ces demiers, ainsi queles merfs 
(рui se rendent au pied; ces derniers sont quelquefois voluminows el en grand nombre.

On trouve encore cle\% plusicurs gasléropoles des ganglions

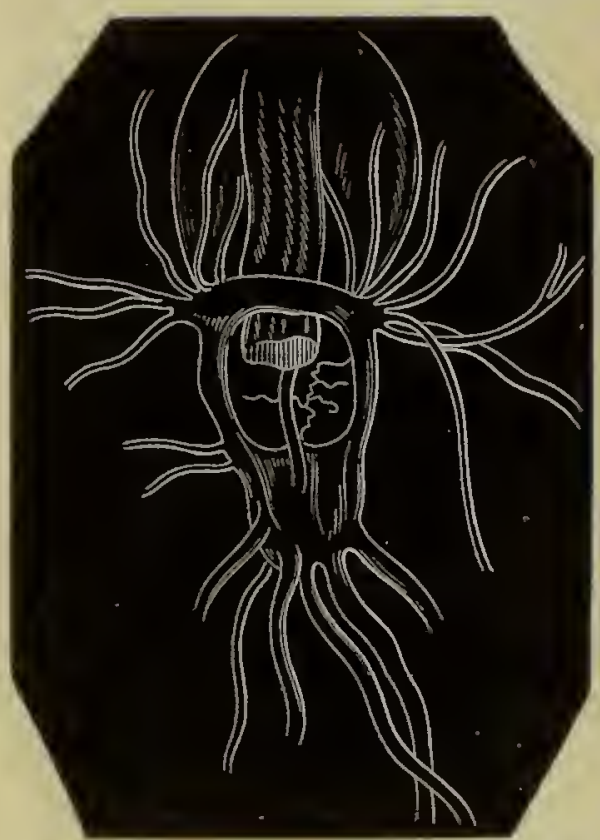

Pig. 282. - Systemse merveux de f'fólix pomatia. ordinairemeut impairs et qui se rapportent ì l'apprareil sexuel ; oll ell voil ilaus les aplysiesel les hullis (fig. 285).

Clez plusieurs de ces mollusques, et particulièrement ceux connus sous le nom de pléroprodes, les ganglions cervicaux manquent, ct toute la partie latérale el sipérieure du collicr est formée par une eomınissure.

O॥ a observé clıez presiue tous ces mollusques un systimestomato-gastrique consistant le plus sonvent daus deux gallylious Irès-petits, situis en dessous de la cavité de la bonclie el collés i ses parois. Clıez les ptéropodes, ces ganglions soul fondus cu un seul, qui est silué sur la ligne médiane.

Acéphales. - Ces mollusques out cncore un collier asoplagien (fig. 284); mais les ganglious sous-œsoplatgieus s'ceartcut fortement des autres et soul logés daus l'inlérieur du pied; on les a nommés ganglions pédieux, parce qu'on ne s'est pas aperçu d'ahord qu'ils forment le dessous du collser. Les gauslions cervicaux ou supérieurs sont sitnes ì l'angle de la bouche. Ils fournissent, outre la conmissure ordinaire, une autre commissure qni se rend an gangrlion situe au-dessous du muscle anducteur postérieur of que nous considerons comme une. seconde partie de la nasse sous-asophagienne. II u'existe pas 
de systeme stomalogastrigue propre; mais on a consideré

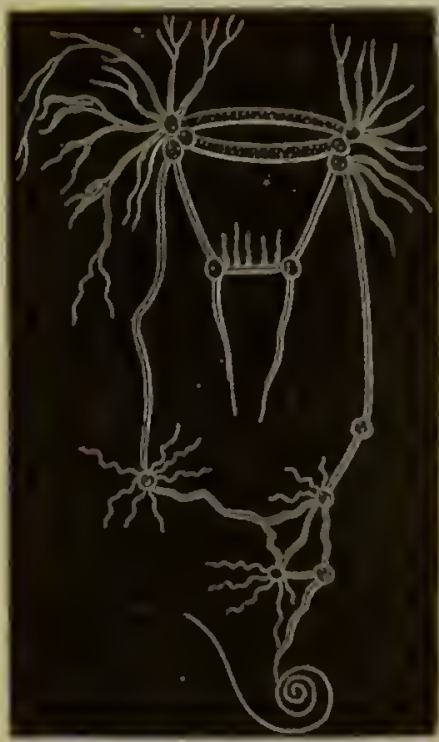

Fig. y8s - systemenerveux de hulla lignaria.

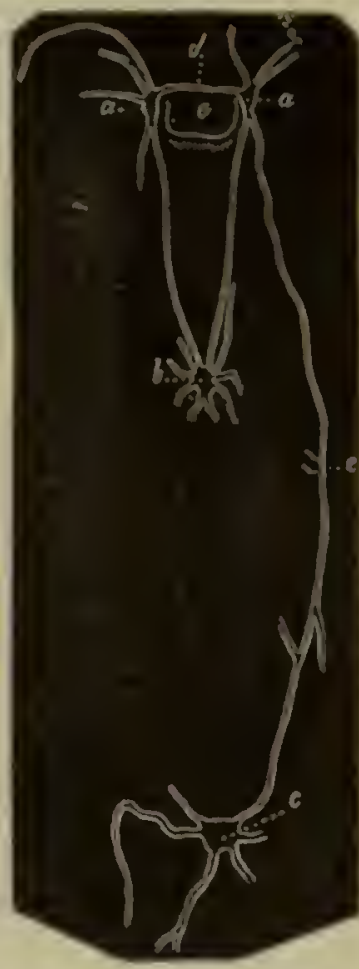

Fik. Zys - Sistame nersent

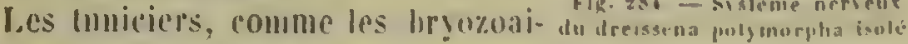
res, ont ee systime à l'ital mulimen- a.tapremièrepairedeganlanre. On distingue mo on deme comps

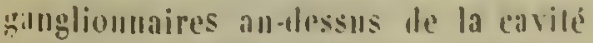
lumeale (lig. 28ii), un collier rudimenhireon ineomplet el quelpues lilets mui partent de la masse (eentrale. Las galuflions. - b. La seconde parre (ganglions sonsinuphagrens). - c. l.al truisième paire.--tl. Cism missure sus-assphagien. ne. - e. Bouche. glioms at les nerfs sont extranolimairement mons el deficals.

Vers. - Ce's animanx muns présentent des difierences tres. wambes sons le rapport de leur systime nerveux, et e'est ell accorliulume imporlance exagéré à eel appareil quel'on a si peu compris jusqü ì présut lenrs afimités. Quelques-uns, en effel, out une chaine zangliomnaire sons-intestinale, comme les arti- 
culés, tandis que d'autres ont cet applareil extrèmement sinılle, ct d'autres encore scmblent en ètre entièrement privés.

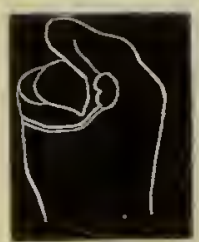

Fig. 285. - Ganglion nerveux d'aleyonella fun. gusa.

Les annélides, sans en excepter les hirudinées (figr. 286), sont pourvis d'un collier osofluagien complet, à deux ganglions sous-aesophiagriens, souvent assez voluminenx, quelquefois réunis par coalescence, et d'une claine de ganglions qui ne correspond pas toujours au nombre apparent d'anneaux du corps (fig. 286); souvent anssi les deux ganglions pairs sont confondus en un seul comme les ganglions cervicaus, de manicre qu'on ne trouve qu'un clapelet simple ou un collier saus renflements.

Le plus important a noter, e'est le passige de la chainc gangliomaire, fol'méc par deux érdons l'éunis, aux deux cortons complétement sépalrés el sans gannglious.

On tronve elicz quelques anmélides des nerfs el des ganylions qui haissent des ganglions eervieaux, se répandent sur' l'aesophlige en dessus el ell dessous, el qui reprèsentent évidemment le systeme stomialo-galstrique.

Dans l'albione et d'aulres hirulinees, les corpuseules des ganglions sont tris-volumineux et places avee symétrie (fig . 287).

Les némitoülles out souvent sur le coted du corps decux eordons longitudimanx sans lentlement gangliommare, et mis ell avanl par une eommissure sans eollier asopluagien.

Plusieurs frémtatodes oul un sys-

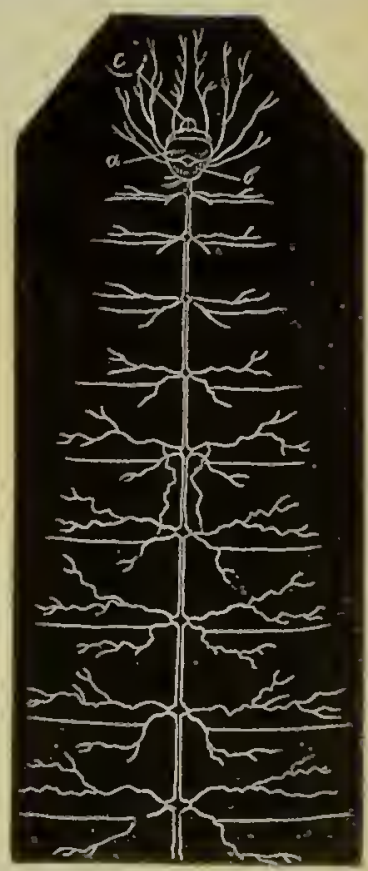

Fig. 2860 - Systeme neweux ate sangsue (di" (jualrefingos).

a. Cervean. - b. Ganglions sons-o'sophatgiens. c. Gianglionsfiontanx. tème nervenx un pen moins développé que les pricidents; il 


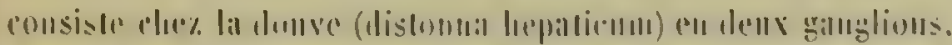

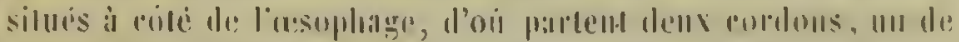

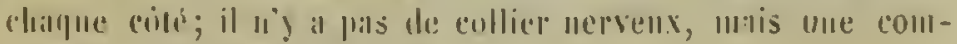

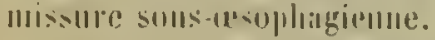

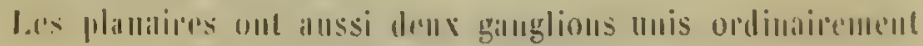
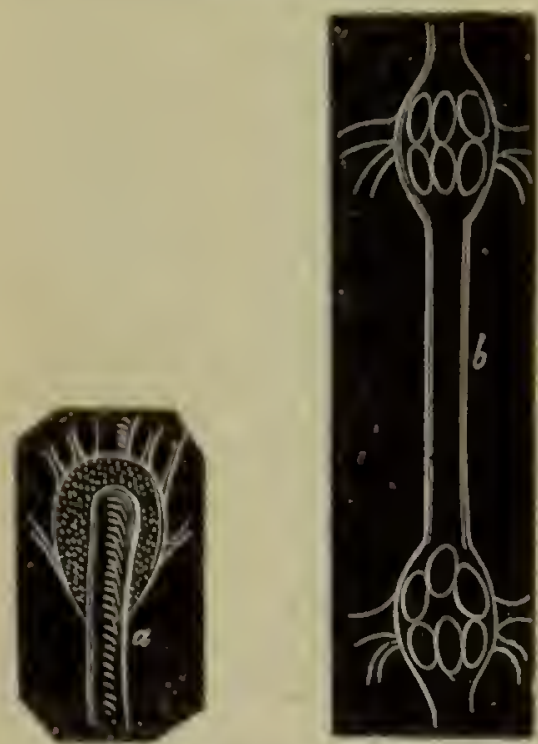

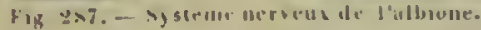

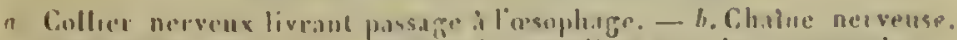

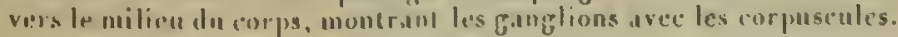

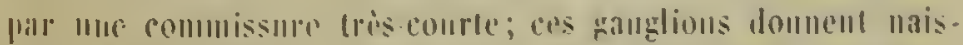

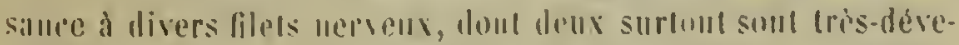
loppes ol se reudeut sur le coté du corps d'avalut ell arriere. (fiv. 288).

On a nuserve anssi des ganglions emtre les rentonses des: solex do costojdes. Dalls les epstouldes il liblill de proglollis, il

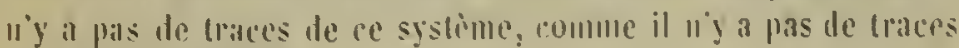

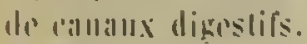

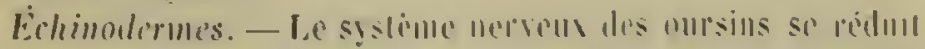

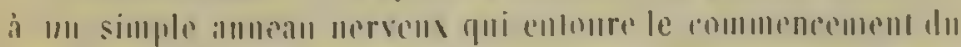

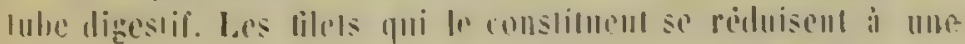


témuité extrême. A claque rayon correspondent deux filets qui portent un rentlement ganglionnaire à leur origine (fig. 289).

Polypes. - On voit des ganglions isolés chez quelques-uns:

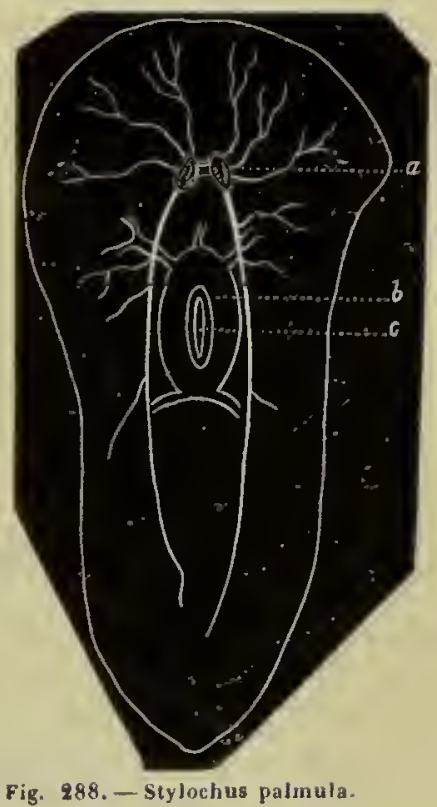

a. Ganglions. - b. Estomac. - c. Bouche.

de ees animaux el des filets nerveux, mais point de collier asophagien.

Dans le béroë pileus, il n'existe pas de collier nerveux, quoi

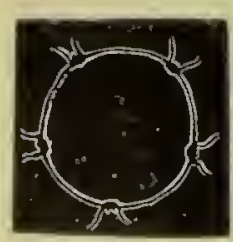

Fig. 289 . qu'on en ail dit, mais bien un ganglion mullilobé, à côté de l'otolithe el qui donne quelques filets nerveux aux côtes.

Chez les polypes proprement dits, toute trace de ganglions ou de cordon nerveux a disparu.

Ces animaux et ceux placés encore plus las dans l'échelle manifeslent évidemment l'exisrence de sensations, mais on ne recomnail plus une substance orrveuse isoléc. 


\section{APPAREIIS IOES SIENS.}

Les organes des sens se divisent en deux caligories : la pre' miere comprend ceux qui sont impressionnés à distance, "uui ne demamdent pas le contact immédiat pour percevoir ; ce sout l'wil et l'oreille; la seconde catergorie renferme ceux qui ont besoin du coutact imméliat pour itre impressionnęs; ce sout les fosses masales pour l'ulfaclion, la langue pour le goût el la surface de la pean ou quelquefois les soies pour le loncher.

L.a vue el l'oüe ont cela de commun qu'elles s'exerecul l"une "tlautre à la suite d'ondulations produites par les corps a une distance, plus ou moins grante; les truis anures sens ont ceci le remarquable que celui de lolfartion doit juger les qualites les corps gazeux; le sens du goù, les qualités des corps liquides; el le towelier, les corps solides. Il y il aimsi un organe de sens particulier correspondant i chacun des états dans lesquels peuvent se trouver les corps, el deux autres organes qui sonl impressiounés par les corps ell mourement.

Tout le moude sait qüil existe une grande difference entru l'eil et l'oreille, et cependant chez les animanx simples leur ressemblance est si grande, que nous avons eru devoir admellre la possibilité d'un seul el mime or zane pourant servir à la fois l'meil el d'oreille. 
Ces denx organes, si parfaits dans les animinx supericurs, sonl formes de parties essentielles et de partics actessoires on de perfectionnement, el les différenees qui surgissent provicunent surtout des modifications que subissent ees dernieres.

Dins l'ocil eomme dans l'oreille eomplètement developpee, il existe une capsule renlermant un liquide au milieu duqnel mage un eorps solide, et autour de ee corps solide s'épanouit un filet nerveux. Celte eapsule est nommée sclerotique dans l'ocil, veslibule membrameux dans l'ureille; le corps solide de l'oeil est le cristallin, relui de l'oreille est l'ololitle. Le nelf est nommé optique el forme, par sou épanonissement, la rétiue; il es nomme acoustigue dans l'oreille. On pourrait pousser celle allalagie eneore beacoup filus loin.

Dans l'weil, une sorte de convercle transparent vient se placer

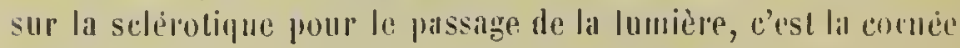
transparente; mu repli de ha pean se forme an devant de l'oeil el premal le unm de panpières. Une glande apparait pour sécréler un liquile qui doil lubritier la surface de la rormée el lui conscrver lonte sa transparence; e'est la glande lacrymale, et un conduit du même nom transporte dans les fosses masiles le liquide qui a servi à la lubréfaetion. Des museles partieuliers se forminl pour mouvoir le globe daus inus les sens et unc cavile osseuse existe pour l'abriter; enfin daus l'interieur, cel'laines parties se moditient pour produire une phos grande metteté de l'image qui vient frapper la rétine, ot un diapluragume" apparail avec une ouverture mobile pour ne laisser pénćtrep dins le globe de l'oeil que certains rilyons et une certiline qualltité de lumière. Ce diaphluagme, e'est l'iris avee sa pupille.

Il faut un pareil organe, bien eonditionné quant à ses partics essentielles, pour reecroir une image, pour distinguer les objets; mais il ne faut pis nécessairement un wil pour voir. Pour distingurer le jour de lil nuil, eet apjareil nptique u'est pas iudispensible; il suffit de quelques platues de pigment, et même quelques animanx sout seusibles a la lumiore, sallis pigment, palr toule la surface de la pean; c'est dans tee sens que l'on peut dire yo'il ne faut pas d'yeux pour voir.

I.'animal vivanl dims l'all on dims l'eau doil neessimrement 
montrer daus la eomposition de l'ail des modifieations en rapport avec le mitieu dans lequel it est appele i vivre; si la cornéce transparente devient tris.bombe dans les niseanx,

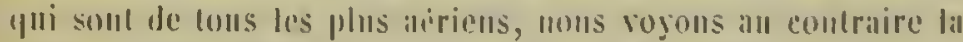

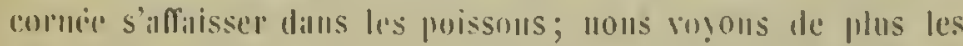

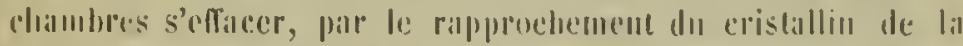
enruée, et par la spluéricité que ce cristallin aequiert. Et ces modilicalions s'blemelent elleore anx partie's alcessoires, par

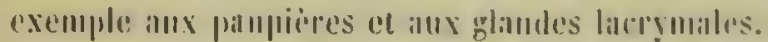

Si dims les animans supipienrs it u'y a jamais plus de deus

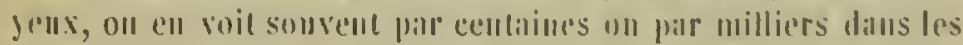

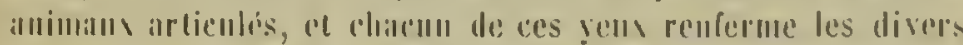

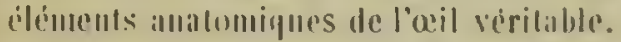

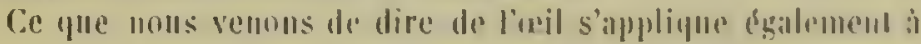
l'oreille. Dans les vertihres supérienrs, ì l'ureille interme yui compremel les organe's essentiels, liement so joindre nue oreille

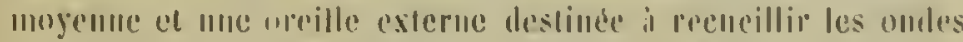
somores. Aux paldies essemlielles s'ajontent des orgalles de per-

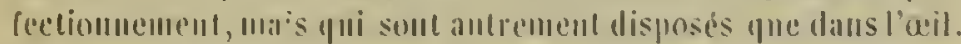
Ainsi a ce vestihnte viennent se joindre dems on trois eantux,

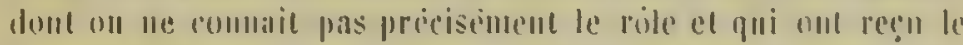

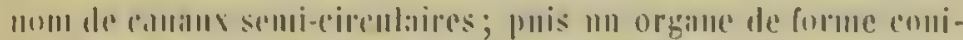

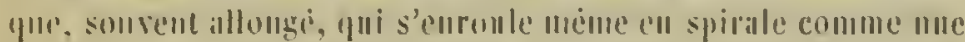

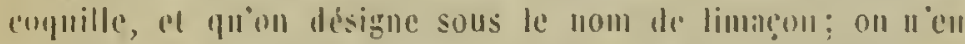

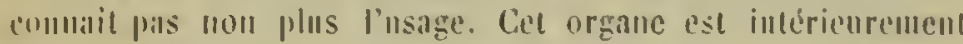

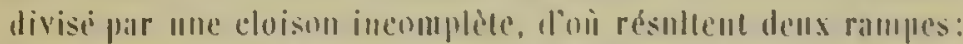

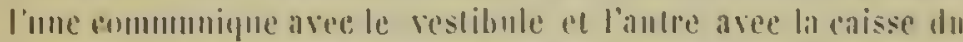
lympan. Le verstibute al un conserele comme la conjonedive de

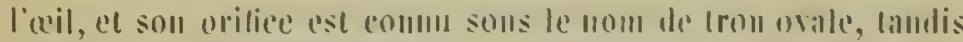
fue l'antre, communiquant avee le limilen, est le trou rond. Cet ensemble est disigne sous te mont doreitte interne.

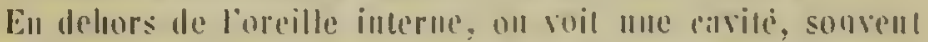

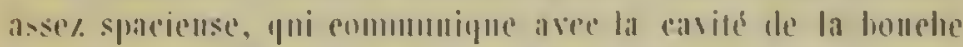

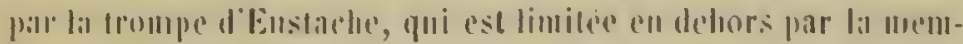

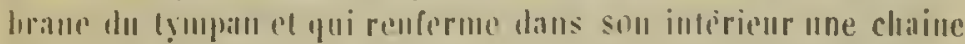

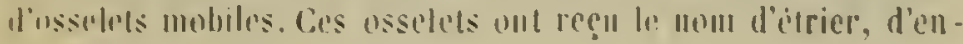

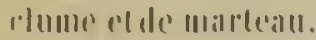


L'orerlle externe est formée par la conque auditive ou le pavillon de l'oreille et eomprend tout ce yue l'on voit de l'oreille a l'extérieur. C'est une partie de perfectionnement comme la précédente, ear on peut très-bien entendre en ne possédallt, comme les poissons, que l'oreille interme.

Les trois autres oryanes de sens ne montrent point de modifications importantes; li peau qui tapisse les fosses nasales et devient organe de l'olfaction n'a besoin, pour acquérir une grande perfeetion, que de très-légères modifications; il filut selllement que cette peau s'amincisse légèrement, qu'elle se lubrifie el qu'elle offre une très-grande surface pour recevoir le contact des gilz. Lai délicatesse de ee sens est ell raison de l'étendue que peut prendre la surface dans les cavilés qui lui sont assignées.

La pean, ‘ui est le siége du sens dı goût, ne demande que l'apparilion de quelques prolongements connus sous le nom de papilles, el un certilin degré de mollessc.

Enfin le sens du toucher pent s'exereer par toute la surface de la peau, pourvu que celle-ci soit molle el non couverte l'appendices qui empêchent le contact immédiat. 


\section{APPAREII, IOE I.I VISI(ON}

Il existe des yeux jusyue dams les animanx les plus simples: mais, daus ce cils, ces orgintes sorvem simphloneml pour distiufourer la lumiere; il falut un apprareil uptique frour reresoir l'inturession d'une intigre.

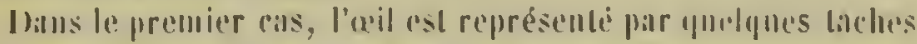
de piguent; dilus le second eas il existe une calpsule on mumlume selérntipur, mu corps solide et transpiarent nagerant an milien d'un lipuide, un filet mervenx el me purtion amincie de lenveloppe, qui devieul transparente, pour le passage de la lumiire, Ime tornie.

Les parties essemticlles de l'xil sont donc : le merl oplique,

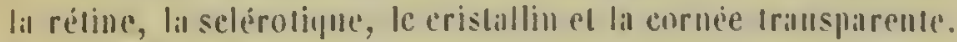
.l ces parties essentielles vieunenl se juindre des parlies de per. fectionnement qui sout : une menbrane noirc derribe la rálium

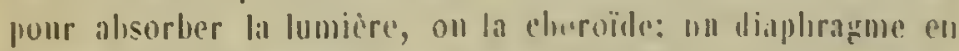

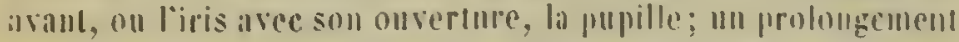
le lit proul, qui peut oblifirer complétement l'ouserture, on les paupieres; me glande lacerymale arec son conduil, pour lubri-

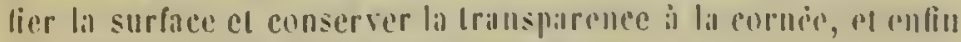
les muscles pour monvoir le globe de linil. 
Les yeux sont simples et au nombre de deux seulement dans les vertehrés; ils sout souvent composés, ct ou ell compte alors par cenlaimes ou par milliers, dans les animaux articulés.

Mammiferes. - Presque tous les mamuifires sont pourvus "l'yenx (figr. 290); ils manq̨uent seulement clıez quelques-u॥s

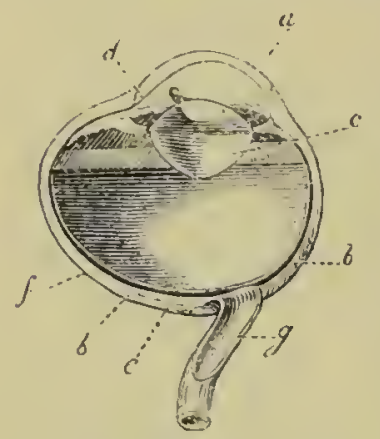

Fig 290. - OEil de mammifëre d'apue. Eschricht.

a. Cornee Iransparente. b. Sclérotique. - c. Cristallin. - d. Iris. - e. Réline. - f. Choroïde. g. Nerfoplique. l'entre eux, qui vivent sons terre. Ils soul le plus grands chez quielques quadrunanes nocturnes. Ils sont toujours logés, comme clicz tous les vertébrés, dans la cavité orbitaire, qui est formée supéricurement par l'os froutil. Ces yeux sont dirigés tons les deux en avant chez les quallumanes, qui se rapproclientle plus de l'lomme; généralement, ils sout silues sur le côtć de la tête, chez les autres mammifères.

L'oul se meul, comme dans lous les vertébrés, par qualie muscles droits el deux obliques; mais quelquefois un muscle cn forme d'entomnoir culoure le uerf optique, s'insìrc au fond de la fosse orbitaire d'un còté, tout autour dn globe de l'ocil te l'autre, et reçoil le nom de musele cloanoïle. 11 double l'action des museles iroits.

Ontre les deux pampières supéricure el inféricure, il part sonvent de l'angle interne de l'neil une troisieme panpière, yni correspond à la membrane clignolante des oiscamx, mais qui ne I'ecouvre janais qu'unc parlie du globe de l'neil; elle est tout à fail rudimentaire daus l'honme. Une glamble, siluce à l'angle interne de l'xil, la glande d'Harderus, s'ourre à la face postépieure de celte troisieme panpière.

Che\% toms les mammiferes, on trouve l'appareil lacrymal an complet : me gramle, deux points lacrylume sur le bord des paupières a un canal en communication avec les fosses nasilles.

Le grobe de l'oeil a mue forme sphérique; la sclribolique est 
extraordinairement épaisse dans les cétaeés soufleurs; le foud lu globe de: l'exil présente, elıez, plusieurs mammifìres, un éclit métallique que l'on désigne sous le nom de tapis; ce tapis est ceaillenx dins le chat el s'observe surtout dans les ruminants.

En dessous de la clioroïde, II. Rainey a observé un musele à lihres strices, yni a pour effel d'iéalter la choroĩde de la selérotique; il l'appolle musele choroïle.

La fache jante u'existe que chez quelques puadrumanes. Lal cavité yui s'étend llu eristillin à la cornée transparente est séparé pirr un diaplıragme; la partie antérirure est désignte sous le nom de e.tamhre antéricure, l'autre, derriere le diaphrag'me, sous le nom de chambre postírieure; la eapareile de ces chambres ne varie guere d'une maniere imporlante dans celte classe. Ce diaplurigme on l'iris est très-mohile; son onverture on la fupille est générillement ronde; lontefois daus quedques mammifires elle s’allonge verlicalement, d'tes borls peurent se rilpprocter au point l'oblitérer complétement le passage de la lumiere; les clials nous montrent un exponple de eette disposition. Chez, l'autres, la pupille est allongére lorizontalement commo dius beaneoup) de ruminants et de parliỵlermes, mais les bords soml arrondis au lien ditre angenlems.

Le eristallin est sultérịne dans les mammiferes aquatiques; cluez les antres il est aplati.

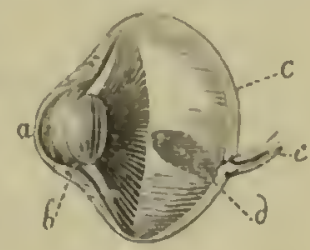

Fig. 291. - Of.il d"aigle. a. Cornese. b. Cristallin. - c. Silerolique. d. Peigne. - e. Nerf optique.

Lientre-croisement des lleux nerfs optiques est partiel dins les mammiferes.

Oiserax. - L'exil des oiseaux (lim. 29) differe notablement quant ì sa forme; son diamctre antéro-posterieur est beaucoup plus petit que le diametre vertieal. I.es yeux ne manquent lans aucun oiseau, et ils sont proportionnellement plus volu. mineux que dans les manmilires. Dans les rapares nocturnes, ils sont diriges en arant comme dans les qnadrumanes; daus tous les autres, ils sont dirigés sur le côte. La truisirme panpicere ou la membrane niclitante exisle chez lons les 
oiseaux; elle passe commc un ridcau au devant du globe de l'ocil, de manière à le rccouvrir complétement; elle est mince, demi-transparente el pourve de deux muscles qui sont situés derrière le globe de l'ocil. 11 existe également une glande d'Harderus avec soll canal excrétcur.

Dans l'épaisseur de la sclérotique il se forme en avant chez quelqucs oiseaux (les rapaces nocturnes) un cercle osseux (fig. 292) qui s'atlaclie à la cavilé de l'orbite; ce cercle est formé de plusieurs osselets qui sont imbriqués et dont le nombre s'élève quelquefois jusqu'à trente. La clıorö̈de forme inl fond du globe un repli dont la surface est régulièrement strice et que l'on désigne sous le nom de peigne. Ce prolongement s'étcnd en avant au milicu du corps hyaloüde. La cornée

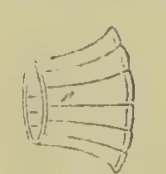

Fig. 292. - Cercle

ossenxde la sclerolique de grantdue.

transparentc est très-bombée, ce qui rend les clıambres très-spacieuses. L'iris est très-mobile: la pupille est toujours arrondie; la couleur jaune de l'iris cst due à des goultelettes d'luile de celtc couleur. La couleur de l'iris est très-variable. On distingue des fibres musculaires cntrc la paroi extcrne du canal de Fontana et le ccrcle osscux; c'cst le musele de Crampton ou le muscle ciliaire ou bien encorc l'anneau fibrenx de Treviranus. Malgré la différence de position, H. Rainey le considère comme analogue du muscle choroïdc. Le cristallin est aplati à sa face antérieure, surtout clıez les oiseaux de proie diurmes, tandis que clıez les nocturues il est très-colvexe.

Reptiles. - Lesorganes de la vision sont tout à fait rudimentaires cliez quelques animaux de celte classe et ils nc sout jamais très-développés. Ils sont situés sur le côté de la tète; outre les six muscles qui mcuvent ces organes, oll trouve souvent encore un muscle choanoïde comme cliez les mammifères.

Les paupières manquent souvent et nc sont en géuẻral que médiocrcment développées quand elles cxistcul. On en voil généralement une cn dessus et une ell dessous, mais clic\% les caméléons la paupière est circulaire et les ycux sc menvent indé. pendamment l'un de l'antre. Ou trouve souvent encore unr membrane nictitantc, chla glande d'Irarderus dans l'angle iutcrne 
de l'urloite. On trouve aussi, du moins daus les reptiles prepure-

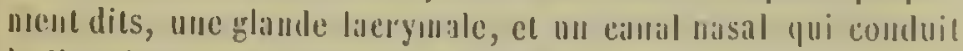
le liquide laterymal dans la cavité du nez.

Cliez les serpents, la peau passe an devant du grolie de l'inl et laisse cutre elle et la corme transparente un elrtain espate pour le pissage du fhuide lacrymal. Quand le serpent mue, l'epi.

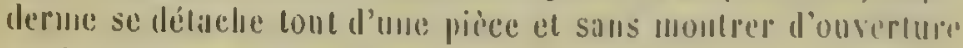
all devaill dug golole de l'aril.

Le globe de l'ail des reptiles (tig. 2!) J) proprement dit. ressemble à eelui des viscaux, tiundis que celui des batraciens se rapproclec plutul des poissolls.

Lá seléruligne devient sourent ciarlilagineuse ou mime montre daus sou éprisseur un cerele osseux; la eloroulda est tre's-épaisse el furme un repli qui penetre dams le eorp's lyaluille commer le peigne el qui mérite re mente mon. l'iris est moluile, diversement colorie; la pupille est tantót arrundic, tanthit feudue transversalement on vertiealomerul; on distingue encore les diux bianlures, le eorps cl les proces eiliaires, ele.
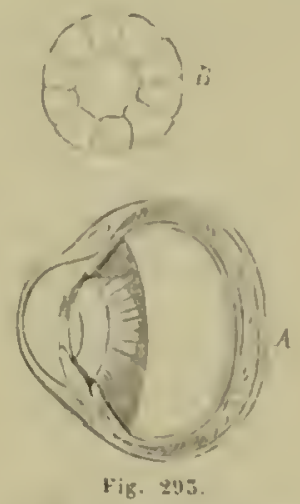

d. (1)ill d'emys ruropas - B. Cercle asseus

l'oissons. - Les poissons portent quelquefois de tris-grands yeux, surtout ceux qui vivent d de grandes profondeurs. Orilinairementils sout situés sur le còté de la téte, mils se trourent aussi quelquefois a u-dessus, comme dans l'uranoseope. Dans les pleuronectes, les deux yeux sont silués du méme cúte du corps dans l'ige adulte. Le globe de l'ueil, ordinairement un peu aplati ell avaut el leémisplérique en arrière, se meut à laide des mèmes muscles, au nombre de six, que nous avons vus dans les autres classes. Ces muscles manquent dans un tris-pelit mombre. Le branchiostome n’a pour tout organe de la vision que deux taclics de pigntent, et tout oregalle accessoire manque daus ces singuliers poissons. l, axil est aussi rudimentaire dins les mỵ.Anö̈les. Clrez quelques animaux de celle classe (les plagiostomes). l'eeil est portésur un pédicule el reproduit la disposition que lon vit dans la classe de's crusticés. 
Il n'y a pas d'appareil laerymal.

Ce qui distingue surtout l'oeil des poissons (fig. 294), c'est le peu de convexité de la eornée transparente, la splıérieité du

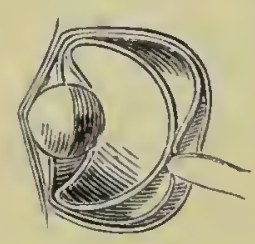

Fig. 294.-OEil de perche (Cuvier). eristallin et le peu de mobilité de la pupille. Les clambres sont presque nulles, tellement le cristallin est rapproché de la cornée transparente; les paupières et la glande laerymale manquent.

C'est exceptionnellement que l'un observe cliez quelques poissons (certains plagiostomes) des paupières el même une membrane nictilante.

L'inmobilité de la pupille est corrigée par un prolongement sous forme de voile qui descend du bord supérieur de la pupille et qui peut ainsi oblitérer l'ouverture (les raies). L'iris présente elı geénéral des couleurs très-vives et très-variées.

L'iris des poissons est mobile el se contracte sous l'exeitation dircete de la lumière el sans intervention de la rétine et de l'encéphale. Cette mobilité est un peu plus grande chez les plagiostomes que clıez les autres; elle est due à des fibres musculaires.

La sclérotique est en génélal fort épaisse et d'une grande durcté; la choroïle se sépare en deux eouclıes qui s'éloignent notablement l'une de l'antre el entre lesquelles se trouve un réseau vasculaire qui a reçu le nom de glande clıoroülale.

La tissure de la rétine livre souvent passage à la membrane interne de la clıoroïde (la ruyschienne), qui plonge dans le corps vitréquelquefois jusqu'au cristallin el forme ce que l'on nomme la campanule de Haller.

Articulés ou épicotylédones. - Dans cet embranchement, on voil des yeux simples eomme dans tes vertébrẻs (on les désigne sous le nom de stenmates), el des yeux composés ou à ficelles dont le nombre peut s'élever à plusieurs milliers. On trouve quelquefois les deux sortes dans le mème animal (fig. 2!y).

Les yeux simples on stemmates se composent d'une cormce transparente, d'un cristallin spluérique ou cylindrique, d'une rétine formée par le merf optique el d'une coucle de piǵnent qui représente la rhoroülle. 
Les yeux composès ou à faceltes sont formés dautam de cones on de cylindres qu'il y a de facelles à la surface. Chaque facelte représente une cornée; derrière celle cornée on trouve

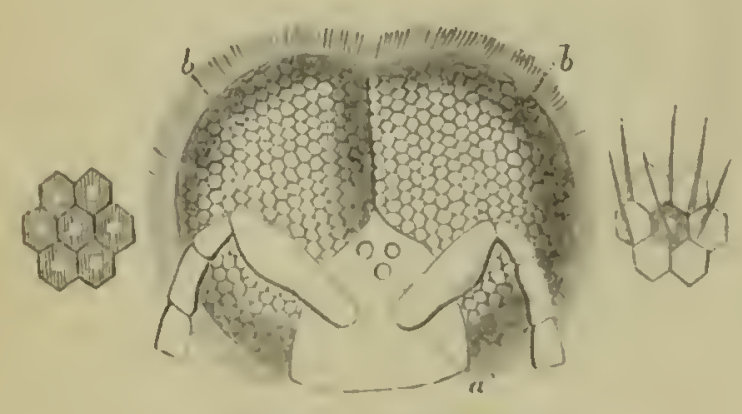

Fig. 295. - Tetc aburill,

a. Veux simples. - b. Veux à facettes.

III) eorps solide, allongé, l'une forme pyramilale, qui reprisente le cristallin; lerriire celui-ei on trouve un corps vitré yui est eutouré d'un caliée formé par un filet optique, la réline: ee filet opthipue provient du renflement ganghom remarque sur le uerfoptique. Ces divers orgines sunt entoures d'une choroüle qui tapisse le cylindre el laisse derrière la corméc une ouverture qui sert de pupille.

Une sorte d'yeux interméliaires, formés d'yeux simples rémnis el couverts l'une cornée commune s'observe chez quelques crustacés.

Ces deux sortes dyenx présentent dans les diverses classes de cet embranchement de nolables changements, surtout quant il leur position el lemr nombre.

Il y a des arlieulés qui onl des vem dans le jeune ige, lorsqu'ils virent librement, mais qui, il l'itint adulte, les perdent, lorsqu'ils leviemuenl parasites.

Insectes. - Les yeux simples sont places sur le cold de ha lète quand ils existent senus; on en troure qunelquefois jusqu’a soixaule de chaque còte el ils font le passage aux yeux composis; yuand les leux sortes dienx evistent simultanément, les 
stemmates sont placés ou sur le froml, souvent an nombre de trois, ou biell sur la nuque.

Le's yeux ì faceltes (fig. $293 \mathrm{et} 296$ ) sont toujour's places sur'

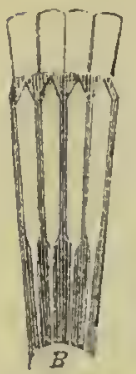

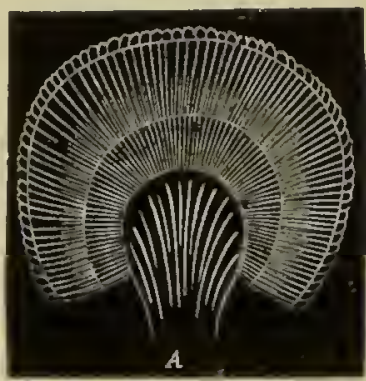

Fig. 296 . le cólé de la tête; ils forment deux globes bombés qui se touehent par. fois au-rlessus; le nombre de ficelles ainsi que lenl grandeur varient beaueoup d'ulı groupe à un autre. C'est chez les libellutes et les diptères que ces yeux solit le plus développés. On compte plusieurs milliers de facettes ou d'yeux daus quelques insectes.

Arachnides. - Il n'existe que des yeux simples daus eetle classe (fig. 297), êt souvéut ees organes manqquent complétement, surtout eliez eeux qui oceupeut l'extrémité de ectle classe el qui vivent en parasites. Ils sont très-variables quant au nombre et quall à la situation. On en voit quelquefois deux à la partie antéricure du dos, d'au-

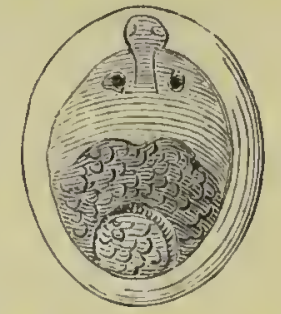

Pig * 297. Larve d'acarus pourvue d'yeux.

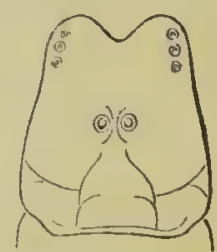

Fig. 298. - Scurpion spinifer (Gervais)

tres fois sur le eôté et qui sont portés sur des pédonentes. Chę d'autres on ell voit quatre, mais dans la pluparl des aranéides, il y en a luit près du bord antérieur du ećphalothorax. Its sont toujour's groupés avee symétrie ct montrent le même arran. gement dans la mème espèee. C'est elıez les seorpious qu'ou en trouve le plus grand nombre; ils en out souvent denx gralnds ¡u milieu et plusicurs petils sur le eôlé (figr. 298).

Myriapodes. - Ils sont simples et placés sur le côté de lia lìte en formantdes séries régulières composées de quatre, sir, luit ou un 
nombre plus consilderable, qui s'ellive quelquefois jusqu' il qua-

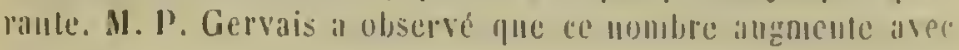
l'ilige.

Crustucis. - Dims cèlle rlasse nu tromve lus dems smites

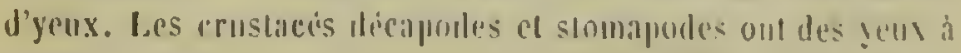
facelles, portés sur deus péduncules (fï. $\overline{5}(00)$ silues sur le

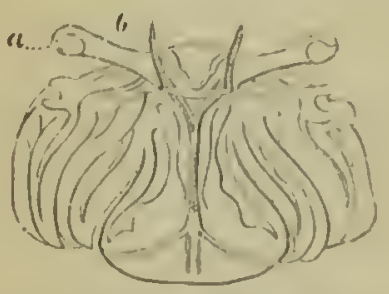

Fig. 300 - Pent priliculis d. ci ustices dienporidr.

a. Youx.-6. Tige mubila.

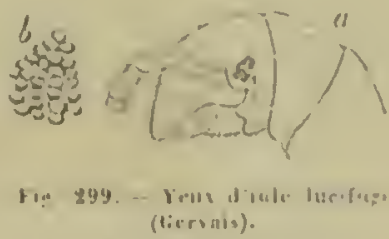

a. Jeuren plune b. Yous isulés

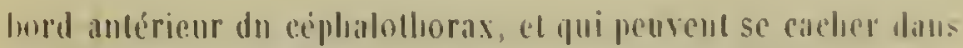
des fosseltes partirnlieres oi ils sont parfinitement alurilis. Clart plusicurs erustilers un trouve des yeux composis sans facelles; ils consistant a un cerlain mombre l'yems simples qui sont eourers par une corme commun

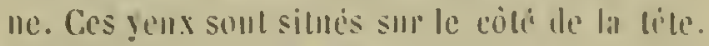
Quelyues erristaces ne montrent plus quinu

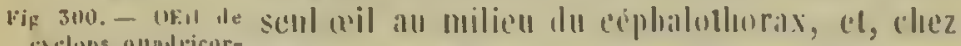
(velups qualicicornis, pherean milieu les crustacés parasites, ces orgumes de sems du front.

manyuent generalement, taudis yüils cxistemt encore dans le jeme age lorsquils menent une vie libre et in. dépendante.

Allocotyledones ou mollusques. - II existe de's yeus dau. les divers uralles, a l'exeeption des brỵuzaires. Cos yeur sonl loujours simples el engenéral ils renferment un appareil optiplue.

Cephalopodes. - Il existe deux yeux très-volumineux el yui sout lugés dans des enviles arbiaires formies par la boite

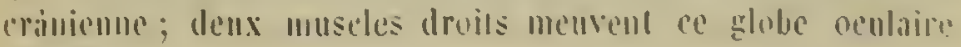
(tig. 301$)$.

Dans le ealmor, au devant dug glebe de licil, evisle unr nu- 
verture qui établit une communication entre le delıors el une

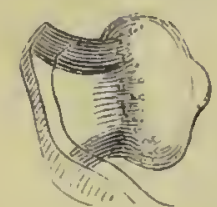
grande cavité qui entourc tout le globe de l'wil (fig. 502); c'est la jocau qui pénètre dans cettc cavité el en tapisse tout l'intérieur, elle reprèsente la conjonctive. La peau devient transparente au devant de la cornée, qui est placée vig. 301, - Globe dans cetle cavité.

Je l'oil el muscles du calmar.

Cet organe de sens se distingue surtout par l'élsorme l'enflement du nerf optique; de ce renflement partent un nombre considérable de filets nerveux, qui pénètrent séparément dins la sclérotique par des ouver-

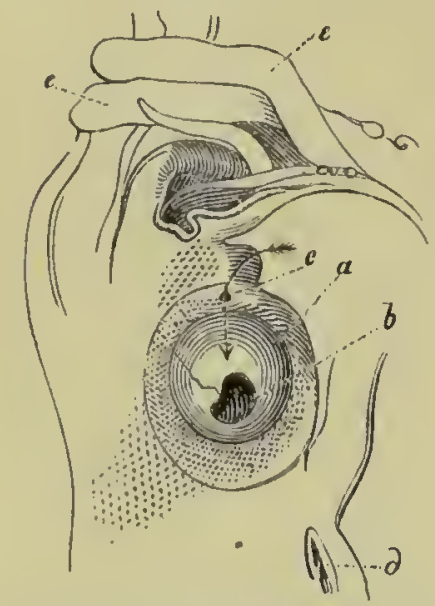

Fig. 302.-Tete de calmar, vue de colus.

a. Globe de l'œil. - $b$. Peau transparente. - c. Orifice. - d. Cartilage articulaire. - c. Bras. tures distinctes, et qui, en se réunissant du côté interne et du côté exterue de la choroïde, forment pour ainsi dire unc rétine internc (la rétine propre. ment ditc) et unc rétine extcruc. En dcdans de cetle rétine extcrue, on voit ume épaisse cou-

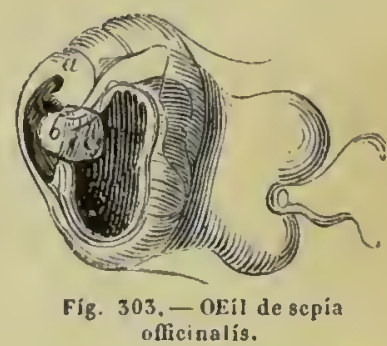

a. Peau. $-b$. Cornée transparente. - c. Cristallin.

clıe de pigment tapissce par unc pulpe nerveuse très-nolle qui se réduit au moindre attouchcment. C'est la rétine véritable qui a longtemps échappé à l'examen des anatomistes. On trouve ensuite la membrane hyaloide ct le corps vitré. I.cs filets nerveux à la sortic du ganglion se croisent dans quclques céphalopodes.

II existe une cornée transparente, mais clle est tcllement 
rapprochice ulu cristallin, qu'elle ne forme avec lui qu'un seul urgalle, que l'on a regariléce comme un cristallin composé le deux seğments (fig. 305). D'après celle délermination, l'uil des céplulopodes reutre dans la conformaltion ordiuaire; il fail simplement par ses filets optioues le passage de l'weil simple à l'œil compose. La peau et non l'iris forme souvent un voile audevant de la cornée pour rétréceir l'ouverture qui livre passage i) la lumicice.

Gístéropodés. - I.es gastéropoles (fig. 50.4 el $50:$ ) sont aussi pourvis de deux yeux assez simples et petits; on leur recommit les divers éléments essenticls du grolue de l'ail. Une selérolipue au-devaul de laumelle passe la pean anineie, une corné, un eristallin, une pupille avec iris, une choroïle el mee rétinte. (Guand il existe deux paires le tentacules, comme daus les limaces, ee sollt les supéricurs qui portent ces Flg. 306. - ofir

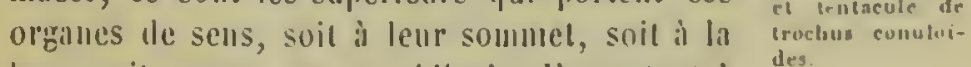
bise, suit encore sur un pedicule. Ils portent is cet ellet le wom de tentacules oculiares. Le nefl qui se rend

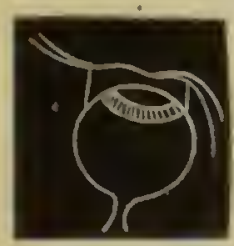

Fig. mo. - (16iil do. tritun, dtiprés J. Muller. anx lenticules est accole au nerf oplipule sur une parlic de son trajel. Che\% quelques gasteropoles, les yenx sont formés d'un amas de piğuent saus selérotique uj cornée, el soul elirectement, sans l'intermede de nerf ophiqur, ell lapport avec les gillghlious sus-aesupha giens.

Aréphales. - Les aceplailes soul ell géuiral pourvis d'yeux; ils sont situés sur le bord du mantean el sont formés d'une selérotinue, d'une coruce converte pare la pean, d'une couclue pigmentaire, diun eristallin el diune réline yni entunre min eorps vitri. Ils sont quelquefois peidumeules el en tris-gramel nomber logeis an milien des pilpilles. Ils sont assez pelits mais nombrem dans les lututres, mais rest dans les peizutes quoils sollt le plus développes.

Tunicirrs. - Chez les ascidies a l'ital de litards, lorsipuils vivot librement, nous avons vusur le cole de la tite des poiuts uoirs salls appareil "plipue; ces points noirs disparaissent lors- 
qu'ils deviennent alultes, el, elıez quelques-uns, de nouveaux yeux apparaissent au pourtour de elsacun des tubes; on voil uı eristallin au milieu d'un amas de pigment.

Bryjozoaires. - Il u’existe plus d'organe de sens spécial.

Vers. - Parmi les vers, il y en a qui sont eomplétement privés d'yeux; d'autres out autour de la tête plusieurs taehes pig. mentaires, et d'autres enfin ont des yeux à appareil optique en nombre trés-variable autour de la tète, par

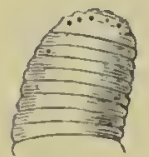

Fig. $506 .-$ Yeux d"une harudiace. exemple les sangsues (fig. 506). Cet appareil optique eonsiste, clıez quelques-uıs, eı uı globe distinet avec une eouche de pigment, une pupille, un corjss transparent el une rétine. Les ner's optiques viennent des ginglions sus-assoplagiens et forment quelquefuis un renflement ganglionmaire avant de pénétrer dans le globe de l'oul.

On trouve des talies de pigment dans divers helmintlies (fig. 507), mais toujours seulement dans le jeune age.

Échinodermes. - On voil dans les astérides des taches pigmentaires rouges i l'extrémilé et en dessous des rayous (fig. 508); 0 II en voit de pareilles silr les pliques qui alterment avec les plaques géuitales daus les oursins, el que l'on appelle pour eelle raison plaques ocellaires; mais il u'y al pas d'appareil oplique.

\section{Polypes. - Les organes} de sens que l'on voit tout autour de l'ombrelle dans

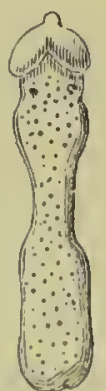

Fig. 307. - Taches de pigment de scolex de eestuide

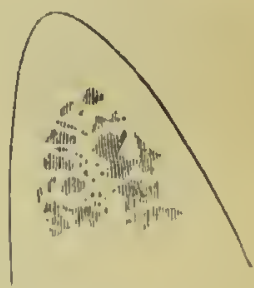

Fig. 308 . - Frétendus yeux des astíries. les miéduses (fig. 509) sout regarlés tantôt comme des yeux, tantôt comme des oreilles; quelyuefois il y en a de deux sortes, et ceux que l'on regarde comme yeux sont silués à côté des vésicules supposées auditives. Ces yeux consistent dans des cellıles à pigment, dans lesquelles est enfoncée à moitió mur. lentille splsérique. 
les polypes de la division des sertulaires, les gorgones ot des hyilraires n'ont plus de taches de pigment, et cependant

1

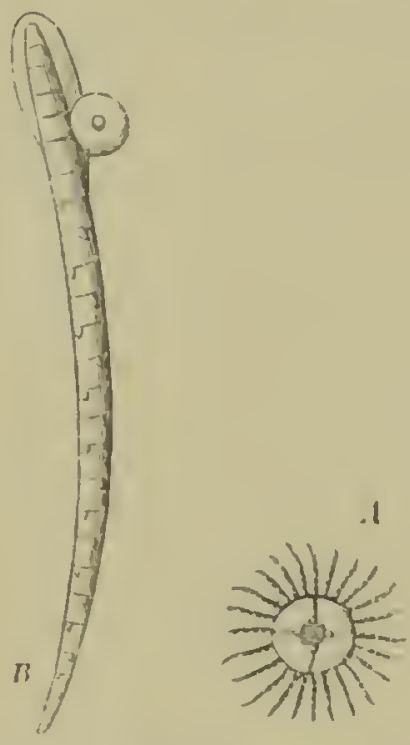

Fing 3019.

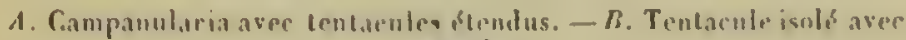
cipusule.

quelques-uns d'entre eux, comme les liyilres, snnt sensibles i lit lumière, puisudils changent de place dius un verre d'eau et cherclent le jour. 


\section{APPAREIL DE L'AUDITION.}

L'organe de l'ouïe est an moins inssi répandu que eelni de la vue, el si, dans les animaux vertélorés, alticulés el mollusques, on ne trouve que dems oreilles, il en existe un nombre beaucoup plus grand dans la plupart des acalèphes.

Dans tous les vertébrés, l'oreille est logée dans l'épaisseur d'un os trìs-dur qui est caclıé en dessous du cràne; il n'a d'abord aucune eommunieation directe avee l'extérieur, puis une cavilé qui eommunique an dehors se forme sous le nom d'oreille moyenne, et enfin, dans les mammifères, il se forme de plus à l'extérieur un eornet acoustique sous le nom de eonque anditive.

Mammifères. - L'oreille interne (fig. 510) se eompose l'u॥ vestibmle el de deux saes qui subissent peu de molifications

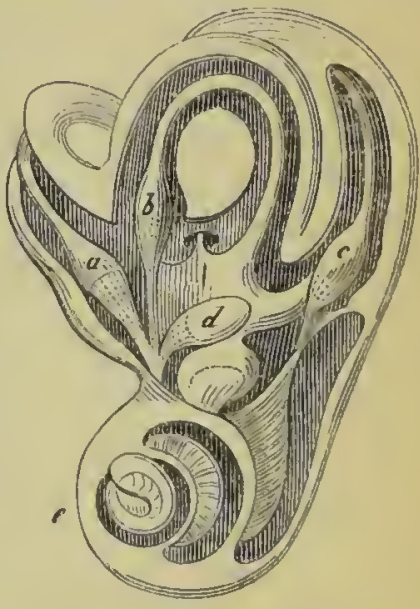

Fig. 310, Oreille interne. Festibulu, ennaur semi-circulaures ossetix, 1:tlyrinthe uembraneux et Ierminaisu des nerfs.

$a, b, c$. Ampoules des canaux semicirculaires. - d. Ololithe. e. Libagon montrant la ramper vestibulairc et la rampe lyonpanique.

dans celte classe. Il existe tonjours trois cananx semi-circulni- 
res qui sont en commonicalion avee le vestibule et un limngon;

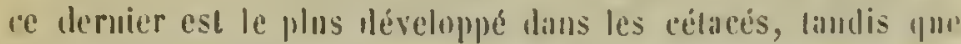
che\% les monotrimes il ressemble ì relui des oiseanx. Le nombre le tours de spire qu'il forme s'ilève justu'il cimp; it ue filit qu’un tour el demi dans les célacés el le hérisson. Lal cavité du limacon est divisée en deux par une cloison incomplete; the commmniyne d'un cotté avee l'oreille moyenne, de l'autre avee fé veslilule.

Les cimaux semi-eirculaires prisententsur leur trajed des aupoules qui sonl grimles chez les mus, les calmassiers, par parm.

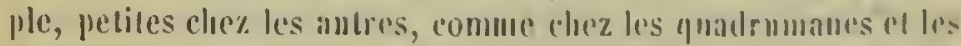
ruminants. Ces divers oryanes de l'ureille interme forment le

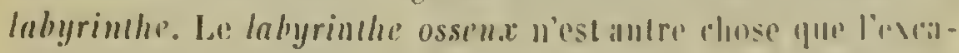

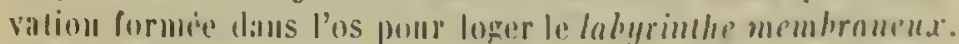

L'oreille moyenue est géméralement assez spacionse: l'us Iympanal forme souvent une forte sullie it la base du crionr, sous furme l'un lanbour. Celle calvite est tris-dibeluppe daus les carnassiers; elle est en commonnicalion arece l'ar-

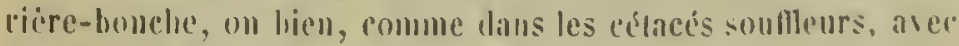
le canal de l'évent, par mu conduil cartilaginemx comun soms le nom de trompe d' linstache. I.a eavilé du lympan est limilée en dehors par la membrane do lympall et présente des sims aceessoires dians l'éprisseur des os. Il eviste deur trous du cúté du vestilule; loun, qui sonvre direetement dans colle cavile, est la fompre orule; l'antre, yni communique aree le limaçon, est la fenétro ronde. Entre la fenctre ovale el la memlirane du lympan, il existe une chaine d'osselets (fig. 3II) geméralement au nombre de trois, l'élrier, l'cuclumé el le marlean, el quelquefois on roil des osselels accessoires. Ces osselets sont mis en monrement par

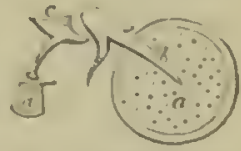

Fig. ill. - rliaine d"uscertes

a. Membrane du Iympan.-B. Marlenin. - r. Enelume. - d. Eericr. deux museles : le muscle de litrier of le tensenr du Iympan. la forme de ees osselels varie buacomp, lout en conservan! leurs caraclires propres. L'élrier est loujours appliqué sur l. fencitre ovale.

Loreille externe se compose d'un à trois carlilages qui for- 
ment avec la peau un pavillon plus ou moins grand qui se dirige dans différents sens au moyen de museles propres; on ch compte jusqu'a vingt-lıuit dans le cheval et le cliat. Les diverses par-

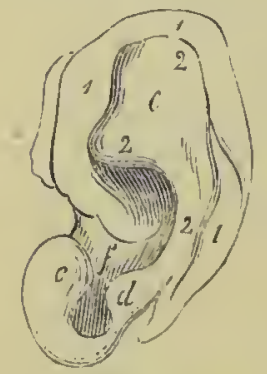

Fig. 312. - Oreille externe.

1. 1. 1. IIflix. -

2. 2. 2. Intluélix. c. Tragus. - d. Antitragus, $-e$.linsse naviculaire. f. Conque.

lies de ee pavillon ont reçu des dénominations diverses, comme on peut le voir par la figure 512. C'est dans quelques cliauves-Souris, l'oreillard, par exemple, que ce pavillon a acquis le plus grand développement. II man-

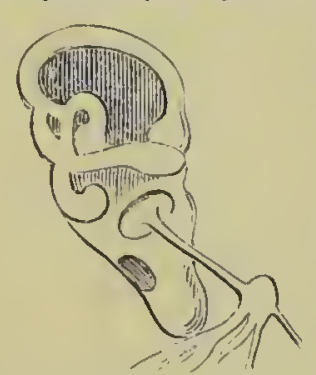

Fif. 513. - Orcille interne d'albitros. que dans les mammifères aquantiques et sollterrains.

Oiseanx.-On troulve encore dans l'orelle interue(fig. 515 ) le veslibule, les camaux semicirculaires an nombre de trois, el le limaçoll. Dans le vestibule on trouve, au milieu du

liquide qui le remplit, deux concrétious calcaires qui représentent l'ololitlie. Le limaçon, au lieu de former des tours de spire, consiste dans un tube ligè rement courbé et obtus, qui est iggalement divisé à l'inlérieur en une rampe vestibulaire el une rampe tympanique.

La caisse du tympan est trisirreguliere; elle communiq̨u: avec divers os, el, par lune trompe d'Euslaclie souvenl osseuse, avec l'arriere-jouche; les deux trompes s'unissent souvent en un seul couduil. II ॥'y it qu'un seul osselel de la chaine bien développé, e'est

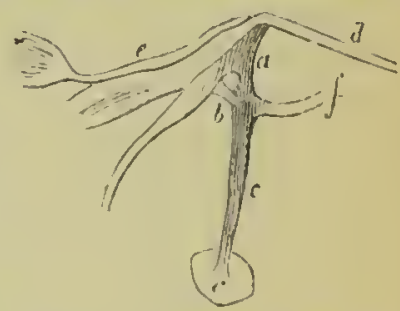

Fig. 314. - Chaine d'osselets de couaril.

a. Marteau. - 6. Enclume. c. Etricr, - d. Muscle interieur du marlean. - e. Mlusrepxlśricur du marteau. f. Muscle de l'etrier.

l'cilrier, qui porte te nom de columelle; les autres sont a l'état rudimentaire (fig. 5l1). Ja membrame du tympan es! convexe ent rlejor's. 
L'oreille externe manulue clıcz tous les niseaux, on distingue toutefois toujours le tron auditif, el, cliez les rapaces nocturnes, les plumes se disposeut antour de celte ouverture ell formant, un pilvillon ( fig. jlij). Lees rilpaces nocturnes ont uII conduil auditif ųui, d'un còté, cst dirigé de haut cul hals, el, du cooté opplosé, de bas en lant.

liepriles el latracions.

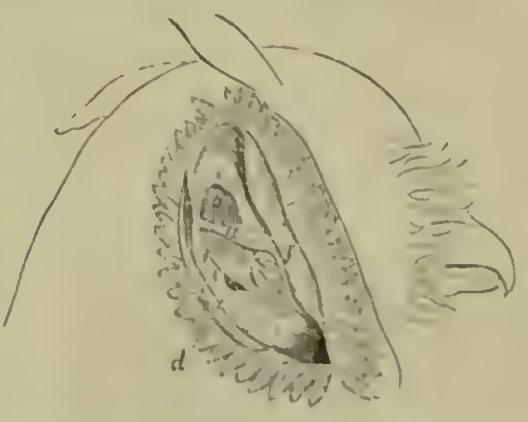
- Les repltiles écailleux Fig. 315.-Parillon el lrou nudurdesirya nlus montrent encore dans leur a. Ifepli médian. - b. Mépression supeoreille interne un vestibulc avec nne on plusieurs comeritions ealeirires (otorieure.-e. lospression inférieure. d. Tron auditif. - e. Stillie usseuse. - f. Limiles antérimires de ce méme pavillou. - g. Limites postétieures.

lilles), trois eanaux semi-eirculaires et un limalon; ce dernier organe, dans les eroeodiles, ressemble parfaitement à celui des oiseaux. La caisse on l'oreille moyenne manţue eomplétement daus les ophidiens el phusieurs sanriens, ainsi que la membrane du tympan. On roit loos columellaire applique sur la fenctre oville. Il existe une trompe d'Eustache guand la caisse ne manque pas.

Le labyrimtle des batraciens montre encore les trois crunau.x semi-circuluires, mais point de véritable limagon. Il existe en. core tonjonrs une fenetre ovale forméc par une columelle quand l'oreille mojenne existe, ou bien celle fentre est couverte par les muscles et la peau quand alle manque. La caisse lu tympan est ponriue anssi d'une trompe d'Eustache.

Il n'existe plus d'oreilte externe.

Poissons. - Les branchiostomes sont les seuls parmi les poissons qui nont point d'oreille. Cet appareil est tonjours rédult an labyrinthe daus celle classe: il $1 \mathrm{y}^{\circ}$ a ni oreille moycune ni comdnil anditif externe.

Ce labyrinte est formé diun restibule avee deux saes latemanx renfermant des otolithes, de cantur semi-cirenlaires, 
généralement au uombre de trois, souvent fort grands et qui portent clacun une ampoule; celles-ci reçoivent elacune un filct nerveux qui ne s'étend pas jusque dans les eanaux. Il n'y a point de limaçon; il u'existe que deux canaux semi-cireulaires daus les lamproies, et ils manquent complétement dans les myxinoïdes. L'otolithe principal est souvent très-développè et dur, par exemple dans les diverses espices de gades. Comme nous venons de le dire, un filet nerveux se rend dans chaque

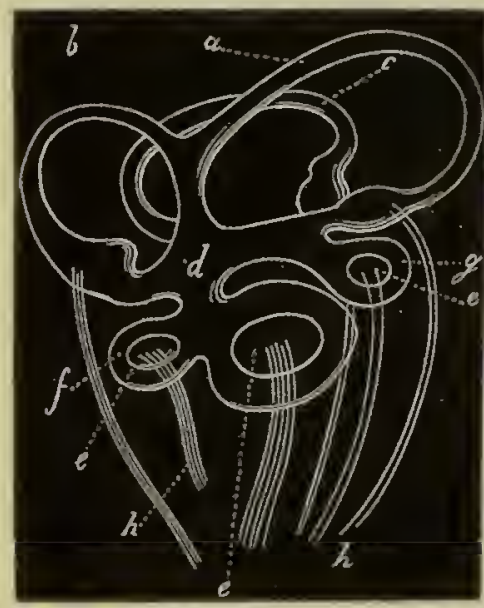

Fig. 316 - Orelle interme de lophius plscatorius (13resehet).

a. Tube antétieur. - - . Tube postérieur. - a. Tube extérieur. d. Sinus médian. - e. e. e. Otolithes. - $f$. Cysticule. - y. Utricule. - h. Nerfs. ampoule, mais il ell existe en outre encore un pour elaque otolillıe; il s'épanouil autour de eelle conerétion.

Cliez quelques poissons, les harengs elitre autres, la vessic natatoire s'étend jusqu'ì l'oreille iuterne el représente jusqu'à un certain point lil eavilé de l'oreille moyenne el une trompe d'Eustache qui s'ouvre dans l'estomac. Il y a, sous ce rapport, des differences trì-graludes dans les poissons.

Le lopidoleprus tracliyrliynchus est pourvu de deux conduits auditifs qui s'ouvrent. sur lc còté du crâne, en arrière el uII peu en dessus.

Articulés. - Tout le nonde

est persuadé que la plupart des animax articules eutendent; mais jusqu'ici on u'est parvenu ì coustater la présenee d'uı organe spcicial que chez quelques insectes el cliez les crustaces décaporles.

Insectes. - Cliez les orthoptères de la famille des acridides, on distingue deux fossettes a milicu d'un anncau corné, el au foud desquelles se trouve une nembrame tendue que l'on regarde pour une uembrane de tympan. Derriere cetle membratue ou lrouve une vésicule: remplie de liquide et à laquelle se rend 
nerf provenant llu troisiène ganglion thorachinue. V. Siebolu a trouvé un organe semblable daus les jambes antérieures des locustides et achélides.

Crustucés. - Les crustacés décapodes moutrent il la base des antennes externes une saillie an centre de laquelle on distimzue une membrane asec une fissure antentre; e'est la membrance du tympan. Derriere cette membrame se trouse une poche, remplie d'un liguide transparent, sur les parois de ladpelle s'épinonit un uerf yui nait avec le nerf antenuaire exterme, sur le colte des ganglions sus-uesophlagiens; c'est le vestibule. Cet organe ne s'ubserve pas dians l'antres erustacés.

Mollusques. - On trouve eet orgine de sens daus tous les mollusques, cxeepte dans les deux derniers ordres: les tuniciers it les bryozonaires.

Cluez les céplailopodes (fig. 317 ), il part des ganglions soms-

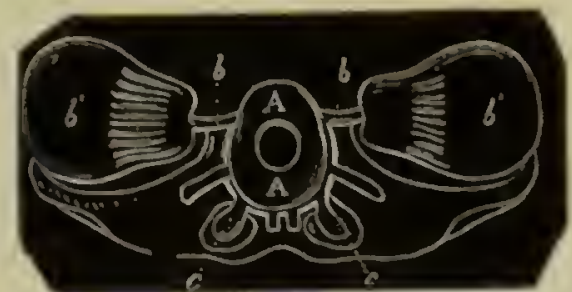

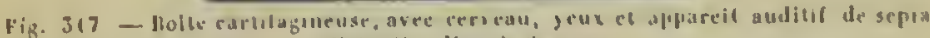
ollicinalis, d'apres searpa.

A. Cervean. - b. Xerf oplique. - b'. OEil. - c. Nerf auditif avec le vestibule.

asophagiens deux filets nerseux qui plongent dans les parois de la boite crintenne et se remlent il un sac membraneux qui, rempli dun liquide, est situé au milien d"une excavation formée lans lépasseur du cartilige crimien. Dans ce sac on trouve une otolitle blanche de lexlure cristilline. C'est toute l'oreille. II n's a alleunc communieation asce l'exlérieur.

Dills les gastiropolles, on rencontre lenx capsules auditives. dellirant aux ganglions osophlagiens, remplies d'un liquide dans lequel on trouve un ololitle unique on un amas de granulations

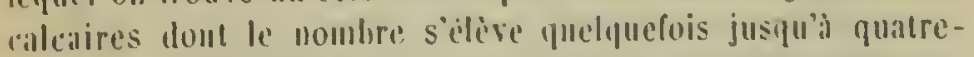

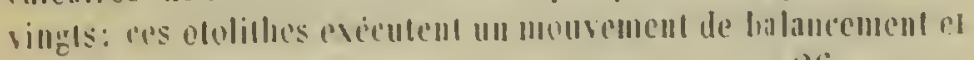
$2(i$. 
de rolation qui semble du il li presenee de cils vibratiles, qui tapissent la cavilé (fig. 518 ) .

Ces capsules sont siluées en dessous des ganglions œsopluil-

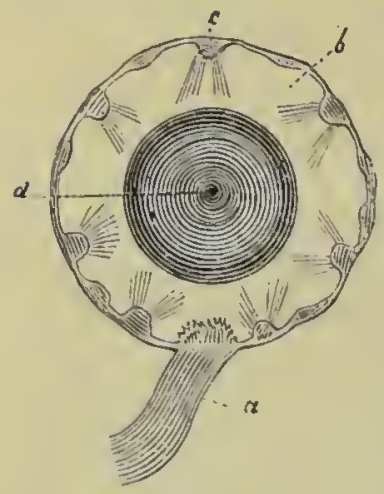

Fig. 318. - Orcille de la carinaire.

"Nerf. - b. Capsule. c. Faisceaux de cils viluratiles. - $d$. Otolitlic. giens cliez les uns (fir. 519); lerriere les yeux el à la partie supérieure du eorps eliez les autres.

Les acépliales ont une oreille sem blable, située dans l'intèrieur du pied, au-devant des ganglions pédicux qui correspondent aux ginglions sousasoplıagiens. Les otolithes sont spluériques, d'une structure cristalline. et exéculent également des mouvements de rotation. On peut fort bien voir cet orgalle dans le pied comprime des cyclas de nos eaux lluviatiles.

Vers. - Dans differents vers a sallg coleré, on lrouve itrilement, a côtê du collier assophagien, des ealpsules auditives dins lesquelles nageut des oblithes. Nul doutr que ce ne soient de véritables oreilles.

Polypes. -Clıcz les cydippes (béroë pileus) (lig. 520), 0แ urouve à côté du gàllglion nerveux unique une eapsule auditive avee un otolitlie qui tourne lentement dans sa cavité; il ne bous paraît pas douteux que ce ne soit une véritable oreille.

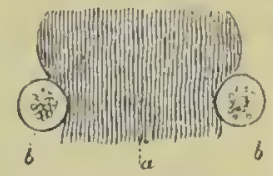

Fig. 319. - Gianglions sous - asoplsagiens de l.ampliseora, avec cajsules auditives et les otolithes.

a. Ganglious - b. Cap. sules. $-c$. Otolithes.

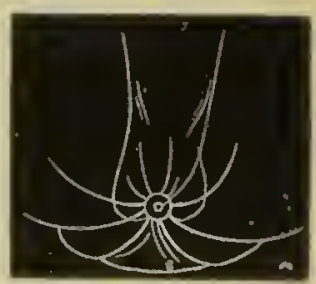

Fige 5zo. - Oreblle ds r) dipe pileus.

Tout antour de l'ombrelle, on voit, daus la plupart des mi duses, des organes que nous ne prowons nous empecher dr

1 M. Kolliker a vu des cils vibratiles dins la capsule mriculaure des molhoques gastéropodes el des embryons de éphalnpodes. I.c mourement particulicr desotolithes est dủ à res organes. 
regarder comme des organes de l'atudition; ce soml des eapusules dans lesquelles on découve des otolithes quelpuefois clairs et

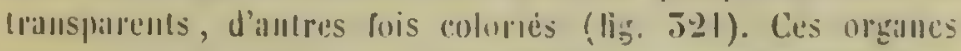
varicut d'use esprece il l'autre fiar le nombre, la dimension, la coulenr el le volume des otolille's. On les ruil exallement dins les pulypes medusiformes.

l.es argines marginaux de:

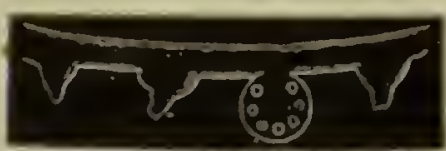

Fig. 3:1. fipsule audirive de thauunatias rymbalmides.

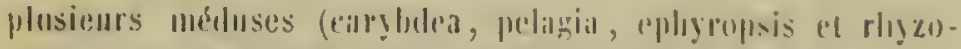

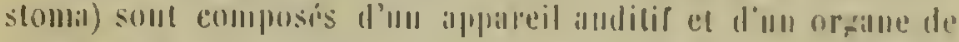
vision. Les yenx consistent dians me masse hemisphiripue de

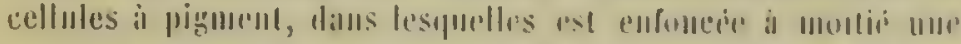

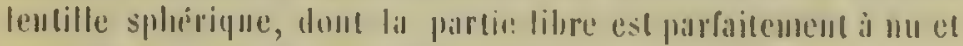
bagguce par l'ean de mer. (Gegenbaluer.) 


\section{APPAREIL DE LOLFACTION.}

Cavilés nasales. - L'organte qui est le sirge de l'olfictien ne consiste que dians une peau légèrenent modifiée par un plus grand développement du résealu vasculaire et parr la présence d'un nerf spécial ; dans l'olfaction, la plus téclicate, la seule modifieation qui survient, consiste dans une ingumentalien de surfice. II s'agil en effet de metre lil plus graule surface possible ell coutact avec l'ail. On ne connail avec cerlitude eet organte que dins les animanx vertébrés el dans les mollusques céphalopodes.

Chez lous les vertébrés, il existe deux fosses qui s'ouvrent ì l'extrémité du museau; ces fosses sont tappissées par la peau qui a reçu le nou de membrance pituitaire; la premiere paire de ner's se perd diuns ses parvis. Ces fosses présentent souvent en avant un réceptacle autour des ouvertures que l'on nomme les narines, et chez tons les vertébrés qui vivent dians l'air, elles communiquent postérieurement avee la bonche. Lees fosses niasiles

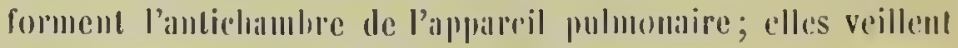

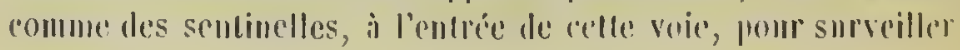


la qualıté lle l'air que l'animal respire. Celte cavité est loujours tapissée par uu epithéliun ciliaire.

Mammifères. - l.es narines sont situes au bout du nuseau, i l'exception des citacés sounleurs; le réceptacle yui les entour. est désigué sous le nom de nez, ou bien il prend une forme allongée et devient un groin, ou bien encore il s'allonge outre mesure pour devenir une trompe, comme chez l'éléphant. Dans quelynes chanves-souris, les ma rines sont entourées d'une conque semblable a me compe auditive, pour concentrer les comanations odorantes. Chez les phopues, les ouvertures lles fosses nisales sont cutourées l'un sphincter yni, en se contractiut, empeche l'eau de pénétrer pendiut la subutersion. Dans les cé lacés sonflleurs, il n'y a point de nez, et les narines, qui sont desigures sous le nou d'évents, sont situérs à la partie superieure de la tete. Il u'y a qu'une senle cavité et une seule uariue? dins les damphins; une cloison les sépare dians les baleines. Dans tous ces animanx, ce tube aérien monlle à sou evtrémitó supuricure une poche assez voluninense, dout les parois sumt de nature musculaire. Dans une espece de phoyue (stemmatopus) de la mer du Nord, les cavités masiles s'étendent sur le cóti, a lat pirtie supérieure de la tite, el l'animal, ful les remplissant d’air, preml une tite volumineuse, d'un aspect mostrucux; c'est le phoupe is capuchon.

Sur le coute de e's cavites nasales, on voit gineralement trois coruets, a replis dautant plus umbreux que loolorat est plus étendu; ils remplissent tellement la cavité, qu'il ne reste que tout juste la place pour le passage de l’air; c'est daus les plioques pue lou voit ces cornets prendre le phus grand développement. Les feullets se divisent et se suludivisent, de maniere que leur coupe présente le mime aspect que l'arbre de vie dans le ecervelet. Le cornet supérieur est géméralement formé par ledhmoide. l.es os frontaux, maxillaires el sphémoidaux so creusint somvent, forment des simns qui portent le nour des os daus lespuels ils se troureut et qui contrihuent rigalement a angmenter la surface de la pituitaire; ces cavites emprisonneut ensuite lair pour quelyue temps at font sentir encore des odeurs quant on ne se trouve plus an milieu des émanations. 
Les simus frontaux s'étendent, ehcz l'éléphant, tout autour de la tête; les spliénoìlaux sont très-grands dans ces mèmes paclydermes, et les sinus maxillaires sont en général assez volumineux. Les sinus froutaux s'étendent, dans les ruminauts à eorne ereuse, jusquc dans l'intérieur de la cheville des cornes.

'Tous les mammifères possèdent cet organe de sens, ì l'exeeption des cétacés sonmeurs, et cneore si les dauphins sont privés de nerfs olfaetifs, et si leur ethmoïde n'est point eriblé d'ouvertures pour leur passagce, au moins Eschricht signalé dans les baleines deux gros nerfs olfactifs.

Dans la plupart des mammiferes, on trouve une glande nasale qui est située de elıaque eôté sur les plarois externes. Son eanal excrétcur est situé au devant ilı cornet inféricur.

L'orgaue de Jacobson (fig. 529) consiste en un tube membraneux, formé d'une enveloppe riche en vais scaux el en nerfs, eld'unc giâine eartilagineuse, situé le long de la branchic intcrne de l'os intermaxillaire, sur le plancher des fosses nasales; à côté de la cloison; ce tube est clos en arrière et s'ou-

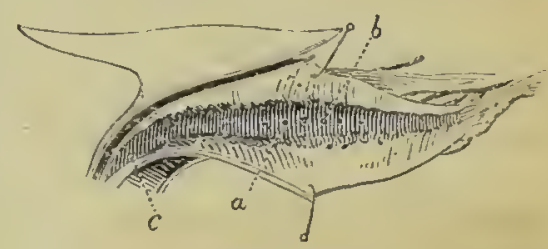

Fig. 322.

Tube de Jacobson ouvert pour nontrer le: bourrelel, a; les eryples, $b$; el l'orifices du tube dans le conduit de Sténun, r (eoction). vre ell avant dans le canal de Sténon.

Cet organe est très-petit chez l'homme et les quadrunanes. un peu plus développé chez les carmassiers, mais il acquicrt ses plus grandes proportions dans les herbivores.

Oisenux. - Cet organc de sens se trouve chez tous les oiseaux, et à très-peu d'exceptions il offre partout la mènıe conposition. Les narines s'ouvrent séparément à la base du bec; il n’y a point de réceptacle, e'est tout au plus si on peut considérèl comne tel les tubes cornés des pétrcls. Les narimes extèrieures paraisseut manquer chez quelques oiseaux. Les fosses Hasiles sout síparées far une cloison du vomer, mais celle cloison est souvent incomplète. On distingune, ch géméral, trois cornels qui 
sont quelynefois ossilics. Selon les oiseaux, l'un ou l'autre de ces trois cornets se développe; c'est le superricur dans les oi seanx alqualiques, e'est le moyen dans les gillinacés el l'iuférieur diuns les passereanx. II existe une glande nasale avec un anal exeréleur; sa place varie selon les ordres. lees fosses na-

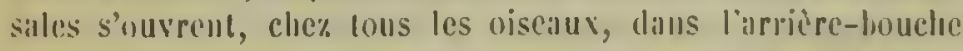
bar une fente longitudinale; des papilles cormes col recourent le boril al font fonction de voile du palais.

liepliles el butruciens. - Les lusse's nasiles sont, comme che\% les oistins, sans réceptarle el s'ouvrent al l'evlrémile du museill. I.es cornets sont rulinentiares. Filles s'vurrent posléricurement dions li cavile de la bouche, mais, a lexception des rrucodiles, pas loin du bord antéricur du palais. On a observe

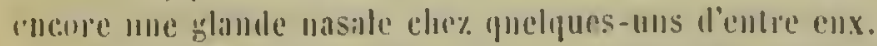

Les marines des protecs sont forl gramles, du moins les cavites, el presentent des replis en forme de branclies, comme (1II roll roil commumuenement dans les poissons.

Polssons. - Cequi distingue surloul les fossesnasalesules poissons, cest yu'au lien de s'unviri dans la cavité de la bouche comme

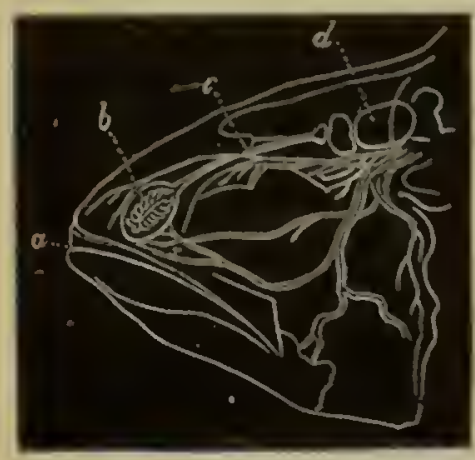

Fig. $325,-$ Nacine de la perelle (ciiviler).

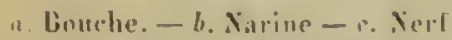
olfalif. - d. Cinvean.

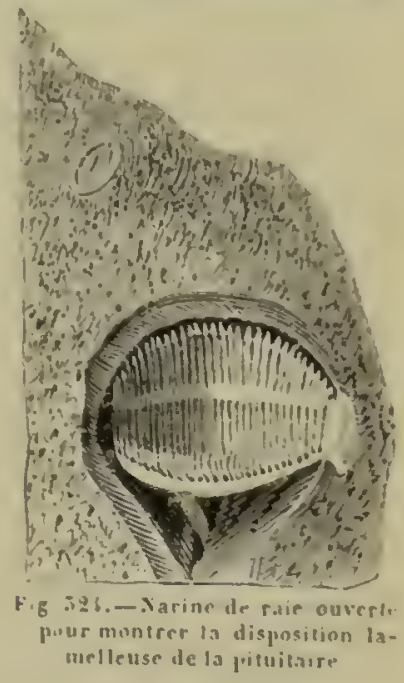

llanslesclasses jucidedentes, clles se lerminent postiricurement en

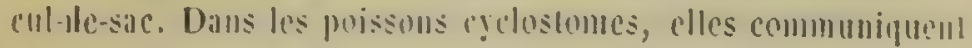


par exception avee la eavité buceale, et les narines sont situées sur la ligne médiane. Celte fosse s'ouvre souvent au delırs par' des ouvertures non loin l'une de l'autre el dont l'antérieure esl souvent prolongée en tube. Chez beancoup de poissons, la eavité olfaelive est fort grande el tapissée par une membrane régulièrement plissée comme une branchic. Souvent les narines se fernent par des replis menubraneux qui agissent eomme des valvules ; e'est ce qu'on voit surtout dans les poissons plagiostomes où les orgines sont lonjours très-éloignés l'un de l'au tre; on les voit à chaque angle de lia lonchic et affectant une fornte différente dans chaque espèee. M. J. Huller a signalé un tétrodon sans narines exléricures, avee des nerfs olfaetifs pénetrant dans des tentacules ou barbilions.

A la base de la première paire d'antennes (petites antennes), il existe dans plusicurs crustaces léeapodes un sac dont Rosentlıal a donné la description, ct qu'il considere comme un organe olfaetif.

Les mollusques eéphalopodes, les seuls parmi les animaux sans vertèbres qui portent ees organes de sens, préscutent dans le voisinage des yeux une fossette, ouverte à l'extérieur et montrant au fond une papille; un nerf naissant des ganglions susaesopllagiens à eôté des nerfs optiques se rend à ees organes. C'est avec raison qu'on les regarde comme un organe de l'odoral.

Il $n$ 'est pas douteux que la plupart des animaux sans vertibres jouissent de ee sens, qu'ils distinguent les odeurs el que la délicatesse de ee sens est même portée à un hatut degré, mais jusqu'iei on n'a pas reconnu un organe que l'on puisse eonsidérer comme en étant le siége. 


\section{APP.MREI, DE: LA GISTTION}

L'organe qui est le siége principal du sens du gout est la langue. Daus tous les vertébrés celte laugrue est farile à recouuaitre, tamlis qu'olle est en général peu distiucte chez. les ani matux saus verteberes. La plupart des aumaux distinguent li mature de leurs alineuts; mais, chez. beancoup dentre eux, il I'y a aucune undification à l'entrée du lube digestif, el cliez. quelques-uus lo tube digestif manque, comue cliez les vers cestoïles el aleanllocéplates.

La laugue de tous les nammifires est molle, musculeuse, batignce par la salive et couverte de papilles. Ces papilles soni
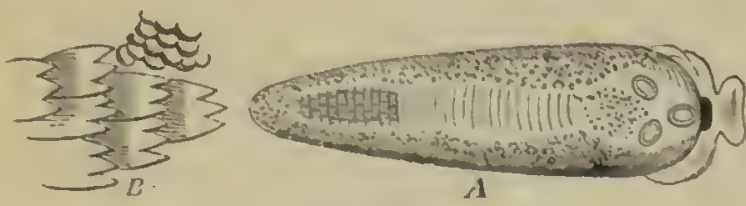

Fig. : :as.

A. Langue de roussette. - B. Papiltes grossies.

cormées chez quelques-uns et servent ì retenir la proie, comme 'liez. plusieurs carmassiers, ou bien elles sont charnues et ne manquent guère que chez. les cétacés.

Le hírisson a la langue en arant couverte dieailles tris- 
dures; chez d'autres manmiferes, ee sont des soies ou des êpines (iig. 523) qui la recouvreut.

Les papilles les plus remarquables par leur taille el la constance de leur nombre sout placées à la lase de la langue en forme de $\mathrm{V}$; on les appelle, à cause de leur forme, papilles calyeiformes.

Elles semblent manıuer dans plusieurs eétacés; on ell voit

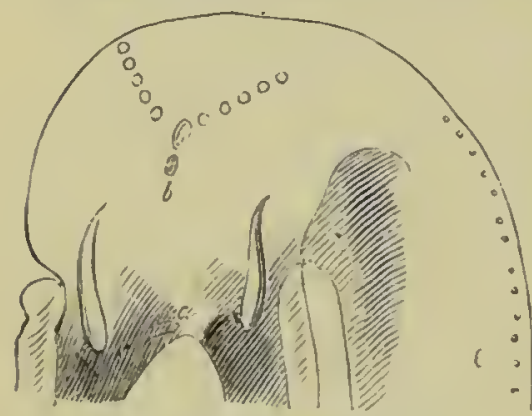

Fig. 326. - Langue d'ours

a. Papilles ordinaires. $-\boldsymbol{b}$. Papilles calyciformes. une seule elez les kanguroos, deux cliez quelques édentés, deux à trois elıez les cliẻiroptères, les cэruassiers e॥ général el les elıevanx; einq dans quelques quadrumanes, et le IIomlure chez d'autres s'éleve parfois jusqu'd dix et douze (fig. 526), par exemple eliez l'ours.

La constance du nombre el de la forme dans claque espèce semble bien iudiquer que ees organes se rattaclient au seus du goùt.

La laugue des oiseaux (fig. 527) est généralenıent peu molle, $\mathrm{et}$, à l'exception de quelques oiseaux, comme les perroquets et les eanards, elle est peu propre à l'exereice de ee sens.

Elle varie bealucoup daus sa forme el sa grandeur; elle est rudimentaire elıez le pélican, très-grande, au contraire, clıez les cygnes, les oies et les eanards; aussi ees derniers oiseaux sout complés parmi le petit nombre de eeux chez lesquels la langue est un organe de gustition.

Elle est généralement rigide, lure et couverte d'un étui corné; clle est quelquefois pénicillée, eomme ehez

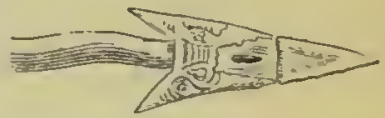

Fig. 327. - Langue de Thalassidroma pelagica. les eolibris, ou en forme le plumes comme cliez les foucans.

Les reptiles out en général une langue molle; sil forme of son développenent sont extrèmement variables. 
Eille est tellement indimentaire chez les crocodiles, yue les aneiens niaient son existence; et, en effet, elle ne consiste que lans nu simple repli qui s'étend le long des deux branches de lat luiclioire.

Lal langue est au contraire extrimement diveloppée chez les camcléous: elle est deveute protractile el sert à la préheusion. On a toutefuis remarnane une partie gustalive an bont; la pointe esl garnic de papilles et se tourne vers le gosier. lal langne des chóloniens est peu levelopree, large: "t plate (fig. 328). Eille est divisece en ivant el sonvent aussi en arriere :

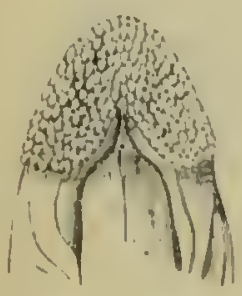

Fig. 328.- Langue de tortue.

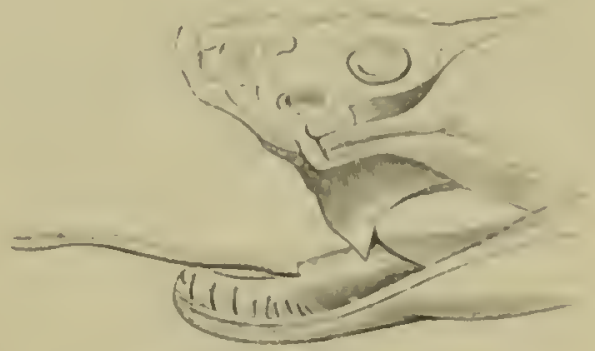

Fig. 349. - I.angue de Irigunuceflate

relle division est filible chez les geehos el les iguanes, plus profoinle eluez. Ies lezarils el semblibble ì rello des serpents chez les varaus. Elle est trís-longue, élloite et profomlément édiancrée elez les serpents; on l'appelle sousent dard. Elle est logice dans une gaine forme anx dépens de la muquense de la bouche (lig. $52 !))$.

La laugue des batraciens est loujours molle; elle est formec ule quelyues rides elez les sirines el les pipas, au point que eet urgane semble manpuer complétement. Elle esi pelite ehez les récilies, proteus el salamimelres; grande, aplatic, trontude et libre en arriere clez la plupart des anoures, à l'exception des rainelles.

La laugue des poissons parail preu propre à l'exercice du sens (lu goùt; clle est rudimentaipe e't salts mourement intrinsedue; souvent elle est dure el girnic de dents.

Ies eyprins el surlout les carpes portent en arriere du palilis 
uII organe contraetile que les gourmets connaissent sous le nom de langue de carpe; ect organe reçoil un grand nombre de uerfs, et si on consilère que ces poissons sont phytophages, il ne pa. raitra pas trop laasardé de le considérer comme étaut le siége de re sells.

Les autres poissons sont earnassiers, avalent leur proie saus la dépecer et sans l'inır'ugner de salive, ce qui est peu favorable à l'exereice de ce seus.

Chez les articules, le sens du goùt s'exerce sans doute dans lit cavité de la bouche, et le uerf spécial provient probablement tes ganglions suns-cesophagiens; mais on ne eonnait rien de certaiu à ee sujet. La cavité de la bouche est tapissée par une membrane délicalte et imprégruée généralement de salive, mais il u'y a pars d'organe special pour l'exercice de ee sens, pas plus que dans les deruières elasses du rèğne animal. 


\section{APPAREIL IOU TOUCHER.}

Considirons d'abord la peau sous te rapporl du sems du louclier, quelle exere quelquefois sur la plus grande parlie de son écendue.

Toucher. - Le siége de ce sens pent risider ditus toutr

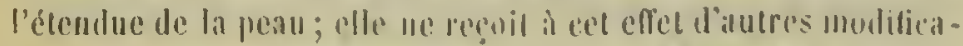
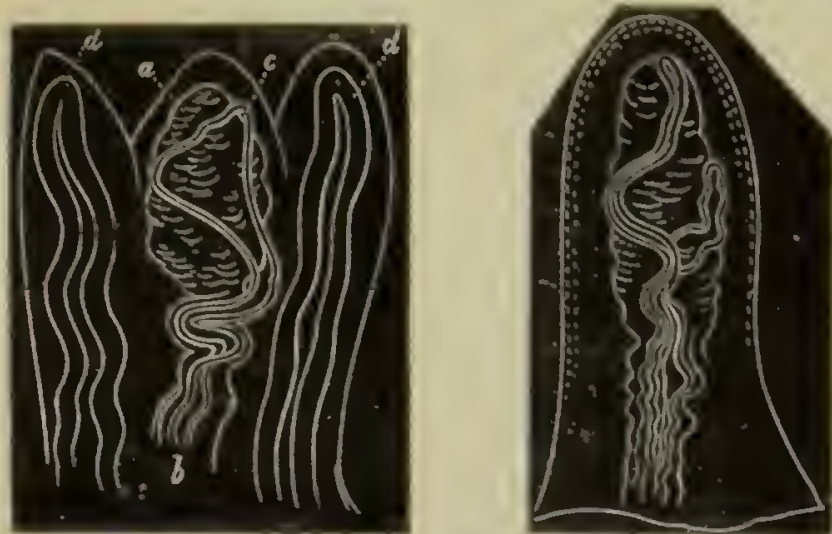

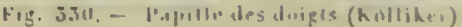

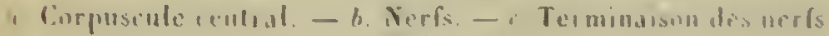
- irissentis

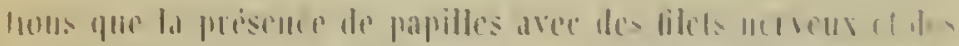
allses visculatires (lï. $3 \overline{3}(0)$. 
Ce u'est que dans ces dernières années que Mcissner a fail connaitre la composition anatomique de ces papilles.

Il existc deux sortes de papillcs, les unes vasculaircs el les autres nerveuses. Les papilles vaseulaires sont plus nombreuses que les autres $\mathrm{ct}$ sont formees d'une ansc vasculaire et d'une enveloppe; les papilles nerveuses sont logécs au milicu des autres et uc reçoivent pas de vaisscaux. Elles sont formécs de mcmbranes horizontalement superposées et ayant cntre ees couches membraneuses de nombreux graius oblougs et d'unc couIenr foucéc.

Depuis, Gcrlach a vu des vaisscaux dans toutes les papilles, qu'elles aient des eorpuseules ou non.

Les principaux organes du toucher sout les lèvres, les doigts, la langue et le nez; e'cst dans ces organes que les papilles sont le plus développées.

C'est le bout des doigls qui cst le siege de ce sciss clrez les quadrumanes en général; le bont de la trompe clicz l'éléplıaut, les feuilles du nez des rhinoloplics, les pavillons de l'oreille ct les ailes des chéiroptères; le boutoir du sanglicr, de la musaraigne, des luérissons et des taupes, surtout des condylures; enliu la levre supéricurc des carmassiers et surtout des phoques; un a eonjparé avee raison les soies qui garnissent ces lèvres aux barbillons des poissons et alux pilpes des inscetcs.

Cliez les oiseaux, le sens du toueher est tris-obtus el ne pent que difficilement s'excrcer, puisque tout le corps est couvert ou d'écailles ou de plumes; aussi scmble-t-il résider chez. quelques oiseaux, par cxemple les pies, dans la languc ou bien au bout du bec, comme cliez les canards el les béeasses. Clicz ces llerniers il se forme cu effet un apparcil spéeial pour discerner les objets pal le bout du bec. On peut cncore eonsidérer le dessous des doigts comme siége de ce scns.

La langue des reptiles scmble cu général servir anssi d'or ganc du toncher. Les serpents et beancoup de samricus dardent constamucut leur langhuc bifide avee liutention évidente d'explorer. On peut considerer encore comme organes de listl les pieds de plusicurs l'eulre enx. 
Les batraciens exereent sans doute le toncher par tonte la surface de leur peau, mais c'est toutefois lans les mains el les pieds pue doit résider surtont ce sens. Les rainelles out des ventouses au bout des doigts; les pipas, les lanirres molles pui soul silns doute propres it l'exercice de ee sens.

On tromve diuns la pean de plusienrs: poissons fluviatiles, surtout aul pourlour de lis bouche, les pappilles singulierement conformés ol pui servent sills antenu doute a l'exereice da sells du tourler. Ces papilles sont ennformées dianès le même plan que celles les mallmiferes (lig. 3.31 ); on en voil surtuul chez les silures, las carpes, plusirurs giales, l'esturguron, ele. Le's trigles mut iो elanpue nagenire pectorale puelentes rayous libres en formic de doigh yui leur servent pour mar. cher an fond de lenal el qui regenivent d’inormes filets merveux; la hamdroie

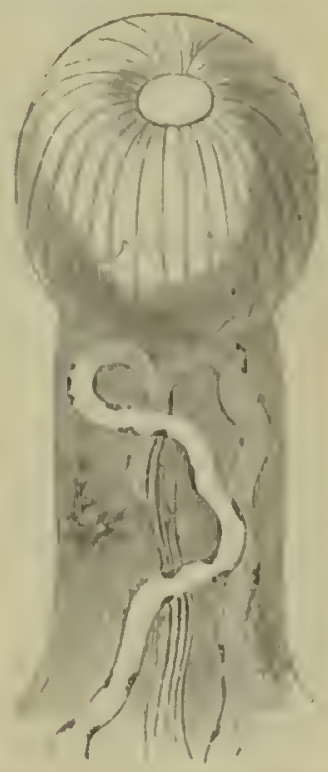

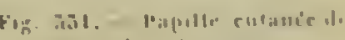
la teste d'un lescisctos

(E. Lepdig.) pétheresse porte une lige molile termince par me feuille membranemse sur la tete, ef des fila. meuls membrineux autour de la mieloire; des seorpines el d'antres poissons portent eneore les appendices membranem qui sorvent probablement an concher.

Clecz les animaux articules, il faut placer ce solus daus les divers appendies eounus sous le nom d'antennes, de pialpes el meme daus les patles; le reste du corpsest emveloppe diune cuirasse solide ordinairement corme on ealeaire el peut propre à lexercice de ce sens.

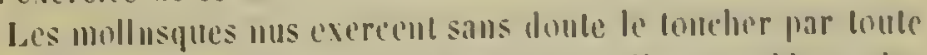
la surface du corps; mais quelijues appendices semblent plus

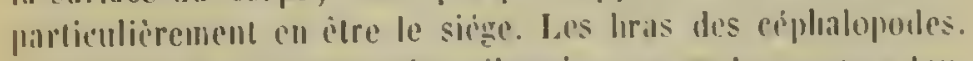

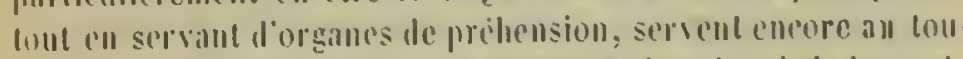

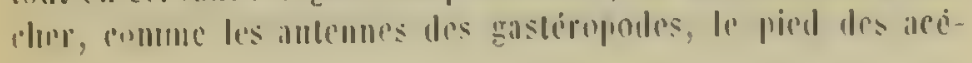


plıales ainsi que les lanières qui bordent le manteau el les lenlacules des bryozoaires.

Les ver's à saug rouge ont souvent de nombrenx appendices autour de la lète pour servir d'organes explorateurs.

Les échinodermes, les polypes et les autres, s'ils u'ont plus

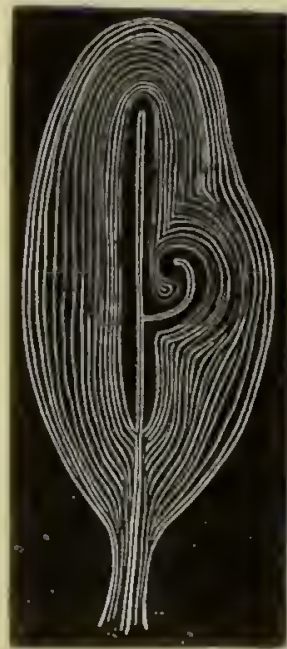

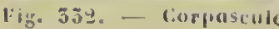
de Pateini (kollih(or).

un organe spécial, ont cependant encore en gèncral quelque prolongement de la peau, comme les cirrlies des acaliphes, les bras des liyilres, les teulacules des polypes, les filaments des rhizoporles qui servent d'organes du loucher.

Les corpuseules de Pacini (fig. 532), que l'on a observés maintenant dans divers organes ef che\% un grand nombre d'animaux, palraissent devoir être considérés commue les papilles exereant le sens du loucher.

Ces corpuscules consistent dans une ciplpsule formée de plusieurs couches, an centre desquelles se termine un filet nerveux.

On les a observes daus la paume le la main el la plante des pieds; dins les frolubérances sous le pied des chieus el des clats; sous le borl supérieur corné dll sabot des rumiIIIIIts, ele.

Ces corpuscules ont aussi été observés daus la langue des oiseaux el a la partie antericure de leurs deux mandibules.

Peau. - Nous venons d'étudier la peau sousle rapport du sens de tact in'elle exerce; eximinous-la mintenaut sous le rapport de sa composition dans les diverses classes du rigune animill.

La pean est forméo de deux couclues parfaitement distinctes: le derne et lípiclerme.

Elle porte des glandes de deur sortes : les mors versent un linuide propre à lubrilien la surface; les andres produsent de: corps solieles, formes de couches juxlapuseces of yutou appelle: poils, nugles, reillles, plumes, cle. Les premiches soul desi 
guées sums le nom te cryptes, les antres sous le num de phanères (de Blaimsille).

Comme fallice accessoire de la pean ou peut eonsillerer ta

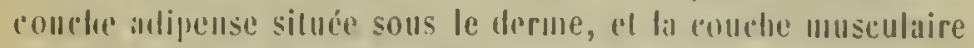
culamin, yui preml yuelquefois une gramde "xlension.

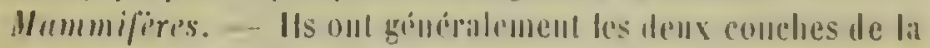

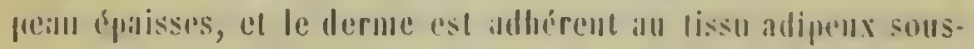

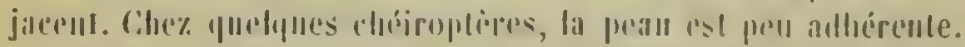

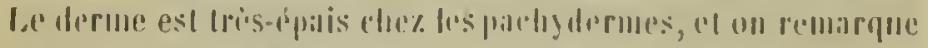

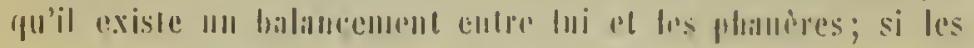

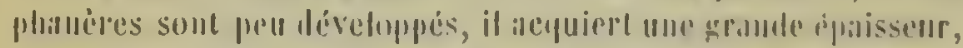

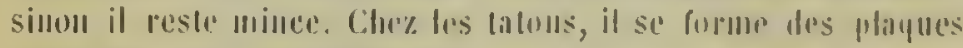

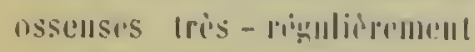
disposées dians l'iprisisceur du derme ot yui constitucut mu syuelelle entame.

L'épiderme varie beancoup selon les réginus des corps qu'it reconvere el selun les minmulfires. Il forme des callhisilessur prossque touth lia salle face du corps, roumme cha! la? hlinocedos, on so developplo semlemint ans fesses, commun chrz les simages de l'anrien bonlimenl, anx gemonx et il la poildiue des chameaux, aux membresdes solipides (clucval, ime) at alux pieds des mamumifirms onguicults. Omehpuefois lépiderme gorme des playues imbringues, soil sul loul lo corps romme diez. les piangulims, soit sur la proue. commor

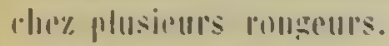

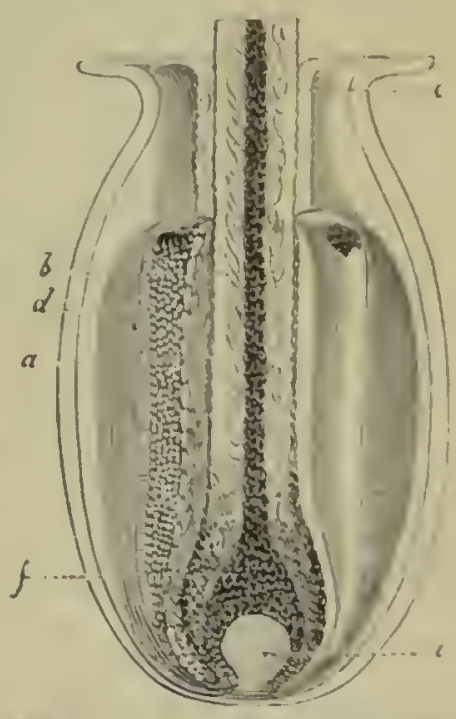

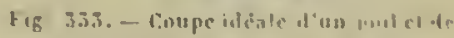

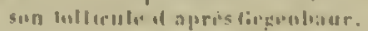

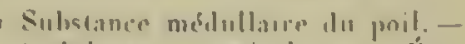

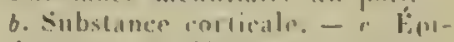
derme - d. Glameles - e. l'apullo pilense. - $f$. Inlpe el elépirt du progenent alus lit racine dis prit.

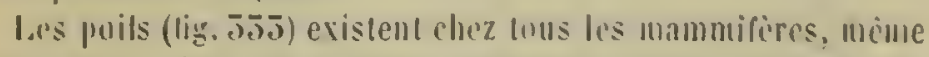
les celatés: clez ces derniers its nipplaraissent toulefois que 
dans le jeune aige. On les appelle piquants quand ils sont gros et pointus, comme elıez les porcs-épics et les liérissons; ils for. ment les vibrisses ou moustaclıes des cliats (ijg. 554); ce sont les soies chez le cochon, des crins au liaul du cou el a la queue du elieval, de la laine ou du duvet ì la base des soies ordiuaires chez les espèces qui vivent dans les pays froills. Ils sont aussi quelquefois cannelés, couverts de tubercules perles (fig. 554)

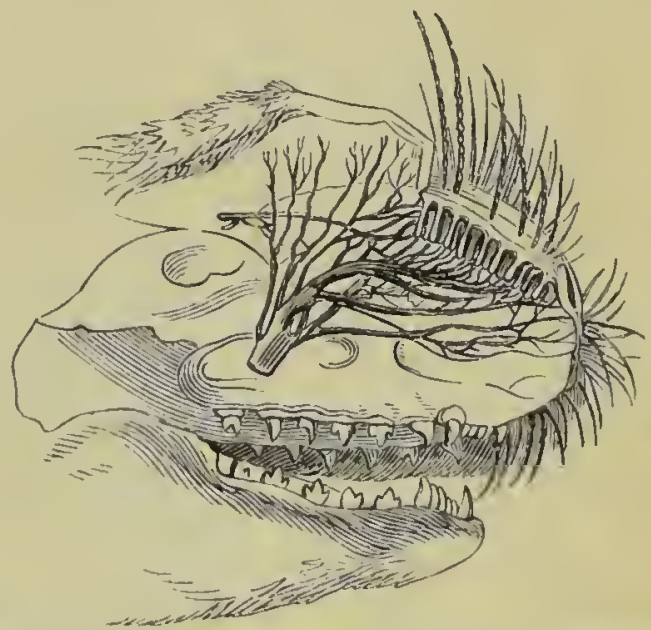

Fib 33 f. - Neri suus-orbitaire de ploque se perdant dans les vibrisses.

ou rantifiés, el semblables à des épines. Dans les piquauts dı porcépic, les vaisseaux pénètrent jusqu'à la substance médul. laire.

Les ongles et les cornes creuses se forment, comme les poils, a l'aide d'une pulpe qui séerèle ees eorps solides.

Nous avons vu plus laut les diverses glandes qui répandent leur produil à la surface de la peau (p. 147).

La couche adipeuse est très-rléveloppée clıcz lous les mantunifères aquatiques. Elle remplace les poils cliez ces animaus. C'est surtout pour cette graisse qu'ou leur fait la cliasse.

La couclı museulaire cutanée est en géméral très-developprée le loug du dos; elle permel au elat de faire le gros dos. Elle recouvre tout le corps et acquiert une grande ipaisseur eliez. 
le liérisson el permet à l'animal de s'enrouler dans sa peau (lig. 53i).

La peau des oiseaux se distingue par la minceur du derme

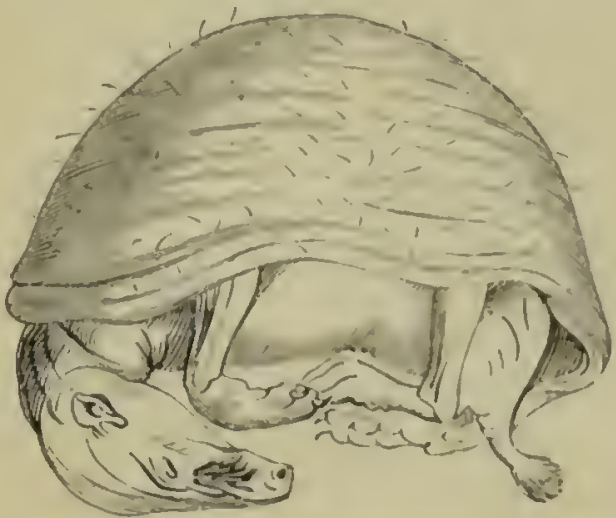

Fig 3.i.i. - Mirisson at derri enroule pour montrer son musele peatucic

et la richesse des vaisseanx qu’il eontient, par le peu de déseloppentent de l'épiderme el entin par la nature particuliere des planires qui sont coumus dans eelle clisse sous le nom de plumes. Tous les oiseaux en out le eorps entièrement couvert, i) l'exception de la falce el des membres postírieurs.

Lépiderme est tris-développé à la face el recouve le bout des deur mandibules; il forne un élui comu sons le nom de hec. Sur le tarse, el quelquefois ì l'aile, il recourre une saillie osseuse el prend le nom d'éperon, on bien eneore il forme des cécilles de formes très-variées el qui reeouvrent le tarse el les loigts.

La peau des oiseaur n'est jamais doullée d'une épaisse eouche adipeuse, el elle n'a jamais non plus une couche musculaire tris-rléveloppée.

La peau se délache quelquefois complétement, ne lient que par quelques brides, el l'air circule tout autour du eorps entre la peau el les muscles (sula alba).

Les plumes se formeut, comme les poils, dans l'épaisseur du lerme: elaque plume est forméc: lo d'un luyau ou partie creuse renfermant souvent la pulpe qui a produit la plume, 
20 d'une lige, et $5^{\circ}$ te barbes. D’apris le développement des barbes, on les divise cn pennes, lorsque les barbes soul plus longucs d'un côté que de l'antre, et eu plumes, lorsqu" celles-ci sont égalcment développécs des dlcux còtés. Souvenı il s'attaclıe de petils museles à la gaiure de la penne pour lia mouvoir.

Les reptiles ont en général la peau ćcailleusc.

Chez les chélonicus, il apparaît, dans l'épaisseur du clıorion, des plaques osseuses qui s'unisscut aux os ct forment, par lcur réunion, la carapace. Ces os cutanés sont placés sur les verlïbres, sur les côtes et puis en dehors des côtes, ils forment une bordure entre la carapace ct le plastron. L'épiderme cs! très-épais et constilue dans certaines espèces dc grandes plaques cornées qui sont connues dans le commerce sous le nom d'écaille.

Les crocodiles portent aussi dans l'épaisseur du derme de grandes plaques osseuses disposées avec symétrie et formant des saillies tlans quelques régions du corps. L'épiderme cst trèsdévcloppé au-dcssus de ces plaques. Le derme est mince clıez Ics autressauriens, et la peau est couverte de rngosités dans les geckotiens et les caméléons.

Les caméléons el d'autres sauriens encorc, qui changent dc

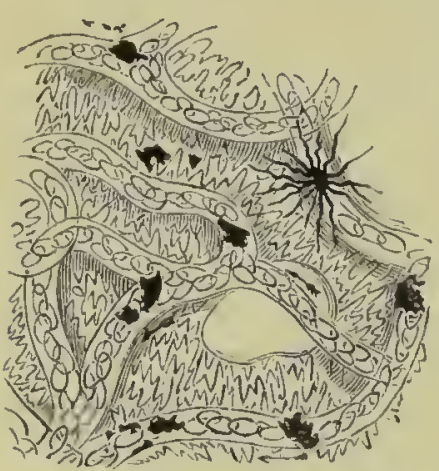

Fig. 336. - Pigurenc de grenouille. couleur, portent des tubercules sous l'épiderme et deux couches de pigment superposécs, de façon à pouvoir sc montrer sous l'ćpiderme simultanément ou sc cacherl'unc au-dessous de l'autre.

Lcs serpents se dépouillent de leur épidcrme en cntier tepuis la bouche jusqu’à la queue. Un nouvel épiderme se montre en dessous après la chute du premier, et scmblc rajeunir l'animal. C'est pourquoi les anciens avaient pris le serpent pour l'embleme de l'immortalité et sans doute aussi pour celui de la médecine. 
Les eryptes sont assez abondanınient répandues sur le eorps de differentes esperes.

Les autres saurieus ont la peau éeailleuse comme les oplriliens; tantot ees écailles sont formées par le derne, tantôt par l'épiderme seulement.

Les hatraeiens out la peau nue et visqueuse. Elle n'est que faiblement adlirrente aux museles et montre entre elle el les Hairs de grimdes lacumes lymphlatiques. Le derme est nimee, convert de plaques de pigment (lig. 556 ) at d'un spiderne dout les eellules montrent distinetement le $10 y$ an (fig. 5.77).

Les siliamandres et les erapauds ont des glandes dans l'épaisseur de la pean qui sécrèlent une visensité îcre qui se répand à la surface de leur eorps.

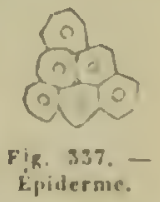

La peau des poissous varie extraordinairement, tant sous le rapport de sil conıposition elle-meme que sous le rapport des écailles qui se forment daus son épaisseur.

Onl distingue partout le derne et l'épiderme. Celle derniere couche, saus nerfs et saus vaisseaux, composíe de eollules juxtaposérs comme daus les autres classes, est lissect généralement visqueuse par la nature mème de ees eellules superficielles.

Le derne diffère de celui des vertébrés supérieurs ell ee que les libres cellulaires qui le eumposent, au lieu de former des faiscraux rutre-croises, forment ici des eouclies de bandes parallèles qui se croisent. Le derme est ordimairement trisadhérent anx muscles et montre quelyuefois à sa faee inféricure une couche de pigment ou une eouelie de graisse.

Les éeailles des poissons (fị. 358 ) ue sont pas des dépenlanees lle l'épiderme; elles sont généralement renfermees dans des poches dépendantes lu derme; leur surface inféricure est couverte de cristaux qui brilleut d'uu vif éclat souvent argenté; une menhorane tris-fine indépendante de la peau les enveloppe ('t ou voit a leur surface des lignes eoncentriques arec un eoutour arrondi ou deutelé. On a signalé des eorpuseules osseux dans quelques exillles qui deviennent dures eomme desos. O॥ doit comprarer ces partics au squelelle eutauc. Les esturgevils et dautres out le eorps couvert de grandes plaques 
osseuses disposées avec symétrie el qqui sont revêtues d'une couclie d'émail comme les dents.

La peau des animaux artieulés est distinctement composée

Fig. 338.

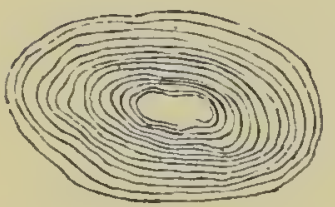

Étaille eycloïde de salmo fario.

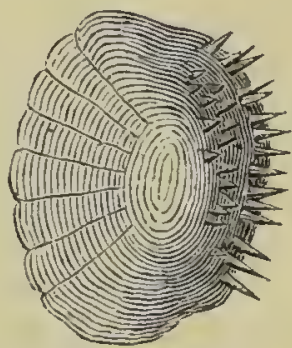

Écaille cténoïde de perea nluviatilis.

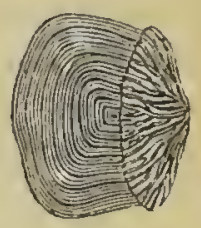

Écaille ganoïde de glyptolepis clegaus.

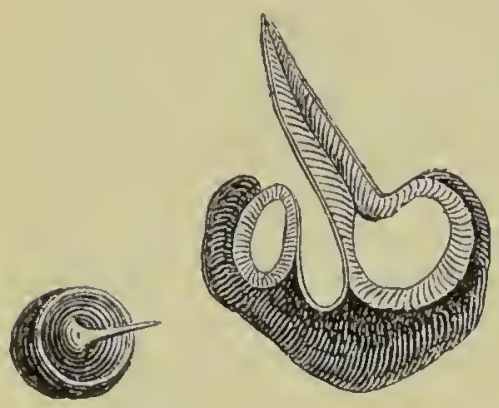

Écailles placoïdes de raia clavata.

eneore d'une conche épidermique el d'un derme ou ehorion. On distingue dans l'épiderme les cellules lamellées ou polyédriques el dans le derme des eouclies fibreuses singulièrement juxlaposćes el formant des dessins réguliers.

On voil souvent des écailles microscopiques formant un duvel autour du corps et qui se délaclıent avec une graude facilité, comme les écailles des papillons.

La peau de ces animaux se divise loujours en anneaux ou segments qqui, avee les appendices cutanés, forment le squelette culané dont nous avons parlé plus liaut.

Elle est ordinairement cornée ou caleaire el montre dans sa eomposition une substance chimique désignée sous le nom de chitine. Celte chitine se combine souvent intimement avee le earbonate el le phosphate de chaux dans les tèts de nature calcaire comme chez les crustacis.

Deux espèces de pigments se présentent quelquefois en cris- 
lanx reguliers de diverses formes el de diverses conleur's thez les crustacés. L'écevisse présente ces denx sulıstances réunies. Au pignent rouge est mêtê un pigment bleu en eristaux prismalipues qui se détruit par l'aetion de la chalemr el des acides, él se dissout lentement dans l'alcool. Voila pourquoi ees auimaux deviennent rouges alpres l'ébullition el après leur séjour dans les lipueurs spirituenses.

Ial eomposition de la peau est la meme daus les cirrlijuides, dont les coynilles ne sont qu'un étal d'incrustation calcaire de l'épiclerme.

La mue n'est que la chute de l'épiderme comme dans les vertibrés.

La peau des mollusques éphahopodes se distiugue sous julusieurs rapports. Dibhord elle est lonte distincte et s'muil aur muscles sons-jacents par dı tissu cellulaire très-liche. Elle montre distinctenent les deux eonches ordinaires, le derme el l'épiderme.

Le derme est assez ejpais el forme de lissu fibreux contraetile; e'est dans ce tissn que se forment les mailles an milieu lesinelles se logent les eellules cliromatophores, dont nous parlerons lout à l'heure.

Il y a des céphalopodes a corps mu, comme les poulpes; d'antres out une coquille iuterne, comme les calmars; d'antres encore ont une eoquille externe dans laquelle ils penvent sabri(e) amplement, eomme les mantiles el les argonaules.

Les bras sont armes de ventouses, et dans ees rentonses on trouve ordinairement, sinen tonjours, un eerele eorne qui depend de la peau.

Dans l'êpaisseur de la peau des céplanlopodes se tronvent des cellules en partic remplies de grannles de pigment; quand les fibres du churion se contraetent, ces cellules paraissent déchiqueties; el lorsqu'elles se relichent, les cellules s'arrondissent par leur propre élasticité. Les gramles de pigment peuvent s'accumuler sers la périphérie en abandonnant le eentre de la cellule; éest ce qui produit alors l'effet d'un noyau au milieu.

On il observé des cellutes chromatophores sur l'autres mollusques, cutre autres chez les cymbulies. 
Le chorion est dense chez les gastéropodes, et, chez tous ceux qui sont aquatiques, il est couvert d'un épithélium vibratile. La couche musculaire est intimement confondue avec le derme et rend la peau très-contractile.

Les gastéropodes sont en général pourvus d'une coquille univalve en spirale et qui sert d'abri à l'animal. Il y en a quelques-uns qui ont une coquille interne, et d'autres en sont com. plétement dépourvus.

La peau des mollusques acéphales montre généralement dans son épaisseur une coquille d̀ deux valves, qui protége puissamment l'animal contre ses ennemis.

Les tuniciers ont une peau membraneuse, coriace ou même d'un aspect cartilagineux e! qui contient de la cellulose.

Les bryozoaires portent dans l'épaisseur de la peau un dépòt membraneux, corné ou calcaire qui constitue le polypier. C'est la coquille d'une colonie. La couclıe musculaire est intimement unie au derme, dans les régions du corps qui ne sont pas incrustées. Une grande partie du corps est couverte, comme chez les précédents, de cils vibratiles.

Les vers ont un épiderme qui offre exactement la structure de l'épithélium à cylindre, et au-dessus de cet épiderme s'étend une membrane finement rayée et pourvuc de nombreux pores, nommée cuticule par quelques naturalistes.

Les couches les plus profondes de l'épiderme contiennent du pigment.

Le derme a souvent plusieurs fois l'épaisseur de l'épidcrme. II consiste en couches nombreuses de fibres entre-croisées, réunies par du tissu cellulaire, et contient des filets nerveux assezabondants, surtout chez les annélides sans tube. Ce derme montre souvent un réseau de vaisscaux sanguins capillaircs assez développés dans les vers à sang rouge.

Plusieurs vers portent dans l'épaisseur de la peau des incrustations calcaires ou cornées, qui deviennent cliez quelques-uns d'entre eux des tubes dans lesquels l'animal peut s'abriter. La surface de la peau cxhale souvent une viscosité qui se transformc en tube membraneux.

La peau des vers intestinaux se distingue par la facilité avec 
laquelle elle absorbe l'eau, el eette faeulté d’absorption continue eneore après la mort. Tout ver intestinal se gonfle daus ce liquide.

Les échinodermes, à très-peu d'exceptions pris, possident IIı squelette cutaıé conformé d’après un plaı particulier.

Plusieurs polypes dans l'épaisseur de la peau possèdent une charpente solide de uature cornée ou calcaire. C'est le polypier.

Dins plusieurs acaliphes, le contael de l'animal avec la surface du corps produit une seisation brülante; e'est que la peau de ces animaux est garnic d'organes particuliers cumus sous le nom d'organes urticaires (fig. 359). Ce sout des ealp. sules renfermant un filament contourné en spirale, qui sort all plus léger contact el se lélaclie avec sa capsule. Il

existe ell outre des organes a. Capsule, - b. Filament urticaire. de préleusiou dans ees mimes

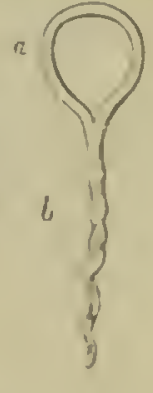

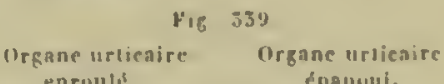
acaliphlies; te sont des anis de soies qui font saillie sur divers organes.

Outre les organes urticaires, plusieurs polyjes out, eomme les liylles, des organes en hamęon, qui eonsistent en vésicules, d'oì sort un filanıent renflé et visqueux à son extrénité libre et qui continue avee le col de la vésicule.

Les polypes ont le corps couvert de cils vibratiles daus le jeure ìge au sortir de l'ceul.

Les infusoires ont une peau lisse el distinete ou enuverte de eils vibratiles serrés et placés régulièrement d la surface du eorps. Elle est gélatineuse el sćerète tantôt une couclic tout autour du corps pour l'enhỵster, tantòt une euirasse ou un étui. 


\section{APPAREIL ELEGTRIQUE.}

Cet appareil établit des rapports entre l'animal qui le porte at le monde extérieur, c'est pourquoi nous en parlons ici après les organes des sens.

Quelques animaux dégagent l'électricité et portent à cet effet uII appareil spécial souvent assez volumincux; d'autres dégigent de la lumière pour éclairer le milieu dans lequel ils vivent.

Jusqu'à présent les poissons sont les seuls animilux cliez lesquels on a trouvé un appareil électrịue, et eeux qui portent ces singuliers organes appartiennent à des ordres divers.

Ces organes n'ont ni le mème volume, ui la même strueture; ils n'occupent pas la mème région du corps, el s'ils sont toujours liches en neris el en vaisseaux, ils tirent leurs nerfs comme leurs vaisseaux d'une souree différente. Les uns en effet recoivent leurs nerfs directement du cerveau (torpille), les auties de la moelle (gymmole).

Oıl a trouvé ect appareil chez les poissons suivants : lorpille, nareine, symnote, mormyre, gymnarclie el malapterure. Dans la queue des raies on a obscrvé un organe particulier construit comme l'appareil ćlectrique, mais yue l'on u'a pas vu 
dégigrer de luide électrinue, malgré les essais qui l'on a tentes. Chez les lurpilles (lis. 310 ), on voil deux organes sur le cule

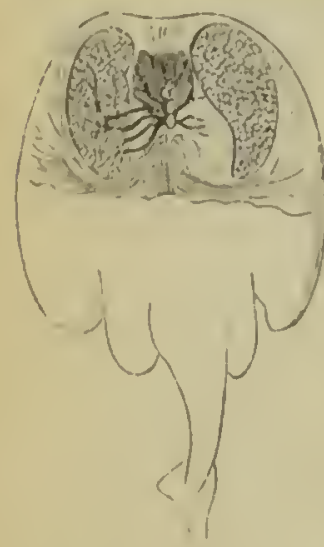

Fig. 360. - Torpille montrane. curaleguis les dent urganes elecirigues et les nerfo qui partent du cerreau jour s'y rendre. de la téle, culre les parois du crine el les membres antericurs, immédiatement en dessous de la peau; ils sont (1) més d'autant de eulontes verlicales yu'ou voil de ligures polygones à la surface. Clanque colonne est composie se nombreux diaplungemes membraneux yni séparenl l'intirieur en un grand nombre de cavilés eloses ou de cliambres, remplies d'un linuide gélatineus ou albumincux. Les libres qui composent ces eolum el ces cloisons tiennemt beaucoup do tissu élastique.

Qualre lrones nerseux sc renden! dans elaneun de ces urganes; le premier mait de la truisieme branche du nerf Irijumean, les trois autres proviennen! les nerfs branchianx du pneumogastrique.

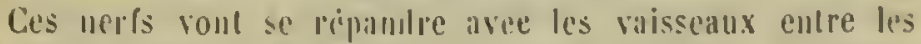
parvis des elvisons "l lipillilimm qui les recoure, au milieu l'une substance transparente.

Les gymmotes blectriques ont quatre organes éleetriques platés dans la queue an-dessus de lia mageoire anale, dont les superrieurs sont plns grands que les autres qui sont placés en dessous. Les colonnes sunt dispostes horizontalement, el les nerfs, au nombre de plus de deux cents, proviennent des nerfs spinaux.

Les phemomenes physico-ploysiologinues de eet appareil si singulier ont ététudies aree le plus grand soin par Malleucci, mais il est bien loin deitle sufisamment comnu dans lout ce yuil ofre d'important. 


\section{POSTFACE.}

Nous avons promis daus l'introduelion (page 7) de donner, dans une note, quelques explications sur la distribution des animaux en trois embranehements et de faire connaitre les modifieations que nous proposons d'introduire dans l'arrangement de quelques classes. Nous publions ici eette note et nous la faisons suivre de divers tableaux qui ont pour objet la réparlition des groupes prineipaux dans ehaeune des classes du règue animal.

Nous profiterons en même temps de eette occasion pour faire eonnaître les prineipaux ouvrages dans lesquels nous avons pris un certain nombre de figures, qui sont interealées dans le texte. Nous avons pensé qu'il serait non-seulement inutile de donnel loutes figures originales daus un ouvrage eonıme celui-ci, mais que souvent même on ne réussirait pas à en donner de meilleures que eelles qui existent.

Ces ouvrages sont: Cuvier, Histoire uaturelle des poissons; Milue Edwarls, Éléments de zoologie; Carus, Erlauterungstafeln, Owen, Todd's Cyelopedia, de Quatrefages, Gervais, Blanchard, Dugès, Kólliker, Eschricht, Vrolik, Brandl. Rusconi, Newport, Wagner, Tréviranus, V. Sichold, Schlegel, ete., divers mémoires. 
Il est inutile, peusous-nous, d'y ajouter que nous n'entendons ancmenent nous attribuer toutes les deseriptions que renferme le texte. Nous avons puisé la pllupart des faits dans les mêmes ourrages et nous les avons coordonnis d'apris le plan que nous avons fait connaitre dans l'introduction.

Nons avous évité autant que possible les eitations; il serait inutile el fastilieux, dans un ouvrage élémentaire, de faire a chanue anteur la part pui lui revient dins les décourertes. Lá mature de eet ouvrage demandait avant tout une exposition sim. ple et preeise des firts qui sonl eutrés définitivenent daus la seience; si, dans quelqunes eas, nous a vons crn deıoir nous cearter le cette rigle, en citant des noms propres, c'rst que utous avous voulu laisser anx auteurs l'bouneur de la léeonverte ou la responsabilité des faits.

Eu parlant de la reproduetion dans les dernieres classes du rigne animal, nous avons employé des expressions dont la rigonteuse exactitule pourrait ètre enutestée. Aiusi, ell faisant mentiou des polypes, nous avons dit que la merlusa anriti est gemmipare daus le jeune àge, scissipare it un ige plus avautei (nous aurions dij dire également gemmipare) et ovipare ì l'êtat adulte. Cela est rrai, en prenant les générations qui se suecident, depuis la sortie de l'œur jusqu'au développenent des organes sexuels, pour des gémérations qui chaugent avee l'àge; la medusa aurita est ovipare, non pas quand liudividu a alteint sou dernier terme de développenent, mais quand les générations qui sont sorties d'un reul sont arrives a ee terme. Dans ees animaux, ee n'est pas l'indivilu qui se métamorphose, ee sont les générations; ce u'est pas la chenille qui se eluange en papillon, c'est une colonie de polypes, sortie d'un seul aur, composée de plusienrs générations engendrées par gemmes, et dont les dernières seules portent des aufs. C'est eomme si une ehenille produisait plusieurs génératious de bourgeons et que les individus, nés par hourgeons, pussent seuls devenir papillons; une génération de elienilles dans cetle supposition serail suivie diune génération de papillons; la ehenille elle-mème ne se transformerait pas en papillon, et celui-ei à son tour ne passerait pas par l'ige ou la forme de la elsenille. La chenille est morte agame 
avant que les ailes el les organes sexuels ne soieut développés.

La premiere génciation, après la sortie de l'œuf, meurt avant l'avoir pris la forme adulte, mais avant de mourir clle a prodnit, par voie gemmipare, une autre génération. Celle-ei commenee son ćvolution moins bas que la premic̀re; en naissant, elle a presque la forme que l'autre avait en mourant el cette seconde genceration devient adulte el sexuée. On peut done dire que les individus de la première généra tion représentent le jeune ìge et que les autres représentent l'age adulte.

Les animaux qui présentent ee phènomène d'une double reproduction par gemmes el par cufs, agame el sexuée, nous les ilppelons digéuèses.

Quand il existe une sueenssion de générations formées d'individus de forme différente, nous disons qu'il y a digénèse avec hétérogonie, comme e'est le cas dans la méduse eitée plus lıaut.

Quand, au contraire, des généralions se sneèdent par oufs et par gemmes et que les individus sont tous semblables, nous disons qu’il y a digénèse avee homogonie.

La digencise avec létérogonie eorrespond à la génération allernante.

On peut eneore ètablir ensuile la digénèse hélérogone complète el incomplète. Quand elle est complète, les générations pareourent eldtierement Icurs métamorphoses; les individus sexués prennent leur rohe de noees; c'est la méduse qui a été engendrée par le polype, comme dans plusieurs campanulaires. La digénèse lıélérogone est au contraire incomplète, quand li robe de noees avorte el que l'animal est réduit à un sae qui porte les culs ou les spermitozoüles. Les sertulaires en général nous en fournissent un exemple.

Les individus de la première génération ou agames, nous les a vons appeles scolex ou proscolex dans la elasse des vers, et on pourrait étendre ee mol aux autres classes; par progloulis, nous designons les individus adultes et eomplets qui portent les organes sexuels. Ainsi les individus qui forment les généraltions entre deux reproduetions sexuelles etqui sout agames, portent, suivant la génération, le nom le scolex, de proscolex on de deutoscolex. 
Let's classes de l'embrancliement des verléliés ou des liypocolylêdones el des épicotylédones sont les mêmes que celles yut sout généralement atures; l'embranchement des allocoḷlèdones demande quelques mots d'explieation.

Nous réunissons daus un seul groupe les mollusques et les ladiaires dle Cuvier, inupuel nous ajoulous encore les vers el les

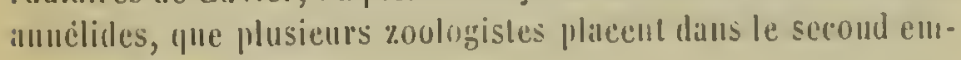
branchement.

On olserve, il est vrai, quelques animaux qui établissent le passage cutre les aumélides el les in lieulés; les vers ì saug rouge ont une chantne nerveuse gangliomaire semblables à celle des articulés, mais l'embryogénic fouruil des cararlines qui doivent primer les autres et qui éu font ul allocolylédone. Du reste, lly a-t-il pas de rrais articules dont les cordons de lit claine gangliomaire se séparent?

La classe des mollusques comprend ciny ordres : les céplialopodes, les gasteropoles, les alephluales, les tuniciers el les bryozoaires. Les ptéropmles de Cuvier doivent prendre plate parmul les gastéropodes, cl plusieurs d'enlre eux, conservant les earactires de l'ige eubryomaire, loirent se tromer a la yueue des gastèropodes.

Daus les deux derniers ordres, ou peut reconuatloe une génération préparatoire (scolex), daus le têtard des tuniciers et dans la larre ciliée des bryozoaires.

Les vers forneut une classe tres-riche qui comprend tous les animaux que le vulgaire désigue sous ce nom. Les ordres principaux sout á peu prìs les mèmes que ceux qui sout géméralement admis, mais ils sout autrement groupes; cetle dasso reuferme deux groupes parallèles dont l'un est diö̈hue et l'autre monoï,pe. La première idie de celte repartition appartient à M. de Quatrefiges.

Lil classe des échinodermes comprend les divisions qui sont généralement admises.

Par coutre, la classe des polynes est complétement bouleverscic; dabordl les bryozoaires en ont cété délinitivement retirés pour fremdre place parmi les mollusques, puis l'embryogénie nous a montre que les polypes el les acalephes sout beaucoup 
plus voisins les uns des autres qu'on ne le eroyait; le même animal est polype à telle génération et aealèplue à telle autre. De quelques-uıs de ces aninaux, on n'a eomın pendant longtemps que les dernières génẻrations; d'aulıes, on n'il connu que les générations préparatoires; et d'un grand nombre, olt ignorait et le développement el l'organisation. Un remanienent général de ees animaux est devenu neeessaire; il y a eneore bien des desiderata, mais nous u'lıésitons pas à réunir dans une seule el ınême elasse les aealèjhes et les polypes de Cuvier.

Les trois premiers groupes cténophorides, siphonophorides et diseophorides sont les mêmes que eeux qui sont génèralement admis, avee eette différenee seulement, que nous avons ajouté a ee dernier les eampanulaites et les tuhulaires qui me peuvent plus en être séparés.

Les siplıonoplıorides eonstituent un groupe extrêmement uaturel; tous ees animaux vivent en communauté el eonstituent des eolonies vivantes, daus lesquelles on reeomnaî les scolex (vessie flottante), les strobila et les proglottis; ees derniers ont la forme de méduse. La division du travail est poussée jusque dans ses dernières limites. Les diverses fonetions sont remplies par des individus d'une forme propre. C'est ici que la digémèse hétérogone est le plus développée; on voit des individus manreurs, nageurs, propragateurs, ete., ete.

Les liydres et les sertulaires forment aussi un groupe distinet, quoiqu'il y ait entre eux celte différenee fondanentale, ıue les hydres ont les bras rétraetiles et une cavité digestive qui s'étend jusqu'au bout de ees appendiees. Ils ont de eommun entre eux qu'ils sont digénèses ineomplets par arrêt de déreloppement.

Lil dernière division ou les anthophorides forment un groupe igalement très-naturel. Ces polypes sont digéuèses incomplets, nou par arrêt de développement, eomme les précédents, mais ì la manière des bryozoaires dout les diverses génẻrations sont semblables, qu'elles proviennent d'un ocuf ou d'un bourgeon. Ces polypes sont gemmipares, vivent en eolonies fixes ou flottantes, ont des parois digestives distinetes et des tentacnles ereux en communication avec lí eavité périgastrique. Les aufs 
se développent toujours dans l'intérieur méme du corps le long fe eloisons menbranenses.

La classe des rlizopodes ou foraminiferes a pour noyau les prétendus céphalopodes microscopiques dont M. Dujardin nous al révéle le premier la simplicité de struclure, amxynels viennent se joindre les noctilugnes, les diflugies et selon toute probilsilité les grégrariues. Ces derniers orgainismes, si problématiques jusque diuss ces deruiers temps, projeltent des filancuts dans

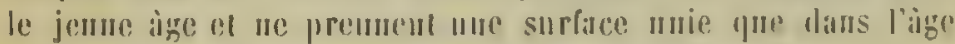
adulte, l'apres les olsservations de M. Licherhilus.

Eumbin lis dermere clisse, cenle des infusoires eorrespond ans infusoires polygastriques dilireubreg; les rutalumes ont pris place parmi les vors el les nombremx végétanx yur lou y avail pliccés r'll sont égalentent retirés. 


\section{REGNE ANIMNL.}

Car. Les animaux sout des îtres orgauisés qui se meuvent spontancment.

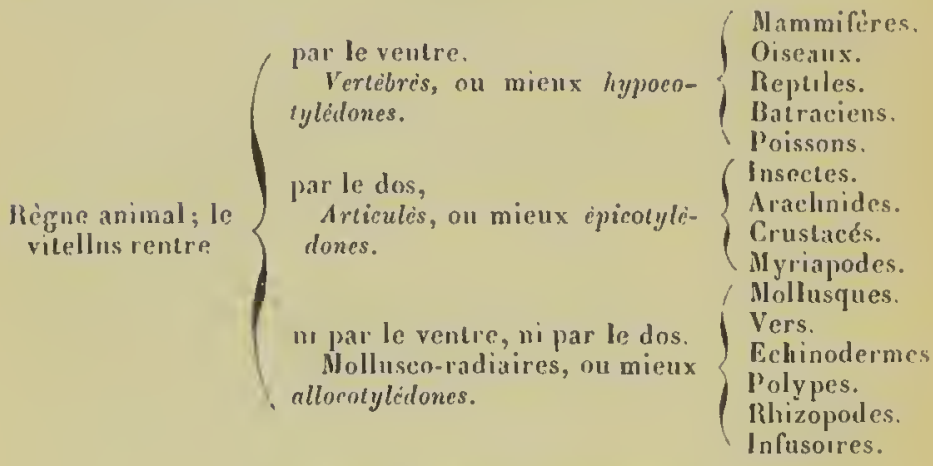

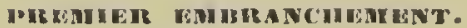

LES HYPOCOTYLENONES.

Car. Animanx domt le vitellus rentre par le ventre ot qui ont un uncelle épinière.

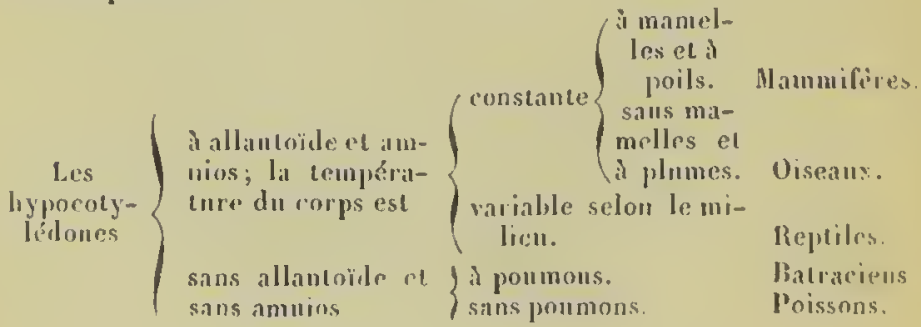


CiASSE 1. - WAMMHERS.

Cill. Des unduelles; le curpes couvert de puils.

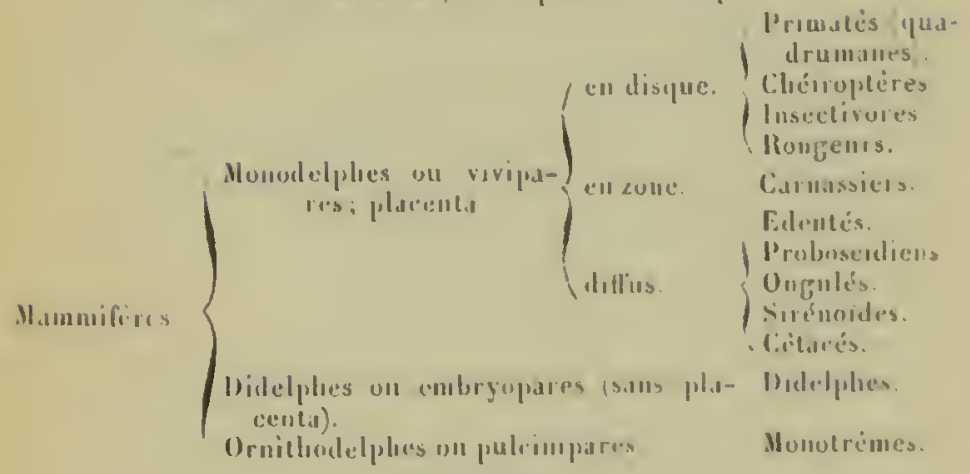

(I) Hlil:.

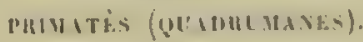

Ciar. Ciéneralement quatre mains; manclles peoturales: pions pendant
\begin{tabular}{l|l}
$\stackrel{\infty}{E} \mid$ Ments \\
E & incisives. \\
$=$ &
\end{tabular}
verticisles.
proselives.
taillés en biain.
e'n preigne.
Simiens.
Limurions.
Clueiromyons.
Galiopitheriens

\section{ClISSF 11. - OISFIt I.}

Car. Vertelurés a lemperature invariable; te corps converl de plumes

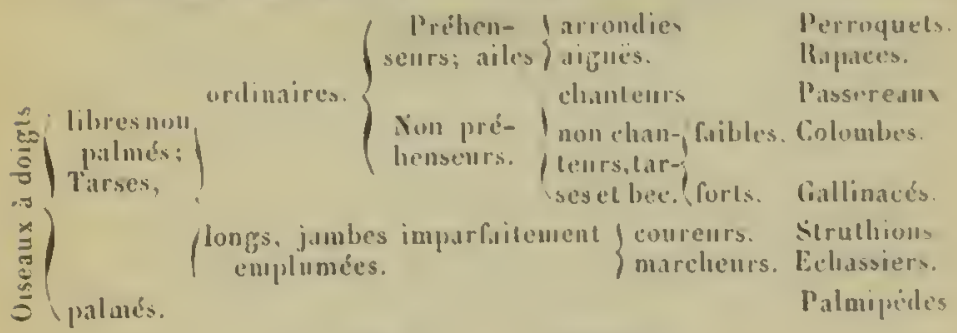

Cl.ASSE III. - HEITILES.

Car. Verfóbres allantodiens a temperature varable.

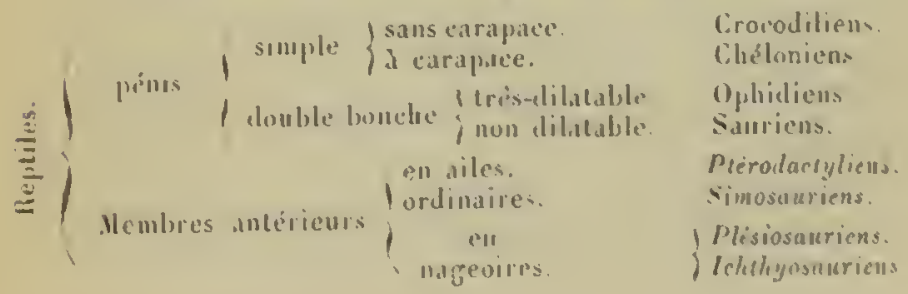




\section{GLASSE IV. - BATRACIENS.}

Car. Vertébrés anallantoìdiens, respirant par des poumons.

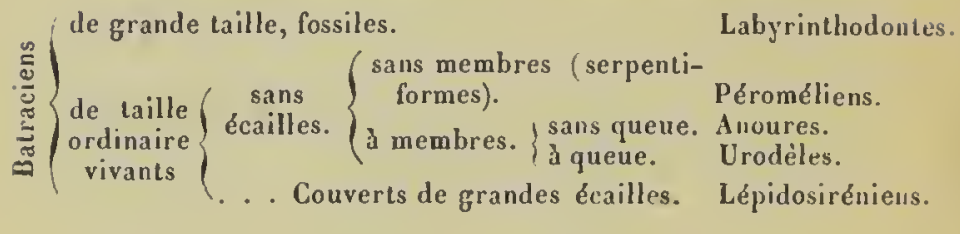

Classe V. - poissons.

Car. Ils respirent par des branchies; le cœur est formé d'une oreillette et d'unventricule, ou remplacé par un vaisseau contractile.

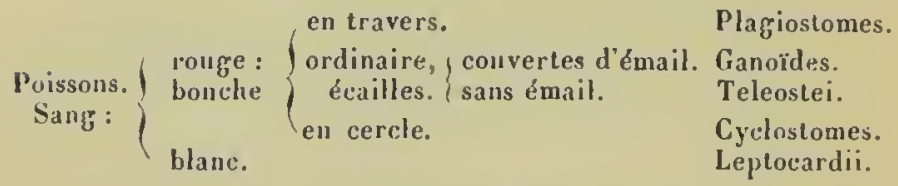

\section{DEUXIEMB EMUIRACURAETT.}

\section{ÉPICOTYLÉDONES OU ARTICULÉS.}

Car. Animaux dout le vitcllus rentrc par le dos; ils nont pas de moelle Épinièrc; leurs paltes sont arliculées.

a l'aicle de tra-(Trois paires de paltes. Irticulés $\left\{\begin{array}{c}\text { chíes ou de } \\ \text { poumons. }\end{array} \quad\left\{\begin{array}{l}\text { Qnatre paires. } \\ \text { Plus de six paires. } \\ \text { sans trachées ni ponmons, à l'aide de bran- } \\ \text { chies en général. }\end{array}\right.\right.$

Insectes. A rachnides. Muriapodes.

Crustaces.

\section{GLASSE 1. INSEGTES.}

Car. Trois paires de pattes; corps divisé en tête, thorax et abdomen; unc paire d'antennes.

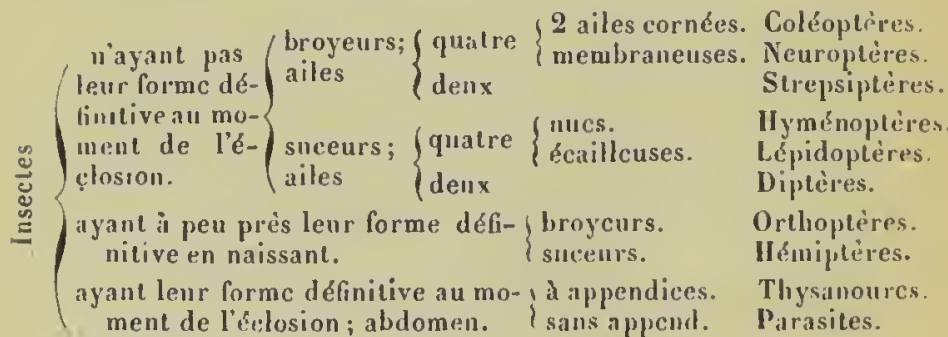




\section{CRASSE II. - ARACHNIDES.}

Car. Quatrc paires de pattes; sans ailes et sans yeux 3 facettes.

\begin{tabular}{|c|c|c|c|}
\hline Arachui- & respirant & $\left\{\begin{array}{c}\text { des ponnons; ab- } \\
\text { domen }\end{array}\left\{\begin{array}{l}\text { articulé. } \\
\text { non artieulé. }\end{array}\right.\right.$ & $\begin{array}{l}\text { Scorpioniens. } \\
\text { Araniens. }\end{array}$ \\
\hline & par & $\begin{array}{l}\text { dess trarliées. } \\
\text { la surface de la pleau. }\end{array}$ & $\begin{array}{l}\text { Acariens. } \\
\text { Tardigradiens }\end{array}$ \\
\hline
\end{tabular}

\section{CLASSE III. - MYRIAPOINS.}

Car. Un grand nombre de paires de pattes.

Myriapodes. $\quad\left\{\begin{array}{c}\text { Pattes a chaque } \\ \text { segunent, }\end{array} \begin{cases}\text { denx paires. Diplopoda. } \\ \text { une paire. }\end{cases}\right.$

\section{ClASSF IV. - CRESTACFS.}

Car. Systéme respiratoire aunatique, se faisant par des branchies ou par la pean; embryons naissant avec des pattes articulées au numbre de deux d einq parres.

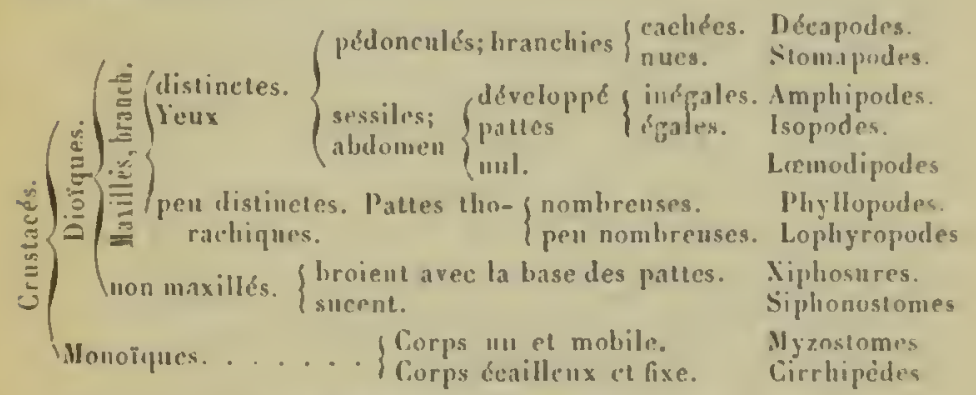

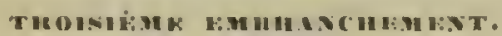

ILIOCUTYLEDONFS OU MULLSCO-RIDIURES.

C.ur. Animaux dont le vitelhus ne rentre plus ni par le dos ni par le ventre; ils nont plus de pattes artientées.

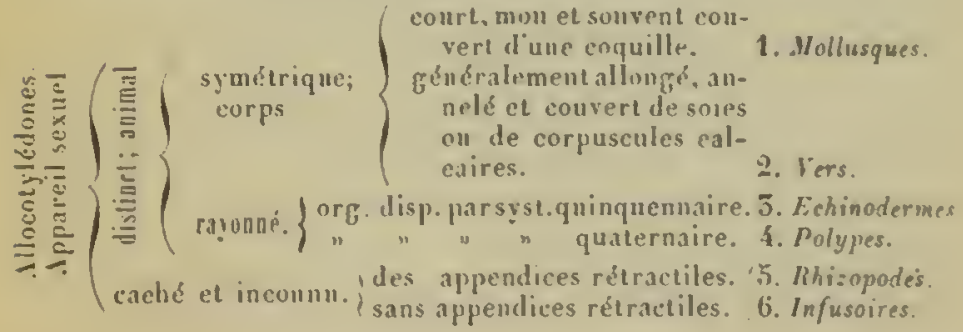

1 Ils urissent avee trois paires de pattes. comme les acariens. 
$I^{\text {TR }}$ Classe. - MOLLUSQUES.

Car. Le corps est euveloppé d'une peau molle, incrustée souveul de plaques calcaires, saus divisions anuularres el sans appendices articulés.

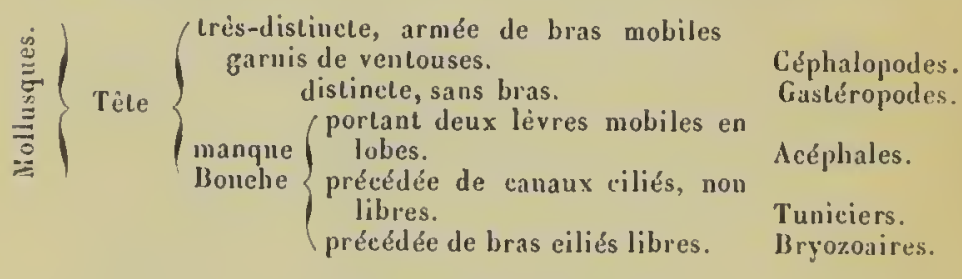

I1 CIIASSE, 一 IERS.

Car. Corpss généralemeut allongé, sans jamais porter des appendiees arlieulés; la peau est souvent incrustée de soies ou de corpuscules ealcaires:

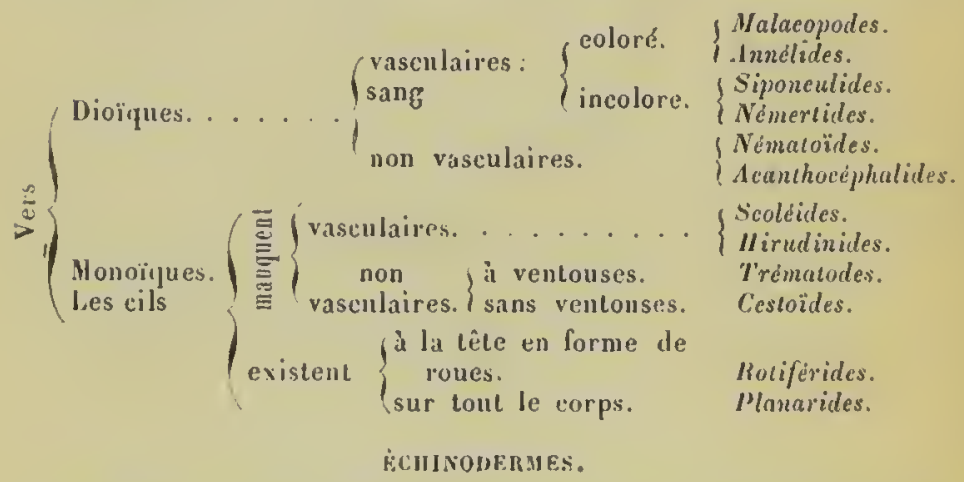

Car. Anımaux rayonnés dans lesquels le nombre quinquenuaire prédomine dans l'arrangemenl de leurs organes; la peau est pénétrée de corpuscules ealcaires qui forment souvent une elarpente dont les picees sont quelquefois mobiles; ils sont généralenıent dioïques et digénèses.

Échinoder-
mes. $\left\{\begin{array}{l}\text { Non pédiculé : } \\ \text { Corps } \\ \text { Pédieulé. }\end{array}\left\{\begin{array}{l}\text { cylindlique. } \\ \text { globuleux, sans rayons. } \\ \text { déprimé, à rayons. }\end{array}\right.\right.$

IJolothurides. Echinides. siellerides. Crinoïdes. 


\section{MVESE ANIMIL}

fititils.

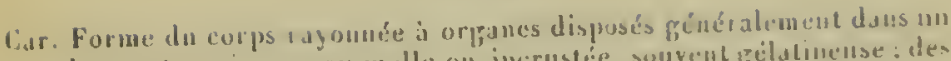
ordre quaternare; pean molle on incrustée, souvent gélalinense des

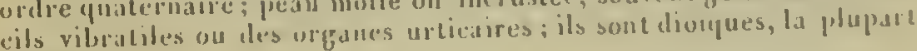
digénéses.

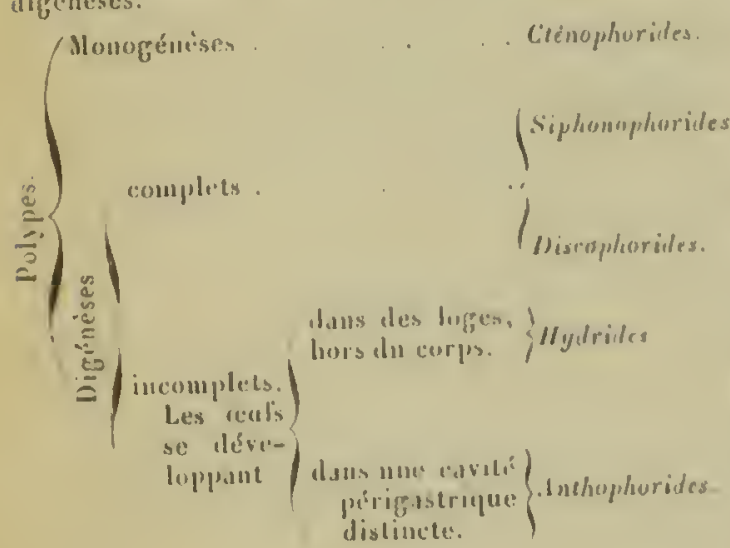

"Cylipp"

dueroé.

vilelle

l'orpite.

liphyses

phiysulie.

Mlinzostume.

Meduse.

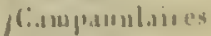

Tubulaires

Illyllen.

fxirtnlaine

Corail.

(ii)! gane

liétille.

l'ematul.

Ietime.

Iladrépur.

Ilcyon.

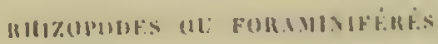

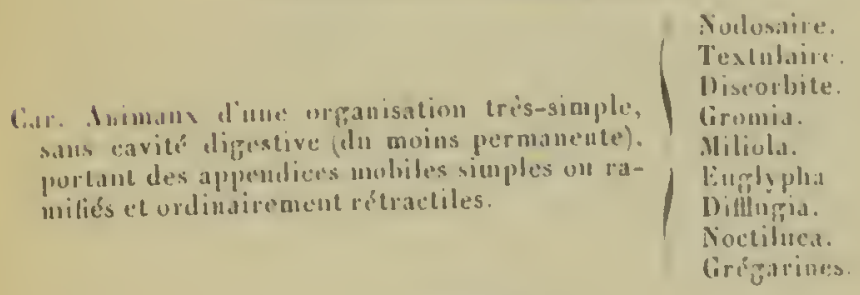

\section{INFP (IIIER}

Car. Comps nu on convert de cils viluratiles: organisation tres-sumple. reproluction par secission

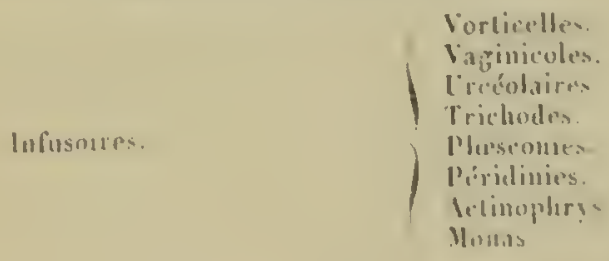




\section{TABLE DES MATIËRES}

Pages.

Introduction ...................... §

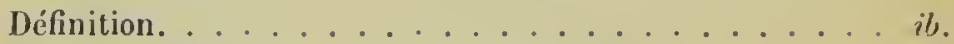

Marche à suivre ................... 6

Tableau du règne animal. . . . . . . . . . . . . 7

Développement comparé . . . . . . . . . . . 8

§ $1 \mathrm{er}^{\mathrm{r}}$ Idée générale sur la structure des animaux. . . . . . 9

Comparaison entre les plantes ct les animaux. . . . . it. Vie végétative. Les organes de nutrition, de respiration, de eireulation, de séerélion, de reproduction........ il.

Vie animale ......................

Organes des sens, système nerveux, appareil de locomotion.

Tableau des appareils .............. I

2. Complication des nrganismes ............. 16

Subordination des caractères. . . . . . . . . . 22

3. Organes analogues ou homologues .......... 27

AppaneILS de ca vik or congervation. 31

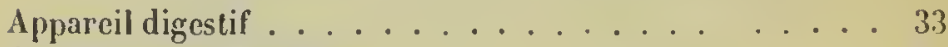

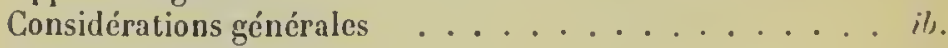

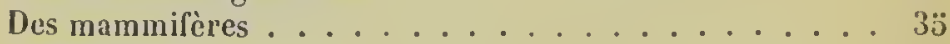

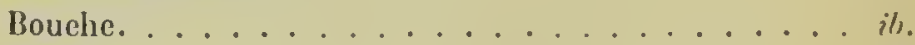

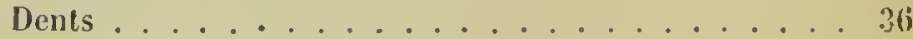

Langue . . . . . . . . . . . . . . . . 38

Estomacs. .................. 40 
Intestins . . . . . . . . . . . . 43

Giandes de Peyer et de Brunner. . . . . . . . . 46

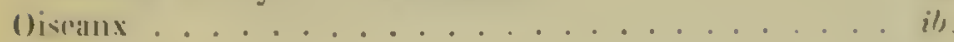

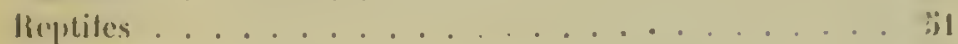

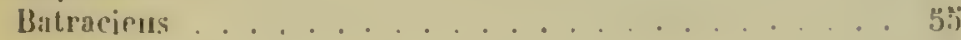

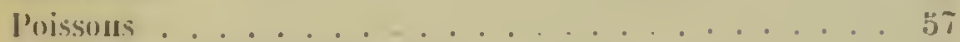

Arteculio on épicotylélonos . . . . . . . . . . bio

Ineretes, arachnides, my riapodes et crustaces. . . . (i)

Aftocotylofones . . . . . . . . . . . . (if

Mullusgutes. . . . . . . . . . . . . . ils.

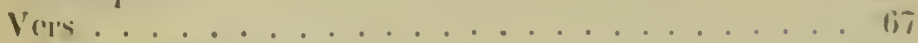

lichinodermes et polypes . . . . . . . . . it.

foraniniferes, infusoires ............ i (0)

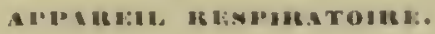

Considérations gruninales. . . . . . . . . . I1

Manmiferes. . . . . . . . . . . . is

Oiseanx .......................

Reptiles . . . . . . . . . . . . . . .

Batraciens. . . . . . . . . . . . . . . s so

Poiswous. . . . . . . . . . . . . . . . . . .

Articulis on eppeotylétones . . . . . . . . . . sti

lusectes . . . . . . . . . . . . . . il

Arachuides, myriapoles . . . . . . . . . . . s s

Crustaces . . . . . . . . . . . . . . s!

Mllocotylidones. . . . . . . . . . . . . ! !

Mollusiques. . . . . . . . . . . . . il.

Vers........................ 94

lichnodermes, polypes, infusoires. . . . . . . . 96

\section{APPAHEII. CIICELATOIRN.}

Considerations generrales. . . . . . . . . . . . .

Sang et globules de sang . . . . . . . . . . . (111

Manmulieres . . . . . . . . . . . . . 102

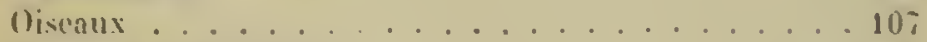

Reptiles et batraciens ............. . . 1t0

Poissons. . . . . . . . . . . . . 114

Irticules on épicotylédones . . . . . . . . . . . 120

Insertes ....................... ib.

Arachuides, myriapudes crustaces . . . . . . . $|2|$ 
Pages.

Mollusques................ 123

Vers.......................... 19;

Échinodermes, polypes. ................. 130

Infusoires .................. 132

Ganglions vasculaires ............... I33

La rate. . . . . . . . . . . . . . il.

Thymus et corps thyroïde . . . . . . . . . . 155

Capsules surrénales ................ I36

APPAIRILE GLANDUKAIRES.

Considérations générales . . . . . . . . . 137

Glandes salivaires . . . . . . . . . . . 139

Foic .................... 14

Pancréas . . . . . . . . . . . . . . 14.5

Glandes spéciales .................. 147

IDPIREMT UTINAMER.

Considérations générales . . . . . . . . . 150

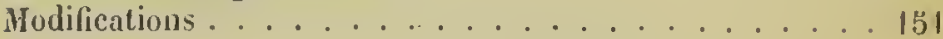

APPAREIL GENGMTEUR.

Considérations générales. . . . . . . . . . . 1300

Composition de l'appareil. . . . . . . . . . . . 160

OEufs ............................. 161

Spermatozoüles ................ 165

Mammifères. . . . . . . . . . . . . . 16i

Oiseaux................................... 179

Reptiles, batraciens................... 17t

Poissons . . . . . . . . . . . . 175

Inscetes. . . . . . . . . . . . . . 178

Myriapodes....................... 180

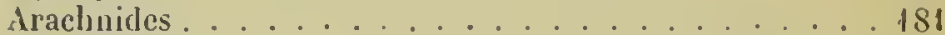

Crustacés ................. . . 182

Spermatophores. . . . . . . . . . . . 184

Mollusques................. $185^{\circ}$

Céphalopoles. . . . . . . . . . . . . . . . 18!

Gastéropodes. .................... (9)

deéphales et tuniciers . . . . . . . . . . 19.'

Bryozoaires ....................... 1!5

Yers. ........................ 196 


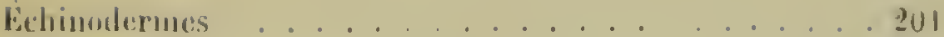

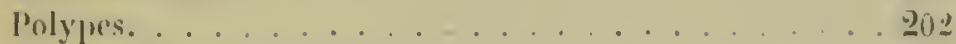

Mlizopodes el inlusoires. . . . . . . . . 2060

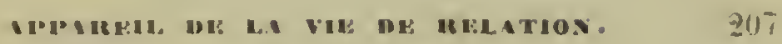

Appareil locommanu . . . . . . . . . . . . 2018

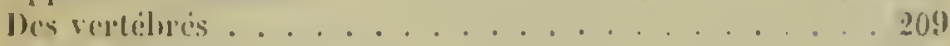

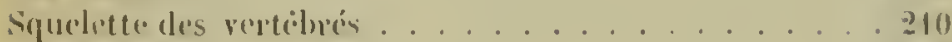

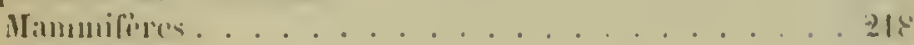

()isealus . . . . . . . . . . . . . 2. 20

Rupliles. . . . . . . . . . . . 23$\}$

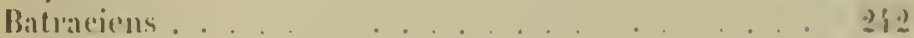

Poiscons. . . . . . . . . 21't

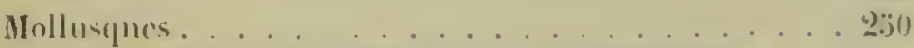

Sipuelette t'ntane des artienlés at des allocotylédours. . ih.

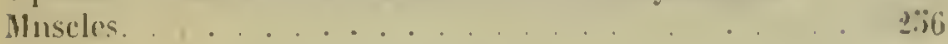

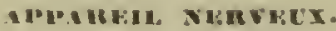

Inimats vertiblex ou liypontyledones. . . . . 200

Mammilèes . . . . . . . . . . . . 20,3

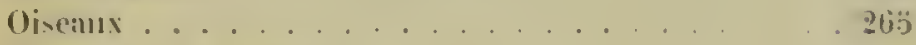

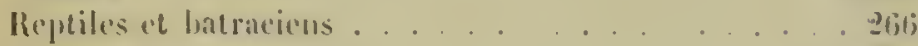

Joissons . . . . . . . . . . . . 2. 267

Inimans sint vertelores . . . . . . . . . 270

Artienlis on épreotyledonten . . . . . . . . . ils.

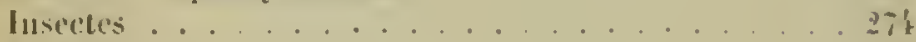

Alindmides. . . . . . . . . . . . il.

Myriapodes. . . . . . . . . . . . . . . ib.

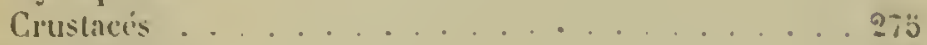

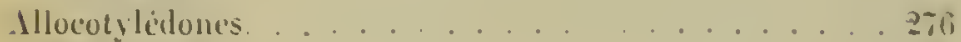

Mhllusques. . . . . . . . . . . . . . . . ij.

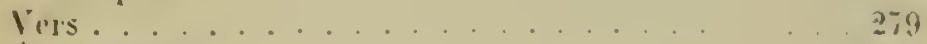

Fininedermes . . . . . . . . . . . . 281

Polypes . . . . . . . . . . . . . . . . . . 282

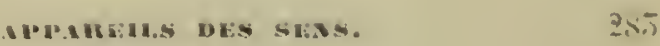

Apparcil d. la vision. . . . . . 285

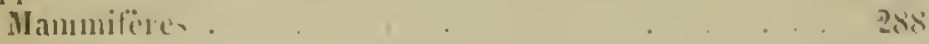

niscalus.............. . . . . . . . . 
Reptiles ............... . . 290

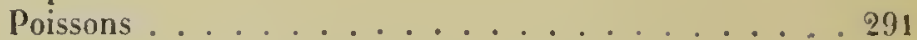

Inseetes . . . . . . . . . . . . . . 293

Araehnides et myriapodes . . . . . . . . . 294

Crustaès . . . . . . . . . . . . . . 295

Mollusques. . . . . . . . . . . . . . . 293

Vers et polypes . . . . . . . . . . . . . . 298

Appareil de l'audition . . . . . . . . . 300

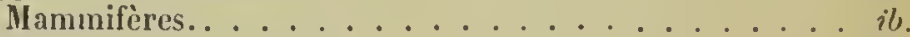

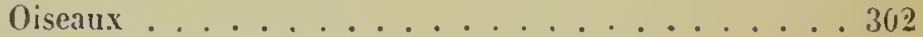

Reptiles et batraeiens . . . . . . . . . . 303

Poissons . . . . . . . . . . . . ib.

Insretes. . . . . . . . . . . . . 304

Mollusques . . . . . . . . . . . . . 30s

Vers et polypes .... . . . . . . . . . . 306

Appareil de l'olfaetion . . . . . . . . . . . 308

Mammifères . . . . . . . . . . . . . . 309

Oiseatux. . . . . . . . . . . . . . . 310

Reptiles et batraeirns. .............. . . 311

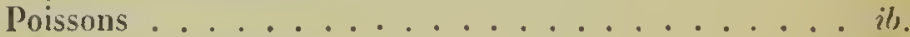

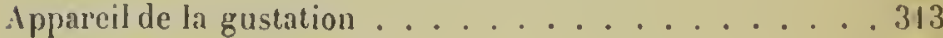

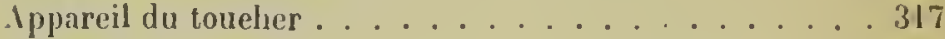

Peau .................... ib.

Papilles. ....................... 318

Cryptes et phanères. . . . . . . . . . . 321

Mammifères ................ il.

Oiseaux . . . . . . . . . . . . . . 323

Reptiles.................. . 324

Batraeiens . . . . . . . . . . . . . 32z

Poissons . . . . . . . . . . . . . ib.

Appareil élcetrique. . . . . . . . . . . . 330

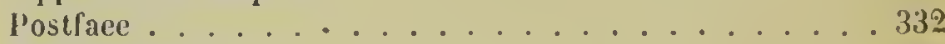

Règne animal. . . . . . . . . . . . . 338

FIN DE LA TABLE DES MATIËRES.

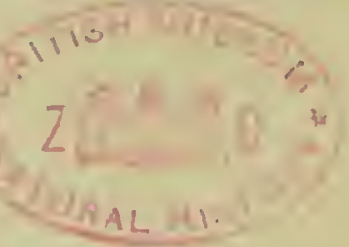




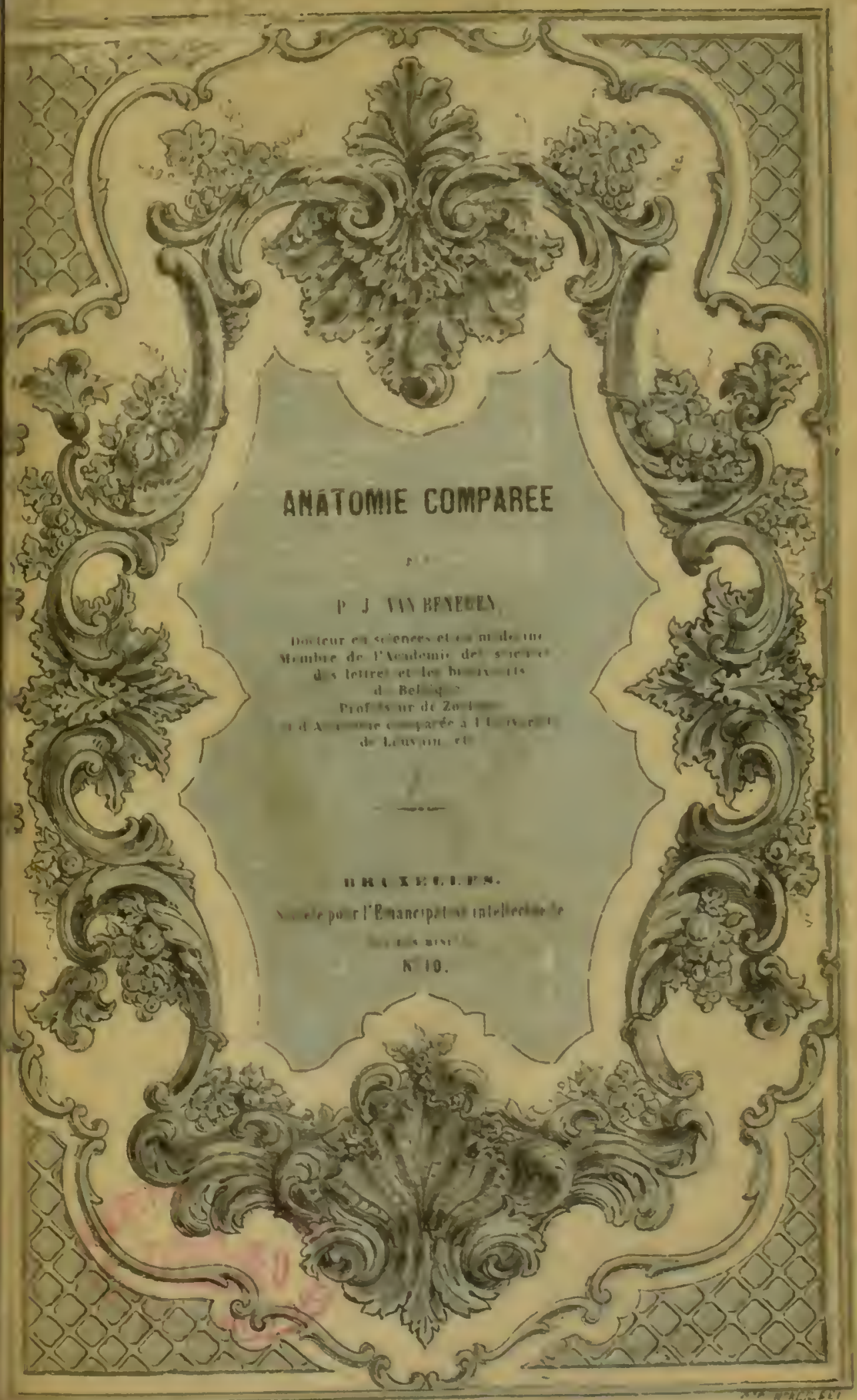






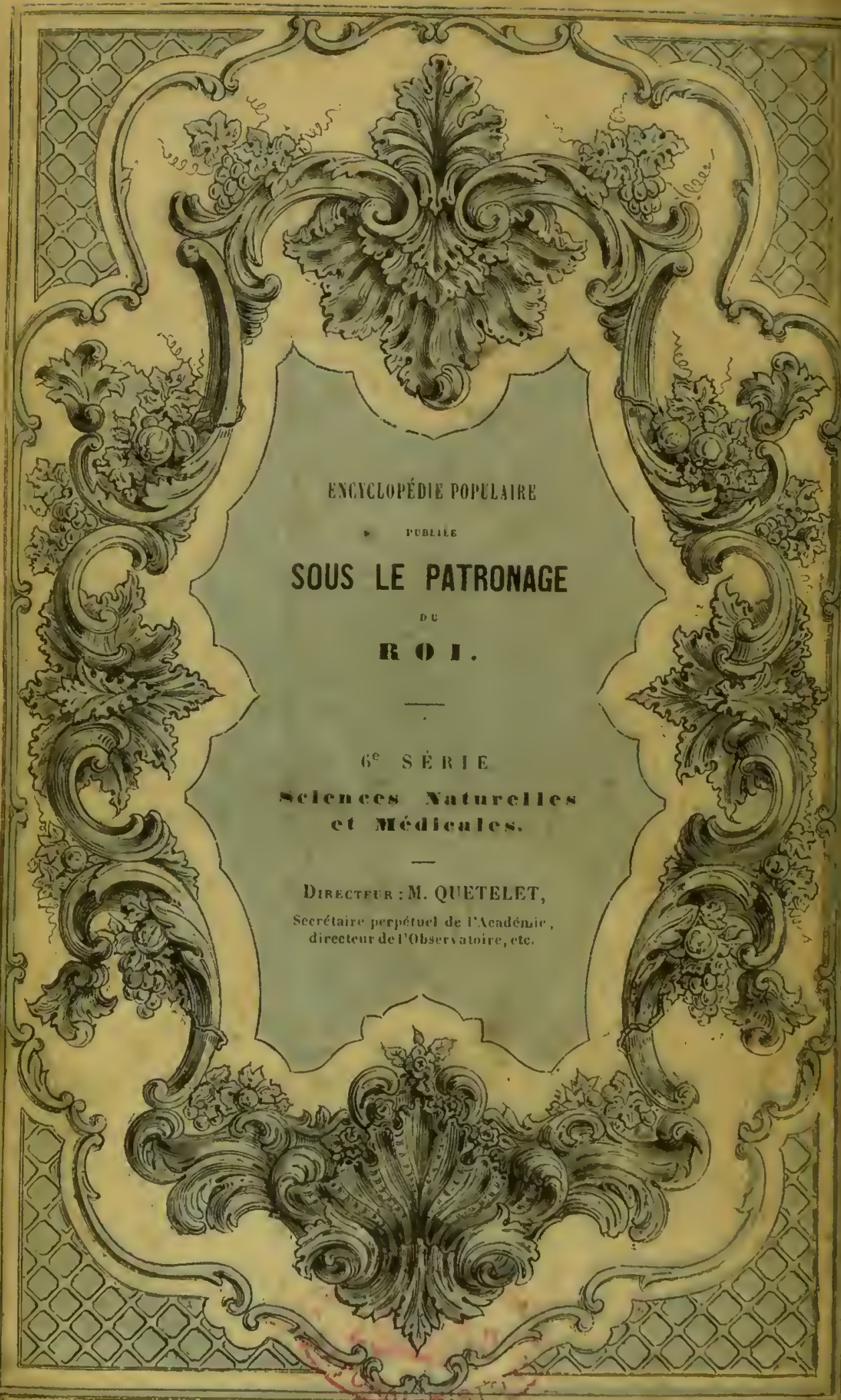




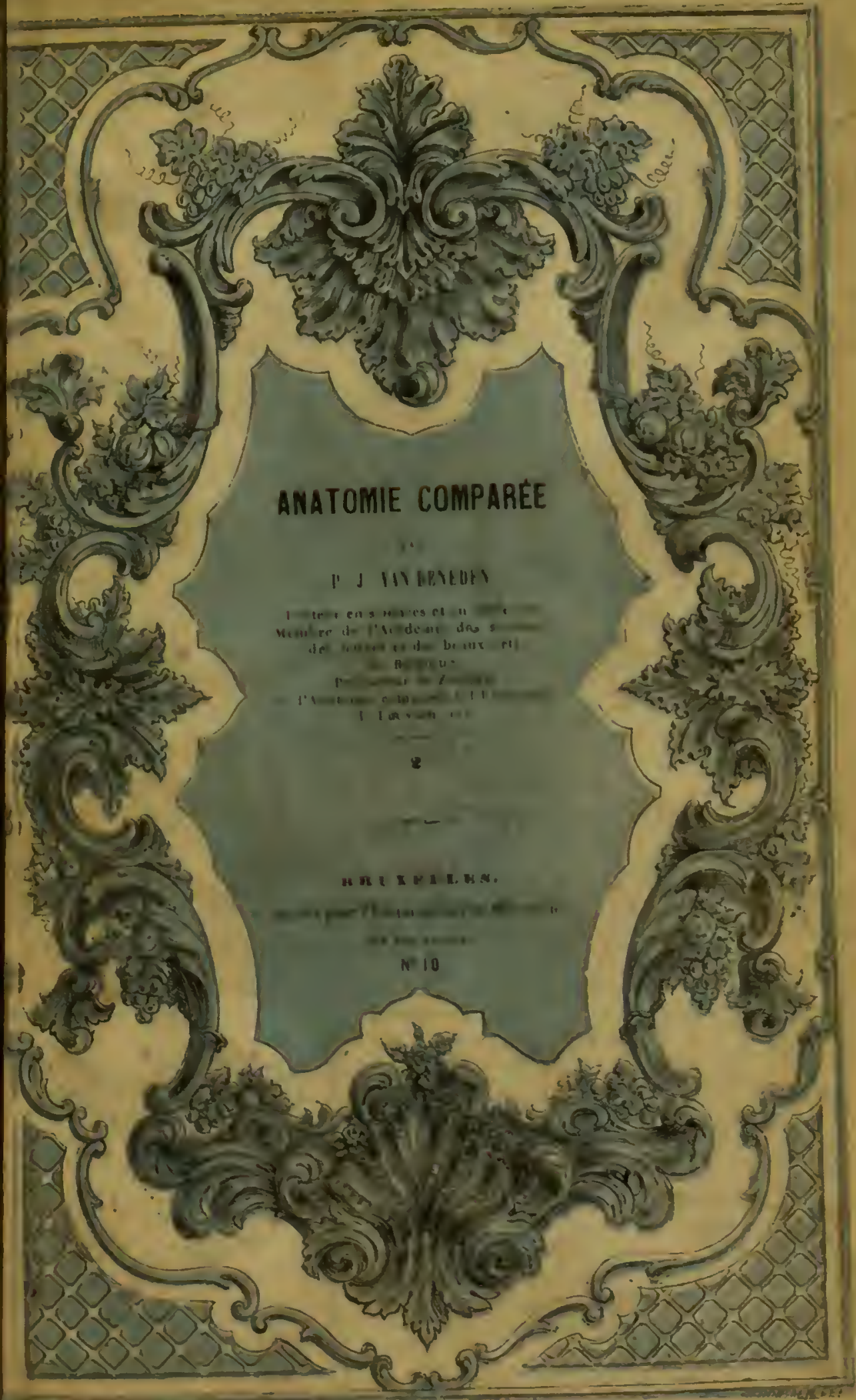






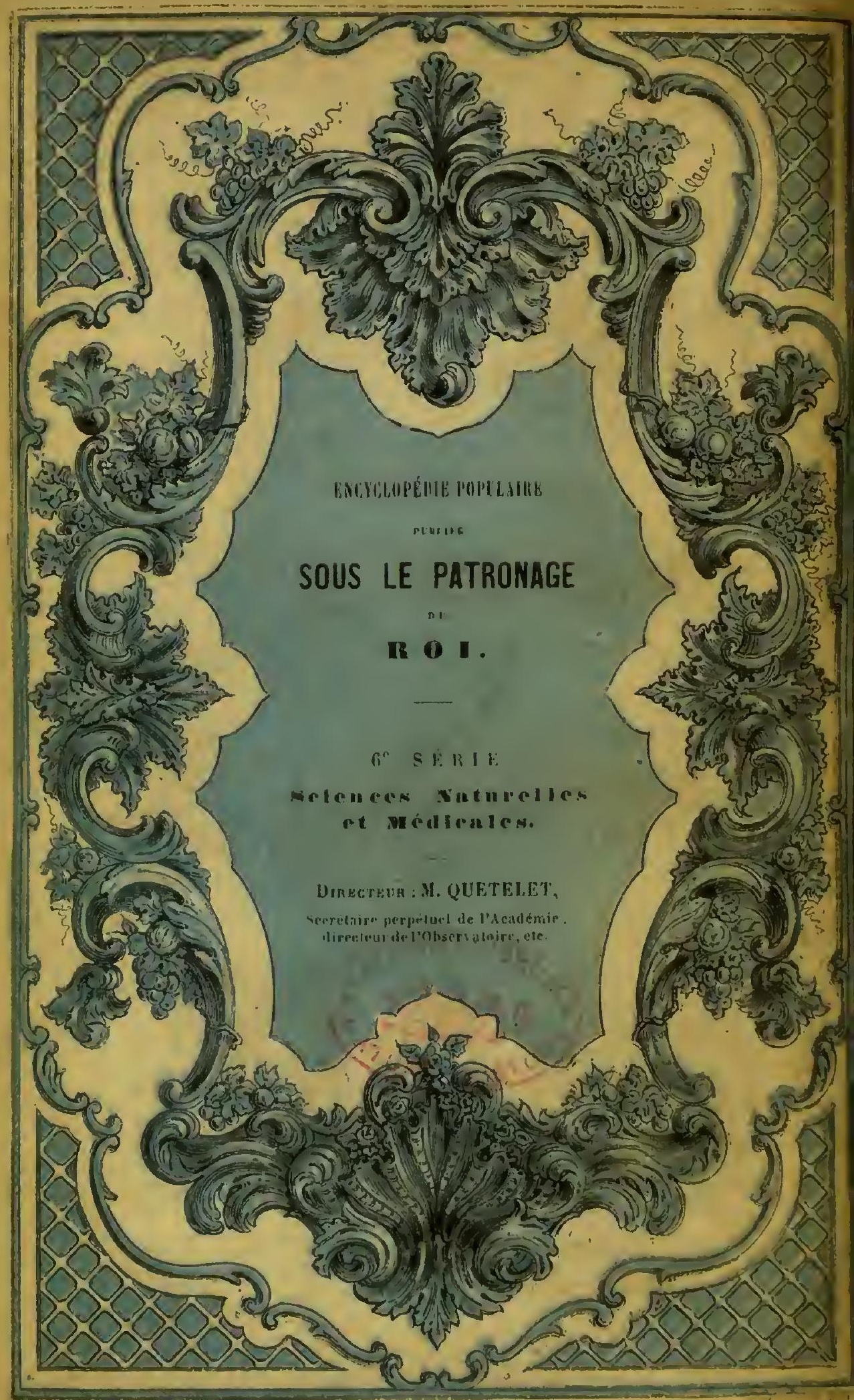




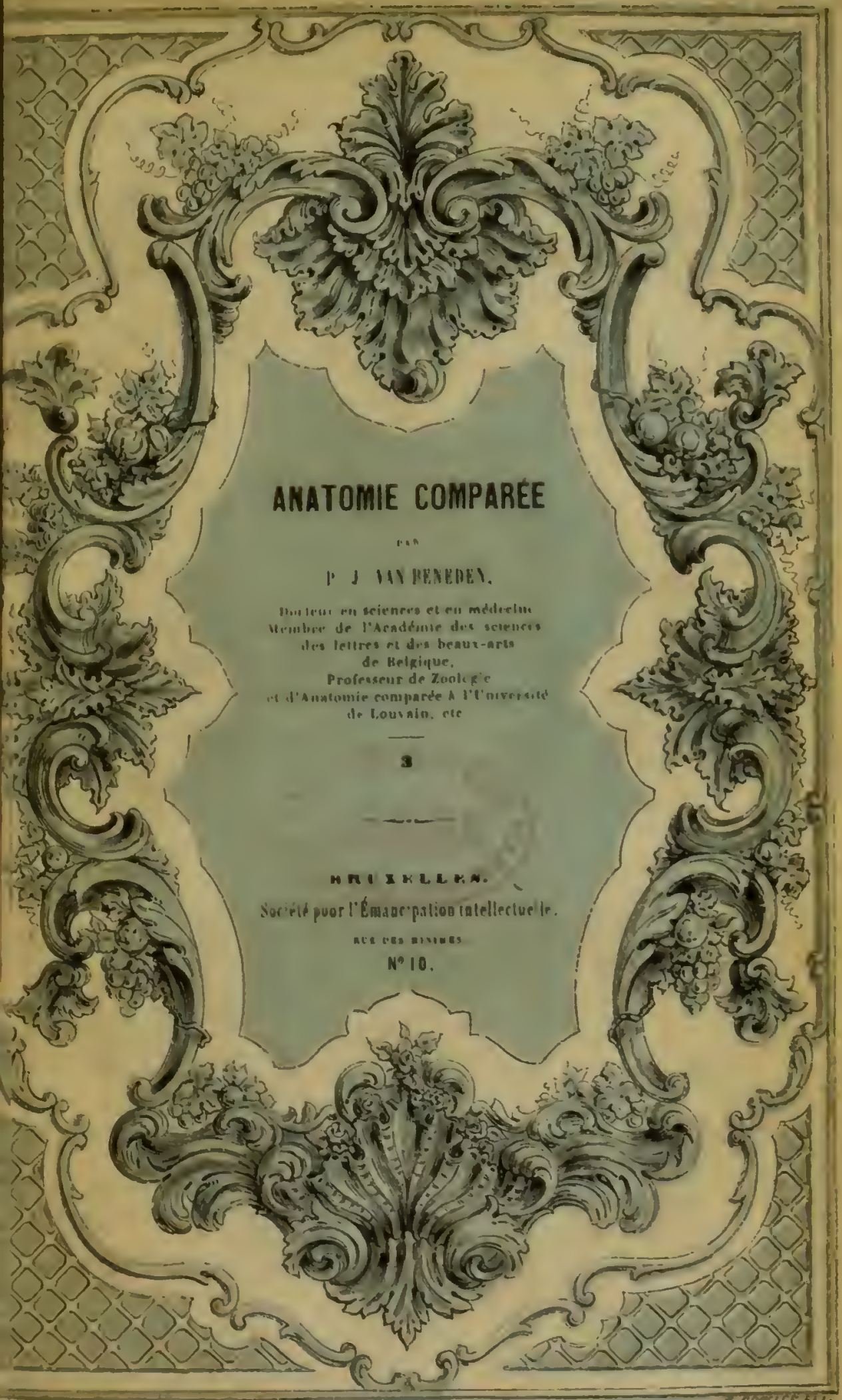





\section{,}

. 


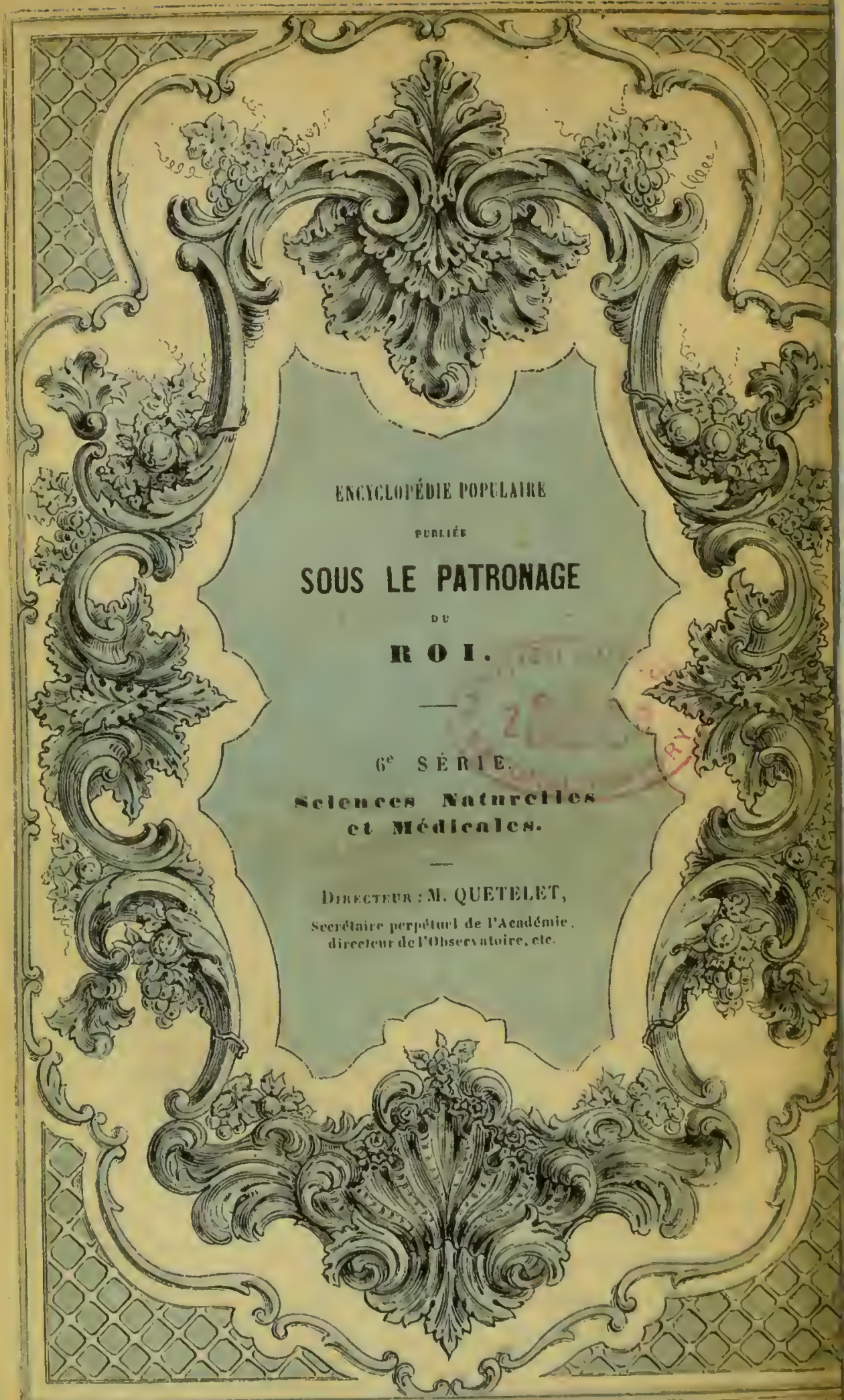





$F^{2}=3$ 